\title{
Risikoaspekte in der Fleischerzeugung
}

-Ergebnisse einer Expertenbefragung-

\author{
Dissertation \\ zur Erlangung des Doktorgrades \\ der Fakultät für Agrarwissenschaften \\ der Georg-August-Universität Göttingen
}

vorgelegt von

Ute Röbken

Diplom-Ingenieurin agr.

geb. am 24. Juli 1967 in Oldenburg (Oldb.)

Göttingen, 2006 
D7

Referent: Prof. Dr. M. Wicke

Koreferent: Prof. Dr. W. Branscheid (Bundesforschungsanstalt für Ernährung und Lebensmittel, Standort Kulmbach)

Tag der mündlichen Prüfung: 04. und 05.Juli 2006 
1

Einleitung .1

1.1

Zielsetzung der Arbeit .2

2

Literatur 3

2.1

Definitionen 3

2.1.1

Verbraucherbild. 3

2.1.2

Expertenbild 3

2.1.3 Risikobegriff, Risikowahrnehmung und Risikobewertung ............................ 4

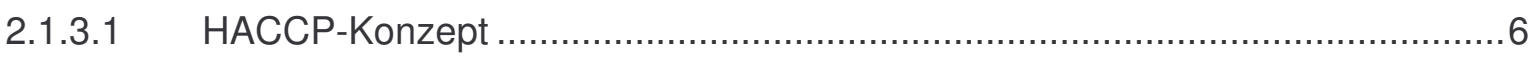

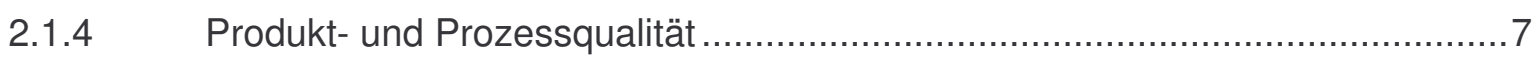

2.2 Die Sicherheit der Fleischproduktion aus der Sicht des Verbrauchers.....8

2.2.1 Wahrnehmung von Risiken bei Lebensmitteln ......................................... 8

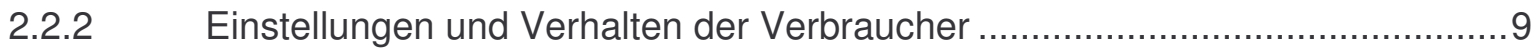

2.2.3 Politische Veränderungen .................................................................... 11

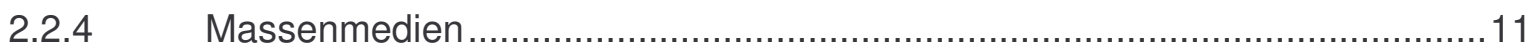

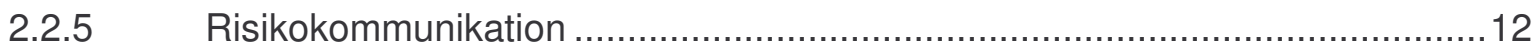

2.3 Die Sicherheit der Fleischproduktion: Initiativen der Wirtschaft ..............13

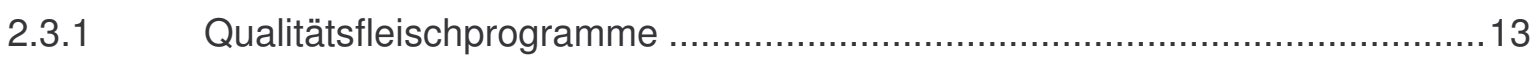

2.3.2 Qualitätsmanagementsysteme ............................................................... 14

$2.4 \quad$ Potentielle Risiken in der Fleischerzeugungskette ...................................16

2.4.1 Darstellung der Fleischerzeugungskette ..................................................16

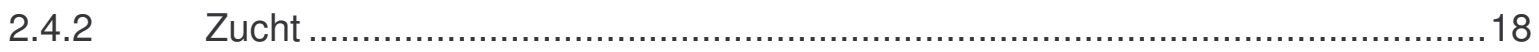

2.4.2.1 Mängel in der Produktqualität ........................................................... 18

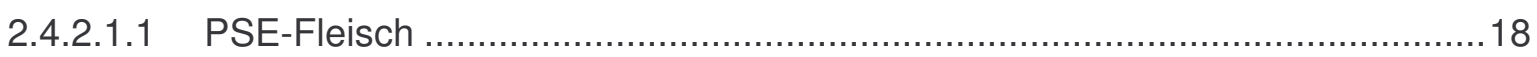

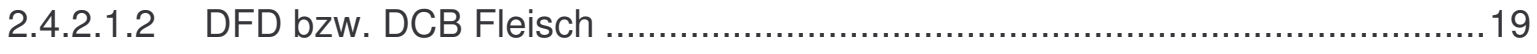

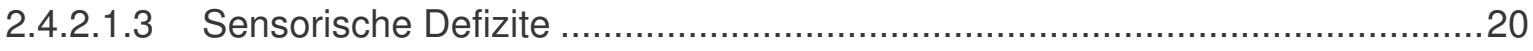

2.4.2.2 Ethische Risiken durch einseitige Zucht .................................................20

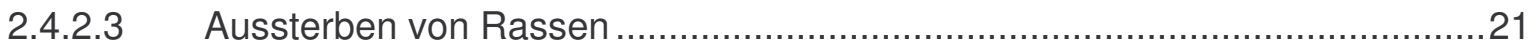

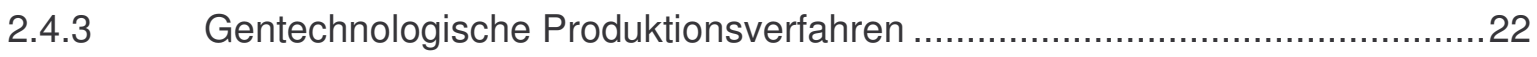

2.4.3.1 Einsatz und Verbreitung von gentechnischen Verfahren ..............................22

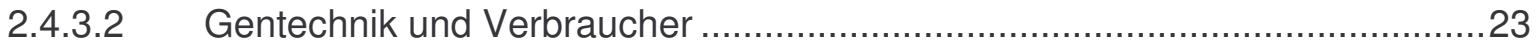


2.4.3.3 Ziele, Vorteile und Risiken der Gentechnik .............................................24

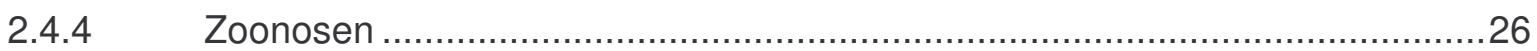

2.4.4.1 Eintragswege von Zoonoseerregern in die Nahrungskette am Beispiel von Salmonellen .27

2.4.4.2 Beherrschbarkeit mikrobieller Risiken in der Fleischproduktion am Beispiel von

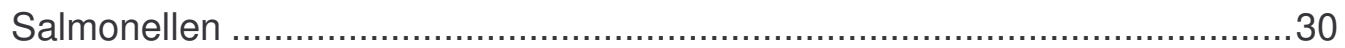

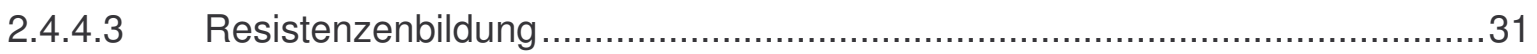

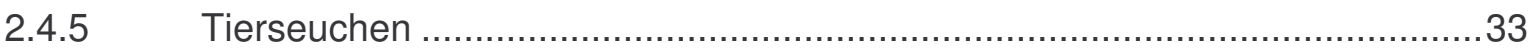

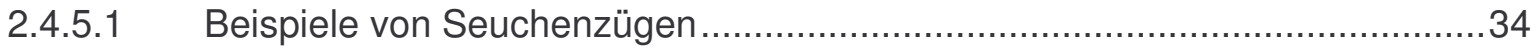

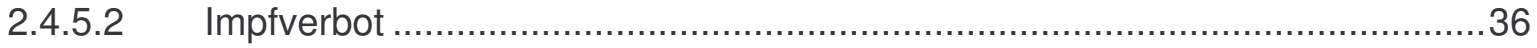

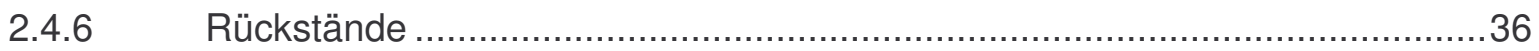

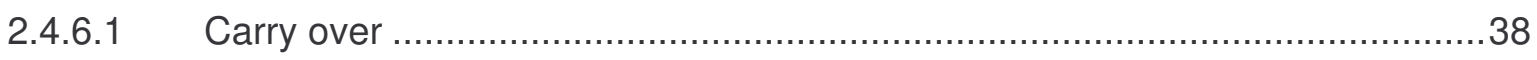

2.4.6.2 Kontaminationen durch Mykotoxine in der Lebensmittelkette ..........................38

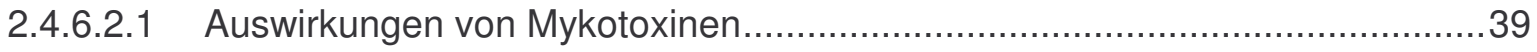

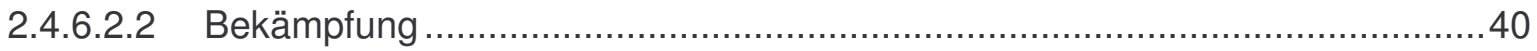

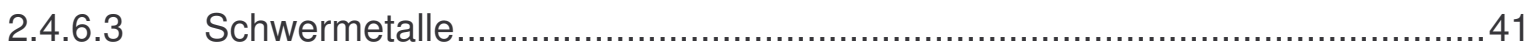

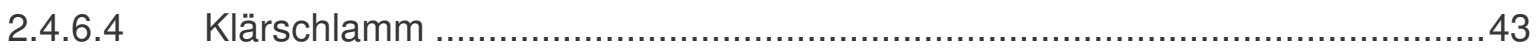

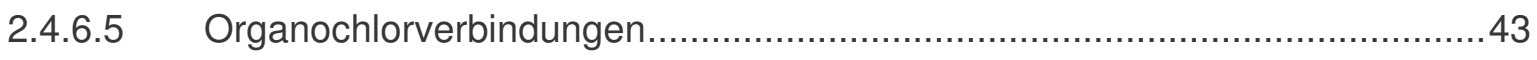

2.4.6.6 Futterzusatzstoffe zur Leistungssteigerung und zu therapeutischen Zwecken

2.4.7 Verfahrenstechnische Risiken von Futtermitteln ........................................46

2.4.7.1 Herstellung und Verfütterung von Tiermehlen ........................................... 49

2.4.8 Verfahrenstechnische Risiken in der Tierproduktion...................................50

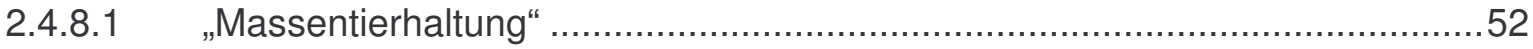

2.4.8.2 Technopathien, Ethnopathien und Haltungsfehler ......................................52

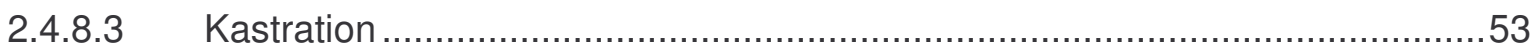

2.4.8.4 Ökologischer Landbau/Freilandhaltung ..................................................... 54

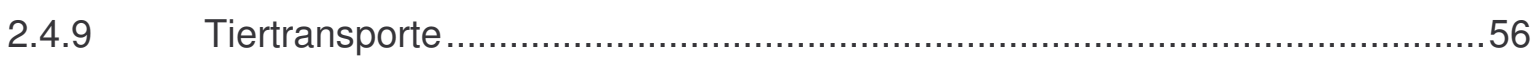

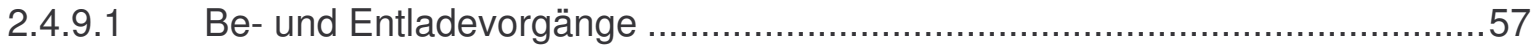

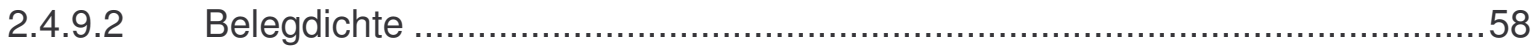

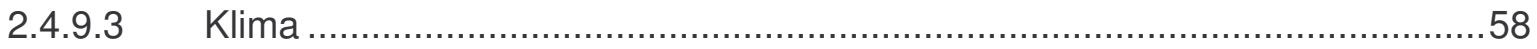

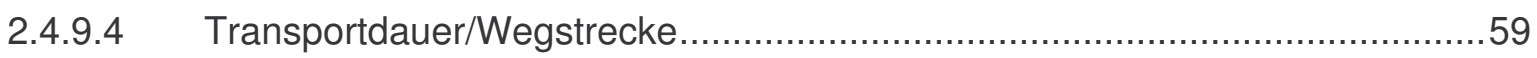

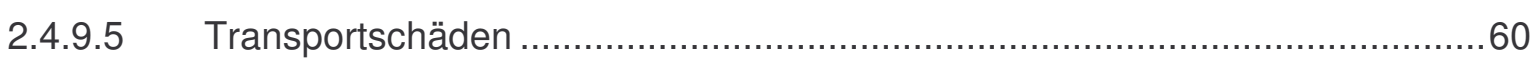

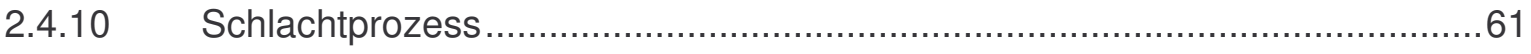

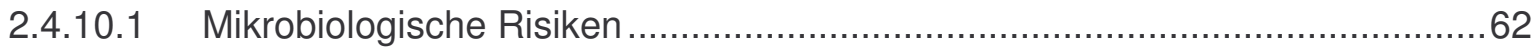




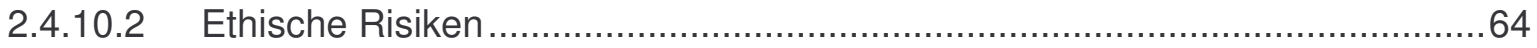

2.4.10.3 Übertragungswege von BSE-Risikomaterial in der Schlachtung....................66

2.4.11 Hygienemaßnahmen und Hygienekonzepte .............................................67

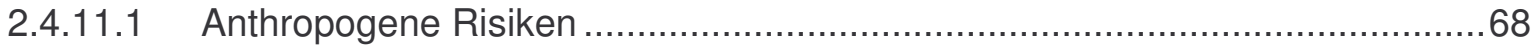

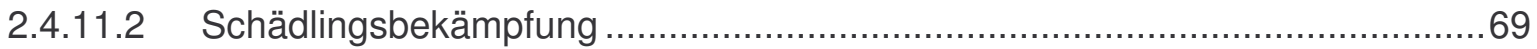

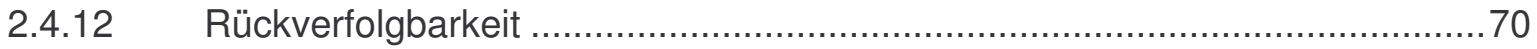

2.4.13 Systeme zur Rückverfolgbarkeit ......................................................... 72

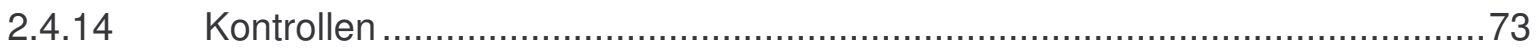

Methoden .78

4.1

Delphi-Methode .78

4.2 Methoden der Expertenbefragung ...................................................................

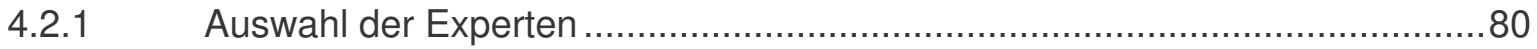

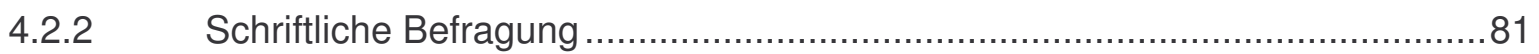

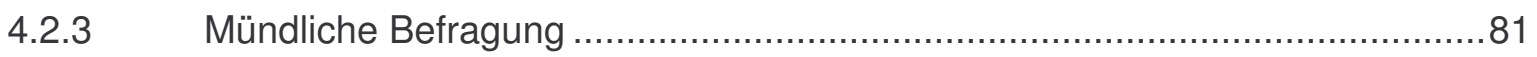

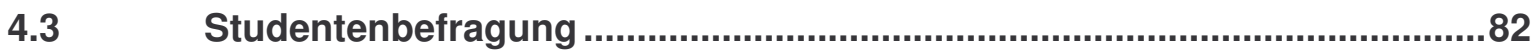

5

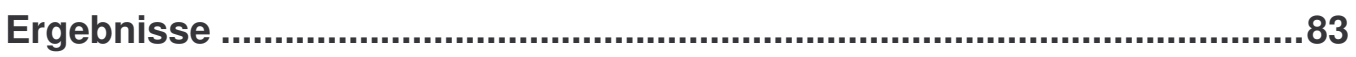

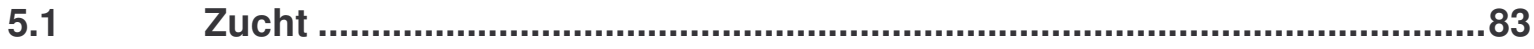

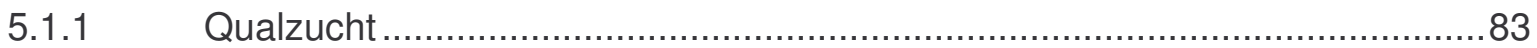

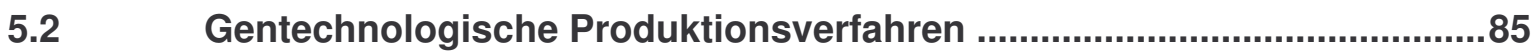

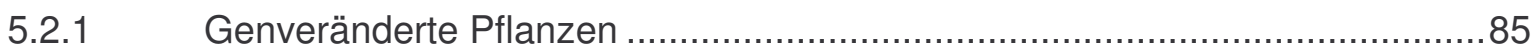

5.2.2 Einsatz von genetisch veränderten, geklonten Tieren .................................86

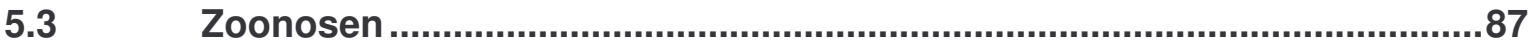

5.3.1 Schädlinge, Vögel und Haustiere in Futtermittel herstellenden Betrieben .......89

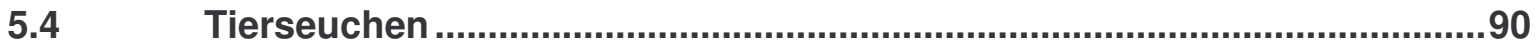

5.4.1 Eintrag von Keimen und Krankheitserregern aus dem Ausland .....................90

5.4.2 Reste aus der Lebensmittelproduktion.....................................................

5.4.3 Einsatz von Prophylaxen/Schutzimpfungen ...............................................

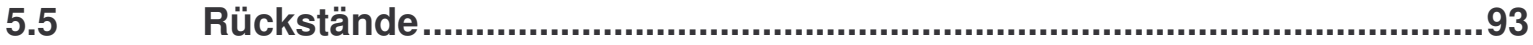

5.5.1 Risiken durch Feldkeime und speziell Mykotoxine in der Lebensmittelkette ..93

5.5.2 Rückstände von Pflanzenschutzmitteln ......................................................95

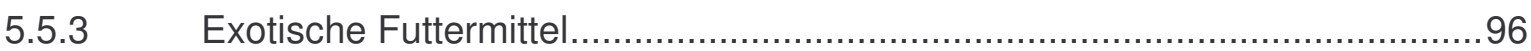


5.5.4 Rückstände aus dem Einsatz von Mineraldüngern

5.5.5 Rückstände aus dem Einsatz von Müllkompost, Klärschlamm und Altlasten .97

5.5.6 Mittel/Futterzusatzstoffe, die sich zur Leistungssteigerung eignen.................99

5.5.7 Rückstände im Bereich der Schlachtung ................................................ 101

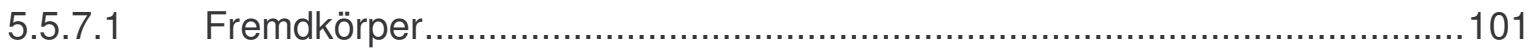

5.6 Herstellung, Lagerung und Transport von Futtermitteln ........................102

5.6.1 Einsatz von tierischen Futtermitteln .................................................... 104

5.7 Tierproduktion .................................................................................... 107

5.7.1 Kupieren, Enthornen, Schnabelkürzen ................................................ 107

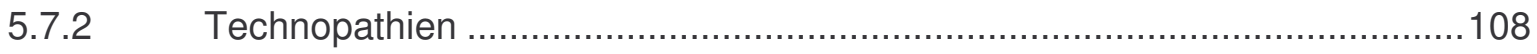

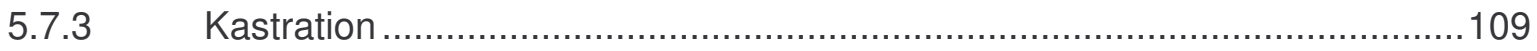

5.7.4 Kritische Punkte für die Produktqualität .....................................................110

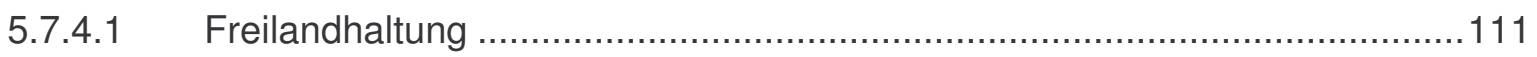

5.7.5 Anzahl der Tiere pro Stall und/oder Betrieb ............................................ 112

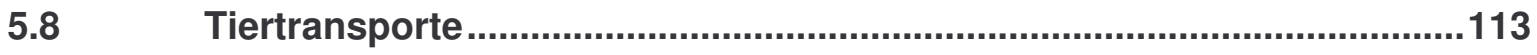

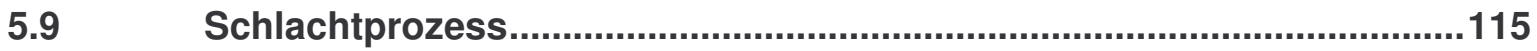

5.9.1 Tierannahme/Wartestall des Schlachthofes und Zuführung zur Betäubung.115

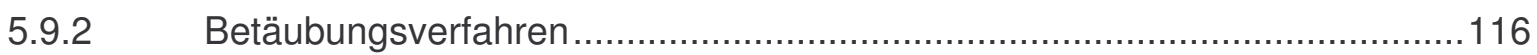

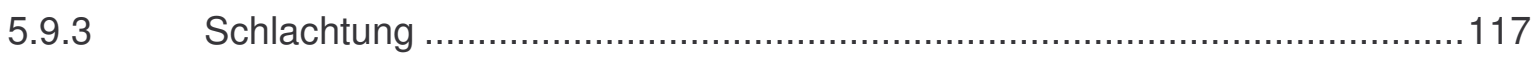

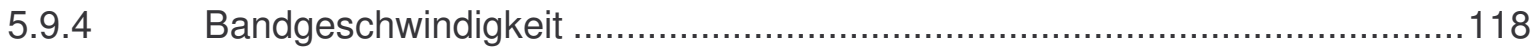

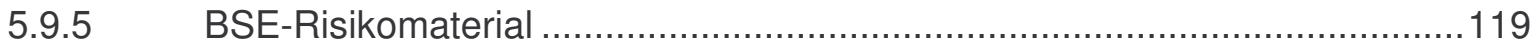

5.9.6 Nebenprodukte und Innereien............................................................ 120

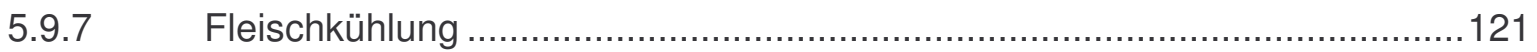

5.9.8 Fleischverladung/Transport/Fleischzerlegung ...................................... 121

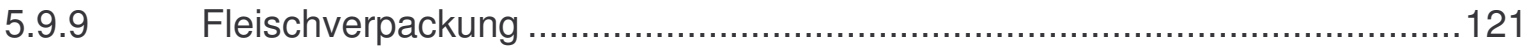

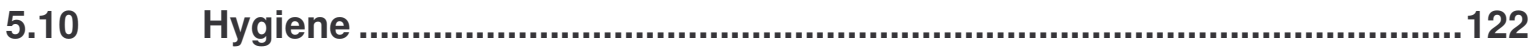

5.10.1 Hygiene in der Futtermittelproduktion .................................................. 122

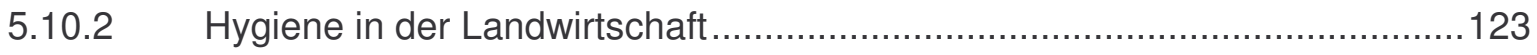

5.10.2.1 Kritische Punkte in der Fütterungshygiene ............................................... 125

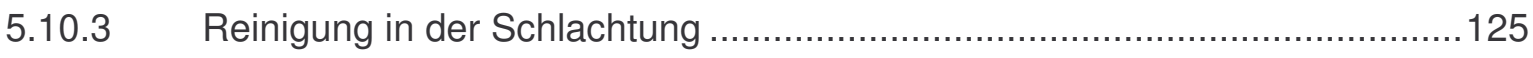

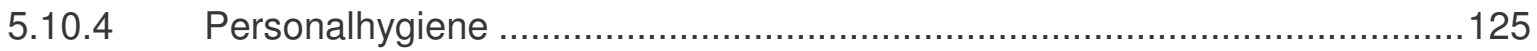

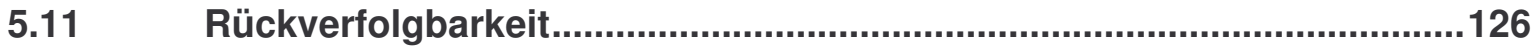

5.11.1 Rückverfolgbarkeit von Futtermitteln .................................................... 126

5.11.2 Dokumentation/Rückverfolgbarkeit Landwirtschaft ..................................127 
5.11.3 Rückverfolgbarkeit in der Schlachtung............................................... 128

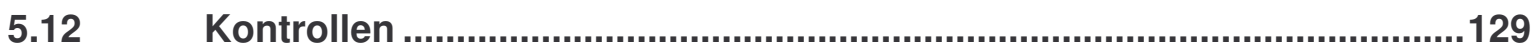

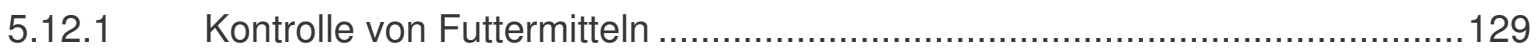

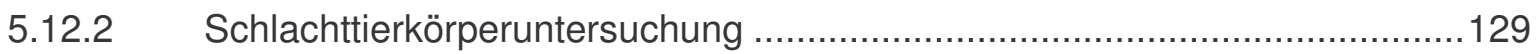

5.12.3 Fleischskandale im Ausland und Kontrollen bei Waren aus Drittländern......131

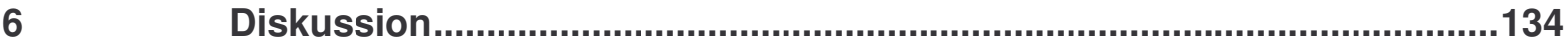

6.1 Methodendiskussion ..............................................................................134

Zucht ............................................................................................................. 135

6.3 Gentechnologische Produktionsverfahren ..........................................137

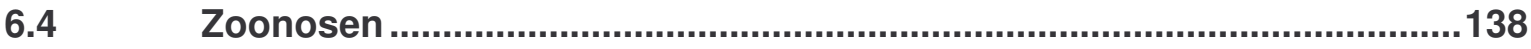

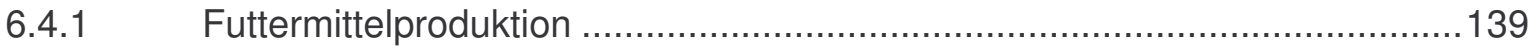

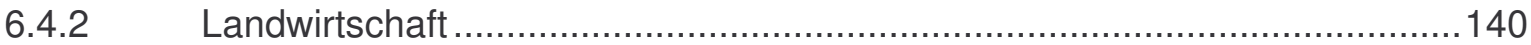

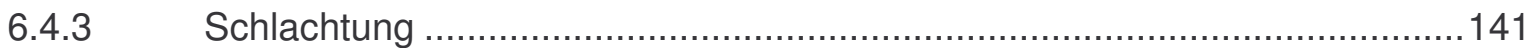

6.5 Tierseuchen ................................................................................................ 142

6.6 Rückstände und Kontaminanten ............................................................... 144

6.7 Landwirtschaftliche Tierproduktion ...........................................................147

6.8 Umgang mit dem lebenden Tier vor der Schlachtung ..............................150

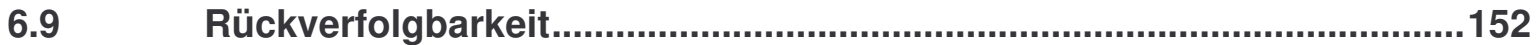

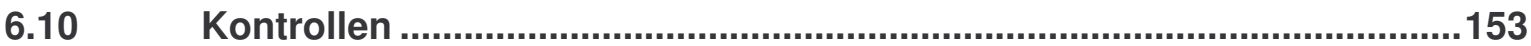

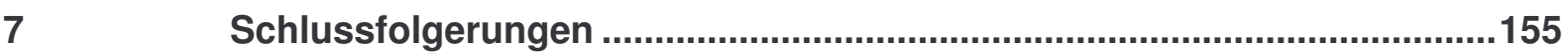

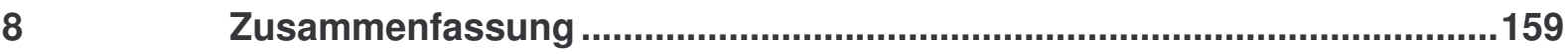

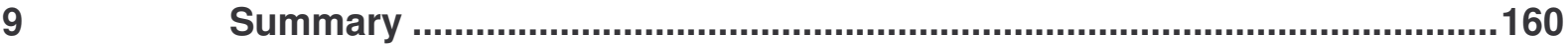

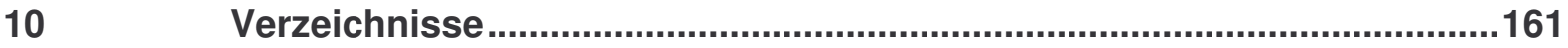

$10.1 \quad$ Literaturverzeichnis ............................................................................ 161

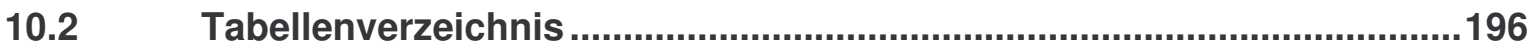

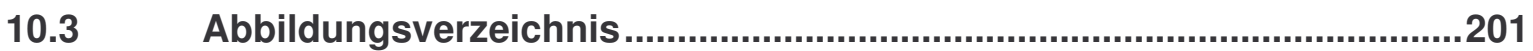

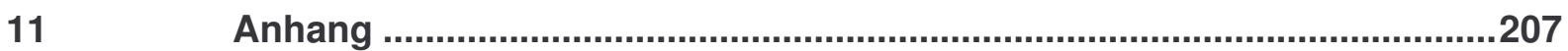

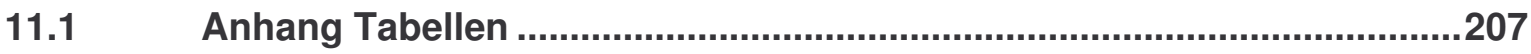

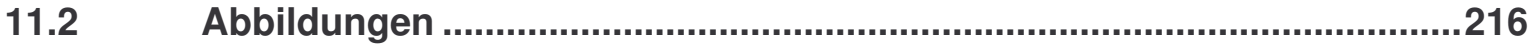




\section{Abkürzungsverzeichnis}

\begin{tabular}{|c|c|}
\hline & allgemein \\
\hline ASP & Afrikanische Schweinepest \\
\hline $\begin{array}{l}\text { aw-Wert } \\
\text { baul }\end{array}$ & $\begin{array}{l}\text { Wasseraktivität } \\
\text { baulich }\end{array}$ \\
\hline DF & Bund deutscher Fleischrinder \\
\hline EAU & Bundesforschungsanstalt für Viruserkrankungen der Tiere \\
\hline $\mathrm{BfR}$ & Bundesinstitut für Risikobewertung \\
\hline BgVV & $\begin{array}{l}\text { Bundesinstitut für gesundheitlichen Verbraucherschutz und } \\
\text { Veterinärmedizin }\end{array}$ \\
\hline BHV 1 & Bovines Herpesvirus 1 \\
\hline HZP & Bundes Hybrid Zucht Programm \\
\hline Bio. & biologisch \\
\hline BLL & Bund für Lebensmittelrecht und Lebensmittelkunde \\
\hline BMU & Bundesministerium für Umwelt, Naturschutz und Reaktorsicherheit \\
\hline BMVEL & Bundesministerium für Verbraucherschutz, Ernährung und Landwirtschaft \\
\hline BRC & British Retail Consortium Global Standard \\
\hline SE & Bovine Spongiforme Enzephalophatie \\
\hline -Mais & genveränderte Maissorte \\
\hline VD & Bovine Virus Diarrhoe \\
\hline BVL & Bundesamt für Verbraucherschutz und Lebensmittelsicherheit \\
\hline CCP & Critical Control Point \\
\hline CMA & Zentrale Marketingorganisation der Agrarwirtschaft \\
\hline $\mathrm{CO}_{2}$ & Kohlendioxid \\
\hline & das heißt \\
\hline ANISH & Danske slagterier \\
\hline $\mathrm{CB}$ & Dark cutting beef \\
\hline DE & 1,1-Dichlor-2,2-bis-(4-chlorphenyl)ethen \\
\hline DR & Deutsche Demokratische Republik \\
\hline DT & 1,1,1-Trichlor-2,2-bis-(4-chlorphenyl)ethan \\
\hline FD & Dark, firm, dry \\
\hline $\mathrm{N}$ & Deutsches Institut für Normung \\
\hline$-G$ & Deutsche Landwirtschafts-Gesellschaft e.V. \\
\hline VA & Desoxyribonucleic Acid \\
\hline $\mathrm{DN}$ & Desoxynivalenol \\
\hline thl x Duro & Deutsche Landrasse $\times$ Duroc \\
\hline $\mathrm{U}(\mathrm{NN})$ & Duroc (stressunempfindlich) \\
\hline$\sqrt{\mathrm{T}}$ & Deutscher Verband Tiernahrung e.V. \\
\hline Coli & Escherichia-coli Bakterium \\
\hline ESA & European Food Safety Authority \\
\hline HEC & enterohämorrhagische Escherichia-coli Bakterien \\
\hline$P$ & Europäische Schweinepest \\
\hline EU & Europäische Union \\
\hline J RL & Richtlinie der Europäischen Union \\
\hline J VO & Verordnung der Europäischen Union \\
\hline JREP GAP & Euro-Retailers Produce Working Group - Good Agricultural Practice \\
\hline$Z G$ & Erzeugergemeinschaft \\
\hline $\mathrm{AL}$ & Bundesforschungsanstalt für Landwirtschaft \\
\hline HO & Food and Agriculture Organization of the United Nations \\
\hline & Futtermittel \\
\hline MEA & Fehlermöglichkeits- und Einflussanalyse \\
\hline HP & Good Hygienic Practice \\
\hline & Good Manufactoring Practice \\
\hline & Qualitätsmanagementsystem für Futtermittel der Niederlande \\
\hline
\end{tabular}




\begin{tabular}{|c|c|}
\hline IV & genverändert \\
\hline GVO & Genveränderte Organismen \\
\hline HACCP & Hazard Analysis Critical Control Point \\
\hline Ht-Raps & genveränderte Rapssorte \\
\hline IBR & Infektiöse bovine Rhinotracheitis \\
\hline IFS & International Food Standard \\
\hline IfSG & Infektionsschutzgesetz \\
\hline IKB & Integrierte Kettenüberwachung (Integrale Keten Beheersing) \\
\hline ILN & Internationale Lokationsnummer \\
\hline ISO & International Organization for Standardization \\
\hline KSP & Klassische Schweinepest \\
\hline LM & Lebensmittel \\
\hline LMHV & Lebensmittelhygiene-Verordnung \\
\hline $\begin{array}{l}\text { LMU-München } \\
\text { mang. }\end{array}$ & $\begin{array}{l}\text { Ludwig-Maximilian-Universität München } \\
\text { mangelhaft }\end{array}$ \\
\hline MHD & Mindeshaltbarkeitsdatum \\
\hline mikrob. & mikrobiologisch \\
\hline Mio. & Millionen \\
\hline MKS & Maul- und Klauenseuche \\
\hline MPA & Medroxyprogesteron Acetat \\
\hline $\mathrm{N}$ & Nennungen \\
\hline NASA & National Aeronautics and Space Administration \\
\hline $\begin{array}{l}\text { N-Gaben/N- } \\
\text { Dünger }\end{array}$ & Stickstoffdünger \\
\hline NN-Sauen & stressunempfindliche Sauen \\
\hline NRKP & Nationaler Rückstandskontrollplan \\
\hline NVE & Nummer der Versandeinheit \\
\hline OIE & Office International des Epizooties \\
\hline Öko & ökologisch \\
\hline org. & organisch \\
\hline OTA & Ochratoxin A \\
\hline PAK & Polycyklische aromatische Kohlenwasserstoffe \\
\hline PCB & Polychlorierte Biphenylen \\
\hline $\mathrm{PCDD} / \mathrm{F}$ & Polychlorierte Dibenzodioxine und -furane \\
\hline PDV & Productschap Diervolder \\
\hline $\mathrm{Pg}$ iTE/Tag & $\begin{array}{l}\text { Pikogramm (pg), international abgestimmte ToxizitätsÄquivalenzfaktoren } \\
\text { (iTE) }\end{array}$ \\
\hline PI & Pietrain \\
\hline PIC & Hybrid-Schweine Zuchtorganisation \\
\hline PP & stressanfällig \\
\hline PSE & Pale, soft, exudative \\
\hline PSM & Pflanzenschutzmittel \\
\hline PVE & Wirtschaftsgruppe für Vieh, Fleisch und Eier, Niederlande \\
\hline QM & Qualitätsmanagement \\
\hline QS & Qualität und Sicherheit \\
\hline RKI & Robert Koch Institut \\
\hline$R L$ & Richtlinie \\
\hline RNA & Ribonuclein Säure \\
\hline Sal. & Salmonellen \\
\hline Studenten (mL) & Studenten deren Eltern einen landwirtschaftlichen Betrieb besitzen \\
\hline Studenten (oL) & Studenten deren Eltern keinen landwirtschaftlichen Betrieb besitzen \\
\hline TDI-Wert & $\begin{array}{l}\text { Angabe zur tolerierbaren täglichen Zufuhr von Stoffen ("tolerable daily } \\
\text { intake") }\end{array}$ \\
\hline TierSchTr-VO & Tierschutztransportverordnung \\
\hline TSE & Transmissible spongiforme Enzephalopathien \\
\hline & unter anderem \\
\hline
\end{tabular}




$\begin{array}{ll}\text { UNO } & \text { United Nations Organisation) } \\ \text { US-Miliär } & \text { United States of America - Militär } \\ \text { VerfVerbG } & \text { Verfütterungsverbotsgesetz } \\ \text { vergl. } & \text { vergleiche } \\ \text { ViehVerkV } & \text { Viehverkehrverordnung } \\ \text { VO } & \text { Verordung } \\ \text { WHO } & \text { World Health Organization } \\ \text { ZDS } & \text { Zentralverband der deutschen Schweineproduktion } \\ \text { ZEA } & \text { Zearalenon } \\ \text { ZNS } & \text { Zentrales Nervensystem } \\ \text { Zutriebsys. } & \text { Zutriebssystem }\end{array}$




\section{$1 \quad$ Einleitung}

Erwartungen von Verbrauchern und der Wirtschaft an das Lebensmittel Fleisch sind hoch. Neben guten sensorischen Eigenschaften hat der Verbraucher auch immer mehr ethische Anforderungen an das Produkt. Diese beginnen in der Regel schon bei der Futtermittelproduktion und sind besonders ausgeprägt bei allen Aspekten, die das lebende Tier betreffen. Um einen dauerhaft gesicherten Absatz von Fleisch und Fleischwaren zu gewährleisten, ist es für die beteiligten Partner der Fleischproduktion notwendig, die Anforderungen, Bedenken und Vorbehalte der Verbraucher zu kennen.

Diese Bedürfnisse werden häufig stark von den Medien geprägt. Immer wieder gelangen Teile der Kette (Futtermittelproduktion, Züchtung, Haltung, Tiertransport, Schlachtung und Produktion) in die öffentliche Kritik. Dem Verbraucher wird über die Medien häufig ein negatives Bild vermittelt. Im krassen Gegensatz zu den negativen Bildern stehen Werbeaussagen der Wirtschaft, Kalender und Bilderbücher. Hier wird häufig die Landwirtschaft von gestern und nicht von heute oder morgen gezeigt (BARTUSSEK, 2001; ALVENSLEBEN, 2002D).

Allein die Aussage, „die deutsche Fleischwirtschaft verfügt im Grundsatz über ein hochwertiges und sicheres Produkt“ (siehe: FeHLHABER, 1998; PudEL, 2002; AlvensLeben, 2002B; BLAHA, 2004), reicht nicht, um das Vertrauen der Verbraucher zu gewinnen. Das Problem bei der Vertrauensbildung besteht allerdings darin, dass es schwierig und kompliziert ist, einen wenig sachkundigen Verbraucher von der Unbedenklichkeit des Konsums von Fleisch zu überzeugen, nachdem sich bei diesem durch die oft verkürzte und undifferenzierte Berichterstattung der Medien ein ganz anderes Bild der Problematik verankert hat.

Die an der Fleischproduktion beteiligten Partner Landwirt, Futtermittelproduzent, Schlachthof und Lebensmittelhandel sowie die Wissenschaft haben ihre Bemühungen in der Vergangenheit darauf konzentriert, wirtschaftlich zu produzieren sowie auch in den einzelnen Bereichen die Produktionsbedingungen zu verbessern. In den letzten Jahren ist aber immer deutlicher geworden, dass zur nachhaltigen und wirklichen Verbesserung nicht nur einzelne Produktionsabschnitte, sondern die gesamte Produktionskette sowie auch die ethischen Anforderungen der Verbraucher im Mittelpunkt der Qualitätssicherung stehen müssen.

Aufgrund der Entwicklung der letzten Jahre hat sich der Fokus der Diskussion unter den Fachwissenschaftlern und Experten des Bereiches Fleischproduktion verschoben. Dies findet seinen konkreten Ausdruck vor allem in der EU-Gesetzgebung. Erstmals wird der integrierte Ansatz im Weißbuch zur Lebensmittelsicherheit (1999) definiert und durch die VO EU 178/2002 für die gesamte Produktionskette festgeschrieben. Insbesondere Fragen wie die der Rückverfolgbarkeit haben stark an Bedeutung gewonnen. Aber auch die Etablierung von Qualitätsmanagementsystemen und deren Forderungen nach HACCP-Konzepten haben dazu geführt, dass in den letzten Jahren eine Reihe von weiteren Risikopunkten erkannt und angegangen worden sind. Neben dem alten Schwerpunkt, Identifizierung und Minimierung der realen Risikopunkte der Fleischproduktion ist ein neuer Forschungs- und Diskussionsschwerpunkt, Bedenken der Verbraucher, hinzugekommen. Im Rahmen dieses Schwerpunktes wird diskutiert, welche Punkte in der Produktionskette vom Verbraucher als kritisch gesehen werden und unter Umständen zu einem Meideverhalten führen könnten und wie darauf zu reagieren ist. 


\section{$1.1 \quad$ Zielsetzung der Arbeit}

Das Ziel ist die Darstellung der Risiken in der Produktionskette Fleisch für die Fleischgruppen Rind, Schwein und Geflügel. Hierbei sollen die Risiken dargestellt werden,

- die real und wissenschaftlich nachweisbar sind oder

- die real keine Gefahr für das Produkt Fleisch darstellen, aber als Risiko vom Verbraucher gesehen werden und somit sein Konsumverhalten beeinflussen.

Die Ziele der Studie sind im Einzelnen:

- Erfassung der Risiken auf der Erzeugerstufe mit Fütterung, Haltung, Tiertransport,

- Erfassung der Risiken in der Schlachtung einschließlich der parallel laufenden Kontrolltätigkeiten,

- Erfassung von Risiken in der Endproduktqualität,

- Erfassung der Risiken in der Herkunftssicherung und Rückverfolgbarkeit über die Produktionsstufen hinweg. 


\section{$2 \quad$ Literatur}

\subsection{Definitionen}

\subsubsection{Verbraucherbild}

Der Europäische Gerichtshof geht in seinem Verbraucherleitbild von einem durchschnittlich informierten, aufmerksamen und verständigen Verbraucher aus (BENNER, 2004B). Das entspricht dem Bild eines souverän agierenden Verbrauchers, der explizit seine eigenen Interessen am Markt verfolgt und um die Besonderheiten der Werbung als Instrument der Informationsvermittlung weiß. Dieses Verbraucherbild findet sich in der wissenschaftlichen Marketingliteratur im so genannten Persuasion Knowledge Model wieder, in dem der Verbraucher im Marktgeschehen seine eigenen Ziele verfolgt. Die hierfür notwendigen Informationen erfasst der Verbraucher auf der Basis zeitpunktunabhängiger, umfassender Reflexionen (BENNER, 2004B).

Das Bild des europäischen Verbrauchers ist nicht mit jenem Verbraucherbild identisch, das zum Grundbestand des Marketing gehört und sich beispielsweise im Elaboration-LikelihoodModel widerspiegelt (PETTY u. CACIOPPO, 1986; STAHLBERG u. FREY, 1993). Das ElaborationLikelihood-Model zeigt zwar auf, wie sich beim Verbraucher Einstellungen gegenüber Produkten bilden; das zugrunde liegende Verbraucherbild unterstellt jedoch, dass sich der Verbraucher lediglich mit Produkteigenschaften auseinander setzt und auf Reize reagiert, die inm etwa durch Werbung dargeboten werden (FRIESTAD u. WRIGHT 1994 u. 1995). Auf das Elaboration-Likelihood-Model bezieht sich auch ALVENSLEBEN (1999B u. 2001C) wonach der Verbraucher die Informationen über Lebensmittel in erster Linie auf dem peripheren Weg verarbeitet. Hierunter werden Einstellungsänderungen durch Reize (z.B. Bilder, Geräusche u.a.) in Verbindung mit positiven oder negativen Gefühlen verstanden. Die Wirkung von Informationen hängt stärker von der Glaubwürdigkeit und Attraktivität des Kommunikators, der Häufigkeit der Wiederholung und weiteren emotionalen Schlüsselinformationen ab. Die Qualität der Schlüsselinformationen hat hierbei einen geringen Einfluss. Sachinformationen stoßen schnell an ihre Grenzen (Alvensleben, 1999B u. 2001C). AlvensLeben (2002C) beruft sich auf eine englische Untersuchung, der zur Folge nur 11\% der Verbraucher aktiv nach Informationen suchen. 51\% der Verbraucher haben hiernach zwar gute Vorsätze, aber tun nichts, und 38\% der Verbraucher haben kein Interesse an neuen Informationen.

$\mathrm{Da}$ es kein einheitliches Bild des Verbrauchers gibt, soll in dieser Arbeit der Verbraucher bzw. Konsument als ein solcher gesehen werden, der seine Informationen über Lebensmittel hauptsächlich auf dem peripheren Weg verarbeitet und für den häufig Schlüsselinformationen/Schlüsselreize zur Meinungsbildung wichtiger sind als Sachinformationen.

\subsubsection{Expertenbild}

In den Lexika KNAURS (1981) und CORON (1992) ist der Experte als „Sachverständiger, Fachmann, Kenner" definiert. Weiter findet man im Internet eine Definition von WIKIPEDIA (2005). „Fachmann oder Fachfrau (auch Spezialist oder Experte) bezeichnet unscharf eine Person, die über umfangreiches Wissen auf einem oder mehreren bestimmten Fachgebieten oder über spezielle Fähigkeiten verfügt. Auch ein Wissensvorsprung gegenüber dem Durchschnitt kann einen Fachmann definieren. Neben dem theoretischen Wissen ist auch eine kompetente Anwendung desselben, also praktisches Handlungswissen, kennzeichnend. Solche Fertigkeiten gründen sich meist auf Training sowie Talent." Hierbei eignet sich die 
Person das Wissen in der Regel durch eine Ausbildung oder ein Studium an, es kann jedoch auch durch Forschung oder auch autodidaktisch erworben werden.

Im Rahmen der Arbeit gelten als Experten Wissenschaftler, Vertreter von Behörden in leitender Stellung sowie Vertreter der Wirtschaft und Dienstleister der Wirtschaft. Diese Personen verfügen über jahrelange Erfahrungen auf ihrem Fachgebiet. Sie haben durch wissenschaftliche Veröffentlichungen ihre Arbeiten dokumentiert oder sich in ihrem jeweiligen Bereich Kompetenzen erworben. Dadurch ergibt sich zwangsläufig, dass es sich bei der berücksichtigten wissenschaftlichen Literatur auch um Expertenmeinungen handelt.

\subsubsection{Risikobegriff, Risikowahrnehmung und Risikobewertung}

Nach Holzheu u. WiedmanN (1993) bezieht sich der Begriff "Risiko“ auf potentielle Ereignisse, deren Ausprägungen im Einzelnen im Voraus ungewiss sind. Sie definieren "Risiko” als ein Konstrukt. Dieses heißt, dass „Risiko" ein Beobachtungskonzept ist, das man mit einer Art Brille vergleichen kann, durch die man die Welt betrachtet. Ein „Risiko“ kann dabei je nach dem, durch welche „Brille“ man es betrachtet, unterschiedlich bewertet werden. Zum Beispiel werden innerhalb der verschiedenen wissenschaftlichen Disziplinen unterschiedliche „Risikobrillen“ getragen. Dadurch wird bei der Betrachtung desselben Gegenstandes Unterschiedliches gesehen. Dieses trifft auch auf die Bewertungen von Risiken durch Verbraucher und Experten zu. Das Risiko, an Fleisch von hormonbehandelten Tieren zu erkranken, wird z.B. vielfach vom Verbraucher als ein größeres Risiko angesehen als eine mikrobiologische Kontamination, die von den Fachleuten als das größere Risiko bewertet wird (vergleiche hierzu HILDEBRAND, 2002).

In der EU-Verordnung 178/2002 wird Risiko als „eine Funktion der Wahrscheinlichkeit einer die Gesundheit beeinträchtigenden Wirkung und der Schwere dieser Wirkung als Folge der Realisierung einer Gefahr" beschrieben.

HENSEL (2003) definiert Risiko als eine Art „Vorstufe der Gefahr“. Durch das Risiko wird das Potenzial eines Stoffes oder Keims charakterisiert, der die Gesundheit unter bestimmten Bedingungen schädigen kann. Durch Risikoerkennung, -bewertung und -management können Schadpotenziale frühzeitig erkannt werden und Rahmenbedingungen festgelegt werden, um das Eintreten der Gefahr zu minimieren bzw. auszuschließen.

In der vorliegenden Arbeit wird der Begriff „Risiko“ als ein Konstrukt, wie er von HOLZHEU u. WIEDMANN (1993) beschrieben wird, verwendet. Der Begriff „Risiko“ wird nicht nur im Zusammenhang mit einer bestimmten Gefahr, durch die es zu einer gesundheitlichen Gefährdung kommen kann, benutzt. Als Risiken werden auch Sachverhalte verstanden, die Schwachpunkte in der Kommunikation darstellen, also einzelne Aussagen der Werbung oder Ereignisse bzw. Aussagen, die das Produktimage Fleisch insgesamt in Frage stellen können. Das können zum einen Risiken sein, die real vorhanden sind. Zum anderen können es aber auch Risiken sein, die real keine Gefahr für das Produkt Fleisch oder den Verbraucher darstellen, aber als Risiko vom Verbraucher gesehen werden und deshalb sein Konsumverhalten beeinflussen (Abbildung 2.1-1).

Wichtig bei dem Empfinden bzw. der Wahrnehmung von Risiken sind nach JUNGERMANN und SOLVIC (1993) folgende Elemente: 
- Das Katastrophenverhalten: Das Empfinden der Katastrophe ist größer, wenn auf einmal viele Menschen betroffen sind, als wenn dieselbe Anzahl an Menschen über einen längeren Zeitrum betroffen ist.

- Die Betroffenheit: Ist jemand direkt von einem Risiko betroffen, bewertet er es stärker, als wenn jemand ein Risiko beurteilen soll, von dem er nicht unmittelbar betroffen ist.

- Die Freiwilligkeit: Freiwillig in Kauf genommene Risiken werden eher akzeptiert und als weniger gefährlich beurteilt als Risiken, mit denen jemand unfreiwillig, gezwungenermaßen konfrontiert wird.

- Die Kontrollierbarkeit: Risiken, bei denen der Mensch der Meinung ist, diese vermeintlich kontrollieren zu können, werden als weniger gefährlich eingestuft als Risiken, die sich der Kontrollmöglichkeit des Einzelnen entziehen.

- Die Verantwortlichkeit: Natürliche Risiken werden als weniger gefährlich eingestuft als Risiken, die aufgrund von technischen Errungenschaften entstehen. Bei natürlichen Risiken wird häufig davon ausgegangen, dass diese unvermeidbar und somit nicht kontrollierbar sind. Risiken, die aufgrund von Entwicklungen durch die Industriegesellschaft entstehen, werden als vermeidbar und kontrollierbar eingestuft und damit unabhängig von ihrer Auswirkung als gravierender bewertet.

- $\quad$ Undeutlichkeit von Risiken: Bei unbekannten Techniken (z.B. Gentechnik) spielt dieser Aspekt eine große Rolle. Hier werden in die Risikobewertung mögliche soziale und politische Fehlentwicklungen mit einbezogen. Dazu kommt die Unsicherheit, dass zum Zeitpunkt der Beurteilung nicht bekannt ist, ob sich z.B. aus einem harmlosen Organismus ein gefährlicher human- oder tierpathogener Krankheitserreger entwickeln kann.

Entsprechende Risikomerkmale werden auch von ALVENSLEBEN (2002B) benannt.

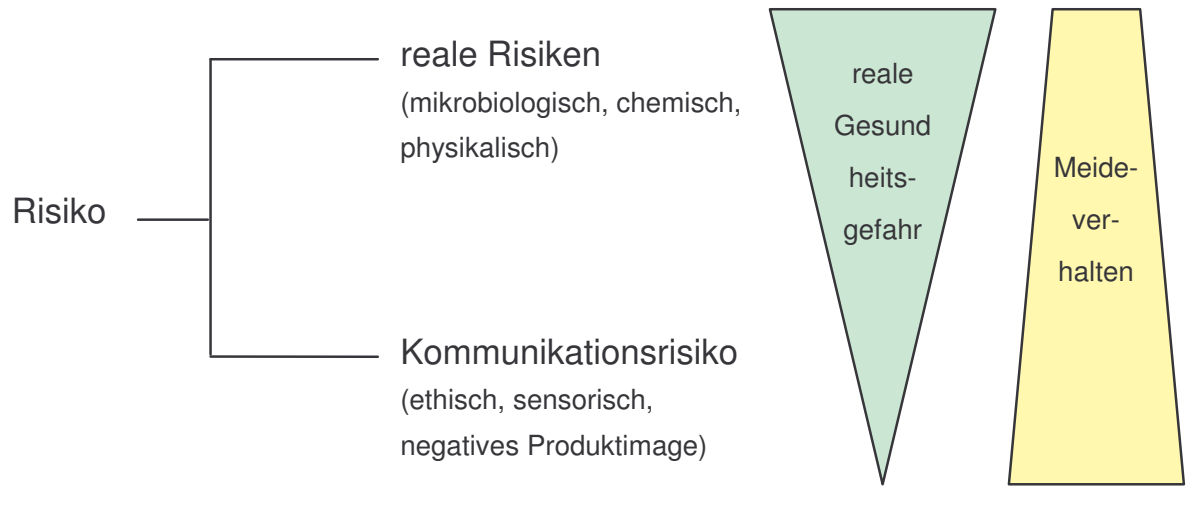

Abbildung 2.1-1: $\quad$ Risikobegriff, Risikowahrnehmung und Risikobewertung.

Risikomerkmale und deren Empfinden spielen gerade bei der Risikobewertung eine entscheidende Rolle. Die Risikobewertung durch Experten befasst sich in erster Linie mit Risiken als Produkt von Wahrscheinlichkeit und Konsequenzen (KEMP, 1993). Experten erfassen Risiken nach Schadendimension, Sachschäden bzw. Gesundheitsschäden (HAMPEL u. RENN, 1999). Experten neigen dazu, Risiken mit hoher Wahrscheinlichkeit, aber geringen Folgen unterzubewerten (KEMP, 1993). 
Die Verbraucher erfassen zusätzlich ökonomische, ökologische, soziale, rechtliche und ethische Auswirkungen (HAMPEL u. RENN, 1999). Beim Verbraucher sind die Schadenfolgen, die sich im ungünstigsten Fall ergeben, wichtiger für die Risikobewertung als die Wahrscheinlichkeit eines möglichen Eintretens des Risikos. Das hat zur Folge, dass Risiken mit geringer Wahrscheinlichkeit, aber hoher Auswirkung oft überbewertet werden (KEMP, 1993).

Dieses wird auch aus der Abbildung 2.1-2 deutlich. Von den Experten werden die ernährungsabhängigen Risiken wesentlich höher bewertet und die Risiken durch BSE wesentlich niedriger bewertet als durch den Verbraucher.

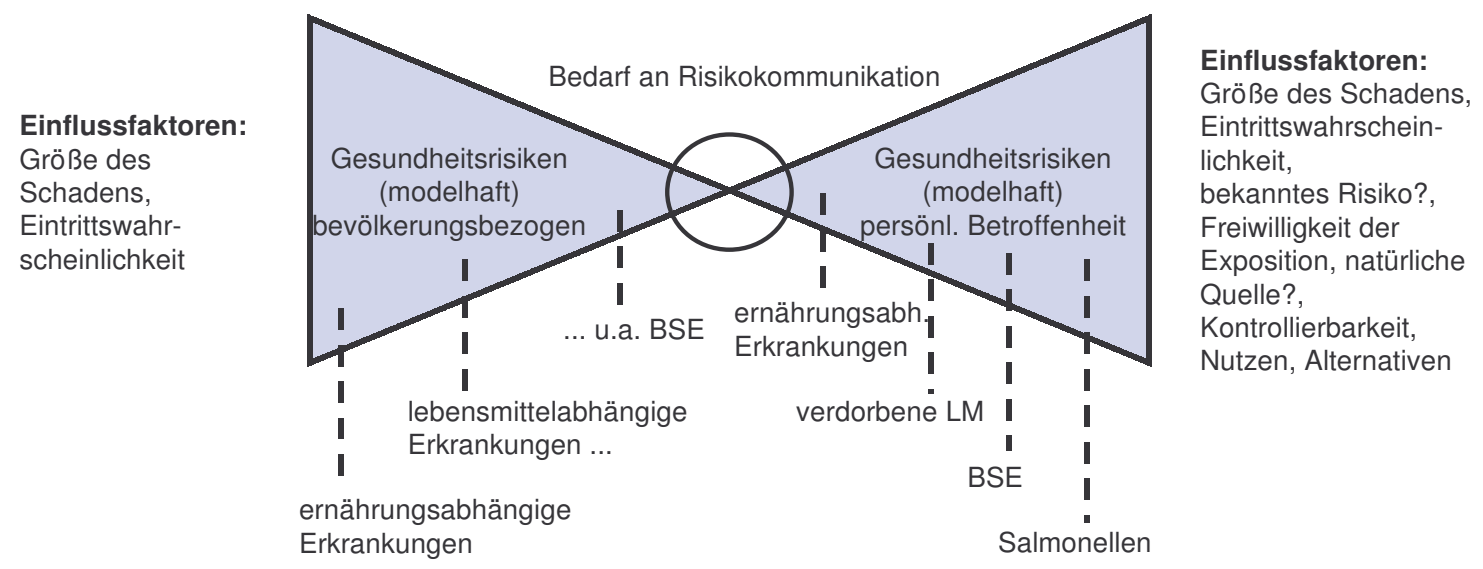

Abbildung 2.1-2: Unterschiedliche Beurteilungen von Risiken durch Experten und Verbraucher (RÖHR ET AL., 2002B).

\subsubsection{HACCP-Konzept}

HACCP-Konzept ist die Abkürzung für Hazard Analysis Critical Control Point-Konzept. Es dient dazu, bedeutende gesundheitliche Gefahren durch Lebensmittel zu identifizieren, zu bewerten und zu beherrschen. Entwickelt wurde das HACCP-Konzept im Jahr 1959, als die NASA ein Lebensmittelunternehmen beauftragte Astronautennahrung herzustellen, die hundertprozentig sicher ist. Das HACCP-Konzept basiert auf der vom US-Militär für technische Anwendungen geschaffene Fehler-Möglichkeits-und-Einfluss-Analyse (FMEA-Methodik). Die FMEA-Methodik wird genau wie das HACCP-Konzept präventiv zur Risiko- und Fehleranalyse von Produkten und Prozessen in der Entwicklung, Konstruktion und Prozessplanung eingesetzt (MORTIMORE u. WALLACE, 2000; WIKIPEDIA, 2006).

Seit 1993 wird die Anwendung von HACCP-Konzepten durch die Food and Agriculture Organisation der UNO empfohlen. Im deutschen Recht wurde das HACCP-Konzept erstmals mit der Lebensmittelhygieneverordnung von 1998 verankert. Es ist seit dem 1. Januar 2006 in der gesamten Lebensmittelproduktion vorgeschrieben.

Im Rahmen des HACCP-Konzeptes werden chemische, physikalische und mikrobiologische Gesundheitsgefahren identifiziert. Anschließend werden die Wahrscheinlichkeit und die Bedeutung der Gesundheitsgefahren bewertet. Aufgrund dieser Analysen sind die notwendigen vorbeugenden Maßnahmen festzulegen. Das HACCP-Konzept liegt primär in der Verantwortung des Unternehmers (HOLZAPFEL ET AL., 2004). Das HACCP-Konzept umfasst eine konsequente Prozessüberwachung. Hierbei geht es um vorbeugende Maßnahmen und we- 
niger um die Untersuchung von Endprodukten (WEBER, 1996). Die HACCP-Analyse erfolgt dabei nach sieben Grundsätzen, die in Tabelle 2.1-1 dargestellt sind. In der Tabelle sind die Schritte aufgeführt, die bei der Erstellung eines HACCP-Konzeptes durchgeführt werden (Weber, 1996; SinelL, 1998B; Mortimore u. WALlace, 2000; HensGen, 2004). Regelungen und Weiterentwicklungen des HACCP-Konzeptes erarbeitet die Codex Alimentarius Kommission (MORTIMORE u. WALLACE, 2000).

Tabelle 2.1-1:

Die 7 Grundsätze des HACCP-Konzeptes nach dem Codex Alimentarius.

\section{HACCP Konzept Codex Alimentarius}

1. Durchführen einer Gefahrenanalyse.

2. Bestimmung der kritischen Kontrollpunkte (CCPs).

3. Festlegung der kritischen Grenzwerte.

4. Festlegung eines Systems zur Überwachung (Monitoring) der CCPs.

5. Festlegung von Korrekturmaßnahmen, wenn die Überwachung anzeigt, dass ein CCP-Punkt nicht mehr beherrscht werden kann.

6. Festlegung eines Verfahrens zur Verifikation des Konzeptes, das bestätigt, dass das System richtig arbeitet.

7. Dokumentation der eingeführten Maßnahmen, anhand derer das System nachzuvollziehen ist.

\subsubsection{Produkt- und Prozessqualität}

Im Rahmen vorliegender Arbeit werden die Begriffe Produktqualität und Fleischqualität synonym verwendet. Die Fleischqualität wird durch folgende Eigenschaften definiert (HOFMANN, 1973):

- sensorisch (Aussehen, Aroma, Textur),

- ernährungsphysiologisch (Nährwert, Vitamine),

- hygienisch-toxikologisch (u.a. Rückstände, Mikroorganismen).

- verarbeitungstechnologisch (u.a. pH-Wert, Wasserbindungsvermögen).

Unter Prozessqualität werden die Einflüsse verstanden, die durch die Produktionsverfahren auf das Tier und auf das Produkt wirken. Hierunter fallen Punkte wie:

- Futterqualität,

- Haltungsbedingungen (u.a. Spaltenboden, Anzahl der Tiere/Stall),

- Transportbedingungen (u.a. Art, Dauer, Klima),

- Betäubungsverfahren und Blutentzug,

- hygienische Voraussetzungen (u.a. Schlacht- und Verarbeitungshygiene),

- Rückverfolgbarkeit,

- Umgang mit den Tieren,

- Verhalten der Tiere. 


\subsection{Die Sicherheit der Fleischproduktion aus der Sicht des Verbrauchers}

\subsubsection{Wahrnehmung von Risiken bei Lebensmitteln}

Die Diskrepanzen bezüglich der Wahrnehmung von Risiken bei Lebensmitteln werden deutlich in den unterschiedlichen Meinungen von Wissenschaft, „Unsere Lebensmittel sind so sicher wie noch nie“, (vergl. FehlHABER, 1998; BergmanN, 2000; PuDEL, 2002; ALVENSLEBEN, 2002B) und der Meinung des Verbrauchers, „Die Lebensmittel werden immer unsicherer" (vergl. REWERTS, 1998; WALLENBURG, 1998). Ursachen für die zunehmende Verbraucherverunsicherung bei Lebensmitteln begründet ALVENSLEBEN (1998B) anhand von fünf möglichen Ursachen:

a) Sättigungsproblem: Die physiologischen Grundbedürfnisse der Verbraucher sind durch das Überangebot an Nahrungsmitteln gesättigt. Für den Verbraucher werden andere Aspekte in der Nahrungsversorgung wichtiger.

b) Sinkendes Grundvertrauen: In der gesamten Gesellschaft ist ein abnehmendes Vertrauen gegenüber etablierten gesellschaftlichen Institutionen und Autoritäten zu beobachten. Dadurch werden wissenschaftliche Ergebnisse und staatliche Kontrollinstanzen in Frage gestellt.

c) Wachsende Entfremdung: Die Verbraucher haben den Bezug zur Urproduktion (Landwirtschaft) verloren. Dazu kommt, dass die immer weiter fortschreitende Technisierung von vielen Verbrauchern nicht erfasst/verstanden wird.

d) Wahrnehmungsverzerrungen: Es kommt bei vielen Risiken zu einer Über- oder Unterschätzung. Diese Beurteilung hängt meist individuell von dem Einzelnen und vom Image des Nahrungsmittels ab.

e) Medienberichte: Medienberichte haben häufig eine Verstärkerfunktion.

Ebenso spielen ethische Aspekte bei der Wahrnehmung eine wichtige Rolle. Am Beispiel der Gentechnik wird deutlich, dass sich die Einwände gegen unbekannte Technologien oder Sachverhalte nicht nur gegen Sicherheitsprobleme, sondern ebenso gegen die ethische Zuverlässigkeit und gegen einen drohenden Missbrauch richtet (VAN DEN DAELE, 1993).

Ein anderes Beispiel zur Risikowahrnehmung ist der Nitrofenskandal im Mai 2002. Hierdurch gelangte erstmals die ökologische Landwirtschaft in das negative Interesse der Medien. Ein ehemaliges Depot für Pflanzenschutzmittel wurde als Getreidezwischenlager genutzt. Über das Getreide war das „krebserregende“ Herbizid in Futtermittel und von dort unter anderem in Bio-Putenfleisch und Bio-Eier gelangt (HEESCHEN, 2003). Nach ALVENSLEBEN (2002B) dürfte das derzeitige Krebsrisiko durch Nitrofen belastete Lebensmittel nicht sehr viel größer sein als das Krebsrisiko, das durch den Genuss von Fleisch in der laufenden Grillsaison entsteht. Dennoch besteht in der subjektiven Risikowahrnehmung ein großer Unterschied. Denn Letzteres ist ein freiwillig eingegangenes Risiko und Ersteres ein unfreiwilliges Risiko, das obendrein durch die umfangreiche, dramatisierende Medienberichterstattung verstärkt in das Verbraucherbewusstsein gerückt worden ist (ALVENSLEBEN, 2002B). 


\subsubsection{Einstellungen und Verhalten der Verbraucher}

Der Wandel des Verständnisses des Verbrauchers in Bezug auf Nahrungsmittel wird in der Geschichte deutlich. War nach dem Krieg noch das Hauptproblem der Menschen in Deutschland satt zu werden, spielt heute in einer Überflussgesellschaft vor allem der Genuss eine Rolle. Um aber reuelos genießen zu können, treten Aspekte wie Sicherheit und Ethik in den Vordergrund (Abbildung 2.2-1).

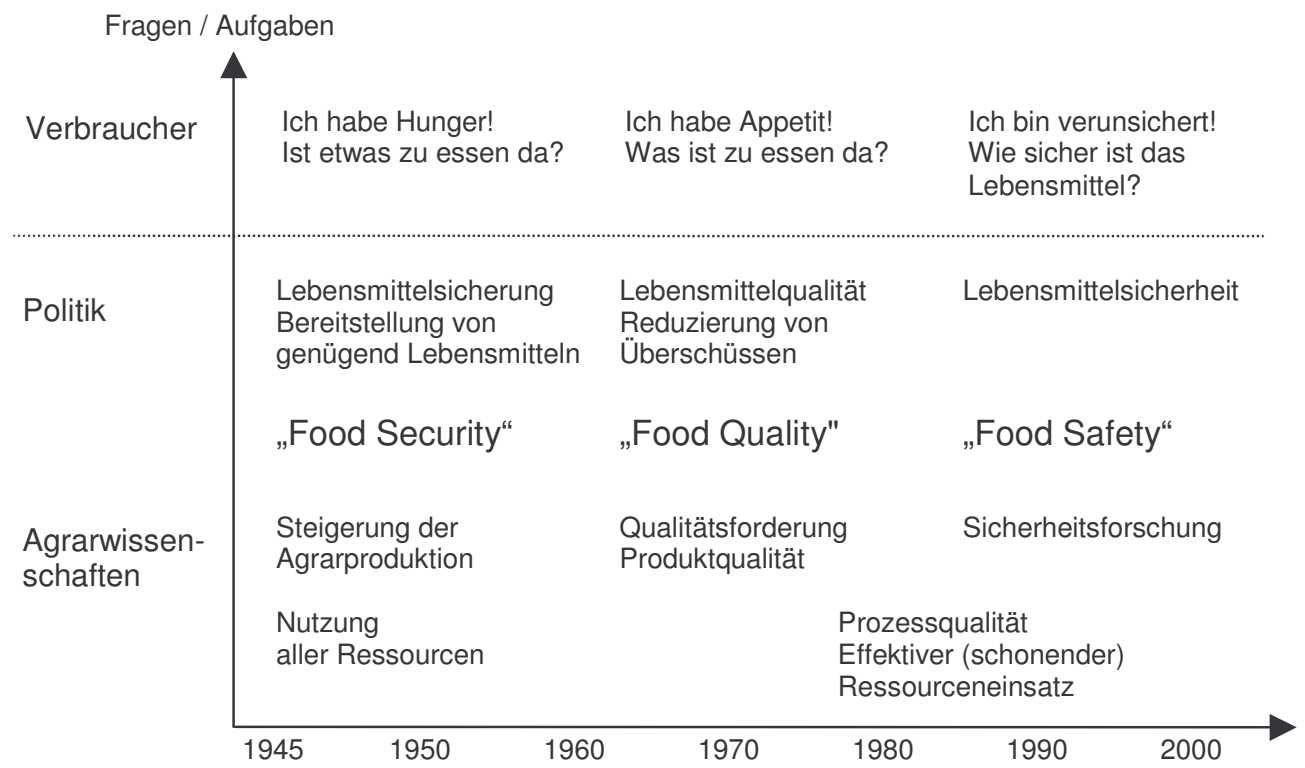

Abbildung 2.2-1: $\quad$ Dominierende Fragen in Bezug auf Lebensmittel sowie Aufgaben für Politik und Agrarforschung nach den 2. Weltkrieg (FLACHOWSKY, 2002 ergänzt).

Hinzu kommt, dass der Anteil der landwirtschaftlichen Bevölkerung an der Gesamtbevölkerung in den letzten Jahrzehnten kontinuierlich abgenommen hat. Hierdurch ist eine wachsende Entfremdung der Bevölkerung von der Landwirtschaft zu beobachten. Dieses hat dazu geführt, dass

- viele Menschen kaum noch wissen, wie landwirtschaftliche Produkte erzeugt werden,

- die Vorstellungen der Verbraucher über die Landwirtschaft und die Lebensmittelproduktion immer häufiger durch die Massenmedien und die Werbung geprägt werden,

- die heutigen Techniken und Produktionsverfahren den meisten Verbrauchern fremd sind,

- eigene Erfahrungen und Anschauungen immer weniger eine Rolle bei der Meinungsbildung über die Landwirtschaft spielen,

- Vertrauen in die Produkte eine sehr wichtige Rolle spielt.

Ebenso werden häufig aus der Sicht des Verbrauchers Lebensmittelsicherheit und Lebensmittelqualität nicht getrennt (ALVENSLEBEN, 1999A). Ein hoher Genusswert eines Lebensmittels wird häufig gleichgesetzt mit einer hohen Qualität bzw. Sicherheit (MELCHERS, 2003).

Die Ansprüche der Verbraucher umfassen neben einer hygienischen und gesundheitlichen Unbedenklichkeit der Produkte auch immer mehr Aspekte wie Tier- und Umweltschutz. Hier- 
durch rücken Aspekte wie Prozess- oder Produktionsqualität immer mehr in den Vordergrund (ADAM, 2001).

Der Verbraucher beurteilt seine Lebensmittel nach verschiedenen Qualitätsfaktoren (FEHLHABER, 1997):

- Geschmack,

- Aussehen,

- Frischegrad,

- gesundheitliche Unbedenklichkeit,

- Relation Preis/Leistung,

- Bequemlichkeit der Zubereitung (Convenience),

- Nährstoffzusammensetzung,

- Transparenz der Herstellungsprozesse,

- ausreichende Kennzeichnung,

- Garantien für gesundheitliche Unbedenklichkeit,

- Herkunftsangaben zu den Rohprodukten,

- Qualitätsgarantien,

- ethische Aspekte,

- Umweltaspekte,

- Haltbarkeit.

Hierbei zieht nicht jeder Verbraucher alle der hier genannten Kriterien für seine Beurteilung des Fleischeinkaufs heran. Die Wahl hängt stark von den persönlichen Präferenzen ab.

Trotz der genannten Beurteilungskriterien werden Lebensmittel von den meisten Verbrauchern gewohnheitsmäßig als Güter des täglichen Bedarfs oder im Rahmen von Impulsentscheidungen gekauft. Sachinformationen erreichen die meisten Verbraucher nur selten. Die Produktwahrnehmung erfolgt bei Lebensmitteln weniger „über den Kopf“ als vielmehr „über den Bauch" (peripherer Weg) und hängt eher von der Glaubwürdigkeit und Attraktivität des Kommunikators oder von anderen emotionalen Schlüsselreizen ab (ALVENSLEBEN, 2001C).

Das Einkaufsverhalten des Verbrauchers steht häufig auch im Widerspruch zu Umfrageergebnissen. Der Absatz von Geflügelfleisch ist in den letzten Jahren stark gestiegen. Im Jahr 2003 stieg der Verbrauch von Geflügelfleisch je Einwohner in Deutschland um ca. 1kg auf 18,2kg gegenüber 2002 (ZMP, 2004). Dieser Anstieg des Pro-Kopf-Verbrauchs von Geflügelfleisch steht allerdings im Widerspruch zum wachsenden Anspruch der Verbraucher an eine tier- und umweltgerechte Produktion von Lebensmitteln (GARTUNG ET AL., 2004). Geflügelfleisch wird überwiegend in großen Tierbeständen in Bodenhaltung produziert, der Verbraucher lehnt aber die Massentierhaltung ab. Ein weiterer Widerspruch im Einkaufsverhalten des Verbrauchers ist der klare Trend zu Convenienceprodukten. Ihr Anspruch an die Produkte ist allerdings, dass diese möglichst naturbelassen und wenig behandelt sind (FEHLHABER, 1997). Nach einer Studie der BEE-UNTERNEHMENSBERATUNG (2002) stufen 70 Prozent der Verbraucher Fertiggerichte als besonders wertvoll ein. In der Studie wird davon ausgegangen, dass Convenience-Food zum schnellen Erhitzen zu Hause deutlich zunehmen wird. 
Bei den ökologisch erzeugten Produkten ist es den Vermarktern gelungen die emotionalen Schlüsselreize positiv zu nutzen (ALVENSLEBEN, 2001C). Da ökologisch erzeugte Produkte unter strengen Regelungen bezüglich Medikamente, ohne Chemie und in anderen Haltungsformen erzeugt werden, werden diese vom Verbraucher für besonders sicher und gesund gehalten (ALVENSLEBEN, 2002B). Bezüglich der Produktsicherheit ist bei Untersuchungen jedoch objektiv kein wesentlicher Unterschied zwischen ökologisch und konventionell hergestellten Produkten festzustellen (TAUSCHER ET AL., 2003).

Vertrauen in die Produkte spielt bei Lebensmitteln eine sehr wichtige Rolle. Gerade die ökologische Produktionsweise ist eine Vertrauenssache; sie kann weder dem Produkt angesehen noch beim Verzehr geschmeckt werden (BENNER, 2004A). Das gestiegene Vertrauen oder das Misstrauen zeigen deutlich die Beispiele BSE-Krise in 2001 und Nitrofen-Skandal in 2002. Während der BSE-Krise in 2001 kam es zu einem Anstieg des Konsums von BioLebensmitteln, da diese für sicherer gehalten werden. Der Nitrofen-Skandal in 2002, von dem hauptsächlich Bio-Lebensmittel betroffen waren, hatte dann einen Nachfragerückgang von Bio-Lebensmitteln zur Folge (BRUHN, 2003).

\subsubsection{Politische Veränderungen}

Auf dem Agrarsektor werden die Rahmenbedingungen immer mehr durch politische Regelungen auf der EU-Ebene (178/2002), aber auch auf nationaler Ebene (Preisausgleichszahlungen, Umweltrecht, Flächennutzungspläne usw.) bestimmt.

Durch ihren verstärkten Einfluss auf den Agrarsektor hat das Meinungsbild der Politiker in Bezug auf die Landwirtschaft eine entscheidende Bedeutung für die zukünftige Ausrichtung der Agrarpolitik. Dies ist zurzeit in Deutschland zu beobachten, wo z.B. von der Politik verstärkt eine Förderung des ökologischen Landbaus propagiert wird. Politikerentscheidungen werden zunehmend durch das Image der Landwirtschaft beeinflusst (ALVENSLEBEN, 1998A; 2002D; EMNID, 2002).

Bei einer Befragung von Politikern konnte festgestellt werden, dass deren Meinung über die Land- und Ernährungswirtschaft im Wesentlichen das Meinungsspektrum der Bevölkerung widerspiegelt, d.h. sie unterliegen etwa den gleichen Urteilen und Vorurteilen wie die Bevölkerung (SCHEPER, 1999; ALVENSLEBEN, 2002 B).

Typische Fehleinschätzungen und Vorurteile von Verbrauchern im Lebensmittelbereich sind nach FEHLHABER (1997):

- chemische Rückstände sind die hauptsächliche gesundheitliche Gefahr: „Chemie ist grundsätzlich schädlich“,

- undifferenzierte Ablehnung von Gentechnik und Bestrahlung,

- $\quad$ kritiklose Sympathie für alternative Lebensmittelerzeugung (Öko- und Biokost),

- übermäßiges Misstrauen gegen Lebensmittelzusatzstoffe,

- blinder Glaube, dass unbehandelte, naturbelassene Lebensmittel ein hohes Maß an Sicherheit bieten.

\subsubsection{Massenmedien}

Durch die Medienberichte werden die offenen, häufig aber nur latent vorhandenen Zweifel über die Sicherheit unserer Nahrungsmittel immer wieder aktiviert (ALVENSLEBEN, 2002C). 
Insbesondere erfolgt dieses über die Bildkommunikation. Bilder prägen sich schneller und auch unbewusst beim Betrachter ein und es ist häufig schwer sich der Wirkung der Bilder zu entziehen (ALVENSLEBEN, 2002C; RÖHR ET AL., 2002B). Gerade in den Medien erhält der Verbraucher nur selten ein reales Bild von der Landwirtschaft und der Lebensmittelproduktion. Hier werden entweder idealisierte Bilder (heile Welt) zu Werbezwecken eingesetzt oder Skandale mit Negativbildern publiziert (MELCHERS, 2003).

Eine Risikoabschätzung spielt bei der Darstellung von Schadensfällen in den Medien kaum eine Rolle. Hier werden die Schadensgrößen in den Mittelpunkt der Betrachtung gestellt. Aussagen zur Schadenswahrscheinlichkeit fehlen häufig (KEPPLINGER u. KNIRSCH, 2002). Es wird vor allem über solche Sachverhalte berichtet, die eine hohe Aufmerksamkeit hervorrufen und ein Interesse an zusätzlichen Informationen wecken. Diese Sachverhalte werden auch als „Schlüsselereignisse“ bewertet. Diese Schlüsselereignisse bewirken häufig, dass nicht nur über den ursprünglichen Sachverhalt, sondern auch über verwandte Themen berichtet wird. Ebenso stimuliert die verstärkte Medienpräsenz von Sachverhalten Aktivitäten von Interessengruppen. Diese sehen eine Chance ihre Anliegen in die Öffentlichkeit zu bringen. Durch die fast permanente Präsenz der Sachverhalte in den Medien wird ein Entscheidungsdruck auf Politik, Wirtschaft und Verwaltung ausgeübt (KEPPLINGER u. Habermeier, 1996; Alvensleben u. KAFKA, 1999).

Die BSE-Krise kann als ein solches Schlüsselereignis angesehen werden. Im Rahmen der BSE-Krise wurde nicht nur vermehrt über dieses Risikoereignis gesprochen, sondern gleichzeitig wurden auch z.B. die Tierhaltungsformen und die moderne Landwirtschaft in Frage gestellt (ALVENSLEBEN, 2002C).

\subsubsection{Risikokommunikation}

Die sachliche Information über Risikopotenziale, die Minimierung von Bewertungsdifferenzen und die Vermeidung von Konflikteskalationen bei Auseinandersetzungen über Risiken sind die Ziele einer Risikokommunikation (WiedemanN u. ClaUbeRG, 2003). Im Rahmen der Risikoanalyse erfolgt hierbei der interaktive Austausch von Informationen und Meinungen über Gefahren und Risiken, risikobezogene Faktoren und Risikowahrnehmung (EU VO 178/2002). Insgesamt ist die Risikokommunikation stark abhängig vom Kommunikationsziel, dem Kommunikator, dem Kommunikationsinhalt, dem Kommunikationskanal (Medium) und dem Kommunikanten (Zielperson/-gruppe). Weitere Ausführungen hierzu sind bei Alvensleben u. KAFKA (1999) zu finden.

Schwierigkeiten entstehen bei der Risikokommunikation, wenn die Beteiligten bei einzelnen Vorkommnissen (sachliche Sicht) von unterschiedlichen Risikokonzepten und -perspektiven ausgehen. Dieses tritt vor allem bei der unterschiedlichen Einstellung von Experten und Verbrauchern auf (vergl. Kapitel 2.1.3). Weitere Probleme können sich aufgrund von organisatorischen Problemen (Ressourcenmängel, mangelnde Absprachen) ergeben sowie aufgrund von Beziehungsproblemen (WIEDEMANN u. ClaUBERG, 2003). Das heißt: Der Kommunikator kann den Sachverhalt nicht entsprechend eindeutig oder glaubwürdig vermitteln oder er ist kein Sympathieträger (ALVEnSLEBEN u. KAFKA, 1999; WiEDEMANN u. CLAUberg, 2003). Die Glaubwürdigkeit des Kommunikators sinkt, je stärker diesem kommerzielle Antriebe unterstellt werden. Besonders schlecht schneiden in Bezug auf die Glaubwürdigkeit bei Lebensmitteln die Lebensmittelhersteller, die Politiker, die Medien und die landwirtschaftlichen Interessenvertreter ab. Positiv schneiden dagegen die Verbraucherverbände, 
Umweltschutzorganisationen, Ärzte, Familienmitglieder und Freunde ab (ALVENSLEBEN u. KAFKA, 1999).

In der Lebensmittelkette Fleisch gibt es große Defizite in der Risikokommunikation. Probleme werden in folgenden Punkten gesehen:

- Die Risikokommunikation ist besonders schwierig, wenn die Beteiligten von unterschiedlichen Risikokonzepten ausgehen (WIEDEMANN, 1999; RENN ET AL. 2000).

- Die Kommunikation baut auf einem Freund-Feind-Schema auf. Das eigene (soziale, ökonomische oder faktische) Überleben darf nicht durch die andere Seite bedroht werden (WIEDEMANN, 1999; RENN ET AL. 2000).

- Die Öffentlichkeit ist nicht damit zufrieden, am Ende des Prozesses im Umgang mit Risiken zu stehen (SANDMAN, 1993).

- Der Verbraucher erwartet bei Lebensmitteln ein „Null-Risiko“ (ALVENSLEBEN, 2002B; HENSEL, 2003).

- $\quad$ Aufgrund von Erkenntnislücken erfolgt die Kommunikation häufig mit moralischen und mit ideologischen Argumenten (WIEDEMANN, 1999).

- Vorliegende Interessendissense bedienen sich der Risikokommunikation, ohne sich jedoch auf Risikoargumente reduzieren zu lassen (WIEDEMANN, 1999).

Betrachtet man die BSE-Krise und den Nitrofenskandal wird ersichtlich, dass es schwierig war, dem Verbraucher die Größe und die Gefährlichkeit des Risikos in verständlicher Form zu erklären. Ein Null-Risiko bei Lebensmitteln gibt es aber nicht (ALVENSLEBEN, 2002B; HENSEL,2003). Wichtig ist, dass zwischen kleinen und großen Risiken unterschieden wird und die Einstufung für den Verbraucher nachvollziehbar und transparent dargestellt wird. Die Kommunikation sollte grundsätzlich so gestaltet sein, dass dem Verbraucher ein transparentes Bild der Risiken vermittelt wird, dass er in die Risikobewertung einbezogen wird und seine Stimme beim Risikomanagement berücksichtigt wird (WIEDEMANN u. CLAUBERG, 2003). Hierdurch sollen Eskalationen verhindert und Bewertungsdifferenzen minimiert werden (WIEDEMANN, 1999).

\subsection{Die Sicherheit der Fleischproduktion: Initiativen der Wirtschaft}

Die Fleischwirtschaft ist heute durch eine starke Umsetzung von Qualitätsprogrammen und/ oder Qualitätsmanagementsystemen geprägt. Die Qualitätsprogramme haben hierbei einen starken Fokus in der Produktqualität in Verbindung mit einer guten Prozessqualität. Qualitätsmanagementsysteme haben dagegen ihren Schwerpunkt in der Prozessverbesserung und der Prozesssicherheit. Die Produktqualität ist in diesen Programmen im Rahmen der Produktsicherheit (z.B. mikrobiologische Qualität) berücksichtigt. Die sensorische Qualität des Fleisches spielt hier keine oder nur eine sehr untergeordnete Rolle.

\subsubsection{Qualitätsfleischprogramme}

Seit Anfang der 80er Jahre gibt es in der Fleischwirtschaft Bestrebungen, Produkte über zusätzliche Informationen am Markt abzugrenzen. Hieraus haben sich verschiedene Qualitätsfleischprogramme mit unterschiedlichen Strategieansätzen entwickelt. Nach BRANSCHEID (1998) lassen sich hierbei zwei Typen unterscheiden:

- Programme, die eine stufenübergreifende Qualitätssicherung (vertikale Integration) gewährleisten, und 
- $\quad$ Programme, die besondere Haltungsverfahren (Ökologie, Tierschutz) herausstellen.

Im Besonderen unterscheiden sich beide Typen in den festgelegten Kontrollparametern. Bei den stufenübergreifenden Programmen sind sowohl Vorgaben für die Produkt- als auch für die Prozessqualität definiert. Bei Programmen, die besondere Haltungsverfahren herausstellen, erfolgt die Sicherung der Produkteigenschaften nur sekundär (BRANSCHEID, 1998).

Heute beinhalten die meisten Qualitätsprogramme eine Kombination aus definierten Parametern für die Produkt- und Prozessqualität. In einer Veröffentlichung der IFAV (2003) wurden in Europa für Schweine ca. 35, für Rind und Kalbfleisch ca. 60 und für Geflügel ca. 4 Markenfleischprogramme identifiziert. Im Rahmen vorliegender Arbeit soll nicht auf die einzelnen Programme eingegangen werden, sondern auf die in der Veröffentlichung der IFAV (2003) herangezogenen Bewertungskriterien. Die nachfolgend genannten Bewertungskriterien geben einen guten Überblick über die Forderungen in den Programmen, wobei natürlich nicht alle Forderungen für alle Programme gelten:

Herkunft der Tiere, Herkunft der Futtermittel, Zusammensetzung und ökologische Produktion des Futters, Tiermehle ${ }^{1}$, gentechnisch veränderte Futtermittel, Einsatz von Düngemitteln, Pestizide, Leistungsförderer und Tierarzneimittel, Haltungsvorschriften (z.B. Licht, Liegefläche, Bodenbeschaffenheit, Auslauf, Weidehaltung bzw. Stallhaltung), Transport, Schlachtung, Behandlung des Fleisches im Schlachthof, Kontrollen wie chemische, physikalische und mikrobiologische Messungen, Geschmack, Geruch und Vorschriften für den Verkauf und Kontrollen des Programms.

\subsubsection{Qualitätsmanagementsysteme}

Auf den Stufen der Futtermittelproduktion und der Schlachtung gehören Qualitätsmanagementsysteme heute zum Standard. In die landwirtschaftliche Urproduktion halten solche Systeme zum Teil erst Einzug (Tabelle 2.3-1).

Der Schwerpunkt der Ausrichtung ist in allen Qualitätsmanagementsystemen gleich. Ein besonderes Gewicht liegt auf den Präventivmaßnahmen zur Vermeidung von Fehlern und der Initiierung von kontinuierlichen Verbesserungsprozessen. Die Sicherheit der erzeugten Produkte soll erhöht werden. Hierbei haben sich drei Ansätze herausgebildet, um Qualitätsmanagementsysteme in der Ernährungs- und Landwirtschaft zu etablieren (SCHRAMM u. SPILLER, 2003):

- Die Integration bzw. Kooperation von Unternehmen entlang der Wertschöpfungskette unter einer gemeinsamen Kontrollstruktur. Die Initiierung erfolgt meistens durch ein dominantes Unternehmen in der Wertschöpfungskette wie bei dem dänischen System DANISH (Danske Slagterier) und dem niederländischen IKB System. Ein weiteres Beispiel ist die Netzwerkbildung der deutschen Geflügelwirtschaft.

- Etablierung eines geeigneten institutionellen Rahmens durch die Entwicklung von kooperativen Zertifizierungsstandards (quality management metasystems). Das einzelne Unternehmen kann sich frei für eine Zertifizierung entscheiden. Hierbei kann es sich um eine branchenübergreifende (DIN EN ISO 9001-2000) oder um eine branchenspezifische (QS, BRC, IFS) Zertifizierung handeln.

1 Das Verfüttern von Tiermehlen ist seit 2000 in Deutschland verboten. 
- Der Staat erlässt übergreifende Zertifizierungsstandards. Hierbei werden häufig Eigenschaften abgesichert, bei denen sich der Konsument nicht selbst von der Richtigkeit der Qualitätsangaben überzeugen kann. Ein Musterbeispiel ist die EU-ÖkoZertifizierung.

Tabelle 2.3-1: $\quad$ Beispiele für Qualitätsmanagementsysteme und ihre Einsatzbereiche.

\begin{tabular}{lcccc}
\hline & Branchensp. Anforderungen & Landwirtschaft & Futtermittel & Schlachtung \\
\hline DIN EN ISO 9001-2000 & $\mathbf{X}$ & & $\mathbf{X}$ & $\mathbf{X}$ \\
DIN EN ISO 22000-2005 & $\mathbf{X}$ & $\mathbf{X}$ & $\mathbf{X}$ & $\mathbf{X}$ \\
GMP+ & $\mathbf{X}$ & & & $\mathbf{X}$ \\
BRC & $\mathbf{X}$ & & $\mathbf{X}$ & $\mathbf{X}$ \\
IFS & $\mathbf{X}$ & & $\mathbf{X}$ \\
DANISH & $\mathbf{X}$ & $\mathbf{X}$ & $\mathbf{X}$ \\
IKB & $\mathbf{X}$ & $\mathbf{X}$ & & \\
EUREP GAP & $\mathbf{X}$ & $\mathbf{X}$ & $\mathbf{X}$ & $\mathbf{X}$ \\
QS & & &
\end{tabular}

Durchgesetzt hat sich hierbei die Normenreihe der DIN EN ISO 9000. In vielen Branchen ist das Qualitätsaudit nach ISO 9001/2000 zum Marktstandard geworden. Die Nachteile dieses Standards sind die zum Teil geringe Eignung für Kleinbetriebe und die fehlenden Branchenspezifika (SCHRAMM u. SPILLER, 2003). Um die branchenspezifischen Defizite aufzufangen ist im November 2005 die DIN EN ISO 22000 als Zertifizierungsnorm für die Lebensmittelindustrie in Kraft gesetzt worden.

Neben den international gültigen DIN-Normen gibt es verschiedene sektorbezogene Ansätze, die sich am Markt etabliert haben. Dieses sind z.B. der Internationale Food Standard (IFS), der British Retail Consortium Global Standard (BRC) oder der GMP+ Standard für Futtermittel. Diese sektorbezogenen Ansätze benutzen als Grundlage für ihre Systeme die Normenreihe der DIN EN ISO 9000.

Nachteilig bei der Entwicklung der verschiedenen Qualitätsmanagementsysteme ist die zum Teil noch fehlende Anerkennung der Systeme untereinander. Dieses führt dazu, dass sich die Unternehmen häufig nach verschiedenen Standards zertifizieren lassen müssen. Neben einer Auditierung nach den offiziellen Standards werden die Unternehmen zusätzlich von ihren Kunden im Rahmen von Lieferantenaudits überprüft. Dieses hat dazu geführt, dass einzelne Unternehmen zum Teil über 50mal pro Jahr auditiert werden (KIEFER ET AL., 2003).

Ob Qualitätsmanagementsysteme einen großen Beitrag zur Schaffung von Verbraucherpräferenzen und zur Erhöhung der Verbraucherzufriedenheit leisten können, stellt ALVENSLEBEN (2001A) in Zweifel, da solche Systeme die eigentlichen Ursachen von Unzufriedenheit kaum beseitigen können. Diese Ursachen sind zumeist nicht objektive Qualitätsmängel, sondern häufig subjektive Wahrnehmungsverzerrungen. Qualitätsmanagementsysteme an sich bewirken noch keine bessere Prozessqualität. Um eine bessere Prozessqualität zu erhalten, müssen die Parameter identifiziert bzw. die Ausrichtung klar festgelegt werden, die für den jeweiligen Prozess von entscheidender Bedeutung sind. Das heißt, neben den eigentlichen Prozessparametern (Menge, Kosten, Ausschuss u.a.) müssen auch Parameter in die Systeme integriert werden, die nicht zur Erhöhung der Prozessleistung beitragen. Diese Parameter müssen das Potenzial besitzen das Vertrauen des Verbrauchers zu steigern. Beispiele sind Rückverfolgbarkeit, Hygiene, Produktsicherheit und Kennzeichnung. 


\subsection{Potentielle Risiken in der Fleischerzeugungskette}

In den nachfolgenden Kapiteln werden mögliche Risiken in der Fleischerzeugungskette, wie sie in der wissenschaftlichen Literatur dargestellt bzw. diskutiert werden, aufgezeigt. Um die Risiken im Rahmen vorliegender Arbeit greifbar darzustellen, werden Themenschwerpunkte gebildet. Hierdurch soll ein Überblick über mögliche Risiken bzw. Entwicklungen in der Fleischerzeugung gegeben werden. Eine umfassende und tief gehende Behandlung aller Themenbereiche kann nicht erfolgen, da ansonsten der Rahmen der Arbeit gesprengt werden würde. Die angesprochenen Themen geben aber einen allgemeinen Überblick über mögliche Risiken. Bei einigen der genannten Themenbereiche spielen ethische Belange teilweise eine ebenso große Rolle wie die realen Risiken. Soweit hierzu Angaben in der wissenschaftlichen Literatur zu finden sind, werden ethische Belange in den nachfolgenden Kapiteln berücksichtigt. Dies hängt mit dem dieser Arbeit zu Grunde liegenden Risiko-Begriff zusammen, der sowohl reale Risiken als auch das Meideverhalten der Verbraucher umfasst.

\subsubsection{Darstellung der Fleischerzeugungskette}

Wie das Beispiel Rindfleisch zeigt, ist die Produktionskette der Fleischerzeugung sehr stark verzahnt. Dadurch wird die Kette sehr unübersichtlich und einzelne Wege sind nur schwer nachzuvollziehen (Abbildung 2.4-1). Innerhalb der einzelnen Tierarten Rind, Schwein und Geflügel sind je nach Integrationsgrad die Ketten unterschiedlich verzweigt. Am unübersichtlichsten ist die Produktionskette beim Rind und am übersichtlichsten beim Geflügel.

Die Verzahnungen in der Mischfutterproduktion sind für alle Tierarten größtenteils gleich. Diese zeichnen sich aus durch:

- $\quad$ eine Vielzahl von Ackerbaubetrieben, die Futtermittelausgangserzeugnisse (z.B. Getreide, Ölfrüchte, Leguminosen) produzieren,

- den mehrfachen Verkauf bzw. Handel ein und desselben Futtermittelausgangserzeugnisses bzw. Futtermittels,

- Importe,

- $\quad$ zum Teil relativ große Vermarktungsgebiete der Futtermühlen,

- die Produktion, den Einsatz und den Handel von Grundfuttermitteln (Gras, Grassilage, Maissilage) in der Rindfleischproduktion.

In der Rindfleischerzeugung verläuft die Kette ähnlich wie bei den Futtermitteln. Hier zeichnet sich die Kette aus durch:

- $\quad$ viele Milchviehhalter, aber auch Züchter von Fleischrindern, die meist in sehr kleinen Betrieben eine Vielzahl an Kälbern erzeugen,

- $\quad$ eigene Mast, Verkauf bzw. Handel der Kälber an Zwischenhändler, Erzeugergemeinschaften oder andere landwirtschaftliche Betriebe,

- das Fehlen von geschlossenen Systemen in der konventionellen Rindermast,

- Verkauf bzw. Handel der Masttiere an Zwischenhändler, Erzeugergemeinschaften und in seltenen Fällen direkt an den Schlachthof,

- Abnahmeverträge zwischen Erzeugergemeinschaften und Schlachthöfen, die zunehmend an Bedeutung gewinnen. 


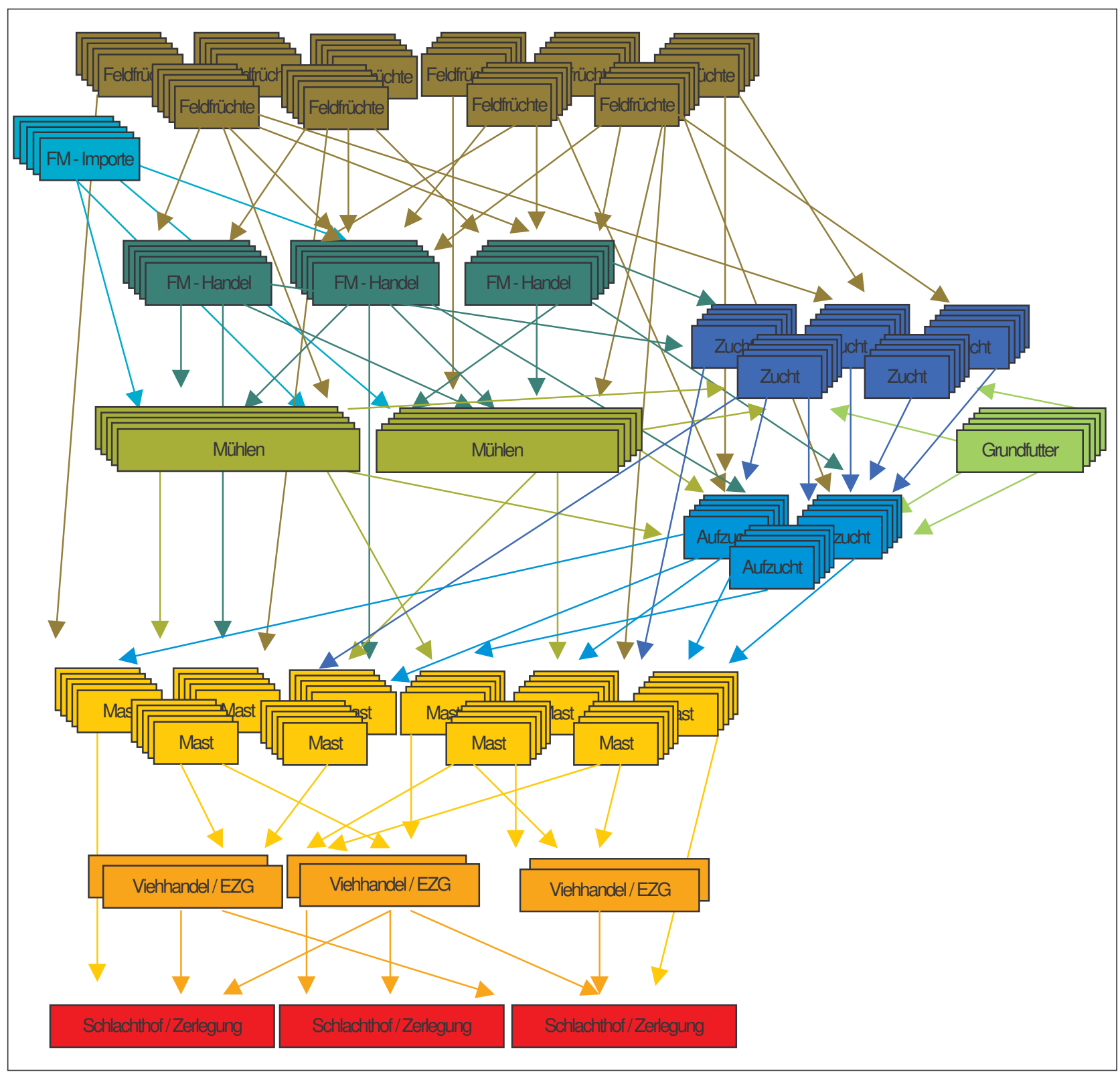

Abbildung 2.4-1: Produktionskette Rindfleisch.

Betrachtet man hierzu im Vergleich die Produktionsketten Schweinefleisch und Geflügelfleisch, stellt sich die Situation ähnlich dar wie bei der Rindfleischproduktion:

- Die Futtermittelproduktion ist bis auf den Einsatz von Grundfuttermitteln genauso kompliziert und vielschichtig wie bei den Rindern.

- $\quad$ Die Bereiche der Tierhaltung und Schlachtung sind straffer organisiert.

- Geflügelfleisch wird fast ausschließlich über organisierte Ketten, die von der Zucht (wenige international tätige und spezialisierte Zuchtunternehmen), der Elterntiererzeugung bis zur Schlachtung vertraglich abgesichert sind, produziert (PINGEL ET AL., 1998).

- In der Schweinefleischerzeugung werden nur bestimmte Zucht- bzw. Kreuzungslinien eingesetzt.

- Der Zukauf der Mastferkel und der Verkauf der fertigen Mastschweine erfolgt meist willkürlich über den Preis. 
- Vertragliche Verbindungen mit Erzeugern und/oder Schlachthöfen sind eher selten. Ähnlich wie bei den Rindern gewinnen Abnahmeverträge zwischen Erzeugergemeinschaften und Schlachthöfen zunehmend an Bedeutung.

\subsubsection{Zucht}

Die Anforderungen des Marktes zwingen die moderne Landwirtschaft wenige, besonders leistungsstarke Nutztierrassen einzusetzten (GEH, 2001). Vielfach kann der Landwirt nur so den ökonomischen Zwängen und den Anforderungen der Fleischwarenindustrie gerecht werden. Aus den Schlachttieren sollen möglichst einheitliche Teilstücke gewonnen werden, die sich standardisiert verarbeiten lassen und somit niedrige Kosten hervorrufen. (vergl. GenRES, 2003; MATHES u. IDEL, 2004). Aber auch der Verbraucher hat heute andere Ansprüche an das Fleisch als noch vor 60 Jahren. Heute wird Fleisch als günstiges Massenprodukt angesehen, dass, im Gegensatz zu früher, möglichst mager sein soll.

Beide Entwicklungen haben über die Jahre zu einer Änderung der Zuchtziele von Masttieren in Richtung mageren, fleischreichen Tieren geführt. Hierbei wurde der Konstitution und der Fleischqualität der Masttiere zu wenig Aufmerksamkeit geschenkt. Beide Aspekte sind erst in den letzten Jahren wieder in das Interesse der Zucht gerückt.

Als Folgen können in der Zucht von Tieren drei Risikoaspekte identifiziert werden, die sich ggf. auf den Fleischkonsum auswirken:

- Mängel in der Produktqualität,

- $\quad$ ethische Risiken durch extreme Ausprägung von bestimmten Fleischteilen,

- Aussterben von Rassen.

\subsubsection{Mängel in der Produktqualität}

Mängel in der Produktqualität sind heute mit hohen finanziellen Einbußen verbunden. Dieses hat dazu geführt, dass die Zucht heute wieder verstärkt den Aspekt der Produktqualität berücksichtigt. Denn neben dem Schlachtertrag, der Schlachtkörperqualität hat die genetische Konstruktion des Tiermaterials auch einen erheblichen Einfluss auf die Fleischqualität (ENDER u. AUGUSTINI, 1998). Der Einfluss der Zucht ist am stärksten beim Rind und am geringsten beim Geflügel (KüNZI u. STANZINGER, 1993). Weitere Einflüsse gehen von der Fütterung, der Haltung, dem Alter und dem Geschlecht aus (SCHEEDER, 1992). Aufgrund des Merkmalsantagonismus zwischen zunehmendem Magerfleischanteil und zunehmender Fleischqualität (GLODEK, 1988; WICKE ET AL. 1991) erfolgt eine Verbesserung der Produktqualität häufig zu Lasten des Schlachtertrags und der Muskelausprägung.

\subsection{PSE-Fleisch}

Bei Schweinen und beim Geflügel tritt häufig ein Qualitätsmangel durch ein sehr schlechtes Wasserbindevermögen auf (PINGEL ET AL., 1998). Bei beiden Tierarten ist dieser Qualitätsmangel genetisch bedingt. Auch bei Rindern ist das Vorkommen von PSE-Fleisch ebenfalls bekannt. Allerdings ist noch unklar, ob eine der Ursachen eine genetisch bedingte Disposition ist. Vermutet wird allerdings, dass das Auftreten von PSE beim Rind mit der Muskelfülle zusammenhängt (FISCHER, 1987).

Bei Schweinen wird PSE-Fleisch durch eine erbliche Prädisposition, eine Fehlregulation im Stoffwechsel des Muskels sowie eine erhöhte Anfälligkeit des Kreislauf- und Nervensystems 
bedingt. In Stresssituationen kommt es bei anfälligen Tieren zu einem überstürzten Glykogenabbau und es entsteht PSE-Fleisch (pale, soft, exudative) (V. LENGERKEN, 1998). Der Anteil an Schweinen, die nach der Schlachtung einen Qualitätsmangel in Form von PSE aufweisen, liegt in Deutschland nach Untersuchungen von ALTMANN ET AL. (2005) zwischen 1,2-8,8 \%. In den Jahren 1996 bis 2003 ist in den USA der Anteil an PSE bei Schlachtschweinen von 10,2\% auf 15,5\% gestiegen (SCANGA ET AL., 2003).

In den letzen Jahren versucht man durch eine Selektion der Mutterlinien auf das Defektallel (MHS-Gen) den PSE-Anteil zu reduzieren. Aufgrund der erwünschten Fleischleistung erfolgt die Sanierung der Linien in erster Linie bei den Sauen. In Hinblick auf die Fleischqualität ist der Erfolg allerdings nur begrenzt. Um den Prozentsatz der PSE-Schweine weiter zu senken, ist auch eine Reduzierung des MHS-Gens in den Vaterrassen notwendig (V. LENGERKEN ET AL., 1998; WICKE ET. AL., 2001).

Bei Geflügel wird vermutet, dass die Abnahme der Fleischqualität durch Muskelfaserdefekt und Veränderungen der Muskelmorphologie hervorgerufen wird. Die Geschwindigkeit des pH-Wert-Abfalls sowie der End-pH-Wert bei Hähnchen ist signifikant von der Genetik beeinflusst. Bei Puten ist der Einfluss nicht festgestellt worden. Bei diesen Tieren wurde ein genetischer Einfluss zwischen frühpostmortalem pH-Wert und der Fleischhelligkeit festgestellt. Der Brustmuskel der Puten besteht fast ausschließlich aus weißen Muskelfasern und besitzt eine gewisse Disposition für niedrige $\mathrm{pH}$-Werte und erhöhte Leitfähigkeitswerte. Die Grundursache liegt in den im Sinne der Muskelhypertrophie vergrößerten Muskelfaserdurchmessern und in den daraus resultierenden Problemen mit dem Zellstoffwechsel (PINGel ET AL., 1998; WiCKE ET AL., 2001; VelLemANN ET AL., 2003; Bihan-Duval, 2004; BRANSCHEID ET AL., 2004).

Bei Puten ist der züchterische Antagonismus zwischen Fleischqualität und Fleischansatz nicht mehr rückgängig zu machen. Qualitätsabweichungen müssen hier bis zu einem gewissen Grad akzeptiert werden. Die wichtigste Lösung zur Verbesserung der Situation ist, die physiologischen Belastungen (z.B. Nüchterungszeiten, Verladen, Transport, Ausladen, Einhängen) der Tiere vor der Schlachtung zu minimieren (BRANSCHEID ET AL., 2004).

\subsection{DFD bzw. DCB Fleisch}

Als weitere Qualitätsmängel treten beim Rind DCB-Fleisch (dark, cutting beef) und beim Schwein DFD-Fleisch (dark, firm dry) auf. DCB-Fleisch bzw. DFD-Fleisch treten in erster Linie aufgrund von Handhabungsfehlern im Umgang mit den Tieren auf. Bei Tieren, die vor dem Töten einer längeren Belastung ausgesetzt werden, kommt es zu einer Glykogenverarmung der Muskulatur. Die Folge ist eine unzureichende $\mathrm{pH}$-Wertabsenkung im Fleischmuskel. Dieses führt zu einer geschlossenen Mikrostruktur mit verringerter Lichtstreuung (dunkler Farbe) und mit hoher Wasserbindung (FISCHER, 1994, 1997; V. LENGERKEN ET AL., 1998). Ebenso ist das Auftreten von DCB-Fleisch beim Rind geschlechts- und rassespezifisch unterschiedlich. Der Anteil bei Bullen (11\%-5\%), die den Fleischqualitätsmangel DFD aufweisen, liegt wesentlich höher als bei Kühen, Färsen oder Ochsen (5\%-1\%). Die rassespezifischen Unterschiede lassen eine genetische Disposition vermuten. Ererbtes Stoffwechselverhalten oder ein unterschiedliches, häufig rassespezifisches Temperament der Tiere kann die zum DCB-Fleisch führenden Vorgänge begünstigen bzw. erschweren (FISCHER, 1987). 


\subsection{Sensorische Defizite}

Sensorische Defizite im Fleisch werden am Beispiel Schweinefleisch dargestellt.

Der Fettgehalt in Fleisch beeinflusst maßgeblich die sensorische Qualität. Die starke Entwicklung in der Zucht zu mageren, fleischreichen Tieren in der Fleischproduktion hat zu einer starken Reduzierung des Fettgehaltes im Fleisch und zu sensorischen Einbußen beim Genusswert von Fleisch geführt (WICKE ET AL., 2001).

Der in der Schweinemast stetige Anstieg der Schlachtgewichte, bei einem erhöhten Proteinansatz und einem geringeren Fettansatz, hat bei den meisten Gebrauchskreuzungen eine erhebliche Verringerung des intramuskulären Fettgehaltes auf bis zu $<1,5 \%$ zur Folge. Aufgrund der sensorischen Einbußen, die durch den niedrigen intramuskulären Fettgehalt bedingt sind, werden heute in der Zucht intramuskuläre Fettgehalte von mindestens $2 \%$ angestrebt. Erreicht werden diese Werte zurzeit nur von der Rasse Duroc. Duroc-Schweine werden in der Zucht sowohl zur Verbesserung der Mutterrassen als auch als Endstufeneber in der Hybridschweineerzeugung eingesetzt (z.B. Dänemark), (V. LENGERKEN ET AL., 1998; WICKE ET AL., 2001). Allerdings sieht BRANSCHEID (2004) aufgrund der Entwicklung zu Convenienceprodukten und der dadurch zunehmenden Bedeutung der Verarbeitungseigenschaften, dass die Steigerung des intramuskulären Fettgehalts in Zukunft keine praktische Bedeutung mehr erlangt. Eine Steigerung des intramuskulären Fettgehalts auf 2,5\% wird es nach seiner Meinung in Zukunft allenfalls bei Nischenprodukten geben.

Ein weiter wichtiger Qualitätsmangel in der Schweinefleischproduktion ist der Ebergeruch im Fleisch. Ebergeruch wird von vielen deutschen Verbrauchern als unangenehm und abstoBend empfunden. Er wird vor allem von den Stoffen Skatol und Androstenon hervorgerufen, die sich vor allem im Fleisch von unkastrierten männlichen Tieren finden lassen (LUY $u$. HILDEBRAND, 2003). Der Ebergeruch wird als urinartig, nach Schweiß oder Fäkalien riechend beschrieben (EFSA, 2004). Im Auftreten (regionale Häufigkeit) von Ebergeruch und in der Sensibilität des Konsumenten gegenüber dem Geruch bestehen erhebliche Unterschiede (BAUMGARTNER ET AL., 2004; EFSA, 2004). Zum Beispiel wird in den Ländern Deutschland und Österreich Fleisch mit Ebergeruch nicht vom Verbraucher akzeptiert. Dagegen gibt es in Großbritannien kaum Unterschiede in der Bewertung von Eberfleisch und dem Fleisch von Kastraten. In den Ländern Spanien und Portugal ist die Ebermast ebenfalls üblich (vergl. EFSA, 2004). Untersuchungen zeigen, dass in Spanien 38\% aller Schweine-Schlachtkörper hohe Androstenon-Konzentrationen, 26\% hohe Skatol-Konzentrationen und 17\% sowohl hohe Androstenon- als auch Skatol-Konzentrationen aufweisen (KÜHNE, 2003). Aus diesen Ausführungen wird ersichtlich, dass eine Akzeptanz von Eberfleisch sehr stark von den Essgewohnheiten in den einzelnen Ländern abhängig ist.

\subsubsection{Ethische Risiken durch einseitige Zucht}

Zur Darstellung der Problematik extremer Ausprägung von einzelnen Körperteilen wird in dieser Arbeit der Begriff „Qualzucht“ gewählt. Diesen Begriff gibt es zurzeit nur in der Zucht von Heimtieren. Qualzucht im Sinne des Paragraphen 11b des Tierschutzgesetzes ist die durch Zucht gezielt geförderte oder geduldete Ausprägung von Form-, Farb-, Leistungs- und Verhaltensmerkmalen, die zu Minderleistungen bezüglich Selbstaufbau, Selbsterhaltung und Fortpflanzung führen und sich in züchtungsbedingten und physiologischen Schäden oder Verhaltensstörungen äußern (BMVEL, 1999). 
Durch die Entwicklung, viel Fleisch, und zwar von den wertvollen Teilstücken, zu extrem günstigen Preisen zu produzieren, wurden immer mehr Tiere mit einem hohen Fleischanteil gezüchtet. Hierbei wird vor allem darauf geachtet, dass der sogenannte Massezuwachs an den wertvollen Teilstücken erfolgt. Dieses hat zur Folge, dass bei bestimmten Tieren extreme Ausprägungen bestimmter Teilstücke zu beobachten sind. Als Beispiele sind bei den Puten der enorme Brustmuskelanteil, beim Schwein eine extreme Schinkenausbildung und beim Rind, z.B. bei dem weiß-blauen Belgier, die doppelte Lende zu nennen. Die Reinzucht von Piétrain-Tieren stößt ebenfalls auf starke ethische Bedenken (WICKE ET AL., 2001). Eine tierschutzrelevante Folge der Zucht auf hohe Fleischanteile ist, dass die weißblauen Belgier schon vor der Geburt so viel embryonale Muskelmasse an den Hinterschenkeln bilden, dass zur Vermeidung von Geburtsproblemen der Kaiserschnitt die Regel ist. Aber auch eine gestiegene Anfälligkeit für sozialen Stress und Leistungsstress, oder Fundamentprobleme, insbesondere bei Schweinen und Geflügel, sind Folgen einer auf hohe Leistungen zielende Zuchtform (BIZERAY ET AL., 2002; IDEL u. MATHES, 2004).

Nach WICKE ET AL. (2001) sind die Hochleistungstiere in der Regel kaum mehr in der Lage, Fehler in der Haltung (z.B. Fütterung, Einstreu, Klima oder Infektionsdruck) zu kompensieren.

\subsubsection{Aussterben von Rassen}

Weltweit sind in den vergangenen 100 Jahren 1.000 der anerkannten 6.400 Nutztier-Rassen ausgestorben, 300 davon während der letzten 30 Jahre. Die FAO warnt vor dem Aussterben von 2.000 weiteren, hoch bedrohten Rassen (BARTH ET AL., 2004; MATHES u. IDEL, 2004). Die Abnahme der Rassen und der genetischen Vielfalten werden z.B. gefördert durch:

- $\quad$ Fortpflanzungstechniken, die eine räumlich und zeitlich unabhängige weltweite Verbreitung von Erbgut ermöglichen (künstliche Besamung),

- den zum Teil überdurchschnittlichen Einsatz weniger „Spitzenvererber“,

- den Einsatz von Hybriden, die die gewünschten Eigenschaften optimal realisieren,

- $\quad$ der bedingten Unabhängigkeit der tierischen Produktion vom Standort durch den weltweiten Futtermittelhandel.

In Deutschland werden ca. 54 Rinderrassen gezüchtet, davon haben 10 Rassen eine wirtschaftliche Bedeutung. Nach Herbucheintragungen werden in der Rindermast vor allem die Rassen Limousin, Fleckvieh, Charolais, Angus, Galloway und Highland eingesetzt (BDF, 2003). Eine weitere Einschränkung der Rassenvielfalt beim Rind sieht BRANSCHEID (2004) nicht. Begründet wird dieses durch eine zu erwartende Öffnung des Milchmarktes in der EU und damit einer sinkenden Dominanz der Milch. Dieses kann zu einem Trend in Richtung Mutterkuhhaltung und damit zu einer Verwendung von mittelrahmigen Fleischrassen führen.

Beim Schwein werden ca. 16 Rassen durch Zuchtvereinigungen betreut, wovon 3 eine wirtschaftliche Bedeutung haben (GENRES, 2003). In der Schweinezucht haben 2002 die Rassen Deutsche Landrasse $(60,8 \%)$, Piétrain $(19,4 \%)$, Deutsches Edelschwein $(13,4 \%)$ und Large White (3,4\%) 97 Prozent Anteil an der Anzahl der ins Herdbuch eingetragenen Eber und Sauen (ZDS, 2003). Die Muttertiere in der europäischen Schweinemast basieren z.B. bis zu $66 \%$ auf Kreuzungen zwischen "Large White und Landrasse“ (ANONYMUS, 2004D). In Deutschland sind etwa $93 \%$ der verkauften Jungsauen Hybrid- oder Kreuzungstiere aus Zuchtunternehmungen oder Züchtervereinigungen (GENRES, 2003). Betrachtet man die Aus- 
gangsrassen in den Ausgangslinien zur Erstellung der Sauen- und Eberlinien in der Hybridzucht, findet man neben den oben genannten Rassen noch die Rassen Hampshire, Duroc und Leicoma (ZDS, 2003). Nach der Einschätzung der FAO bezüglich gefährdeter Nutztierrassen wurden die Rasse Duroc mit "gefährdet“ und die Rasse Hampshire mit "kritisch“ eingestuft (TGRDEU, 2005). Nach BRANSCHEID (2004) erfüllen die derzeit genutzten Genotypen ziemlich gut die Anforderungen des Marktes und der Verbraucher, und es wird in den nächsten 25 Jahren keine durchgreifenden Änderungen geben.

In der Geflügelproduktion spielen Rassen kaum noch eine Rolle. Die Rassen wurden durch kommerzielle Hybriden der Zuchtunternehmen verdrängt. Bei Legehennen wird z.B. der Markt von drei großen Zuchtunternehmen bestimmt (ANONYMUS, 2004D). In der Geflügelmast ist die Situation ähnlich.

\subsubsection{Gentechnologische Produktionsverfahren}

$\mathrm{Da}$ die Entwicklungen der Gentechnik in der Pflanzenproduktion wesentlich weiter fortgeschritten sind als in der Tierproduktion, wird der Fokus auf den Pflanzenbau gelegt.

Im Kompendium Gentechnologie UND LeBensmitTEL (2000) wird Gentechnik als eine Weiterentwicklung der seit Jahrtausenden bestehenden Züchtungs- und Produktionsverfahren angesehen. Die grüne Gentechnik und die klassische Züchtung haben das gleiche Ziel: die Optimierung der Nutzorganismen (JANY u. KIENER, 2000A, 2000B). Sie wird hierbei als ein Hilfsmittel für die klassische Züchtung betrachtet, kann diese aber noch nicht ersetzen (QAIM u. VIERCHOW, 1999). Durch die Gentechnik wird die Möglichkeit geschaffen Methoden einzusetzen, die das gesamte genetische Potenzial unabhängig von Kreuzungsbarrieren nutzbar machen.

Im Agrar- und Lebensmittelsektor werden die eingesetzten Methoden als grüne Gentechnik bezeichnet. Die Einsatzgebiete der grünen Gentechnik sind laut JANY u. KIENER (2000A):

- Pflanzen zur Lebensmittelgewinnung und nachwachsende Rohstoffe,

- transgene Tiere (Forschung steht am Anfang),

- Mikroorganismen als Starter- und Schutzkulturen für die Veredelung von Rohstoffen,

- Mikroorganismen zur Gewinnung von Hilfs- und Zusatzstoffen oder isolierte Produkte aus transgenen Pflanzen, z.B. Öle aus Soja.

\subsubsection{Einsatz und Verbreitung von gentechnischen Verfahren}

Der Anbau von gentechnisch veränderten Pflanzen nimmt weltweit rasant zu. 2002 wurden insgesamt 58,7 Mio. Hektar GVO-Pflanzen angebaut. 2003 waren es 67,7 Mio. Hektar und 2004 stieg die Anbaufläche auf 81 Mio. Hektar (JAMES, 2002, 2004; TRANSGEN, 2005A). Dieses entspricht einer Steigerung von ca. 15\% in 2003 und 20\% in 2004. Beim Anbau dominieren Soja, Mais, Raps und Baumwolle. Bei diesen Pflanzen stammen ca. 23\% (2002) der weltweit produzierten Mengen von GVO- Pflanzen. Ungefähr 96\% der GVO-Flächen befinden sich in den Ländern USA (47,6 Mill. ha), Argentinien (16,2 Mill. ha), Kanada (5,4 Mill. ha), Brasilien (5 Mill. ha) und China (3,7 Mill. ha) (JAMES, 2004). In Europa wird GVOMais in Spanien (25.000 ha, ca. 4-5\% der spanischen Maiserzeugung) und auf verschiedenen Versuchsfeldern u.a. in Deutschland (BROOKES, 2002) angebaut. In Rumänien wurden 2003 ca. 70.000 ha GVO-Sojabohnen angebaut (ANONYMUS, 2004B). 
Gentechnische Verfahren in der Tierproduktion befinden sich zurzeit noch im Versuchsstadion (z.B. Klonschaf Dolly). Ein kommerzieller Einsatz ist zurzeit noch nicht abzusehen. So weit erkennbar zählen neben anderen Faktoren züchterische Probleme etwa hinsichtlich der Stabilität der genetischen Veränderungen zu den Ursachen des Forschungs- und Entwicklungsrückstands (ANONYMUS, 2004B). Nach JANY (2000B) werden transgene Tiere in der Landwirtschaft voraussichtlich in den nächsten 20 Jahren keine Bedeutung in der Lebensmittelproduktion erlangen. Es wurden zwar verschiedene Projekte bezüglich der Veränderung von Eigenschaften durchgeführt (fettärmeres Fleisch, größere oder krankheitsresistente Tiere), die meisten dieser Projekte scheitern jedoch oder wurden aufgegeben (TRANSGEN, 2005B). Eine Ausnahme bildet die Fischzucht. In den USA liegt seit einiger Zeit ein Antrag zur kommerziellen Zucht von gentechnisch veränderten Lachsen vor, die deutlich schneller wachsen. Eine Genehmigung durch die Behörden steht noch aus. Zulassungen für die EU liegen nicht vor und sind in den nächsten Jahren nicht zu erwarten (TRANSGEN, 2005C).

In der Lebensmittelproduktion sind bereits aus gentechnisch veränderten (Mikro-) Organismen gebildete Substrate (Enzyme, Aromen usw.) im Einsatz. Gentechnisch veränderte (Mikro-) Organismen, z.B. als Starterkulturen bzw. zur Fermentation von Lebensmitteln, sind verfügbar oder befinden sich kurz vor der Marktfähigkeit. Eine Zulassung dieser Produkte erfolgte bisher nur vereinzelt und auf nationaler Ebene. In Deutschland ist eine solche Zulassung noch nicht erteilt worden (ANONYMUS, 2004B).

\subsubsection{Gentechnik und Verbraucher}

Vom Verbraucher werden gentechnische Verfahren eher kritisch gesehen und abgelehnt. Die Ängste der Verbraucher können in gesundheitliche, ökologische und diffuse Ängste eingeteilt werden (RIEDER u. ANWANDER PHAN-HUY, 1998).

Vor allem in den vom Überfluss geprägten Industrieländern ist für den Verbraucher der Nutzen der Gentechnik nicht ohne weiteres erkennbar (JANY u. KIENER, 2000B). In Deutschland haben 2002 52\% gentechnische Verfahren in der Lebensmittelproduktion abgelehnt (GASKELL ET AL., 2003). Die Ablehnung ist aber je nach Einsatzbereich unterschiedlich. Kritisch gesehen wird vor allem der Bereich der grünen Gentechnik (Agrar- und Lebensmittelsektor). Gentechnische Anwendungen in der Futtermittelproduktion und Tierzucht, die zur Verbesserung der Produktqualität, zur Ertragssteigerung bzw. zur Leistungssteigerung und zur Erzeugung von Resistenzen gegen Krankheitserreger und Herbizide eingesetzt werden, werden abgelehnt. Ebenso werden gentechnische Verfahren beim Tier (u.a. Klonen) abgelehnt. Akzeptiert werden am ehesten Anwendungen im Non-Food Bereich. Gentechnische Verfahren zur Herstellung von Arzneimitteln und Impfstoffen sowie der Einsatz der Gentechnik zur Heilung von Tierkrankheiten (unter strenger Berücksichtigung des Tierschutzes) werden dagegen eher von der Mehrheit der Verbraucher unterstützt. Dies gilt ebenso für gentechnisch veränderte Pflanzen zur Gewinnung von Energie und Rohstoffen (BECKER, 1999; Hampel, 1999; Hampel u. Renn, 1999; Hampel u. Pfenning, 2001; RenN u. Hampel, 2002; GASKELL ET AL., 2003).

In statistischen Erhebungen zur Akzeptanz von gentechnischen Verfahren ist ein positiver Trend zu beobachten. Nach einer Veröffentlichung des INSTITUTS FÜR DEMOSKOPIE IN ALLENSBACH (2002) waren 199825 \%, Anfang 200136 \% und Ende 200144 \% der Bevölkerung der Meinung, dass der Nutzen der Gentechnologie gegenüber den Risiken überwiegt. Dieser Trend lässt sich auch 2002 erkennen. Im europäischen Vergleich steht Deutschland 
bei der Akzeptanz von gentechnischen Anwendungen an viertletzter Stelle. Lediglich in den Ländern Dänemark, Großbritannien und Griechenland ist die Akzeptanz noch schlechter (GASKELL ET AL., 2003).

Noch drastischer ist die Einschätzung des Einsatzes von genveränderten bzw. geklonten Tieren. In Umfragen sind die Einschätzungen bzw. die Akzeptanz negativ. 88\% der Befragten befürworten einen Stopp der Forschung bezüglich geklonter Tiere (INSTITUTS FÜR DEMOSKOPIE ALLENSBACH, 2002). In einer anderen Studie werden genveränderte Nahrungsmittel als „nicht sinnvoll“, „risikobehaftet“ und „moralisch nicht akzeptabel“ bewertet. (GASKELL, 2003).

\subsubsection{Ziele, Vorteile und Risiken der Gentechnik}

Im Gegensatz zum Verbraucher sieht die Wissenschaft in der grünen Gentechnik kaum Gefahren (RENN u. HAMPEL, 2002). In der Tabelle 2.4-1 sind die Argumente für Nutzen/Vorteile und Risiken/Nachteile von gentechnischen Verfahren gegenübergestellt. Die Aspekte sind eingeteilt nach Wirkungen auf die Allgemeinheit und auf die Wirtschaft.

Durch den Einsatz von gentechnischen Verfahren in der Nutzpflanzenproduktion wird vor allem eine Steigerung der Rentabilität angestrebt. Hierdurch sollen die Produktionskosten gesenkt und die Erträge gesteigert werden. Die Kostenminimierung soll insbesondere durch einen geringeren Aufwand an Pestiziden und Herbiziden bewirkt werden (BODENMÜLLER, 2000; ANONYMUS, 2004B). Dass der Anbau von gentechnisch veränderten Pflanzen zu einer Reduzierung der eingesetzten Pflanzenschutzmittel führt, kann allerdings noch nicht eindeutig beurteilt werden. Pflanzenschutzmaßnahmen hängen von zu vielen Parametern (z.B. Klima, Schädlings- oder Wildkräuterbefall, Niederschlag, Bodenverhältnisse u.a.) ab. Bei gentechnisch veränderter Baumwolle und Soja hat man sowohl starke Einsparungen bei Pflanzenschutzmaßnahmen als auch erhöhte Einsatzmengen festgestellt (JANY u. KIENER, 2000A). Ebenso ist eine Steigerung der Rentabilität nicht unumstritten. Erträge von Ht-Raps und Ht-Mais konnten nicht signifikant gesteigert werden und laut einer sechsjährigen Anbaustudie in den USA ist der Anbau von Bt-Mais langfristig nicht wirtschaftlich (NISCHWITZ, 2004).

Erste praxisfähige Verfahren zur Verbesserung der Produktqualität gibt es heute schon, die haben noch keine wirtschaftliche Bedeutung erlangt. Durch gentechnische Verfahren bei Nutzpflanzen sollen Verbesserungen der Produktqualität/Inhaltsstoffe, z.B. durch die Erhöhung erwünschter und Verminderung unerwünschter Inhaltsstoffe, der Produktion neuer Inhaltsstoffe (gesünderer Fettsäuregehalt, Impfstoffe, Vitamine etc.) erreicht werden (FLACHOWSKY ET AL., 2000; ANONYMUS, 2004B). Auch sind im Anbau von Pflanzen unter nachteiligen klimatischen Bedingungen und auf Grenzstandorten Erfolge in der Forschung erzielt worden (ANONYMUS, 2004B).

Eine Grundvoraussetzung für das Inverkehrbringen von transgenen Pflanzen sowie Erzeugnissen der 1. Generation (Pflanzen mit Herbizidtoleranz bzw. einer Resistenz gegenüber Insekten, Viren, Pilzen, oder Bakterien) ist eine umfassende, langjährige Prüfung auf gesundheitliche Unbedenklichkeit. Mögliche gesundheitliche Gefährdungen bei zugelassenen transgenen Pflanzen wurden bislang nicht beobachtet (JANY U. KIENER 2000B). 


\begin{tabular}{|c|c|c|}
\hline & Chancen / Vorteile & Risiken / Nachteile \\
\hline $\begin{array}{l}\text { Allgemein- } \\
\text { heit }\end{array}$ & $\begin{array}{ll}\text { - } & \text { Entfernung von Allergenen } \\
\text { Wachstum auf Grenzstandorten } \\
\text { (karg, salzig, kalt) } \\
\text { schadstoffabbauende und tollerante } \\
\text { Pflanzen }\end{array}$ & $\begin{array}{ll}\text { - } & \text { Entstehung neuer Allergene } \\
\text { - } & \text { Wangelnde Transparenz/fehlende } \\
\text { - } & \text { Verbraucherempfinden (Unnatürlichkeit, } \\
& \text { Unsicherheit) } \\
\text { - } \quad \text { Entstehung neuer, unbeabsichtigter } \\
\text { toxischer Stoffwecheselprodukte } \\
\text { - } \quad \text { Missbrauch von Methoden } \\
\text { ethische Gründe }\end{array}$ \\
\hline Beides & $\begin{array}{ll}\text { - } & \text { pos. technol. Eigenschaften durch } \\
\text { - } & \text { Entlastung von Boden und Umwelt } \\
\text { weniger Pflanzenschutzmaßnahmen und } \\
\text { - } \begin{array}{l}\text { Düngung } \\
\text { - }\end{array} \text { - wedikamente aus Pflanzen Zusatzstoffe im Lebensmittel } \\
\text { - Ausschaltung toxischer oder hygenischer } \\
\text { Risiken im Produkt oder } \\
\text { Herstellungsverfahren } \\
\text { bessere sensorische Eigenschaften }\end{array}$ & $\begin{array}{l}\text { - Mögliche Auswirkungen auf die Umwelt } \\
\text { (Auskreuzungen, Auswildern, resistente } \\
\text { Insekten, Viren, Bakterien, Abnahme der } \\
\text { Agrobiodiversität) } \\
\text { - Antibiotikaresistenz }\end{array}$ \\
\hline $\begin{array}{l}\text { Wirtschaft- } \\
\text { lichkeit }\end{array}$ & $\begin{array}{ll}\text { - } & \text { Erhöhter Nährwert / Inhaltsstoffe } \\
\text { - } & \text { längere Haltbarkeit von Lebensmitteln } \\
& \text { höhe Rentabilität }\end{array}$ & $\begin{array}{l}\text { - } \quad \text { Abhängigkeit von einigen } \\
\text { Großkonzernen (Saatgut) } \\
\text { - Benachteiligung der Landwirtschaft in } \\
\text { der dritten Welt }\end{array}$ \\
\hline
\end{tabular}

Tabelle 2.4-1:

Nutzen/Vorteile und Risiken/Nachteile von gentechnischen Verfahren (LeHNER, 1999; QUAIM U. VIRCHOW, 1999; FLACHOWSKY ET AL., 2000; JANY, 2000A, 2000B; Kompendium GenteChNOLOGIE UND LebenSmitTEL, 2000; NISCHWITZ, 2004).

Nach der Meinung verschiedener Wissenschaftler gehören gentechnisch modifizierte Lebensmittel zu den mit am besten untersuchten Erzeugnissen (z.B. JANY u. KIENER, 2000B; ENGEL, 2002; JAMES, 2002; SCHIEMANN, 2002). Die auftretenden Risiken sind nicht höher oder andersartiger als bei konventionellen Erzeugnissen. Da es sich aber um eine neue Technologie handelt, ist trotzdem eine umfassende und sorgfältige Sicherheitsbewertung notwendig (JANY U. KIENER, 2000B). Risikoanalysen und Experimente haben gezeigt, dass transgene Pflanzen Risiken in sich bergen können (GAISSER ET AL. 2000). Zum Beispiel wird aus den USA von sogenannten „Superunkräutern“ berichtet, die gegen den Einsatz von Pestiziden resistent sind (NISCHWITZ, 2004). Es wird aber auch ein möglicher negativer Einfluss von genveränderten Pflanzen auf Insekten nicht ausgeschlossen. In 4 von 24 Studien sind negative Einflüsse festgestellt worden (BODENMÜLLER, 2000).

Der horizontale Gentransfer von Antibiotika-Resistenzgenen in transgenen Pflanzen auf Mikroorganismen ist ein in der Öffentlichkeit diskutiertes Risikoszenario. In der Verwendung von Antibiotika-Resistenzgenen (Kanamycin und Ampicillin) wird keine zusätzliche Gefährdung gesehen. Die menschliche Darmflora weist bereits zu 2-30\% eine Resistenz gegen die eingesetzten Antibiotika auf (JANY u. KIENER, 2000B). Ein Gentransfer von der transgenen Pflanze auf Bakterien oder andere Organismen wird zwar als extrem gering eingestuft, aber auch nicht ausgeschlossen (FLACHOWSKY ET AL., 2000; SCHIEMANN, 2002). Unter bestimmten Bedingungen kann transgene Pflanzen-DNA von Bodenbakterien aufgenommen und stabil in das Bakteriengenom eingebaut werden (SMALLA, 2003). Auf die Gefahren von Antibiotikaresistenzen wird ausführlicher in dem Kapitel 2.4.4.3 „Resistenzbildung“ eingegangen. 
Nach heutigem Wissenstand konnte eine Gefahr neuer Lebensmittelallergien nicht festgestellt werden. Genveränderte Pflanzen werden, hinsichtlich Allergien, als gut untersuchte und sichere Erzeugnisse dargestellt. Trotzdem kann das Auftreten von Allergien durch genveränderte-Enzyme, wie bei allen anderen klassisch gewonnenen Proteinen, grundsätzlich nicht ausgeschlossen werden. Enzyme, die traditionell in der Lebensmittelverarbeitung eingesetzt werden, bergen auch nach ihrer Gewinnung aus GVO kein neues allergenes Potenzial (JANY u. KIENER, 2000A, 2000B). Allerdings gibt es keine „echten“ wissenschaftlich abgesicherten Testverfahren oder zellphysiologische Systeme, die eine Vorhersage zum allergenen Potenzial eines Proteins ermöglichen JANY (2000A). Nicht auszuschließen ist das Auftreten von Lebensmittelallergien durch den vermehrten Einsatz von Enzymen, deren Einsatz vor der gentechnischen Gewinnung nicht rentabel war. (JANY u. KIENER, 2000A, 2000B).

Aufgrund der derzeitigen Wissenslage ist es nicht möglich, Risiken, die durch transgene Pflanzen entstehen können, vollständig festzustellen. Gegensätzliche Meinungen, wie mit diesen Risiken umgegangen wird, müssen sachlich und auf wissenschaftlicher Basis geführt werden. Bei der Risikoanalyse muss immer wieder überprüft werden, ob auch alle Aspekte, d.h. naturwissenschaftlich-technische wie auch ethisch-moralische, mit einbezogen worden sind (GAISSER ET AL., 2000). Dieses sind wichtige Grundsätze, die bei der Zulassung neuer gentechnisch veränderter Organismen eingehalten werden müssen, denn gentechnische Methoden sind aus der heutigen Lebensmittelproduktion nicht mehr wegzudenken. In der Zukunft wird der Einsatz eher noch zunehmen (vergl. KOMPENDIUM GENTECHNOLOGIE UND LEBENSMITTEL, 2000; ANONYMUS, 2004B).

\subsubsection{Zoonosen}

Jährlich erkranken ca. 30\% der Bevölkerung in Industrienationen an Lebensmittelinfektionen (WHO, 2000). Diese durch Mikroorganismen verursachten Lebensmittelinfektionen und intoxikationen stellen ein zentrales Problem für die öffentliche Gesundheit dar (GAREIS, 2000). Hierbei gehören die Zoonosen zu den größten gesundheitlichen Risiken, die von Lebensmitteln tierischer Herkunft ausgehen können (SINELL, 1998A; BAARS, 2003; FEHLHABER, 2003A). Die Anzahl der Zoonosen ist sehr vielfältig. Weltweit sind mehr als 50 Parasiten, knapp 40 Virusarten, ca. 25 Bakterienarten, ca. 15 Pilze und ca. 10 Clamydienund Rickettsienarten als Zoonoseerreger bekannt (HAMMER, 2001). Die meisten Fälle an Infektionen und Intoxikationen (ca. 90\%) werden aber durch weniger als zwei Dutzend Mikroorganismen hervorgerufen (WHO, 2000).

Nach FEHLHABER (2002) werden ca. 60\% der Zoonosenerkrankungsfälle durch kontaminierte Lebensmittel tierischen Ursprungs hervorgerufen. Veröffentlichungen von STEINBACH $u$. KROELL (1999) und KREUZER (2002) zufolge lag der Anteil bei 70\%. Hierbei spielen die Erkrankungen, die durch Bakterien hervorgerufen werden, die größte Rolle. Viruserkrankungen durch kontaminierte Lebensmittel sind dagegen eher gering. Nach Schätzungen liegt der Anteil bei ca. 2\% (MeTTEnLEITER, 1998). Erkrankungen, die durch Parasiten wie Trichinellen oder Toxoplasmen hervorgerufen werden, spielen in Deutschland heute eher eine untergeordnete Rolle.

Wie vorab beschrieben gibt es eine Vielzahl an Zoonoseerregern, auf die im Rahmen dieser Arbeit nicht im Einzelnen eingegangen werden kann. Ein Überblick über verschiedene Keime gibt die Tabelle 2.4-2. 
Bei den bakteriellen Enteritiserkrankungen (RKI, 2004) lagen 2003 die Erkrankungen durch Salmonellen, gefolgt von den durch Campylobacter, Yersinien, darmpathogene E-Coli und EHEC an erster Stelle. In anderen Ländern sieht die Reihenfolge zum Teil schon anders aus. In machen Regionen der USA und in Kanada ist die Yersinose inzwischen hinter der Salmonellose die zweithäufigste Ursache klinischer Enteritiden (NEUBAUER ET AL., 2001). Eine andere Keimgattung die sich stark verbreitet, sind die Campylobacter. In den Niederlanden, USA, und England treten mehr Erkrankungen durch Campylobacter als durch Salmonellen auf (DEFRA, 2002; KREUZER 2002; HEESCHEN, 2003). In den USA werden Salmonellen und Campylobacter für 2-4 Millionen der Lebensmittelinfektionen verantwortlich gemacht (OLAH ET AL., 2004). Keime, deren natürliches Vorkommen, Erkrankungsfälle und deren Bedeutung als Übertragungsweg Fleisch sind in Tabelle 2.4-2 dargestellt.

Die Haupteintragswege der Keime sind nicht ausreichend gegarte oder durch rohes Fleisch kontaminierte Lebensmittel. Zum Beispiel können alle verzehrfertigen Lebensmittel, die Salmonellen auch nur in geringer Menge enthalten, ein gesundheitliches Risiko für den Menschen darstellen (KREUZER, 2002). Die Enteritis-Salmonellose beim Menschen und EHEC-Infektionen treten weltweit vor allem in Ländern mit einer hochentwickelten Landwirtschaft auf (FRANK UND ALPERS, 2002). Bei Geflügel konnte in Großbetrieben bei $74,3 \%$ der Herden und in Kleinbetrieben bei 9,7\% der Herden eine Belastung von Salmonella Enteritidis nachgewiesen werden (ELLERBROEK ET AL., 1999).

Tabelle 2.4-2: Bedeutende Keime für lebensmittelbedingte Erkrankungen (MüLLER, 2002; HARTUNG, 2003; RKI, 2004).

\begin{tabular}{|c|c|c|c|}
\hline Keim & natürliches Vorkommen & $\begin{array}{l}\text { Erkrankung in } \\
2003\end{array}$ & $\begin{array}{l}\text { Bedeutung als } \\
\text { Fleisch- Risiko }\end{array}$ \\
\hline Salmonellen & $\begin{array}{l}\text { Geflügel } \\
\text { Rind } \\
\text { Schwein }\end{array}$ & 62.836 & hoch \\
\hline Campylobacter & $\begin{array}{l}\text { Darm von Mensch und Tier } \\
\text { (insbesondere Rind, Geflügel) }\end{array}$ & 47.566 & hoch \\
\hline Yersinien & $\begin{array}{l}\text { Schwein } \\
\text { Geflügel }\end{array}$ & 6.548 & hoch \\
\hline E-Coli & $\begin{array}{l}\text { Rind } \\
\text { Schwein } \\
\text { Geflügel } \\
\text { Mensch }\end{array}$ & 5.415 & hoch \\
\hline EHEC & Rind & 1.135 & hoch \\
\hline Rota-Viren & Mensch (fäkal oraler Infektionsweg) & 45.919 & niedrig \\
\hline Nowalk-like-Viren & Kalte Fleischwaren & 41.250 & niedrig \\
\hline
\end{tabular}

\subsubsection{Eintragswege von Zoonoseerregern in die Nahrungskette am Beispiel von Salmonellen}

Durch vielfältige Eintragsquellen in der Produktionskette Fleisch ist eine völlige Eliminierung von pathogenen Keimen schwer umzusetzen. Häufige Eintragsquellen in die Nahrungsmittelkette sind (GAREIS, 2000; KRIETER, 2001; HARTUNG, 2002; BAARS, 2003):

- Ausbreitung durch fäkal-orale Infektion,

- Zukauf von infizierten Tieren (z.T. ohne Symptome),

- Futtermittel, vor allem eiweißreiche Mehle tierischen Ursprungs,

- Fütterungstechnik (Flüssigfütterung), 
- weltweiter Handel mit Tieren und Futtermitteln,

- belebte Vektoren (Schadnager, Vögel, Mensch),

- unbelebte Vektoren (kontaminierte Stiefel, Kleidung, Gerätschaften),

- Transport zum Schlachthof,

- unzureichende Betriebshygiene (Reinigungs- und Desinfektionsmaßnahmen),

- $\quad$ veränderte Produktionsverfahren.

Mit Zoonoseerreger infizierte Futtermittel gelten als eine Infektionsquelle der Tiere bzw. der tierischen Erzeugnisse (SAULI, 2004). Im Rahmen der Programme zur Salmonellenreduzierung in Geflügelbeständen wurde die Bedeutung der Futtermittel als Infektionsquelle untersucht. Deren epidemiologische Bedeutung wird aber unterschiedlich gesehen. Da die derzeit bedeutsamen Salmonella-Serovare in der Humanmedizin nur in Ausnahmefällen im Tierfutter nachgewiesen werden können, warnen BISPING (1993) und DORN U. SCHWARZER (1991) vor einer Überschätzung der epidemiologischen Bedeutung von Futtermitteln. KLARMANN (2001) konnte in seinen Untersuchungen zum Nachweis von Salmonellen in Futtermitteln von 1997-1999 keinen direkten Zusammenhang zwischen dem Nachweis von Salmonellen in Futtermitteln und dem Auftreten von Erkrankungen in Tierbeständen (Allgemeininfektionen, Durchfallerkrankungen) feststellen. Dagegen sehen KöHLER (1993) und SCHLÜTER ET AL. (1992) in mit Salmonellen kontaminierten Futtermitteln eine der Hauptinfektionsquellen für Geflügelbestände.

Im Rahmen des Qualitätsmanagementprogramms GMP+ werden seit 1997 bei Geflügelfutter und seit 2002 bei Rinder- und Schweinefutter von der Productschap Diervoeder (PDV) Untersuchungen auf Salmonellen gefordert. So waren 2003 0,4\% der Geflügelfutterproben ( $n=6126), 0,7 \%$ der Rinderfutterproben $(n=1375)$ und $0,6 \%$ der Schweinefutterproben $(n=2857)$ positiv (PDV, 2004). PIETSCHMANN u. HAFEZ (2002) gehen sogar davon aus, dass zirka 1-3\% der eingesetzten Futtermittel bakteriell kontaminiert sind.

Aber nicht nur bei den verschiedenen Fertigfutterarten, sondern auch bei den Rohkomponenten sind starke Unterschiede in der Salmonellenkontamination festzustellen. Nach Angaben der PDV gelten als salmonellenkritisch Raps-, Soja- und Sonnenblumenextraktionsschrot, dampferhitzte Sojabohnen, Fischmehl und Weizenkleie. Insgesamt wurden von der PDV (2004) für das Jahr 2003 eine Salmonellenbelastung der Futtermittelausgangserzeugnisse von 3,6\% ( $n=14903$ Proben) festgestellt.

Aber auch die Rekontamination spielt beim Vorkommen von Salmonellen in Futtermitteln eine Rolle. Durch die bei der Herstellung der Futtermittelkomponenten angewandten Erhitzungsverfahren werden in der Regel die Salmonellen abgetötet. Die Rekontamination erfolgt während des Transportes, der Lagerung und der Verarbeitung zu Mischfutter unter Einwirkung von Staub, Nagetieren, Insekten und Menschen (BISPING, 1993).

In der Bekämpfung von pathogenen Keimen hat die Urproduktion eine Schlüsselstellung. Allerdings sind die Infektketten sehr unterschiedlich und schwer zu unterbrechen (SINELL, 1998A; FEHLHABER, 2003A). Die systematische Bekämpfung von pathogenen Keimen (Salmonellen, Campylobacter u.a.) ist zurzeit als unbefriedigend einzustufen. Am Beispiel der Salmonellen wird deutlich, dass es in den letzten Jahren keine nennenswerten Fortschritte bei der Verdrängung von pathogenen Keimen aus den Tierbeständen gegeben hat (FEHLHABER, 2003A; BAARS, 2003). 
Sowohl die konventionellen als auch die ökologischen Haltungssysteme bergen Probleme einer konsequenten Bekämpfung. Tiere in Haltungssystemen, bei denen Kontaktmöglichkeiten zur Außenwelt bestehen, sind z.B. stärker durch eingeschleppte Keime gefährdet (BAARS, 2003). Aber auch die konzentrierte Haltung vieler Tiere auf engem Raum begünstigt die Ausbreitung von pathogenen Keimen (KREUZER, 2002).

Große Probleme werden in den baulichen Unzulänglichkeiten zahlreicher Ställe gesehen (LÖHREN, 2004). Aufgrund von nicht optimalen baulichen Gegebenheiten wie Ritzen und (Dehnungs-) Fugen in Fußböden sowie Wänden und Luftschächten lassen sich Ställe vielfach nur schwer reinigen und desinfizieren. Ein weiterer Schwachpunkt bei der Salmonellenbekämpfung ist die häufig nicht ausreichende Schadnagerbekämpfung. Es hat sich aber auch gezeigt, dass eine vollständige Freiheit des Stalles und des Stallbiotopes von Salmonellen auch nach lehrbuchmäßiger Reinigung und Desinfektion nahezu unmöglich ist (LÖHREN, 2004).

Die Fütterungshygiene spielt ebenfalls bei der Reduzierung der Salmonellengefahr eine wichtige Rolle (MEYER, 2004). Gezielte Reinigungen und Desinfektionen der Futterbehälter und Fütterungsanlagen sind häufig allerdings nur schwierig durchführbar. In modernen Flüssigfutteranlagen (Schweinefütterung) ist eine Spülung mit desinfektionshaltigem Wasser möglich. Hier besteht jedoch das Risiko der Veränderung des Futtergeschmackes. Futtersilos, aber auch Transportwege für Trockenfutter und der Futtertrog müssen in sämtliche Maßnahmen mit einbezogen werden, da sich dort leicht bakterienhaltige Rückstände bilden (VAN DER WOLF ET AL. 1999).

Eine weitere Gefahr besteht während der prämortalen Phase. Bei Stress (z.B. während des Transportes zum Schlachthof oder im Wartestall) können Mikroorganismen die Darmschranke passieren und in die Organe und in das Muskelfleisch gelangen. Auf diese Weise können unerkannt Zoonoseerreger das Fleisch kontaminieren (LAMBOOIJ, 2000; FEHLHABER, 2003B; BAARS, 2003).

Ein Eintrag von Zoonoseerregern in den Stufen der Schlachtung, Zerlegung und weiterverarbeitenden Industrie kann aus heutiger Sicht nicht verhindert werden. Darum ist es wichtig, dass auf diesen Stufen eindeutige Konzepte vorhanden sind, die eine Kreuzkontamination reduzieren bzw. verhindern (vergl. SINELL, 1998A). Eine Verbreitung über tierunabhängige, indirekte Wege während Schlachtung, Verarbeitung, Handel und Zubereitung ist häufig zu beobachten. Weitere Ausführungen hierzu sind in Kapitel 2.4.10.1 zu finden. Um die Möglichkeiten der Kreuzkontamination auf dem tierunabhängigen, indirekten Weg zu minimieren, ist es aber auch notwendig den Primäreintrag möglichst gering zu halten. Denn bereits kleinste Einträge können zu dauerhaften Kontaminationen im Verarbeitungsbetrieb führen (HAMMER, 2001).

SAULI (2004) (Tabelle 2.4-3) hat den Sicherheitsstandard bezüglich des Eintrags von Zoonoseerregern in die Lebensmittelkette in der Schweiz bei den verschiedenen Tierarten ermittelt. So konnten große Unterschiede bei den Sicherheitsstandards entlang der Produktionskette festgestellt werden. Bei der Produktion von Geflügelfutter und punktuell bei der Herstellung von genussfertigen Lebensmitteln wurden weitreichende Maßnahmen beobachtet. Dagegen wies die Produktion von Rinder- und Schweinefleisch mit Ausnahme des Schlachtprozesses auf allen Stufen einen niedrigen Sicherheitsstandard auf. Mit zunehmendem Sicherheitsstandard wurde eine Abnahme der Prävalenz von pathogenen Keimen beobachtet. 
Tabelle 2.4-3: $\quad$ Verlässlichkeit der Bewertung und Prävalenz von Salmonellen (1998 2000) in jedem Produktionsschritt (SAULI, 2004).

\begin{tabular}{|c|c|c|c|c|c|}
\hline Produktionsschritt & Eier & Geflügel & Schwein & Rind & Milchprodukte \\
\hline $\begin{array}{l}\text { Futtermittel } \\
\text { Sicherheitsstandard } \\
\text { Verlässlichkeit } \\
\text { Prävalenz }\end{array}$ & $\begin{array}{l}\text { Sehr gut } \\
\text { Hoch } \\
1,8 \%{ }^{1}\end{array}$ & $\begin{array}{l}\text { Sehr gut } \\
\text { Hoch } \\
1,8 \%{ }^{1}\end{array}$ & $\begin{array}{l}\text { Niedrig } \\
\text { Mittel } \\
2 \%{ }^{2}\end{array}$ & $\begin{array}{l}\text { Niedrig } \\
\text { Mittel } \\
2 \%{ }^{2}\end{array}$ & $\begin{array}{l}\text { Niedrig } \\
\text { Mittel } \\
2 \%{ }^{2}\end{array}$ \\
\hline $\begin{array}{l}\text { Tierproduktion } \\
\text { Sicherheitsstandard } \\
\text { Verlässlichkeit } \\
\text { Prävalenz }\end{array}$ & $\begin{array}{l}\text { Gut } \\
\text { Hoch } \\
-\end{array}$ & $\begin{array}{l}\text { Mittel } \\
\text { Hoch } \\
-\end{array}$ & $\begin{array}{l}\text { Niedrig } \\
\text { Hoch } \\
\text { - }\end{array}$ & $\begin{array}{l}\text { Niedrig } \\
\text { Hoch } \\
\text { - }\end{array}$ & $\begin{array}{l}\text { Niedrig } \\
\text { Hoch } \\
\text { - }\end{array}$ \\
\hline $\begin{array}{l}\text { Schlachthof } \\
\text { Sicherheitsstandard } \\
\text { Verlässlichkeit } \\
\text { Prävalenz }\end{array}$ & & $\begin{array}{l}\text { Mittel } \\
\text { Hoch } \\
0,9 \%\end{array}$ & $\begin{array}{l}\text { Mittel } \\
\text { Hoch } \\
0,2 \%\end{array}$ & $\begin{array}{l}\text { Mittel } \\
\text { Hoch } \\
0,2 \%\end{array}$ & \\
\hline $\begin{array}{l}\text { Lebensmittel } \\
\text { Sicherheitsstandard } \\
\text { Verlässlichkeit } \\
\text { Prävalenz }\end{array}$ & $\begin{array}{l}\text { Gut } \\
\text { Hoch }^{7} / \text { Mittel }^{8} \\
2,8 \% \%^{7} / 0,40 \%^{8}\end{array}$ & $\begin{array}{l}\text { Gut }^{3} / \text { Sehr gut } \\
\text { Hoch } \\
5,4 \%\end{array}$ & $\begin{array}{l}\text { Niedrig }{ }^{3} / \text { Mittel }^{4} \\
\text { Mittel } \\
0,6 \%{ }^{10} / 0,1 \%{ }^{11}\end{array}$ & $\begin{array}{l}\text { Niedrig }^{3} / \text { Mittel }^{4} \\
\text { Mittel } \\
0,6 \%{ }^{10} / 0,1 \%{ }^{11}\end{array}$ & $\begin{array}{l}\text { Gut }^{5} / \text { Sehr gut } \\
\text { Hoch }^{9} / \text { Mittel }^{9} \\
0 \%{ }^{12} / 0,5 \%{ }^{13}\end{array}$ \\
\hline \multicolumn{6}{|c|}{$\begin{array}{l}\text { 1) Futtermittel für Legehennen oder Masthühner, 2) Futtermittel für Schweine und Rinder, 3) Frisches Fleisch, 4) Fleischerzeugnisse oder } \\
\text { gekochte Produkte, 5) Frische Produkte und Hart- und Halbhartkäse, 6) Milchpulver oder Säuglingsnahrung, 7) Konsumeier, 8) Eiprodukte, 9) } \\
\text { Frischprodukte und Hart- und Halbhartkäse sowie Milchpulver und Säuglingsnahrung, 10) Frisches Schweine- und Rindfleisch, 11) } \\
\text { Fleischerzeugnisse oder gekochte Produkte aus Schweine- oder Rindfleisch, 12) Milchpulver oder Säuglingsnahrung und Frischprodukte, 13) }\end{array}$} \\
\hline
\end{tabular}

\subsubsection{Beherrschbarkeit mikrobieller Risiken in der Fleischproduktion am Beispiel von Salmonellen}

Die Effektivität eines Programms gegen die Salmonelleninfektion ist stark davon abhängig, dass Maßnahmen in der gesamten Produktionskette, das heißt beginnend beim Futtermittelwerk über den Tierbestand, der verarbeitenden Industrie, dem Handel, und den Gaststätten bis hin zum Verbraucher, durchgeführt werden. Der Bekämpfungsschwerpunkt ist allerdings die Urproduktion. (GERIGK, 1992; BLAHA, 1993; FEHLHABER, 2003A).

Nach SMULDERS und UPMANN (2000) lassen sich die Einzelmaßnahmen zur Gewährleistung einer geringen bakteriellen Belastung in drei Kategorien einteilen:

- Präventivmaßnahmen bei der Produktion zur Kontaminationsverhütung,

- Verhinderung des bakteriellen Wachstums,

- Entfernung/Abtötung vorhandener Mikroorganismen.

In Deutschland stehen Maßnahmen bezüglich der Reduzierung eines Erregereintrages aus der Urproduktion erst am Anfang. Durch die EU-rechtlichen Bestimmungen (EU VO 2160/2003 und 178/2002) werden die Pflichten der Landwirtschaft zur Mitwirkung am Prozess der Gewährleistung der Lebensmittelsicherheit in den nächsten Jahren steigen (FEHLHABER, 2003A). Besonders schwierig macht die Bekämpfung, dass Tierbestände meistens als klinisch gesund erscheinen. Auch lassen sich bei der Fleischuntersuchung keine erkennbaren Veränderungen diagnostizieren. Auf diese Weise gelangen die Keime unweigerlich in die weitere Produktionskette. Der Keimstatus der einzelnen Tierbestände, die zur Schlachtung gelangen, ist zurzeit noch weitgehend unbekannt (SINELL, 1998A; FEHLHABER, 2003A). 
In Deutschland erfolgt eine Erhebung über den Salmonellenstatus der Schlachtschweine im Rahmen des QS-Programms (QS, 2005D). In den skandinavischen Ländern Schweden, Norwegen, Finnland und Dänemark werden Programme zur Reduzierung der Salmonellenbelastung in den Tierbeständen schon seit vielen Jahren erfolgreich umgesetzt. Zum Beispiel existiert in Schweden seit 1963 ein staatliches Programm zur Salmonellenbekämpfung und in Dänemark wurde 1995 der erste gemeinsame Salmonellenhandlungsplan eingeführt (KREITMEIR, 2004). In Deutschland wurden von 1996 bis 1998 Untersuchungen in ausgewählten Schlachthöfen Deutschlands zur Abschätzung des Salmonellenbefallgrades in den Mastschweinebeständen durchgeführt. Diese Untersuchungen haben einen Salmonellenbefall von etwa 5-10 \% der zur Schlachtung angelieferten Schweine ergeben. Außerdem sind etwa 30-60 \% der Schlachtschweine liefernden Bestände salmonellen-positiv (BLAHA, 2001).

Dass sich mit einer konsequenten Bekämpfung Erfolge in der Zoonosenbekämpfung erzielen lassen, zeigt das Beispiel der Salmonellenbekämpfung bei Schweinen in Dänemark und in den Niederlanden. Vor der Einführung der Salmonellenbekämpfung in diesen Ländern waren dort ca. 2/3 aller Betriebe mit Salmonellen kontaminiert (STEINBACH u. KROELL, 1999). Durch die Kontrolle der gesamten Erzeugerkette (Futtermittel, Ferkel, Mast, Schlachtung) konnte der Infektionsdruck gesenkt werden (FEHLHABER, 1998). Durch strikte Regularien müssen für infizierte Bestände Handlungspläne zwischen Produzent, Berater und Tierarzt innerhalb von 35 Tagen aufgestellt und ausgearbeitet werden, ansonsten drohen Geldbußen. Hierdurch konnte die Anzahl der Salmonellen positiven Fleischproben in Dänemark auf 0,8\% im Jahr 2000 gesenkt werden (KREITMEIR, 2004).

Erfolge sind auch in den Geflügelbeständen in Deutschland sichtbar. Hier haben vorgeschriebene Impfungen zu einer Absenkung der Salmonellenbelastung geführt (FEHLHABER, 1998). Aber auch die konsequente Umsetzung von Hygienemaßnahmen hat Erfolge gezeigt.

Die Grundprinzipien der Bekämpfung sind in allen Programmen ähnlich. Sie beruhen auf folgende Bekämpfungsmaßnahmen:

- All-in-all-out-Systeme,

- Optimierung der Betriebshygiene,

- Wechsel der Kleidung zwischen verschiedenen Ställen,

- Entmisten, Mistlagerung, Entsorgung,

- Desinfektion,

- Maßnahmen im Außenbereich,

- $\quad$ konsequente Schadnagerbekämpfung

- Impfungen

- $\quad$ Malus-Systeme (FEHLHABER, 1998; BLAHA, 2001; LÖHREN, 2004).

\subsubsection{Resistenzenbildung}

Durch die medikamentöse Bekämpfung von Zoonoseerregern in den Tierbeständen haben sich Resistenzen gegen antimikrobielle Wirkstoffe entwickelt (BFR, 2003A). Antibiotisch wirksame Stoffe sind Arzneimittel, die einen besonderen Einfluss auf die Beschaffenheit, den Zustand und die Funktion des Körpers ausüben (SANTARIUS, 2004). Zu den antibiotisch wirksamen Stoffen gehören z.B. Penicilline, Tetracycline, Sulfonamide und Chloramphenicol. 
Sie wurden in der Tiermast zur Vorbeugung gegen Infektionen und als Mittel zur Leistungssteigerung eingesetzt. Die eingesetzten antimikrobiologischen Mittel in der Tierhaltung sind teilweise dieselben oder ungefähr dieselben, die auch in der Humanmedizin verwendet werden (MCKELLAR, 1999; MüLLER, 2001; FAO ET AL., 2004). Seit dem 01.01.2006 ist der Einsatz antimikrobiologischer Mittel zur Leistungssteigerung verboten.

Die Ausbreitung von antibiotikaresistenten pathogenen Bakterien führt kontinuierlich zu einer Reduktion des anwendbaren Spektrums von Antibiotika und stellt zusehends eine Bedrohung für eine erfolgreiche Therapie in der Humanmedizin dar (MCKELLAR, 1999). Die Entwicklung von Resistenzen bei bakteriellen Infektionserregern gegen Antibiotika wird durch zwei Hauptkomponenten bestimmt: dem genetischen Potenzial zur Resistenzentwicklung und dem Selektionsdruck durch den Antibiotikaeinsatz (WITTE u. KLARE, 1999).

Resistente Infektionserreger gelangen durch den Übertragungsweg Lebensmittel oder direkt vom Tier zum Menschen (BFR, 2003A). Laut einer Veröffentlichung der FAO ET AL. (2004) werden die meisten Antibiotika resistenten Bakterien über kontaminierte Lebensmittel auf den Menschen übertragen. Ein anderer Übertragungsweg verläuft über den Kot der Tiere. Die resistenten Bakterien gelangen in den Boden und schließlich ins Grundwasser (MÜLLER, 2001). Inwieweit der Übertragungsweg über die Umwelt zurück zum Menschen ebenfalls zum Problem der Resistenzbildung bei Krankheitserregern beiträgt, ist noch nicht ausreichend untersucht worden, wird aber als wahrscheinlich angenommen (SZEWZYK ET AL., 2001).

Das Bundesinstitut für Risikobewertung (BfR) stellte in einem Forschungsprojekt fest, dass resistente Zoonoseerreger aus den Ställen über die Nahrung zum Menschen gelangen können (BFR, 2003B). OLAH ET AL. (2004) halten die resistenten Stämme für eine potentielle Quelle für Lebensmittelinfektionen beim Menschen. Nach BFR (2003B) konnten bei den aus Lebensmitteln und Tierbeständen isolierten resistenten Keimen weder im Hinblick auf die genetischen Eigenschaften noch in Bezug auf die Häufigkeit des Vorkommens der Resistenzen Unterschiede festgestellt werden.

Tabelle 2.4-4:

Wichtige Zoonoseerreger und deren Resistenzen auf bestimmte Stoffe (BFR, 2003B).

\begin{tabular}{ll}
\hline Erreger & Resistenzen \\
\hline Salmonellen & Fluorchinolonresistenz, Apramycin-Gentamicinresistenz, Multiresistenz \\
Campylobacter & Fluorchinolonresistenz, Makrolidresistenz, \\
E-Coli & Fluorchinolonresistenz, Apramycin-Gentamicinresistenz, \\
& Streptothricinresistenz \\
Enterokokken & Glycopeptidresistenz, Streptograminresistenz, Makrolidresistenz \\
\hline
\end{tabular}

Während beim Geflügel nur bei $24 \%$ der isolierten Salmonellen Mehrfachresistenzen nachgewiesen werden konnten, sind es beim Schwein ca. $77 \%$ der isolierten Salmonellen, die gegen 2-3 und mehr Antibiotika resistent sind (HAMMER, 2001). Insgesamt ist das Resistenzverhalten von Salmonellen-Isolaten verschiedener Herkünfte in den Jahren 2000-2002 (Tabelle 2.4-5) deutlich zurückgegangen, liegt aber insgesamt noch zu hoch (BFR, 2003C).

Zur Reduzierung der Gefährdung wird empfohlen (BFR, 2003B):

- Präventive Maßnahmen durch Verringerung des Einsatzes ohne vollständige Kenntnis der wissenschaftlichen Beweiskette, 
- Antibiotika der neueren Generation nur zur Einzeltierbehandlung und nur dann, wenn der Erreger gegen andere Antibiotika resistent ist, einzusetzen,

- Verringerung des Einsatzes antimikrobieller Substanzen durch Verbesserung der tierischen Produktion und der Haltungsbedingungen,

- Einschränkung des Einsatzes antimikrobieller Substanzen bei Lebensmittel liefernden Nutztieren.

Tabelle 2.4-5: Anteil der resistenten Salmonellen-Isolate verschiedener Herkünfte 2000-2002 (SCHROETER ET AL., 2002 gekürzt).

\begin{tabular}{llll}
\hline Herkunft & \multicolumn{2}{c}{ Gesamt resistent in \% der untersuchten Proben } \\
& $\mathbf{2 0 0 0}$ & $\mathbf{2 0 0 1}$ & $\mathbf{2 0 0 2}$ \\
\hline Tier & 77,5 & 72,0 & 48,0 \\
Rind & 83,8 & 90,3 & 63,6 \\
Schwein & 94,3 & 89,8 & 83,3 \\
Geflügel & 75,7 & 63,3 & 43,5 \\
LM & 81,1 & 63,3 & 50,0 \\
FM & 76,9 & 55,6 & 12,4 \\
Umwelt & 82,8 & 43,4 & 40,8 \\
Total & 78,9 & 65,9 & 45,2 \\
\hline
\end{tabular}

\subsubsection{Tierseuchen}

Eine wichtige Bedeutung bei der Ausbreitung der Tierseuchen haben Viren (KREISSLDöRFLER, 2002). In den letzten Jahren sind in Europa immer wieder Fälle der Schweinepest, der Maul- und Klauenseuche (MKS) und der Geflügelpest aufgetreten. Risiken durch Tierseuchen entstehen durch:

- $\quad$ eine extrem schnelle Übertragung der Erreger und der dadurch bedingten Erkrankung der Tiere mit Todesfolgen,

- hohe finanzielle Einbußen (Produktionsausfall, Handelsverbote),

- $\quad$ eine mögliche Übertragung auf den Menschen mit Krankheits- oder Todesfolgen,

- Konsumverzicht, aufgrund der Verunsicherung der Verbraucher,

- Konsumverzicht, aufgrund ethischer Ablehnung der Verbraucher von Massentötungen.

Das Hauptproblem der Tierseuchen ist das hohe pathogene Potenzial der Erreger. Die Viren werden von infizierten Tieren über die Atemluft ausgeschieden und können vom Wind verbreitet werden. Die Übertragung ist hierbei abhängig von Luftfeuchtigkeit, Temperatur, Windstärke und -richtung (MüLLER, 2001). Hierbei ist die Ausscheidung je nach Tierart unterschiedlich, z.B. scheiden infizierte Schweine durch Atemluft 1000 bis 3000 mal mehr MKS-Viren aus als ein infiziertes Rind (MÜLLER, 2001). Aber auch Übertragungen durch Schadnager, Insekten oder den Menschen sind nachgewiesen.

Die Übertragungsrisiken sind vielfältig und werden u.a. begünstigt durch (BGVV, 1998; ANONYMUS, 2002A; KREISSL-DÖRFLER, 2002):

- den zunehmenden globalen Handel mit Tieren und Nahrungsmitteln,

- einen Warenaustausch zwischen den Ländern, auf einem unterschiedlichen hygienischen Niveau und durch länderspezifische Erregerspektren, 
- den Handel mit Nachrungsmitteläbfällen,

- $\quad$ keine konsequente Meldung von Seuchenfällen in Drittländern,

- $\quad$ nicht ausreichende Grenzkontrollen bzw. begrenzte Mittel für Kontrollen/ Kontrolleinrichtungen,

- gemeinschaftliche Unzulänglichkeiten bei der Kennzeichnung und Rückverfolgbarkeit der Tiere,

- Anwendungsausnahmen der Richtlinie 72/462/EWG, insbesondere für Fleischtransporte geringer Mengen (weniger als $1 \mathrm{~kg}$ ) durch Reisende,

- die starke Zunahme des Nutztierbestands und der Besatzdichte in einigen Gebieten der EU.

Die staatliche Tierseuchenbekämpfung ist seit ca. 150 Jahren in Europa etabliert. Als weltweite Organisation hat das Internationale Tierseuchenamt (OIE, Office International des Epizooties) in Paris eine zentrale Rolle bei der Sicherung des Gesundheitsschutzes des Verbrauchers. Durch die Entwicklung gesundheitspolizeilicher Regeln und Normen für den internationalen Handel mit Tieren und mit Produkten tierischen Ursprungs werden durch das OIE wichtige Regelungen für die Gewährleistung der gesundheitlichen Sicherheit innerhalb des Welthandels aufgestellt. Diese Regelungen sind wichtige Grundsteine für die Bekämpfung von Tierseuchen.

Das OIE führt einen aktuellen Überblick über die weltweit bestehenden Tierseuchen und deren Ausbrüche in den verschiedenen Ländern (www.oie.int). Unterstützt wird das OIE durch länderspezifische Organisationen, in Deutschland durch die Bundesforschungsanstalt für Viruserkrankungen der Tiere (BFAV).

Die Vorbeugemaßnahmen, um eine Übertragung einzugrenzen, sind in den letzten Jahren ständig weiterentwickelt worden. Drittländer, aus denen Tiere bzw. Waren in die EU eingeführt werden, verfügen heute größtenteils über gut harmonisierte Kontrollmaßnahmen und über ein gutes Informationsnetz bei den Veterinär- und Gesundheitsdiensten. (ANONYMUS, 2002A). Innerhalb der EU sind Notfallpläne für das Verhalten und Maßnahmen im Fall von Seuchenausbrüchen vorhanden.

Besonders anfällig für Tierseuchen sind Gebiete mit hohen Bestandsdichten. Gerade aufgrund der Anfälligkeit dieser Gebiete gibt es Forderungen, die Bestandsdichten zu reduzieren (NORTHOFF, 1998). Die niederländische Regierung hat 1998 per Gesetz die Schweinebestände in den Niederlanden gesenkt. Hierzu wurde unter anderem die höchstzulässige Anzahl an Schweinen pro Betrieb („Schweinerechte“) festgeschrieben. Ebenso wurden Regelungen zum Ankauf von „Schweinerechten“ erlassen. Alle Bestimmungen haben zusammen zu einem etwa 25 \%igen Rückgang des Schweinebestands in den Niederlanden geführt (IKB, 2004B).

\subsubsection{Beispiele von Seuchenzügen}

Der Seuchenzug der Maul- und Klauenseuche in England hat zu noch nicht dagewesenen negativen Bildern in der Presse geführt. Die Maul- und Klauenseuche ist eine Viruserkrankung der Klauentiere (Wiederkäuer, Schweine). Das die Krankheit übertragende Virus ist hochinfektiös und außerordentlich leicht übertragbar (KREISSL-DÖRFLER, 2002). Es wird geschätzt, dass bereits 1-10 MKS-Virus-Partikel für die Infektion eines Rindes ausreichen (MüLLER, 2001). Für den Menschen gilt die Seuche allerdings als ungefährlich, Übertra- 
gungsfälle sind selten und verlaufen gutartig. Ebenfalls stellt auch der Verzehr von Fleischwaren verseuchter Tiere nach derzeitigem Wissensstand keine Gesundheitsgefährdung dar (ANONYMUS, 2002E).

Der letzte große Seuchenzug der Maul- und Klauenseuche in der Europäischen Union hatte 2001 in England ihren Ursprung. Hervorgerufen wurde der Seuchenzug durch die unrechtmäßige Einfuhr von verseuchtem Fleisch und eine nicht ordnungsgemäße Wiederverwertung von Küchenabfällen in der Tiernahrung (Schweine) (ANONYMUS, 2002E; KREISSL-DÖRFLER, 2002). Man vermutet, dass von einer infizierten Farm das Virus auf Schafe einer nahe gelegenen Farm übersprang und vermutlich über verschiedene Schafmärkte im ganzen Land weiter nach Frankreich und Irland übertragen wurde. Durch indirekte Kontakte mit infizierten Anlagen und durch eventuelle Tierkontakte wurde die Seuche auch in die Niederlande verschleppt (KREISSL-DÖRFLER, 2002).

Um dauerhaft der weltweiten MKS-Gefährdung entgegenzuwirken, ist ein geschlossenes System von Vorbeuge- und Bekämpfungsmaßnahmen innerhalb der EU unerlässlich. Die schnelle Verbreitung der MKS im Vereinigten Königreich und die Verschleppung in drei weitere Mitgliedstaaten der EU hat gezeigt, wie anfällig das gesamte System der Tierhaltung ist (BMVEL, 2001).

Insgesamt wurden, nach Schätzungen, im Rahmen der Seuchenbekämpfung 2001 bis zu 10 Millionen Tiere (Rinder, Schweine, Schafe, Ziegen u. Wildtiere) getötet (KREISSL-DÖRFLER, 2002). Sollte es aber zu einer gezielten Einschleppung (Tierhandel oder kriminelle Hintergründe) der MKS in die EU kommen, kann davon ausgegangen werden, dass die Ausbreitung der Seuche noch ganz andere Dimensionen erreicht (BMVEL, 2001).

Nachfolgende Maßnahmen werden vom BMVEL, 2001, zur Verhinderung der Einschleppung und Weiterverbreitung des MKS-Erregers empfohlen:

- $\quad$ einheitliche Maßnahmen an den Außengrenzen der EU zur wirksamen Kontrolle des Verbots der Einfuhr von relevanten Tieren und tierischen Erzeugnissen aus MKSRisiko-Ländern,

- restriktive einheitliche Maßnahmen an den Außengrenzen der EU bei MKS-Ausbrüchen in benachbarten Ländern,

- Regelungen zur Einschränkung der Anzahl und Dauer von Tiertransporten, zum Abbau von Anreizen für Tiertransporte, zur Vermeidung von Tierkontakten während des Transportes, zur Residenzpflicht für Klauentiere sowie zur Einzeltierkennzeichnung auch für Schafe und Ziegen.

Die Gefügelpest ist ebenfalls ein aktuelles Thema, das in der Presse diskutiert wird. Vor allem seit im Januar 2006 die ersten erkrankten Wildtiere in Deutschland gefunden wurden.

Die Geflügelpest, umgangssprachlich auch Vogelgrippe genannt, wird durch hochpathogene Vogel-Influenza-A-Viren vom Subtyp $\mathrm{H} 5$ oder $\mathrm{H} 7$ hervorgerufen. Alle anderen aviären Influenzaviren anderer Subtypen (es gibt insgesamt 15) sind gering pathogen und gelten nicht als Geflügelpest. Influenzaviren kommen ebenfalls beim Menschen und anderen Nutztierrassen vor. Nicht eindeutig geklärt ist zurzeit, ob und wie schnell die GeflügelgrippeViren mutieren können. Dass ein direkter Übergang auf den Menschen möglich ist, ist bekannt, aber bisher nur in wenigen Fällen vorgekommen, wobei es allerdings auch zu Tödesfällen gekommen ist (HEESCHEN, 2006). Eine direkte Übertragung des Influenzavirus von Mensch zu Mensch konnte nicht nachgewiesen werden. Sollte es zu einer Kreuzung 
eines normalen menschlichen Grippe-Virus mit dem Erreger der Geflügelpest sowie zu einer direkten Übertragung von Mensch zu Mensch kommen, kann dieses zu einer sogenannten Pandemie führen. Bei den Ausbrüchen der Geflügelpest 2004 und 2005 in Asien wurde von der EFSA (2005) keine direkte Gefahr für den Menschen gesehen. Ebenso wird keine Gefährdung durch den Verzehr von Geflügelfleisch oder Eiern gesehen. Es ist aber nicht ausgeschlossen, dass es unter Umständen dazu kommen kann. Allerdings führen Spekulationen über eine mögliche Übertragungsgefahr auf den Menschen und Bilder von Massentötungen von Geflügel zu einer Verunsicherung beim Verbraucher.

\subsubsection{Impfverbot}

Innerhalb der EU gilt ein Impfverbot bei Tierseuchen wie Maul- und Klauenseuche, Schweinepest und Geflügelpest, das sich an den handelspolitischen Empfehlungen des Internationalen Tierseuchenamtes (Office International des Epizooties - OIE) orientiert (KREISSL-DöRFLER, 2002). Nach dem Ausbruch der Maul- und Klauenseuche in Europa werden Regelungen bezüglich Notimpfungen neu diskutiert. Gerade die massenhafte Keulung der Tiere und die damit verbundenen Bilder haben Unmut ausgelöst. Auch ist es aus ethischen Gründen fraglich, ob Unmengen an Fleisch vernichtet werden müssen.

Ein Impfverbot ist ausgesprochen worden weil:

- durch den Impfstoff sich der Seuchenvirus weiter im Tier vermehren kann und nicht ausgeschlossen ist, dass es in geringen Maßen über den Kot ausgeschieden wird (HEESCHEN, 2004),

- in vereinzelten Fällen Impfdurchbrüche (MKS-Ausbruch in Folge unzureichend inaktivierter Erreger in der Vakzine) festgestellt wurden (BAUMGARTNER, 2001; ANONYMUS, 2002E),

- $\quad$ alle Serotypen nicht vollständig durch eine Impfung abgedeckt werden (KREISSLDÖRFLER, 2002),

- Länder, die nicht als erregerfrei gelten, starken Handelsbeschränkungen auf dem Weltmarkt unterliegen.

\subsubsection{Rückstände}

Rückstände und Kontaminanten werden immer wieder in tierischen Lebensmitteln gefunden. Hierbei kann es sich um Mykotoxine, Pestizide, Rückstände aus Tierarzneimitteln und Umweltkontaminanten wie Schwermetalle, Dioxine und andere Rückstände aus Verbrennungsprozessen handeln. Sie kommen in der Pflanzenproduktion wie in der Tierproduktion gleichermaßen vor, wobei unumstritten ist, dass viele Rückstände in der Tierproduktion ihre Ursprünge in der Pflanzenproduktion haben.

HONIKEL (1998) definiert Rückstände, Kontaminationen und Verunreinigungen wie folgt:

- Rückstände sind Schadstoffe, die vom Menschen verursacht werden und deren Anwesenheit im Lebensmittel billigend in Kauf genommen wird.

- Kontaminationen oder Verunreinigungen sind Schadstoffe, die unabsichtlich in Lebensmitteln auftreten.

- Rückstände sind immer vom Menschen verursacht (anthropogen). Kontaminationen können vom Menschen wie von der Natur ausgehen (geogen). 
Das FLEISCHHYGIENEGESETZ (2003 § 4) sieht als Rückstände Stoffe mit pharmakologischer Wirkung und deren Umwandlungsprodukte sowie Rückstände von anderen Stoffen, die in Lebensmittel übergehen und gesundheitlich bedenklich sein können.

Werden Rückstände und Kontaminanten in Lebensmitteln gefunden, erfolgt die anschlieBende Diskussion häufig auf einer sehr emotionalen und weniger auf einer wissenschaftlichen Basis (KROKER, 2003). Dies hat teilweise dazu geführt, dass Verbraucher die Gefahr durch Rückstände, Verunreinigungen und Zusatzstoffe als hoch bewerten. Experten sehen eher in der mikrobiologischen Gefährdung und in natürlich enthaltenen Toxinen bei Lebensmitteln ein hohes Risiko (BERGMANN, 2000). Bei den Diskussionen um Rückstände und Kontaminanten in Lebensmitteln ist es häufig eher nebensächlich, inwieweit es durch den Rückstand bzw. durch die Kontamination zu einer Gesundheitsgefahr kommt. Als Beispiele seien der MPA- (Medroxyprogesteron Acetat) und der Nitrofenskandal 2002 genannt. Eine akute Gesundheitsgefahr für den Verbraucher, trotz Überschreiten der Grenzwerte, bestand weder bei den Funden von MPA in Glukosesirup und Futtermitteln noch bei Nitrofen in Biogetreide (2002) (KUNDKE, 2003).

Bei der gesamten Diskussion über Rückstände und Kontaminanten muss beachtet werden, dass die Nachweisverfahren immer feiner werden. Heutige „Null-Werte“ können durch modernere Geräte morgen zu messbaren Größen und dadurch zu einer „potentiellen“ Gesundheitsgefahr werden. Wird in einem Lebensmittel ein Rückstand gefunden, ist dieses nicht gleichbedeutend mit einem Risiko. Nur das gesundheitsgefährdende Potenzial des Rückstandes ist maßgeblich, und es muss bei der Bewertung berücksichtigt werden, ob dieser Rückstand in einem Lebensmittel toleriert werden kann (POTTHAST, 2002; SANTARIUS, 2004).

Bei der Vielzahl an Rückständen ist es nicht immer einfach die richtigen Untersuchungen durchzuführen. Die durchgeführten Kontrollen müssen also so gestaltet werden, dass zum einen eine Kontrolle bezüglich der vorgeschriebenen Höchstmengen bzw. Grenzwerte bei Rückständen und Kontaminanten erfolgt. Zum anderen muss aber auch überwacht werden, dass keine verbotenen Substanzen im Bereich der Tier- und Pflanzenproduktion eingesetzt werden. Durch sogenannte Multimethoden (pro Untersuchung 100 Stoffe aus Pflanzenschutzmitteln) lassen sich Stoffe qualitativ und quantitativ nachweisen (KUNDKE, 2003). Neben der Auswahl des zu analysierenden Stoffes spielt bei der Überwachung von Rückständen auch die Probennahme eine Rolle. Die Schritte Probennahme, Lagerung, Probenvorbereitung und instrumentelle Analytik haben auf die Genauigkeit der Ergebnisse einen entscheidenden Einfluss (POTTHAST, 2002).

Bei vielen Umweltkontaminanten ist eine sichere Risikoabschätzung kaum möglich, da keine repräsentativen Untersuchungen existieren (TEUFEL ET AL., 2000).

Neben den erwarteten Kontaminationen, Rückständen bzw. Risiken kann es immer wieder zu unerwarteten Funden von Rückständen bzw. Risiken kommen, wie dies z.B. die schwedischen Acrylamidfunde (BGVV, 2002B) in frittierten, gebackenen und gerösteten Lebensmitteln mit einem hohen Kohlenhydratgehalt zeigen. Die schwedischen Acrylamidfunde zeigen, dass bei Lebensmitteln Stoffe zu einem Problem werden können, die man als solche dort nicht erwartet. Hier ist es wichtig, dass sich die Analytik schnell auf solche überraschende Befunde einstellt und geeignete Methoden zum qualitativen und quantitativen Nachweis findet (KUNDKE, 2003). Ebenso ist es wichtig sichere Schnellmethoden zu 
entwickeln, um in möglichst kurzer Zeit Ergebnisse von möglichst vielen Proben zu erhalten (KUNDKE, 2003).

\subsubsection{Carry over}

Unter Carry Over wird der Übergang eines Stoffes vom Futtermittel in das Muskelfleisch bzw. in die Organe und Produkte von Nutztieren bis zum Menschen verstanden. Diese Übergänge können beim Menschen zu Erkrankungen führen. Carry Over Vorgänge sind z.B. für Mykotoxine, Schwermetalle, Organochlorverbindungen, Futterzusatzstoffe und Hormone bekannt (KUiPer-GoodmanN, 1999; Weidenbörner, 1999, EU, 2000A, Petersen, 2001; SchenkEL, 2001, SCHWIND, 2004). In der Abbildung 2.4-2 sind die Carry Over Vorgänge dargestellt.

Insgesamt ist die Belastung von Fleisch durch Rückstände und Kontaminanten in den letzten Jahren klar rückläufig (SCHWIND, 2004). Hierzu haben Maßnahmen zur Beschränkung der Einträge in die Umwelt (Regelungen bezüglich Düngung, Pflanzenschutz, Abfall, Emissionen u.a.) geführt.

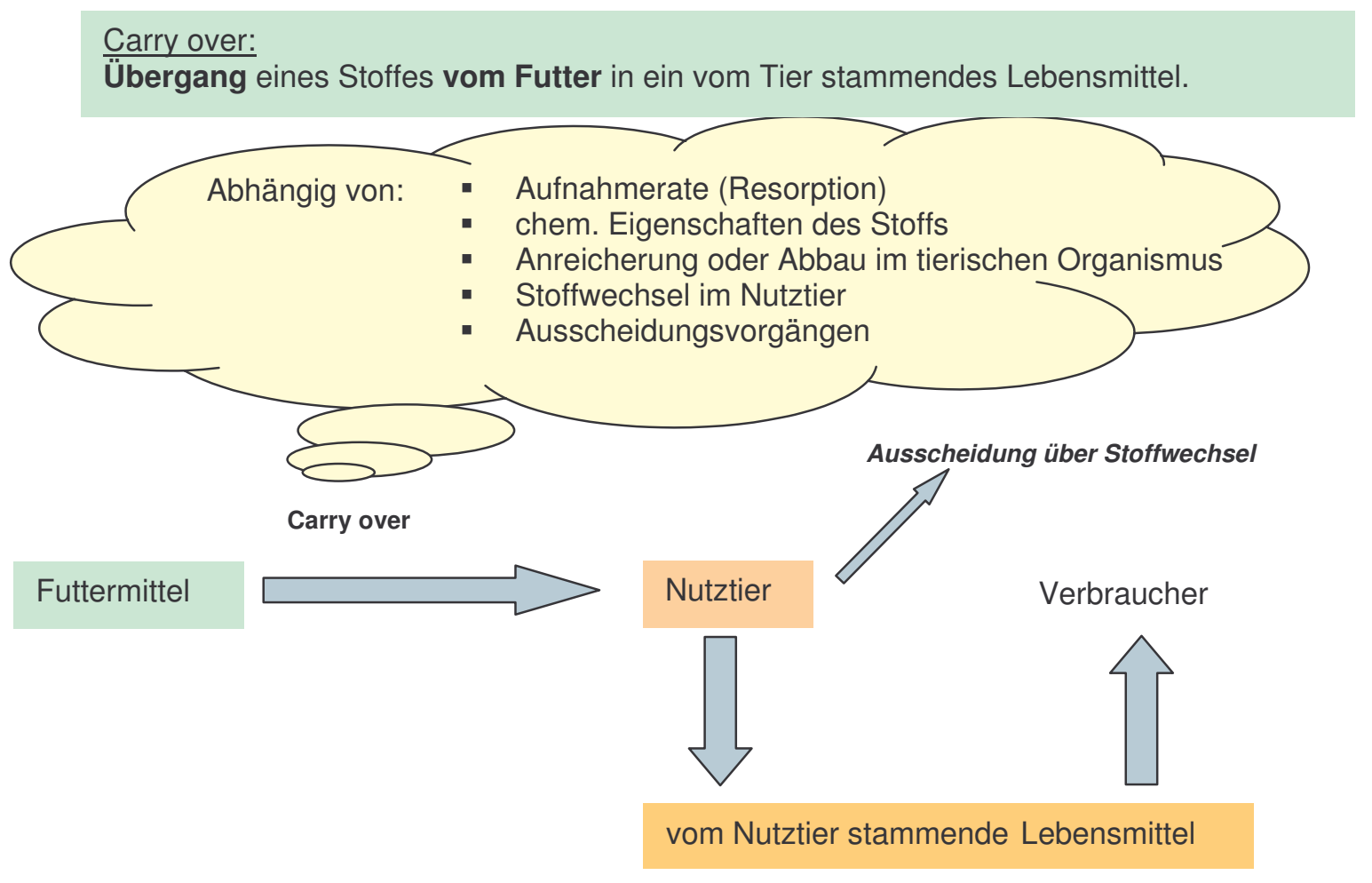

Abbildung 2.4-2: $\quad$ Carry over in im vom Tier stammenden Lebensmitteln (SCHWIND u. HECHT, 2004).

\subsubsection{Kontaminationen durch Mykotoxine in der Lebensmittelkette}

Schimmelpilzgifte (Mykotoxine) sind seit Jahrzehnten bekannt. Sie spielen vor allem in pflanzlichen Produkten (Getreide, Nüsse, Körnerfrüchte, Bier usw.) eine Rolle (SINELL, 1998A). Hierbei handelt es sich um Stoffwechselprodukte bestimmter Schimmelpilze, die in Abhängigkeit von den vorherrschenden Konditionen (z.B. Feuchtigkeit, Temperatur, Substrat, konkurrierende Mikroflora) in unterschiedlichem Umfang gebildet werden können. Aufgrund des zum Teil hohen Toxizitätspotenzials einiger dieser Toxine stellen mykotoxin- 
kontaminierte Nahrungs- und Futtermittel weltweit ein ernsthaftes Problem sowohl in der Landwirtschaft als auch in der Lebensmittelindustrie dar (ANONYMUS, 2002C).

Zearalenon (ZEA), Desoxynivalenol (DON) und Fumonisin B1 sind in Mitteleuropa die bedeutendsten Mykotoxine, die Ertragsverluste beim Getreide hervorrufen (VERREET u. AUMANN, 2001). Nach Untersuchungen von ELLNER (2002) überschritten in dem sehr feuchten Jahr 1998 69\% der untersuchten Proben den festgelegten Orientierungswert von $500 \mu \mathrm{g}$ Desoxynivalenol pro kg Weizen. Dagegen lag der Anteil an überhöhten DON-Konzentrationen bei Weizen in den Jahren 1999-2001 (9\%, 24\%, 17\%) deutlich niedriger.

Eine höhere Belastung von biologisch angebautem Getreide mit Mykotoxinen sieht WoLFF (2001) nicht. WOLFF begründet dies damit, dass auf Halmverkürzer verzichtet wurde und dass Mais in der Fruchtfolge nicht eingesetzt wird. Ein Risiko der verstärkten Bildung von Mykotoxinen bei der Lagerung wird allerdings in der Nichtanwendung von Vorratsschutzmitteln und in den zum Teil unzureichenden Möglichkeiten der Belüftung und Kühlung gesehen.

Neben den oben genannten Mykotoxinen spielen in der Nahrungsmittelproduktion vor allem Aflatoxine und Ochratoxine eine Rolle. Diese Mykotoxine werden vorwiegend durch unsachgemäße Lagerung gebildet (ENGELHARD, 1999).

\subsection{Auswirkungen von Mykotoxinen}

Mykotoxinhaltige Futtermittel können bei Tieren allgemein Reproduktionsstörungen, verminderte Tageszunahmen, Abnahme der Lebendmasse, Senkungen der Milchleistung, Senkungen der Legeleistung sowie erhöhte Krankheitsanfälligkeiten auslösen. Hierbei bestehen je nach Tierart Unterschiede hinsichtlich der Empfindlichkeiten gegenüber den verschiedenen Mykotoxinen (MÜNSTER ET AL., 2002B; YIANNIKOURIS u. JOUANY, 2002). Schweine und Geflügel scheinen die höchste Anfälligkeit für gesundheitliche Schädigungen durch aufgenommene Mykotoxine zu haben. Wiederkäuer sind im Vergleich zu den anderen Tierarten weniger empfindlich gegenüber oral aufgenommenen Mykotoxinen, was auf den umfangreichen mikrobiellen Abbau in den Vormägen (z.B. Ochratoxin A) zurückzuführen ist (MüNSTER ET AL., 2002B).

Beim Menschen kommt es insgesamt nur in seltenen Fällen zu akuten Intoxikationen. Unter mitteleuropäischen Bedingungen stellt die Langzeitwirkung kleiner Dosen die größere Gefährdung für die Gesundheit dar. Die toxischen Wirkungen der einzelnen Mykotoxine sind sehr unterschiedlich, sie reichen von genotoxischen Wirkungen bis zu kanzerogenen, hormonähnlichen, immunsuppressiven oder allgemein toxischen Effekten (KöHLER, 2002).

Ein Carry Over ist für die wichtigsten Mykotoxine (Aflatoxine, Ochratoxin A, T-2 Toxin und Zearalenol) nachgewiesen worden (KUIPER-GOODMANN, 1999; WEIDENBÖRNER, 1999; WOLFF, 2001; FUCHS ET AL., 2002; KÖHLER, 2002). Über die Aufnahme von mykotoxinhaltigen Futtermitteln können einzelne Mykotoxine in unveränderter oder metabolisierter Form in verschiedenen Organen beim Nutztier abgelagert (Fleisch, Innereien) oder ausgeschieden (Milch) werden (SINELL, 1998A; KUIPER-GOODMANN, 1999; WEIDENBÖRNER, 1999; MÜNSTER ET AL., 2002B). Auf diese Weise können Lebensmittel tierischer Herkunft Mykotoxine enthalten, ohne dass eine solche Kontamination äußerlich erkennbar ist (MÜNSTER ET AL., 2002B). Der Carry Over ist bei den einzelnen Mykotoxinen unterschiedlich. 
Bei Ochratoxin A (OTA) und Aflatoxine sind Carry Over in Lebensmittel nachgewiesen worden. Beide Mykotoxine werden über das Futter aufgenommen und im Gewebe oder den Organen abgelagert. Aflatoxine werden aber auch direkt über die Milch ausgeschieden (WEIDENBÖRNER, 1999; MÜNSTER ET AL., 2002B; RANDEL, 2002).

Das Vorkommen und die Höhe der Rückstände ist aber je nach Tierart unterschiedlich (MÜNSTER ET AL., 2002B). Erhöhte Gehalte an OTA lassen sich vor allem beim Schwein und beim Geflügel, jedoch weniger bei Wiederkäuern nachweisen (ELMADFA U. BURGER, 1999; MÜNSTER ET AL., 2002B). Beim Wiederkäuer wird Ochratoxin A im Rinderpansen durch die Aktivität der Mikroorganismen abgebaut. Aus diesem Grund wird bei ausgewachsenen Wiederkäuern nicht mit nennenswerten Rückständen von OTA in Fleisch und Milch ausgegangen (ELMADFA u. BURGER, 1999). Erhöhte OTA-Werte werden vor allem bei Schweinenieren, Schweineblut und Blutplasma sowie bei Wurstwaren, in dem Schweineblut und Plasma verarbeitet werden, gefunden (WOLFF ET AL., 1996).

Bei Fusarientoxinen (DON, ZEA) wurden in Futtermittelversuchen keine nennenswerten Carry Over in Lebensmitteln tierischer Herkunft nachgewiesen, die Hinweise auf eine Gefährdung des Menschen geben. Rückstände können nur durch sehr hohe Konzentrationen von Fusarientoxinen im Futter erreicht werden, wie sie bei einer normalen Fütterung nicht vorkommen (WEIDENBÖRNER, 1999; DÄNICKE u. OLDENBURG, 2000; DÄNICKE ET. AL., 2002).

\subsection{Bekämpfung}

Um erfolgreich den Eintrag von Mykotoxinen in die Nahrungskette entgegenzuwirken, müssen die Bekämpfungskonzepte in der Urproduktion ansetzen (SINELL, 1998A). Hierbei ist zu unterscheiden, ob es zu einer Kontamination während des Wachstums auf dem Feld oder nach der Ernte bei der Lagerung gekommen ist (Abbildung 2.4-3).

Die Belastung des Getreides durch Mykotoxine während des Wachstums ist vor allem vom vorherrschenden Wetter während der Vollblüte abhängig, in der die Ährenfusarien die Pflanzen infizieren. Eine hohe Feuchtigkeit und hohe Niederschläge fördern die Entwicklung der Mykotoxine (AUMANN u. VERREET, 2002). Neben den Witterungseinflüssen haben aber auch die Sorte, die chemische Bekämpfung, die Fruchtfolge, die Bodenbearbeitung, der Einsatz von Halmverkürzern und die Düngung einen Einfluss auf die Befallanfälligkeit (AUMANN u. VERREET, 2002; MAIER u. SCHIER, 2004A, 2004B).

Die rechtzeitige chemische Bekämpfung ist eine der wichtigsten Bekämpfungsmaßnahmen. Diese ist nur während eines sehr kurzen Zeitfensters während der Vollblüte und nur während eines Zeitraums zwischen einem Tag vor und zwei Tagen nach einer massiven Infektion möglich (AUMANN u. VERREET, 2002). In der Fruchtfolge bewirkt Körnermais als Vorfrucht von Weizen das höchste Infektionsrisiko. Bei einer Minimalbodenbearbeitung überleben Fusarien auf Pflanzenrückständen und können so zu einer Infektion des neuen Bestandes führen (AUMANN u. VerReEt, 2002; MAIER u. SCHIER, 2004A, 2004B). Ebenso fördern Halmverkürzer die Bildung von Mykotoxinen an der Pflanze. Optimierte bedarfsangepasste N-Gaben reduzieren dagegen die Befallwahrscheinlichkeit (AUMANN u. VERREET, 2002).

Eine Kontamination der Futtermittel während der Lagerung ist abhängig von der Kornfeuchte (aW-Wert), der Temperatur, der Verfügbarkeit von Sauerstoff und der Einlagerungsdauer. Hinzu kommen Parameter wie mechanische Beanspruchung (Bruchkorn), Kontamination des Lagers, der Luft oder des Produktes mit Schimmelpilzsporen (DRUSCH ET AL., 2002). Durch 
eine optimale Steuerung der Parameter kann die Bildung von Toxinen gering gehalten werden.

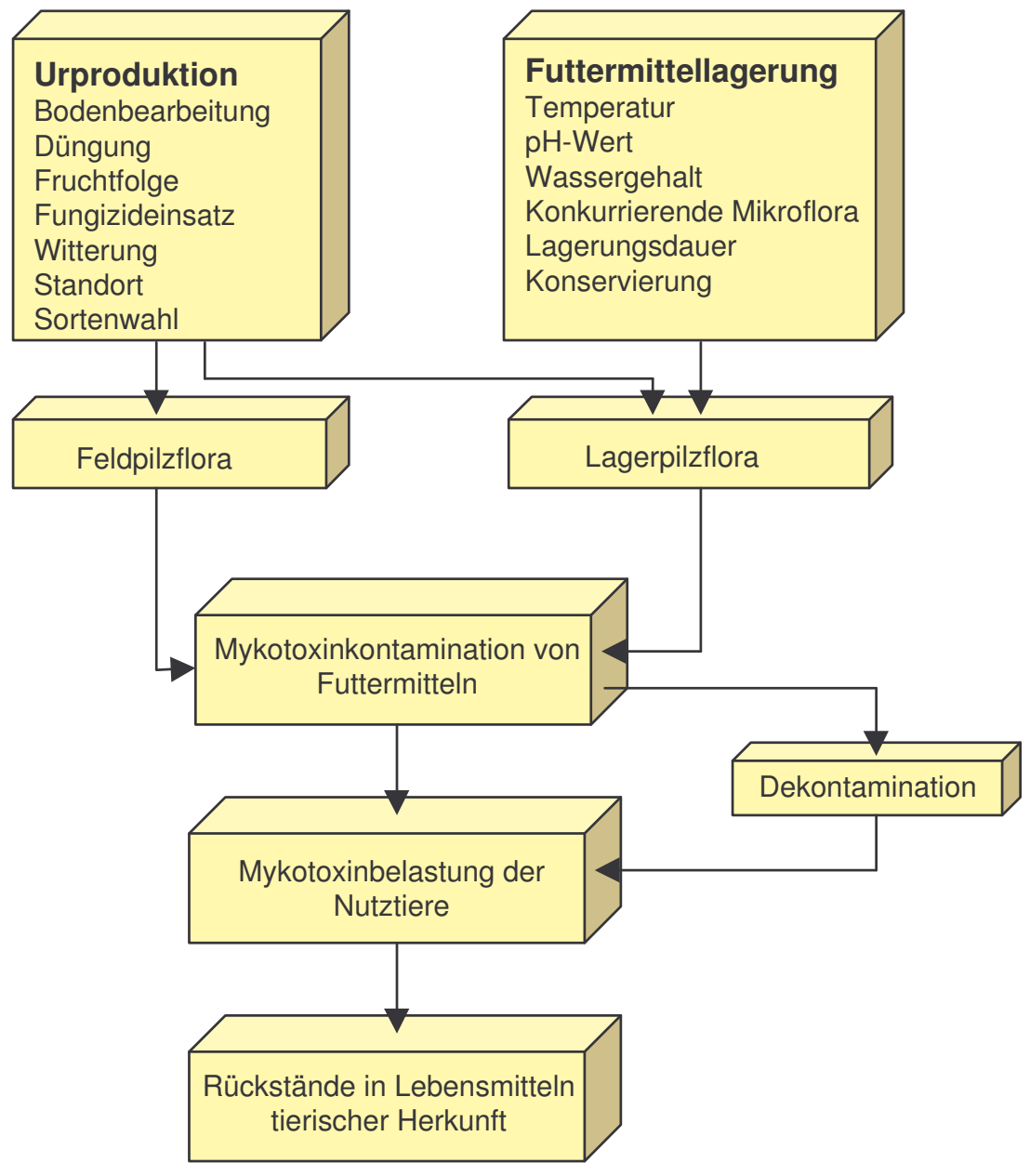

Abbildung 2.4-3: Einflüsse auf die Mykotoxinproduktion in Futtermitteln, die Mykotoxinbelastung von Nutztieren sowie mögliche Rückstände in den Lebensmitteln tierischer Herkunft (DÄNICKE U. OLDENBURG, 2000).

\subsubsection{Schwermetalle}

Unter den Begriff „Schwermetall“ fallen laut Definition die Elemente, die eine Dichte größer als $5 \mathrm{~g} \mathrm{~cm}^{3}$ aufweisen. Insofern wird auch Arsen (Dichte: $5,3 \mathrm{~g} \mathrm{~cm}^{3}$ ) häufig dieser Gruppe zugeordnet, obwohl es sich dabei eigentlich um ein Halbmetall handelt" (KüHNEN u. GOLDBACH, 2004). Schwermetalle sind natürliche Bestandteile der Umwelt. Sie begleiten die Geschichte der Menschheit und haben historische Perioden geprägt (Eisen- und Bronzezeit). Probleme sind hauptsächlich in Folge der industriellen Nutzung von Schwermetallen entstanden (SCHENKEL, 2001).

Nach SCHENKEL (2001) gelangen Schwermetalle hauptsächlich über Industrieemissionen in die Umwelt und dann über das Futter in den tierischen Organismus und dadurch in die Nahrungskette. Neben den Industrieemissionen spielen aber auch Düngung, Pflanzenschutz, Beregnung, Bodenbearbeitung, Medikamente, Desinfektionsmittel, Futterzukauf, Stalleinrich- 
tung und andere Faktoren eine Rolle beim Eintrag von Schwermetallen in die Nahrungskette (KÜHNEN u. GOLDBACH, 2004). Ähnliche Faktoren sind auch in der Tabelle 2.4-6 aufgeführt.

Die Verteilung der Schwermetalle innerhalb des Organismus ist mehr oder weniger charakteristisch für die einzelnen Elemente, (vergleiche auch Tabelle 2.4-7). Zum Beispiel weisen Blei und Cadmium eine ausgeprägte Affinität zu einzelnen Organen auf. Cadmium wird vor allem in der Leber und Niere angereichert. Der Übergang in die Muskulatur oder in tierische Produkte ist meist nur gering (SCHENKEL, 2001). Quecksilber wird dagegen auch in beachtlichen Mengen in der Muskulatur einlagert (KÜHNEN u. GOLDBACH, 2004).

Tabelle 2.4-6:

Eintragspfade Schwermetalle (SCHENKEL, 2001).

Mischfutter und zugekaufte Einzelfuttermittel überregionaler Eintrag

betriebseigene Futtermittel regionaler Eintrag
Arzneimittelanwendung inkl. Desinfektionsmaßnahmen Fütterungs- und Stalleinrichtungen verunreinigte mineralische Komponenten Ausgangsprodukt Einzelfutter Herstellung Einzelfutter (z.B. Aufkonzentration in einem Nebenprodukt) Herstellung Mischfutter/Abrieb bzw. Mahlen/Mischen) Aufnahme Wurzel/Auflagerung Kontamination bei Ernte/Konservierung, Lagerung und Aufarbeitung Aufnahme von Erde Trinkwasser Klauenbäder Elementverbindungen als Trägerstoff Mühlen, Mischer, Fördereinrichtungen, Anstriche, Verzinkungen, Hilfsmittel

Tabelle 2.4-7: $\quad$ Ablagerungsorte verschiedener Schwermetalle im Organismus landwirtschaftlicher Nutztieren (SCHENKEL, 2001 abgeändert).

\begin{tabular}{l|l}
\hline Element & Organe \\
\hline Blei & Knochen, Leber, Niere (Gehirn) \\
Cadmium & Niere (Cortex), Leber (Gastrointestinum) \\
Chrom & Leber, Niere \\
Kupfer & Leber, Niere \\
Nickel & Niere, Knochen \\
Quecksilber & Niere (Cortex), Leber \\
Zink & Knochen, Leber, Niere, Pankreas \\
Arsen & Leber, Niere, Haut \\
Thallium & Niere (Medulla), Leber, Knochen, Muskulatur \\
Vanadium & Niere, Knochen, Leber \\
\hline
\end{tabular}

In den letzten Jahren hat sich der Schwermetalleintrag in die Umwelt deutlich reduziert. Regelungen wie Benzinbleigesetz, stärkere Kontrollen von Emissionen, Einleiterkontrollen in Abwässer u.a. haben dazu beigetragen (SCHENKEL, 2001). Die Beanstandungsquote bei Schwermetallen in der amtlichen Futtermittelüberwachung lag in den Jahren 2002-2004 im Bereich von 0,2\%-0,5\% (BMVEL, 2004D).

Nach HONIKEL U. HECHT (1999) kann die Belastung von Fleisch und Fleischerzeugnissen mit Schwermetallen als gering bezeichnet werden und ist weiterhin rückläufig. Quecksilber als Rückstand im Fleisch besitzt heute keinerlei Bedeutung mehr (HECHT, 1997). 


\subsubsection{Klärschlamm}

Kompost oder Recyclingdünger sind Dünger pflanzlicher, tierischer, mikrobieller und mineralischer Herkunft. Kompost ist unter Luftzutritt verrottetes pflanzliches oder tierisches Material. Klärschlamm ist Schlamm aus den kommunalen Abwasserreinigungsanlagen in aufbereiteter oder nicht aufbereiteter Form. In der Schweiz ist die Ausbringung von Klärschlamm ab Ende September 2006 verboten. In Deutschland darf auf Gemüse- und Obstanbauflächen sowie in Wasserschutzgebieten und auf 10 Meter breiten Uferrandstreifen ebenfalls kein Klärschlamm ausgebracht werden (Klärschlammverordnung).

Das Klärschlammaufkommen in Deutschland lag 2001 bei ca. 2.425 Mio. Tonnen Trockensubstanz, wovon 57\% stofflich verwertet wurden (BERGS, 2004).

Die Klärschlamm- und Kompostverwertung auf landwirtschaftlichen Flächen wird von verschiedenen Seiten, aufgrund der nicht einwandfrei geklärten Rückstandsfrage, in Frage gestellt (HERTER ET AL., 2001). Nach einem gemeinsamen Beschluss der Agrar- und Umweltminister vom 13.06.2001 darf es durch Düngemaßnahmen zu keiner Schadstoffanreicherung im Boden kommen. Probleme bei der Verwertung von Klärschlamm, Kompost oder Recyclingdünger sind vor allem Rückstände von Schwermetallen und organischen Schadstoffen. Während der Anteil an Schwermetallen im Klärschlamm stark rückgängig verläuft (HERTER ET AL., 2001; BERGS, 2004; KÜHNEN u. GOLDBACH, 2004), ist die Situation bei den organischen Schadstoffen eher unklar (STADELMANN, 2001; BERGS, 2004). Hier ist die Datenlage eher unzureichend und eine Vielzahl der Schadstoffe, obwohl die Gehalte teilweise stark abnehmend sind, machen Sorgen (BERGS, 2004).

Die Klärschlamm- und Kompostverwertung auf landwirtschaftlichen Flächen ist aber auch ein wichtiger Bestanteil der Kreislaufwirtschaft. Hierbei sind aber die Forderungen bezüglich der Eignung der landwirtschaftlichen Flächen und der strengen Einhaltung der Schadstoffgrenzwerte zu beachten (KUMMER, 2001). Nach STADELMANN (2001) werden dadurch Nährstoffreserven geschont, z.B. konnten 1999 rund 7 Mio. Franken für Düngekosten eingespart werden.

\subsubsection{Organochlorverbindungen}

Organochlorverbindungen werden bzw. wurden in zahlreichen Insektiziden oder Pflanzenschutzmitteln eingesetzt. Eine besondere Bedeutung haben die polychlorierten Biphenylen (PCBs) und polychlorierte Dibenzodioxine und -furane (PCDD/F, Dioxine). Diese Stoffe sind in der Umwelt sehr persistent und es erfolgt in den Ökosystemen eine Anreicherung der teilweise sehr toxischen Isomere. Diese Rückstände in den Ökosystemen können sich in der Nahrungskette kumulieren (HEESCHEN, 2003).

Innerhalb der Nahrungsmittelkette werden immer wieder Rückstände von Organochlorverbindungen gefunden. Mögliche Eintragswege sind:

- $\quad$ Stoffe, die in der EU verboten sind, werden teilweise noch in Drittländern eingesetzt und über Futtermittelimporte besteht die Gefahr, dass sie in die Nahrungskette gelangen (SCHWIND, 2000).

- Bei der unvollständigen Verbrennung organischen Materials entstehen polycyklische aromatische Kohlenwasserstoffe (PAKs). Durch fehlerhafte Trocknungstechnologien oder durch ungeeignete Brennstoffe ist es in den letzten Jahren immer wieder zu Einträgen in die Nahrungskette gekommen. Als Beispiel sei der Dioxineintrag durch 
Trockengrün 1999 in Brandenburg genannt, wo kontaminiertes Abfallholz zur Trocknung von Grünfutter verwendet wurde.

- Durch Unwissenheit werden Materialien eingesetzt, die Dioxinrückstände enthalten (z.B. natürlicher Kaolinit-Ton) (FLACHOWSKY, 2002).

Nach der nationalen Berichterstattung über Pfanzenschutzmittelrückstände kann kein klarer Trend bei der Abnahme von Pflanzenschutzmittelrückständen im Getreide festgestellt werden (vergl. Tabelle 2.4-8). Im Getreide sind 2003 in ca. 33\% der Proben Rückstände festgestellt worden. Davon lagen bei ca. 1\% der Proben die Werte über dem Grenzwert. Deutliche Unterschiede zwischen in Deutschland angebautem Getreide und importiertem Getreide liegen nicht vor.

Tabelle 2.4-8:

Pfanzenschutzmittelrückstände in Getreide (BVL, 2002, 2003; EU, 2001A, 2002c, 2003C).

\begin{tabular}{c|ccccccccc}
\hline & \multicolumn{3}{|c}{ keine Rückstände } & \multicolumn{3}{c}{ Rückstände unterhalb der } \\
Grenzwerte & \multicolumn{3}{c}{ Rückstände oberhalb der } \\
Grenzwerte \\
Getreide & alle & Ausland & Inland & alle & Ausland & Inland & alle & Ausland & Inland \\
\hline 2003 & 67 & 74 & 64 & 32 & 25 & 35 & 1,0 & 0,9 & 1,1 \\
2002 & 75 & 69 & 76 & 23 & 29 & 22 & 2,1 & 2 & 2,1 \\
2001 & 67 & 38 & 86 & 31 & 63 & 36 & 1,3 & 0 & 1,8 \\
2000 & 63 & 64 & 63 & 33 & 30 & 35 & 4,1 & 6,7 & 2,8 \\
1999 & 61 & 73 & 57 & 38 & 26 & 42 & 1,2 & 0,9 & 1,3 \\
\hline
\end{tabular}

Es gehen über $90 \%$ der Dioxindispositionen des Menschen auf Lebensmittel zurück, wovon circa $80 \%$ der Gesamtexposition Lebensmittel tierischen Ursprungs beteiligt sind. Hochrechnungen haben ergeben, dass die derzeitige Belastung der Bevölkerung mit PCDD/F (Gesamtzufuhr) kaum 50\% des WHO-TDI-Wertes erreichen dürfte (HEESCHEN, 2003). Die Dioxinbelastung in den Lebensmitteln ist in erster Linie auf die Belastung von Futtermitteln zurückzuführen (BMU, 2001). Die Anreicherung der Dioxine erfolgt im Fettgewebe. Nahm der Mensch 1992 noch pro Tag ca. 39 pg iTE/Tag polychlorierte Dibenzodioxine und -furane aus Fisch- und Fleischerzeugnissen auf, so sank die Aufnahme 1999/2000 auf ca. 5,8 pg iTE/Tag (BLÜTHGEN ET AL., 2000). Trotz der gesunkenen Werte stellt Dioxin nach wie vor ein Problem dar.

Folgende Maßnahmen sieht HEESCHEN (2003) zur Belastungsreduktion von Organochlorvebindungen:

- Reduktion der Emissionen in Verbrennungsanlagen,

- Emissionsminderung in der Metallindustrie,

- $\quad$ kein Einsatz von verbleitem Benzin (obsolet),

- keine Klärschlammausbringung auf landwirtschaftliche Flächen,

- $\quad$ keine Chlorbleiche bei der Papierherstellung,

- $\quad$ keine Verwendung von Pentachlorphenol im Holzschutz,

- ordnungsgemäße Anwendung der Trocknungstechnologie bei der Herstellung von Futtermitteln,

- Festlegung von „Aktionswerten“/Grenzwerten für Futter-/Lebensmittel. 


\subsubsection{Futterzusatzstoffe zur Leistungssteigerung und zu therapeutischen Zwecken}

Die EU-Verordnung 1831/2003 regelt die Zulassung und Verwendung von Futterzusatzstoffen. Laut Verordnung müssen Futterzusatzstoffe den Ernährungsbedarf der Tiere decken, die Beschaffenheit des Futtermittels und der tierischen Erzeugnisse sowie die ökologischen Folgen der Tierproduktion positiv beeinflussen. Ebenso sollen sie die Leistung oder das Wohlbefinden der Tiere, insbesondere durch Einwirkung auf die Magen- und Darmflora oder auf die Verdaulichkeit der Futtermittel, erhöhen oder eine kokzidiostatische oder histomonostatische Wirkung haben. Futterzusatzstoffe lassen sich in vier Hauptgruppen unterteilen:

- technologische Zusatzstoffe (Konservierungsmittel, Antioxidationsmittel, Emulgatoren, Stabilisatoren, Verdickungsmittel, Geliermittel, u.a.),

- $\quad$ sensorische Zusatzstoffe: Farbstoffe, Aromastoffe,

- $\quad$ ernährungsphysiologische Zusatzstoffe: Vitamine, Provitamine und chemisch definierte Stoffe mit ähnlicher Wirkung; Verbindungen von Spurenelementen, Aminosäuren,

- zootechnische Zusatzstoffe: Verdaulichkeitsförderer, Darmflorastabilisatoren, Stoffe, die die Umwelt günstig beeinflussen, sonstige zootechnische Zusatzstoffe (VO EU 1831/2003).

Aufgrund der möglichen Bildung von Resistenzen ist der Einsatz von antimikrobiellen Stoffen als Wachstumsförderer ab dem 1.1.2006 in der gesamten EU verboten (EU VO 1831/2003).

Rückstände von Tierarzneimitteln oder anderen Substanzen, die zur Leistungssteigerung eingesetzt werden, werden immer wieder im essbaren Gewebe gefunden. Eine Rückstandsfreiheit im absoluten Sinne wird auch aus Gründen der modernen Analytik bei behandelten Tieren kaum zu erreichen sein. Aus der Sicht des Verbraucherschutzes ist es sinnvoll, akzeptable und nicht akzeptable Rückstandskonzentrationen im essbaren Gewebe festzulegen (KROKER, 2003).

Man kann die Medikamente, die in der Tierproduktion eingesetzt werden können, in vier Gruppen einteilen: Antiparasitika, Antiinfektiva, Hormone und hormonell wirksame Substanzen und Psychopharmaka (KROKER, 2003). SANTARIUS (2004) sieht in den meisten Arzneimittelrückständen im Vergleich zu eventuell cancerogen wirkenden Kontaminanten (Schwermetallen, Pestiziden), technologischen Hilfsstoffen (Nitrit/Nitrat) oder in der Prozesstechnik entstehenden Stoffen (Nitrosamine, Benzpyren aus Räucherrauch etc.) kaum Risiken. Der Anteil der jährlich beanstandeten Proben bei der Untersuchung von Tieren und tierischen Erzeugnissen liegt bei weit unter 1\%. SANTARIUS, (2004) gibt aber auch zu bedenken, dass Fragen bezüglich bakterieller Resistenzauslösungen durch Antibiotikarückstände sowie die Risikoeinschätzung von Arzneimittelrückständen oder bedenklichen pflanzlichen Inhaltsstoffen in Lebensmitteln, mit hinreichender Sicherheit für den Verbraucher, nie endgültig abgeschlossen sein können. Ebenfalls werden in der Tiermast freie und gekapselte Säuren, Probiotika (lebende Mikroorganismen), Prebiotika (spezielle Zuckerfraktionen), Enzymkomplexe, Aromen und Kräuter zur Leistungssteigerung eingesetzt. Durch deren Wirkung auf den Verdauungstrakt oder durch die positive Reizung der Geruchs- und Geschmacksnerven sollen die Futterverwertung und die täglichen Zunahmen der Tiere gesteigert werden (ANONYMUS, 2002C). Pflanzen- bzw. Kräuterextrakte, z.B. aus Oregano, werden im Geflügelbereich als fleischqualitätsverbessernd diskutiert (SCHMITZ ET AL. 2003). 
In der Europäischen Union ist der Einsatz von hormonell wirksamen Stoffen in der Nutztiermast seit 1988 verboten (COUNCIL OF THE EUROPEAN COMMUNITIES, 1996). Seit 1989 gilt das Verbot auch für importiertes Rindfleisch und Rindfleischprodukte. Dieses führt regelmäßig zu politischen Auseinandersetzungen mit den USA, wo hormonell wirksame Stoffe in der Tiermast erlaubt sind. Wissenschaftlich konnten weder die sichere Unbedenklichkeit noch klare Risiken bezüglich des Hormoneinsatzes nachgewiesen werden (MISSURA, 2001; EU, 2002B). Bei einer bestimmungsgemäßen Anwendung von natürlichen Sexualhormonen werden kaum erhöhte Hormongehalte im essbaren Gewebe gefunden. MEYER (1999) berichtet, dass Fleisch, das in Europa durch übliche Bullenmast erzeugt wird, höhere Hormongehalte aufweist als Fleisch von Ochsen aus den USA, das unter dem Einsatz von Hormonen produziert wurde. Fleisch hat immer einen Anteil an natürlichen Hormonen. Dieser Anteil an natürlichen Hormonen im essbaren Gewebe ist abhängig vom Alter und Geschlecht der Tiere. Probleme durch Hormonrückstände entstehen vor allem bei einer Fehlplatzierung der Implantate.

Rückstände von verbotenen und nicht zugelassenen Stoffen werden nach wie vor gefunden (BVL, 2004). Insgesamt haben Untersuchungen im Rahmen des Nationalen Rückstandskontrollplans (NRKP) in Deutschland von 2004 (Stoffe mit anaboler Wirkung, Tierarzneimittel, Kontaminanten) ergeben, dass bei Rindern zwischen 0,2-0,4\%, bei Schweinen 0,1\% und bei Mastgeflügel 0\% der untersuchten Proben positiv waren (ZERL, 2005). In Dänemark wurden bei Untersuchungen von Schweinefleisch (1991-2001) in 0,04\% bis 0,005\% der Proben Rückstände von Antibiotika/Chemotherapeutika gefunden. Eine Begründung kann hierbei der seit 1998 freiwillige Verzicht auf antibiotische Leistungsförderer bei Mastschweinen sein (DANISH, 2003). Durch das Verbot der letzten antibiotischen Stoffe als Wachstumsförderer in der Mast ist zu erwarten, dass die Rückstandsfunde auch in Deutschland weiter sinken werden.

Nach den Jahresberichten zum Nationalen Rückstandskontrollplan hat sich die Rückstandssituation bei hormonell wirkenden Stoffen zur Leistungssteigerung stark verbessert. Sind 1998 noch in 1,38\% (BVL, 2001) der untersuchten Proben Rückstände von Chloramphenicol gefunden worden, lag der Anteil an positiven Proben 2004 bei 0,1\% (BVL, 2004).

\subsubsection{Verfahrenstechnische Risiken von Futtermitteln}

Die Futtermittelproduktion kann in zwei große Bereiche untergliedert werden: in die Mischfutterindustrie und die landwirtschaftliche Urproduktion mit der Grundfutterproduktion und der Erzeugung von Feldfrüchten für die Mischfutterindustrie.

In der Futtermittelproduktion hat es von Seiten des Gesetzgebers eine Verschärfung des Rechtes gegeben. Durch die VO EU 178/2002 sind Futtermittel mit Lebensmitteln gleichzusetzen. Die wesentlichste Änderung im Futtermittelrecht erfolgte durch das Inkrafttreten der EU VO 183/2005. Durch diese Verordnung werden erstmals Regelungen über die gute Fütterungspraxis für Lebensmittel-Tiere festgelegt, die die Vorschriften über eine gute Fütterungspraxis des Codex Alimentarius in die Praxis umsetzt. Wesentliche Inhalte der neuen Verordnung sind (KRUSE, 2005):

- die allgemeinen Bestimmungen über die Futtermittelhygiene auf allen Stufen der Herstellung von Futtermitteln, des Verkehrs mit Futtermitteln und der Verwendung von Futtermitteln,

- die Anforderungen an die Sicherstellung der Rückverfolgbarkeit von Futtermitteln und

- die Registrierung und Zulassung von allen Betrieben. 
Zu mehr als zwei Drittel werden die Nutztiere über wirtschaftseigenes Futter (Grünfutter, Silagen, Heu und wirtschaftseigene Kraftfuttermischungen) ernährt (FLACHOWSKY, 2002). Das Hauptrisiko, das von der landwirtschaftlichen Futtermittelproduktion ausgeht, ist die Gefahr von Rückständen. Hier spielen vor allem Rückstände in der Folge von Altlasten und Pflanzenschutz eine wichtige Rolle (vergl. Kapitel 2.4.6). Die Bodenbearbeitungs- und Anbaumethoden, der Pflanzenschutz- und Düngemitteleinsatz sowie das Ernteverfahren haben einen wesentlichen Einfluss auf die Produktsicherheit.

Ansatzpunkte für Maßnahmen zur Verbesserung der Futtermittelqualität im landwirtschaftlichen Betrieb sind (DLG, 2001):

- Sorgfalt und Kenntnisse des Personals,

- verbesserte Erfahrungen mit Eigenkontrollen (Betriebsblindheit),

- Spezialisierungsgrad (Vielfalt erhöht Risiken),

- Intensität der Produktionsverfahren,

- qualitätsfördernde Maßnahmen im Anbau, bei der Lagerung/Konservierung,

- Verarbeitung betriebseigener Futtermittel,

- $\quad$ stärkere Berücksichtigung der Qualität beim Einkauf von Futtermitteln,

- Anzahl und Eignung der Futterlager (Silos),

- Kennzeichnung der verschiedenen Futtermittel,

- $\quad$ sensorische und analytische Qualitätskontrollen,

- technischer Zustand der Fütterungsanlagen,

- Aufzeichnungen.

Die Lagerung spielt sowohl beim wirtschaftseigenen Futter als auch im Handel und der Mischfutterproduktion eine entscheidende Rolle zur Gesunderhaltung der Produkte. Probleme können hier entstehen durch:

- Kontaminationen durch Reste vorheriger Ware (siehe Nitrofenskandal),

- $\quad$ Pilzbefall (Wassereintritt, mangelnde Belüftung, Einlagerung von feuchter Ware),

- mikrobiologische Kontamination (Schadnager, Vögel).

Welche Probleme sich aus einer nicht ordnungsgemäßen Lagerung ergeben können, hat der Nitrofenskandal gezeigt. Mit Nitrofen belasteter Weizen wurde an verschiedene Mischfutterwerke verkauft. Neben der direkten Verbreitung des Nitrofens über den Weizen erfolgte auch eine Verbreitung über die Reinigungs- und Verarbeitungsanlagen der Mischfutterindustrie. Bei langzeitiger Verarbeitung von kontaminiertem Getreide kam es zu einer Akkumulation des Schadstoffes in der Verarbeitungsanlage und somit zu einer Belastung im Mischfutter kommen. Die Verbreitung des belasteten Weizens hat zu umfangreichen Rückrufaktionen auf allen Stufen der Kette geführt (PESTEMER, ET AL., 2003).

In der Mischfutterproduktion werden, aufgrund von Kundenwünschen, eine Vielzahl von Rezepturen für Vormischungen und Mischfutter produziert. Gerade in klein- und mittelständischen Unternehmen erfolgt die Produktion ,just in time“ in Einstrang-Produktionsanlagen, bei der alle Futtermittel über eine Linie produziert werden. Die ,just in time“ Produktion ist vielfach mit einem häufigen Rezepturwechsel verbunden. Hierdurch steigt die Gefahr der Verschleppung kritischer Komponenten und der Kreuzkontamination nachfolgender Chargen (STRAUCH, 2002). Um ein sicheres Produkt zu erzeugen, spielen die Mischgenauigkeit und 
die Verschleppung eine Rolle. Die Mischgenauigkeit ist hauptsächlich abhängig von der Partikelgrößenverteilung und der Dichte der eingesetzten Mikrokomponenten (IFF, 1998). Unter einer Verschleppung der Anlage wird die Abtrennung eines Mengenanteils einer Mischung oder Einzelkomponente verstanden, die in der Anlage oder in Elementen der Anlage verbleibt. Dieses können zum Beispiel Reste, Anbackungen, Verkrustungen oder Ablagerungen in Toträumen sein (STRAUCH, 2002). Zur Minimierung der Verschleppung wird das Verschleppungsniveau der Anlage ermittelt. Aufgrund des ermittelten Verschleppungsniveaus der Anlage, der zugegebenen Menge an kritischen Stoffen und einem Sicherheitsfaktor für den eingesetzten Stoff kann die Anzahl der Spülchargen berechnet werden, die notwendig sind, um das Verschleppungsrisiko zu minimieren. Eingesetzte Spülchargen können die Verschleppung nur reduzieren, aber nicht verhindern (PDV, 2005B).

Alle Betriebe, die Vormischungen, Medikamente oder Leistungsförderer beimischen, müssen in Deutschland anerkannt und registriert sein. Die Entwicklung in der Futtermittelproduktion geht immer weiter in die Richtung, Futtermittel für eine Tierart auf einer eigenen Linie zu produzieren. In großen Werken werden Geflügel- und Schweinefutter nicht mehr zusammen mit Rinderfutter produziert. Das Einmischen von Arzneimitteln in Mischfutter ist nur noch in Betrieben erlaubt, die über eine Herstellungserlaubnis nach §13 Arzneimittelgesetz verfügen (HELLWIG, 2003; BODE, 2004).

Eine weitere prozessbedingte Gefährdung ist der Eintrag von pathogenen Keimen in das Mischfutter. Dies betrifft vor allem die Verschleppung von Salmonellen. Kritisch ist z.B. der Kühlungsprozess in den herkömmlichen Kraftfutterwerken. Zur Kühlung werden große Mengen Luft durch das erhitzte Futter geblasen. Durch den Entzug von Wärme und Feuchtigkeit besteht das Risiko, dass es in bestimmten Bereichen des Kühlers zur Kondensatbildung kommt. Das hier anhaftende Material hat die für die Salmonellenvermehrung notwendige Feuchte und Temperatur. Wichtig ist es daher, Maßnahmen zur Salmonellenreduzierung durchzuführen, wie z.B.:

- Untersuchung der eingehenden Rohware auf Salmonellen (Ausschluss der Lieferanten, deren Rohware häufiger mit positiven Proben aufgefallen ist),

- Hitzebehandlung des Futters mittels Heißdampf in einem Konditionierer über 3 Minuten bei mindestens $83^{\circ} \mathrm{C}$,

- eine ausschließliche Verwendung gefilterter Luft von der Westseite (Hauptwindrichtung) für den nachfolgenden Kühlprozess, damit beim Kühlen eine Rekontamination vermieden wird und

- $\quad$ saubere nachfolgende Förder- und Transporteinrichtungen (LÖHREN, 2004).

Durch den Transport von Futtermitteln kann es ebenso wie durch den Herstellungsprozess zu Verschleppungen kommen. Striktes Hygienemanagement und die Einhaltung von Transportreihenfolgen sind notwendig, um die Verschleppung zu beherrschen. Die zurzeit weit reichendsten Regelungen bezüglich Transporte sind in dem Regelwerk GMP B4.1 festgeschrieben. Transportgüter werden hier in vier Kategorien, je nach deren Gefahrenpotenzial, eingeteilt:

- $\quad$ Produkte mit sehr hohem Risiko (tierischer Dung, Asbest, Faulschlamm u.a.),

- mikrobiologisch verunreinigte Materialien,

- Materialien mit physikalischem und/oder chemischem Risiko oder Produkte mit verarbeiteten tierischen Eiweißen (Steinkohle, Aluminiumsulfat, Braunkohle u.a.), 
- $\quad$ neutrale Materialien (z.B. Futtermittelausgangserzeugnisse u.a.).

Für jede Kategorie sind spezifische Reinigungsvorschriften vorgegeben, an die sich der Transporteur zu halten hat (PDV, 2005A).

Weitere Probleme in der Produktion von Futtermitteln gehen von den temperaturgesteuerten Prozessen aus.

Trocknungsverfahren führen immer wieder zu Rückständen in Futter- und Lebensmitteln (PETERSEN, 2001). Die Hauptrisikofaktoren bei der Trocknung sind der Brennstoff, der Brennertyp, die Verbrennungsbedingungen und das Temperaturprofil im Trockner (DE BOT ET AL., 2004). Vor allem beim Einsatz von festen Brennstoffen (z.B. Holz, Kohle) oder schwerem Heizöl besteht ein Risiko des Eintrags von unerwünschten Stoffen (Dioxine, Furane, PAKs). Dagegen verursachen der Einsatz von Gas und schwefelarmes Heizöl kaum Rückstände (HECHT, 2003). Gerade die Risiken der Trocknung durch falsches Brennmaterial ist auch ein Problem, das bei importierten Futtermitteln aus Drittländern nicht unterschätzt werden sollte. Die Wichtigkeit einer ordnungsgemäßen Handhabung der Trocknungsanlagen wird zunehmend in den verschiedenen Qualitätsmanagementsystemen (GMP+, QS) und auch auf der gesetzgebenden Ebene gefordert. Die EU empfiehlt, dass indirekte Trocknungsverfahren zu bevorzugen sind, da hier die Gefahr einer Belastung durch Rückstände aus dem Verbrennungsprozess nicht besteht (RL 95/53/EG).

\subsubsection{Herstellung und Verfütterung von Tiermehlen}

Eine Änderung der Herstellungsbedingungen von Tiermehlen in England in den 70er Jahren gilt als eine mögliche Ursache für die BSE-Erkrankung von Rindern. Als Quelle wurden Schafe, die an Scrapie erkrankten und zu Tiermehl verarbeitet wurden, vermutet (HONIKEL, 2001; FRIES, 2002A). Aus Arbeitsschutzgründen wurden organische Lösungsmittel, die zum Entzug von Fetten dienten, nicht mehr verwendet. Damit sind neben der Lösungsmittelwirkung auch Erhitzungsschritte, die zum Ziel hatten, die Entzugschemikalien zu verflüchtigen, weggefallen. Aufgrund des Wegfalls dieser Schritte wurde der infektiöse Erreger nicht mehr ausreichend inaktiviert (RKI ET AL., 2001; DAHMS, 2003). Es werden auch noch andere Wege diskutiert. (HONIKEL, 2001; BORCHERS, 2002A, 2002B). Bei Rindern, die nach dem Verfütterungsverbot von Eiweißen tierischen Ursprungs geboren wurden, ist der BSE-Erreger festgestellt worden. Diese Tiere sind als sogenannte „Born-after-Ban-Fälle“ bekannt geworden (SCHLENKER, 2003; KIENZLE ET AL., 2004). Über die Übertragungswege von „Born-after-Ban-Fällen“ gibt es zurzeit nur Vermutungen. Ursachen könnten sein:

- Das Prionprotein ist mutiert (StAUFENBIEL, 2001; SCHLENKER, 2003).

- Verwendete Tierfette sind zum Teil bis Mitte 2002 nur bei $80^{\circ} \mathrm{C}-100^{\circ} \mathrm{C}$ erhitzt worden (BORCHERS, 2002A; 2002B).

- $\quad$ Fleisch- und Knochenmehle sowie tierische Fette gelangten trotz Verfütterungsverbot in die Wiederkäuerfütterung, z.B. durch Kreuzkontamination (SCHLENKER, 2003; KIENZLE ET AL., 2004). Untersuchte Wiederkäuerfuttermittelproben enthielten zwischen 1996-2000 um 70\% tierische Bestandteile. Im Zuge des umfassenden Verfütterungsverbots für tierische Bestandteile reduzierte sich dieser Anteil im Jahr 2001 auf $7 \%$ und im Jahre 2002 auf 0,3\% (KIENZLE ET AL.2004).

- Eine Übertragung erfolgt auf vertikalem bzw. maternalem Weg (FRIES, 2002B; SCHLENKER, 2003; HEESCHEN, 2004). 
- Chemische Substanzen und Umweltnoxen können prädisponierend für die Entstehung infektiöser Prionen wirken (STAUFENBIEL, 2001; SCHLENKER, 2003).

- Risikomaterial oder Risikotiere werden nicht sachgemäß transportiert, gelagert oder aufbewahrt (SCHLENKER, 2003).

Neben den gesundheitlichen Risiken hat das Verfütterungsverbot von tierischen Eiweißen in der Tiermast kurzzeitig eine verstärkte Diskussion in der Wirtschaft über die möglichen Veränderungen in der Fettsäurenzusammensetzung bzw. der Oxidationsstablität des Fettes ausgelöst. Für die Herstellung von Dauerwaren wird ein kerniger Speck benötigt, dessen Fett eine feste Konsistenz und eine hohe Oxidationszahl aufweist (v. LENGERKEN ET AL., 1998). Dieser Speck zeichnet sich durch einen hohen Anteil an gesättigten Fettsäuren aus. Gesättigte Fettsäuren machen das Fett fest. Sie werden in der Verarbeitung bevorzugt, da gesättigte Fettsäuren chemisch generell stabiler sind, so dass sie nicht so schnell ranzig werden wie ungesättigte Fettsäuren (POTTHAST, 1981; WARNANTS ET AL., 1998).

Im Fahrplan für die TSE-Bekämpfung der EU (2005) werden Überlegungen angestellt, die eine Aufhebung von Bestimmungen des Verfütterungsverbotes von Tiermehlen an Nichtwiederkäuer betreffen. Hierbei wird aber darauf verwiesen, dass das Verbot der Verwertung innerhalb derselben Tierart gemäß der EU VO 1774/2002 eingehalten werden muss. Eine Lockerung der vorhandenen Maßnahmen soll sich nach dem Fahrplan am Risiko orientieren und den technologischen und wissenschaftlichen Fortschritt berücksichtigen (EU, 2005).

Nach MÄNNER und SIMON (2001) kann auf Grund des derzeitigen Wissenstandes ein Risiko durch Fleisch- und Fleischknochenmehl nahezu ausgeschlossen werden, wenn es von BSEgetesteten und für den menschlichen Verzehr freigegebenen Tieren stammt. Allerdings muss sichergestellt sein, dass Hight-Risk-Material, wie Hirn, Rückenmark und Darmtrakt, sauber entfernt ist und das Tiermehl nur an Nichtwiederkäuer verfüttert wird. Um ein Restrisiko auszuschließen müssen diese Erzeugnisse vor der Verwendung einem Autoklavierungsverfahren unterzogen werden. Wenn in landwirtschaftlichen Betrieben Tiermehle wieder verfüttert werden, muss sichergestellt sein, dass diese über keine Wiederkäuerhaltung verfügen. Futtermittel mit Tiermehlen dürfen bei einer Wiederzulassung nur in garantiert von Wiederkäuerfutter getrennten Produktionwegen produziert werden (ANONYMUS, 2001). MÄNNER u. SIMON (2001) vertreten die Meinung, dass ein Totalverbot der Tierkörper- und Schlachtabfallverwertung zur Futtermittelherstellung weder aus seuchenhygienischen Gründen erforderlich noch aus ökonomischen Gründen sinnvoll ist.

\subsubsection{Verfahrenstechnische Risiken in der Tierproduktion}

Die heutige Tierpoduktion ist stark ausgerichtet nach der Wirtschaftlichkeit (Kosten, Preise) und dem Tierschutz. Trotz des hohen Stellenwertes des Tierschutzes hat es in den letzten Jahren Entwicklungen gegeben, die zunehmend hinterfragt werden. Durch einen ständig wachsenden Kostendruck haben sich Tierhaltungssysteme entwickelt, die Mängel aufweisen. Mängel werden teilweise durch den Einsatz von Medikamenten zur Prophylaxe und zur Leistungssteigerung oder durch Eingriffe am Tier (Schwänze kupieren, Schnabel kürzen) ausgeglichen. Dieses ist sowohl aus der Sicht des Tierschutzes als auch aus der Perspektive des vorsorgenden gesundheitlichen Verbraucherschutzes und der Lebensmittelsicherheit kritisch zu sehen (BMVEL, 2002). In den meisten Fällen werden Risiken, die sich negativ auf das Tier auswirken, auch vom Verbraucher als ethisch bedenklich angesehen. Andere Risiken in der Tierproduktion haben zwar keinen negativen Einfluss auf das Tier, passen 
aber nicht zum Bild des Verbrauchers über Tierhaltung und werden deshalb negativ gesehen (WILDNER, 1998). Verfahrenstechnische Risiken in der Tierproduktion sind in der Tabelle 2.4-9 dargestellt.

Diskussionen über die heutigen Tierhaltungsverfahren werden in ganz Europa geführt (AlVENSLEBEN, 2001B; HARPER, 2001). Da der Informationsstand der Verbraucher zum Teil sehr gering ist, besteht die Neigung zur Generalisierung, d.h. er vermutet, dass die Nutztiere in den konventionellen Haltungsformen generell schlecht gehalten werden („Massentierhaltung") (AlvensLeben, 2001B, 2002D; HARPER u. HenSON; 2001). SieS und MAHLAU (1997) befragten Verbraucher bezüglich ihrer Assoziation zu dem Wort Tierhaltung. Von den gegebenen Antworten waren 3 positiv, 17 neutral und 40 negativ. Häufige negative Aussagen bezüglich der Tierhaltung sind Assoziationen wie Masse, nicht artgerecht (Käfig, wenig Platz) und Verhaltensstörungen. Nach folgender Rangfolge akzeptieren Verbraucher Haltungssysteme (absteigende Reihenfolge): Milchviehhaltung, Schafhaltung, Schweinehaltung, Rindermast, Eiererzeugung, Kälbermast, Geflügelmast. Wichtig für das Tierwohl ist aus der Sicht der Befragten Fütterung, Platz, Auslauf ins Freie, Transport, natürliches Verhalten und Schlachtung (HARPER, 2001; HARPER u. HENSON, 2001; AlVENSLEBEN, 2001B, 2002D).

Durch die Verankerung im Grundgesetz Artikel 20a hat der Tierschutz in Deutschland seit August 2002 einen noch höheren Stellenwert erlangt. Umfangreiche Regelungen, die den Schutz der Tiere gewährleisten, hat es aber auch schon vor dem 1. August 2002 (siehe Tierschutzgesetz) gegeben und diese gehören nach wie vor zur guten landwirtschaftlichen Praxis (BMVEL, 2002).

Laut dem Tierschutzbericht 2003 des BMVEL sind Haltungsbedingungen dann tiergerecht, wenn:

- $\quad$ sie den spezifischen Eigenschaften der Tiere Rechnung tragen,

- die körperlichen Funktionen nicht beeinträchtigt werden,

- die Anpassungsfähigkeit der Tiere nicht überfordert wird,

- $\quad$ essentielle Verhaltensmuster nicht so eingeschränkt und verändert werden, dass dadurch Schmerzen, Leiden oder Schäden am Tier entstehen.

Vom Verbraucher werden Haltungsformen der ökologischen Tierhaltung bevorzugt. Die ökologische Tierhaltung nimmt in Deutschland allerdings nur eine Nische ein. Zwar steigt der Anteil an Tieren, die in Systemen nach ökologischen Vorgaben gehalten werden. Der Anteil ist jedoch noch sehr gering. 2001 wurden 2,8\% der Tiere in Betrieben, die nach ökologischen Vorgaben produzieren, gehalten, 2003 waren es 3,3\% (STATISTISCHES BUNDESAMT, 2005).

Tabelle 2.4-9: $\quad$ Verfahrenstechnische Risiken in der Tierproduktion.

\begin{tabular}{|c|c|}
\hline & Risiken \\
\hline Risiken für das Tier & - Fütterungsfehler \\
\hline beide & $\begin{array}{l}\text { - } \text { bauliche Mängel } \\
\text { - } \text { mangelndes Platzangebot } \\
\text { - } \quad \text { Eingriffe am Tier (z.B. kupieren, schnabelkürzen, enthornen u.a.) } \\
\text { - } \quad \text { fehlende Tierhygiene }\end{array}$ \\
\hline ethische Risiken & - Großbestände (Massentierhaltung) \\
\hline Verbraucher Risiken & - Rückstände von Medikamenten \\
\hline
\end{tabular}




\subsubsection{1 „Massentierhaltung“}

„Massentierhaltung“ ist ein Begriff, der von Verbraucherverbänden oder Tierschutzorganisationen herangezogen wird, um die heutigen Strukturen in der Landwirtschaft, subjektiv und stark negativ belastet, zu beschreiben (BLAHA, 2004). Es gibt keine klare Definition des Begriffs „Massentierhaltung“, aber er beinhaltet negative produktionstechnische Sachverhalte wie z.B.:

- $\quad$ ein hoher Tierbesatz pro Stallfläche,

- höchster Mechanisierungsgrad,

- Gefährdung von Tieren beim Ausfall technischer Einrichtungen,

- $\quad$ eine große regionale Tierdichte,

- Umweltbelastungen.

Die Strukturen in der Tierhaltung sind innerhalb Deutschlands und innerhalb der Tierarten (Rind, Schwein, Geflügel) sehr unterschiedlich. Besonders bei Schweinen und Geflügel gibt es Gebiete mit enormen Viehkonzentrationen und großen Beständen (z.B. Niedersachsen, Nordrhein-Westfalen). Vergleicht man die Zahlen Tiere pro Viehhalter, so lag Deutschland 2001 mit durchschnittlich 233,2 Schweine/Halter in der EU erst an 10. Stelle und beim Rindvieh mit 67,0 Rinder/Halter an 6. Stelle (ZMP-BILANZ, 2004A; 2004B).

Das Wohlbefinden der Tiere (in den Beständen) ist aber unter optimalen Bedingungen nicht abhängig von der Größe der Tierbestände und der Tierdichte in der Region. Die Bedingungen der Tierhaltung wie Management, Stallausstattung (Boden, Wände), Klimaregelung und Platzangebot pro Tier haben einen wesentlich stärkeren Einfluss. Auch anhand der Anzahl der gehaltenen Tiere in einem Betrieb kann keine Aussage über die Umweltgerechtigkeit der Haltungsmethode gemacht werden. Diese Anforderungen lassen sich vielfach in größeren Strukturen leichter realisieren, da häufig umfangreiche finanzielle Investitionen notwendig sind. Für kleine landwirtschaftliche Betriebe rechnet sich der Aufwand häufig nicht.

Beanstandungen, die im Rahmen von Kontrollen festgestellt wurden, liegen zwischen 14\% und 28\% (WITTKOWSKI u. HERMÜLHEIM, 2001; BMVEL, 2005A). Vor allem baulich Defizite wurden beanstandet. Mangelnder Platzbedarf oder kranke Tiere spielen bei den Nennungen keine Rolle. Genannt wurden:

- keine Beschäftigungsmöglichkeiten,

- fehlende Alarmanlagen,

- fehlende Notstromaggregate,

- fehlende oder mangelhafte veterinärmedizinische Aufzeichnungen bei Behandlungen,

- bauliche Mängel und

- Mängel bei der Tierkontrolle.

\subsubsection{Technopathien, Ethnopathien und Haltungsfehler}

Technopathien sind durch Haltungsverfahren bedingte Erkrankungen, Verletzungen bzw. Verhaltensstörungen. Ethnopathien sind Verletzungen, die infolge von Verhaltensabweichungen auftreten (WIESNER u. RIBBEK, 1991; DLG, 2000). Sowohl Technopathien als auch Ethnopathien entstehen als Folge von Bewegungsarmut bzw. Langeweile der Tiere. Typische Verhaltensstörungen oder Schädigungen bei den einzelnen Tierarten sind: 
Kannibalismus durch Schwanzbeißen, Verletzungen bei Rangkämpfen, Federpicken, Beinschäden u.a.

Tabelle 2.4-10: Ursachen und Maßnahmen bei Technopathien, Ethnopathien und Haltungsfehlern.

\begin{tabular}{|c|c|c|c|c|c|}
\hline & Tierart & Ursache & Maßna & imen & Literatur \\
\hline $\begin{array}{l}\text { Schwanz- } \\
\text { beißen } \\
\text { Rang- } \\
\text { kämpfe }\end{array}$ & $\begin{array}{l}\text { Rinder } \\
\text { Schweine }\end{array}$ & $\begin{array}{l}\text { Genetik } \\
\text { Klima } \\
\text { Besatzdichte } \\
\text { Fütterung } \\
\text { Nachahmung } \\
\text { Langeweile }\end{array}$ & $\begin{array}{l}\text { Kupieren der } \\
\text { Schwänze } \\
\text { Enthornen } \\
\text { (Rinder) } \\
\\
\text { Schnabel- } \\
\text { kürzen }\end{array}$ & $\begin{array}{l}\text { Optimierung von Stallklima, } \\
\text { Fütterung, Besatzdichte, } \\
\text { Beschäftigung, } \\
\text { Änderung der Zuchtziele } \\
\text { (vermindertes } \\
\text { Aggressionsverhalten). }\end{array}$ & $\begin{array}{l}\text { BARTUSSEK (2001);. } \\
\text { BADERTSCHER (2003); } \\
\text { HUNTER ET AL. (2001); } \\
\text { BROOKES (2004) } \\
\text { BEZIRKSREGIERUNG } \\
\text { WESER-EMS (2005); } \\
\text { HAFEZ (1997); } \\
\text { BERK U. COTTIN (2003), } \\
\text { BESTMAN, 2002; } \\
\text { MCADIE U. KEELING, 2002; } \\
\text { KJAER ET AL., 2001 }\end{array}$ \\
\hline $\begin{array}{l}\text { Bein- } \\
\text { schäden }\end{array}$ & $\begin{array}{l}\text { Rinder } \\
\text { Schweine } \\
\text { Geflügel }\end{array}$ & $\begin{array}{l}\text { Klima } \\
\text { falsche Einstreu } \\
\text { bzw. Bodenge- } \\
\text { staltung } \\
\text { hohe Wachs- } \\
\text { tumsleistung } \\
\text { Fütterungsfehler }\end{array}$ & & $\begin{array}{l}\text { Optimierung von Stallklima, } \\
\text { Fütterung, Besatzdichte, } \\
\text { Beschäftigung, } \\
\text { Bodengestaltung, } \\
\text { Änderung der Zuchtziele } \\
\text { (langsameres Wachstum, } \\
\text { weniger Massenzuwachs), } \\
\text { Förderung der } \\
\text { Bewegungsaktivität }\end{array}$ & $\begin{array}{l}\text { FinCHER ET AL. (1956); } \\
\text { FAULL ET AL. (1996); } \\
\text { GRANDIN (2000) WICKE ET } \\
\text { AL. (2001); BIZERAY ET AL. } \\
\text { (2002); }\end{array}$ \\
\hline
\end{tabular}

Aber nicht nur mangelhafte Haltungsbedingungen sind Ursachen für Verhaltensstörungen. Jahrzehnte wurden hauptsächlich Fehler im Management als Ursachen von Problemen, und somit auch Zucht bedingten Problemen, angesehen. Zuchtziele wurden nicht in Frage gestellt, sondern Haltungsbedingungen verändert. Beispiele, wie auf ein aggressives Sozialverhalten reagiert wurde, sind: Stromdrähte, Dämmerlicht und Rotlicht, aber auch Schnabelkürzen und Schwanzkupieren (MATHES u. IDEL, 2004). Ursachen und Maßnahmen bei Technopathien, Ethnopathien und Haltungsfehlern sind in Tabelle 2.4-10 aufgeführt.

Um die Verluste in der Tierhaltung zu reduzieren wurden Maßnahmen wie das Kupieren der Schwänze, das Schnabelkürzen beim Geflügel und das Enthornen bei den Rindern durchgeführt. Hierbei handelt es sich um massive Eingriffe am Tier, die mit Schmerzen verbunden sind. Schmerzempfindungen der Tiere beim Schnabelkupieren von Legehennen (HESTER u. SHEA-MOORE, 2003) und dem Enthornen (FAULKNER u. WEARY, 2000) wurden festgestellt. Typische Schmerzreaktionen beim Enthornen sind Kopfschütteln, Ohrenzucken und Kopfreiben (FAULKNER u. WEARY, 2000).

\subsubsection{Kastration}

Die Kastration ist ein weiterer massiver Eingriff am Tier und hat somit ein hohes ethisches Risikopotenzial. Kastrationen werden aufgrund von Produktverbesserungen (z.B. beim Schwein der Ebergeruch vergl. Kapitel 2.4.2.1.3) durchgeführt. Ebenso wie bei den vorab beschriebenen Eingriffen am Tier ist die Kastration von jungen Tieren ohne Betäubung ebenfalls mit Schmerzen verbunden (TAYLOR ET AL., 2001; HAY ET AL., 2003; BINDER ET AL., 2004A). Untersuchungen von TAYLOR ET AL., 2001, haben ergeben, dass der Eingriff der 
Kastration für sehr junge Ferkel genauso belastend ist wie für Ferkel, die erst nach 14 oder mehr Tagen kastriert werden. Eine Betäubung ist nach dem Tierschutzgesetz -TierSchG §5 Abs. 3 Nr.1 erst für das Kastrieren von über vier Wochen alten männlichen Rindern und Schweinen vorgeschrieben.

Die Kastration von männlichen Ferkeln ist z.B. in Deutschland, Frankreich oder der Schweiz weit verbreitet. In Irland, Großbritannien, Portugal und Spanien werden die Ferkel fast gar nicht mehr kastriert (LUY u. HILDEBRAND, 2003), und Norwegen hat ab 2009 ein Kastrationsverbot erlassen (BVET, 2003). Nach BRANSCHEID (2004) wird die Entwicklung in Deutschland dahin gehen, dass die Kastration zukünftig nur noch durch Tierärzte vorgenommen werden darf.

Alternativen zur Kastration sind:

- $\quad$ eine Kastration mit Betäubung,

- die Immunokastration,

- die Jungebermast bis zu einem Schlachtgewicht von $80 \mathrm{~kg}$,

- $\quad$ eine Aussortierung der Schlachtkörper am Schlachtband durch Geruchsprüfung,

- die Züchtung von Schweinen mit einem geringen Ebergeruch,

- $\quad$ Spermien-Sexing, Mast ausschließlich weiblicher Tiere (DUNSHEA ET AL., 2001; LUY u. HILDEBRAND, 2003; GutzWILleR, 2003; BaumgartNeR ET AL., 2004; BINDER ET AL., 2004B).

Alle diese Verfahren werden zurzeit in verschiedenen Ländern erprobt. Eine befriedigende Marktreife für den deutschen Markt hat bis jetzt noch kein Verfahren erreicht.

\subsubsection{4 Ökologischer Landbau/Freilandhaltung}

Der ökologische Landbau gilt allgemein als eine besonders ressourcenschonende und umweltverträgliche Wirtschaftsweise. Als besondere Kennzeichen gelten die artgerechten Haltungsverfahren, weitgehend geschlossene betriebliche Stoffkreisläufe und vielfältige Fruchtfolgen (BMVEL, 2002, 2003). Geregelt werden die Anforderungen für den ökologischen Landbau in der EG-Öko-Verordnung. Die Anforderungen der einzelnen Organisationen des ökologischen Landbaus in Deutschland gehen zum Teil weit über die Forderungen der EG-Öko-Verordnung hinaus (BMVEL, 2002).

Die extensive Tierhaltung und Freilandhaltung sind vom ökologischen Landbau geforderte Haltungsformen für Tiere. Auch der Verbraucher bevorzugt diese Haltungsformen; sie entsprechen am ehesten seinen ethischen Bedürfnissen. Untersuchungen haben jedoch ergeben, dass die Freilandhaltung nicht nur Vorteile birgt. Da die meisten Untersuchungen die Freilandhaltung von Legehennen betreffen, sollen diesbezügliche Veröffentlichungen als Beispiel für mögliche Probleme in dieser Haltungsform herangezogen werden. Die dort gemachten Feststellungen sind zum Teil auch für andere Produktionsformen und Tierarten gültig.

Bei der Freilandhaltung ist zu beachten, dass Haltungssysteme, in denen Tiere viele Kontaktmöglichkeiten zur Außenwelt haben, eine verstärkte Gefährdung bezüglich der Kontamination mit Keimen besteht (BAARS 2003; METHNER, 2004). Die Infektionsgefahr durch extern eingeschleppte Mikroorganismen, aber auch die interne Anreicherung der Mikroorganismen im Boden ist höher (PIETSCHMANN u. HAFEZ, 2002). Aufgrund der Zoonosenproblematik und der damit verbundenen Verbrauchergefährdung (bzw. eines durchzu- 
führenden Verbraucherschutzes ) sind die ökologischen Haltungssysteme kritisch zu hinterfragen und deren Vor- und Nachteile sorgfältig zu prüfen (FEHLHABER, 2003A).

Das Management in der Freilandhaltung hat sich in den letzen Jahren stark verbessert; trotzdem sind die durchschnittlichen Tierverluste in alternativen Hennenhaltungssystemen hoch. Ursachen sind bakterielle (z.B.Coliseptikämie, Pasteurella multocida Infektionen oder Rotlauf) und parasitäre Erkrankungen (z.B. mit Spul-, Haar- oder Rachenwürmern). Insgesamt werden Erkrankungen beobachtet, die jahrelang nicht mehr vorgekommen sind. Umfassende prophylaktische Maßnahmen sind unverzichtbar, um einen vertretbaren Gesundheitszustand der Hennen aufrechtzuerhalten (PETERMANN U. MAIWORM, 2004). Einen erhöhten Bedarf an Medikamenten bei der Boden- und Volierenhaltung konnten auch KREIENBROCK ET AL. (2004) in ihren Untersuchungen zur Legehennenhaltung feststellen. Zur Stabilität der Tiergesundheit in diesen Systemen sind weitere Anstrengungen erforderlich (METHNER, 2004). Der Kannibalismus untereinander ist im Gegensatz zu der Käfighaltung ausgeprägter (Pietschmann u. Hafez, 2002).

ELLENDORFF (2003) stellt im Rahmen einer Modellstudie zwischen hochintensiven und niedrigintensiven Masthähnchenproduktionssystemen fest, dass „hohe Produktionsintensitäten der Forderung nach Nahrungsmittelzuwachs bei gleichzeitigem Umweltschutz wesentlich besser nachkommt als niedrigintensive Produktionssysteme." Begründet wird diese These mit der geringeren Ausscheidung von Stickstoff und Phosphor, der besseren Energieeffizienz und dem geringeren Flächenbedarf in den intensiven Produktionssystemen (ELLENDORFF, 2003).

Untersuchungen in der Schweiz bei Mastschweinen, die in Ställen mit Vollspaltenbodenbuchten sowie Mehrflächensystemen mit eingestreutem Liegebereich und Auslauf gehalten wurden, haben gezeigt, dass Tiere, die in Vollspaltensystemen gehalten werden, signifikant mehr Hautwunden und Verletzungen durch Schwanzbeißen aufweisen. Zudem zeigten die Tiere in diesem Haltungssystem mehr Augenausfluss und Ohrenkratzer. In Mehrflächensystemen wurden signifikant mehr Tiere mit Lahmheiten und einer höheren Inzidenz von Husten und Infektionen mit Hämophilus parasuis (Glässersche Krankheit) beobachtet. Ebenso waren die Schweine teilweise von Sonnenbrand betroffen. Bei den Schlachttierkörpern aus Mehrflächensystemen wurden wesentlich mehr Beanstandungen bei Hüften und Gelenken festgestellt als bei Tieren auf Vollspaltenböden. Insgesamt lagen diese Beanstandungen aber auf einem tiefen Niveau ( $<0.5 \%$ der Tiere). In Mehrflächensystemen konnten eine kürzere Mastdauer, eine bessere Tageszunahme und eine geringere Tierverlustrate nachgewiesen werden, andererseits wurden eine schlechtere Futterverwertung und höhere Tierarztkosten festgestellt (BADERTSCHER, 2003).

Im Rahmen der Projekte der FAL zum Themenbereich Wechselwirkungen zwischen "Tier und Technik" sowie "Tier und Umwelt" wurden die Haltungssysteme (Schwein) Außenklimastall und dem Vollspaltenverfahren untersucht. In beiden Haltungsformen gab es gleich gute Lebendmassezunahmen. Im Gegensatz zu den Untersuchungen von BADERTSCHER (2003) konnte kein signifikanter Einfuss auf die Tiergesundheit festgestellt werden (HESSE, 1999).

Bei der Freilandhaltung von Rindern gibt es kaum Probleme. Freilandhaltung bei Rindern ist ein gängiges Haltungsverfahren. Bei einer ganzjährigen Freilandhaltung von Rindern kommt es im Winter zu einer Verdichtung des Bodens rund um den Fütter- und Tränkeplatz. Die Flächen in diesem Bereich sind meistens feucht und durch Exkremente belastet. Hier kann es zu einer Belastung von nahen Gewässern kommen (WASSMUTH ET AL., 2001). 


\subsubsection{Tiertransporte}

Tiertransporte geraten in regelmäßigen Abständen durch negative Schlagzeilen in das Interesse der Öffentlichkeit. Häufig zeigen die Bilder schwere Verstöße, aber auch in diesem Bereich werden Bilder gerne aus dem Zusammenhang gerissen. Hier kommt es dann zu einer einseitigen, bewusst negativen, Darstellung (TROEGER ET AL., 1998).

Trotz schon vorhandener umfangreicher gesetzlicher Regelungen (z.B. EU-Transportrichtlinie, Tierschutztransportverordnung, Viehverkehrsverordnung) in Europa werden von Seiten der Politik und der Tierschutzverbände weitere strengere und umfassendere Regelungen gefordert. Die Forderungen beziehen sich auf Transportzeiten, Wartezeiten, erlaubte Zuladungen, Ausstattung der Fahrzeuge sowie die Qualifikation des Personals. Einheitliche gesetzliche Regelungen in den unterschiedlichen Ländern gibt es nicht. In Europa sind relativ umfangreiche gesetzlichen Regelungen für den Transport von Tieren verfasst worden (vergl. v. RICHTHOFEN, 2003).) Allerdings sind nicht alle Bereiche geregelt und die vorhandenen Regeln noch nicht in allen EU Ländern umgesetzt. Außerhalb der EU gibt es nur wenige Regelungen. Zum Beispiel gibt es in Australien, Neuseeland und in den USA lediglich Empfehlungen für den Transport von landwirtschaftlichen Nutztieren (GRANDIN 2001B; STAMPER, 2004).

Eine Durchführung tierschutzgerechter Tiertransporte ist nicht nur für das Wohlbefinden der Tiere notwendig, sondern es sprechen hierfür auch starke wirtschaftliche Interessen. Es ist heute unumstritten, dass die Tiertransporte einen unmittelbaren Einfluss auf die Fleischqualität (PSE-, DFD-Fleisch, Hämatome) haben. Die Tiere werden unter den spezifischen Bedingungen des Transportes mit einer Vielzahl an Belastungsfaktoren (Stressoren) konfrontiert (TROEGER ET AL., 1998). Nach FISCHER (1994) und TROEGER ET AL. (1998) sind dies:

- Trennung von Artgenossen und gewohnter Umgebung,

- mechanische Belastungen (Schwachstellen bei den Verlade- und Transporteinrichtungen sowie dem Personal),

- $\quad$ physische bzw. motorische Belastung (Muskelarbeit) durch nicht gewohnte Ortsveränderungen,

- $\quad$ psychische/emotionale Belastungen (Angst vor unbekannten Situationen und Zusammenkommen mit fremden Tieren),

- Einschränkung der Bewegungsmöglichkeit auf dem Fahrzeug,

- Belastungen des Wärmehaushaltes (Kälte, Zugluft, Hitze, Schwüle),

- Belastungen durch Verdauungstätigkeiten (Transport unmittelbar nach der Futteraufnahme) und

- $\quad$ Aussetzen der Futter- und Wasseraufnahme.

Monogastriden wie Schwein und Geflügel reagieren wesentlich empfindlicher auf diese Belastungen als Wiederkäuer (Rind, Schaf), (TROEGER ET AL., 1998).

Die Belastung der Tiere macht sich in der Veränderung von biochemischen und physiologischen Kriterien bemerkbar, die heute auch messbar sind (TROEGER ET AL., 1998). Die Herz- und Atemfrequenz erhöht sich stark. Zum Beispiel kann die Herzfrequenz beim Heraustreiben von Schweinen aus der Bucht auf mehr als das doppelte des Ruhewertes (ca. 80 Schläge/Min.) ansteigen. Beim weiteren Beladevorgang (Treiben zum Fahrzeug u. Aufladen) steigt die Herzfrequenz teilweise bis weit über 200 Schläge/Min. Befindet sich das 
Tier auf dem Fahrzeug, sinkt die Herzfrequenz auf ca. 150 Schläge/Min. (FISCHER, 1994; TROEGER ET AL., 1998). Die Belastungsintensität beim Entladen kann je nach der Gestaltung des Abladevorgangs ähnlich hoch sein (FISCHER, 1994). Andere Faktoren, mit denen die Belastung gemessen werden kann, sind z.B. die Körpertemperatur, die Höhe der belastungsabhängigen Enzyme, der Gehalt an Cortisol und B-Endorphinen und die Veränderung des Hämatokritwertes (TROEGER ET AL., 1998).

In Deutschland wurden nach Mitteilung der für die Durchführung des Tierschutzgesetzes zuständigen obersten Länderbehörden $2001 \mathrm{ca}$. 640.000 Transportkontrollen durchgeführt (BMVEL TIERSCHUTZBERICHT, 2003). Festgestellt wurden ca. 40 verschiedene Arten der Zuwiderhandlungen. Hierunter fielen z.B.: Überladung; Überschreitung der Transportzeit; Überschreitung der vorgesehenen Tränk-, Fütter- und Ausruhzeiten; Transport transportunfähiger Tiere; gemeinsamer Transport unverträglicher Tiere; Mängel bei der Belüftung; fehlender Witterungsschutz; lange Standzeiten bei heißer Witterung; Mängel am Transportmittel/Fahrzeugmängel; fehlende Abtrennung; unzureichende Einstreu; unzureichende Reinigung und Desinfektion; fehlendes Desinfektionsbuch; u.a. Über die Anzahl der gefunden Beanstandungen sind keine Angaben vorhanden.

\subsubsection{Be- und Entladevorgänge}

Be- und Entladevorgänge sind bei einem ordnungsgemäß durchgeführten Transport die größte Belastung für die Tiere (FISCHER, 1994; SCHÜTTE ET AL., 1994). Die heutigen Masttiere sind aufgrund ihrer physischen Konstitution (hoher Muskelansatz, geringer Fettansatz und schnelles Wachstum bei Bewegungsarmut) nur in Grenzen belastbar. Durch die Be- und Entladevörgänge werden durch nervale und lokomotorische Belastungen Stoffwechselzustände induziert, die die Adaptionsfähigkeit des Tieres bis zur Grenze der Regulierbarkeit beansprucht (TROEGER, ET AL., 1998). Dieses kann unter Umständen den Tod der Tiere zur Folge haben.

Gerade die Be- und Entladevorgänge sind stark abhängig vom Verhalten der Treiber und den baulichen Gegebenheiten (Ställe, Treibwege, Verladeeinrichtungen und Fahrzeuge). In Deutschland darf nur derjenige gewerbsmäßig Tiere transportieren, der über einen Sachkundenachweis verfügt (Tierschutztransportverordnung, §13). Ursachen für Fehler bei der Verladung sind häufig (FISCHER, 1994; TROEGER ET AL., 1998; LAMBOOIJ, 2000; OfROU, 2001; GRANDIN, 2002A):

- Zeitdruck (fester Schlachttermin, nächster Transporttermin, weitere Zuladestellen),

- mangelnde ethologische Kenntnisse und praktische Erfahrungen (Ausnutzung des natürlichen Erkundungsverhaltens, des Herdentriebes und der Fluchtzone der Tiere),

- Hindernisse beim Ausstallen aus den Stallungen (z.B. Übersteigen des Troges),

- Treibwege mit scharfen Kanten oder Ecken (Verletzungsgefahr), schlechte Beleuchtung und scharfe Richtungsänderungen,

- fehlende Rampen oder mangelnde Ausstattung der Rampen bezüglich Breite, Stabilität, Rutschfestigkeit, Tierführung und Neigungswinkel (max. $20^{\circ}$ bei Schweinen, max. $25^{\circ}$ bei Rindern).

Auf die Benutzung von Elektrotreibern sollte verzichtet werden (TROEGER ET AL., 1998; LAMBOOIJ, 2000). Eine Anwendung des Elektrotreibers ist immer für das Tier mit Schmerzen verbunden und somit belastend (FISCHER, 1994; TROEGER ET AL., 1998). In Ausnahmefällen 
jedoch, wenn durchdachte und sanftere Techniken versagen, kann der besonnene Gebrauch des Elektrotreibers das kleinere Übel sein (FISCHER, 1994).

\subsubsection{Belegdichte}

Der Einfluss der Belegdichte auf das Stressempfinden der Tiere ist wissenschaftlich nachgewiesen. Zu wenig Platz bedingen häufig Hitzestress, Müdigkeit, eine schlechtere Fleischqualität, Verletzungen und eine höhere Anzahl an Transporttoten (BERG, 1998). Wenn sich nicht alle Schweine hinlegen können, werden diese in ihrem Ruheverhalten gestört. Tiere verbringen sitzend die Fahrt oder weichen nach oben aus. Sie legen sich auf andere Tiere oder treten auf deren Gliedmaßen. Rinder können die Kräfte, die durch Anfahren, Bremsen und Richtungsänderungen entstehen, nur schwer ausbalancieren. Sie geben diese Kräfte an ihre Nachbarn weiter. Tiere können dabei stürzen und haben keine Möglichkeit wieder aufzustehen. Diese Vorgänge führen zu vermehrtem Stress, zu Verletzungen und Schmerzen (FISCHER, 1994; TROEGER ET AL., 1998). Zu viel Platz ist ebenfalls zu vermeiden, da bei plötzlichen Stopps und Kurven die Tiere das Gleichgewicht verlieren und stürzen können, was zu vermehrten Verletzungen führen kann (BERG, 1998).

Ladedichten für alle Tierarten sind in den EU-Richtlinien 91/628/EWG, 95/29EWG und in der TierSchTr-VO für den Straßen-, Schienen- und Schiffstransport geregelt.

\subsubsection{Klima}

Das Klima hat zum einen Einfluss auf den Belastungsstatus der Tiere während der Fahrt, und zum anderen bestimmt es auch mit, in welcher Kondition die Tiere überhaupt zum Transport gelangen und wie gut sie sich während der Wartezeit am Schlachthof erholen können (TROEGER ET AL., 1998). Fehlende oder unzureichende Möglichkeiten der Klimasteuerung führen zu Verlusten bei Tieren. In vielen Tiertransportern fehlen Ventilatoren zum Temperaturausgleich. Die Belüftung erfolgt alleine durch die Bewegung der Fahrzeuge (LAMBOOIJ, 2000).

Negativ wirken sich zu hohe als auch zu niedrige Temperaturen aus. Einen Anstieg der Mortalität in Abhängigkeit von der Temperatur ist sowohl für Schweine als auch für Geflügel nachgewiesen (DEWEY ET AL., 2004; NIJDAM ET AL., 2004; WERNER ET AL., 2005). Rinder sind nicht so temperaturempfindlich. Sie besitzen ein besseres Thermoregulationsvermögen (starkes Schwitzen) und werden dadurch nicht so stark belastet (TROEGER ET AL., 1998).

Aber nicht nur die Temperatur allein hat einen Einfluss auf die Verluste bzw. Fleischqualitätsmängel. Steigt neben der Außentemperatur auch der sogenannte Schwülefaktor (Temperatur + zweifacher Dampfdruck), wird es für die Tiere immer schwieriger überschüssige Wärme abzugeben (FISCHER, 1994). Bei Schweinen wurden durchschnittliche Verluste im Jahr von ca. $0,17 \%$ festgestellt. Mit steigender Temperatur erhöhen sich die Verluste bei Temperaturen über $20^{\circ} \mathrm{C}$ auf $0,4 \%$, bei Temperaturen zwischen $26^{\circ} \mathrm{C}$ und $28^{\circ} \mathrm{C}$ auf $0,6 \%$ und über $28^{\circ} \mathrm{C}$ auf $0,76 \%$ (DEWEY ET AL., 2004). Bei Geflügel bewirken Temperaturen $>15^{\circ} \mathrm{C}$ signifikant höhere Transportverluste (NIJDAM ET AL., 2004).

Ebenso haben niedrige Temperaturen einen negativen Einfluss auf das Wohlbefinden der Tiere während des Transportes. Niedrige Temperaturen tragen dazu bei, dass die Tiere während des Transportes nicht zur Ruhe kommen und überdurchschnittlich hohe Herzfre- 
quenzwerte aufweisen (TROEGER ET AL., 1998). Deutlich höhere Transportverluste bei Geflügel wurden bei Temperaturen von $<5^{\circ} \mathrm{C}$ festgestellt (NIJDAM ET AL., 2004).

Standards für Klimabedingungen bei Tiertransporten sind 1999 von der EU erarbeitet worden. In ihren Empfehlungen fordert der wissenschaftliche Ausschuss z.B. (EU, 1999):

- Überwachungssysteme, mit denen die Temperatur und die Luftfeuchtigkeit erfasst werden und bei Abweichungen Alarm ausgelöst wird,

- $\quad$ elektrisch betriebene Belüftungssysteme bei Standzeiten,

- regelbare Belüftungssysteme zur Sicherstellung von Klimabedingungen unterhalb der festgelegten Grenzwerte, ggf. im Winter Heizsysteme,

- Belüftungssysteme, die eine ausreichende Luftbewegung im Fahrzeug (aber keinen Zug) sicherstellen (Minimalluftfluss $10 \mathrm{~m}^{3} / \mathrm{h} / 100 \mathrm{~kg}$ Körpergewicht).

\subsubsection{Transportdauer/Wegstrecke}

Ein Einfluss auf das Wohlbefinden der Tiere ist sowohl bei kurzen $(\varnothing 1 \mathrm{~h})$ sowie auch bei langen ( $\varnothing$ 8h) Transportzeiten nachgewiesen. In einer Untersuchung von WERNER ET AL. (2005) wurden die Transport- und Wartestallverluste, Kreislaufschwächen und Frakturen in Abhängigkeit von der durchschnittlichen Fahrzeit ermittelt (Abbildung 2.4-4). Sowohl kurze als auch lange Transporte führen bei Schweinen zu erhöhten Verlusten. Negative Einflüsse (Tote, erhöhter PSE-Anteil, Frakturen) in Abhängigkeit von den Transportzeiten haben auch SCHÜTTE ET AL. (1994), LEHESKA ET AL. (2003) und GUARDIA ET AL. (2004) festgestellt. Dagegen haben WARRISS U. BROWN (1994) und DEWEY ET AL. (2004) keinen Einfluss der Fahrstrecke auf z.B. die Anzahl der toten Tiere festgestellt.

Rinder werden zum Teil über längere Strecken transportiert. Die deutsche Tierschutztransportverordnung vom 25. Februar 1997 (BGBI. I, S. 348) schreibt auf der Grundlage der Transportrichtlinie der EU bei Rindertransporten mit Spezialfahrzeugen nach einer Transportzeit von 29 Stunden (14 h Transport, $1 \mathrm{~h}$ Versorgungspause, $14 \mathrm{~h}$ Transport) ein Ausruhen der Tiere für 24 Stunden vor. Dabei sind die Tiere abzuladen und in einer Versorgungsstation zu versorgen. Pausen während der Tiertransporte werden von verschiedenen Wissenschaftlern unterschiedlich beurteilt. Nach GRANDIN (2001B) muss darauf geachtet werden, dass Pausen nicht zu Belastungen der Tiere führen. In den USA durchgeführte Untersuchungen haben gezeigt, dass die Gesundheit von $250 \mathrm{~kg}$ schweren Weidekälbern nach einem 32 stündigen Transport besser war, als wenn diese zwischenzeitlich abgeladen wurden und ausgeruht waren. Bei Transportzeiten über 32 Stunden war allerdings ein positiver Effekt des Abladens und Ausruhens feststellbar. Bei Schweinetransporten sind Pausen durchaus förderlich. Die Schweine sollten aber auf dem Fahrzeug verbleiben und sich alle hinlegen können. Bei hohen Temperaturen ist es allerdings wichtig, dass die Tiere beim Stopp gekühlt werden (GRANDIN, 2001B).

Nach dem Vorschlag der EU über den Schutz von Tieren beim Transport (2003) darf im Falle von Straßentransporten die Fahrtzeit neun Stunden nicht überschreiten. Die Ruhezeiten müssen mindestens 12 Stunden betragen. Die neunstündige Fahrtzeit schließt eine 45minütige Pause für den Fahrer, während der das Fahrzeug steht, nicht ein (ANONYMUS, 2003A; EU, 2003B). Hinsichtlich der absoluten Tiertransportzeiten gibt es innerhalb der EU unterschiedliche Ansichten. Etwa die Hälfte der Mitgliedstaaten will die absolute Tiertransportzeit auf acht Stunden begrenzen. Die übrigen EU-Länder wollen an der derzeitigen 
Zyklusregelung festhalten (ANONYMUS, 2004C). Die oben genannte Verordnung wird deshalb aufgrund von Meinungsverschiedenheiten zwischen den einzelnen EU-Ländern in naher Zukunft nicht in Kraft treten (WERNER ET AL., 2005).

Der 23. Deutsche Tierärztetag (2003) fordert, dass alle Schlachttiertransporte mit einer voraussichtlichen Dauer von mehr als 4 Stunden oder über eine Entfernung von mehr als $300 \mathrm{~km}$, unabhängig von ihrem Zielort, durch einen amtlichen Tierarzt vor Transportbeginn überprüft und das Ergebnis in einer amtlichen Transportbescheinigung dokumentiert werden soll. Folgende Vorteile werden darin z.B. gesehen:

- Der Transport mit einer Dauer von mehr als 4 Stunden ist als Ausnahme anzusehen.

- $\quad$ Die Belastung der Tiere während der Beladung kann durch die Anwesenheit des amtlichen Tierarztes auf das unvermeidliche Ausmaß reduziert werden.

- $\quad$ Es erfolgt eine ständige Kontrolle der Höchstladedichten.

- Die Transportfähigkeit der Tiere für den konkreten Transport wird zweifelsfrei und unabhängig von wirtschaftlichen Interessen festgelegt.

- $\quad$ Es wird sichergestellt, dass geeignete Transportmittel eingesetzt werden (BTK, 2003).

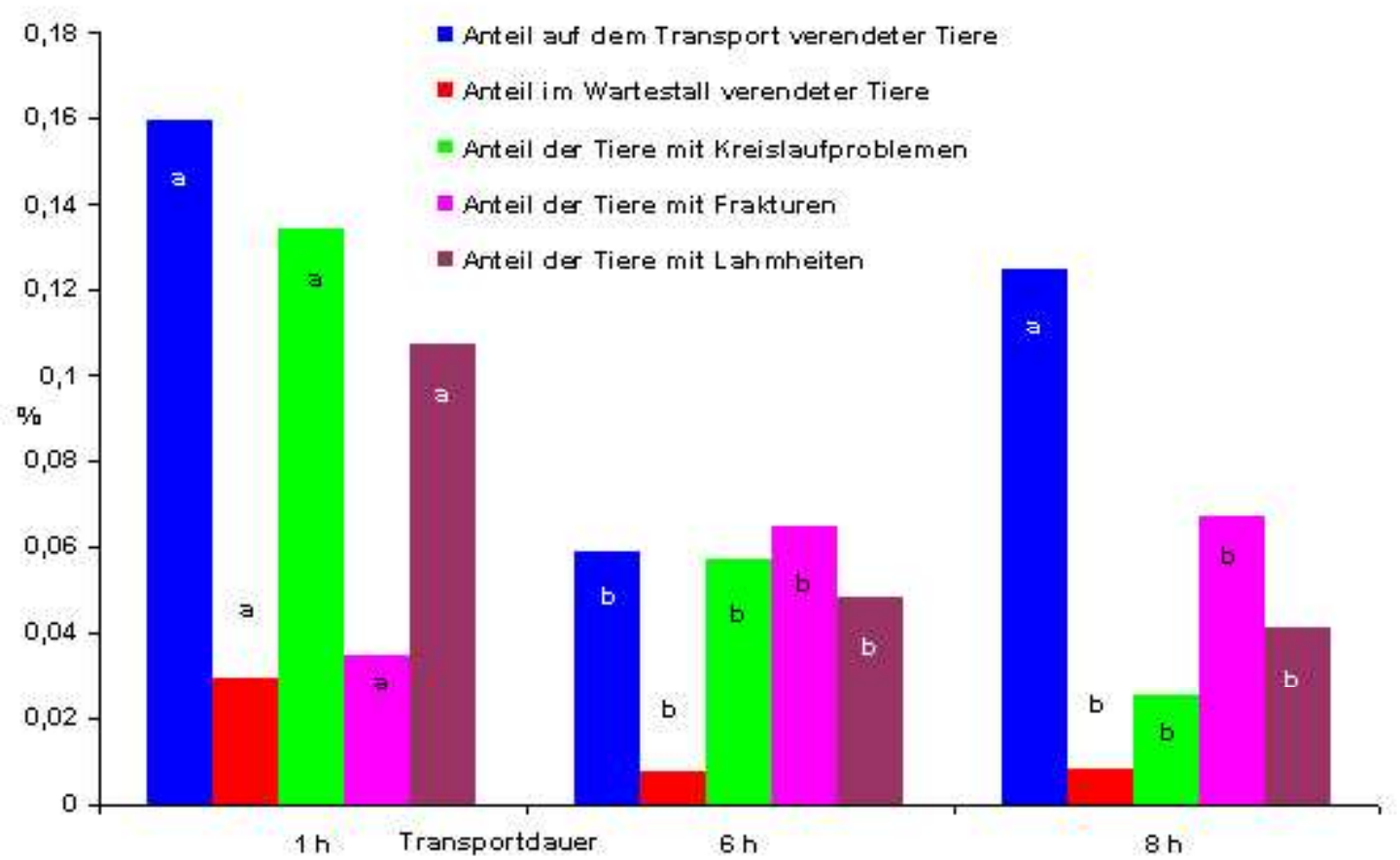

Abbildung 2.4-4: Mittelwerte der Anteile verendeter Schweine oder von Tieren mit Kreislaufproblemen, Frakturen oder Lahmheiten im Jahr 2003 in Abhängigkeit von der durchschnittlichen Transportdauer $(n=319.005)$. Säulen mit unterschiedlichen Buchstaben $(a, b)$ zwischen den Jahren unterscheiden sich signifikant $(P<0,05)$ (WERNER ET AL., 2005).

\subsubsection{Transportschäden}

Unter den Transportschäden sind die Tierverluste besonders wichtig. Betroffen sind häufig stressanfällige Schweine, Sauen und alte Kühe (GRANDIN, 2001B). DEWEY ET AL. (2004) berichten, dass 2001 0,17\% der transportierten Schweine in Ontario während des Transportes gestorben sind. In einer Veröffentlichung von WARRISS u. BROWN (1994) sind 0,072\% 
von 2,9 Millionen transportierten Schweinen beim Transport gestorben. LAMBOOIJ (2000) berichtet von 0,1 bis $0,4 \%$ transporttoten Schweinen in den USA, Kanada und Europa. In Europa liegen nach WARRISS (1998) die transportbedingten Mortalitäten von Schlachtschweinen unterschiedlich hoch. In Deutschland waren 0,5\% der Schlachtschweine auf dem Transport verendet. Dagegen lag die Anzahl der Transporttoten in Dänemark nur bei 0,03\%. Nach einer Veröffentlichung von DANISH (2004) liegt in Dänemark die Streblichkeitsrate während des Transportes 2003 sogar nur bei 0,0017\%. Mehr als 95\% der dänischen Mastschweine werden weniger als 3 Stunden transportiert. WERNER ET AL. (2005) stellten bei ihren Untersuchungen fest, dass in dem von ihnen ausgewerteten Datenmaterial der Anteil an verendeten Schweinen im Zeitraum von 1999 bis 2003 signifikant von 0,15\% auf 0,08\% gesunken ist. GRANDIN (2002B) berichtet von einer Reduzierung der toten Tieren, wenn LKW-Fahrer für eine geringe Rate an toten Tieren prämiert werden. Ebenso gingen Prellungen zurück, wenn Mitarbeiter finanzielle Abzüge dafür erhielten.

Bei Broilern findet man in der Literatur Zahlen über transporttote Tiere, die zwischen 0,19\% und 0,56\% liegen (Alvarez, 1981; Bayliss u. Hinton 1990; Gregory u. Austin, 1992). GRAY ET AL. (2004) berichten, dass eins von vier in den USA produzierten Hähnchen Prellungen an Beinen, Brust oder Flügeln während des Fangens und des Transportes erleiden.

Weitere Verluste entstehen durch die Belastung während des Transportes durch Hämatome, Verletzungen und Fleischmängel wie PSE, DFD, DCB, (GRANDIN, 1990; BIDNER 2003). Neben der genetischen Veranlagung der Tiere zu PSE (siehe Kapitel, 2.4.2.1.1) hat gerade die Handhabung der Tiere vor, während und nach dem Transport einen wesentlichen Einfluss auf die Ausprägung.

\subsubsection{Schlachtprozess}

Die Schlachtung gehört zu den am besten geregelten bzw. untersuchten Bereichen in der Produktionskette Fleisch. Zahlreiche Untersuchungen befassen sich mit der Schlachthygiene, der Gewinnung von hygienisch einwandfreiem Fleisch. Bewertungsverfahren wie die Schlachtkörperuntersuchung, Kühlzeiten, Keimzahlbestimmung u.a. sind seit Jahren etabliert. Hinzu kommen Audits bzw. Kontrollen durch Kunden, Zertifizierungsgesellschaften und Behörden. Diese gehören in diesem Teil der Produktionskette zum Standard. Seit 1994 sind Schlachtbetriebe in Deutschland gesetzlich dazu verpflichtet ein Eigenkontrollsystem zu implementieren. Die im $\S 4$ LMHV aufgeführten 5 Grundsätze der Eigenkontrollen folgen dem HACCP-Konzept, es fehlt aber die Forderung der Verpflichtung zur Dokumentation. Dies wird durch die Verordnungen EU 178/2002 und EU 852/2004 geändert. Hier wird ein HACCP-Konzept nach den Grundsätzen des Codex Alimentarius ab dem 01.01.2006 in der gesamten EU gefordert.

Innerhalb des Schlachtprozesses bergen die einzelnen Arbeitsschritte unterschiedliche Risiken. Es kann zu physikalischen Gefahren (z.B. abgebrochene Messer oder Maschinenteile, Glassplitter, Kunststoff- und Plastikteile oder Knochensplitter) oder zu chemischen Gefahren (z.B. Farbanstriche, Rohrbahnfett, Lösungsmittel, Schädlingsbekämpfungs-, Reinigungs- und Desinfektionsmittelrückstände) kommen. Das Risiko der physikalischen, aber auch der chemischen Gefahren ist unter Beachtung der Einhaltung der allgemeinen Sorgfaltspflicht (z.B. regelmäßige Wartung von Anlagen, Geräten usw., sorgfältige Anwendung und Einhaltung der Reinigungs-, Desinfektions- und Schädlungsbekämpfungsmaßnahmen) als gering einzustufen (LEITLINIE BADEN-WÜRTTEMBERG, 2004). Eine wichtigere 
Rolle spielen in der Schlachtung die mikrobiologischen Gefahren, die aufgrund der üblichen Schlachttechnologie nicht auszuschließen sind. Die Menge der Keime ist dabei abhängig von der Einhaltung der guten Hygienepraxis, aber auch von der Kontamination der Schlachttiere (SeidleR, 1998; ReUter, 2003; Leitlinie BAdEN-WüRTTEMBERG, 2004). Einmal auf das Fleisch gebrachte Keime können im Verlauf des Schlachtprozesses und der nachfolgenden Lagerung der Tierkörper weder beseitigt noch auf ein annehmbares Maß reduziert werden. Allerdings kann die Einhaltung einer guten Hygienepraxis, die unverzügliche Kühlung des Fleisches und die gute Abtrocknung der Oberfläche, der weiteren Vermehrung von Keimen entgegenwirken. Unter der Annahme, dass die vorab genannten Punkte eingehalten werden, kann das Risiko als gering eingestuft werden (LEITLINIE BADEN-WÜRTTEMBERG, 2004). Neben den biologischen, physikalischen und chemischen Risiken gibt es in der Schlachtung auch noch Punkte, die ein ethisches Potenzial beinhalten. Da sich diese Punkte auf den Fleischkonsum auswirken können, sollen diese mit behandelt werden.

Nachfolgend sollen die biologischen und die ethischen Risiken betrachtet werden, da sich hier eine Auswirkung nachträglich nicht beseitigen oder auf ein annehmbares Maß reduzieren lässt.

\subsubsection{Mikrobiologische Risiken}

In jedem Schlachtprozess gibt es Schwachstellen, an denen die vorgeschriebene Hygiene nicht eingehalten werden kann und die Gefahr der Übertragung von pathogenen Keimen besteht. Diese sind:

- Eintrag über das lebende Tier und die Kreuzkontamination der Tiere in Stall untereinander,

- Kreuzkontamination durch die Schlachttechnologie,

- Übertragung durch Schlachtfehler,

- $\quad$ mangelnde Kühltechnologie.

Die Kontaminationsgefahr der Tiere in den Warteställen untereinander oder über die Einrichtungen ist ein nicht zu unterschätzendes Hygienerisiko (SEIDLER, 1998; REUTER, 2003; WEISE, 2003). Nach WEISE (2003) werden gerade bei der Anlieferung von Geflügel auf dem Schlachthof massenhaft fäkale Keime gefunden. Käfige mit Tieren werden übereinander gestapelt. Durch Transportkäfige mit perforierten Deckeln und Böden und der Stapelung der belegten Käfige in mehreren Lagen übereinander kommt es zu einer Verunreinigung der Tiere durch Kot. Durch mangelnde Nüchterungszeiten vor der Schlachtung wird dieses verstärkt.

Die EU VO 853/2004 Abschnitt 3 regelt, dass Schlachtbetriebe Schlachttiere nur dann zur Schlachtung annehmen dürfen, wenn der Herkunftsbetrieb höchstens 24 Stunden vor der Ankunft der Tiere im Schlachtbetrieb die „Informationen zur Lebensmittelkette“ übermittelt hat (STETTER, 2004). Diese Daten umfassen z.B. Informationen über den Gesundheitszustand der Tiere, eventuelle medikamentöse Behandlungen, Ergebnisse von Zoonosen und Rückstandsuntersuchungen u.a. Bei Geflügel ist, im Gegensatz zu Schweinen und Rindern, ein Begleitattest des Tierarztes schon gesetzlich vorgeschrieben (WEISE, 2003). Aber auch auf die Sauberkeit der angelieferten Tiere hat das neue Hygienepaket einen Einfluss. Gemäß der EU VO 853/2004 hat der Lebensmittelunternehmer dafür zu sorgen, dass die Haut oder das Fell von Tieren, die geschlachtet werden, sauber ist. Es dürfen keine Tiere 
geschlachtet werden, bei denen ein nicht vertretbares Risiko einer Kontaminierung des Fleisches während der Schlachtung besteht. Zur nationalen Umsetzung fehlen zurzeit noch die Umsetzungsverordnungen. Übergangsregelungen sind bis Dezember 2009 möglich (BERTLING, 2006).

Waschungen von Tieren, direkt vor oder während der Schlachtung, zur Reduzierung der Keimzahlen sind unzweckmäßig. Die Zufuhr von Wasser begünstigt die Vermehrungsbedingungen der Mikroorganismen oder sich im Ruhestadium befindliche Mikroorganismen werden zum Wachstum angeregt (REUTER 2003).

Eine weitere Kontaminationsquelle besteht in den sich anschließenden Prozessen, z.B. Brühen bei Schweinen und Geflügel, dem Fellabzug bei den Rindern oder durch eine Übertragung von Schlachtkörpern untereinander bzw. über die Schlachteinrichtungen. Diese sogenannten Kreuzkontaminationen kommen in allen Schlachtprozessen vor. Ursächlich hierfür sind meistens Maschinen oder Geräte, die sich aufgrund ihrer Konstruktion nicht richtig reinigen und desinfizieren lassen (z.B. Peitschenmaschine, Kratzmaschine) bzw. eine Reinigung zwischen den einzelnen Schlachtkörpern nicht möglich ist. Aufgrund der hohen Technisierung und den fehlenden Möglichkeiten, die Maschinen ausreichend nach jedem Tier zu desinfizieren, gibt es in der Geflügelschlachtung kaum Produktionsschritte, an denen eine Kreuzkontamination während der Schlachtung wirksam verhütet werden kann. Eine andere Ursache ist das Brühwasser (Brühkessel oder Wasserbad), das während des Schlachtprozesses nie komplett erneuert wird (REUTER, 2003).

Ein weiterer Weg ist die Übertragung bei Schlachtfehlern. Hier ist bei allen drei Tierarten besonders der Prozessschritt Entnahme des Magen-Darmtraktes kritisch zu bewerten. In diesen Organen befinden sich eine Vielzahl von pathogenen Keimen. Wenn diese Organe bei der Entnahme verletzt werden, beim Rind (Speiseröhre und Darm) oder Schwein (Darm) nicht richtig verschlossen werden, kann es zu einer Kontamination des Schlachtkörpers und somit des Fleisches kommen (PINGEL ET AL., 1998; SEIDLER, 1998; TROEGER, 1998; ReUTER, 2003; WEISE, 2003). Beim Rind ist ein weiterer Kontaminatonsschwerpukt das Vorschlachten und der Fellabzug. Durch unsaubere Tiere und/oder einer mangelhaften Schlachttechnik des Mitarbeiters kommt es zur Übertragung von pathogenen Keimen vom Fell auf den Schlachtkörper.

Die Kühlverfahren haben sich in den letzten Jahren immer weiter verbessert. Bei Rindern und Schweinen werden tiefe Temperaturen und Luftbewegungen genutzt, um die Schlachtkörper in relativ kurzer Zeit herunterzukühlen. Es darf allerdings zu keinem Gefrieren der oberflächlichen Gewebeschichten mit der Ausnahme der Ohren kommen. Entscheidend ist, dass die Schlachtkörper möglichst schnell heruntergekühlt werden, um so die Vermehrung der vorhandenen Mikroorganismen zu verhindern. Abtrocknung der Oberflächen, niedrige Temperaturen und ein Absenken des pH-Wertes während der Kühlung spielen dabei eine wichtige Rolle (KRÖCKEL u. HECHELMANN, 1998; REUTER, 2003).

Im Gegensatz zu der Kühlung bei Rindern und Schweinen ist der Kühlvorgang beim Geflügel ein wesentlich sensiblerer Prozess. Für die Kühlung werden Luftkühl- und Luftsprühkühlverfahren oder Tauchkühlverfahren eingesetzt. Luftkühl- und Luftsprühkühlverfahren bergen hygienische Risiken durch Aerosolbildung sowie durch mangelnde Reinigungs- und Desinfektionsmöglichkeiten der Kühlräume im Mehrschichtbetrieb. Im Tauchkühlverfahren werden die Schlachtkörper mechanisch und kontinuierlich entgegen der Kühlwasserströmung transportiert. Der Nachteil dieses Verfahrens ist der sehr hohe Wasserverbrauch, der bei ca. 4 
Liter pro Tierkörper liegt und das Risiko der Kreuzkontamination. Aus diesem Grunde muss in der EU bei Tauchkühlverfahren mit mikrobiologischen Methoden kontrolliert werden, ob diese einwandfrei funktionieren (WEISE,2003).

Insgesamt sind Prozessuntersuchungen erforderlich, um die für die Weiterverbreitung von pathogenen Keimen relevanten Schlachtabschnitte zu identifizieren und Interventionsmaßnahmen zu erarbeiten. Allerdings sind die kritischen Hygienepunkte, die eine mögliche mikrobielle Kreuzkontamination fördern, je nach angewendeter Schlachttechnologie und baulicher Einrichtung der Schlachtanlage von Betrieb zu Betrieb an unterschiedlichen Stellen zu finden (BFR, 2005B; MetHNER, 2004).

\subsubsection{Ethische Risiken}

Im Bereich Schlachtung spielen neben den hygienischen Aspekten aber auch ethische Aspekte eine entscheidende Rolle. Gerade die Bereiche, bei denen mit dem lebenden Tier umgegangen wird (Tierannahme, Wartestall, Betäubung und Tötung), bergen ein hohes Risikopotenzial (WOLTERSDORF,1994; TROEGER ET AL., 1998; GRANDIN, 2001B, 2004; u.a.).

Beim Umgang mit dem lebenden Tier auf dem Schlachthof können nachfolgende Punkte zu Beeinträchtigungen der Tiere bzw. zu Problemen führen:

- Lärm,

- Beleuchtung,

- Klima (Zugluft, Luftfeuchtigkeit, Temperaturen),

- Versorgung der Tiere (fehlende Tränken bzw. Fütterungsmöglichkeiten bei liegen gebliebenen Tieren),

- Bauliche Gegebenheiten (Treibgänge mit scharfem Richtungswechsel, rutschiger Boden u.a.),

- Buchtenbelegung,

- Treibhilfen (Elektrotreibstab),

- Fehltötungen.

Die Warteställe in den Schlachthöfen bieten den Tieren vielfach keine Möglichkeiten sich von den Strapazen des Transportes zu erholen. In den Ställen ist es vielfach laut und eine Möglichkeit zur Klimaregelung besteht selten (WOLTERSDORF, 1994; SCHÄFFER u. V. BORELL, 2002). Aufgrund der Bedeutung der Lebendtierhandhabung für die Fleischqualität sind die Stallanlagen vermehrt in das Interesse der Schlachthofbetreiber gerückt. Umbaumaßnahmen und neue Konzepte werden an verschiedenen Schlachthöfen umgesetzt. Ein wesentliches Manko in dem Bereich der Lebendtierhandhabung ist, dass kaum objektive Kontrollkriterien bzw. Bewertungsverfahren in den Bereichen Viehannahme und Wartestall vorhanden sind (SCHÄFFER u. V. BORELL, 2002). Zur Beurteilung der Bereiche Viehannahme und Wartestall bieten sich an (GRANDIN, 2001A; SCHÄFFER u. V. BORELL, 2002; 2005):

- Lautstärke im Stall bzw. Vokalisation der Tiere und

- bauliche Voraussetzungen (Treibganggestaltung, Lichtquellen u.a.).

Lärm, falsche Beleuchtung, Zugluft und/oder Fehler bei der Treibganggestaltung hindern Schlachttiere sich ohne Probleme in eine gewünschte Richtung zu begeben (GRANDIN, 1996; SCHÄFFER u. v. BORELL, 2002). Dieses führt zu Stress und damit zu Abweichungen in der 
Fleischqualität. Um die Tiere in die gewünschte Richtung zu bewegen, werden häufig Elektrotreiber eingesetzt ${ }^{2}$. Der Elektrotreibstab wird dann nicht wie gesetzlich vorgeschrieben ausschließlich im Bereich der Hinterbeinmuskulatur eingesetzt, sondern am gesamten Tier. Der Einsatz des Elektrotreibers ist eine der Hauptursachen für eine verstärkte Vokalisation der Tiere. Bauliche Mängel und fehlerhaftes Treibmanagement führen zu einer verstärkten Vokalisation (GRANDIN, 2001A; SCHÄFFER u. BORELL, 2004A u. 2005).

Der ethisch sensibelste Bereich auf dem Schlachthof ist die Betäubung und Tötung der Tiere. Denn alle eingesetzten Betäubungsverfahren sind beim Tier mit physiologischen Stress verbunden (PERES, ET AL., 2005). Die Betäubung stellt in erster Linie eine Tierschutzmaßnahme dar, mit dem Ziel das Wahrnehmungs- und Empfindungsvermögen der Schlachttiere auszuschalten, bis der Tod durch Blutentzug eingetreten ist (MÄNNL, 1994). Die verschiedenen Betäubungs- bzw. Tötungsverfahren werden vom Verbraucher zurzeit meistens verdrängt. Allerdings werden zukünftig die Betäubung und Entblutung eine zunehmende Bedeutung erlangen (MOJE, 2004).

In Deutschland ist bei Rindern die Betäubung per Bolzenschuss am weitesten verbreitet. Nach vON HOLLEBEN ET AL. (2002) gilt die Betäubung per Bolzenschuss als ein bewährtes und sicheres Betäubungsverfahren, wenn sie korrekt durchgeführt wird. Risiken des Bolzenschusses sind:

- Fehlschüsse,

- Verletzungsgefahr des Personals,

- Übertragung von Gehirnmaterial in den Körper (ANIL u. HARBOUR, 2001; HILDEBRAND, 2002; FRIES, 2003).

Als zweite Methode ist in Deutschland die Elektrobetäubung bei Rindern zugelassen. Diese hat sich aufgrund fehlender technischer Umsetzung aber nicht durchgesetzt. Sie bietet gerade im Hinblick auf die Problematik des spezifischen Risikomaterials eine wichtige Alternative zur Betäubung mit dem Bolzenschuss (ANONYMUS, 2002D, TROEGER, 2002). Eine Elektrobetäubung bei Rindern erfolgte in Deutschland Ende 2004 an vier Schlachthöfen (MOJE, 2004). In anderen Ländern wie Neuseeland, Australien und Russland ist die Elektrobetäubung bei Rindern wesentlich mehr verbreitet (ANONYMUS, 2002D).

Bei Schweinen erfolgt die Betäubung durch Gas oder elektrischen Strom. Über die Vorteile und Nachteile beider Methoden gibt es umfangreiche Publikationen. Es gibt in der Branche einen allgemeinen Streit darüber, welche der Methoden die bessere ist. Wie unterschiedlich die Bewertungen sind, zeigt ein Vergleich zwischen den Niederlanden und Dänemark. In den Niederlanden war die $\mathrm{CO}_{2}$-Betäubung bis vor kurzem verboten. Dieses wird begründet mit einer unzureichenden Betäubungseffektivität, den Kosten und den negativen Einflüssen auf die Fleischqualität. Heute werden $\mathrm{CO}_{2}$-Anlagen in den Niederlanden eingesetzt, z.B. bei Boxtel/VION/DUMECObv (RANGNICK, 2005). In Dänemark hat man sich dagegen für die $\mathrm{CO}_{2}$-Betäubung entschieden. Als positive Kriterien werden hier z.B. aufgeführt: eine sichere Betäubung, wenig Unbehagen der Tiere, keine Beeinträchtigung der Qualität des Fleisches u.a. (SCHÄFFER u. V. BORELL, 2004B).

\footnotetext{
${ }^{2}$ Nach Richtlinie 93/119/EG ist die Verwendung von Elektroschockgeräten möglichst zu vermeiden.

Die Geräte müssen sich nach maximal einer Sekunde abschalten.
} 
Ein Kritikpunkt der $\mathrm{CO}_{2}$ - Betäubung ist, dass die Zeit vom Ende des Betäubungsvorganges bis zum Stechen von 20 Sekunden (Tierschutz-Schlachtverordnung), bei den sogenannten Backloder-Systemen nicht eingehalten werden. Die Tiere werden hier gruppenweise betäubt und das Stechen erfolgt bei den letzten Tieren unter Umständen erst nach 60 Sekunden (HOLST, 2001). Von MACHOLD ET AL. (2003) wird das $\mathrm{CO}_{2}$-Gas aufgrund seiner schleimhautreizenden und atemstimulierenden Wirkung (subjektives Erstickungsgefühl) kritisch gesehen. Dagegen stellten VELARDE ET AL. (2001) in Untersuchungen in Spanien fest, dass der Anteil an PSE-Fleisch nach der Elektrobetäubung (36\%) signifikant höher lag als bei der $\mathrm{CO}_{2}$ Betäubung (4,5\%). Allerdings lag der Anteil an DFD-Fleisch nach der $\mathrm{CO}_{2}$-Betäubung signifikant höher $\left(6,1 \% \mathrm{CO}_{2}\right.$ - und 0,6\% Elektrobetäubung). Besser als die Elektrobetäubung schnitt auch die $\mathrm{CO}_{2}$-Betäubung im Hinblick auf punktförmige Einblutungen und kleinflächige Hautblutungen ab. Dagegen wurden bei der $\mathrm{CO}_{2}$-Betäubung signifikant mehr großflächige Hämatome gefunden.

Die gängigste Methode in der industriellen Geflügelschlachtung ist die Betäubung im Wasserbad. Der Betäubungserfolg hängt von der Stromstärke, der Einwirkzeit und der Tauchtiefe ab. Tierschutzrelevante Probleme durch den Wasserbadbetäuber sind (SCHÜTTABRAHAM, 1999):

- das Einhängen der Tiere in den Schlachtbügel,

- $\quad$ ein fehlerhaftes Eintauchen der Köpfe (Fehlbetäubungen, vorzeitige Stromstöße).

Umfangreiche Informationen zur Elektrobetäubung bei Geflügel hat SCHÜTT-ABRAHAM (1999) publiziert.

Eine Alternative zum Wasserbadbetäuber ist die Betäubung mit Gas. Von BARTON GADE ET AL. (2001) und GRANDIN (2005) wird diese Methode als tiergerechter angesehen. Die Tiere müssen nicht mehr lebend aufgehangen werden, und bei einer ordnungsgemäßen Funktion der Anlage kommt es auch nicht zu Fehlbetäubungen. Die positive Wirkung dieser Betäubung hängt aber sehr stark davon ab, welches Gas zur Betäubung eingesetzt wird. So haben LAMBOOIJ ET AL. (1999) u. BARTON GADE ET AL. (2001) festgestellt, dass je nach Gasmischung Reaktionen wie Keuchen, Kopfschütteln, Flügelschlagen, Zuckungen und Krämpfe unterschiedlich sind. Der große Nachteil dieser Anlagen sind die hohen Installations- und Betriebskosten (GRANDIN, 2005).

Insgesamt wird die Schlachttierbetäubung aufgrund der steigenden Bedeutung des Tierschutzes immer kritischer hinterfragt (MACHOLD ET AL., 2003). Angesichts der immer wieder vorkommenden Vorfälle von Fehlbetäubungen ist nach VON HOLLEBEN ET AL. (2002) das Argument, dass es sich hierbei um unvermeidbare Einzellfälle oder um sogenannte schwarze Schafe handelt, nicht akzeptabel. Ebenso sehen auch LUY U. HILDEBRAND (2004), dass nachlässiges Betäuben nicht als „Kavaliersdelikt“ betrachtet werden darf. Um hier Verbesserungen zu erlangen ist aber, nach der Meinung von VON HOLLEBEN ET AL. (2002), ein Höchstmaß an Courage bei den für die Ahndung zuständigen Personen notwendig.

\subsubsection{3 Übertragungswege von BSE-Risikomaterial in der Schlachtung}

Unsicherheiten bezüglich der Übertragungsgefahr von BSE-Erregern durch technologische Prozesse bei der Fleischgewinnung sind vorhanden. Es wird bei der penetrierenden Bolzenschussbetäubung nicht ausgeschlossen, dass es zu einer Übertragung von Gehirnmaterial in den Körper kommt (ANIL u. HARBOUR, 2001; HILDEBRAND, 2002; FRIES, 2003). LÜCKER veröf- 
fentlichte 2002, dass der vermutete Nachweis von ZNS-Partikeln im Blut und in den Lungen infolge der Betäubung mit dem konventionellen Bolzenschuss noch nicht abschließend bestätigt werden kann.

Bei der Zweiteilung des Schlachtkörpers nach der traditionellen Methode (Sägen) ist eine Kontamination der Schnittflächen mit Rückenmarksubstanz praktisch nicht vermeidbar (HILDEBRAND, 2002; FRIES, 2003). Bei der Durchtrennung des Rückenmarkkanals wird ein nicht unerheblicher Anteil an ZNS-Material (ca. 18g) weitergetragen (TROEGER ET AL., 2002). Aus diesem Grunde wurden neue Ansätze getestet, bei denen die Wirbelsäule ohne Spaltung aus dem Schlachtkörper entfernt werden kann (TROEGER ET AL., 2002). Alle Methoden, die eine Entfernung der Wirbelsäule ohne Spaltung bewirken, haben sich zurzeit noch nicht durchgesetzt.

Als weitere mögliche Gefahrenpunkte, an denen ZNS-Material übertragen werden kann, werden die an Risikoarbeitsplätzen verwendeten Messer und das Reinigungswasser beim Absetzen des Kopfes (BANDICK, 2003), sowie unzureichende Reinigungs- und Desinfektionsmaßnahmen angesehen. Bei Reinigungs- und Desinfektionsmaßnahmen wirken nur bestimmte Mittel, z.B. Natronlauge und Natriumhypochloridlösung. Reinigungs- und Desinfektionsmittel, die üblicherweise in der Schlachtung eingesetzt werden, inaktivieren den BSEErreger nicht (LIPPMANN ET AL., 2004).

\subsubsection{Hygienemaßnahmen und Hygienekonzepte}

Hygienemaßnahmen in der Futtermittelproduktion, Landwirtschaft und Lebensmittelproduktion sind heute in allen Bereichen selbstverständlich und fallen unter den Begriff gute Herstellungspraxis (GMP Good Manufacturing Practice) bzw. unter gute Hygiene-Praxis (GHP Good Hygienic Practice). Neben GMP und GHP haben Hygienekonzepte auf der Grundlage des HACCP eine große Bedeutung in der Lebensmittelhygiene und Lebensmittelsicherheit gewonnen.

Definiert wird der Begriff Lebensmittelhygiene in der DIN 10503 als „Die Gesamtheit der Maßnahmen, durch welche die gesundheitliche Unbedenklichkeit und der einwandfreie Zustand von Lebensmitteln in allen Stufen des Herstellens, Behandelns und Inverkehrbringens sichergestellt werden." Im Einzelnen werden unter Lebensmittelhygiene Maßnahmen in den Bereichen Produkthygiene, Produktionshygiene und Personalhygiene verstanden. Je nach Produktionsstufe sind diese unterschiedlich gestaltet.

Nach HENSGEN (2004) werden nachfolgend Faktoren aufgezählt, die einen Einfluss auf die einwandfreie hygienische Beschaffenheit von Lebensmitteln haben:

ungeeignete Behandlungs- und Zubereitungsverfahren, Mikroorganismen, Verunreinigungen, tierische Schädlinge, Temperaturen, Witterungseinflüsse, menschliche und tierische Ausscheidungen, Abfälle, Abwässer, Reinigungs-, Desinfektions-, Pflanzenschutz- und Schädlingsbekämpfungsmittel, Aerosole, Gase, Gerüche, Dämpfe und Rauch.

Trotz der umfangreichen Maßnahmen von Wirtschaft und Überwachungsbehörden kommt es aber immer wieder zu Vorfällen. Diese Vorfälle können verschiedene Ursachen haben. Einerseits ist es häufig das Nichtverstehen von Zusammenhängen (mangelnde Schulung, neue Prozesse) oder Ignoranz (HENSGEN, 2004). Andererseits spielt aber auch der Aspekt mangelnde finanzielle Aufwendungen eine Rolle. 
Allgemeine Hygienekonzepte, die Maßnahmen wie Personalhygiene, Sauberkeit, Instandhaltung, Desinfektion usw. beinhalten, sind die Grundbausteine für die Lebensmittelsicherheit (FEHLHABER, 1998). Fehlende Hygienekonzepte führen zu Risiken (mikrobiologische, chemische und physikalische) auf allen Stufen der Kette. Hierbei ist es wichtig, dass die Konzepte individuell für jeden Betrieb erarbeitet werden. Aufgrund von baulichen und technischen Gegebenheiten sind, trotzt gleicher Produkte, die Risiken in den Unternehmen unterschiedlich. Zusätzlich zu allgemeinen Hygieneregeln wird von Lebensmittel erzeugenden Betrieben ein Konzept der Gefahrenidentifizierung und Bewertung (HACCPKonzept) gefordert (VO EU 178/2002, Hygiene-Paket“ (EU VO 852/2004; 853/2004; 854/2004). Ausgenommen hiervon ist allerdings die Primärerzeugung (landwirtschaftliche Betriebe). Trotz dieser Ausnahme bezüglich der Erstellung eines HACCP-Konzeptes ist der landwirtschaftliche Betrieb aber dazu verpflichtet, Risiken zu identifizieren und innen vorzubeugen (ANONYMUS, 2004A, EU VO 852/2004 u. 853/2004). Die konsequente Umsetzung der Basishygiene ist gerade im landwirtschaftlichen Betrieb besonders wichtig, um eine Einschleppung von Krankheiten und Seuchen zu verhindern. Außerdem können Hygienefehler zu einer Kontamination auf den nachfolgenden Stufen führen. In der Anlage 1 der EU VO 852/2004 im Teil A „Allgemeine Hygienevorschriften für die Primärproduktion und damit zusammenhängende Vorgänge“ und im Teil B „Empfehlungen für die Leitlinien für die gute Hygienepraxis“ festgelegt.

In der Futtermittelproduktion gibt es erst ab dem 1.01.2006 (EU VO 852/2004, EU VO 183/2005) eine Verpflichtung zur Durchführung einer Risikoanalyse und der Einführung eines HACCP-Konzeptes. Aufgrund der Anforderungen der Wirtschaft (geforderte Zertifizierungen nach QS und GMP+) sind HACCP-Konzepte in der industriellen Futtermittelproduktion aber weitreichend eingeführt. Grundvoraussetzung für eine Zertifizierung ist ein HACCP-System. In Deutschland gab es Mitte 2003 ca. 408 Mischfutterbetriebe (DVT, 2004A). Im Rahmen des QS-Systems waren am 01.01.2005 486 Mischfutterbetriebe QS zertifiziert, wobei nicht alle Betriebe in Deutschland ansässig sind (QS, 2005A). Laut dem Jahresbericht des DVT 2003/ 2004 stammt das in Deutschland hergestellte Mischfutter zu ca. 95\% aus QS-zertifizierten Betrieben. Zusätzlich zu der QS-Zertifizierung verfügen viele dieser Betriebe auch über eine Zertifizierung nach GMP+.

In dem Bereich der Schlachtung sind die Hygienevorschriften sehr detailliert beschrieben und in den meisten Betrieben umfassend eingeführt. Seit 1993 ist die europäische Lebensmittelwirtschaft durch die Richtlinie 93/43/EG dazu verpflichtet, ein Eigenkontrollsystem in Anlehnung an das HACCP-Konzept zu implementieren. Zusätzlich ist auch die Überwachung der allgemeinen Hygienebedingungen durch betriebseigene Kontrollen im AMTSBLATT DER EU 2001/471/EG und der RICHTLINIE 64/433/EWG klar geregelt.

Insgesamt sind HACCP-Konzepte ein wichtiges Instrument zur Gefahrenidentifikation und Vorbeugung. Gänzlich werden durch diese Konzepte aber die Risiken nicht ausgeschaltet. Dieses liegt an dem unterschiedlichen Detaillierungsgrad und dem nicht einheitlichen Verständnis bzw. der Umsetzung von HACCP.

\subsubsection{Anthropogene Risiken}

„Anthropogene Risiken sind Gefahren, die durch den Menschen, meist durch menschliches Fehlverhalten, ausgelöst werden. Dazu zählen: mangelnde Motivation, mangelnde Qualifikation, mangelnde Aufsicht, Überlastung der Mitarbeiter" (HENSGEN, 2004). 
Mangelnde Personalhygiene ist eine Gefahr, die immer wieder zu negativen Vorfällen in der Lebensmittelbranche führt. Diese Gefahr ist besonders hoch auf der Stufe der Schlachtung. Sie darf aber ebenfalls auf den Stufen der Primär- und Futtermittelproduktion nicht unterschätzt werden. Durch Personenverkehr können z.B. Krankheiten oder Seuchen im landwirtschaftlichen Betrieb übertragen werden. Aber auch eine Inaktivität bei Unfällen (z.B. beim Platzen von Ölschläuchen) oder die ungenügende Reinigung von Geräten, Fahrzeugen kann zu Gefahren führen. Um Gefahren zu vermeiden, ist es notwendig, dass alle Personen, die im landwirtschaftlichen Betrieb tätig sind, die Grundregeln einer ausreichenden Betriebshygiene kennen (PFANNHAUSER 2005). Dazu gehören Kenntnisse über eine richtige Lagerung, die persönliche Hygiene und die Dokumentation der Bearbeitungsschritte.

Auf der Stufe der Schlachtung kann es bei Fehlverhalten, z.B. durch den direkten Kontakt zum Lebensmittel (Hände, Arbeitskleidung, Arbeitsgeräte), zu einer Kontamination der Lebensmittel mit Mikroorganismen kommen. Hierbei spielen vor allem die Hygienehandlungen bei der Arbeit (Händewaschen, Messerwechsel) eine Rolle. Im Gegensatz zu der Rinder- und Schweineschlachtung spielen Hygienefehler durch das Personal in der Geflügelschlachtung, aufgrund des hohen Grades an automatischen Prozessen, nur an wenigen Stellen eine Rolle (WEISE, 2003). Bei entsprechenden Hygienemaßnahmen (z.B. Hände und Arbeitsgeräte waschen und desinfizieren, regelmäßiger Kleidungswechsel, Einhalten der vorgeschriebenen Hygieneregeln) kann eine Übertragung weitgehend vermieden werden (WEBER, 1996; HENSGEN, 2004).

Aufgrund von Erkrankungen oder als Träger von pathogenen Keimen kann der Mensch selbst eine Gefahr für Lebensmittel sein. Auf der Stufe der Schlachtung sind die Mitarbeiter verpflichtet, sich nach dem Infektionsschutzgesetz (IfSG) (2000) eigenverantwortlich beim Arbeitgeber zu melden, wenn sie Symptome einer Erkrankung nach §42 IfSG bei sich feststellen.

\subsubsection{Schädlingsbekämpfung}

Die Schädlingsbekämpfung gehört zu den vorbeugenden Hygienemaßnahmen auf allen Stufen der Lebensmittelproduktion. Zum einen sind Schädlinge Ekel erregend und zum anderen übertragen diese oftmals pathogene Keime. Je nach Schädling können die Lebensmittel z.B. mit Haaren, Exkrementen, Eiern und Kadavern verunreinigt werden (HENSGEN, 2004).

Das Eindringen von Schädlingen kann aufgrund der unterschiedlichen Ausbreitungswege nicht immer ausgeschlossen werden. Schädlinge können aktiv in den Betrieb einwandern bzw. fliegen oder passiv durch den Befall von Rohstoffen, Verpackungsmaterialien, Transportmitteln u.a. in den Betrieb gelangen (HENSGEN, 2004). Um den Befall aber zu begrenzen oder zu kontrollieren sind Präventionsmaßnahmen erforderlich. Mögliche Risiken des Befalls entstehen z.B. durch:

- $\quad$ offene Türen oder Fenster (auch kleine Schlitze),

- Rückzugsmöglichkeiten (z.B. Schächte, Abdeckungen, still gelegte Maschinen, Materialien, die längere Zeit an Hauswänden usw. gelagert werden),

- mangelnde Wareneingangskontrolle auf Schädlingsbefall,

- mangelhaftes bzw. fehlendes Monitoring- und Bekämpfungsprogramm. 
In der Lebensmittelproduktion werden meistens externe Dienstleister (Fachunternehmen) zur Schädlingsbekämpfung und zum Schadnager-Monitoring eingesetzt. In der Futtermittelproduktion und in der Landwirtschaft erfolgt die Schädlingsbekämpfung in der Regel durch eigene Mitarbeiter. Diese Mitarbeiter dürfen die Schädlingsbekämpfung nur durchführen, wenn sie über die dazu notwendige Sachkunde verfügen (SCHADE ET AL., 2003). Neben der Sachkunde (Umgang mit Giftstoffen) sollten die Mitarbeiter aber auch Befallsspuren erkennen können. Ebenso ist es wichtig, die Besonderheiten der einzelnen Schädlinge und die gefährdeten Betriebsbereiche zu kennen (SCHADE ET AL., 2003). Hierzu ist eine Schulung der Mitarbeiter erforderlich.

\subsubsection{Rückverfolgbarkeit}

Bei der Rückverfolgbarkeit handelt es sich um die Schaffung der organisatorischen Voraussetzungen, produktrelevante Informationen und Daten in geeigneter Weise mit definierten Produkteinheiten zu verknüpfen und verfügbar zu machen (BLL, 2001). Um eine umfassende Rückverfolgbarkeit zu gewährleisten, sind Aufzeichnungen auf allen Stufen notwendig. Hierzu müssen Bezugswege und Verarbeitungsschritte (z.B. Erstellen einer Futtermischung) des Futters, die Futterzuteilung, die Herkunft der Tiere, Einzelheiten des Herdenmanagements, tiermedizinische Applikationen, Absatzwege usw. auf der ganzen Kette vom Futter über das (einzelne) Tier letztendlich sogar bis zum Endverbraucher erfasst und dokumentiert werden (SCHÖPE, 2005). Situationen, in denen ein Produkt vom Markt zurückgerufen werden muss, oder Vorfälle, bei denen es wichtig ist, den Ursprung der Produkte zu ermitteln, werden immer wieder vorkommen. Eine 100\% Sicherheit gibt es bei Lebensmitteln nicht.

Risiken durch mangelhafte bzw. nicht funktionierende Systeme der Rückverfolgbarkeit entstehen durch:

- unzureichenden Rückruf,

- lange Reaktionszeiten bei der Identifikation der Vermarktungswege,

- Schnittstellenprobleme zwischen den einzelnen Stufen bzw. Marktpartnern.

Durch das Inkrafttreten der EU VO 178/2002 ist die Rückverfolgbarkeit ab dem 01.01.2005 für die gesamte Lebensmittelkette gesetzlich vorgeschrieben. Unter Artikel 18 sind die Regelungen bezüglich der Rückverfolgbarkeit festgelegt. Insbesondere der Absatz 2 regelt: „Die Lebensmittel- und Futtermittelunternehmer müssen in der Lage sein, jede Person festzustellen, von der sie ein Lebensmittel, Futtermittel, ein der Lebensmittelgewinnung dienendes Tier oder einen Stoff, der dazu bestimmt ist oder von dem erwartet werden kann, dass er in einem Lebensmittel oder Futtermittel verarbeitet wird, erhalten haben“. Bei der Rückverfolgbarkeit ist es wichtig, dass beide Richtungen „upstream“ (vom Produzenten zum Verbraucher) und „downstream“ (vom Produzenten zum Ursprung) gewährleistet sind. Durch die EU VO 178/2002 wird die Rückverfolgbarkeit „one step up“ und „one step down“, also die Identifizierung des unmittelbaren Abnehmers bzw. Lieferanten, gefordert (BLL, 2003). Anforderungen hinsichtlich der internen (innerbetrieblichen) Rückverfolgbarkeit gibt es keine. Der Detaillierungsgrad und die Aufbewahrungszeit der Daten unterliegen der Sorgfaltspflicht des Unternehmers (BLL, 2003; EU, 2004, WEGNER-HAMBLOCH, 2004). Allerdings hat der Unternehmer sein System der Rückverfolgbarkeit so aufzubauen, dass er eine gezielte und präzise Rücknahme vornehmen kann (Erwägungsgrund 28 der EU VO 178/2002). 
Umfangreiche detaillierte Regelungen zur Rückverfolgbarkeit und zum Rückruf (innerbetrieblich sowie nach außen) werden ebenfalls von verschiedenen Lebensmittel- und Futtermittelstandards (PDV, 2003A, 2003B; HDE, 2004; BRC, 2005) gefordert.

Probleme werden nach SCHÖPE (2005) vor allem in der Datenerfassung auf allen Stufen landwirtschaftlicher Arbeiten gesehen. Ebenso ist der Detaillierungsgrad der Rückverfolgbarkeit auf den verschiedenen Stufen der Lebensmittelproduktion sehr unterschiedlich. Je höher der Zerkleinerungs- und Verarbeitungsgrad sowie die Zahl der Verteilungs- und Verarbeitungsstufen ist, um so schwerer wird die Rückverfolgbarkeit bis zu den ursprünglichen Rohstoffquellen (BLL, 2001).

Besonders bei Futtermitteln ist die Rückverfolgbarkeit sehr schwierig. In der Futtermittelproduktion werden in der Regel aus kleinen Mengen größere Chargen gebildet, die dann auf den weiteren Verarbeitungsstufen vermischt werden. Erschwerend kommt hinzu, dass sowohl inländische und ausländische Waren verwendet werden (vergl. auch Kapitel 2.4.1). Klare Vorgaben für die Festlegung von Chargen schreibt z.B. der GMP-27-Standard für die Mischfutterhersteller vor. Mindestens alle drei Monate hat eine Leermeldung der Silos zu erfolgen. Erfolgt diese Meldung aus organisatorischen oder anderen Gründen nicht, muss dieses begründet werden (PDV, 2003B).

Die Probleme bei der Rückverfolgbarkeit von Futtermitteln zeigte auch ein Fall von Anfang 2003, bei dem ein Thüringer Trockenwerk ungeeignetes Brennmaterial zur Trocknung eingesetzt hatte. Hierdurch war es bei den getrockneten Produkten zu einer Dioxinkontamination gekommen. Schwierigkeiten gab es vor allem bei der Ermittlung der in Betracht kommenden Menge und deren Verbleib bzw. Weiterverarbeitung auf den weiteren Stufen (HEESCHEN, 2003).

Einen wichtigen Part spielt die Kennzeichnung und Rückverfolgbarkeit bei gentechnisch veränderten Produkten. Durch die EU VO 1829/2003 und EU VO 1830/2003 sind klare Regelungen für die Kennzeichnung und Rückverfolgbarkeit verabschiedet worden. Unklarheit herrscht zurzeit noch bei der Kennzeichnung von Futtermitteln, die gentechnisch veränderte Zusatzstoffe, Vitamine, Enzyme oder andere Zusatzstoffe enthalten. Diese Zusatzstoffe werden bewusst zugesetzt und fallen damit nicht unter die Vorgabe „zufällig und technisch vermeidbar“. Laut der Definition im Gesetz müssten sie demnach gekennzeichnet werden (DVT, 2004B).

Ein effektives System zur Rückverfolgbarkeit von Rindfleisch und Rindfleischerzeugnissen vom Einzelhandel bis hin zum Ursprungsbetrieb ist in der EU durch die Verordnung 1760/2000 vorgeschrieben. Nach dem Bericht der EU-KOMISSION (2003A) zur Kontrolle der Rückverfolgbarkeit in den Mitgliedsstaaten in 2002 sind bis auf sechs Mitgliedsstaaten die einschlägigen Vorschriften vollständig umgesetzt worden. Die Herkunftssicherung von Fleisch vom Schlachtbetrieb zurück bis zum Ursprungsbetrieb wird von der EU-Komission als zufriedenstellend bewertet. Probleme werden in den späteren Stadien der Produktionskette von den Zerlege- und Verarbeitungsbetrieben des Großhandels bis hin zur Lagerung, zum Vertrieb und Einzelhandel gesehen. Die Verbringung und der Ursprung des Fleisches konnte hier häufig nicht mit ausreichender Sicherheit zurückverfolgt werden (EU, 2003A). Nach der Feinzerlegung ist eine Rückverfolgbarkeit auf das einzelne Tier nur noch in den seltensten Fällen möglich, da hierzu im Zerlegebetrieb eine Einzeltierzerlegung notwendig wäre. Im Normalfall werden aber in den Zerlegebetrieben Tageschargen gebildet. Optimal wäre es, wenn die Tiere nach Anlieferer zerlegt werden. In der Regel setzt sich aber eine 
Tagescharge aus den Produkten verschiedener Anlieferer (z.B. Schlachthöfen) zusammen. Dieses kann dazu führen - führt man eine Rückverfolgbarkeit auf den Mastbetrieb durch dass in einer Charge ggf. mehr als 200 Anlieferer von Tieren zusammengefasst sind (SCHÜTT-ABRAHAM, 2003).

Bei Schweinen existiert zurzeit noch kein Tierpass. Nach der Viehverkehrsverordnung (VIEHVERKV) sind aber alle Schweinehalter, Viehhandelsunternehmen, Transportunternehmen, Schlachtstätten und Sammelstellen verpflichtet, ihre gehandelten Schweine zu melden. Ein wichtiges Ziel der Schweinedatenbank ist es, im Seuchenfall Kontaktbetriebe zu ermitteln und diese Informationen den zuständigen Veterinärbehörden zur Verfügung zu stellen. ADAM (2001) sieht in der Rückverfolgbarkeit vom Schlachtbetrieb zum Mäster in den meisten Schlachtbetrieben keine Probleme. Sollen diese Daten dann jedoch auf den Ferkelerzeuger zurückverfolgt werden, ist dieses nach seiner Meinung kaum zu realisieren, so lange die Schweine nur mit den vorgeschriebenen Ohrmarken gekennzeichnet sind. Um hier eine sichere Rückverfolgbarkeit zu gewährleisten, müssten die Tiere über eine elektronische Einzeltierkennzeichnung verfügen. Diese ist aus Kostengründen zurzeit noch nicht realisierbar.

Beim Geflügel ist die Rückverfolgbarkeit auf den Erzeuger aufgrund der relativ großen Einheiten schon seit längerem gegeben.

Wie wichtig eine konsequente Rückverfolgbarkeit ist, hat der Ausbruch der Maul- und Klauenseuche im Vereinigten Königreich gezeigt. Bei Schafen hat man durch die Maul- und Klauenseuche festgestellt, dass hier die Forderungen der Richtlinie 92/102/EWG12 des Rates vom 27. November 1992 über die Kennzeichnung und Registrierung von Tieren nicht konsequent umgesetzt wurde. Fehlende Kennzeichnung von Tieren (Schafe), fehlende Registrierung der Verkäufe und die dadurch bedingten schwer nachzuvollziehenden Handelsbewegungen haben ein Ausbreiten der Maul- und Klauenseuche begünstigt (ANONYMUS, 2002E; KREISSL-DÖRFLER, 2002).

\subsubsection{Systeme zur Rückverfolgbarkeit}

Zurzeit werden zwei verschiedene Ansätze verfolgt, die die Rückverfolgbarkeit erleichtern sollen. Der eine Ansatz beruht auf der Grundlage von technischen Kennzeichnungsmöglichkeiten die per Datenverarbeitung erfasst werden können. Der andere Ansatz beruht auf der Erfassung von biometrischen Daten.

Als technische Kennzeichnungsmöglichkeiten werden zurzeit Systeme mit EAN-Codes, DOT-Codes oder Transpondern erprobt bzw. eingesetzt. EAN-Codes sind international standardisierte und zentral verwaltete Nummernsysteme für Artikel (EAN), Adressen (ILN) und Versandeinheiten (NVE). Weitere Informationen sind auf der Internetseite www.ean.de zu finden (GS1, 2005). Diese EAN-Systeme werden vor allem auf den Stufen der Zerlegung und der Weiterverarbeitung eingesetzt. Ein ähnliches System, das auf einem Punktemuster beruht, ist der DOT-Code. Durch dieses System können die Tiere oder Schlachtkörper direkt gekennzeichnet werden. Die Daten können später mit dem EAN-Code verbunden werden. Das System der DOT-Codes wird von der Hendrix Meat Group eingesetzt (ANONYMUS, 2004E).

Transponder zur Identifikation von Tieren und Warenströmen sind eine weitere technische Möglichkeit. Bei den Tieren befindet sich der Transponder z.B. in den Ohrmarken. Diese Informationen werden am Schlachthof übernommen und in die dortigen Systeme integriert. 
Auf den nachfolgenden Stufen kann sich der Transponder in der Verpackung oder am Transportbehältnis bzw. am Fahrzeug befinden (BOLSCHER, 2001).

Biometrische Verfahren verwenden zur Identifizierung unverwechselbare physiologische Eigenschaften der Tiere. Systeme, die auf der Erfassung von biometrischen Daten beruhen, sind die DNA -Analyse oder das Retina-Scanning. So wird zum Beispiel durch die DNAAnalyse in Verbindung mit einer Datenbank die Zuordnung eines Stück Fleisches zu einem Einzeltier möglich. Dieses Verfahren wird zurzeit bei Rindern erprobt. Verschiedene Unternehmen bieten hierzu Lösungen an (ANONYMUS, 2002B; ANONYMUS, 2004F).

\subsubsection{Kontrollen}

Die ursprünglichen Aufgaben der Lebensmittelüberwachung sind die Überwachung des Gesundheitsschutzes und der Schutz vor Täuschung. Nach den Ausführungen von FEHLHABER (2000) hat die Schlachttieruntersuchung wichtige, für den Verbraucherschutz relevante Aufgaben zu erfüllen:

- „Schutz des Menschen vor gesundheitliche Gefahren, die vom Fleisch der zu schlachtenden Tiere ausgehen können,

- Schutz vor wirtschaftlicher Übervorteilung (Täuschungsschutz),

- Mithilfe in der Tierseuchenprophylaxe und -bekämpfung,

- Überwachung des Tierschutzes in der Tierhaltung, beim Transport und Handling auf dem Schlachthof."

Die Wirksamkeit der Schlachttieruntersuchung hat dazu geführt, dass Zoonosen wie Tuberkulose, Brucellose, Trichinellose u.a. in Deutschland entweder vollständig eliminiert oder nahezu lückenlos unter Kontrolle sind (SAMITZ, 2000; HILDEBRAND, 2002). Allerdings können Zoonoseerreger (Salmonellen, E-coli, Campylobacter), mit denen ein Schlachttier infiziert sein kann, solange keine klinischen Symptome am Tier sichtbar sind, von der Schlachttieruntersuchung zurzeit noch nicht erkannt werden (FEHLHABER, 2000). Sowohl FEHLHABER (1999) als auch PÖCKER ET AL. (2004) sehen hier in der Eigenkontrolle eine wichtige Unterstützung der Lebensmittelüberwachung. In der Neuordnung des europäischen Lebensmittelrechts wird diese Einschätzung umgesetzt. Eine wesentliche Änderung bei der amtlichen Kontrolle in den Bereichen Tierschutz, Tiergesundheit, Futtermittel- und Lebensmittelrecht wird sich durch das Inkraftreten der EU weit gültigen EU VO 882/2004 ergeben. Nach HORN (2005) wird mit dieser Verordnung ein umfassender, integrierter Ansatz der amtlichen Kontrollen der Sicherheit und Qualität von Lebensmitteln umgesetzt. Bewährte Regelungen aus dem Bereich der Lebensmitteluntersuchung werden auf die Bereiche Tiergesundheit und Futtermittel übertragen. Einfuhrbedingungen sowie auch die Einfuhrkontrollen werden für Futter- und Lebensmittel gemeinschaftsweit einheitlich geregelt (Horn, 2005). Es ist wichtig, Kontrollen in der gesamten Kette und vor allem in der Urproduktion durchzuführen. Denn viele der aufgetretenen Probleme haben ihren Ursprung in der Urproduktion. Ein Schritt in diese Richtung ist durch das Inkraftsetzen der EU VO 1782/2003 erfolgt. Die Gewährung von Direktzahlungen ist ab dem Jahr 2005 u.a. an die Einhaltung von Vorschriften in den Bereichen Umwelt, Futtermittel- und Lebensmittelsicherheit sowie Tiergesundheit und Tierschutz (Cross Compliance) geknüpft (ANONYMUS, 2005).

Aber nicht nur staatliche Ansätze sollen zu einer Verbesserung des Gesundheitsstatus in den Tierbeständen führen. Maßgeblich zur Verbesserung des Gesundheitsstatus in den 
Tierbeständen in der Schweinemast hat die Erfassung der Befunddaten am Band per PC beigetragen. Durch die Zuordnung der Daten zu den Ohrmarken ist es den Mästern möglich, besser die Ursachen für die gesundheitlichen Probleme zu ergründen. Gegebenenfalls lassen sich sogar Rückschlüsse auf den Ferkellieferanten ziehen (STRACKE, 2000).

Aufgrund von hohen Bandgeschwindigkeiten sind zum Teil Verunreinigungen und gesundheitlich bedeutsame Mängel in der Geflügelschlachtung nur schwer erkennbar. Als weiteres Manko kommt hinzu, dass sich die Untersuchungsplätze häufig den technischen und räumlichen Bedingungen der bestehenden Schlachtanlagen unterordnen, so dass nicht immer der sinnvolle und nötige Untersuchungsplatz zur Verfügung steht (KÖLLING, 1997; WEISE, 2003). Es erfolgt im Wesentlichen nur eine Auslese nach visuellen Qualitätsmerkmalen. Dies kann für Herden, die ein geringes Beanstandungsprofil aufweisen, durchaus angemessen sein. Bei Herden, die ein anderes Profil zeigen und bei denen mehrere Merkmale gleichzeitig zu berücksichtigen sind, können für den Verbraucher relevante Merkmale visuell nicht erkannt werden (KöLLING, 1997). Um dieses Manko auszugleichen, wird in der Geflügelschlachtung eine erweiterte Schlachtgeflügeluntersuchung vor der Ausstallung gesetzlich geordert. Des Weiteren sind die Tierhalter von Schlachtgeflügel dazu verpflichtet, Nachweise über alle Fakten und Vorgänge zu führen, die zur Beurteilung des Gesundheitszustandes der Tiere oder der Genusstauglichkeit beitragen können (WEISE, 2003). Aber auch eine weitere Einbindung von Daten bzw. Überwachungen von pathogenen Erregern bzw. von hygienischen Bedingungen in die Geflügelfleischuntersuchung ist notwendig, um eine mögliche Übertragung pathogener Erreger zu reduzieren (ELLERBROEK,1997).

Aufgrund von aufgetretenen Probleme bei Lebensmitteln ist das Vertrauen der Öffentlichkeit in die gegenwärtig praktizierten Formen der Lebensmittelkontrolle stark erschüttert. Festzustellen ist, dass das Thema BSE trotz umfangreicher Regelungen und Gesetze immer wieder in der Presse auftaucht (vergl. HILDEBRAND, 2002). Beispiele hierfür sind: falsche BSE-Tests (ANONYMUS 2004G, 2004H) und Risikomaterial, welches trotz Verbots in Fleischwaren gefunden wurde (HILDEBRAND, 2002). Inwieweit es sich hierbei um vorsätzliches Verhalten, Fehler im Verfahrensablauf oder Fehler bei den Kontrollen handelt, ist offen. 


\section{Schlussfolgerungen aus dem Literaturteil}

Im Literaturteil wurden die unterschiedlichen Standpunkte der Verbraucher, der Industrie und der Wissenschaft, wie und welche Risiken zu bewerten sind, dargestellt. Dabei ist deutlich geworden, dass es nicht nur zwischen Verbrauchern und Experten stark voneinander abweichende Sichtweisen/Standpunkte gibt. Auch unter den Experten und Wissenschaftlern existieren in vielen Bereichen unterschiedliche Auffassungen darüber, wie Risiken zu bewerten sind und wie mit ihnen umgegangen werden sollte.

Die Literaturübersicht kann folgendermaßen thesenartig zusammengefasst und Zielsetzungen für die eigenen Untersuchungen formuliert werden.

- Die Rasse bzw. die genetische Konstruktion hat einen Einfluss auf die Produktqualität des Fleisches und die Wirtschaftlichkeit. Wirtschaftliche Ziele der Fleischwirtschaft lassen sich häufig nur zu Lasten der Konstitution und der Produktqualität der Tiere verwirklichen. Der Begriff „Qualzucht“ wird in dieser Arbeit bewusst gewählt, um extreme Zuchtrichtungen deutlich zu machen. Die negativen Auswirkungen der intensiven Fleischproduktion haben allerdings auch zu entgegengesetzten Entwicklungen in der Fleischindustrie und Züchtung geführt, bei denen mehr Wert auf Produktqualität gelegt wird.

Angesichts dieser widersprüchlichen Entwicklung soll festgestellt werden, welches derzeit und zukünftig die bevorzugten Rassen/ genetischen Konstruktionen sind, die sich positiv auf die Produktqualität auswirken. Zugleich soll aber auch diskutiert werden, inwieweit die Zucht auf einen hohen Fleischanteil negativ zu sehen ist und ob die damit oft verbundenen negativen Ausprägungen messbar sind.

- Eine abschließende Bewertung der möglichen Risiken und Chancen des Einsatzes gentechnischer Verfahren kann im Moment nicht getroffen werden. Trotz der Bedenken der Verbraucher wird ein vermehrter Einsatz der Gentechnologie in Zukunft aber kaum noch aufzuhalten sein. Zurzeit erfolgt der Einsatz kommerziell im Bereich der Pflanzenproduktion.

Im Rahmen dieser Arbeit sollen die Risiken und Vorteile des Einsatzes von Gentechnik in der Fleischerzeugung diskutiert werden. Zum einen soll erörtert werden, wie der Einsatz von genveränderten Pflanzen in der Futtermittelindustrie und deren möglichen Auswirkungen auf das Fleisch zu bewerten sind. Zum anderen soll aber auch diskutiert werden, ob und ab wann auch mit dem Einsatz von genveränderten bzw. geklonten Tieren in der Fleischproduktion zu rechnen sein wird.

- Laut der wissenschaftlichen Literatur stellen die Zoonosen in der Produktionskette Fleisch die größte Gefährdung für den Menschen dar. Die Bedeutung einzelner Zoonosen wechselt, und es besteht die Gefahr, dass neue Erreger eingeschleppt werden.

Es soll erörtert werden, welche Zoonoseerreger heute und in Zukunft eine Rolle spielen und wie sich die Situation in Deutschland entwickeln wird. Ferner soll diskutiert werden, welche geeigneten Vorkehrungen zu treffen sind, um die Eintragsschwerpunkte bzw. der Verbreitung von Zoonosen in den einzelnen Teilen der Produktionskette Fleisch noch besser identifizieren zu können.

- Tierseuchen sind in den letzten Jahren immer wieder in der EU als auch weltweit aufgetreten (MKS, Schweinepest, Vogelgrippe). Durch den internationalen Waren-, Tierund Personenverkehr besteht die Gefahr, dass sich Seuchen in der Zukunft noch 
schneller ausbreiten und auch neue Keime nach Deutschland gelangen können. Angesichts dieser Problematik, vor allem aber auch unter Berücksichtigung des Seuchenzugs der Maul- und Klauenseuche in England, hat die Diskussion über den Einsatz von Prophylaxen und Schutzimpfungen an Brisanz gewonnen. Inwieweit der Einsatz von Prophylaxen und Schutzimpfungen in Zukunft notwendig sein wird, ist umstritten. Außerdem gibt es unterschiedliche Meinungen darüber, welche Probleme durch einen grundsätzlichen Verzicht auf Impfungen entstanden sind bzw. neu entstehen könnten.

Im Rahmen dieser Arbeit soll zum einen eingeschätzt werden, welche Tierseuchen in Deutschland in den nächsten Jahren relevant werden können. Zum anderen sollen aber auch die Risiken und Chancen von Impfungen genauer diskutiert werden.

- Rückstände spielen in der Nahrungskette zunehmend eine wichtige Rolle, sowohl in der fachwissenschaftlichen als auch in der öffentlichen Diskussion. Werden Rückstände und Kontaminanten in Lebensmitteln gefunden, erfolgt die anschließende öffentliche Diskussion häufig auf einer sehr emotionalen und weniger auf einer wissenschaftlichen Basis. Dieses Diskussionsverhalten wird häufig durch das Verhalten der Medien gefördert. Hinzu kommt, dass heute Stoffe festgestellt werden, die vor wenigen Jahren aufgrund der fehlenden Analytikinstrumentarien und -verfahren noch nicht bekannt waren oder noch nicht nachgewiesen werden konnten.

Angesichts der Bedeutung der zum Teil kontroversen und sehr emotional geführten Debatte in der Öffentlichkeit soll das Problem der Rückstände auf mehreren Ebenen diskutiert werden. Es sollen mögliche Quellen von Rückständen und Kontaminanten und, inwieweit das Tier als Filter zum Schutz des Menschen ausreicht oder nicht, erörtert werden. Ebenso soll diskutiert werden, wie mit den Ergebnissen durch die immer feiner werdenden Analysemethoden umgegangen werden soll. Ob und wie soll eine Kommunikation mit dem Verbraucher erfolgen.

- In den letzten Jahren hat sich gezeigt, dass die Futtermittelproduktion vielfach verantwortlich ist für Skandale auf den nachgelagerten Stufen. Mögliche Ursachen hierfür sind die Verfahren in der Futterproduktion (Verschleppung in der Anlage, thermische Verfahren, Lagerung).

Im Rahmen dieser Arbeit soll untersucht werden, welche Punkte bei der Herstellung, bei der Lagerung und dem Transport von Futtermitteln in erster Linie kritisch zu bewerten sind.

- Es ist deutlich geworden, dass die Landwirtschaft eine wichtige Rolle bei der Bekämpfung von Zoonosen spielt. Angesichts der vorhandenen Defizite muss auf dieser Stufe der Produktion vieles verbessert werden. Die Landwirtschaft besitzt aber auch ein hohes ethisches Risiko. Der Umgang mit den Tieren, die Haltungsverfahren und -bedingungen sowie auch der Transport von Tieren werden immer wieder kritisch hinterfragt. Die Bedenken und Vorurteile der Verbraucher sind gerade in diesem Bereich besonders groß und können leicht zu einem Meideverhalten beim Kauf bestimmter Fleischarten führen. Positiver werden vom Verbraucher die Verfahren der ökologischen Tierhaltung beurteilt.

Defizite in den Haltungsformen, deren Ursachen und deren mögliche Auswirkung auf das Verhalten der Verbraucher sollen diskutiert werden. Schwerpunkte sind:

die Begrenzung der Anzahl der Tiere pro Stall oder Betrieb, 
die Berechtigung von Maßnahmen wie Kupieren, Enthornen, Schnabelkürzen und Kastration,

mögliche Risiken der Freilandhaltung.

- Auf der Stufe der Schlachtung ist vor allem die Einhaltung strenger hygienischer Regelungen für die Produktsicherheit entscheidend. Hier kommt es trotzdem immer wieder zu Problemen. Neben den hygienischen Aspekten spielen aber auch ethische Aspekte eine Rolle. Der Schlachttiertransport, die Behandlung der Tiere vor der Betäubung sowie Betäubungs- bzw. Tötungsverfahren stehen immer wieder im Fokus der Diskussionen.

Es soll erörtert werden, inwieweit die voranschreitende Technik der Schlachthöfe und der Personaleinsatz auch weiterhin Probleme bei der Durchsetzung einer vorbildlichen Hygiene bereiten werden. Hierbei interessiert es besonders herauszufinden, an welchen Stellen weiterhin Schwachstellen zu finden sein werden. Aber auch die ethischen Aspekte wie der Schlachttiertransport, die Behandlung vor der Betäubung und die Betäubung sollen vor dem Hintergrund diskutiert werden, inwieweit alternative Methoden und Verfahren tierschutzgerechter und damit leichter vom Verbraucher zu akzeptieren wären.

- Hygienemaßnahmen und -konzepte halten vermehrt Einzug in die Lebensmittelproduktion. Im Jahr 2002, dem Jahr der Expertenbefragung, sind Hygienekonzepte, die über die allgemeinen Anforderungen nach GMP + hinausgehen, nur ab der Stufe der Schlachtung gesetzlich vorgeschrieben. Nachvollziehbare Hygienekonzepte fehlen vor allem auf den Stufen Futtermittelproduktion und Landwirtschaft. Ebenso ist das Verständnis von ausreichender Hygiene auf den verschiedenen Stufen sehr unterschiedlich.

Im Rahmen der Arbeit soll der Hygienestatus der einzelnen Stufen beurteilt und Schwachstellen identifiziert werden.

- Der Ansatz vom „Acker bis zum Teller“ oder vom „Stable to table“ stellt hohe Ansprüche an die Rückverfolgbarkeit. Innerhalb der verschiedenen Produktionsstufen fallen jedoch immer wieder Schwachstellen auf, wenn es zu Rückrufaktionen kommt. Zum Detaillierungsgrad der Rückverfolgbarkeit gibt es teilweise sehr unterschiedliche Meinungen. Die Forderungen reichen von relativ großen Tageschargen über Anlieferungschargen bis zum einzelnen Tier.

Anhand der Befragung sollen Schwachstellen bei der Rückverfolgbarkeit und der notwendige Detaillierungsgrad genauer identifiziert werden.

- Kontrollen werden aufgrund von neuen Analysemethoden immer genauer. Rückstände, die noch vor wenigen Jahren nicht nachzuweisen waren, sind heute ein Thema. Insgesamt nimmt der Kontrollumfang auf den einzelnen Stufen zu. Hierbei variiert die Sicherheit der Kontrollen. Diese ist z.B. abhängig von der Person, der Probennahme oder der Methode.

Unter dem Aspekt "Sicherheit lässt sich nicht erkontrollieren" soll die Kontrollsituation auf den einzelnen Stufen betrachtet werden. Es soll erörtert werden, wo es Schwachstellen bei der Kontrolle auf den einzelnen Stufen der Fleischproduktion gibt und wie die Kontrollen in der Futtermittelproduktion, bei Lebensmittelimporten und bei der Schlachtkörperuntersuchung am Schlachtband verbessert werden können. 


\section{$4 \quad$ Methoden}

Eigene Untersuchungen erfolgten im Rahmen einer Expertenbefragung als Risikoanalyse für deutsches Qualitätsfleisch. Die Studie wurde in Kooperation mit der heutigen Bundesforschungsanstalt für Ernährung und Lebensmittel (BFEL), Standort Kulmbach, Herr Prof. Dr. Branscheid und dem Forschungs- und Studienzentrum für Veredelungswirtschaft WeserEms der Fakultät für Agrarwissenschaften der Georg-August-Universität Göttingen, Prof. Dr. Wicke, durchgeführt. Um die Ergebnisse der damaligen Befragung genauer nachvollziehen zu können, werden in diesem Teil zunächst die dieser Umfrage zu Grunde liegenden Methoden sowie der Ablauf der Befragung dargestellt und erläutert.

\subsection{Delphi-Methode}

Die Expertenbefragung ist eine Methode aus der empirischen Sozialforschung. Hiermit wird nach GLÄSER und LAUDEL (2004) ein bestimmter Ausschnitt der sozialen Welt beobachtet, um mit diesen Beobachtungen zur Weiterentwicklung von Theorien beizutragen. Aufgrund von Expertenmeinungen können Unternehmen Vorhersagen zu Sachbereichen erhalten, die unklar sind oder bei denen widersprüchliche Meinungen vorliegen. Hierzu werden z.B. Experten gebeten, Sachverhalte einzuschätzen, die dann ausgewertet und den Experten zur möglichen Revidierung oder Verfeinerung der Einschätzung ein weiteres Mal vorgelegt werden (KOTLER U. BIEMEL, 1992). Eine Expertenbefragung kann zur Verkürzung von aufwendigen Beobachtungsprozessen dienen, wenn die Experten als „Kristallisationspunkte“ praktischen Insiderwissens betrachtet und stellvertretend für eine Vielzahl zu befragender Akteure interviewt werden (BOGNER U. MENZ, 2002).

Als Methode bei der Expertenbefragung wurde die Delphi-Methode gewählt. Nach AICHHOLZER (2002) und HÄDER u. HÄDER (1994) ist die Delphi-Methode ein relativ stark strukturierter Gruppenkommunikationsprozess, in dem Fachleute Sachverhalte beurteilen, über die ein unsicheres und unvollständiges Wissen vorhanden ist. Die Grundidee von Delphi ist, in mehreren Wellen Expertenmeinungen zur Problemlösung zu nutzen und sich in diesem Prozess eines anonymen Feedbacks zu bedienen (HÄDER U. HÄDER, 1998). In der Regel ist die Delphi-Technik eine in zwei oder mehreren Runden durchgeführte Befragung einer Gruppe von Fachleuten. Der Fragebogen der jeweils nachfolgenden Befragung enthält dabei die Ergebnisse aus der vorherigen Fragerunde (AICHHOLZER, 2002).

Die Merkmale von Delphi sind nach HÄDER U. HÄDER (1994):

- die Verwendung eines formalisierten Fragebogens,

- die Befragung von Experten,

- die Anonymität von Einzelantworten,

- die Ermittlung einer statistischen Gruppenantwort,

- $\quad$ die Information der Teilnehmer über die Gruppenantwort und

- $\quad$ eine mehrfache Wiederholung der Befragung.

Bei der Methode wird davon ausgegangen, dass Experten in der Lage sind, in ihrem Fachgebiet sehr gute Schätzungen über zukünftige Entwicklungen abzugeben. Denn Experten sind sich der verschiedenen Einflüsse bewusst, die die Entwicklung des eigenen Fachgebietes bestimmen. Die Auswahl der Experten für die Anlage einer Delphi-Befragung ist sehr wichtig. Die Kompetenz der zu befragenden Experten beeinflusst die Qualität der 
Problemlösung wesentlich. Für eine Delphi-Befragung sind im Normalfall 15-25 Teilnehmer ausreichend. Werden bei der Befragung sehr unterschiedliche Themenkomplexe behandelt, so ist die Teilnehmerzahl auf 30-40 zu erhöhen (HÄDER U. HÄDER, 1994).

Nach HÄDER (2000) haben sich bei der Strukturierung der Expertengruppen folgende Kriterien durchgesetzt:

- Zuordnung der Experten zu dem jeweiligen Fachgebiet,

- Herkunft der Experten aus verschiedenen Bereichen wie etwa Hochschule, öffentlicher Dienst, privater Sektor,

- Herkunft aus unterschiedlichen geographischen Regionen,

- $\quad$ ein unterschiedlich ausgeprägter Grad der Fachkenntnisse.

Insgesamt ist es allerdings sehr schwierig, Experten für eine Befragung zu gewinnen. Im Normalfall liegt die Bereitschaft der ausgewählten Experten an einer Befragung teilzunehmen bei 25\%-40\%. Die Bereitschaft sinkt dabei mit jeder Befragungsrunde. Das beste Mittel dem entgegenzuwirken besteht z.B. darin, durch neue Formulierungen oder veränderte Fragerichtungen, durch Zusatzfragen oder zusätzliche objektive Informationen den Befragungsprozess über alle Runden hinweg lebendig zu halten und kreative Stellungnahmen zu fördern (GESCHKA, 1977). Nach HÄDER U. HÄDER (1994) müssen nicht alle Experten dazu bewegt werden, bis zur letzten Befragungsrunde dabeizusein, da der Zusammenhang zwischen der Expertenzahl und der Validität der Ergebnisse nicht direkt proportional sein muss. Wichtiger kann es sein, dass die Experten mit der höchsten individuellen Kompetenz an allen Befragungsrunden teilnehmen. Hierbei bleibt offen, wie die Einschätzung der Kompetenz festgelegt wird.

Die Statements in einem Delphi-Fragebogen sollten nicht mehr als 20 Worte haben und möglichst leicht verifizierbar bzw. konkret sein. Ebenfalls sollte der Fragebogen für eine Expertengruppe 25 Statements nicht überschreiten. Nach HÄDER U. HÄDER (1998) ist es nicht sinnvoll, Fragen, bei denen in der ersten Befragungsrunde Konsens besteht, im Fragebogen der zweiten Befragungsrunde zu belassen.

Die Rückinformation der Ergebnisse kann durch die Rückmeldung von Mittelwerten und geeignet erscheinenden Streuungsmaßen, graphischen Darstellungen, Tabellen usw. erfolgen. Bisher existieren keine Standards für die Gestaltung des Feedbacks (HÄDER U. HÄDER, 1998).

\subsection{Methoden der Expertenbefragung}

Die Befragung der Experten gliederte sich in zwei Runden. In der ersten Runde wurden Experten angeschrieben und anhand von drei Bögen (Landwirtschaft, Futtermittel, Schlachtung/Zerlegung) schriftlich befragt. Die zweite Befragungsrunde erfolgte als Interview.

Die Auswahl der Themen für die Expertenbefragung basierte auf folgenden Grundlagen:

- den Prüfplänen und Lastenhefte des ehemaligen CMA-Prüfsiegels,

- der niederländischen und dänischen nationalen Markenprogramme,

- den Regelungen von QS,

- den Regelungen von EUREP GAP, 
- den Regelungen von GMP+,

- aufgrund eigener Erfahrungen.

Zusätzlich sind die Ergebnisse einer Auswertung von abgelaufenen Fleischkrisen berücksichtigt worden. Die Auswertung erfolgte nach einem Verfahren, das dem des HACCPKonzeptes entspricht. Es wurden folgende Punkte identifiziert:

Herkunft der Tiere; Herkunft der Futtermittel; Zusammensetzung und ökologische Produktion des Futters; Tiermehle; gentechnische veränderte Futtermittel; Einsatz von Düngemitteln; Pestizide; Leistungsförderer und Tierarzneimittel; Haltungsvorschriften wie Licht, Liegefläche; Bodenbeschaffenheit; Auslauf; Weidehaltung bzw. Stallhaltung; Transport; Schlachtung; Behandlung des Fleisches im Schlachthof; Kontrollen wie chemische, physikalische und mikrobiologische Messungen; Geschmack; Geruch; Gewebequalität; Vorschriften für den Verkauf und Kontrollen des Programms.

Da viele Risiken stufenübergreifend eine Rolle spielen, bezieht sich der Umfang der Studie auf die Stufen der Futtermittelproduktion, der landwirtschaftlichen Tierproduktion und der Schlachtung. Da alle Produktionsstufen eigene spezifische Risiken bergen, wurden aus den identifizierten Punkten jeder Stufe eigene Thesen erarbeitet. Ein weiterer Grund für einen Fragenkatalog nach Stufen ist, dass einige der ausgewählten Experten zum Teil nur in einer Produktionsstufe über Expertenwissen verfügen.

\subsubsection{Auswahl der Experten}

Die Experten stammten aus den verschiedenen Bereichen der Erzeugungskaskade Landwirtschaft, Futtermittelwirtschaft und Schlachtung. Sie setzten sich aus Mitarbeitern in führenden Positionen der Wirtschaft, Wissenschaft, Verwaltung und Beratung zusammen. Alle Befragten verfügten über eine akademische Ausbildung und/oder waren auf ihrem Gebiet als Fachleute anerkannt. Die Befragung wurde in ganz Deutschland durchgeführt. Die regionale Verteilung geht aus Abbildung 11.2-27 hervor.

In der ersten Runde wurden 89 Experten angeschrieben. Insgesamt antworteten 40 Experten. Davon stammten 20\% der Experten aus der Beratung, 12,5\% aus der Verwaltung, 47,5\% aus der Wirtschaft und $20 \%$ aus der Wissenschaft. Einige Experten beantworteten nur einen, andere alle drei Fragebögen. Bei jeder Frage wurde der Experte gebeten, seine eigenen Fachkenntnisse einzuschätzen (hoch=3, mittel=2, gering=1). Die Antworten schlüsseln sich wie folgt auf (Tabelle 11.1-56):

- 22 Antworten aus dem Bereich Fütterung ( $\varnothing$ Fachkenntnisse: 1,99),

- 25 Antworten aus dem Bereich Landwirtschaft ( $\varnothing$ Fachkenntnisse: 2,16),

- 29 Antworten waren aus dem Bereich Schlachtung/Zerlegung, ( $\varnothing$ Fachkenntnisse: 2,20).

Die Auswahl der Experten für die zweite, mündliche Befragungsrunde, erfolgte aufgrund der Selbsteinschätzung der Experten aus der schriftlichen Befragung. Aus der Gesamtheit der 40 Experten wurden für die zweite Befragungsrunde 25 Personen anhand ihrer Fachkenntnis ausgewählt. Es wurden nur Experten mit hoher und mittlerer Kompetenz befragt. Aus den Bereichen Beratung und Verwaltung wurden jeweils 3 Experten, aus dem Bereich Wissenschaft 6 Experten und aus dem Bereich Wirtschaft 13 Experten befragt. 22 Experten nahmen teil. Je nach Kompetenz wurden den Experten zwischen 9 und 20 Fragen gestellt. 


\subsubsection{Schriftliche Befragung}

In den Fragebögen wurden geschlossene und offene Fragen verwendet. Jeder Fragebogen bestand aus ca. 22 offenen und/oder geschlossenen Fragen. Durch die geschlossenen Fragen sollten verschiedene Sachverhalte nach den Kriterien einer Risikobeurteilung eingeschätzt werden. Hierbei wurde Wert darauf gelegt, dass bei den Fragen sowohl eine Einschätzung zu realen aber auch zu Risiken, die der Verbraucher als kritisch sehen könnte, erfolgte. Die Fragen richteten sich nach den Beurteilungskriterien des HACCP-Konzeptes: Wahrscheinlichkeit und Auswirkung einer Gefährdung sowie der Gefahrenkategorie. Als Gefahrenkategorien wurden zusätzlich zu den klassischen Kriterien des HACCP-Konzeptes (chemisch, mikrobiologisch, physikalisch) die Punkte sensorisch, ernährungsphysiologisch und ethisch definiert. Des weiteren hatten die Experten den Einfluss auf die jeweilige Tierart, die Gesundheitsgefahr, vorhandene Standards und ein mögliches Meideverhalten eingeschätzt. Bei allen geschlossenen Fragen hatten die Experten, die Möglichkeit, eigene Anmerkungen oder Begründungen für ihre Einschätzungen anzugeben. Bei den offenen Fragen wurden die Experten gebeten, konkrete Kriterien zu nennen, die ihrer Meinung nach für den jeweiligen Inhalt der Frage von Relevanz waren.

Die Auswertung der geschlossenen Fragen erfolgte anhand der Erfassung der absoluten und der relativen Häufigkeiten. Die Darstellung der Ergebnisse erfolgte über Balkendiagramme und Tabellen. Balkendiagramme wurden zur Darstellung von Fragen mit genau einer Antwortmöglichkeit verwendet, die Tabellenform wurde für Fragen, bei denen Mehrfachnennungen gewünscht waren, eingesetzt.

Bei offenen Fragen wurden die Häufigkeiten nach der Anzahl der Nennungen einzelner Begriffe ermittelt. Nicht eindeutige Nennungen wurden nicht zugeordnet und nicht extra aufgeführt. Hier erfolgt die Darstellung wie bei den geschlossenen Fragen als absolute oder relative Häufigkeiten in Form von Balkendiagrammen oder als Aufzählung im Text.

\subsubsection{Mündliche Befragung}

Die Antworten aus der schriftlichen Befragung wurden als Ausgangsmaterial für die zweite Befragungsrunde genutzt. Aus dem Bereich Schlachtung wurden 10, aus dem Bereich Landwirtschaft 12 und aus dem Bereich Futtermittel 9 Themenbereiche ausgewählt.

Die Auswahl der Fragen für die zweite Befragungsrunde erfolgte dabei nach folgenden Gesichtspunkten:

- Führt nach der Einschätzung der Experten der Sachverhalt zu einem Meideverhalten?

- Gibt es eine Diskrepanz bei der Bewertung des Meideverhaltens und der Einschätzung der Gesundheitsgefahr?

- Fehlen Standards für Kontrollen?

- Hat der Auftraggeber ein direktes Interesse bekundet, den Sachverhalt weiter zu verfolgen?

- Wurden von Seiten der Experten Kommentare oder Antworten gegeben, die interessant erschienen, weiter verfolgt zu werden?

- Antworten aus offenen Fragen, die verdichtet werden sollen.

Die Ergebnisse ausgewählter Fragen der ersten Runde wurden den Experten per PowerPoint Präsentation vorgestellt. Anschließend wurden sie gebeten, einzelne Fragen zu 
spezifizieren oder eine erweiterte Fragestellung zu beantworten. Zusätzlich wurden ihnen Fragen zu den Sachverhalten aus dem ersten Fragebogen gestellt, deren Bedeutung sie für das Jahr 2015 einschätzen sollten. Um die Antworten der offenen Fragen anschließend ohne Datenverlust auswerten zu können, wurden alle Interviews, mit Einverständnis der befragten Personen, auf einem Diktiergerät aufgezeichnet. Die Antworten zu den geschlossenen Fragen wurden in schriftlicher Form erfaßt.

Die Auswertung der geschlossenen Fragen verlief identisch zu der schriftlichen Befragung.

\subsection{Studentenbefragung}

Die Studentenbefragung wurde gewählt, um die Einschätzungen der Experten zu einer möglichen Meinung der Verbraucher zu prüfen.

Insgesamt wurden 64 Studenten befragt. Alle befragten Studenten studierten Agrarwissenschaften, hauptsächlich im 3. und 5. Semester. Die Hauptstudienrichtungen der Studenten waren Tierproduktion, Agribusiness und Pflanzenproduktion. Das Durchschnittsalter der Studenten lag bei ca. 23 Jahren. Über 56\% der Studenten verfügten über keine berufliche Ausbildung vor dem Studium. 62,5\% der Studenten kommen von einem landwirtschaftlichen Betrieb.

Im Rahmen der Umfrage wurden auch bei den Hintergrunddaten die Geschmackspräferenzen abgefragt. Insgesamt bevorzugten die Studenten Schweinefleisch. An zweiter Stelle folgte bei den Studenten, die von einem landwirtschaftlichen Betrieb stammten, das Rindfleisch. Bei den Studenten hingegen, die nicht von einem landwirtschaftlichen Betrieb stammten, stand Geflügelfleisch an zweiter Stelle (Tabelle 11.1-57).

Den Studenten wurden ähnliche Fragen bzw. teilweise die gleichen Fragen gestellt wie den Experten in der zweiten Befragungsrunde. Die Fragen für die Studenten wurden dahingehend ausgewählt, dass sie auch ohne besondere Fachkenntnisse und auch von Verbrauchern beantwortet werden könnten. In der Auswertung wurden nur Fragen berücksichtigt, die einen direkten Bezug zu den an die Experten gestellten Fragen haben.

Bei der Ergebnisdarstellung und der anschließenden Diskussion wird teilweise zwischen Studenten, deren Eltern einen landwirtschaftlichen Betrieb besitzen, und Studenten, deren Eltern keinen landwirtschaftlichen Betrieb besitzen, unterschieden. Hierfür sollen nachfolgende Begriffe verwendet werden:

alle Studenten

Studenten deren Eltern einen landwirtschaftlichen Betrieb besitzen

Studenten deren Eltern keinen landwirtschaftlichen Betrieb besitzen
= Studenten,

= Studenten $(\mathrm{mL})$,

= Studenten (oL). 


\section{$5 \quad$ Ergebnisse}

Die Darstellung der Ergebnisse aus der Experten- und Studentenbefragung erfolgt nach Themenschwerpunkten. Ein Teil der Abbildungen, Tabellen und befindet sich im Anhang und es erfolgen Verweise hierauf.

\section{$5.1 \quad$ Zucht}

Nach Meinung von ca. 63\% der Experten werden die Rassen/Genetikvielfalt, die zur Fleischproduktion eingesetzt werden, abnehmen. Allerdings sehen auch $22 \%$ eine Tendenz zur Zunahme (Tabelle 5.1-1). Bei Rückfragen in der mündlichen Befragung wurden kaum Unterschiede zwischen den einzelnen Arten genannt. Bei Geflügel (heute werden nur noch wenige Rassen/Genetikvielfalt eingesetzt) wird es die wenigsten Veränderungen geben.

Tabelle 5.1-1: $\quad$ Entwicklung der zur Fleischproduktion eingesetzten Rassen/Genetikvielfalt zum Jahr 2015.

\begin{tabular}{ccccccc}
\hline & & stark abnehmend & abnehmend & wie heute & zunehmend & stark zunehmend \\
\hline Experten & $\mathrm{N}=19$ & 4 & 8 & 3 & 2 & 2 \\
& $\%$ & 21 & 42 & 16 & 11 & 11 \\
\hline
\end{tabular}

Bei Rindern wurden als bevorzugte Rassen Limousin, Angus/Anguskreuzungen, Herford und Fleckvieh; bei Schweinen wurden Duroc, Landrasse x Duroc, allgemein Kreuzungstiere und Hybriden aus stresssanierten Linien genannt. Auffällig ist die geringe Nennung des BHZP. (Tabelle 11.1-1 und Tabelle 11.1-2). Bei Geflügel konnten keine Schwerpunkte ermittelt werden. Genannt wurden hier zu gleichen Teilen: Bronzepute, BUT BIG 6, COBB, ROSS, französische Genetiken und langsam wachsende Hähnchenhybriden.

\subsubsection{Qualzucht}

Der Fleischanteil ist nicht nur eine Frage des Genotyps, sondern auch des Tierschutzes. Die befragten Experten schließen nicht aus, dass die Zucht auf einen hohen Fleischanteil eine Form der Qualzucht ist (für 71\% der Experten mit „hoher“ bzw. „mittlerer“ Wahrscheinlichkeit). Sie sehen hier auch mögliche Auswirkungen auf die Produktqualität des Fleisches (für 75\% der Experten mit "hoher" bzw. „mittlerer" Wahrscheinlichkeit). Eine Gesundheitsgefahr für den Verbraucher wird aber eher ausgeschlossen (Abbildung 5.1-1).

Dass züchterische Fehlentwicklungen zu einem Meideverhalten führe, wird nur von $29 \%$ der Experten sicher angenommen. 17\% schließen dieses aus (Abbildung 5.1-1). Diese Bewertung deckt sich mit der Einschätzung der Studenten. Zirka 30\% der Studenten würden bei dem Bekanntwerden von Missständen sicher oder höchstwahrscheinlich mit einem Meideverhalten reagieren. Allerdings hätte Qualzucht bei zirka 26,5\% keinen Einfluss auf deren Fleischkonsum (Tabelle 5.1-2). Splittet man das Ergebnis der Studenten in Studenten (mL) und in Studenten (oL), würden ca. doppelt so viele Studenten (oL) mit einem Meideverhalten reagieren als das bei Studenten $(\mathrm{mL})$ der Fall wäre.

Das Problem einer sogenannten Qualzucht wird von den meisten Experten für die Arten Schwein $(90,9 \%)$ und Geflügel $(95,5 \%)$ gesehen. Hier spielen die stark ausgeprägten Teilstücke bei der Bezahlung die entscheidende Rolle. Lediglich 59,1\% der Experten sehen einen Einfluss für Rinder (Tabelle 11.1-4). 
Ein Problem bei der Beurteilung von Qualzucht ist das Fehlen von entsprechenden Standards (Abbildung 5.1-1). In der mündlichen Befragung sollte versucht werden hierfür Messparameter zu finden.

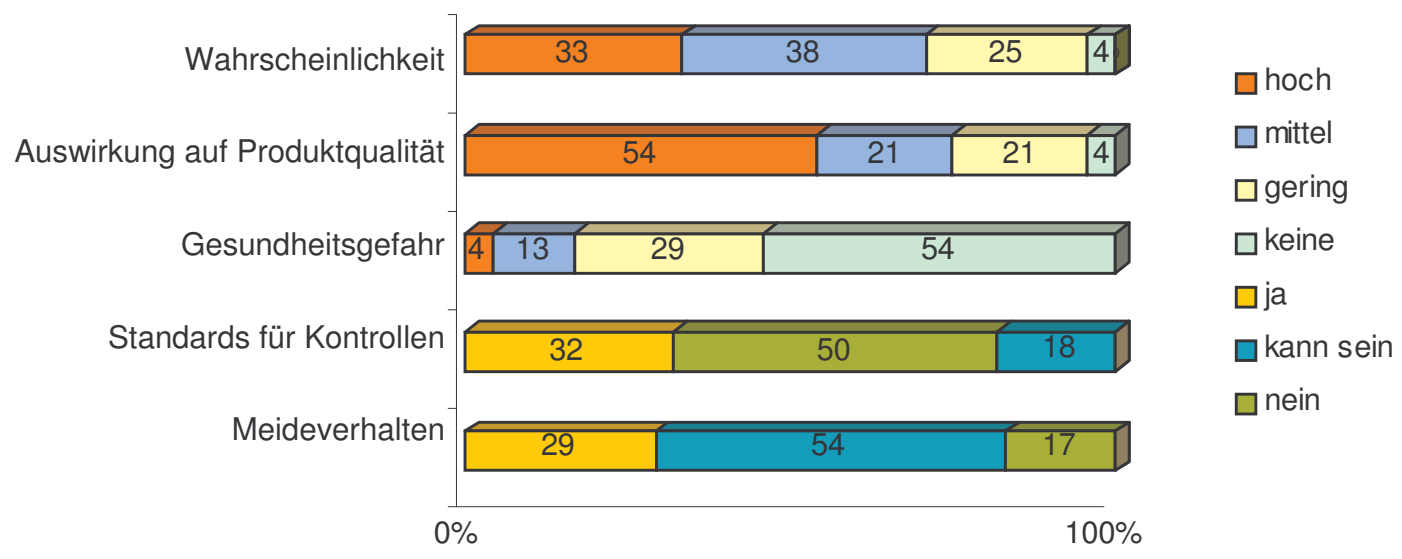

Abbildung 5.1-1: $\quad$ Antworten auf die These: Die Zucht auf einen hohen Fleischanteil ist eine Form der Qualzucht.

Tabelle 5.1-2: $\quad$ Verzichtverhalten von Studenten auf Fleisch bei Bekanntwerden von Missständen.

\begin{tabular}{ccccccc}
\hline & & nein & eher nein & neutral & eher ja & ja \\
\hline Studenten & $\mathrm{N}=64$ & 17 & 19 & 9 & 13 & 6 \\
& $\%$ & 27 & 30 & 14 & 20 & 9 \\
Studenten & $\mathrm{N}=24$ & 1 & 9 & 3 & 9 & 2 \\
(oL) & $\%$ & 4 & 38 & 13 & 38 & 8 \\
Studenten & $\mathrm{N}=40$ & 16 & 10 & 6 & 4 & 4 \\
(mL) & $\%$ & 40 & 25 & 15 & 10 & 10 \\
\hline
\end{tabular}

Genannt werden: Verluste, Fortpflanzungsfähigkeit, Gentests (MHS-Sanierung), Veränderungen an Knochen und Gelenken sowie am Körperbau, das Verhältnis zwischen Knochenbau/Alter und Fleischansatz, Bewegungsverhalten und Herz-Kreislaufprobleme.

Diese Punkte müssen aber sehr kritisch betrachtet werden, da sie auch andere Ursachen, z.B. mangelhafte Haltungsbedingungen oder Infektionskrankheiten, haben können. Ein Experte war der Meinung, dass es das Problem der Qualzucht nicht gibt. Die Tiere würden sonst nicht die entsprechenden Zunahmen realisieren. Ebenso müsste die Ausfallquote dann so hoch sein, dass sich eine Produktion nicht rechnet. Er sieht mehr Probleme in schlechten Haltungsbedingungen. Ein anderer Experte sieht generell Probleme in der Definition von Standards. Nach seiner Meinung spielen subjektive Wahrnehmungen eine starke Rolle und die Vorstellungen zwischen Verbraucher und Landwirtschaft gehen zu weit auseinander.

Tendenziell gehen sowohl die meisten Experten als auch die meisten Studenten davon aus, dass der Verbraucher bis zum Jahr 2015 in der Tierzucht verstärkt ein Problem der Qualzucht sehen wird (Tabelle 5.1-3). 
Tabelle 5.1-3: $\quad$ Entwicklung des Problembewusstseins der Verbraucher im Hinblick auf Qualzucht zum Jahr 2015.

\begin{tabular}{ccccccc}
\hline & & stark abnehmend & abnehmend & wie heute & zunehmend & stark zunehmend \\
\hline Experten & $\mathrm{N}=21$ & 0 & 6 & 5 & 9 & 1 \\
& $\%$ & 0 & 29 & 24 & 43 & 5 \\
Studenten & $\mathrm{N}=64$ & 3 & 13 & 14 & 33 & 1 \\
& $\%$ & 5 & 20 & 22 & 48 & 2 \\
\hline
\end{tabular}

\subsection{Gentechnologische Produktionsverfahren}

\subsubsection{Genveränderte Pflanzen}

Die Experten halten Auswirkungen von gentechnisch veränderten Pflanzen auf das Endprodukt Fleisch und auf die Produktqualität (gesundheitliche Risiken) für wenig wahrscheinlich. Es werden keine oder nur geringe Gesundheitsgefahren für den Verbraucher gesehen. Dem gegenüber steht die Einschätzung, dass der Verbraucher höchstwahrscheinlich bei dem Einsatz genveränderter Futtermittel mit einem Meideverhalten reagieren wird. Insgesamt halten 78\% der Experten die Wahrscheinlichkeit eines Meideverhaltens für hoch oder mittel (Abbildung 5.2-1).

Obwohl keine Einflüsse auf die Qualität erwartet werden (Abbildung 5.2-1), ordnen die Experten das Risiko nicht nur der ethischen Kategorie zu, sondern sehen auch ernährungsphysiologische und mikrobiologische Komponenten (Tabelle 11.1-5).

Experten sowie auch die Studenten gehen bis zum Jahr 2015 von einer Zunahme bzw. einer starken Zunahme des Einsatzes von genveränderten Pflanzen aus (Tabelle 5.2-1).

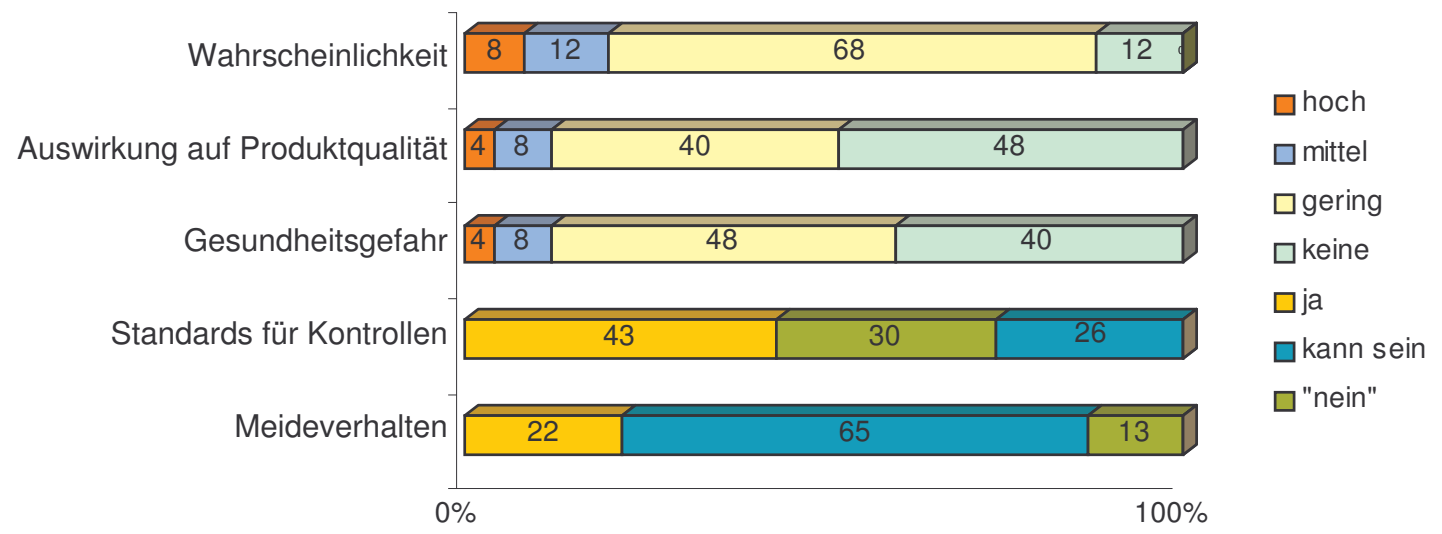

Abbildung 5.2-1: $\quad$ Ergebnisse zur These: Der Verbraucher steht heute genveränderten Pflanzen sehr kritisch gegenüber. Inwieweit diese Veränderungen eine Auswirkung über das Futtermittel auf das Produkt Fleisch haben, ist heute noch ungewiss. Bitte nennen Sie Ihre Einschätzung zu genveränderten Pflanzen. 
Tabelle 5.2-1: $\quad$ Einschätzung der Entwicklung des Einsatzes von genveränderten Pflanzen in der Fütterung von Masttieren zum Jahr 2015.

\begin{tabular}{ccccccc}
\hline & stark abnehmend & abnehmend & wie heute & zunehmend & stark zunehmend \\
\hline Experten & $\mathrm{N}=19$ & 0 & 1 & 0 & 8 & 10 \\
& $\%$ & 0 & 5 & 0 & 42 & 53 \\
Studenten & $\mathrm{N}=64$ & 0 & 1 & 3 & 44 & 16 \\
& $\%$ & 0 & 2 & 5 & 65 & 28 \\
\hline
\end{tabular}

\subsubsection{Einsatz von genetisch veränderten, geklonten Tieren}

Dass in der Zukunft auch genveränderte/geklonte Tiere in der Fleischproduktion eingesetzt werden, wird von den Experten als wahrscheinlich eingestuft. 25\% der Experten sind der Meinung, dass die Wahrscheinlichkeit des Einsatzes hoch ist. Keiner der Experten schließt den Einsatz aus. Der Einfluss auf die Produktqualität wird dabei eher positiv gesehen. 38\% der Experten beurteilen den möglichen Einfluss auf die Produktqualität mit hoch und $46 \%$ mit mittel. Lediglich 13\% der Experten sehen keinen Einfluss auf die Produktqualität. Eine Gefahr für den Verbraucher sehen sie eher nicht. Sie gehen aber im Gegensatz dazu bei dem Bekanntwerden des Einsatzes solcher Tiere von einem Meideverhalten seitens des Verbrauchers aus. Bei den Standards für Kontrollen sind die Meinungen der Experten sehr unterschiedlich. 29\% der Experten sind eindeutig der Meinung, dass Standards vorhanden sind, und 58\% der Experten sind der Meinung, dass Standards fehlen (Abbildung 5.2-2). Ebenso wie bei den gentechnisch veränderten Pflanzen wird in dem Einsatz von genveränderten/geklonten Tieren vor allem eine ethische Gefahr $(90,9 \%)$ gesehen. Daneben werden aber auch die Kategorien ernährungsphysiologische $(27,3 \%)$ und sensorische (22,7\%) Gefahren genannt (Tabelle 11.1-6).

Der Einfluss der Verfahren der Gentechnik und des Klonens wird bei allen drei Tierarten (Rind, Schwein und Geflügel) als hoch (>60\%) angesehen. Mit $87 \%$ wird ein Einfluss zuerst beim Schwein und am wenigsten (65\%) beim Rind erwartet (Tabelle 11.1-7).

Auf die ergänzende Frage, wie sich 2015 das Problembewusstsein der Verbraucher in Hinblick auf genveränderte Tiere entwickeln wird, sehen 57\% der Experten eine zunehmende bzw. eine stark zunehmende Tendenz, und nur $24 \%$ gehen von einer Abnahme aus. Ähnlich ist hier auch die Bewertung der Studenten. 52\% gehen von einer zunehmenden bzw. einer stark zunehmenden Tendenz und 30\% gehen von einer Abnahme aus (Tabelle 5.2-2).

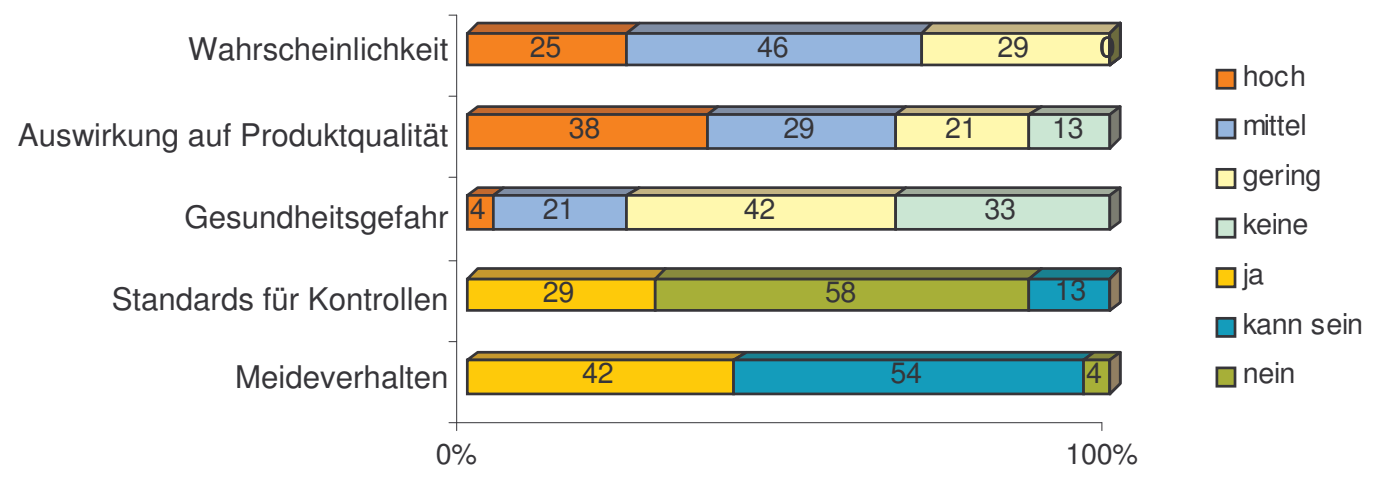

Abbildung 5.2-2: Ergebnisse zur These: Genveränderte/geklonte Tiere werden in der Zukunft für die Fleischproduktion eingesetzt. 
In der mündlichen Befragung wurden die Experten gebeten eine Einschätzung abzugeben, wie der Umgang mit dem Verbraucher (Information/Aufklärung) erfolgen soll. Schwerpunktmäßig wurden hierzu nachfolgende Antworten gegeben:

- Die Kommunikation darüber braucht nicht geführt werden, bevor nicht wissenschaftlich fundierte Aussagen darüber vorliegen, welchen sinnvollen Nutzen der Einsatz Gentechnik birgt und ob Gefahren davon ausgehen.

- Es muss ein offensiver Umgang mit der Problematik Gentechnik erfolgen. Vor allem muss transparent kommuniziert werden, in welchen Bereichen es zu einer Gefahr kommen kann oder wie sich die Veränderung auf die Produkte niederschlägt.

- Es müssen die positiven Aspekte der Verfahren für das Tier herausgestellt werden, wie: bessere Gesundheit, mehr Wohlbefinden, Tierschutz, Einflüsse auf das Produkt und geringere Gefahren für den Menschen (Zoonosen).

- Die Problematik muss immer im Zusammenhang mit der Humanmedizin gesehen werden. Wenn hier Fortschritte erzielt werden, kann die Akzeptanz leichter erreicht werden.

- Eine höhere Produkt/Genussqualität wird als Argument höchstwahrscheinlich nicht akzeptiert werden.

- Klare Standards müssen festgelegt werden, die für alle Ebenen und Länder (national und international) gelten. Es muss offen hierüber mit dem Verbraucher und den Verbänden diskutiert werden. Es müssen klar die Vor- und Nachteile dargelegt werden. Die festgelegten Standards müssen dabei mit einem hohen Aufwand kontrolliert werden.

Ebenso sollten die Experten dazu Stellung nehmen, welche Gefahren sie für einen Vermarkter von Fleisch sehen. Insgesamt wird vom Meideverhalten der Verbraucher und somit von Umsatzrückgängen ausgegangen. Eine Akzeptanz kann nur dann erreicht werden, wenn der Kunde umfassend informiert wird, wenn zugesagte Garantien (genveränderte und nicht genveränderte Produkte) kontrollierbar eingehalten werden und alle Beteiligten nach einer einheitlichen Strategie verfahren.

Tabelle 5.2-2: Einschätzung der Entwicklung des Problembewusstseins der Verbraucher in Hinblick auf genveränderte/geklonte Tiere zum Jahr 2015.

\begin{tabular}{ccccccc}
\hline & & stark abnehmend & abnehmend & wie heute & zunehmend & stark zunehmend \\
\hline Experten & $\mathrm{N}=21$ & 0 & 5 & 4 & 9 & 3 \\
& $\%$ & 0 & 24 & 19 & 43 & 14 \\
Studenten & $\mathrm{N}=63$ & 0 & 19 & 11 & 27 & 6 \\
& $\%$ & 0 & 30 & 17 & 43 & 10 \\
\hline
\end{tabular}

\section{3}

Zoonosen

Nach der Bedeutung der Zoonosen wurden die Experten auf zwei Ebenen befragt. In einer schriftlichen Befragung wurden die Experten gebeten Zoonosen zu nennen, die gegenwärtig und in der Zukunft eine Rolle spielen. Die genannten Zoonosen wurden von den Experten in der mündlichen Befragung hinsichtlich ihrer Bedeutung für die Zukunft bewertet (Abbildung 5.3-1). Die Salmonellose ist nach der Meinung der Experten heute die wichtigste Zoonose, gefolgt von der Listeriose und Erkrankungen durch Campylobacter und coliforme Keime. Zukünftig wird sich die Bedeutung allerdings verschieben. Salmonellen, Listerien, 
Campylobacter und coliforme Keime werden weiterhin eine wichtige Rolle spielen, aber deren Bedeutung wird gleichmäßiger sein. Neben diesen Zoonosen werden aber Staphylokokken, Botulismus und Influenza an Bedeutung gewinnen. Auffällig ist auch, dass die parasitären Erkrankungen durch Zystizerkosen (Bandwürmer) und Trichinen eher keine Bedeutung mehr haben werden.

Als weitere Zoonosen wurden von einzelnen Experten in der mündlichen Befragung Streptokokken, die Tuberkulose und die Paratuberkulose genannt. Bei der Paratuberkulose wird ein Zusammenhang mit MorbusCrohn beim Menschen vermutet. Als weitere Erkrankung wurde beim Geflügel die Papageienkrankheit Chlamydia psittaci genannt, die für den Menschen die bedeutendste Orniothose ist.

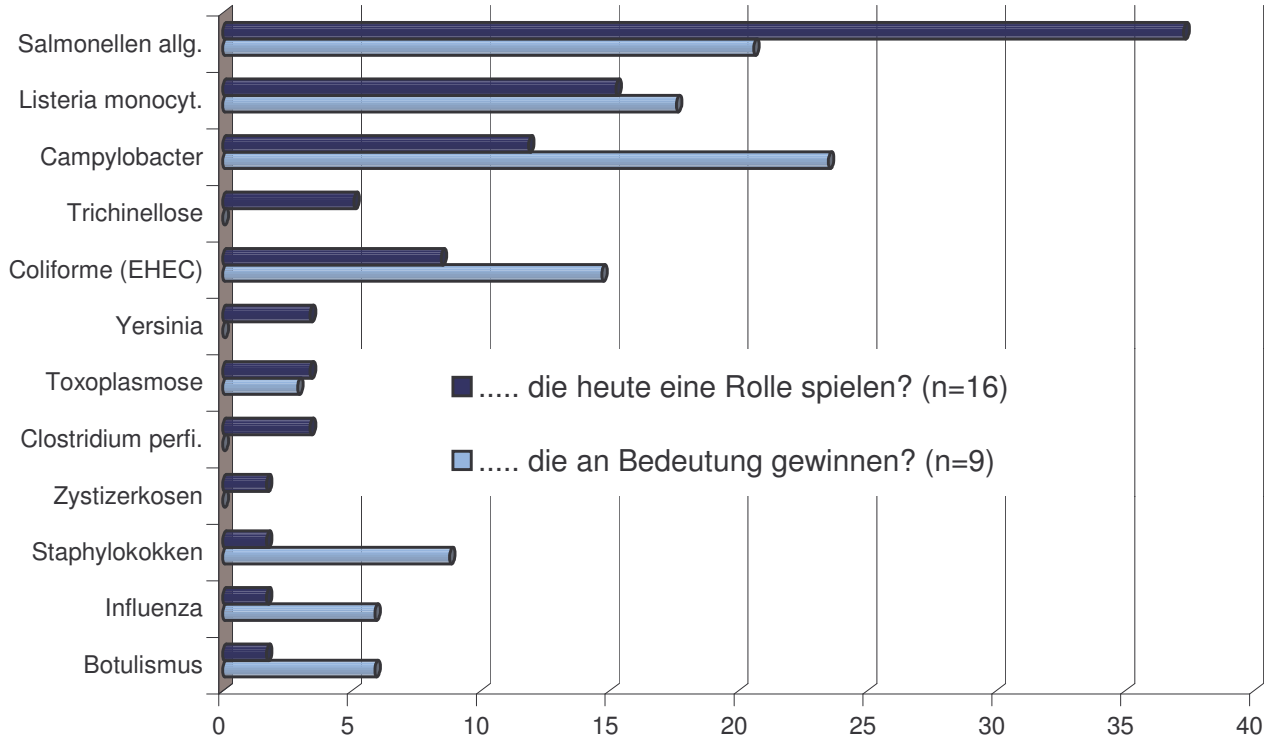

Abbildung 5.3-1: Heutige/zukünftige Bedeutung von Zoonosen: Welche Zoonosen sehen Sie, die heute eine Rolle spielen, bzw. die an Bedeutung gewinnen?

Die Wahrscheinlichkeit, dass das Risiko einer Erkrankung des Menschen durch Zoonosen in Deutschland steigen wird, sehen $13 \%$ der Experten als hoch und 54\% als mittel an. Eine Auswirkung auf die Produktqualität wird dagegen von 38\% der Experten als gering und von $25 \%$ als nicht vorhanden eingestuft. Standards für die Kontrollen sind nach der Meinung von 75\% der Experten vorhanden (Abbildung 5.3-2). Eine Gefahr wird hauptsächlich für die Tierarten Schwein und Geflügel gesehen (Tabelle 11.1-10).

Über die Entwicklung der Erkrankungen durch Zoonosen bis zum Jahr 2015 kann aus den Antworten der Experten keine eindeutige Prognose abgeleitet werden Tabelle 5.3-1). Bezüglich der Einschätzung der Entwicklung des Vorkommens von pathogenen Keimen, die über das Futtermittel das Nahrungsmittel Fleisch kontaminieren, gehen die Experten von einer Abnahme aus (Tabelle 5.3-2).

Tabelle 5.3-1: $\quad$ Einschätzung der Entwicklung der Anzahl der Erkrankungen durch Zoonosen zum Jahr 2015.

\begin{tabular}{ccccccc}
\hline & & stark abnehmend & abnehmend & wie heute & zunehmend & stark zunehmend \\
\hline Experten & $\mathrm{N}$ & 0 & 5 & 7 & 6 & 2 \\
& $\%$ & 0 & 25 & 35 & 30 & 10 \\
\hline
\end{tabular}




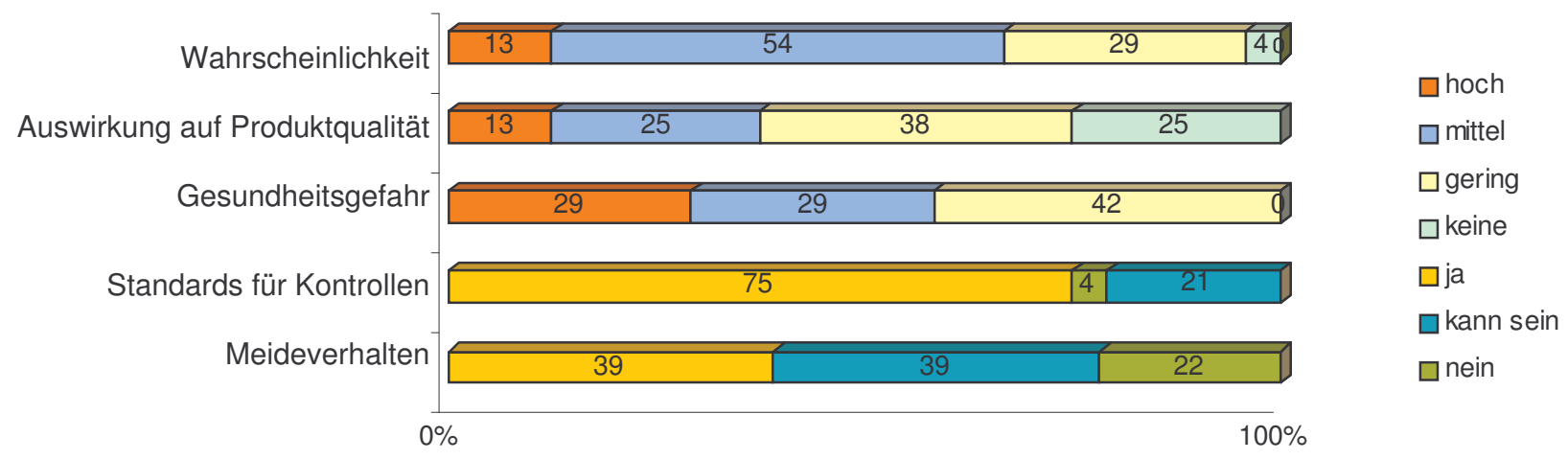

Abbildung 5.3-2: Beurteilung der Frage: Wird das Risiko einer Erkrankung des Menschen durch Zoonosen in Deutschland steigen?

Tabelle 5.3-2: $\quad$ Einschätzung der Entwicklung des Vorkommens von pathogenen Keimen, die über das Futtermittel das Nahrungsmittel Fleisch kontaminieren, zum Jahr 2015.

\begin{tabular}{ccccccc}
\hline \multirow{3}{*}{ Experten } & & stark abnehmend & abnehmend & wie heute & zunehmend & stark zunehmend \\
\cline { 2 - 7 } & $\mathrm{N}$ & 2 & 8 & 6 & 3 & 0 \\
& $\%$ & 11 & 42 & 32 & 16 & 0 \\
\hline
\end{tabular}

\subsubsection{Schädlinge, Vögel und Haustiere in Futtermittel herstellenden Betrieben}

Zurzeit sind Schädlinge, Vögel und Haustiere in fast allen Futtermittel erzeugenden Betrieben zu finden. Die vollständige Einschränkung des Kontaktes ist eher unwahrscheinlich. Gerade als Überträger von pathogenen Mikroorganismen haben Schadnager usw. eine nicht zu unterschätzende Bedeutung in der Futtermittelproduktion. Eine Gefahr für den Verbraucher durch die Übertragung solcher Keime wird von $17 \%$ der Experten als hoch und von $65 \%$ als mittel eingestuft. Von einem Meideverhalten bei Fleisch wird nicht ausgegangen (Abbildung 5.3-3). Hauptsächlich wird durch die Experten ein mikrobiologisches Risiko (95,7\%) gesehen. Neben diesem sehen die Experten auch ein chemisches $(17,4 \%)$ und ein ernährungsphysiologisches (13\%) Risiko (Tabelle 11.1-11). In erster Linie existiert die Gefahr für Schweine und Geflügel (Tabelle 11.1-12).

Als größte Gefahr wird die Übertragung von Salmonellen genannt. Aber auch andere pathogene Keime wie Clostridien, Staphylokokken und Enterobacteriaceae könnten eine Rolle spielen.

Durch eine systematische und gezielte Schädlingsbekämpfung lässt sich aber das Risiko der Übertragung stark reduzieren. Die Experten gehen davon aus, dass die Gefahr der Kontamination mit Krankheitskeimen bis zum Jahr 2015 so bestehen bleibt wie heute, bzw. eher abnehmen wird (Tabelle 5.3-3).

Tabelle 5.3-3: $\quad$ Einschätzung der Entwicklung der Übertragung von Krankheitskeimen durch Schädlingsbefall zum Jahr 2015.

\begin{tabular}{ccccccc}
\hline \multirow{3}{*}{ Experten } & & stark abnehmend & abnehmend & wie heute & zunehmend & stark zunehmend \\
\cline { 2 - 7 } & $\mathrm{N}$ & 0 & 8 & 9 & 2 & 0 \\
& $\%$ & 0 & 42, & 47 & 11 & 0 \\
\hline
\end{tabular}




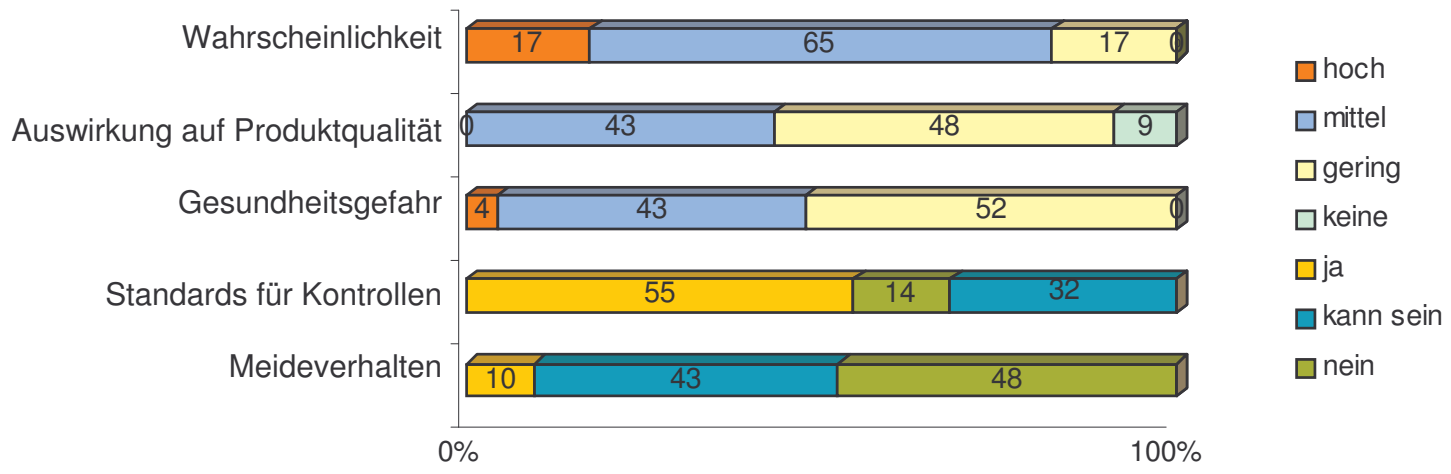

Abbildung 5.3-3: Beurteilung der These: Schädlinge, Vögel und Haustiere sind in fast allen Futtermittel herstellenden Betrieben zu finden.

\section{4}

Tierseuchen

\subsubsection{Eintrag von Keimen und Krankheitserregern aus dem Ausland}

Die Gefahr des Eintrags von Keimen und Krankheitserregern wird nach Ansicht der meisten Experten bis zum Jahr 2015 zunehmen. Allerdings ist keine eindeutige Tendenz in der Bewertung sichtbar, ob anzeige- und meldepflichtige Krankheiten in der Zukunft zu- oder abnehmen werden (Tabelle 5.4-1und Tabelle 5.4-2). Genannt wurden:

Für Geflügel: Geflügelpest, Newcastle Krankheit, Campylobacter, Coccidose, Marek'sche Krankheit, Tuberkulose, Mycoplasmose, Salmonellosen, Listeriose, Influenzaviren.

Für Schweine: MKS, Schweinepest (KSP, ASP, ESP), Aujeszky'sche Krankheit, Salmonellosen, Listeriose, Transmissible Virale Gastroenteritis, Leptospirose, Parasiten, Staphylokokkenerkrankung, Toxoplasmose, Trichinellose.

Für Rinder: $\quad$ MKS, BSE/TSE, Bovines Herpesvirus (BHV1), Bovine Virus Diarrhoe (BVD), Leukose, Brucellose, Rind IBR.

Tabelle 5.4-1: Einschätzung der Entwicklung der Gefahr des Eintrags von Keimen/ Krankheitserregern aus dem Ausland zum Jahr 2015.

\begin{tabular}{ccccccc}
\hline & & stark abnehmend & abnehmend & wie heute & zunehmend & stark zunehmend \\
\hline Experten & $\mathrm{N}=21$ & 0 & 4 & 4 & 12 & 1 \\
& $\%$ & 0 & 19 & 19 & 57 & 5 \\
\hline
\end{tabular}

Tabelle 5.4-2: Einschätzung des Auftretens von anzeige- und meldepflichtigen Krankheiten zum Jahr 2015.

\begin{tabular}{ccccccc}
\hline & & stark abnehmend & abnehmend & wie heute & zunehmend & stark zunehmend \\
\hline \multirow{2}{*}{ Experten } & $\mathrm{N}=21$ & 0 & 8 & 5 & 7 & 1 \\
& $\%$ & 0 & 38 & 24 & 33 & 5 \\
\hline
\end{tabular}

Die Experten sehen eine Gefahr, dass zurzeit in Deutschland nicht vorkommende Keime bzw. Krankheitserreger durch den weltweiten Handel eingeschleppt werden. Eine klare Aussage zur Gesundheitsgefahr kann aus den Ergebnissen nicht abgeleitet werden. 47\% der Experten schätzen die Gesundheitsgefahr mit gering bis keine und $53 \%$ mit hoch bis 
mittel ein. Der Großteil der Experten (52\%) sagt, dass Standards für Kontrollen vorhanden sind. Allerdings sind auch $33 \%$ der Experten der Meinung, dass Standards fehlen. Ein Meideverhalten wird nicht ausgeschlossen. 27\% sagen, dass es sicher zu einem Meideverhalten kommen kann, und $45 \%$ schließen ein Meideverhalten nicht aus (Abbildung 5.4-1). Neben einer mikrobiologischen Gefahr sehen die Experten aber auch eine chemische $(18,2 \%)$, eine ernährungsphysiologische $(22,7 \%)$ und eine ethische Gefahr $(18,2)$ (Tabelle 11.1-13).

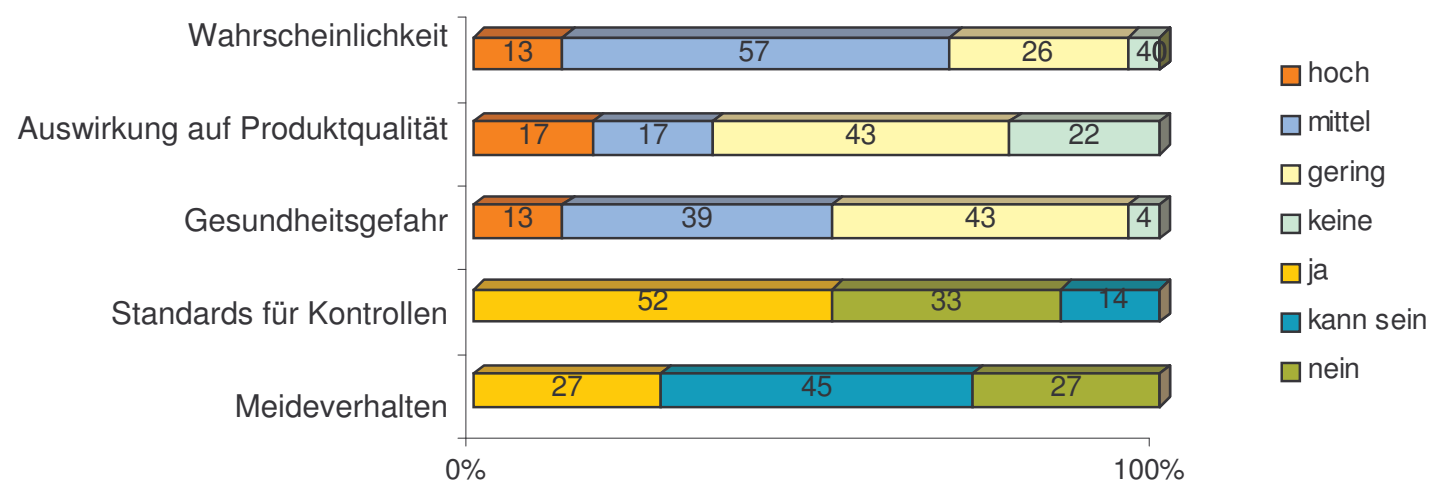

Abbildung 5.4-1: $\quad$ Beurteilung der These: In Deutschland z.Zt. nicht vorkommende Keime/ Krankheitserreger werden durch den weltweiten Handel eingeschleppt.

\subsubsection{Reste aus der Lebensmittelproduktion}

Dass Reste aus der Lebensmittelproduktion als Futtermittel geeignet sind, bestätigen $21 \%$ der Experten und $38 \%$ halten dieses für wahrscheinlich. Auswirkungen auf die Produktqualität und eine Gesundheitsgefahr werden eher nicht gesehen. Allerdings wird nicht ausgeschlossen, dass Misstände zu einem Meideverhalten führen können (Abbildung 5.4-2).

Wenn es über diese Futtermittel zu einer Gefährdung kommen sollte, ist diese in erster Linie mikrobiologischer Natur (77,3\%), aber es werden auch chemische $(27,3 \%)$, ernährungsphysiologische (45,5\%), und ethische (27,3\%) Gefahren gesehen (Tabelle 11.1-15). Die Verfütterung spielt vor allem in der Schweinehaltung eine Rolle (Tabelle 11.1-16). Die meisten Experten gehen allerdings davon aus, dass der Einsatz bis zum Jahr 2015 abnehmen wird (Tabelle 5.4-3).

Der überwiegende Anteil der Studenten hält einen Einsatz von Resten aus der Lebensmittelproduktion für sinnvoll. Die positive Einschätzung liegt aber bei Studenten $(\mathrm{mL})$ um ca. $20 \%$ höher als bei den Studenten (oL) (Tabelle 5.4-4).

Tabelle 5.4-3: $\quad$ Einschätzung der Entwicklung des Einsatzes von Resten aus der Lebensmittelproduktion in der Tierfütterung zum Jahr 2015.

\begin{tabular}{ccccccc}
\hline & & stark abnehmend & abnehmend & wie heute & zunehmend & stark zunehmend \\
\hline \multirow{2}{*}{ Experten } & $\mathrm{N}=21$ & 2 & 11 & 2 & 6 & 0 \\
& $\%$ & 10 & 52 & 10 & 29 & 0 \\
\hline
\end{tabular}




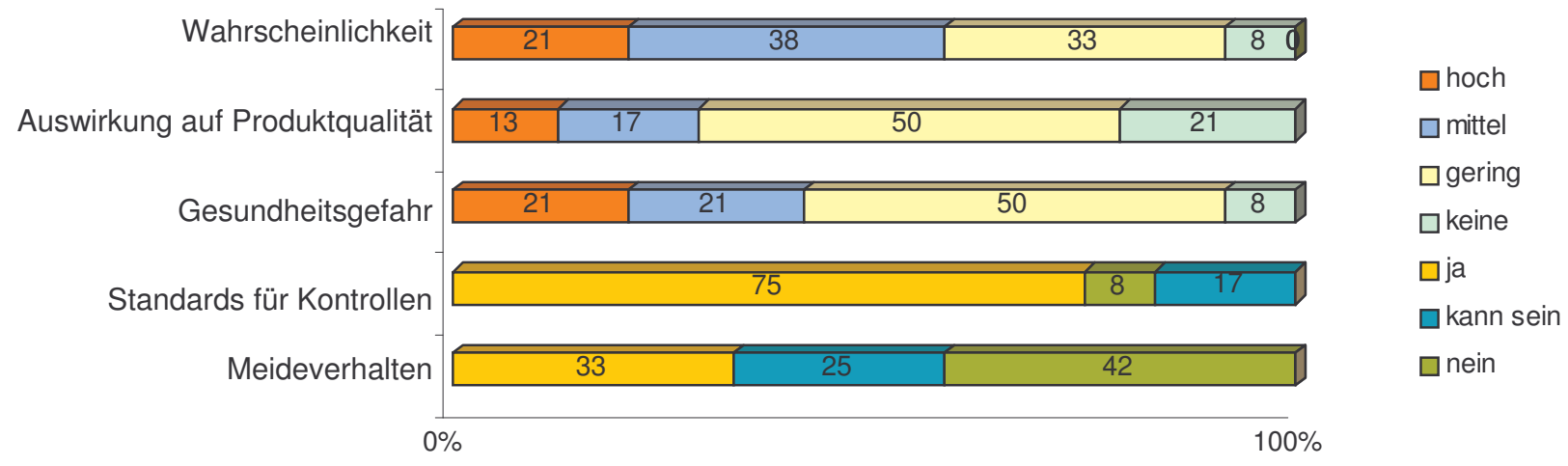

Abbildung 5.4-2: Beurteilung der These: Reste aus der Lebensmittelproduktion sind als Futtermittel geeignet.

Tabelle 5.4-4: Beurteilung der Frage: Halten Sie es für sinnvoll, Reste aus der Lebensmittelproduktion in der Tierfütterung einzusetzen?

\begin{tabular}{ccccc}
\hline Studenten & & beide & Studenten $(\mathrm{mL})$ & Studenten $(\mathrm{oL})$ \\
\hline \multirow{3}{*}{ sinnvoll } & $\mathrm{N}=64$ & 47 & 32 & 15 \\
& $\%$ & 73 & 80 & 63 \\
\hline
\end{tabular}

\subsubsection{Einsatz von Prophylaxen/Schutzimpfungen}

Nach der überwiegenden Meinung der Experten (67\%) darf auf den Einsatz von Prophylaxen und Schutzimpfungen nicht verzichtet werden. Auswirkungen auf die Produktqualität und eine Gesundheitsgefahr werden nicht gesehen. Es wird nicht ausgeschlossen, dass es bei bestimmten Mitteln zu einem Meideverhalten kommen kann (Abbildung 5.4-3). Betroffen sind vor allem die Tierarten Schwein und Geflügel (Tabelle 11.1-17).

Das Ergebnis der schriftlichen Befragung hat ergeben, dass Standards für den Einsatz von Prophylaxen bzw. Schutzimpfungen vorhanden sind (Abbildung 5.4-3) . In der mündlichen Befragung wurden die Experten um eine Einschätzung bezüglich der Umsetzung der Standards für Kontrollen gebeten und gefragt, welche Probleme sich aus einem Verzicht von Prophylaxen bzw. Schutzimpfungen ergeben könnten. Insgesamt gehen die Experten davon aus, dass die Standards umgesetzt werden. Ein Experte ist der Meinung, dass die vorhandenen Standards zwar zum Teil aufgeweicht wurden, aber die noch vorhandenen Standards umgesetzt werden.

Tabelle 5.4-5: Einschätzung der Entwicklung des Einsatzes von Prophylaxen/ Schutzimpfungen zum Jahr 2015.

\begin{tabular}{ccccccc}
\hline & & stark abnehmend & abnehmend & wie heute & zunehmend & stark zunehmend \\
\hline \multirow{2}{*}{ Experten } & $\mathrm{N}=21$ & 0 & 1 & 1 & 17 & 2 \\
& $\%$ & 0 & 5 & 5 & 81 & 10 \\
\hline
\end{tabular}

Probleme sehen die Experten bei einem Verzicht von Prophylaxen bzw. Schutzimpfungen vor allem im Anstieg des Infektionsdruckes und des Seuchenrisikos und dem dadurch bedingten vermehrten Auftreten von Krankheiten. Aber auch Aspekte wie erhöhte Tierverluste und ein Anstieg der Produktionskosten wurden genannt. 
Insgesamt gehen die Experten zum Jahr 2015 davon aus, dass der Einsatz von Prophylaxen bzw. Schutzimpfungen zunehmen werden (Tabelle 5.4-5). Die Studenten halten den Einsatz von Prophylaxen bzw. Schutzimpfungen ebenfalls für notwendig (Tabelle 5.4-6).

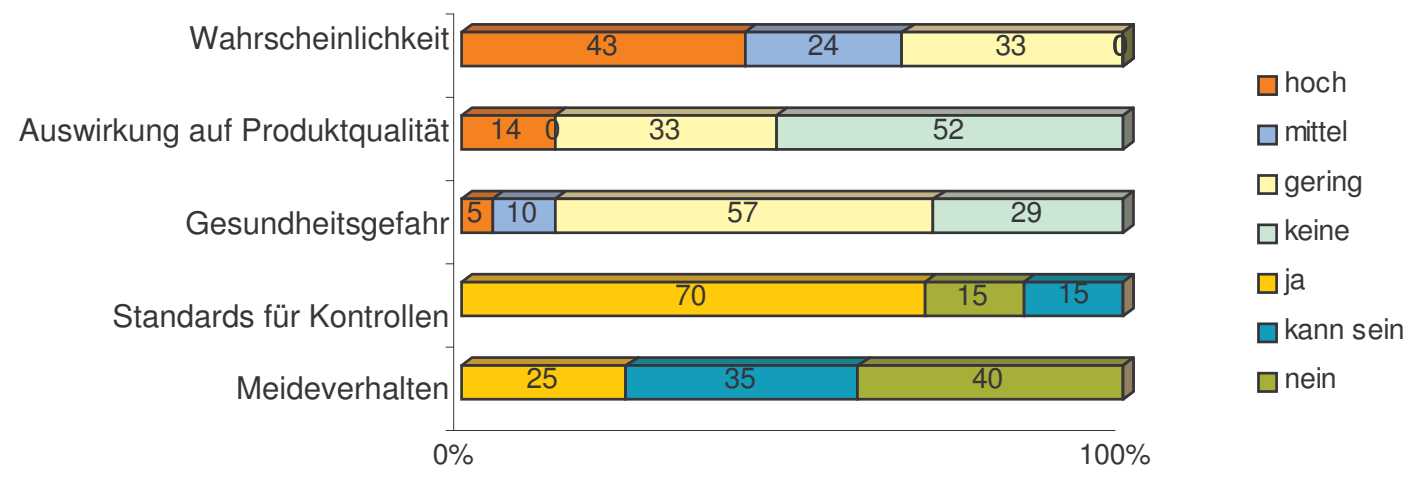

Abbildung 5.4-3: Beurteilung der These: Auf Prophylaxen/Schutzimpfungen zum Erhalt des gesundheitlichen Status darf nicht verzichtet werden.

Tabelle 5.4-6: $\quad$ Einschätzung der Studenten bezüglich der Notwendigkeit des Einsatzes von Prophylaxen/Schutzimpfungen.

\begin{tabular}{ccccc}
\hline Studenten & & Studenten & Studenten $(\mathrm{mL})$ & Studenten $(\mathrm{oL})$ \\
\hline $\mathrm{N}$ & 61 & 39 & 22 \\
$\%$ & 80 & 77 & 86 \\
\hline
\end{tabular}

5.5

Rückstände

5.5.1 Risiken durch Feldkeime und speziell Mykotoxine in der Lebensmittelkette

In der schriftlichen Befragung wurden die Experten zuerst allgemein zu der Rolle von Feldkeimen bei Futtermitteln befragt. Eine Differenzierung zwischen Bakterien, Parasiten und Mykotoxinen ist nicht vorgenommen worden. In diesem Kapitel werden in erster Linie die Ergebnisse zu Mykotoxinen dargestellt. Der Bereich der Bakterien wird ausführlich im nachfolgenden Kapitel betrachtet. Parasiten scheinen nach der Meinung der Experten kaum eine Rolle zu spielen. Parasiten wurden allgemein nur von einem Experten genannt (Abbildung 11.2-1).

Auf alle Feldkeime (Bakterien, Mykotoxine, Parasiten) bezogen schließen die Experten nicht aus, dass Feldkeime in der Nahrungskette eine Rolle spielen werden. 41\% der Experten schätzen die Wahrscheinlichkeit der These, dass Feldkeime in der Nahrungskette eine Rolle spielen werden, mit hoch (6\%) und mittel (35\%) ein. Einen Einfluss auf die Produktqualität wird nicht ausgeschlossen. Eine Gefahr für den Verbraucher wird von über $70 \%$ der Experten für gering gehalten. Insgesamt wird die Gefahr der Feldkeime als gering eingestuft und es wird nicht davon ausgegangen, dass es nicht zu einem Meideverhalten beim Verbraucher kommt. (Abbildung 5.5-1). Hauptsächlich sehen die Experten einen Einfluss der Feldkeime auf das Schwein (75\%). Der Einfluss der Feldkeime auf die Tierarten Rind und Geflügel wird mit 56\% eingestuft (Tabelle 11.1-9). 
10 von 12 befragten Experten sehen einen Übergang („Carry Over“) von Mykotoxinen vom Futtermittel auf das Fleisch. Auf die Frage, ob das Tier als Filter ausreicht, haben 8 der befragten Experten mit nein und 4 mit ja geantwortet.

Als Mykotoxine, die in der Nahrungskette von besonderer Bedeutung sind, werden in von den Experten genannt Abbildung 5.5-2 : Aflatoxine, Ochratoxin, Zeralenon, Desoxynivalenol und das Mutterkornalkaloid. Diese Mykotoxine sind das Ergebnis einer Gewichtung der in der schriftlichen Befragung genannten Mykotoxine (Abbildung 11.2-2). Die Mykotoxine werden in erster Linie als mikrobiologische Gefahr (82,4\%) eingestuft. Daneben wird auch eine ernährungsphysiologische (35,3\%) und chemische $(23,5 \%)$ Gefahr gesehen (Tabelle 11.1-8).

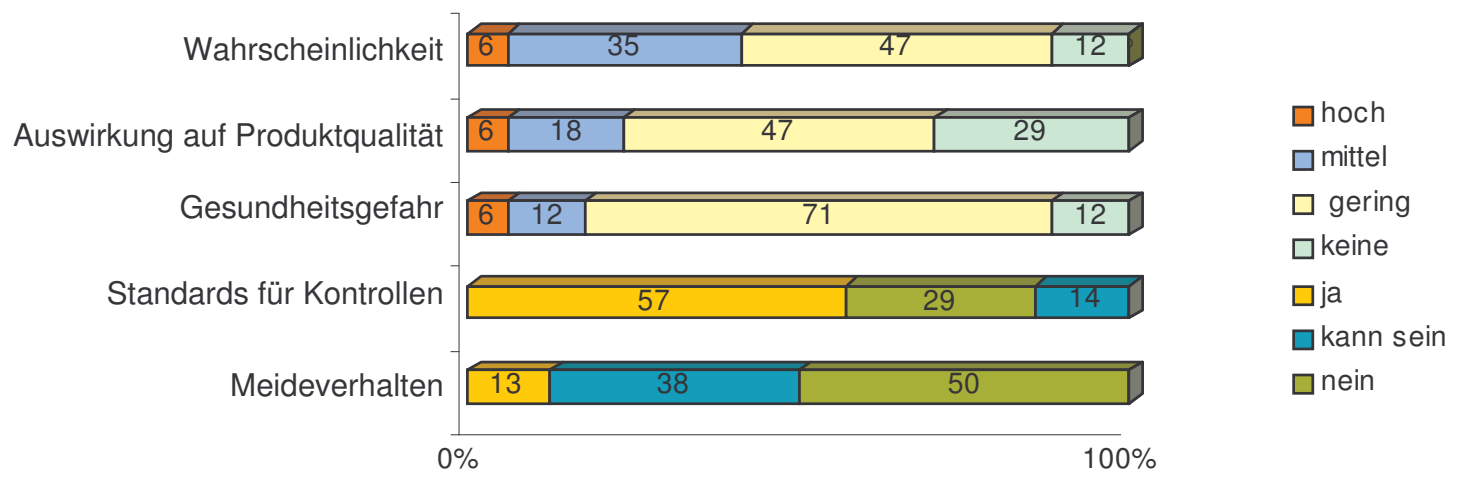

Abbildung 5.5-1: $\quad$ Beurteilung der These: Feldkeime werden in der Nahrungskette eine Rolle spielen.

$\square$ Aflatoxine
$\square$ Ochratoxin
$\square$ Zeralenon
$\square$ Desoxynivalenol DON
$\square$ Mutterkornalkaloid
$\square$ Fusarientoxine
$\square$ Citrinin
$\square$ Trichothecene
$\square$ Schwärzepilztoxine

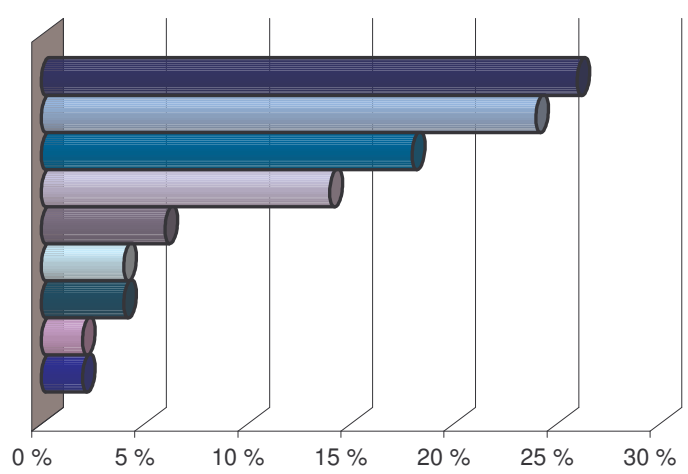

Abbildung 5.5-2

Einschätzung der Mykotoxine, die in der Nahrungskette von Bedeutung sind, in \% der Nennungen.

Die Experten sind sich nicht einig, wie sich das Vorkommen von Mykotoxinen entwickelt, die über das Futtermittel das Nahrungsmittel kontaminieren. Zirka 32\% der Experten gehen von einer Zunahme und zirka 42\% von einer Abnahme aus. (Tabelle 5.5-1).

Tabelle 5.5-1: $\quad$ Einschätzung der Entwicklung des Vorkommens von Mykotoxinen, die über das Futtermittel das Nahrungsmittel Fleisch kontaminieren, zum Jahr 2015.

\begin{tabular}{ccccccc}
\hline & & stark abnehmend & abnehmend & wie heute & zunehmend & stark zunehmend \\
\hline \multirow{2}{*}{ Experten } & $\mathrm{N}=19$ & 1 & 7 & 5 & 6 & 0 \\
& $\%$ & 5 & 37 & 26 & 32 & 0 \\
\hline
\end{tabular}




\subsubsection{Rückstände von Pflanzenschutzmitteln}

Rückstände können auf vielfältigen Wegen in das Futtermittel gelangen. Möglichkeiten sind: Pflanzenschutzmittel, Müllkompost, Klärschlamm, Altlasten, Düngung und Importe von belasteten Futtermitteln. Reste von Pflanzenschutzmitteln werden immer wieder in Nahrungsmitteln gefunden. In vielen Fällen, wie z.B. Nitrofen und Dioxine, gelangen diese Stoffe über die Nahrungskette (Futtermittel - Tier - Fleisch) zum Menschen.

Nach Aussage von Experten gibt es über 300 bekannte Wirkstoffe für den Pflanzenschutz. Untersuchungen durchzuführen, die diese Stoffe vollständig berücksichtigen, ist unmöglich. Gewisse Wirkstoffkomponenten haben sich über den eigentlichen Einsatzbereich hinaus verbreitet und sind z.B. ubiquitär in der Umwelt vorhanden. Sie sind somit zu einem Rückstandsproblem geworden, das sich verselbständigt hat.

Ein besonderes Problem sind die häufigen Änderungen der Zulassung von Pflanzenschutzmitteln. Wenn Pflanzenschutzmittel kurzzeitig die Zulassung verlieren, kann sich die Frage ergeben, wann ein Rückstand Hinweise auf eine illegale Anwendung des Mittels ergibt.

In der mündlichen Befragung war der überwiegende Anteil der Experten der Meinung, dass die eingesetzten Pflanzenschutzmittel zukünftig sicherer sein werden und die Rückstände real abnehmen (Tabelle 5.5-2). Allerdings wird es auch vermehrt Veröffentlichungen bezüglich der Rückstände geben, da die Analysemethoden immer feiner werden.

Der Verbraucher müsste daher durch eine offensive Informationspolitik erfahren, dass es keine Null-Toleranz gibt. Hierzu ist es wichtig, dass durch die Wissenschaft toxikologisch begründete Grenzwerte festgelegt werden. Vorhandene Risiken und die Verbesserung in der Sicherheit der Pflanzenschutzmethoden müssten offen kommuniziert werden. Gerade die Verbesserungen müssten herausgestellt werden.

Die Experten sind aber auch der Meinung, dass sich die Transparenz in der Urproduktion stark verbessern muss. Als Vorschläge wurden hier genannt:

- Einbindung der Urproduktion in Systeme bzw. Verträge und deren Kontrolle (Beispiel QS),

- umfassende Dokumentation aller in der Futterwirtschaft eingesetzten Stoffe (Positivliste).

Tabelle 5.5-2: $\quad$ Einschätzung der Entwicklung über das Vorkommen von Rückständen aus Pflanzenschutzmitteln im Futtermittel zum Jahr 2015.

\begin{tabular}{llcrrrc}
\hline & & stark abnehmend & abnehmend & wie heute & zunehmend & stark zunehmend \\
\hline Experten & $\mathrm{N}=19$ & 0 & 16 & 0 & 3 & 0 \\
& $\%$ & 0 & 84 & 0 & 16 & 0 \\
\hline
\end{tabular}

Die Experten schließen nicht aus, dass Pflanzenschutzmittel, die in Drittländern eingesetzt wurden, zur Anreicherung von Schadstoffen in Futtermitteln führen. Von einer Auswirkung auf die Qualität gehen 16\% nicht aus und $42 \%$ halten diese für gering. Der überwiegende Teil der Experten (58\%) schätzt eine Gesundheitsgefahr ebenfalls als gering ein. Einfluss auf die Qualität bzw. eine Gesundheitsgefahr sehen allerdings $42 \%$ der Experten. Bei den Standards wird überwiegend von deren Existenz ausgegangen, von einem Meideverhalten gehen $42 \%$ aus und ebenfalls $42 \%$ schließen es nicht aus (Abbildung 5.5-3). 
Als Hauptrückstände aus importierten Futtermitteln werden Dioxine und PCB`s sowie andere chlorierte Kohlenwasserstoffverbindungen genannt (Abbildung 11.2-5).

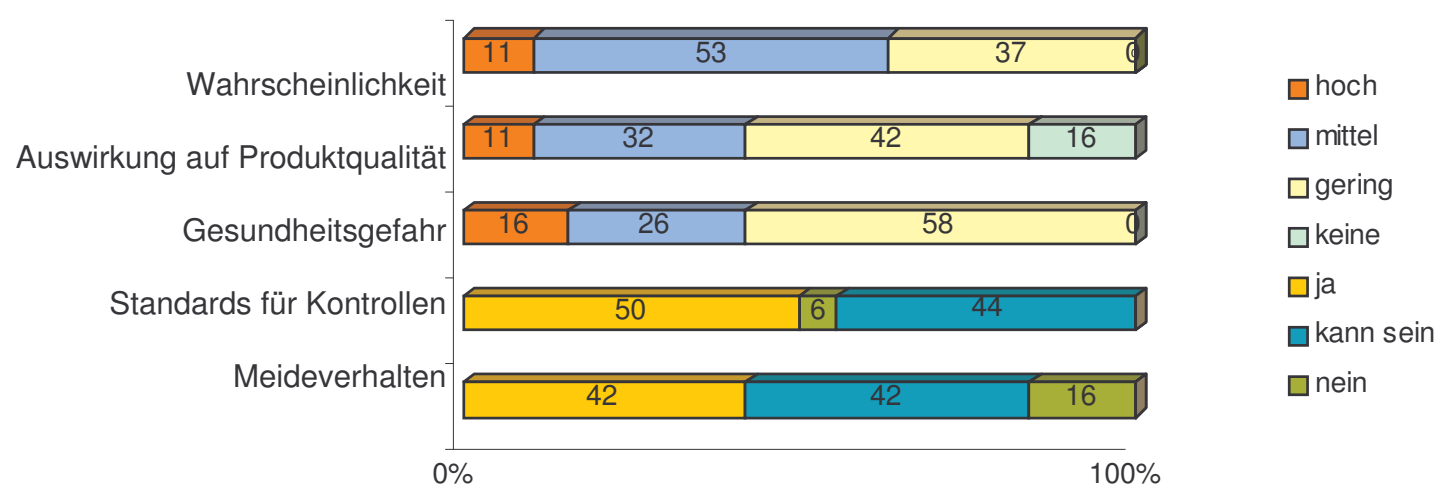

Abbildung 5.5-3: Einschätzung der These: In Drittländern sind häufig Pflanzenschutzmittel und/oder Mittel zur Behandlung von Futtermitteln zugelassen, die in Deutschland verboten sind. Bitte beurteilen Sie die Gefahr, dass sich Rückstände von solchen Mitteln im Futter anreichern.

\subsubsection{Exotische Futtermittel}

Nach der Meinung der Experten kann grundsätzlich von allen in der Tiernahrung eingesetzten exotischen Früchten/Pflanzen eine Gefahr ausgehen. Hierbei ist zu beachten, dass die Gefahr nicht von den Produkten selbst, sondern nur von den Rückständen ausgeht. Diese sind stark abhängig von Herstellung, Weiterverarbeitung und Transport/Lagerverhältnissen. Wird in allen Stufen HACCP angewandt, ist die Gefahr gering.

Als besonders kritische Verfahrensschritte werden mangelnde Hygiene bei der Ernte und Verarbeitung, die Trocknung, die Lagerung und der Transport der Futtermittel genannt (Tabelle 11.1-19).

Nachfolgend genannte Produkte können hiervon betroffen sein :Sojaimporte, Nebenprodukte aus der Verarbeitung von Ölfrüchten, Palmkerne, Tapioka, Kakao, Kokosextraktionsschrot, Citrus, Baumwollextraktionsschrot, Erdnussextraktionsschrot.

Die Frage zur Entwicklung von Rückständen von in der EU verbotenen Stoffen in importierten Futtermitteln wird weder von den Experten noch von den Studenten eindeutig beantwortet. Die Antworten der Experten tendieren zu abnehmenden Rückständen, die der Studenten eher zu zunehmenden Rückständen (Tabelle 5.5-3).

Möglichkeiten den Eintrag von Rückständen durch Pflanzenschutzmittel in die Nahrungskette zu reduzieren, sehen die Experten in:

- der Einhaltung der Wartezeiten,

- der Reduzierung der zugelassenen Mittel bzw. der Verschärfung der Auflagen für die Zulassung von Mitteln,

- der Einbindung der Urproduktion in Systeme, z.B. QM, QS, GMP+,

- der Zunahme des Kontrollumfangs, beginnend mit der Feldkontrolle. Der Landwirt muss dazu verpflichtet werden alle eingesetzten Stoffe zu dokumentieren. 
Tabelle 5.5-3:

Einschätzung der Entwicklung des Vorkommens von Rückständen von in der EU verbotenen Stoffen in importierten Futtermitteln zum Jahr 2015.

\begin{tabular}{lllrccc}
\hline & & stark abnehmend & abnehmend & wie heute & zunehmend & stark zunehmend \\
\hline Experten & $\mathrm{N}=21$ & 2 & 9 & 4 & 6 & 0 \\
& $\%$ & 6 & 39 & 17 & 33 & 6 \\
Studenten & $\mathrm{N}=64$ & 0 & 16 & 20 & 28 & 0 \\
& $\%$ & 0 & 25 & 31 & 44 & 0 \\
\hline
\end{tabular}

Nach der Meinung der Experten ist es wichtig zu kommunizieren, dass es keine NullToleranzen gibt. Die gefundenen Werte/Rückstände müssen nachvollziehbar durch Wissenschaftler interpretiert und bewertet werden. Die Kommunikation mit dem Verbraucher sollte aber nicht erst stattfinden, wenn etwas gefunden wurde, sondern die Problematik Rückstände sollte im Vorfeld diskutiert werden.

\subsubsection{Rückstände aus dem Einsatz von Mineraldüngern}

Probleme bei dem Einsatz von Mineraldüngern werden von den Experten hauptsächlich für die Umwelt gesehen (Belastung des Bodens und des Grundwassers). Diese Probleme würden aber nicht auftreten, wenn die Düngung nach wissenschaftlichen Grundsätzen der „Guten fachlichen Praxis" sowie nach dem Versorgungsgrad des Bodens und des Pflanzenbedarfs erfolgt.

\subsubsection{Rückstände aus dem Einsatz von Müllkompost, Klärschlamm und Altlasten}

Hier werden die Risikopotenziale genau wie bei den Pflanzenschutzmitteln hauptsächlich im chemischen, mikrobiologischen und ethischen Bereich gesehen (Tabelle 11.1-20). Die Experten gehen davon aus, dass eine Belastung der Futtermittel durch mit Altlasten verunreinigte Böden eher weniger wird. Die meisten der Experten sehen keine oder nur eine geringe Auswirkung (68\%) auf die Produktqualität. Die Gesundheitsgefahr schätzen 53\% als nicht vorhanden oder gering ein und $47 \%$ als mittel oder hoch. Dass ausreichende Standards für Kontrollen vorhanden sind, sagen 39\%. Dass Rückstände aus Altlasten zu einem Meideverhalten führen können, sehen 35\% der Experten (Abbildung 5.5-4).

$56 \%$ der Experten schätzen, dass es durch Rückstände im Müllkompost und Klärschlamm, die sich im Fleisch anreichern können, zu keiner oder nur einer geringen gesundheitlichen Gefährdung kommen kann. Es werden überwiegend keine oder nur geringe Auswirkungen auf die Produktqualität (66\%) gesehen. Standards für Kontrollen sehen 62\% der Befragten und ein Meideverhalten wird für wahrscheinlich gehalten (Abbildung 5.5-5).

Tabelle 5.5-4: $\quad$ Einschätzung der Entwicklung über das Vorkommen von Rückständen aus Müllkompost/Klärschlamm im Futtermittel zum Jahr 2015.

\begin{tabular}{lllllll}
\hline \multirow{3}{*}{ Experten } & & stark abnehmend & abnehmend & wie heute & zunehmend & stark zunehmend \\
\cline { 2 - 7 } & $\mathrm{N}=19$ & 3 & 11 & 4 & 1 & 0 \\
& $\%$ & 16 & 58 & 21 & 5 & 0 \\
\hline
\end{tabular}

Die hauptsächlichen Rückstände, die durch Altlasten in die Nahrungskette gelangen, sind nach der Meinung der Experten: Schwermetalle, Dioxine, PCBs, und DDT/DDE (Abbildung 11.2-5). Bei Müllkomposten und Klärschlamm wird die Hauptgefahr bei Schwermetallen, aromatischen Kohlenwasserstoffen und mikrobiologischen Rückständen gesehen (Abbildung 
11.2-7). Insgesamt werden die Rückstände aber bis zum Jahr 2015 abnehmen (Tabelle 5.5-4).

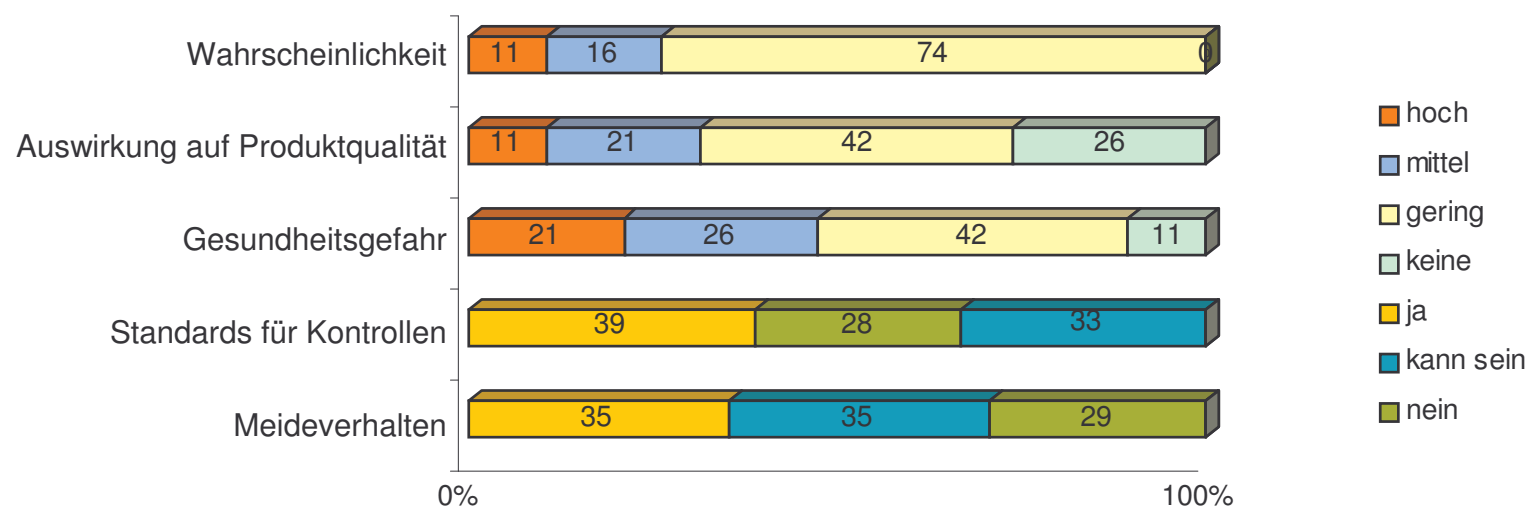

Abbildung 5.5-4: Beurteilung der These: Eine Belastung der Futtermittel durch mit Altlasten verunreinigten Böden wird auch in Zukunft vermehrt vorkommen.

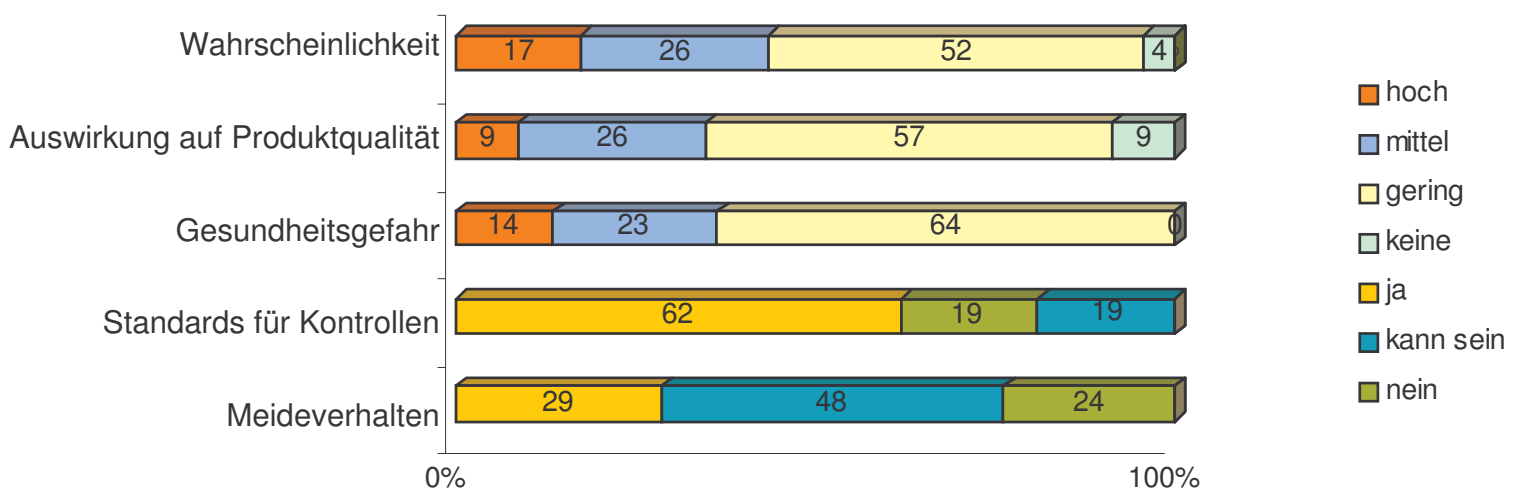

Abbildung 5.5-5: Rückstände im Müllkompost und Klärschlamm führen über eine Anreicherung im Fleisch zu einer gesundheitlichen Gefährdung des Menschen.

Große Unterschiede bezüglich des Vorkommens bei einer Tierart werden von den Experten nicht gesehen. Für das Rind wird die Gefahr bei Altlasten mit ca. 90\% am höchsten und für das Geflügel mit ca. $74 \%$ am niedrigsten eingeschätzt. Bei Klärschlamm wird mit ca. $91 \%$ die Gefahr beim Schwein am höchsten und bei den andern beiden Tierarten mit ca. $76 \%$ eingeschätzt. Ähnlich hoch wird die Gefahr durch Rückstände von Pflanzenschutzmitteln aus importierten Futtermitteln gesehen: Schweine 100\%, Geflügel 95\%, Rind 74\% (Tabelle 11.1-21).

Alle acht der mündlich befragten Experten stimmen der These zu, dass die zunehmende Sensibilität der Verbraucher für Rückstände und die Verbesserung der Laboranalytik das Thema Altlasten im Zusammenhang mit humanmedizinischen Erkenntnisfortschritten neu aufleben lassen wird.

In erster Linie sehen die Experten in der Rückstandsproblematik (hier bei Altlasten) ein Problem in der Kommunikation. Grenzwerte müssen klar, nachvollziehbar und transparent festgelegt und kommuniziert werden. Aber auch der Kontrollumfang in der Produktion und 
die Rückverfolgbarkeit bis auf den Acker muss verbessert werden. Aufgrund der Fülle der Substanzen können allerdings nicht alle möglichen Substanzen überwacht werden.

\subsubsection{Mittel/Futterzusatzstoffe, die sich zur Leistungssteigerung eignen.}

Der analytische Nachweis von Rückständen bestimmter Mittel bzw. Futterzusatzstoffe, die zur Leistungssteigerung eingesetzt werden, hat diese in die Kritik der Verbraucher gebracht. Hierbei spielt es keine Rolle, ob die Stoffe schädlich sind oder nicht.

13 verschiedene Mittel/Futterzusatzstoffe werden von den Experten in der ersten Befragung genannt (Abbildung 11.2-6). In der mündlichen Befragung sollte dann beurteilt werden, ob diese Stoffe im Sinne der Wissenschaft und/oder im Sinne des Verbrauchers unbedenklich sind. Dieselben Stoffe werden auch durch die Studenten beurteilt. Im Sinne des Verbrauchers und der Wissenschaft werden von den meisten Experten, aber auch nach der Einschätzung der meisten Studenten, antibiotische Stoffe, Hormone und Kokzidiostatika für kritisch gehalten. Bei einigen anderen Stoffen (Prebiotika, Enzyme und synthetischen Aminosäuren) schätzen die Experten, dass der Verbraucher diese Stoffe kritisch sehen könnte. Von Seiten der Wissenschaft werden diese Stoffe hingegen eher als unbedenklich eingestuft. Ein starker Unterschied in der Bewertung ist bei den Kräutermischungen zu erkennen. Aus der Sicht der Verbraucher werden sie von allen als unbedenklich eingestuft. Aus der Sicht der Wissenschaft sehen ca. 50\% der Befragten bestimmte Stoffe in den Kräutermischungen als bedenklich an. Alle anderen genannten Stoffe werden aus der Sicht des Verbrauchers und der Wissenschaft eher als unbedenklich eingestuft (Abbildung 5.5-6).

Die Bewertung der Studenten gleicht tendenziell der Bewertung der Experten bezüglich des Verbrauchers. Deutliche Abweichungen in der Bewertung sind bei den Enzymen/Phytasen, den Aminosäuren und den Aromastoffen sichtbar. Enzyme/Phytasen und Aminosäuren werden von den Studenten positiver und Aromastoffe deutlich negativer eingeschätzt als in der Bewertung der Experten.

Deutliche Unterschiede in der Bewertung sind auch zwischen Studenten $(\mathrm{mL})$ und Studenten (oL) vorhanden. Zum Beispiel werden von über $20 \%$ der Studenten $(\mathrm{mL})$, antibiotische Stoffe für unbedenklich gehalten. Deutlich positiver werden von dieser Gruppe auch Hormone, Kokzidostatika, Enzyme/Phytasen und Aromastoffe bewertet (Abbildung 11.2-8).

Grundsätzlich sind fast alle Experten der Meinung, dass heute aus wirtschaftlichen Gründen auf den Einsatz von Leistungsförderern verzichtet werden kann. Vier der Experten schränken allerdings ihre Aussage ein. Nach einem Experten sollte der Verzicht dann für alle EULänder gelten, damit die Chancengleichheit gegeben ist. Die anderen Experten sehen Probleme bei einem Verzicht bei jungen Tieren (Ferkeln und Umstallung von Vor- auf Endmast bei Schweinen u. Geflügel) bzw. bei nicht optimalen Haltungs- und Hygienebedingungen.

Folgende genannte Stoffe, mit denen das Tier im landwirtschaftlichen Betrieb in Berührung kommt, werden nach der Meinung der Experten in der Zukunft vermehrt eine Rolle spielen (Abbildung 5.5-7): organische Verbindungen, Mykotoxine, Bakterien, Dioxine/Furane und Schwermetalle. Bei den Antworten handelt es sich um eine Gewichtung der aus der schriftlichen Befragung erhaltenen Antworten (Abbildung 11.2-9).

Bezüglich des Vorkommens von Rückständen un- bzw. erlaubter Futterzusatzstoffe gehen die Experten von einer Abnahme bis zum Jahr 2015 aus. Bei den Umweltkontaminanten fällt 
die Wertung nicht so eindeutig aus. 50\% gehen von einer Abnahme aus, dagegen gehen $25 \%$ von einer Zunahme bzw. starken Zunahme aus (Tabelle 5.5-5).

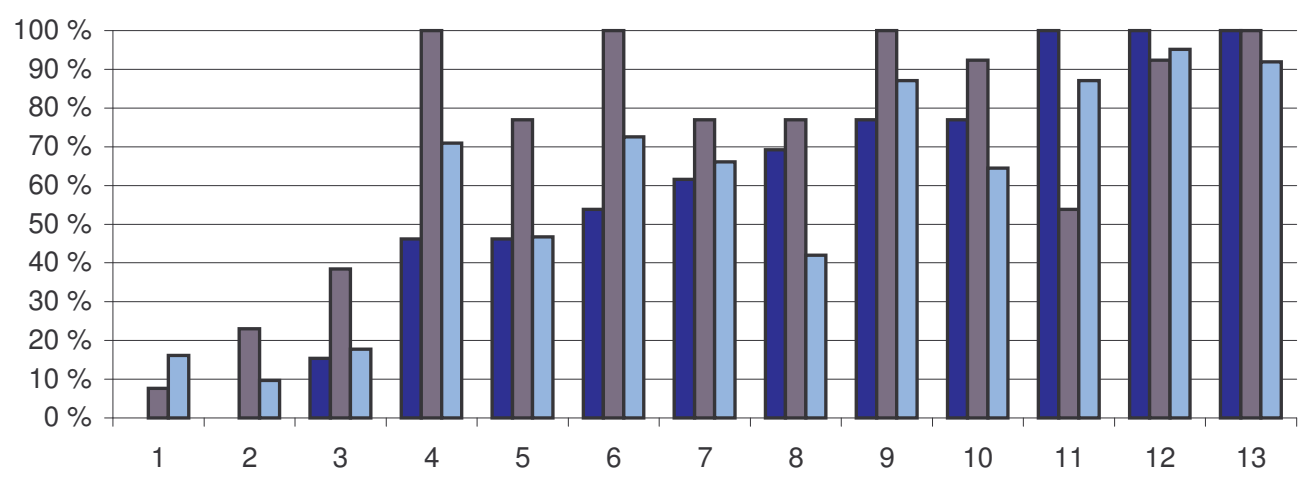

Experteneinschätzung Verbraucher $\square$ Experteneinschätzung Wissenschaft $\square$ Studenten
1. Antibiotische Stoffe
2. Hormone
3. Kokzidostatika
4. Enzyme/Phytasen
5. Prebiotika
6. Aminosäuren (synth.) 7. Probiotika
8. Aromastoffe
9. Spurenelemente
10. Org. Säuren
11. Kräutermischungen
12. Mineralstoffe
13. Vitamine

Abbildung 5.5-6: Einschätzung der Experten $(\mathrm{N}=13)$ und der Studenten $(\mathrm{N}=62)$ bezüglich der Unbedenklichkeit von Futterzusatzstoffen.

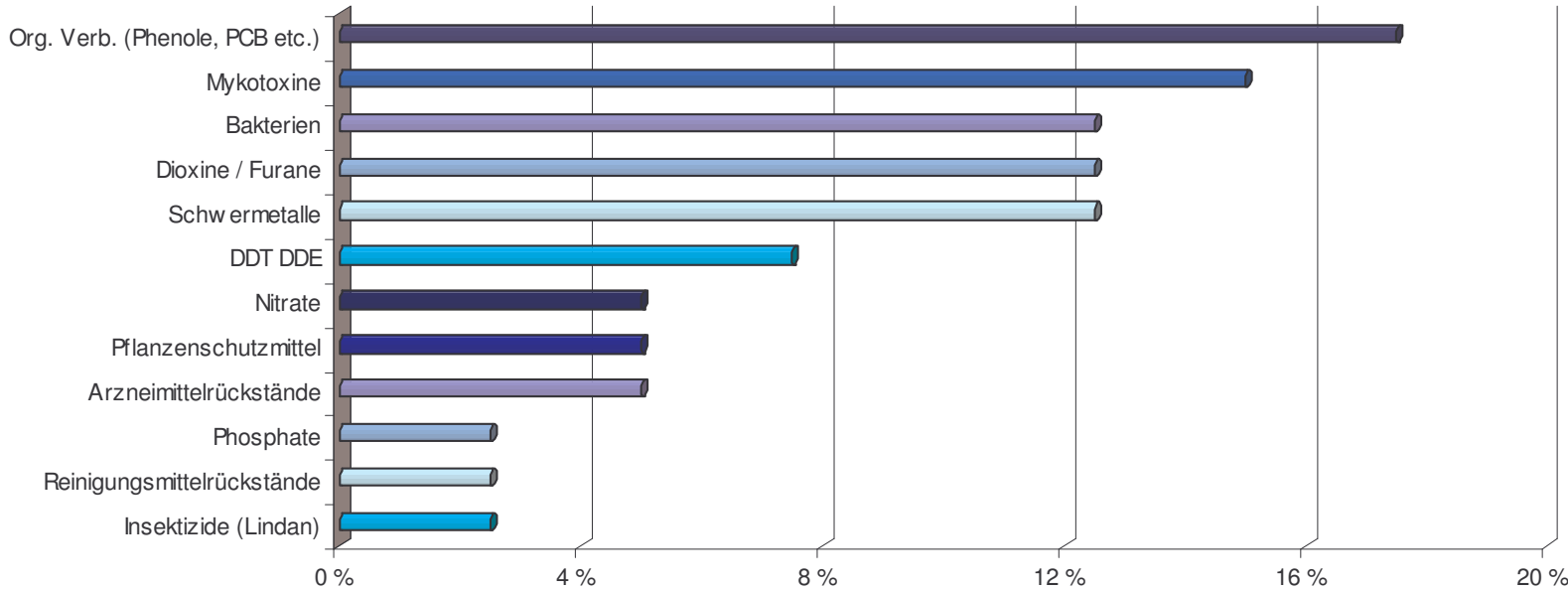

Abbildung 5.5-7: $\quad$ Einschätzung, welche Rückstände von Umweltkontaminanten, mit denen das Tier im landwirtschaftlichen Betrieb in Berührung kommt, in Zukunft vermehrt eine Rolle spielen, in \% der Nennungen.

Tabelle 5.5-5: $\quad$ Einschätzung der Entwicklung des Vorkommens von Rückständen (un-) erlaubter Futterzusatzstoffe und Umweltkontaminanten zum Jahr2015.

\begin{tabular}{lllllll}
\hline Experten & & stark abnehmend & abnehmend & wie heute & zunehmend & stark zunehmend \\
\hline Futterzu- & $\mathrm{N}=21$ & 3 & 12 & 2 & 4 & 0 \\
satzstoffe & $\%$ & 14 & 57 & 10 & 19 & 0 \\
Umweltkon- & $\mathrm{N}=20$ & 0 & 10 & 5 & 3 & 2 \\
taminanten & $\%$ & 0 & 50 & 25 & 15 & 10 \\
\hline
\end{tabular}




\subsubsection{Rückstände im Bereich der Schlachtung}

Rückstände spielen auf den Stufen der Futtermittelproduktion und der Landwirtschaft eine wichtige Rolle, über eine Kontamination auf der Stufe Schlachtung gibt es wenige Erkenntnisse. $12 \%$ der Experten gehen nicht davon aus und 52\% halten die Wahrscheinlichkeit für gering, dass Rückstände aus Kunststoffmaterialien in Lebensmittel gelangen. Von einem Einfluss auf die Produktqualität, einer Gesundheitsgefahr bzw. einem Meideverhalten der Verbraucher gehen die Experten nicht aus (Abbildung 5.5-8).

Widersprüchlich zu den Antworten aus Abbildung 5.5-8 ist die Bewertung der Experten bei der Einschätzung der Gefahrenkategorie. Obwohl die meisten keinen Einfluss auf die Produktqualität sehen, gehen $30,4 \%$ der Experten von einer sensorischen Gefahr aus. $28,6 \%$ sehen eine chemische, $17,4 \%$ eine mikrobiologische und $21,7 \%$ eine physikalische Gefahr (Anhang Tabelle 11.1-22).

Ein Experte fügte in der mündlichen Befragung hinzu, dass eine mögliche Gefahr bestehen kann, wenn Bedarfsgegenstände, z.B. E2-Kisten, über sogenannte graue Kanäle importiert werden. Hier ist die Wahrscheinlichkeit gegeben, dass Billigmaterialien eingesetzt werden.

Eine andere Gefahr wird gesehen, wenn Schneidbretter, E2-Kisten usw. abgenutzt sind und porös werden. Hierbei kann es durch Abrieb zu einer Verunreinigung des Produktes kommen. Bei der Bewertung für das Jahr 2015 gehen die meisten Experten (47\%) davon aus, dass die potentielle Gefahr der Schadstoffabgabe über Bedarfsgegenstände weiter sinkt. Allerdings rechnen $24 \%$ der Experten mit einem Anstieg (Tabelle 5.5-6).

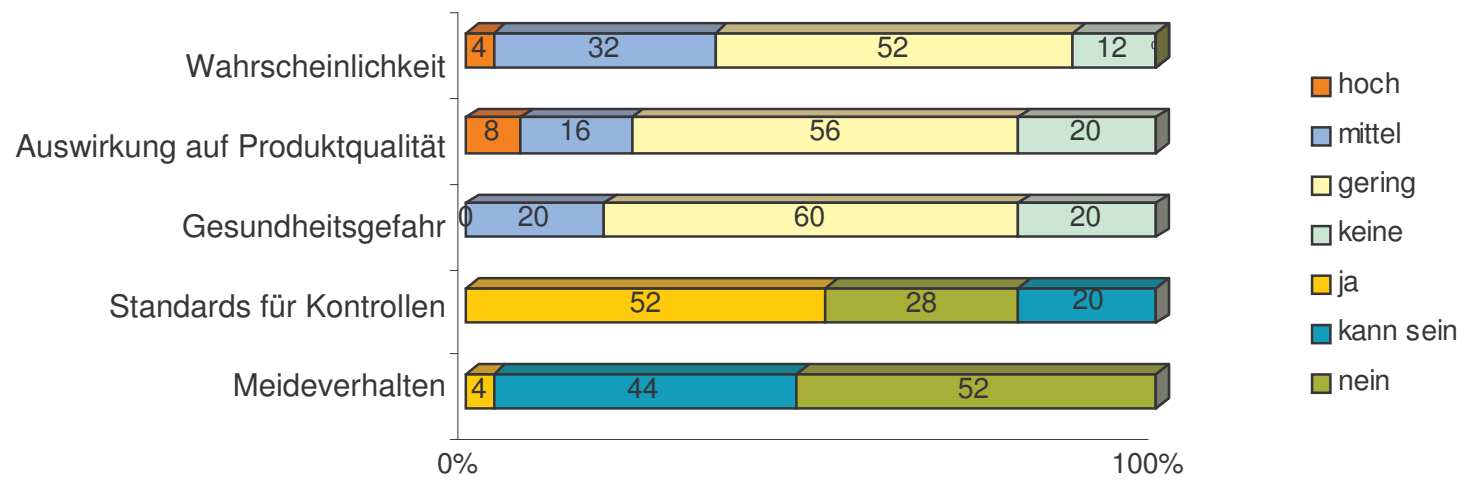

Abbildung 5.5-8: Beurteilung der These: Kunststoffmaterialien können Schadstoffe abgeben und führen beim Einsatz in Lebensmittelbetrieben zu Kontaminationen.

Tabelle 5.5-6: $\quad$ Einschätzung der Entwicklung der potentiellen Gefahr der Schadstoffabgabe durch Bedarfsgegenstände zum Jahr 2015.

\begin{tabular}{ccccccc}
\hline & & stark abnehmend & abnehmend & wie heute & zunehmend & stark zunehmend \\
\hline Experten & $\mathrm{N}=17$ & 1 & 7 & 5 & 4 & 0 \\
& $\%$ & 6 & 41 & 29 & 24 & 0 \\
\hline
\end{tabular}

\subsubsection{Fremdkörper}

Die meisten Experten (64\%) gehen davon aus, dass die Wahrscheinlichkeit, dass die These "Untersuchungen auf Fremdkörper im Produkt Fleisch (Metall, Glas usw.) werden zurzeit gar nicht oder nur begrenzt durchgeführt", zutrifft, gering ist. Die Wahrscheinlichkeit der 
Auswirkung auf die Produktqualität wird von 47\% mit hoch bzw. mittel und die Gesundheitsgefahr von $43 \%$ mit hoch bzw. mittel eingeschätzt. Bezüglich der Standards für Kontrollen gehen 48\% der Experten davon aus, dass Standards vorhanden sind, $22 \%$ sind allerdings der Meinung, dass keine ausreichenden Standards vorhanden sind. Das Meideverhalten wird vom überwiegenden Anteil der Experten für möglich (56\%) bzw. nicht vorhanden (33\%) eingeschätzt (Abbildung 5.5-9). Hauptsächlich wird eine Gefahr bei Rind $(92,3 \%)$ und Schwein $(96,2 \%)$ gesehen (Tabelle 11.1-24).

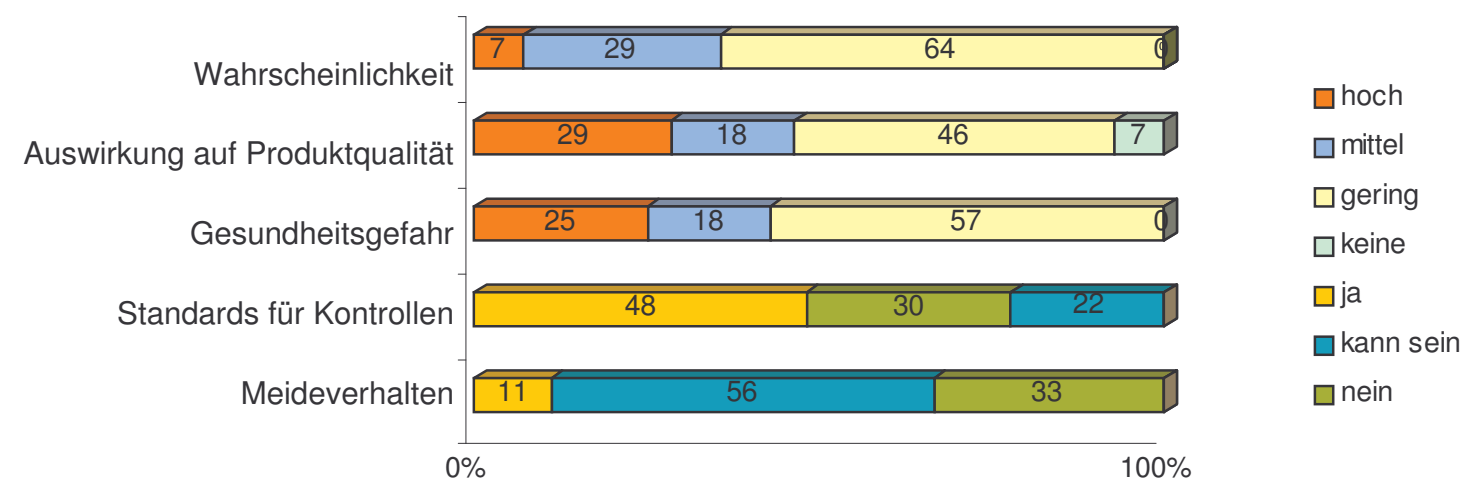

Abbildung 5.5-9: $\quad$ Beurteilung der These: Fremdkörper werden häufiger im Produkt Fleisch gefunden. Untersuchungen auf Fremdkörper im Produkt Fleisch (Metall, Glas usw.) werden zurzeit gar nicht oder nur begrenzt durchgeführt.

Bei der Bewertung für das Jahr 2015 wird davon ausgegangen, dass die potentielle Gefahr durch Fremdkörper im Fleisch weiter sinkt (Tabelle 5.5-7).

Tabelle 5.5-7: $\quad$ Einschätzung der Entwicklung der potentiellen Gefahr der Fremdkörper zum Jahr 2015.

\begin{tabular}{ccccccc}
\hline & & stark abnehmend & abnehmend & wie heute & zunehmend & stark zunehmend \\
\hline Experten & $\mathrm{N}=17$ & 2 & 6 & 6 & 3 & 0 \\
& $\%$ & 12 & 35 & 35 & 18 & 0 \\
\hline
\end{tabular}

\subsection{Herstellung, Lagerung und Transport von Futtermitteln}

Kritische Punkte im Herstellungsprozess für Futtermittel werden vor allem in der Verschleppung und der Trocknung gesehen. Als weitere Punkte werden veraltete Technik, übermäßige Erhitzung, ungeeignete Lagerung, mikrobielle Belastung und Fehler in der Mischgenauigkeit und Stabilität genannt (Abbildung 11.2-10).

Eine Aussage darüber, wie sich die Anlagensicherheit bis zum Jahr 2015 entwickeln wird, kann nicht eindeutig getroffen werden (Tabelle 5.6-1).

Tabelle 5.6-1: $\quad$ Einschätzung der Entwicklung der Anlagensicherheit (Verschleppungsgrad) im Herstellungsprozess Futtermittel zum Jahr 2015.

\begin{tabular}{ccccccc}
\hline & & stark abnehmend & abnehmend & wie heute & zunehmend & stark zunehmend \\
\hline Experten & $\mathrm{N}=17$ & 0 & 7 & 2 & 7 & 1 \\
& $\%$ & 0 & 41 & 12 & 41 & 6 \\
\hline
\end{tabular}

Nach der Einschätzung der Experten werden die Mängel in der Lagerung von Futtermitteln bis zum Jahr 2015 abnehmen (Tabelle 5.6-2). Zurzeit sind die hauptsächlichen Risikopunkte 
bei der Lagerung von Futtermitteln, der Befall durch Schadnager und Ungeziefer, das Eindringen von Feuchtigkeit, mangelnde Reinigung der Lager, Lagerung in ungeeigneten Räumen und der Befall und die Vermehrung von Mykotoxinen (Abbildung 5.6-1).

Tabelle 5.6-2: $\quad$ Einschätzung der Entwicklung der Kontamination des Futtermittels bei der Lagerung zum Jahr 2015.

\begin{tabular}{ccccccc}
\hline & & stark abnehmend & abnehmend & wie heute & zunehmend & stark zunehmend \\
\hline Experten & $\mathrm{N}=18$ & 2 & 11 & 4 & 1 & 0 \\
& $\%$ & 11 & 61 & 22 & 6 & 0 \\
\hline
\end{tabular}

Bei Futtermitteltransporten werden von den Experten die größten Risiken in der ungenügenden Reinigung der Fahrzeuge und der damit verbundenen Verschleppung/ Vermischung gesehen. Aber auch die Vornutzung der Transportfahrzeuge spielt eine wichtige Rolle (Abbildung 11.2-11). Die Sicherheit von Futtermitteltransporten wird nach der Einschätzung der Experten bis 2015 zunehmen (Tabelle 5.6-3).

Rückstände von Reinigungsmitteln, die nach der Reinigung der Fahrzeuge das Futtermittel kontaminieren, sehen die meisten Experten nicht als ein potentielles Problem. Ebenso werden keine oder nur kaum Auswirkungen auf die Produktqualität und keine oder nur eine geringe Gesundheitsgefahr gesehen. Bezüglich der Standards für Kontrollen gehen 38\% der Experten davon aus, dass es Standards gibt, und 48\% der Experten sind sich nicht sicher. Dass Probleme bei der Reinigung von Transportfahrzeugen zu einem Meideverhalten führen kann, wird von 62\% der Experten nicht gesehen (Abbildung 5.6-2).

Tabelle 5.6-3: Einschätzung der Entwicklung des Auftretens von Defiziten beim Transport von Futtermitteln zum Jahr 2015.

\begin{tabular}{|c|c|c|c|c|c|}
\hline Experten & stark abnehmend & abnehmend & wie heute & zunehmend & stark zunehmend \\
\hline \multirow[t]{2}{*}{ Experten } & 2 & 9 & 4 & 3 & 0 \\
\hline & 11 & 50 & 22 & 17 & 0 \\
\hline \multicolumn{6}{|l|}{ 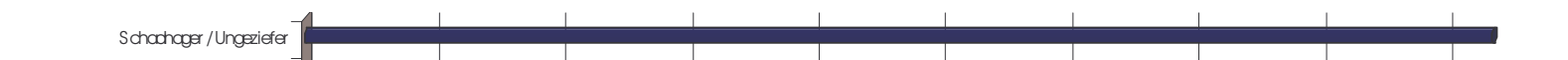 } \\
\hline \multicolumn{6}{|l|}{ 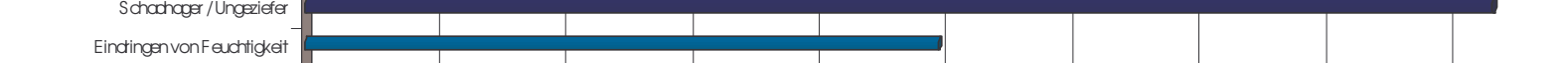 } \\
\hline \multicolumn{6}{|l|}{ unsabaresilos / mang Retiriang } \\
\hline 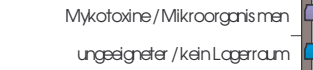 & & & & & \\
\hline fds chekorservieung & 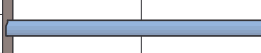 & $\Rightarrow$ & & & \\
\hline Vermis chen mit anderen Chargen & & $\Rightarrow$ & & & \\
\hline Allosten & & & & & \\
\hline mang B elütung Kondenrs woss ertbild ing & & & & & \\
\hline Siloconstriche/Schutz & 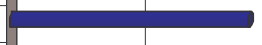 & & & & \\
\hline $\begin{array}{r}\text { Tempadu } \\
\text { Überlogring }\end{array}$ & \begin{tabular}{|l|l|} 
\\
\end{tabular} & & & & \\
\hline Kontamindionnit Res flost töncen & 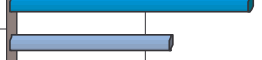 & & & & \\
\hline & ד & & & & \\
\hline & 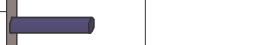 & & & & \\
\hline 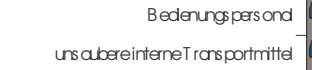 & 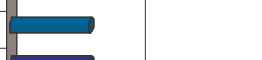 & & & & \\
\hline ungosigntele Logertectrik & 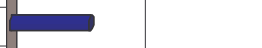 & & & & \\
\hline 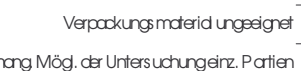 & $\longrightarrow$ & & & & \\
\hline 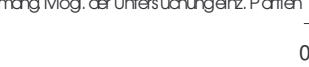 & $2 \%$ & $6 \%$ & $10 \%$ & $14 \%$ & $18 \%$ \\
\hline
\end{tabular}

Abbildung 5.6-1: Einschätzung der kritischen Punkte, die bei der Lagerung von Futtermitteln eine Rolle spielen, in \% der Nennungen. 


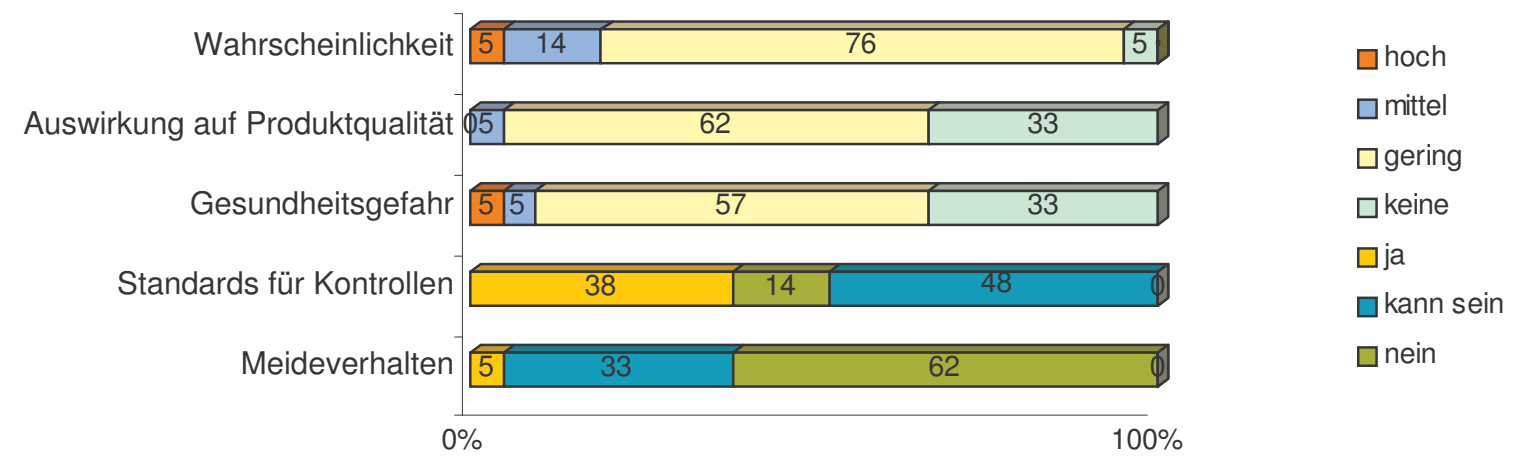

Abbildung 5.6-2: Beurteilung der These: Rückstände von Reinigungsmitteln in Transportfahrzeugen werden zu einem „potentiellen Problem“ in der Tierproduktion.

\subsubsection{Einsatz von tierischen Futtermitteln}

Die These, „Tierische Futtermittel stehen zurzeit stark in der Kritik und deren Verfütterung ist verboten. Bitte nennen Sie Ihre Einschätzung, ob durch geeignete Aufbereitungsmethoden von tierischen Futtermitteln, bei der Verfütterung an Nichtwiederkäuer, keine Gefahr für den Menschen ausgeht" kann nicht ausgewertet werden. Wie sich aus den Kommentaren, in der mündlichen Befragung und in Gesprächen mit einzelnen Experten herausgestellt hat, wurde die Frage falsch gelesen. Viele der Experten haben die Frage so bewertet, dass bei der Verfütterung an nicht Wiederkäuer, eine Gefahr für den Menschen ausgeht. Die Ergebnisse sind im Anhang dargestellt (Abbildung 11.2-18).

Aufgrund der mündlichen Befragung (14 Experten) ist davon auszugehen, dass die meisten Experten keine Gefahr durch tierische Futtermittel sehen. In dieser Befragung wurden die Experten nach möglichen Folgen, die sich aus einer Wiederzulassung tierischer Futtermittel in der Fleischerzeugung ergeben könnten. befragt. Die meisten der Experten sehen keine Probleme, sondern eher Vorteile durch eine Wiederzulassung. Genannt werden: eine bessere Tiergesundheit, Eiweiß- u. Aminosäurenversorgung, Knochenstabilität. Angesprochen werden mögliche Probleme bei der sauberen Trennung zwischen Rind und Schwein und einem eventuellen Kannibalismusverbot. Ansonsten werden in erster Linie Probleme in der Kommunikation mit dem Verbraucher gesehen.

Die Antworten zur Frage, ob sich die Fleischqualität durch tierische Futtermittelkomponenten positiv oder negativ verändern wird, widersprechen den Antworten auf die These, dass der Verzicht auf tierische Futtermittelkomponenten sich auf die Zusammensetzung des Fleisches auswirkt. Nur 32\% der Experten sehen eine mittlere bis hohe Wahrscheinlichkeit, dass die These zutrifft, und $68 \%$ sehen keinen oder nur einen geringen Einfluss auf die Produktqualität (Abbildung 5.6-3). Bei der direkten Frage nach dem Einfluss von tierischen Futterkomponenten auf die Fleischqualität gehen dagegen $62 \%$ der Experten von einem positiven Einfluss aus (Tabelle 5.6-4).

Eine Darstellung der Ergebnisse zur Einschätzung der Gefahrenkategorie bei der These, dass sich der Verzicht auf tierische Futtermittelkomponenten auf die Zusammensetzung des Fleisches auswirkt erfolgt nicht, da die Antworten im Zusammenhang mit der These nicht eindeutig sind. Die Antworten sind im Tabelle 11.1-36 dargestellt. Der Verzicht auf tierische 
Futtermittelkomponenten hat nach der Einschätzung der Experten vor allem einen Einfluss auf Schweine und Geflügel (Tabelle 11.1-37).

Tabelle 5.6-4: $\quad$ Beurteilung der Experten über die Auswirkung tierischer Futtermittelkomponenten auf die Fleischqualität.

\begin{tabular}{lll}
\hline & Einschätzung & Bewertung \% \\
\hline $\mathrm{N}=16$ & positive Auswirkungen & $63 \%$ \\
& keine Auswirkungen & $31 \%$ \\
& negative Auswirkungen & $6 \%$ \\
\hline
\end{tabular}

Insgesamt gehen die Experten davon aus, dass bis zum Jahr 2015 der Einsatz von tierischen Futtermitteln wieder zunehmen und deren Sicherheit in der Produktion steigen wird (Tabelle 5.6-5). Studenten ( $\mathrm{mL}$ ) gehen tendenziell davon aus, dass der Einsatz tierischer Futtermittel wieder zunehmen und die Sicherheit steigen wird. Eine andere Einschätzung wird durch die Studenten (oL) getroffen. Diese Gruppe der Studenten geht davon aus, dass der Einsatz von tierischen Futtermitteln zurückgehen wird und die Sicherheit nicht größer wird (Tabelle 11.1-38).

Tabelle 5.6-5: Einschätzung des Einsatzes und der Sicherheit von tierischen Futtermitteln zum Jahr 2015.

\begin{tabular}{lllllll}
\hline Experten & & stark abnehmend & abnehmend & wie heute & zunehmend & stark zunehmend \\
\hline Einsatz & $\mathrm{N}=19$ & 2 & 3 & 3 & 10 & 1 \\
& $\%$ & 11 & 16 & 16 & 53 & 5 \\
Sicherheit & $\mathrm{N}=19$ & 0 & 0 & 2 & 12 & 5 \\
& $\%$ & 0 & 0 & 11 & 63 & 26 \\
\hline
\end{tabular}

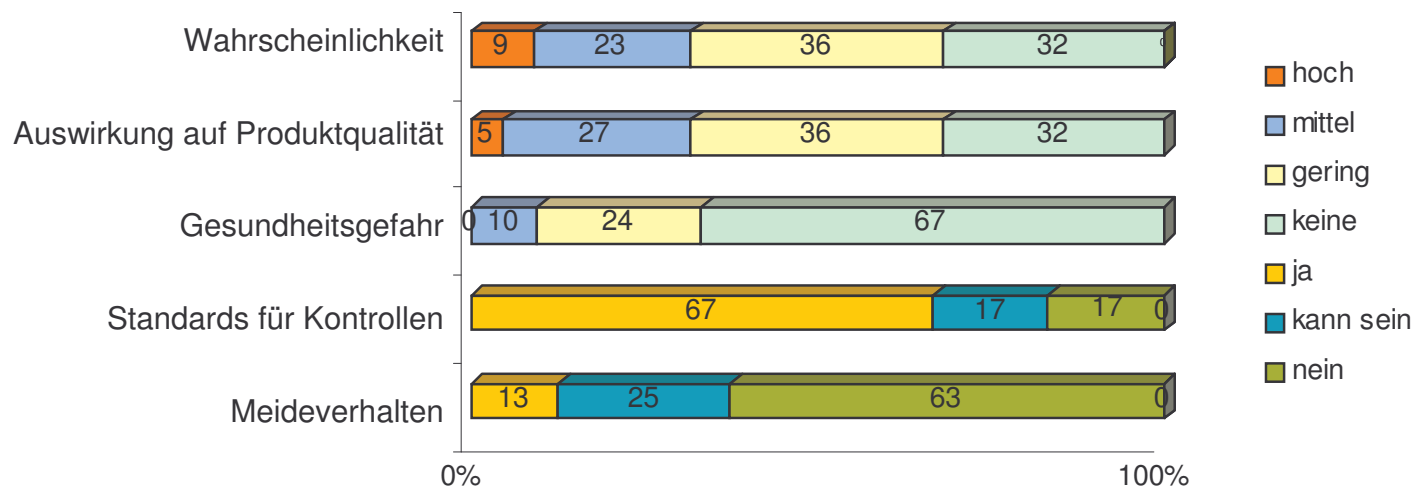

Abbildung 5.6-3: Beurteilung der These: Der Verzicht auf tierische Futtermittelkomponenten wirkt sich auf die Zusammensetzung des Fleisches aus.

Als Risikopunkte eines ungewollten Eintrags von tierischen Futtermitteln in die Nahrungskette werden die illegale Entsorgung, der Import von Fleisch aus Ländern, in denen der Einsatz von Tiermehlen in der Fütterung zulässig ist und die Verschleppungsgefahr bei der Mischfutterherstellung genannt (Abbildung 5.6-4).

In der Entsorgung der Ursprungsstoffe, die zur Herstellung von tierischen Futtermitteln verwendet werden, sehen $20 \%$ der Experten keine und $40 \%$ eine geringe Gefahr. Die 
Gesundheitsgefahr wird von 69\% der Experten als nicht vorhanden oder als gering eingeschätzt. Ausreichende Standards für Kontrollen sehen $57 \%$ der Experten. Bezüglich des Meideverhaltens bei einer unsachgemäßen Entsorgung kann keine Aussage getroffen werden. $27 \%$ der Experten schätzen, dass es zu einem Meideverhalten kommt, und 27\% schätzen, dass es zu keinem Meideverhalten kommt (Abbildung 5.6-5).

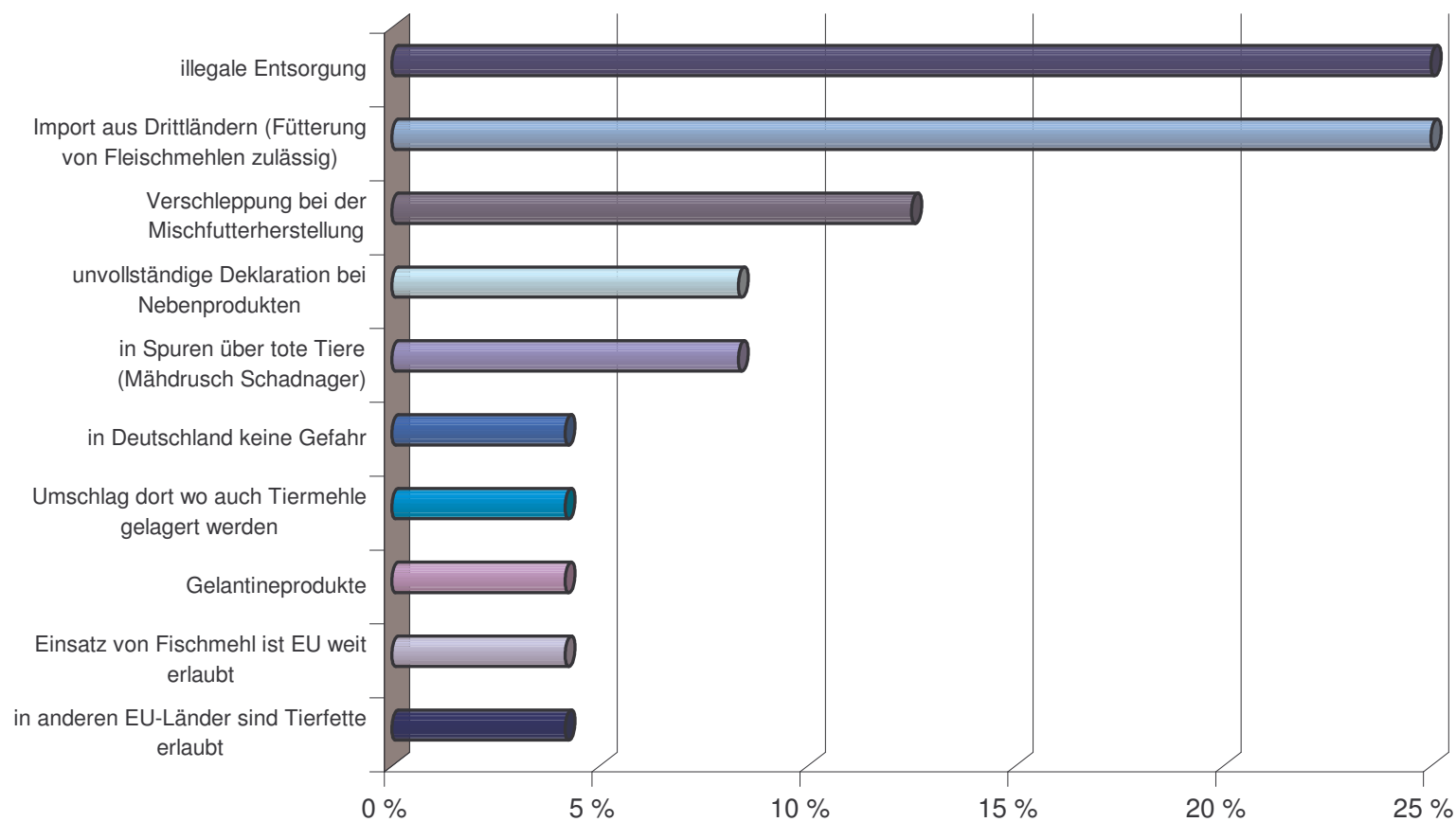

Abbildung 5.6-4: Mögliche Wege, wie tierische Futtermittel in die Nahrungskette gelangen, in \% der Nennungen.

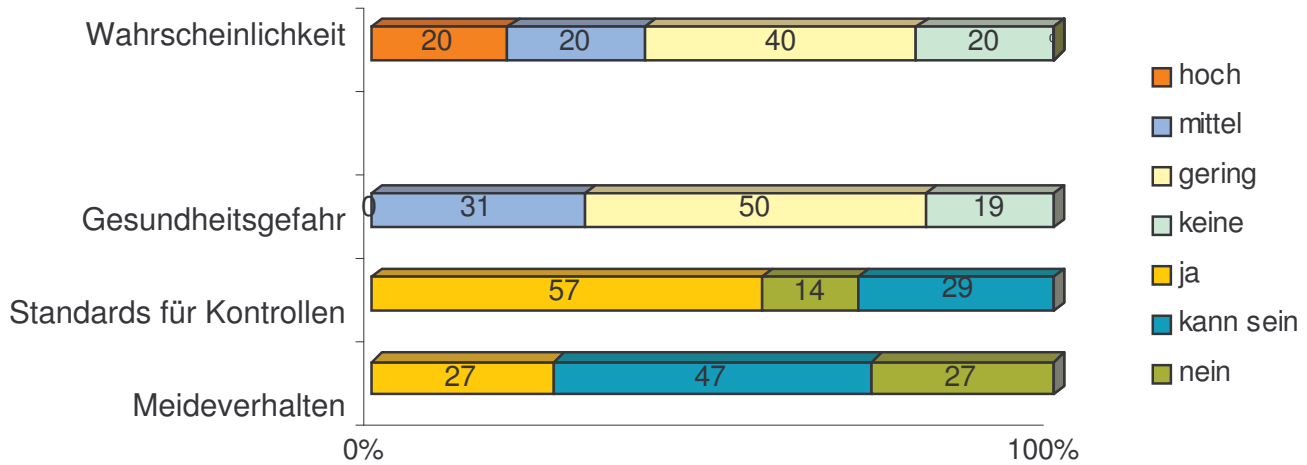

Abbildung 5.6-5: Beurteilung der These: Die Entsorgung der Ursprungsstoffe, die zur Herstellung von tierischen Futtermitteln verwendet wurden, ist nicht ausreichend gesichert. 


\subsection{Tierproduktion}

\subsubsection{Kupieren, Enthornen, Schnabelkürzen}

Die Wahrscheinlichkeit, dass Maßnahmen wie Kupieren, Enthornen und Schnabelkürzen in der Zukunft verboten werden, wird von 54\% der Experten als hoch eingeschätzt. Ein Einfluss auf die Produktqualität und eine Gesundheitsgefahr werden eher nicht gesehen. Standards für Kontrollen sind nach der Meinung von 62\% der Experten vorhanden. 17\% gehen von einem Meideverhalten aus und 48\% schließen ein Meideverhalten bei Missständen nicht aus (Abbildung 5.7-1). Kupieren, Enthornen und Schnabelkürzen werden vor allem als eine ethische Gefahr gesehen (Tabelle 11.1-27).

In der mündlichen Befragung gehen von 11 Befragten 6 davon aus, dass die Standards umgesetzt werden. Von einzelnen Befragten wurden zusätzlich nachfolgende Anmerkungen gemacht:

- Zur Kontrolle von Mängeln beim Schwänze kupieren besteht die Möglichkeit eine Kontrolle auf dem Schlachthof durchzuführen. Nach der Meinung der Experten werden dort ein erheblicher Anteil Tiere angeliefert, die falsch kupiert oder verbotenermaßen kupiert wurden. Die Veterinäre am Schlachthof müssten die Möglichkeit haben hier Maßnahmen zu veranlassen. Das Problem besteht bei Rindern und bei Schweinen.

- Kontrollen in der Landwirtschaft sind nur im Ansatz vorhanden. Die Ämter sollten das umsetzen/durchsetzen, was das Gesetz heute schon vorschreibt und entsprechende Maßnahmen verhängen.

Die bestehenden Standards werden von drei Experten als ausreichend angesehen. Die anderen Experten sagen, dass es in der Zukunft neue Standards geben wird oder bestehende Standards verschärft werden. Die Experten gehen zum Teil davon aus, dass vor allem Kupieren in Zukunft verboten wird. Als Verbesserung oder Weiterführung der bestehenden Standards wird eine bessere und regelmäßige Schulung der durchführenden Personen, ähnlich wie der Sachkundenachweis im Schlachthof, genannt. Es wurde aber auch die Meinung vertreten, dass Kupieren, Enthornen und Schnabelkürzen sich durch produktionstechnische Veränderungen bei den heutigen Produktionsverfahren nur schwer auffangen lassen. Wenn überhaupt, müssen alle Produktionsbedingungen optimal gestaltet sein.

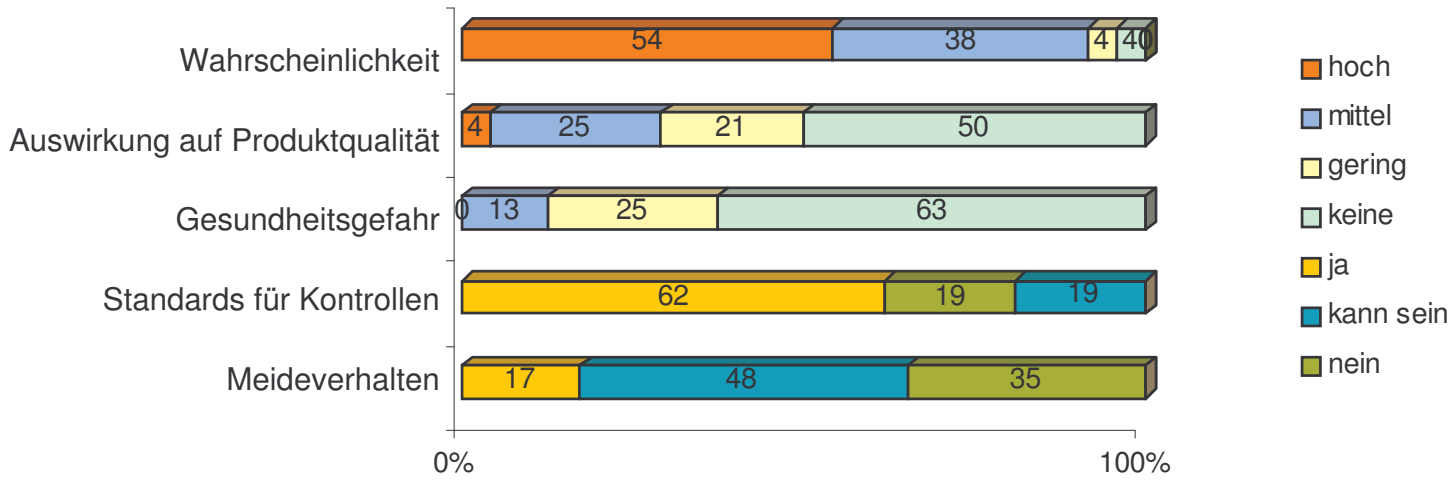

Abbildung 5.7-1: $\quad$ Beurteilung der These: Durch die Verankerung des Tierschutzgedankens im Grundgesetz hat der Tierschutz einen andern Stellenwert erlangt. Hierdurch ist die Wahrscheinlichkeit hoch, dass das Kupieren, das Enthornen, das Schnabelkürzen etc. zukünftig verboten werden. 
Bezüglich der Änderung in der Produktionstechnik bei einem Verbot von Kupieren, Enthornen und Schnabelkürzen haben 7 von 11 Experten die Erhöhung des Platzbedarfes genannt. Bei Schweinen wird von einigen Experten auf mehr Beschäftigungsmöglichkeiten hingewiesen. Der Spieltrieb der Schweine muss stärker befriedigt werden. Weitere Punkte, die genannt wurden, sind eine bessere Klimasteuerung, Optimierung der Futterzusammensetzung und Veränderung der Stallflächen.

Ein Experte merkt an, dass selbst, wenn die gesamten Mastbedingungen optimal gestaltet sind, z.B. Schwanzbeißen nicht auszuschließen ist. Nach seiner Meinung sind die Ursachen für das Schwanzbeißen noch nicht bekannt und einige Rassen haben hierzu eine Veranlagung.

Ein anderer Experte merkt an, dass es bei Geflügel eine wirksame Maßnahme gegen Federpicken gibt. Durch die Verringerung der Lichtintensität wird Federpicken deutlich eingeschränkt bzw. verhindert. Das setzt aber eine Lichtintensität im Bereich von 1-3 Lux voraus. Der Gesetzgeber fordert aber mindestens eine Lichtstärke von 20 Lux.

Für das Jahr 2015 schätzt der überwiegende Anteil der Befragten, dass die Bedeutung der Maßnahmen wie Kupieren, Enthornen und Schnabelkürzen sich im Vergleich zur heutigen Situation nicht ändern bzw. abnehmen wird (Tabelle 5.7-1).

Die Studenten wurden befragt, ob sie Bedenken gegen Maßnahmen wie Kupieren, Enthornen und Schnabelkürzen haben. Hier gab es Unterschiede in den Antworten zwischen Studenten $(\mathrm{mL})$ und Studenten (oL). Zirka $82 \%$ der Studenten $(\mathrm{mL})$ haben keine Bedenken gegen die Maßnahmen. Bei den Studenten (oL) lag dieser Anteil bei ca. 56\% (Tabelle 5.7-2).

Tabelle 5.7-1: $\quad$ Einschätzung der Bedeutung von Maßnahmen wie Kupieren, Enthornen, Schnabelkürzen zum Jahr 2015.

\begin{tabular}{ccccccc}
\hline & & stark abnehmend & abnehmend & wie heute & zunehmend & stark zunehmend \\
\hline \multirow{2}{*}{ Experten } & $\mathrm{N}=21$ & 0 & 7 & 10 & 1 & 3 \\
& $\%$ & 0 & 33 & 48 & 5 & 14 \\
\hline
\end{tabular}

Tabelle 5.7-2:

Antworten zu der Frage: Haben Sie Bedenken gegen Maßnahmen wie Kupieren, Enthornen und Schnabelkürzen?

\begin{tabular}{lcccc}
\hline Studenten & & Studenten & Studenten $(\mathrm{mL})$ & Studenten $(\mathrm{oL})$ \\
\hline \multirow{2}{*}{ Keine Bedenken } & $\mathrm{N}$ & 64 & 40 & 24 \\
& $\%$ & 73 & 82 & 57 \\
\hline
\end{tabular}

\subsubsection{Technopathien}

Die meisten Experten sind der Meinung, dass die heutigen Haltungssysteme Technopathien begünstigen. Ein Einfluss auf die Produktqualität und die Gesundheit des Verbrauchers wird als gering eingestuft. Standards für Kontrollen sind vorhanden. Bezüglich des Meideverhaltens kann keine eindeutige Aussage getroffen werden. 30\% der Experten gehen von einem Meideverhalten aus und 30\% gehen von keinem Meideverhalten aus (Abbildung 5.7-2).

Technopathien werden hauptsächlich als ethische Gefahr (55\%) gesehen und treten überwiegend beim Geflügel und bei Schweinen auf (Tabelle 11.1-32 und Tabelle 11.1-33). Als Technopathien werden Gelenk- und Beinschäden, Klauenschäden, Schwanzbeißen, 
direkte Verletzungen, Hautschäden, Schäden durch das Stallklima usw. genannt (Abbildung 11.2-12).

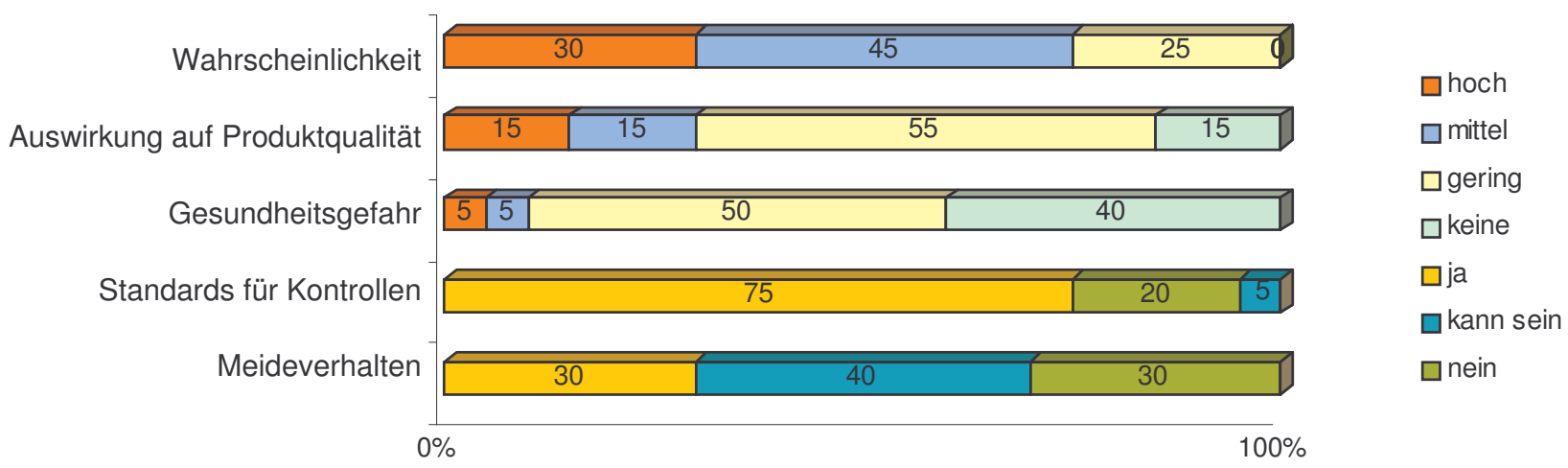

\section{Abbildung 5.7-2: Beurteilung der These: Die heutigen Haltungssysteme begünstigen} Technopathien.

In der mündlichen Befragung werden die Experten gebeten, zu der Frage Stellung zu nehmen, ob hohe Standards vorhanden sind und diese umgesetzt und kontrolliert werden. Nur drei von den 10 befragten Experten gehen davon aus dass diese umgesetzt werden. Fünf Experten gehen von einer teilweisen Umsetzung aus. Allerdings sehen sieben der Experten Mängel bei den Kontrollen. Als konkrete Maßnahmen, die über die vorhandenen Standards hinausgehen, wird eine Steigerung der Beschäftigungsmöglichkeiten und klare Vorgaben für die Bodengestaltung genannt. Insgesamt wird aber darauf hingewiesen, dass die Eigenverantwortung der Tierhalter steigen muss.

Die meisten Experten gehen aber davon aus, dass das Auftreten von Technopathien bis 2015 abnehmen wird (Tabelle 5.7-3).

Tabelle 5.7-3:

Einschätzung des Auftretens von Technopathien zum Jahr 2015.

\begin{tabular}{ccccccc}
\hline & & stark abnehmend & abnehmend & wie heute & zunehmend & stark zunehmend \\
\hline \multirow{2}{*}{ Experten } & $\mathrm{N}=20$ & 0 & 13 & 3 & 3 & 1 \\
& $\%$ & 0 & 65 & 15 & 15 & 5 \\
\hline
\end{tabular}

\subsubsection{Kastration}

Die Kastration spielt in Deutschland nur bei Schweinen eine wichtige Rolle. Ein Beibehalten der Kastration wird von den meisten Experten als ein eher geringes Problem gesehen. 57\% der Experten gehen davon aus, dass die Wahrscheinlichkeit, dass die These, ein Beibehalten der Kastration kann zu Problemen führen, nicht gegeben oder gering ist. Nur 4\% der Experten gehen davon aus, dass die These zutrifft. Auswirkungen auf die Produktqualität werden dagegen von 44\% der Experten gesehen und 36\% halten die Wahrscheinlichkeit von Auswirkungen auf die Produktqualität für möglich. Eine Gesundheitsgefahr wird nicht gesehen (76\%) und Standards für die Kontrollen sind vorhanden (73\%). Zu einem Meideverhalten bei Fleisch kann es kommen (Abbildung 5.7-3). Aus den ergänzenden Antworten geht hervor, dass vor allem eine ethische $(69,6 \%)$ und eine sensorische $(56,5 \%)$ Gefahr gesehen wird und vor allen anderen Arten das Schwein betroffen ist (Tabelle 11.1-27und Tabelle 11.1-28). 
Die Alternativen zur Kastration werden aber von den meisten Experten ebenso kritisch gesehen. Wenn keine Kastration durchgeführt wird, dürften die männlichen Schweine nicht ausgemästet werden (geringere Teilstückausbeute) und es müssten Schnellmethoden entwickelt werden, um den Skatol- und Androsenongehalt im Fleisch zu bestimmen. Sollte solches Fleisch in den Frischfleischmarkt gelangen, hat dieses aufgrund des unangenehmen, zum Teil penetranten Geruchs ebenfalls eine Meidereaktion zur Folge. Eine Immunkastration würde ebenfalls vom Verbraucher kritisch gesehen werden, da er den Vorgang nicht nachvollziehen kann. Eine Kastration würde am ehesten akzeptiert werden, wenn sie unter Vollnarkose und durch einen Tierarzt durchgeführt wird. Dieses ist bei der heutigen Kostenstruktur in der Schweineproduktion nicht zu realisieren.

Die Meinung über die Entwicklung der Fleischproduktion von kastrierten Tieren ist sehr unterschiedlich, die meisten Experten sind der Meinung, dass sich keine Änderungen gegenüber heute ergeben werden. Ca. $17 \%$ nehmen an, dass die Kastration abnehmen und ca. $24 \%$ gehen davon aus, dass sie zunehmen wird (Tabelle 5.7-4).

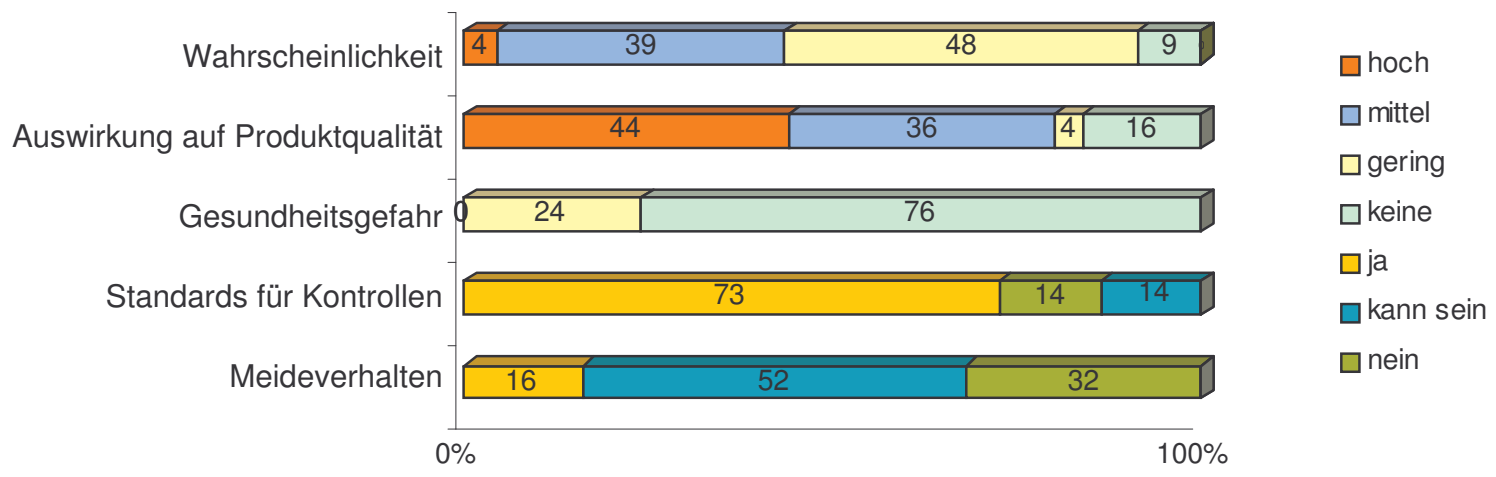

Abbildung 5.7-3: Beurteilung der These: Die Kastration von Tieren wird vom Verbraucher aus Tierschutzgründen sehr kritisch gesehen. Ein Beibehalten der Kastration kann zu Problemen führen.

Tabelle 5.7-4: $\quad$ Entwicklung der Fleischproduktion von kastrierten Tieren zum Jahr 2015.

\begin{tabular}{ccccccc}
\hline & & stark abnehmend & abnehmend & wie heute & zunehmend & stark zunehmend \\
\hline \multirow{2}{*}{ Experten } & $\mathrm{N}=17$ & 1 & 2 & 10 & 4 & 0 \\
& $\%$ & 6 & 12 & 59 & 24 & 0 \\
\hline
\end{tabular}

\subsubsection{Kritische Punkte für die Produktqualität}

Einen Einfluss auf die Produktqualität haben vor allem die Punkte Gesundheitsmanagement/ Hygiene, Fütterung, Klima/Licht, Genetik, Haltung und Mastintensität. Besonders kritisch werden nach Meinung der Experten vom Verbraucher dagegen die Punkte Besatzdichte, Masthilfsmittel, Haltung, Fütterung, Gesundheitsmanagement/Hygiene, Schlachttechnik und Verladetechnik bewertet (Abbildung 5.7-4). 


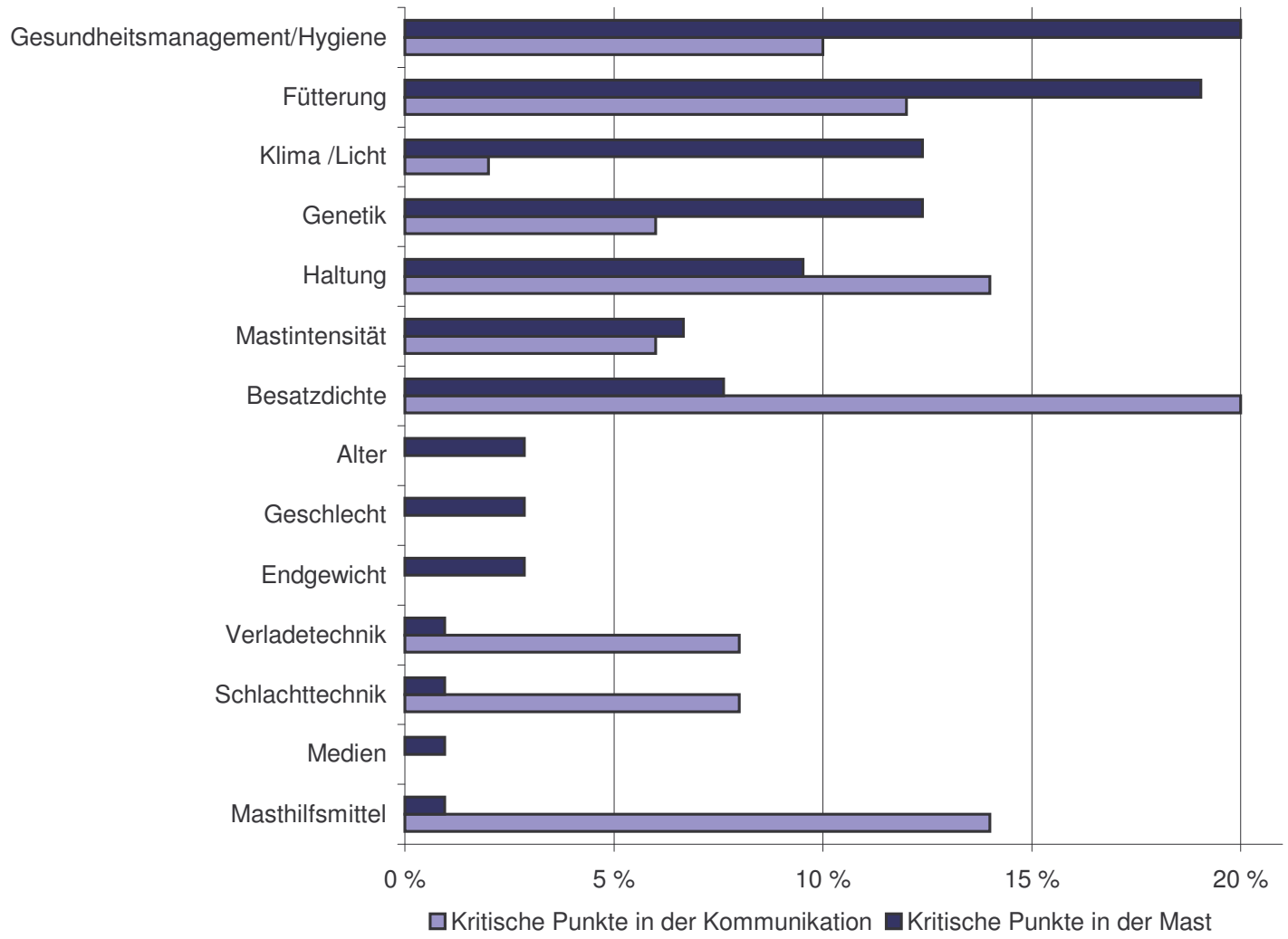

Abbildung 5.7-4: $\quad$ Kritische Punkte in der Mast von Tieren, die einen Einfluss auf die Produktqualität haben, und die Beurteilung, die aus der Sicht des Verbrauchers in der Kommunikation als kritisch zu bewerten sind, in \% der Nennungen.

\subsubsection{Freilandhaltung}

Die Wahrscheinlichkeit der Belastung des Bodens durch die Freilandhaltung von Schweinen und Geflügel wird von 39\% der Experten als hoch und von ca. 35\% der Experten als mittel eingeschätzt. Einen Einfluss auf die Produktqualität und eine Gefährdung für den Verbraucher wird für möglich bis gering gehalten. Über Standards für Kontrollen sind sich die Experten uneinig. 43\% sagen, es sind ausreichend Standards vorhanden und $35 \%$ sind der Meinung, dass Standards fehlen. Ein Meideverhalten wird als eher unwahrscheinlich eingeschätzt (Abbildung 5.7-5). Aus den ergänzenden Antworten geht hervor, dass vor allem eine mikrobiologische (81\%), aber auch eine chemische $(42,9)$ Gefahr gesehen wird (Tabelle 11.1-29).

Managementfehler werden in den Kommentaren der schriftlichen Befragung vermehrt für die Ursache von Gefahren genannt. Aufgrund der Ergebnisse der ersten Befragung wurden die Experten nach den Risiken der Freilandhaltung für die Gesundheit des Menschen und das Wohlbefinden der Tiere auch mündlich gefragt. Diese Frage wurde weitergeführt und es wurden die Experten gebeten zu beurteilen, ob sich eventuell auftretende Risiken in der Zukunft relativieren werden. Kritisch wird vor allem die Anreicherung von Parasiten und pathogenen Keimen und den daraus resultierenden Folgen wie Krankheitsdruck genannt. Weitere wichtige Punkte waren Seuchenrisiko und Kannibalismus. Bei vielen der Antworten wurde immer wieder auch auf die erhöhten Anforderungen an das Management bzw. die Ausbildung hingewiesen. 


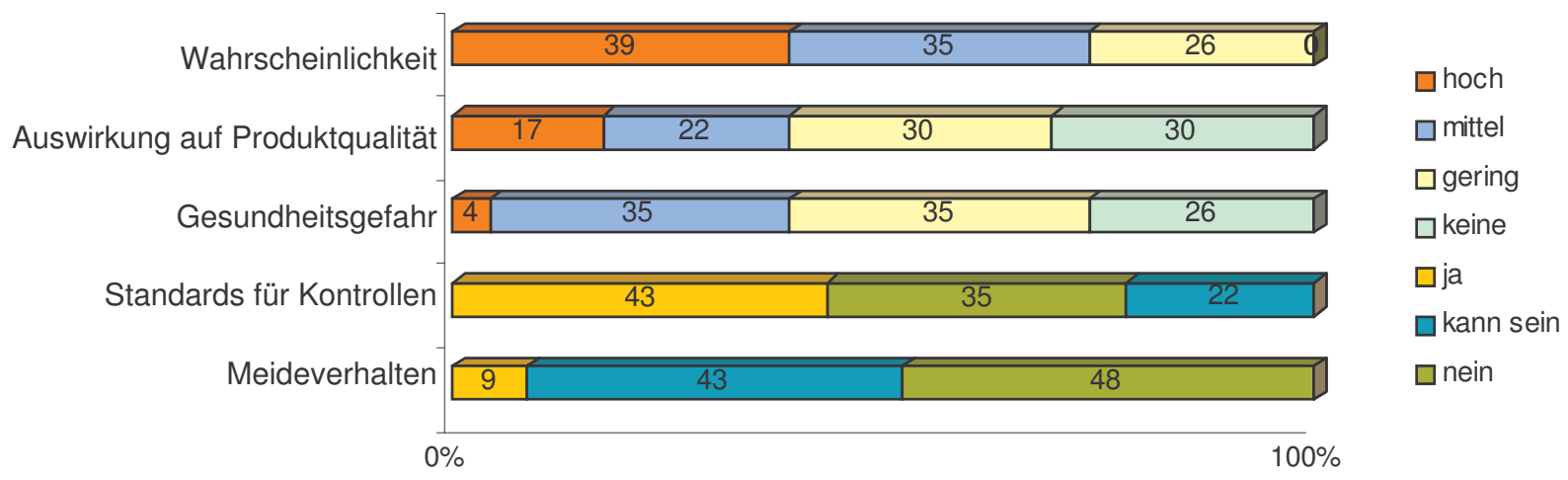

Abbildung 5.7-5: Beurteilung der These: Durch Freilandhaltung bei Geflügel und Schweinen kommt es zu einer starken Belastung des Bodens.

Die zukünftige Entwicklung der Probleme wird von den Experten sehr unterschiedlich bewertet. Ein Teil der Experten (40\%) ist der Meinung, dass durch mehr Erfahrungen die Probleme verringert werden. Der andere Teil der Experten (60\%) ist dagegen der Meinung, dass die Risiken nicht abnehmen, sondern eher zunehmen werden. Gefahren für die Gesundheit von Mensch und Tier durch die Freilandhaltung werden von 62,5\% der Studenten $(\mathrm{mL})$ und von 37,50\% der Studenten (oL) gesehen (Tabelle 5.7-5).

In der zeitlichen Einschätzung für das Jahr 2015 wird der Anteil an Freilandhaltung bei Geflügel und Schweinen wie heute bleiben bzw. leicht zunehmen (Tabelle 5.7-6).

Tabelle 5.7-5:

Antworten zu der Frage: Sehen Sie Gefahren in der Freilandhaltung von Geflügel und Schweinen für den Menschen oder das Tier?

\begin{tabular}{lcccc}
\hline Studenten & & Studenten & Studenten $(\mathrm{mL})$ & Studenten (oL) \\
\hline \multirow{2}{*}{$\mathrm{Ja}$} & $\mathrm{N}$ & 64 & 40 & 24 \\
& $\%$ & 53 & 63 & 38 \\
\hline
\end{tabular}

Tabelle 5.7-6: Einschätzung der Entwicklung des Anteils an Freilandhaltung bei Geflügel und Schweinen zum Jahr 2015.

\begin{tabular}{ccccccc}
\hline & & stark abnehmend & abnehmend & wie heute & zunehmend & stark zunehmend \\
\hline \multirow{2}{*}{ Experten } & $\mathrm{N}=20$ & 0 & 4 & 7 & 9 & 0 \\
& $\%$ & 0 & 20 & 35 & 45 & 0 \\
\hline
\end{tabular}

\subsubsection{Anzahl der Tiere pro Stall und/oder Betrieb}

Die Experten sehen es als sehr wahrscheinlich an, dass die Anzahl der Tiere pro Stallgebäude und/oder pro Betrieb begrenzt wird. Sie sehen durch die Bestandsgrößen aber kaum einen negativen Einfluss auf die Produktqualität und die Gesundheit des Verbrauchers. $\mathrm{Ob}$ es aufgrund der Tieranzahl zu einem Meideverhalten beim Fleischkonsum kommt, wird von den Experten nicht eindeutig beantwortet (Abbildung 5.7-6). Neben einer ethischen Gefahr $(68,4 \%)$ werden noch chemische $(31,6 \%)$ und mikrobiologische $(42,1 \%)$ Gefahren gesehen (Tabelle 11.1-30). Einen Einfluss hat die Beschränkung der Tierzahlen hauptsächlich für Schweine und Geflügel (Tabelle 11.1-31).

Für tiergerecht wird keines der Haltungsverfahren in der Mast von den Studenten gehalten. Am tiergerechtesten wird noch die Haltung von Rindern (48,44\%) angesehen. Auffällig ist, dass Studenten $(\mathrm{mL})$ die Haltungssysteme deutlich positiver einschätzen als Studenten (oL) 
Besonders negativ wird von dieser Studentengruppe die Geflügelhaltung gesehen. Nur $4,17 \%$ halten die Geflügelhaltung für tiergerecht (Tabelle 5.7-7).

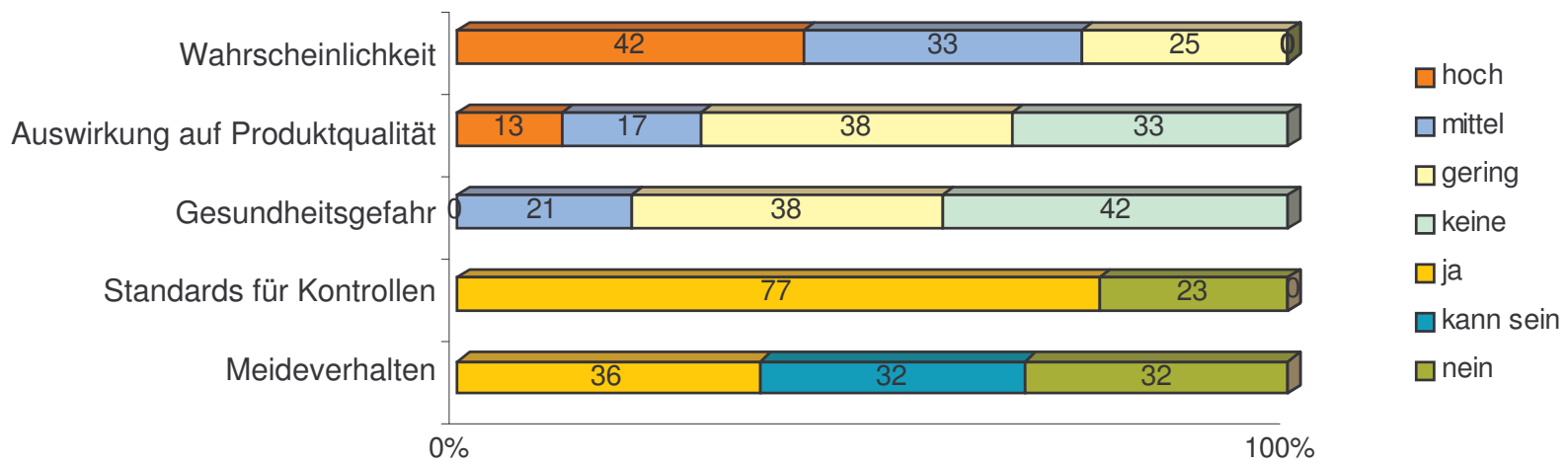

Abbildung 5.7-6: Beurteilung der These: Die Anzahl der Tiere pro Stallgebäude und oder pro Betrieb (Einschränkung der Massentierhaltung) sollte begrenzt werden.

Tabelle 5.7-7: $\quad$ Antworten zu der Frage: Halten Sie die heutigen Haltungssysteme in der Tiermast für tiergerecht?

\begin{tabular}{|c|c|c|c|c|c|c|c|c|c|c|}
\hline \multirow[t]{2}{*}{ Studenten } & & \multicolumn{3}{|c|}{$\begin{array}{c}\text { Studenten } \\
\mathrm{N}=64\end{array}$} & \multicolumn{3}{|c|}{$\begin{array}{l}\text { Studenten }(\mathrm{mL}) \\
\qquad \mathrm{N}=40\end{array}$} & \multicolumn{3}{|c|}{$\begin{array}{l}\text { Studenten (oL) } \\
\qquad \mathrm{N}=24\end{array}$} \\
\hline & & ja & nein & teilweise & ja & nein & teilweise & ja & nein & teilweise \\
\hline Rinder & $\%$ & 48 & 19 & 33 & 60 & 15 & 25 & 29 & 25 & 46 \\
\hline Schweine & $\%$ & 27 & 34 & 39 & 30 & 25 & 45 & 21 & 50 & 29 \\
\hline Geflügel & $\%$ & 13 & 45 & 42 & 18 & 43 & 40 & 4 & 50 & 46 \\
\hline
\end{tabular}

\section{$5.8 \quad$ Tiertransporte}

Die meisten Experten stimmten der These zu, dass Tiertransporte vom Verbraucher zunehmend abgelehnt werden und dass ein langer Transport aus der Sicht der meisten Verbraucher tierschutzwidrig ist. Eine Auswirkung auf die Produktqualität schätzen $41 \%$ mit hoch und $37 \%$ mit mittel ein. Eine gesundheitliche Gefährdung für den Verbraucher durch Transporte wird eher als gering (52\%) bewertet (). Diese Einschätzung der Experten deckt sich mit ihrer Beurteilung bei der Risikokategorie. Tiertransporte werden hauptsächlich als ein ethisches Risiko gesehen, wovon alle der untersuchten Tierarten betroffen sind (Tabelle 11.1-34 und Tabelle 11.1-35). Tiertransporte sind immer wieder Auslöser für negative Berichte in der Presse, obwohl es relativ umfassende gesetzliche Standards für Transporte gibt. Aufgrund dieses Sachverhalts sollten in der zweiten Befragungsrunde Maßnahmen ermittelt werden, wie über die gesetzlichen Regelungen hinaus Tiertransporte sicherer gestalten werden können. Zusammenfassend wurden hier folgende Punkte von den Experten genannt:

- die stärkere Kontrolle/Überprüfung des Transportes,

- Verhängen von konkreten Maßnahmen bzw. Strafen bei ermittelten

Abweichungen/Verstößen, 
- $\quad$ eine regelmäßige Schulung des Transportpersonals (nicht nur theoretisch), ggf. gesetzliche Regelung der Schulung,

- $\quad$ eindeutigere Festlegung der Transportzeiten,

- Sonderregelungen für Tiertransporte im Stau,

- Vorgaben/Verbesserung der Hygiene an den Sammelstellen,

- Begrenzung von Umladen/Zuladen,

- Verbesserung der Pflichtausstattung der Fahrzeuge.

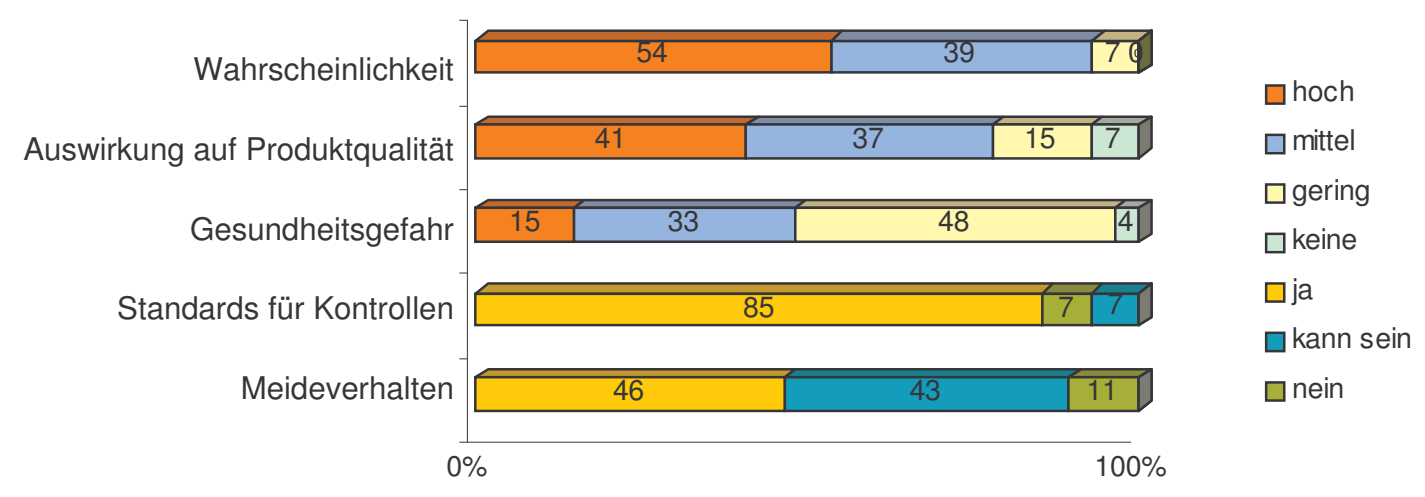

Abbildung 5.8-1: Beurteilung der These: Tiertransporte werden vom Verbraucher zunehmend abgelehnt. Ein langer Transport ist aus der Sicht der meisten Verbraucher tierschutzwidrig.

Für die Experten sind vor allem das Klima, der Be- und Entladevorgang, die Ladedichte, die Transportdauer und die Versorgung der Tiere kritische Punkte beim Transport. Die Studenten halten aus der Sicht des Verbrauchers in erster Linie die Transportdauer, die Ladedichte, die Versorgung und die Hygiene für bedenklich. Die Beachtung des Klimas beim Transport wird von allen Experten als einer der wichtigsten Punkte angesehen. Bei den Studenten wird das Klima erst an 5. Stelle zusammen mit den allgemeinen Transportbedingungen genannt (Abbildung 5.8-2). Nach der Meinung der Experten müssen extreme Klimasituationen vermieden werden. Ein voll klimatisiertes oder hermetisch abgeschlossenes Fahrzeug wird aber nur von 5 von 12 Experten in Abhängigkeit mit der Transportzeit für notwendig befunden.

Die Experten gehen aber davon aus, dass bis 2015 der Anteil an Tiertransporten in klimatisierten Fahrzeugen zunimmt (59\%), bzw. stark zunimmt (9\%) (Tabelle 5.8-1).

Bei den Transportzeiten wird tendenziell davon ausgegangen, dass diese bis 2015 abnehmen werden. $52 \%$ der Experten gehen von abnehmenden bis stark abnehmenden Transportzeiten aus. Allerdings sind auch 29\% der Experten der Meinung, dass die Transportzeiten zunehmen werden (Tabelle 5.8-2). 


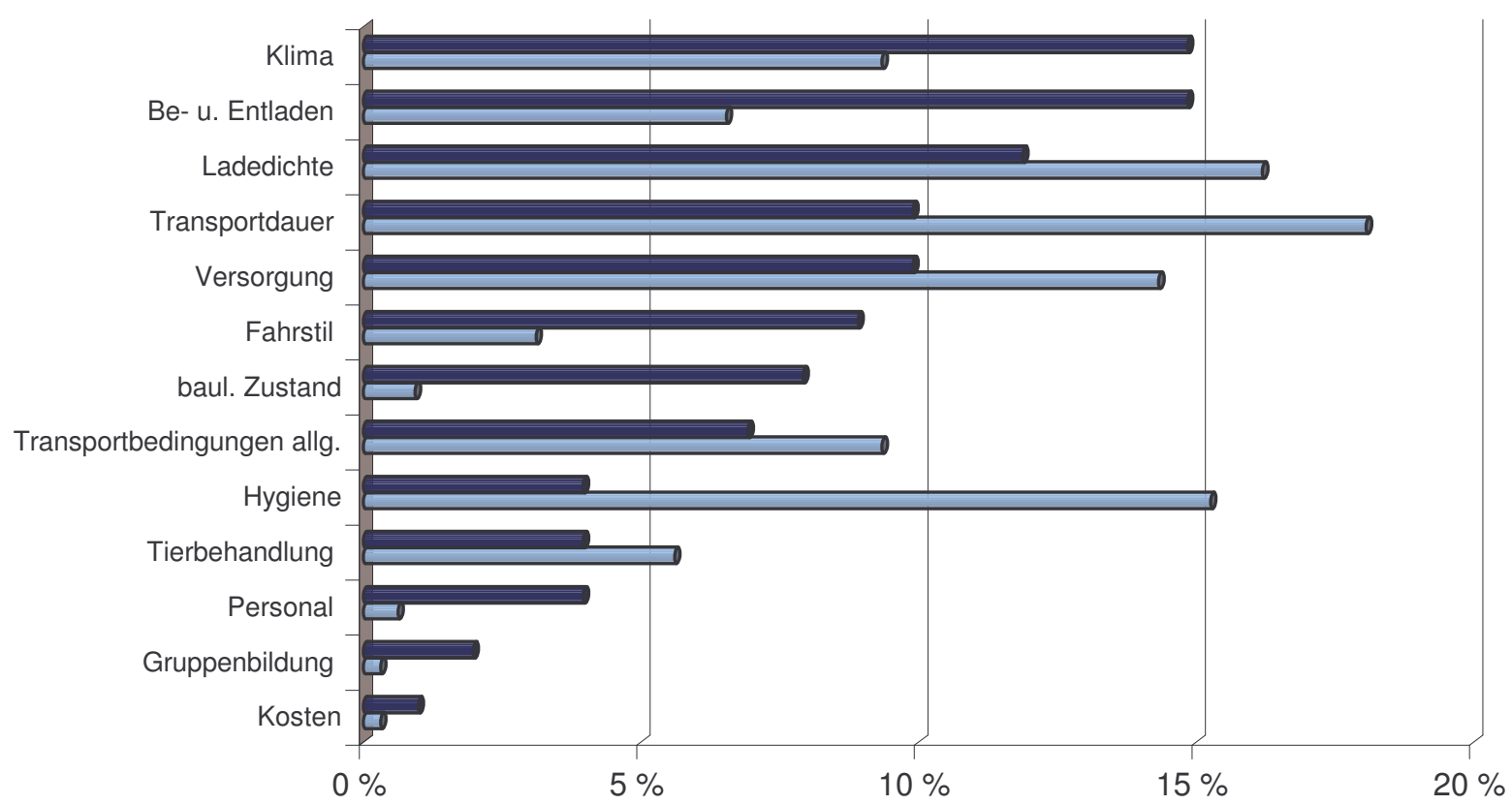

$\square$ Studenten: Kritische Punkte aus der Sicht des Verbrauchers $\square$ Experten: Kritische Punkte

Abbildung 5.8-2: Kritische Punkte beim Transport und die Beurteilung der Punkte durch die Studenten bezüglich der Bedenklichkeit aus der Sicht der Verbraucher, in \% der Nennungen.

Tabelle 5.8-1: $\quad$ Einschätzung der Entwicklung von Tiertransporten in vollklimatisierten Fahrzeugen zum Jahr 2015.

\begin{tabular}{ccccccc}
\hline & & stark abnehmend & abnehmend & wie heute & zunehmend & stark zunehmend \\
\hline \multirow{2}{*}{ Experten } & $\mathrm{N}=22$ & 0 & 0 & 7 & 13 & 2 \\
& $\%$ & 0 & 0 & 32 & 59 & 9 \\
\hline
\end{tabular}

Tabelle 5.8-2:

Einschätzung der Entwicklung der Transportzeit für Schlachttiere zum Jahr 2015.

\begin{tabular}{ccccccc}
\hline & & stark abnehmend & abnehmend & wie heute & zunehmend & stark zunehmend \\
\hline \multirow{2}{*}{ Experten } & $\mathrm{N}=21$ & 2 & 9 & 4 & 6 & 0 \\
& $\%$ & 10 & 43 & 19 & 29 & 0 \\
\hline
\end{tabular}

\section{9}

\section{Schlachtprozess}

\subsubsection{Tierannahme/Wartestall des Schlachthofes und Zuführung zur Betäubung}

Bauliche Mängel, Fehler in der Aufstallung und im Umgang mit den Tieren werden als hauptsächliche Risikopunkte für die Produktqualität von den Experten genannt. Daneben spielen die nicht eingehaltenen Ausruhzeiten und das mangelhafte Einhängen beim Geflügel eine wichtige Rolle. Vergleicht man diese Punkte mit denen, die nach Meinung der Experten beim Verbraucher ein Meideverhalten hervorrufen könnten, kann man eine Verschiebung feststellen. Bauliche Mängel werden hier erst an 6. Stelle genannt. Entscheidender für ein Meideverhalten werden Punkte wie der Umgang mit den Tieren, die Aufstallung, das Einhängen in das Schlachtband bei Geflügel und Lärmeinflüsse auf das Tier angesehen 
(Abbildung 5.9-1). Heute noch vorhandene Mängel im Bereich Tierannahme/Wartestall werden aber nach der Meinung der Experten bis zum Jahr 2015 abnehmen (Tabelle 5.9-1).

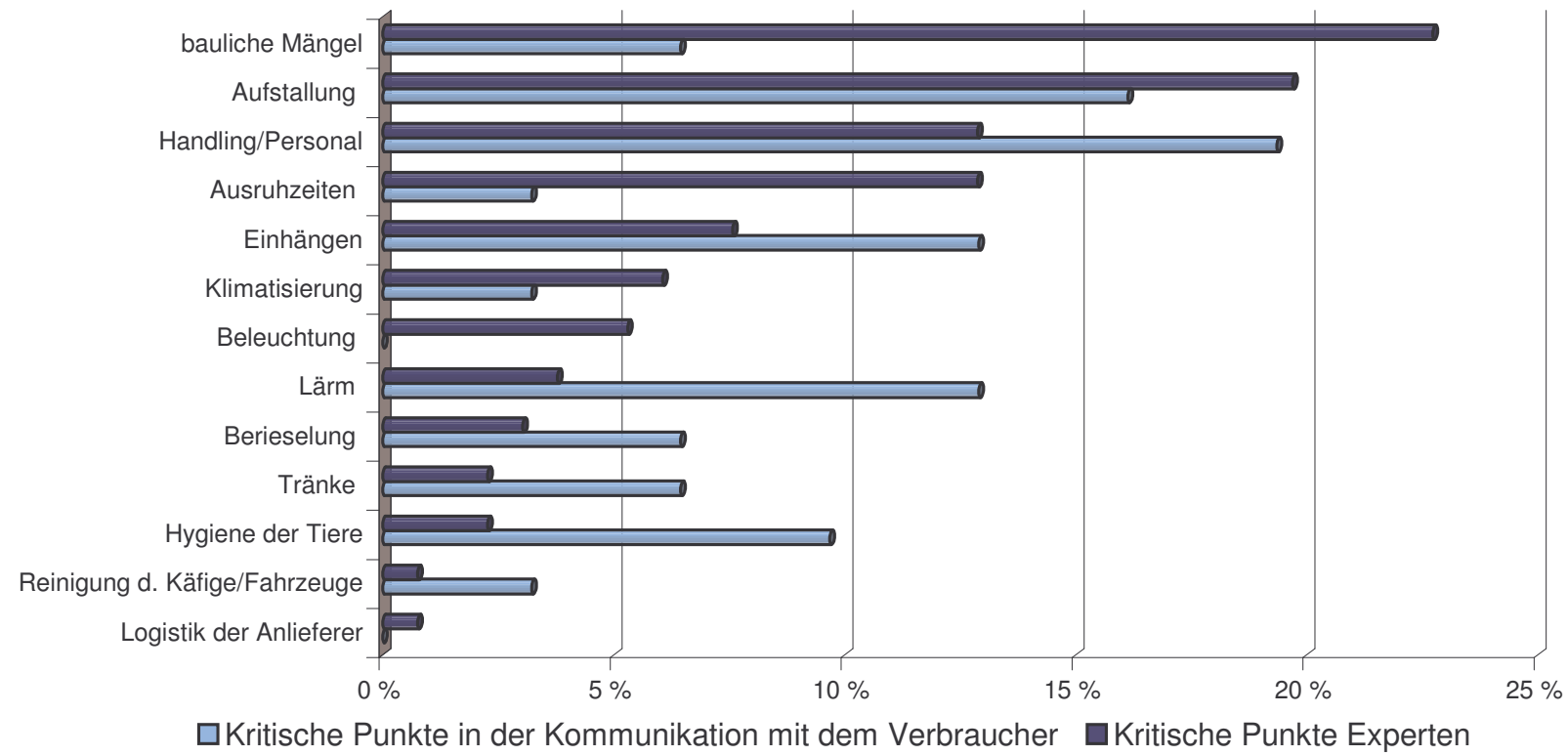

Abbildung 5.9-1: Bauliche Mängel und Managementfehler für den Bereich Tierannahme/ Wartestall, die die Produktqualität negativ beeinflussen und Einschätzung der Punkte, die in der Kommunikation durch den Verbraucher kritisch gesehen werden, in \% der Nennungen.

Tabelle 5.9-1: $\quad$ Einschätzung über das Auftreten von Behandlungs- und Haltungsdefiziten im Bereich Tierannahme/Wartestall.

\begin{tabular}{ccccccc}
\hline & & stark abnehmend & abnehmend & wie heute & zunehmend & stark zunehmend \\
\hline \multirow{2}{*}{ Experten } & $\mathrm{N}=17$ & 2 & 13 & 1 & 1 & 0 \\
& $\%$ & 12 & 76 & 6 & 6 & 0,00 \\
\hline
\end{tabular}

\subsubsection{Betäubungsverfahren}

Der Bereich des Zutriebs zur Betäubung gehört mit zu den sensiblen Bereichen in einem Schlachtunternehmen. Von den Experten werden hier als hauptsächliche Mängelpunkte das Handling durch das Personal und bauliche Mängel gesehen (Abbildung 5.9-2).

Die Betäubungsverfahren werden nach der Meinung der Experten grundsätzlich vom Verbraucher kritisch beurteilt. Hierbei ist nicht das System entscheidend, sondern die ordnungsgemäße Durchführung der Betäubung. Schwerpunkte bezüglich eines Verfahrens konnten nicht festgestellt werden.

Bei einer unzureichenden Betäubung ist jedes Verfahren kritisch zu bewerten (falscher Ansatzpunkt der Elektroden, ungenügende Betäubungszeit bei $\mathrm{CO}_{2}$, falsche $\mathrm{CO}_{2}$-Konzentration usw.). Ebenso ist eine nicht sachgerechte Durchführung der Betäubung (Nichteinhaltung der Bedingungen siehe Tierschutzverordnung) als kritisch zu bewerten und nicht das Verfahren an sich. Eine eindeutige Bevorzugung oder Ablehnung eines Verfahrens konnte nicht festgestellt werden (Abbildung 11.2-13). Die Studenten sehen vor allem beim Schächten die geringste Akzeptanz durch den Verbraucher. Nach der Meinung der Studenten würde der Verbraucher am ehesten die $\mathrm{CO}_{2}$-Betäubung als Verfahren akzeptieren (Tabelle 5.9-3). 
Nach der überwiegenden Meinung der Experten werden die Mängel bei der Betäubung bis zum Jahr 2015 abnehmen (Tabelle 5.9-2).

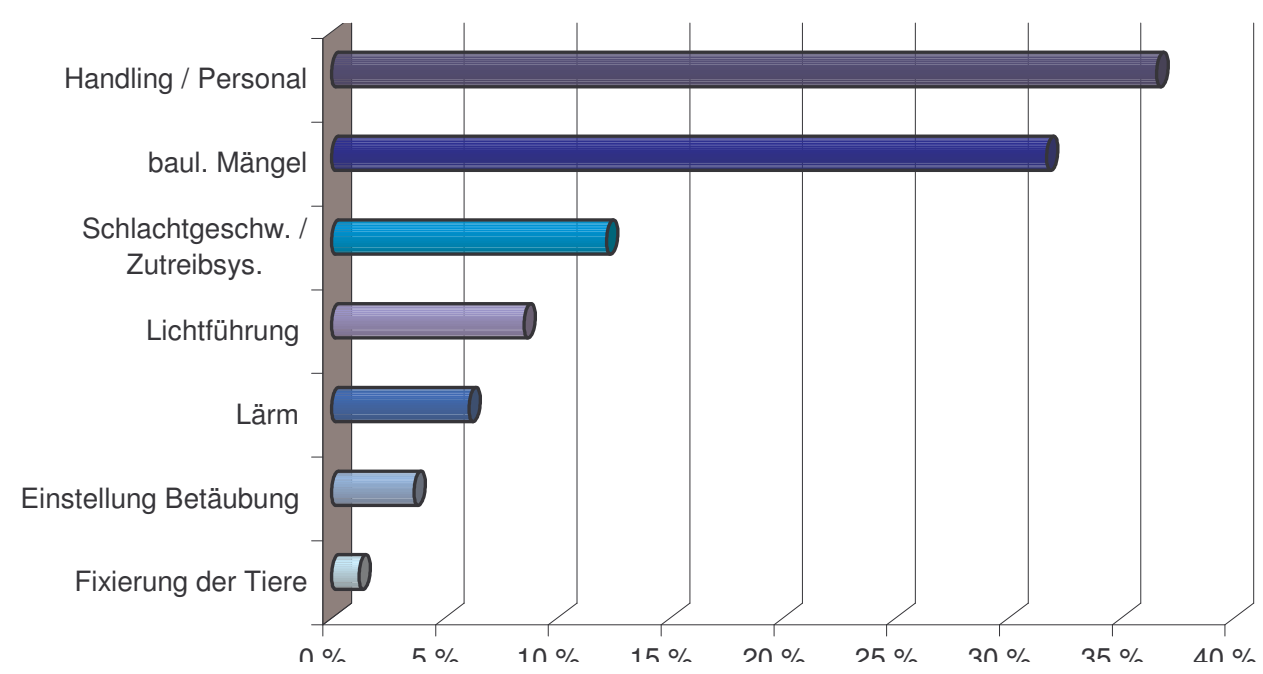

Abbildung 5.9-2: Mängel beim Zutrieb bzw. Einhängen der Tiere zur Betäubung, in \% der Nennungen.

Tabelle 5.9-2:

Entwicklung von Defiziten bei der Betäubung zum Jahr 2015.

\begin{tabular}{ccccccc}
\hline & & stark abnehmend & abnehmend & wie heute & zunehmend & stark zunehmend \\
\hline Experten & $\mathrm{N}=17$ & 4 & 10 & 0 & 3 & 0 \\
& $\%$ & 24 & 59 & 0 & 18 & 0 \\
\hline
\end{tabular}

Tabelle 5.9-3:

Beurteilung der Studenten, welche Betäubungsverfahren der Verbraucher akzeptieren könnte.

\begin{tabular}{ccccc}
\hline Studenten & & $\begin{array}{c}\text { Studenten } \\
\mathrm{N}=64\end{array}$ & $\begin{array}{c}\text { Studenten }(\mathrm{mL}) \\
\mathrm{N}=40\end{array}$ & $\begin{array}{c}\text { Studenten }(\mathrm{oL}) \\
\mathrm{N}=24\end{array}$ \\
\hline Bolzenschuss & $\%$ & 47 & 43 & 54 \\
$\mathrm{CO}_{2}$ & $\%$ & 63 & 73 & 46 \\
Strom & $\%$ & 44 & 50 & 33 \\
Schächten & $\%$ & 5 & 5 & 4 \\
Keines & $\%$ & 11 & 8 & 17 \\
\hline
\end{tabular}

\subsubsection{Schlachtung}

Hygienemängel, Mängel bei der Magen-Darm-Entnahme, beim Brühen, Entbluten und Betäuben werden als hauptsächliche Risikopunkte im Schlachtprozess genannt (Abbildung 5.9-3).

In den Gesprächen bei der zweiten Befragungsrunde wurde deutlich, dass Personal-, Prozess- und Bedarfsgegenständehygiene häufige Schwachpunkte in den Betrieben darstellen. Mängel bei der Entnahme des Magen-Darm-Paketes sind abhängig vom Technisierungsgrad des Schlachtbandes. Vollautomatische Anlagen wurden im Allgemeinen besser bewertet. Insgesamt schätzen die Experten ein, dass der Schlachtprozess im Jahr 2015 sicherer sein wird als heute. Im Einzelnen wird die Gefahr der Kreuzkontamination beim Brühen abnehmen (sowohl bei Schweinen als auch beim Geflügel). Ein Anstieg der Kontamination durch den Fellabzug, durch die Entnahme der inneren Organe und durch das Entfernen der Wirbelsäule wird nicht gesehen. Die Experten gehen zum Teil eher von einer Abnahme aus (Tabelle 5.9-4). 


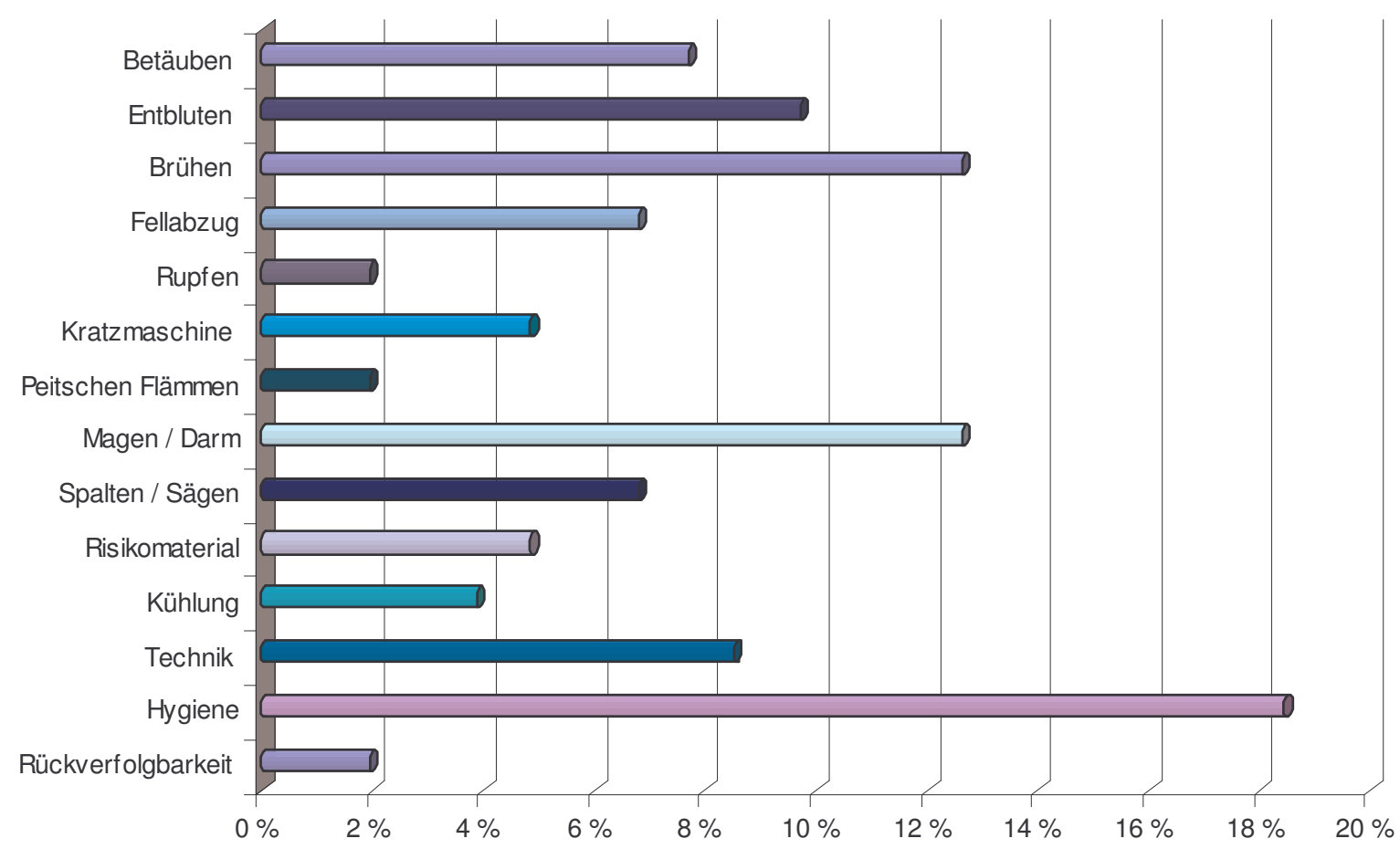

Abbildung 5.9-3: $\quad$ Kritische Punkte im Schlachtband, in \% der Nennungen.

\subsubsection{Bandgeschwindigkeit}

Nach der überwiegenden Meinung der Experten (62\%) trifft die These, dass aufgrund der Bandgeschwindigkeiten beim Geflügel und beim Schwein die einzelne Schlachttierkörperuntersuchung nicht gewährleistet ist, nicht zu. Eine Auswirkung auf die Produktqualität und eine Gefährdung des Verbrauchers durch die Bandgeschwindigkeit wird nicht gesehen. Standards für Kontrollen sind vorhanden und von einem Meideverhalten wird nicht ausgegangen (Abbildung 5.9-4). Hauptsächlich wird in einer hohen Bandgeschwindigkeit eine mikrobiologische Gefahr gesehen (Tabelle 11.1-36). Trotz der mikrobiologischen Gefahr haben die Experten in der mündlichen Befragung verneint, dass die Bandgeschwindigkeit einen gravierenden Einfluss auf die Produkthygiene hat, solange die Bandgeschwindigkeit mit den technischen und personellen Möglichkeiten des Schlachtbandes übereinstimmt.

Tabelle 5.9-4:

Beurteilung einzelner Prozesse in der Schlachtung zum Jahr 2015.

\begin{tabular}{lcccccc}
\hline Experten & & stark abnehmend & abnehmend & wie heute & zunehmend & stark zunehmend \\
\hline Defizite beim Schlacht- & $\mathrm{N}=17$ & 4 & 10 & 1 & 2 & 0 \\
prozess & $\%$ & 24 & 59 & 6 & 12 & 0 \\
Kreuzkontamination beim & $\mathrm{N}=16$ & 0 & 12 & 3 & 1 & 0 \\
Brühen & $\%$ & 0 & 75 & 19 & 6 & 0 \\
Kontamination beim Fell- & $\mathrm{N}=14$ & 0 & 7 & 6 & 1 & 0 \\
abzug & $\%$ & 0 & 50 & 43 & 7 & 0 \\
Kontamination bei & $\mathrm{N}=14$ & 2 & 4 & 7 & 1 & 0 \\
Entfernung der Wirbelsäule & $\%$ & 14 & 29 & 50 & 7 & 0 \\
Kontamination b. Entnahme & $\mathrm{N}=16$ & 1 & 5 & 7 & 3 & 0 \\
der inneren Organe & $\%$ & 6 & 31 & 44 & 19 & 0 \\
\hline
\end{tabular}




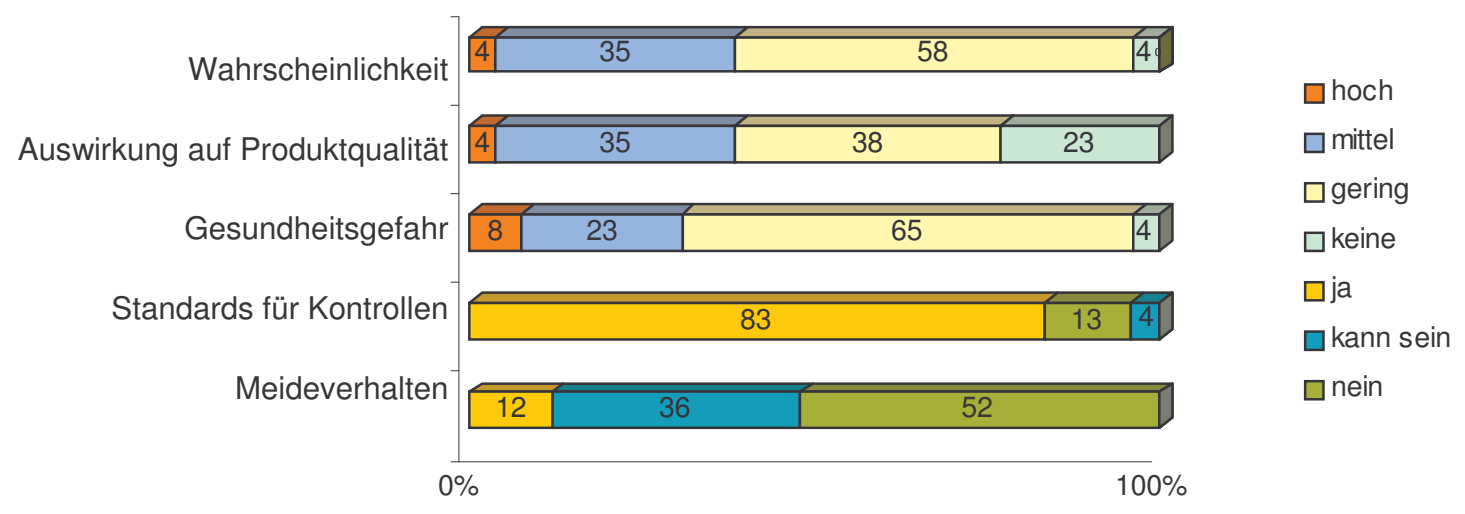

Abbildung 5.9-4: Beurteilung der These: Aufgrund der Bandgeschwindigkeiten ist beim Geflügel und beim Schwein die einzelne Schlachttierkörperuntersuchung nicht gewährleistet.

\subsubsection{BSE-Risikomaterial}

Die Wahrscheinlichkeit, dass die These, rückstandslos lässt sich das BSE-Risikomaterial nicht entfernen, zutrifft, wird von $72 \%$ der Experten mit gering beurteilt. Eine Gefährdung des Verbrauchers durch die Entsorgung wird nicht gesehen. Standards für Kontrollen sind vorhanden. Ein Meideverhalten durch Rückstände von Risikomaterial wird von 54\% der Experten als hoch eingeschätzt (Abbildung 5.9-5). Als Risiken werden hauptsächlich mikrobiologische und ethische Gefahren gesehen. Die Gefahr besteht bei Rindern (Tabelle 11.1-42 und Tabelle 11.1-43).

In absteigender Reihenfolge, werden von den Experten Rückenmark, Kopf, Zunge, Milz, Mandeln, Därme, Lunge, Lymphe + Knoten, Peyersche Platten, alles Nervengewebe, Thymus, Seperatorenfleisch für kritisch gehalten (Tabelle 11.1-44). Als kritische Verfahren werden die Schussbetäubung und die Schlachttierspaltung genannt.

Auf das Jahr 2015 gesehen gehen die Experten aber von einer abnehmenden Bedeutung von BSE-Risikomaterialien in der Sensibilität der Verbraucher aus (Tabelle 5.9-5).

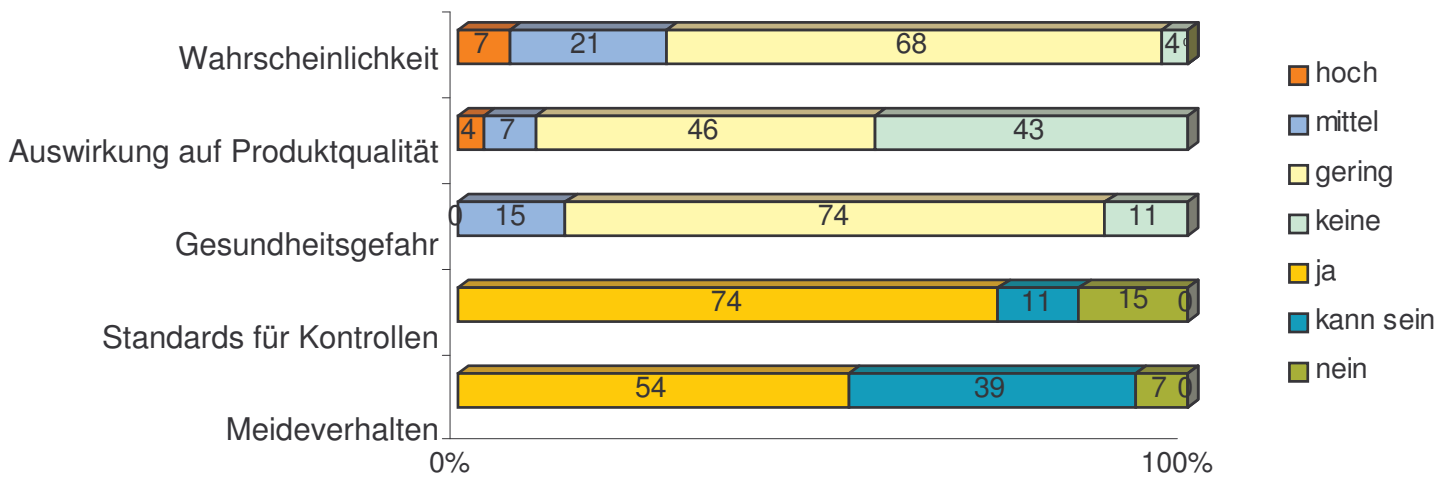

Abbildung 5.9-5: Beurteilung der These: Rückstandslos lässt sich das BSE-Risikomaterial nicht entfernen. 
Tabelle 5.9-5: Einschätzung der Sensibilität des Verbrauchers bezüglich BSERisikomaterial zum Jahr 2015.

\begin{tabular}{lllllll}
\hline & & stark abnehmend & abnehmend & wie heute & zunehmend & stark zunehmend \\
\hline \multirow{2}{*}{ Experten } & $\mathrm{N}=18$ & 6 & 7 & 2 & 3 & 0 \\
& $\%$ & 33 & 39 & 11 & 17 & 0 \\
\hline
\end{tabular}

\subsubsection{Nebenprodukte und Innereien}

Bei den Innereien und den Nebenprodukten werden als hauptsächliche Risikopunkte hygienische Defizite bei der Gewinnung und der Bearbeitung genannt. Neben der Hygiene werden die Temperaturführung und die Qualifikation des Personals als weitere Probleme genannt. Die für die Nebenprodukte Blut, Darm und Kopf genannten kritischen Punkte sind in der nachfolgenden Aufzählung aufgelistet.

Alle Bereiche: Hygiene, Temperatur, ausreichende Vorsorgemaßnahmen, Personalqualifikation,

Blut: Anlagenhygiene, Kühlung, unhygienisches Stechen, Chargengröße, Citratzusatz zu gering, Trennung vom Serum und Blutkuchen, Keimbelastung,

Därme: Reinigung, Konservierung, Wurmbefall, Harnblasenbeschädigungen, Kühlung, Keimbelastung,

Kopf: ethische Gründe, Risikomaterial Einzelkennzeichnung, Gehirnverteilung durch Betäubung, keine kritischen Punkte, Fleischuntersuchung, Finnen, Lymphknoten, Borsten/Schwein, Augen, Ohrmarken, mangelnde Untersuchung.

Insgesamt wird von den Experten davon ausgegangen, dass bestehende Defizite bei der Gewinnung von Nebenprodukten und Innereien bis 2015 eher abnehmen werden (Tabelle 5.9-6).

Sowohl die Experten als auch die Studenten gehen davon aus, dass die Verzehrmengen von Innereien zum Jahr 2015 abnehmen bzw. stark abnehmen werden (Tabelle 5.9-7).

Tabelle 5.9-6: $\quad$ Einschätzung der Entwicklung von Defiziten bei der Gewinnung von Nebenprodukten und Innereien zum Jahr 2015.

\begin{tabular}{ccccccc}
\hline Experten & & stark abnehmend & abnehmend & wie heute & zunehmend & stark zunehmend \\
\hline Neben- & $\mathrm{N}=16$ & 0 & 9 & 6 & 1 & 0 \\
produkte & $\%$ & 0 & 56 & 38 & 6 & 0 \\
Innereien & $\mathrm{N}=15$ & 1 & 8 & 4 & 2 & 0 \\
& $\%$ & 7 & 53 & 27 & 13 & 0 \\
\hline
\end{tabular}

Tabelle 5.9-7:

Entwicklung der Verzehrmengen von Innereien zum Jahr 2015.

\begin{tabular}{ccccccc}
\hline & & stark abnehmend & abnehmend & wie heute & zunehmend & stark zunehmend \\
\hline Experten & $\mathrm{N}=18$ & 5 & 11 & 2 & 0 & 0 \\
& $\%$ & 28 & 61 & 11 & 0 & 0 \\
\multirow{5}{*}{ Studenten } & $\mathrm{N}=64$ & 13 & 41 & 7 & 3 & 0 \\
& $\%$ & 20 & 64 & 11 & 5 & 0 \\
\hline
\end{tabular}




\subsubsection{Fleischkühlung}

Bei der Fleischkühlung wurden als kritische Punkte vor allem Defizite in der Temperatur/Zeitführung, in der Kerntemperatur, der Kühlkette und in der Lagerdichte genannt (Abbildung 11.2-14). Insgesamt gehen die Experten davon aus, dass die Defizite bis 2015 abnehmen (Tabelle 5.9-8).

Tabelle 5.9-8: $\quad$ Einschätzung über das Auftreten von Defiziten bei der Fleischkühlung zum Jahr 2015.

\begin{tabular}{ccccccc}
\hline & & stark abnehmend & abnehmend & wie heute & zunehmend & stark zunehmend \\
\hline Experten & $\mathrm{N}=17$ & 4 & 11 & 2 & 0 & 0 \\
& $\%$ & 24 & 65 & 12 & 0 & 0 \\
\hline
\end{tabular}

\subsubsection{Fleischverladung/Transport/Fleischzerlegung}

Bei der Fleischverladung, dem Transport und bei der Fleischzerlegung werden vor allem Defizite in der Hygiene, Kühlung/Kühlkette und beim Personal als Risikopunkte genannt. Für die Studenten sind aus der Sicht des Verbrauchers beim Transport von Fleisch vor allem die Reinigung, die Überwachung und die Kühlung kritisch zu bewerten. Bei der Zerlegung die Rückverfolgbarkeit, Personal- und Arbeitsplatzhygiene sowie die Reinigung (Abbildung 11.2-15 und Abbildung 11.2-16).

Bei beiden Prozessen gehen die Experten aber davon aus, dass potentielle Defizite bis zum Jahr 2015 abnehmen werden (Tabelle 5.9-9).

Tabelle 5.9-9:

Einschätzung von Defiziten beim Fleischtransport und in der Zerlegung zum Jahr 2015.

\begin{tabular}{ccccccc}
\hline & & stark abnehmend & abnehmend & wie heute & zunehmend & stark zunehmend \\
\hline Fleisch- & $\mathrm{N}=17$ & 3 & 8 & 5 & 1 & 0 \\
transport & $\%$ & 18 & 47 & 29 & 6 & 0 \\
Zerlegung & $\mathrm{N}=17$ & 2 & 10 & 4 & 1 & 0 \\
& $\%$ & 12 & 59 & 24 & 6 & 0 \\
\hline
\end{tabular}

\subsubsection{Fleischverpackung}

Verpackungsfehler, mangelhaftes oder falsches Verpackungsmaterial, Hygienemängel und Mängel in der Kühlung sind die am häufigsten von den Experten genannten kritischen Punkte. Vom Verbraucher werden nach der Meinung der Experten von den vorab genannten Punkten die Kühlung, die Kennzeichnung, die Hygiene, Verpackungsfehler und die Lagerung kritisch gesehen (Abbildung 11.2-17).

Sechs von neun Experten sind der Meinung, dass eine Migration unerwünschter Kontaminanten aus der Verpackung für die Qualität des Produktes eine Rolle spielt. Eingesetzte Verpackungsmaterialien müssen alle zugelassen sein. Nach der Meinung eines Experten gibt es noch zu wenige Erkenntnisse über die bei der Etikettierung eingesetzten Kleber und Farben sowie die Art des Druckes.

Insgesamt sind die Experten aber der Meinung, dass die Defizite in der Fleischverpackung abnehmen werden (Tabelle 5.9-10). 
Tabelle 5.9-10: $\quad$ Einschätzung von Defiziten bei der Fleischverpackung zum Jahr 2015.

\begin{tabular}{ccccccc}
\hline & & stark abnehmend & abnehmend & wie heute & zunehmend & stark zunehmend \\
\hline \multirow{2}{*}{ Experten } & $\mathrm{N}=17$ & 2 & 11 & 3 & 1 & 0 \\
& $\%$ & 12 & 65 & 18 & 6 & 0 \\
\hline
\end{tabular}

\subsection{0}

\section{Hygiene}

\subsubsection{Hygiene in der Futtermittelproduktion}

Über $50 \%$ der befragten Experten gehen von Hygienemängeln in der Futtermittelproduktion aus. Die Wahrscheinlichkeit, dass diese Mängel einen Einfluss auf die Produktqualität haben, halten 59\% der Experten für gering oder nicht vorhanden. Von keiner oder nur einer geringen Gefährdung der Verbraucher gehen ca. 65\% der Experten aus. Hygienestandards in der Futtermittelproduktion sind vorhanden. Dass Hygienemängel in der Futtermittelproduktion zu einem Meideverhalten bei Fleisch führen kann, wird nicht ausgeschlossen (Abbildung 5.10-1). Alle Experten sehen in erster Linie eine mikrobiologische Gefahr. Aber es wird auch eine chemische Gefahr von 53\% der Experten gesehen (Tabelle 11.1-45). In erster Linie haben Hygienemängel Auswirkungen auf Schweine und Geflügel (Tabelle 11.1-46).

Als kritische Punkte werden die Futterlagerung und Lagerungsdauer, die Sauberkeit und die Reinigung der Anlagen, die mikrobielle Kontamination und die Schädlingsbekämpfung genannt. Diese Punkte werden auch als kritisch in der Kommunikation mit dem Verbraucher gesehen. Die Reihenfolge ist hier nur etwas verschoben. Zusätzlich sehen die Experten in der Kommunikation mit dem Verbraucher den Punkt Importe kritisch. Die Studenten halten aus der Sicht des Verbrauchers vor allem die mikrobielle Kontamination, die Sauberkeit und Reinigung, die Schädlingsbekämpfung und die Importe für kritisch. Futterlagerung und Lagerungsdauer werden dagegen weniger genannt (Abbildung 5.10-2).

Insgesamt wird sich nach der Meinung der Experten die Hygiene in der Futtermittelproduktion bis zum Jahr 2015 verbessern (Tabelle 5.10-1).

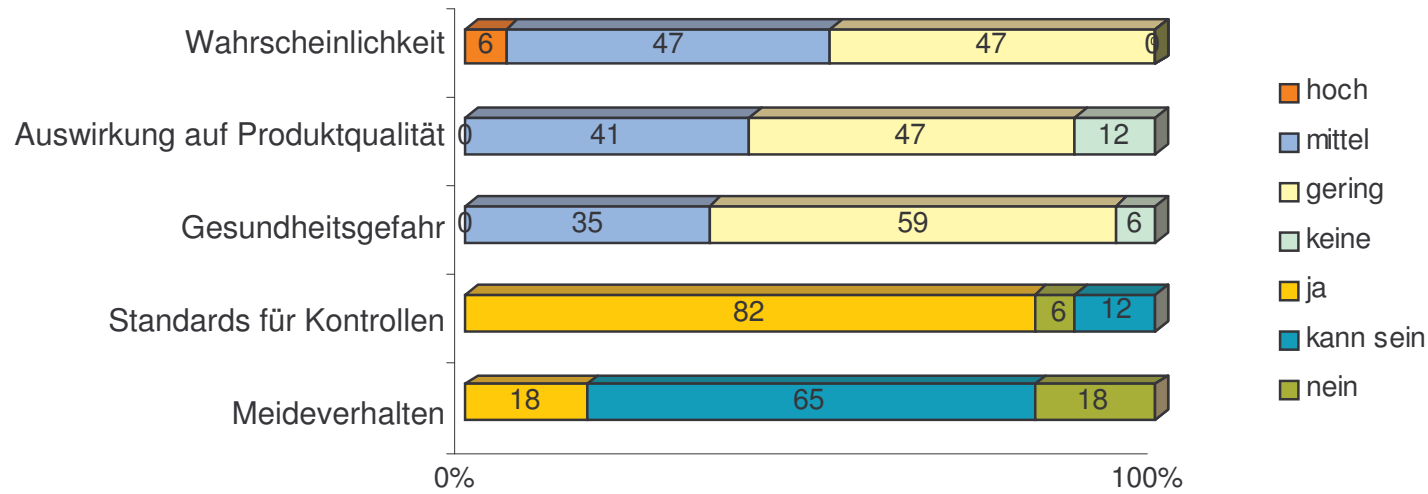

Abbildung 5.10-1: $\quad$ Beurteilung der These: Die Hygiene in der Futtermittelproduktion ist vielfach mangelhaft. 
Tabelle 5.10-1: Einschätzung über das Auftreten mangelhafter Hygiene im Produktionsprozess Futtermittel zum Jahr 2015.

\begin{tabular}{ccccccc}
\hline & & stark abnehmend & abnehmend & wie heute & zunehmend & stark zunehmend \\
\hline \multirow{2}{*}{ Experten } & $\mathrm{N}=19$ & 4 & 14 & 1 & 0 & 0 \\
& $\%$ & 21 & 75 & 5 & 0 & 0 \\
\hline
\end{tabular}

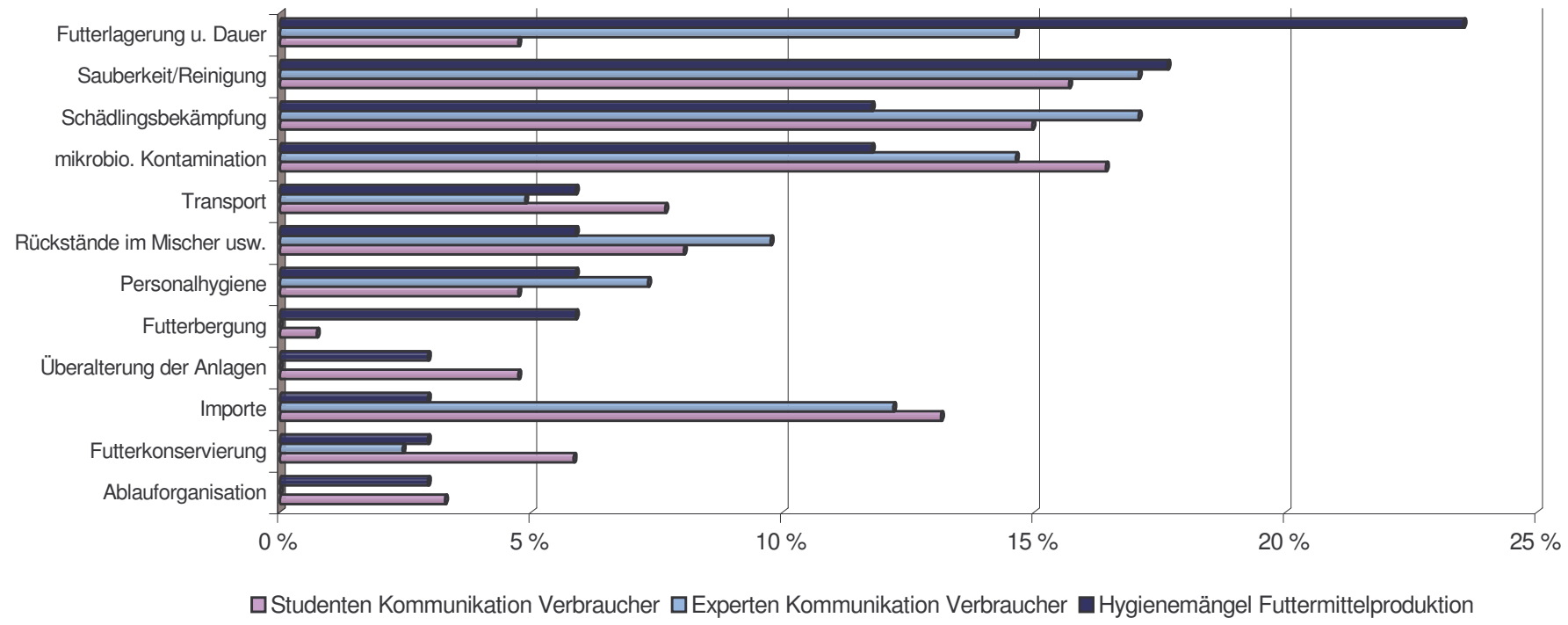

Abbildung 5.10-2: $\quad$ Punkte in der Futtermittelproduktion, bei denen die Gefahr von Hygienemängel besteht, und Einschätzung der Punkte durch die Experten und die Studenten, die durch den Verbraucher kritisch gesehen werden könnten, in \% der Nennungen.

\subsubsection{Hygiene in der Landwirtschaft}

79\% der Experten beurteilen die Wahrscheinlichkeit, dass die These, Hygieneprogramme im landwirtschaftlichen Betrieb sind häufig mangelhaft, zutrifft mit hoch oder mittel. Durch Hygienemängel ist ein Einfluss auf die Produktqualität und die Gesundheit des Verbrauchers möglich. Standards für Kontrollen sind vorhanden. Bei Bekanntwerden von Missständen ist ein Meideverhalten durch den Verbraucher nicht auszuschließen (Abbildung 5.10-3). In erster Linie sehen die Experten in Hygienemängeln eine mikrobiologische Gefahr (95,7\%). Aber ethische (26,1\%), ernährungsphysiologische $(21,7 \%)$, chemische $(17,4 \%)$ und sensorische (13\%) Gefahren werden ebenfalls nicht ausgeschlossen. In erster Linie sind Schweine und Geflügel betroffen (Tabelle 11.1-47 und Tabelle 11.1-48).

Hygienemängel sehen die Experten vor allem bei der Reinigung und Desinfektion, beim Rein-Raus-Prinzip, beim Tierverkehr, bei der Tiergesundheitskontrolle und bei der Futterhygiene. Kritisch in Bezug auf eine Gefährdung des Verbrauchers werden eine mangelhafte Seuchenhygiene, die Reinigung und Desinfektion, die Personalhygiene, die Schadnagerbekämpfung, die Futterhygiene und fehlende Tiergesundheitskontrollen/programme bewertet (Abbildung 5.10-2).

In der mündlichen Befragung wurden die Experten um eine Stellungnahme zu der Frage, „Hohe Standards sind vorhanden, werden diese umgesetzt und kontrolliert?“, gebeten. Drei von neun Experten waren der Meinung, dass die vorhandenen Standards weder ausreichend umgesetzt sind noch kontrolliert werden. Ein Experte war der Meinung, dass in der 
Schweinehaltung die Standards umgesetzt sind und auch kontrolliert werden. Die anderen Experten gehen davon aus, dass die Standards umgesetzt, aber nicht ausreichend kontrolliert werden.

Überwiegend gehen die Experten aber davon aus, dass Hygienemängel bis zum Jahr 2015 abnehmen werden (Tabelle 5.10-2).

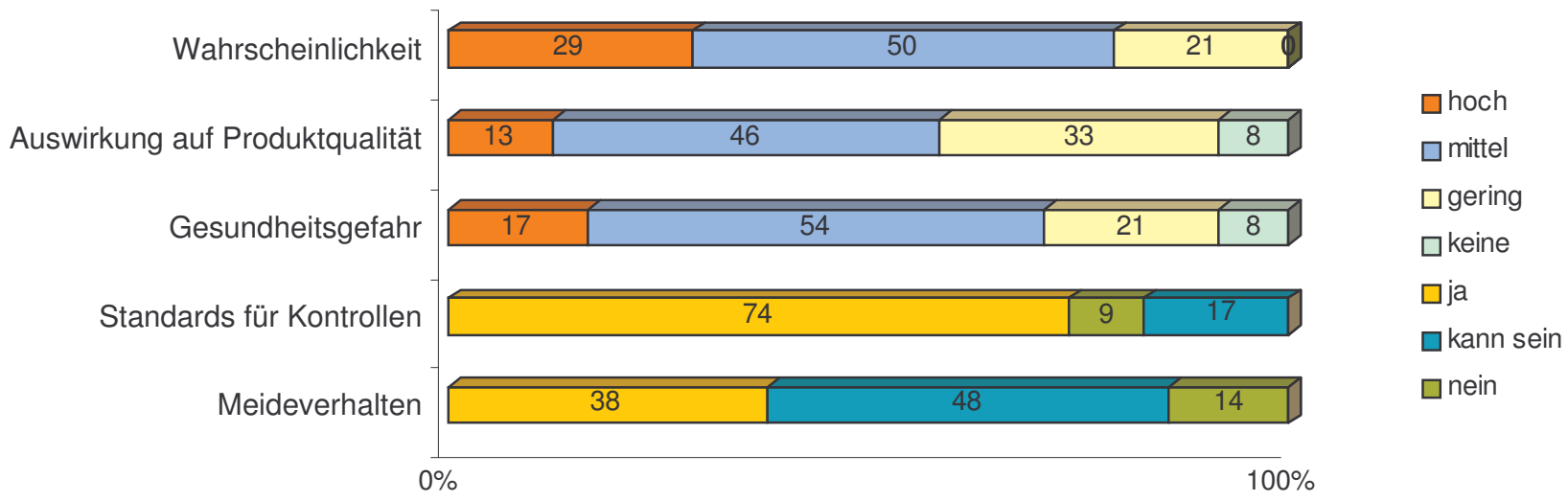

Abbildung 5.10-3: Beurteilung der These: Hygieneprogramme im landwirtschaftlichen Betrieb sind häufig mangelhaft.

Tabelle 5.10-2: $\quad$ Einschätzung über das Auftreten von Hygienemängeln im landwirtschaftlichen Betrieb zum Jahr 2015.

\begin{tabular}{ccccccc}
\hline & & stark abnehmend & abnehmend & wie heute & zunehmend & stark zunehmend \\
\hline \multirow{2}{*}{ Experten } & $\mathrm{N}=20$ & 1 & 13 & 4 & 2 & 0 \\
& $\%$ & 5 & 65 & 20 & 10 & 0 \\
\hline
\end{tabular}

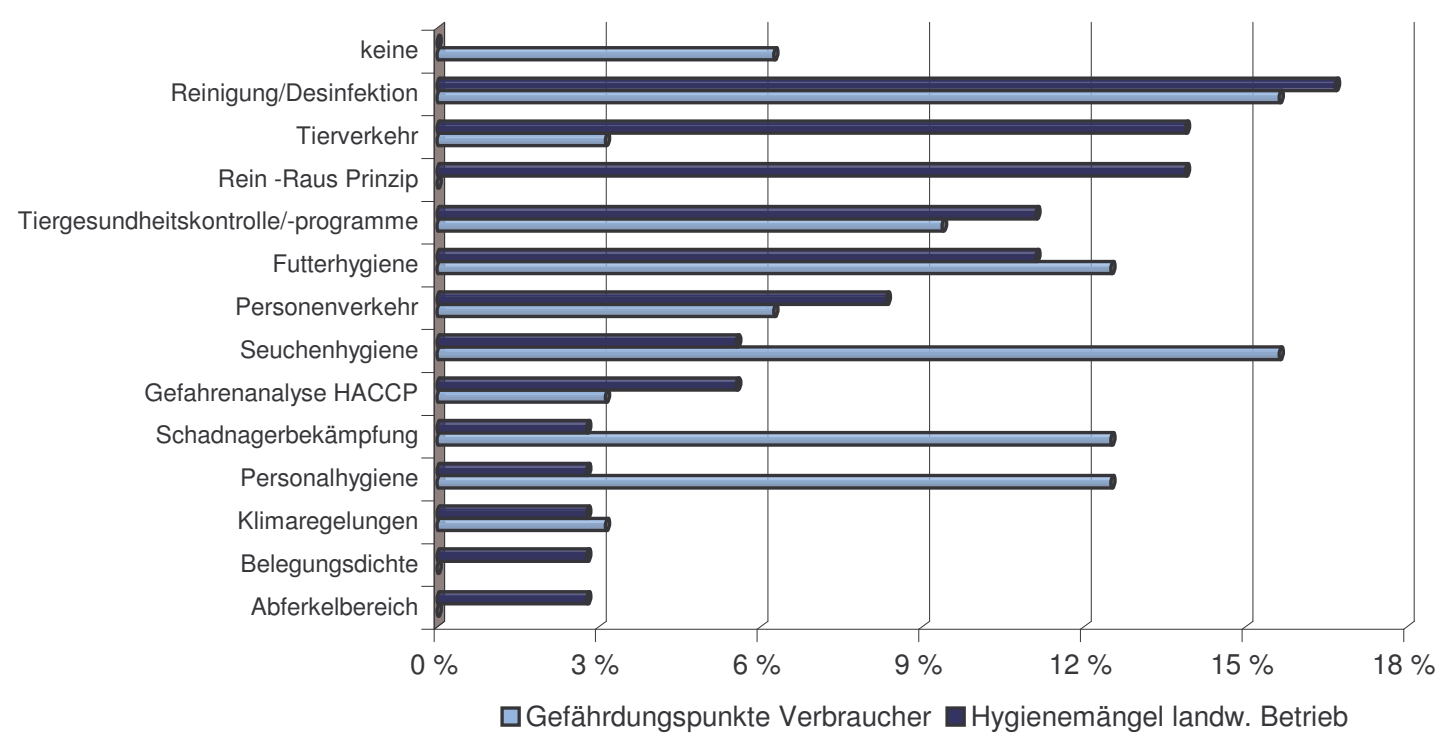

Abbildung 5.10-4: Hygienemängel im landwirtschaftlichen Betrieb und Einschätzung der Punkte durch die Experten, wo es zu einer Gefährdung der Verbraucher kommen kann, in \% der Nennungen. 


\subsubsection{Kritische Punkte in der Fütterungshygiene}

Die Bedeutung der Fütterungshygiene wird nach der Meinung der Experten bis zum Jahr 2015 zunehmen, wenn nicht sogar stark zunehmen (Tabelle 5.10-3). Als kritische Punkte, die sich auf die Futterhygiene auswirken, werden von den Experten die Reinigung der Installationen der Futtertechnik, belastete Futtermittel, die Futterlagerung, die Schadnagerbekämpfung und die Wasserqualität genannt. Zusätzlich zu den vorab aufgeführten Punkten wird von den Experten die Stallhygiene als ein kritischer Punkt genannt, bei dem es zu einer Gefährdung des Verbrauchers kommen kann. Die Studenten sehen vor allem eine Gefahr für den Verbraucher durch belastete Futtermittel und durch eine mangelhafte Stallhygiene. Abweichend von den Punkten, die die Experten genannt haben, werden von den Studenten Managementfehler als kritisch bewertet (Abbildung 11.2-19).

Tabelle 5.10-3: Einschätzung über die Futterhygiene im landwirtschaftlichen Betrieb zum Jahr 2015.

\begin{tabular}{ccccccc}
\hline & & stark abnehmend & abnehmend & wie heute & zunehmend & stark zunehmend \\
\hline \multirow{2}{*}{ Experten } & $\mathrm{N}=21$ & 0 & 2 & 1 & 9 & 9 \\
& $\%$ & 0 & 10 & 5 & 43 & 43 \\
\hline
\end{tabular}

\subsubsection{Reinigung in der Schlachtung}

Die hauptsächlich genannten Risikopunkte sind: unsachgemäße Reinigung, Personalfehler und mangelhafte Reinigungskontrolle (Abbildung 11.2-20). Die Reinigung wird nach Ansicht der Experten bis zum Jahr 2015 besser werden (Tabelle 5.10-4).

Tabelle 5.10-4: $\quad$ Einschätzung über das Auftreten mangelhafter Reinigungshygiene im Schlacht- bzw. Zerlegebetrieb zum Jahr 2015.

\begin{tabular}{ccccccc}
\hline & & stark abnehmend & abnehmend & wie heute & zunehmend & stark zunehmend \\
\hline \multirow{2}{*}{ Experten } & $\mathrm{N}=17$ & 2 & 11 & 3 & 0 & 1 \\
& $\%$ & 12 & 65 & 18 & 0 & 6 \\
\hline
\end{tabular}

\subsubsection{Personalhygiene}

Die Personalhygiene wird als einer der kritischsten Punkte in einem Lebensmittel herstellenden Betrieb gesehen. 89\% der Experten bewerten die Wahrscheinlichkeit, dass die These zutrifft „die Personalhygiene ist einer der wesentlichsten kritischsten Punkte in einem Lebensmittel herstellenden Betrieb" als hoch oder mittel. Der Einfluss auf die Produktqualität und auf die Sicherheit des Produktes halten 25\% der Experten für hoch. Die Standards für diesen Bereich sind sehr hoch (93\%) und umfassend. Dass es aufgrund von mangelhafter Personalhygiene zu einem Meideverhalten kommt, wird nicht ausgeschlossen (Abbildung 5.10-5). Mangelnde Personalhygiene wird von allen Experten als eine mikrobiologische Gefahr eingeschätzt. Zusätzlich werden aber auch noch eine chemische (25\%), eine ethische (25\%) und eine sensorische (25\%) Gefahr gesehen (Tabelle 11.1-49).

Als wichtigste Punkte, die einen Einfluss auf die Personalhygiene haben, werden das Verhalten der Mitarbeiter, die mangelhafte oder mangelhaft getragene Arbeitskleidung, fehlende oder mangelhafte Personalschulungen und erkrankte Mitarbeiter im Produktionsprozess genannt (Abbildung 11.2-21). 
Insgesamt wird die Personalhygiene in der Fleisch verarbeitenden Industrie durch die Studenten als gut $(67,6 \%)$ beurteilt (Tabelle 5.10-6). Sowohl nach der Einschätzung der Experten als auch der Studenten werden die Probleme durch mangelhafte Personalhygiene bis zum Jahr 2015 abnehmen (Tabelle 5.10-5).

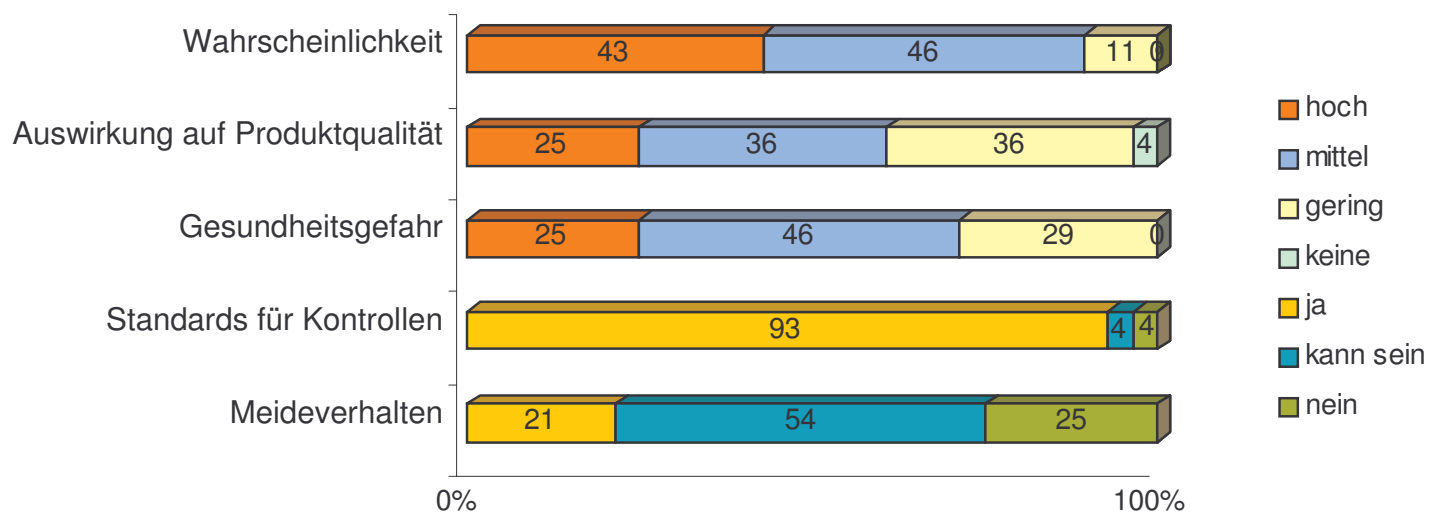

Abbildung 5.10-5: Beurteilung der These: Die Personalhygiene ist einer der wesentlichsten kritischsten Punkte in einem Lebensmittel herstellenden Betrieb.

Tabelle 5.10-5: Einschätzung über das Auftreten mangelhafter Personalhygiene zum Jahr 2015.

\begin{tabular}{ccccccc}
\hline & & stark abnehmend & abnehmend & wie heute & zunehmend & stark zunehmend \\
\hline \multirow{4}{*}{ Experten } & $\mathrm{N}=17$ & 2 & 9 & 4 & 2 & 0 \\
& $\%$ & 12 & 53 & 24 & 12 & 0 \\
Studenten & $\mathrm{N}=64$ & 3 & 41 & 19 & 1 & 0 \\
& $\%$ & 5 & 64 & 30 & 2 & 0 \\
\hline
\end{tabular}

Tabelle 5.10-6: Beurteilung der Personalhygiene in der Fleisch verarbeitenden Industrie.

\begin{tabular}{ccccccc}
\hline & & sehr schlecht & schlecht & durchschnittlich & gut & sehr gut \\
\hline Studenten & $\mathrm{N}=64$ & 0 & 7 & 14 & 37 & 6 \\
& $\%$ & 0 & 11 & 22 & 58 & 10 \\
\hline
\end{tabular}

\subsection{Rückverfolgbarkeit}

\subsubsection{Rückverfolgbarkeit von Futtermitteln}

Die Rückverfolgbarkeit von Futtermitteln wird nach der Meinung der Experten bis zum Jahr 2015 zunehmen bis stark zunehmen (Tabelle 5.11-1). Als wichtigste Risiken in der Rückverfolgbarkeit werden der weltweite Handel, die unterschiedlichen Mischungen und Rezepturen, die unklare Definition von Chargen, die Verarbeitung/Verschleppung von Resten oder Restbeständen und der Mangel an durchgängigen Systemen gesehen. Ein weiterer kritischer Punkt ist die „kriminelle Energie“, die an einer Verdunkelung der Rückverfolgbarkeit mitwirken kann (Abbildung 11.2-22). 
Tabelle 5.11-1:

Entwicklung des Detaillierungsgrades bei der Rückverfolgbarkeit.

\begin{tabular}{|c|c|c|c|c|c|c|c|}
\hline & & & stark abnehmend & abnehmend & wie heute & zunehmend & stark zunehmend \\
\hline \multirow[t]{2}{*}{ Experten } & Futtermittel & $\mathrm{N}=19$ & 0 & 0 & 0 & 11 & 8 \\
\hline & & $\%$ & 0 & 0 & 0 & 58 & 42 \\
\hline \multirow[t]{2}{*}{ Experten } & Schlachtung & $\mathrm{N}=18$ & 0 & 0 & 0 & 11 & 7 \\
\hline & & $\%$ & 0 & 0 & 0 & 61 & 39 \\
\hline \multirow[t]{2}{*}{ Studenten } & Fleisch & $\mathrm{N}=64$ & 0 & 1 & 4 & 39 & 20 \\
\hline & & $\%$ & 0 & 2 & 6 & 61 & 31 \\
\hline
\end{tabular}

\subsubsection{Dokumentation/Rückverfolgbarkeit Landwirtschaft}

Nach Meinung der meisten Experten ist in vielen landwirtschaftlichen Betrieben die Dokumentation mangelhaft. 41\% der Experten schätzen die Wahrscheinlichkeit, dass in den meisten landwirtschaftlichen Betrieben die Dokumentation mangelhaft ist und hierdurch eine schlüssige Rückverfolgbarkeit meist ausgeschlossen ist, als hoch und $32 \%$ als mittel ein. Standards für Kontrollen werden von 92\% der Experten gesehen. Dass es durch Mängel in der Rückverfolgbarkeit zu einem Meideverhalten beim Fleisch kommen kann, schätzen 45\% der Experten mit hoch ein (Abbildung 5.11-1). Auf die ergänzende Frage nach der Gefahrenkategorie wird nicht eingegangen, da die Antworten bezogen auf Abbildung 5.11-1 widersprüchlich sind. In erster Linie sind Schweine und Geflügel betroffen (Tabelle 11.1-51 und Tabelle 11.1-52).

Als besondere Schwachstellen werden fehlende oder unvollständige Bestandsregister, eine unvollständige oder fehlende Kennzeichnung der Tiere, unkoordinierter Zukauf der Jungtiere (nicht bei Geflügel) und persönliche Überforderung des Landwirtes genannt. Kritisch in der Kommunikation mit dem Verbraucher werden dagegen die mangelnde Fälschungssicherheit der Kennzeichnung, die unvollständige oder fehlende Kennzeichnung der Tiere, die fehlende Zuordnung des Futters zum Mastdurchgang und der unkoordinierte Zukauf der Jungtiere genannt (Abbildung 5.11-2).

Die meisten Experten gehen davon aus, dass Maßnahmen bezüglich der Kennzeichnung bis 2015 zunehmen, wenn nicht sogar stark zunehmen werden (Tabelle 5.11-2).

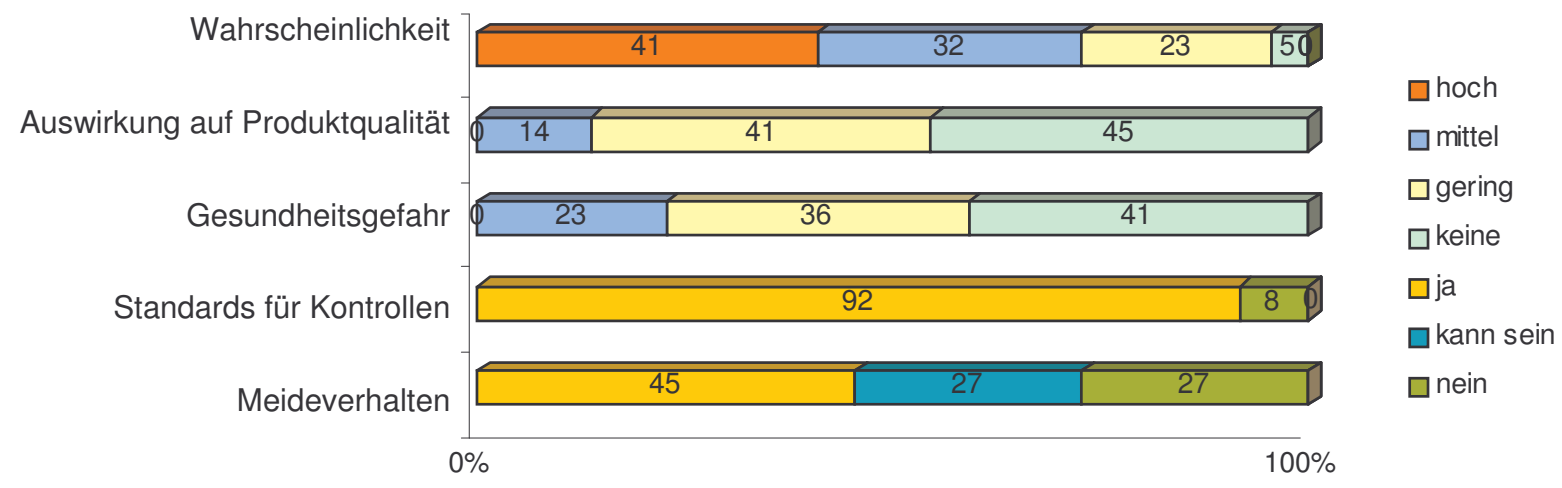

Abbildung 5.11-1: Beurteilung der These: In den meisten landwirtschaftlichen Betrieben ist die Dokumentation mangelhaft. Hierdurch ist eine schlüssige Rückverfolgbarkeit meist ausgeschlossen. 
Tabelle 5.11-2: $\quad$ Einschätzung der Maßnahmen bezüglich der Kennzeichnung im landwirtschaftlichen Betrieb zum Jahr 2015.

\begin{tabular}{ccccccc}
\hline & & stark abnehmend & abnehmend & wie heute & zunehmend & stark zunehmend \\
\hline \multirow{2}{*}{ Experten } & $\mathrm{N}=21$ & 0 & 1 & 0 & 11 & 9 \\
& $\%$ & 0 & 5 & 0 & 52 & 43 \\
\hline
\end{tabular}

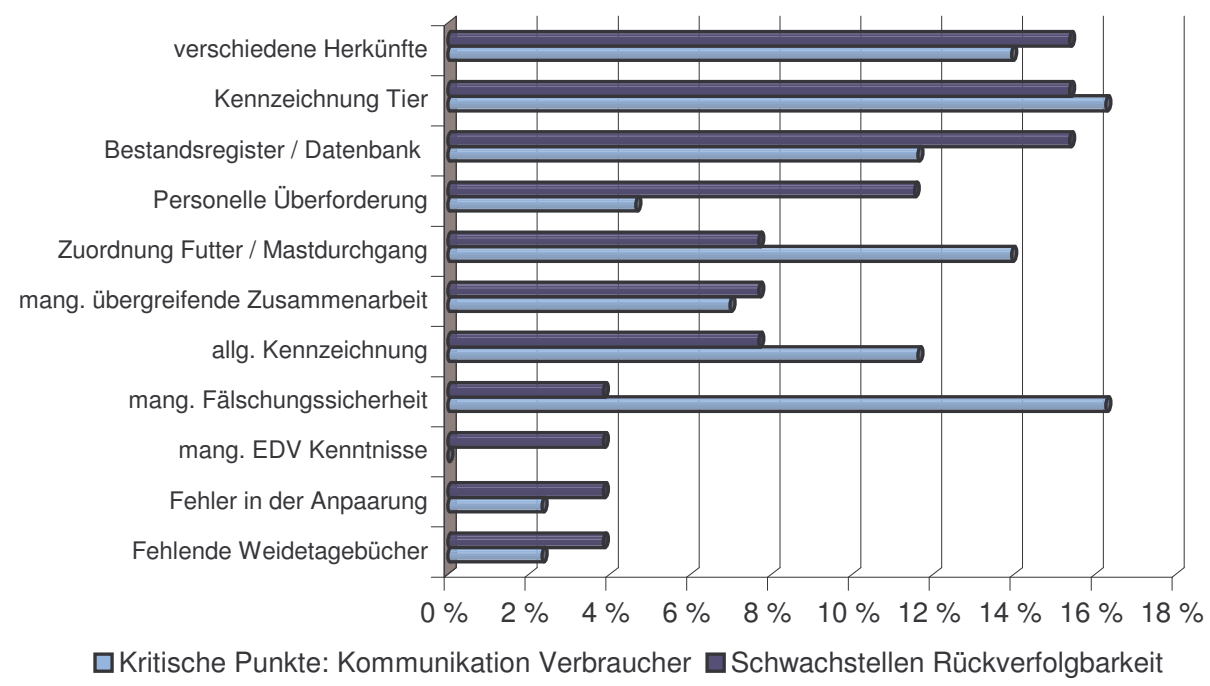

Abbildung 5.11-2: Schwachstellen in der Rückverfolgbarkeit, in der Landwirtschaft und Beurteilung der Punkte, die in der Kommunikation mit dem Verbraucher kritisch zu bewerten sind, in \% der Nennungen.

\subsubsection{Rückverfolgbarkeit in der Schlachtung}

Die Rückverfolgbarkeit wird beim Rind aufgrund der Rindfleischetikettierungsverordnung als relativ gut beurteilt. Beim Schwein und beim Geflügel sind stärkere Defizite vorhanden. Als hauptsächliche Risikopunkte werden die Chargengröße und die Kennzeichnung genannt (Abbildung 11.2-23). Über den Detaillierungsgrad in der Rückverfolgbarkeit werden von den Experten unterschiedliche Aussagen getroffen. Folgende Meinungen sind vertreten:

- eine Rückverfolgung auf die Tagesproduktion ist in der Zerlegung ausreichend,

- eine Rückverfolgbarkeit pro Lieferant ist wichtig,

- die Rückverfolgbarkeit muss bis zum Einzeltier stattfinden.

Bei der Rückverfolgbarkeit sollte vor allem darauf geachtet werden, dass die verwendeten Systeme durchgängig, transparent und nachvollziehbar sind. Hierbei muss den so genannten Schnittstellen eine besondere Aufmerksamkeit in der Kontrolle gewidmet werden. Fehler, die bei der Kennzeichnung erfolgen, müssen dokumentiert werden.

Bis zum Jahr 2015 wird der Detaillierungsgrad in der Rückverfolgbarkeit weiter zunehmen, wenn nicht sogar stark zunehmen (Tabelle 5.11-1). 


\section{$5.12 \quad$ Kontrollen}

\subsubsection{Kontrolle von Futtermitteln}

Kritische Punkte in der internen Kontrolle werden vor allem in einem zu geringen Kontrollumfang, dem Mangel an fehlenden Systemen, einer schlechten Rohkomponentenqualität und einer mangelhaften Selbstkontrolle gesehen. Ähnlich sind die Antworten für die externe Kontrolle. Hier werden als hauptsächliche Risikopunkte genannt, dass staatliche Kontrollen nicht umfassend genug sind, Systeme nur ungenügend umgesetzt werden, zum Teil Fachkenntnisse fehlen und die Prüfer nicht genügend unabhängig sind (Abbildung 11.2-24 und Abbildung 11.2-25).

Bei der Ermittlung von positiven Befunden sollte nach Meinung der Experten wie folgt verfahren werden: Wenn mit anerkannten technischen Mitteln die Kontamination auf ein akzeptables Maß zu reduzieren oder vollständig auszuschalten ist, kann der Rohstoff oder das Futtermittel verwendet werden. Ist dieses nicht möglich, muss der Rohstoff oder das Futtermittel vernichtet werden. Ein Problem ist hierbei immer noch die Risikobeurteilung. Zurzeit gibt es für eine Reihe von Kontaminanten noch keine toxikologisch begründeten Grenzwerte.

Auf Dauer sollten nach der Meinung der Experten die Untersuchungen beim Rohstoff verstärkt werden. Aber selbst dann gibt es keine 100\%-ige Sicherheit, da nur begrenzte Mengen und diese nicht auf alle Stoffe untersucht werden können. Wenn ein Betrieb über ein funktionierendes, systematisches HACCP-System verfügt, können nach der Meinung der Experten viele Risiken begrenzt werden (8 von 9 Experten). Insgesamt gehen die Experten davon aus, dass der Kontrollumfang bei Futtermitteln zunehmen, wenn nicht sogar stark zunehmen wird (Tabelle 5.12-1).

Tabelle 5.12-1: $\quad$ Entwicklung des Kontrollumfangs bei Futtermitteln zum Jahr 2015.

\begin{tabular}{ccccccc}
\hline & & stark abnehmend & abnehmend & wie heute & zunehmend & stark zunehmend \\
\hline \multirow{2}{*}{ Experten } & $\mathrm{N}=19$ & 0 & 0 & 0 & 10 & 9 \\
& $\%$ & 0 & 0 & 0 & 53 & 47 \\
\hline
\end{tabular}

\subsubsection{Schlachttierkörperuntersuchung}

Die Schlachttierkörperuntersuchung wird von den Experten allgemein als sicher eingestuft und sollte sich nach der Meinung eines Großteils der Experten nicht verändern. 33\% der Experten schätzen die Wahrscheinlichkeit, dass die Qualität der Schlachttierkörperuntersuchung leidet, wenn diese in Eigenverantwortung durch den Schlachthof durchgeführt wird, zutrifft, mit hoch und 30\% mit mittel ein. Einen Einfluss auf die Produktqualität und eine Gefährdung für die Verbraucher wird von den meisten Experten mit nicht vorhanden oder gering eingeschätzt. Standards für Kontrollen sind vorhanden.

Bezüglich eines Meideverhaltens kann keine eindeutige Aussage getroffen werden. 20\% der Experten gehen von einem Meideverhalten bei negativen Vorfällen aus und 44\% halten ein Meideverhalten für wahrscheinlich (Abbildung 5.12-1). Durch mangelnde Kontrollen kann es hauptsächlich zu einer mikrobiologischen (85\%) Gefahr kommen. Aber auch die anderen Gefahrengruppen schließen die Experten nicht aus (Tabelle 11.1-53).

Ebenso wie die Experten halten auch die Studenten die Schlachttierkörperuntersuchungen für sicher (Tabelle 5.12-2). Allerdings ist das Vertrauen der Studenten in die staatlichen Kontrollen höher als in die privaten Kontrollen (Tabelle 11.1-55). 


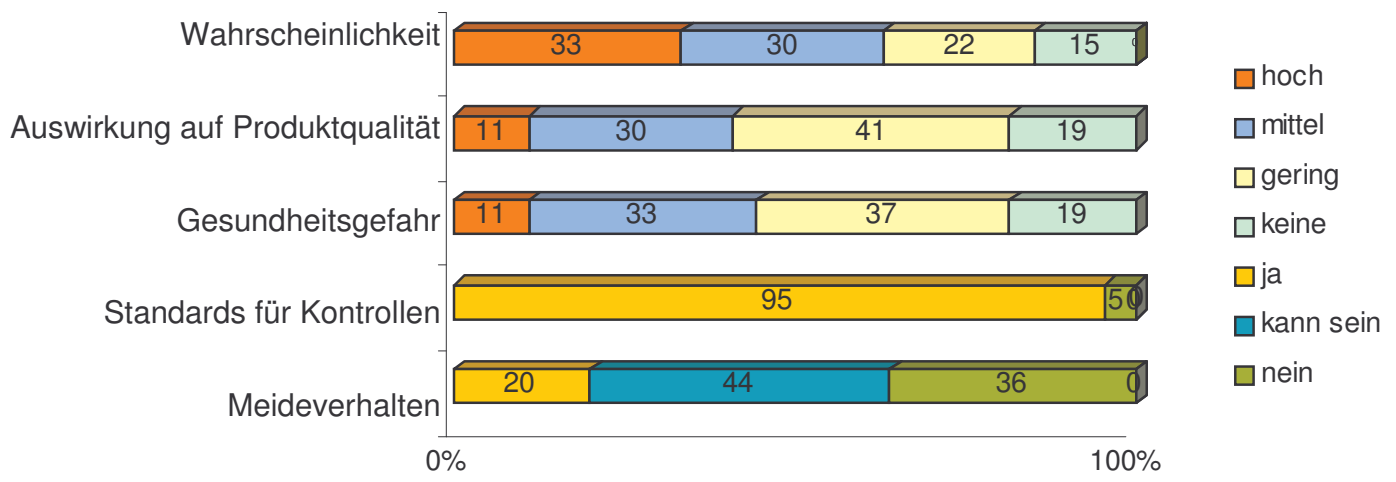

Abbildung 5.12-1: $\quad$ Beurteilung der These: Die Qualität der Schlachttierkörperuntersuchung leidet, wenn diese in Eigenverantwortung durch den Schlachthof durchgeführt wird.

Tabelle 5.12-2: $\quad$ Antworten auf die Frage: Halten Sie die Schlachttieruntersuchung und Fleischuntersuchung in Deutschland für sicher?

\begin{tabular}{lcccc}
\hline Studenten & & Studenten & Studenten $(\mathrm{mL})$ & Studenten $(\mathrm{oL})$ \\
\hline \multirow{2}{*}{ ja } & $\mathrm{N}=62$ & 62 & 40 & 22 \\
& $\%$ & 85 & 83 & 91 \\
\hline
\end{tabular}

Einige Experten sind der Meinung, dass zusätzlich weitere mikrobiologische Untersuchungen und Untersuchungen auf Rückstände durchgeführt werden sollten. Allerdings sind auch $20 \%$ der Experten der Meinung, dass keine weiteren Untersuchungen notwendig sind (Abbildung 5.12-2).

Ein wichtiger Kommentar in Bezug auf die Schlachttierkörperuntersuchung ist, dass sich am Endprodukt keine Qualität „erkontrollieren“ lässt. Aufgrund des Kommentars wurde den Experten in der mündlichen Befragung die Frage gestellt: „Welche Untersuchungen fehlen in den Vorstufen?" Die meisten Experten sind der Meinung, dass bei Rindern und Schweinen eine amtliche Untersuchung im Erzeugerbetrieb (bei Geflügel Pflicht) zwar wünschenswert ist, sich aber aufgrund der Bestandsstruktur nicht realisieren lässt. Der Landwirt sollte aber seine Bestände auf freiwilliger Basis untersuchen lassen. Als wichtige Untersuchungen werden hier solche auf pathogene Keime, Betriebs- und Futterhygiene und Rückstände in Futtermitteln genannt. Diese Punkte sollten durch externe Stellen kontrolliert werden. Ein Experte empfiehlt, hierüber ein regelmäßiges Monitoring anhand von Blut- und Urinproben durchzuführen und dadurch den Status eines Betriebes festzustellen. Hierbei sollte sich auf eine kleine, aber definierte Stichprobe von Untersuchungen geeinigt werden.

Für das Jahr 2015 gehen die meisten Experten davon aus, dass die Anzahl an Untersuchungen, die ergänzend zur Schlachttierkörperuntersuchung durchgeführt werden, zunehmen bis stark zunehmen werden (Tabelle 5.12-3). 


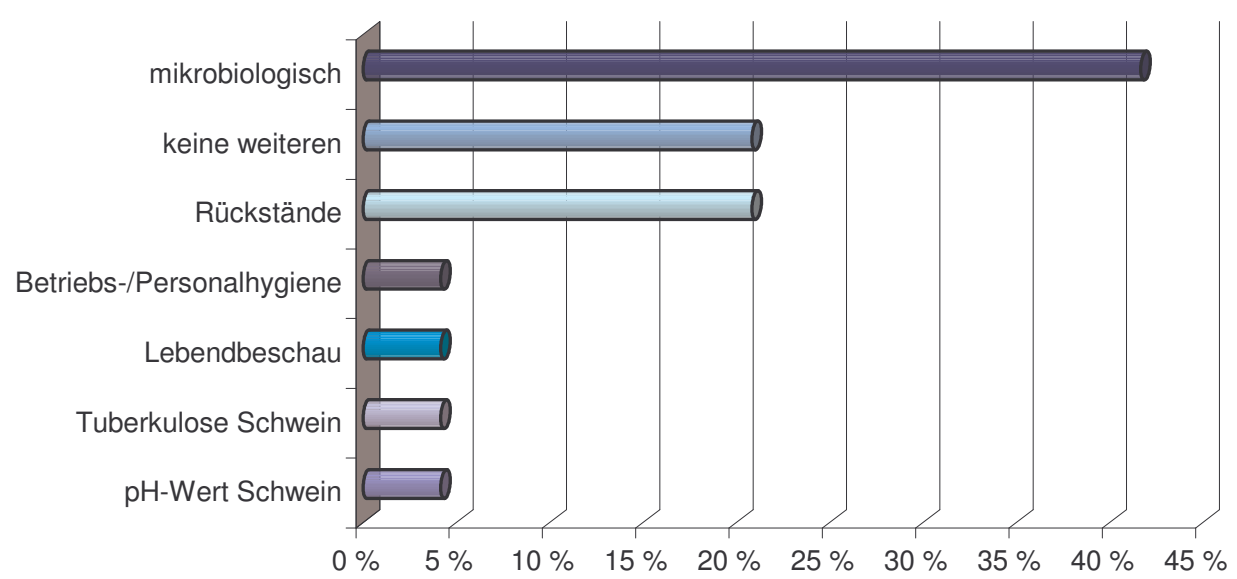

Abbildung 5.12-2: Untersuchungen, die ergänzend zur amtlichen Untersuchung erfolgen sollen, in \% der Nennungen.

Tabelle 5.12-3: $\quad$ Entwicklung des Umfangs von Untersuchungen, die ergänzend zur Schlachtkörperuntersuchung durchgeführt werden, zum Jahr 2015.

\begin{tabular}{ccccccc}
\hline & & stark abnehmend & abnehmend & wie heute & zunehmend & stark zunehmend \\
\hline \multirow{2}{*}{ Experten } & $\mathrm{N}=18$ & 0 & 3 & 0 & 8 & 7 \\
& $\%$ & 0 & 17 & 0 & 44 & 39 \\
\hline
\end{tabular}

\subsubsection{Fleischskandale im Ausland und Kontrollen bei Waren aus Drittländern}

Fleischskandale im Ausland haben einen Einfluss auf den deutschen Fleischmarkt. Die Wahrscheinlichkeit, dass es hierdurch zu einem Meideverhalten auch für deutsches Fleisch kommt, halten 52\% der Experten für hoch und $43 \%$ für mittel. Einen Einfluss auf die Produktqualität und eine Gefahr für den Verbraucher wird von $70 \%$ der Experten mit gering oder nicht vorhanden eingeschätzt. Dass Standards für Kontrollen vorhanden sind, schätzen $55 \%$ der Experten. Von einem Meideverhalten gehen $46 \%$ aus und 54\% halten ein Meideverhalten für wahrscheinlich (Tabelle 5.12-7). Die Risiken werden hier hauptsächlich in den Kategorien chemische, mikrobiologische und ethische Gefahr gesehen (Tabelle 5.12-4). Alle Tierarten sind hiervon betroffen (Tabelle 5.12-5).

Tabelle 5.12-4: $\quad$ Einschätzung der Gefahrenkategorie durch Fleischskandale im Ausland.

\begin{tabular}{ccccccc}
\hline & chemisch & mikrobiologisch & physikalisch & sensorisch & $\begin{array}{c}\text { ernährungs- } \\
\text { physiologisch }\end{array}$ & ethisch \\
\hline $\mathrm{N}=25$ & 13 & 13 & 3 & 7 & 8 & 17 \\
$\%$ & $52 \%$ & $52 \%$ & $12 \%$ & $28 \%$ & $32 \%$ & $68 \%$ \\
\hline
\end{tabular}

Tabelle 5.12-5: $\quad$ Einfluss von Fleischskandalen im Ausland auf die Tierart.

\begin{tabular}{cccc}
\hline & Rind & Schwein & Geflügel \\
\hline $\mathrm{N}=27$ & 24 & 25 & 26 \\
$\%$ & 89 & 92 & 96 \\
\hline
\end{tabular}

Die wichtigsten Kontrollen bei Waren, die aus Drittländern zugekauft werden, sind nach Meinung der Experten: Kontrollen auf Rückstände, auf in der EU nicht zugelassene Stoffe; mikrobiologische Kontrollen sowie Eigenkontrollen und externe Kontrollen im Herkunftsland. 
Kontrollen, die gesetzlich in der EU und in Deutschland für Waren aus dem Inland vorgeschrieben sind, sollten immer durchgeführt werden (Abbildung 5.12-4). 6 von 10 Experten halten im Allgemeinen die Risiken bei Lieferungen aus Drittländern für beherrschbar. Sie weisen aber auch darauf hin, dass Kontrollen aufgrund der Vielzahl an Stoffen und Kontrollmöglichkeiten nie 100\% sicher sein können. Vielfach muss ein hoher Aufwand betrieben werden. Von Vorteil wäre es grundsätzlich, wenn die Importeure sich von der Sicherheit der Bedingungen im Herstellungsland selbst überzeugen würden.

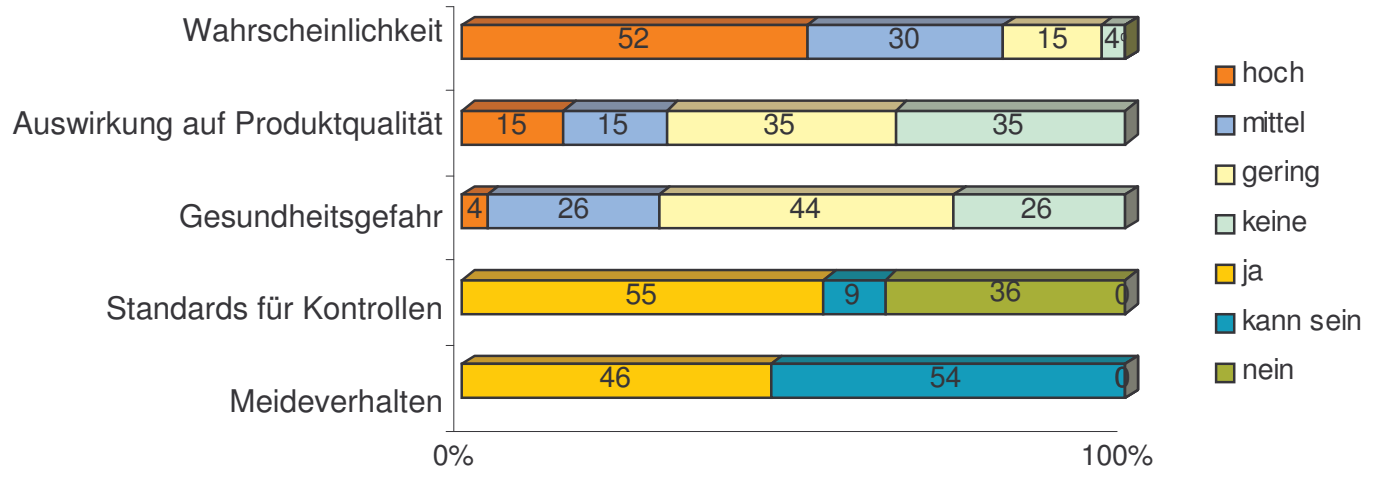

\section{Abbildung 5.12-3: Beurteilung der These: Durch Fleischskandale im Ausland kommt es zu einem Meideverhalten bei Fleisch in Deutschland.}

Länder aus dem osteuropäischen und asiatischen Raum wurden im Allgemeinen als unsicherer eingestuft als Länder aus Amerika. Es wird aber auch hier darauf verwiesen, dass man keine allgemeingültige Aussage für diese Räume treffen kann. Der Sicherheitsstandard kann in den einzelnen Ländern sehr unterschiedlich sein.

Für die Zukunft schätzen die Experten sowie auch die Studenten ein, dass die Probleme bis 2015 zunehmen werden (Tabelle 5.12-6). Fleisch aus Deutschland wird von den meisten Studenten als sicher eingeschätzt, Fleisch aus Drittländern dagegen als eher unsicher (Tabelle 5.12-7).

Tabelle 5.12-6: $\quad$ Entwicklung von Problemen durch Fleischimporte zum Jahr 2015.

\begin{tabular}{ccccccc}
\hline & & stark abnehmend & abnehmend & wie heute & zunehmend & stark zunehmend \\
\hline Experten & $\mathrm{N}=18$ & 0 & 3 & 3 & 11 & 1 \\
& $\%$ & 0 & 17 & 17 & 61 & 6 \\
Studenten & $\mathrm{N}=64$ & 0 & 7 & 22 & 34 & 1 \\
& $\%$ & 0 & 11 & 35 & 53 & 2 \\
\hline
\end{tabular}

Tabelle 5.12-7: $\quad$ Beurteilung der Sicherheit von Fleisch aus Deutschland und von Fleisch, das aus Drittländern zugekauft wurde.

\begin{tabular}{llcccccc}
\hline & & sehr schlecht & schlecht & $\begin{array}{c}\text { durch- } \\
\text { schnittlich }\end{array}$ & gut & sehr gut \\
\hline \multirow{3}{*}{ Studenten } & Fleisch aus & $\mathrm{N}=61$ & 6 & 33 & 19 & 3 & 0 \\
& Drittländern & $\%$ & 10 & 54 & 31 & 5 & 0 \\
Studenten & Fleisch aus & $\mathrm{N}=64$ & 0 & 1 & 8 & 46 & 9 \\
& Deutschland & $\%$ & 0 & 2 & 13 & 72 & 14 \\
\hline
\end{tabular}




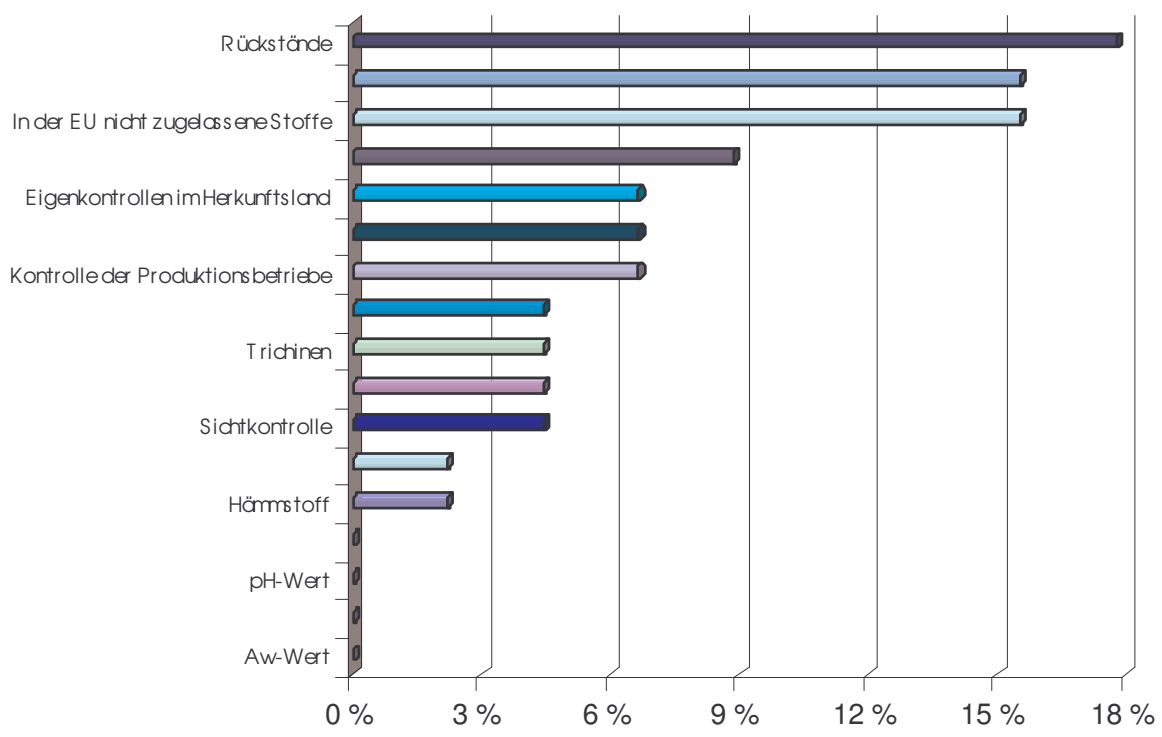

Abbildung 5.12-4: Kontrollen, die bei Waren aus Drittländern durchgeführt werden sollten, bewertet nach Wichtigkeit, in \% der Nennungen. 


\section{Diskussion}

Aufgrund der vielfältigen Fragen können nicht alle Ergebnisse diskutiert werden. Aus diesem Grund werden nur Punkte diskutiert, die mindestens einem der nachfolgenden Kriterien entsprechen:

- die Meinung der Experten und der Studenten weichen voneinander ab,

- die vorliegende wissenschaftliche Literatur ist widersprüchlich,

- es wird Handlungsbedarf gesehen, um vorhandene Risiken zu minimieren.

Ein Teil der Punkte wird anhand der vorab gewählten Struktur diskutiert. In einigen Punkten, z.B. Zoonosen und Rückstände wird aber von dieser Struktur abgewichen. Ursachen für Zoonosen und Rückstände, sind häufig in der Verfahrenstechnik bzw. in den Produktionsmethoden oder aber in den Hygiene- und Kontrollmaßnahmen begründet. Die Diskussion erfolgt an dieser Stelle dann entlang der einzelnen Stationen der Produktionskette Futtermittel, Landwirtschaft und Schlachtung.

Unterschiede bei den einzelnen Tierarten konnten aufgrund der Fragenstruktur nicht eindeutig ermittelt werden. Aus diesem Grund wird auch nur an den Stellen auf einzelne Tierarten eingegangen, an denen eindeutige Unterschiede in der Bewertung vorlagen. Hierbei ist zu berücksichtigen, dass Unterschiede bei einer detaillierteren Betrachtung vorhanden sind.

\subsection{Methodendiskussion}

Im Rahmen der Diskussion über die in dieser Arbeit verwendeten Verfahren und Methoden sollen drei Punkte genauer erörtert werden. Der erste Punkt betrifft die Auswertung der Fragebögen der Expertenbefragung. Der zweite Punkt ist die Frage, inwieweit die Ergebnisse der Studentenbefragung in Bezug auf die Sichtweise der Verbraucher aussagekräftig sind und inwieweit sie damit sinnvoller Weise im Rahmen dieser Arbeit herangezogen werden können bzw. sollten. Der dritte Punkt bezieht sich auf die Frage, welche Probleme und Chancen sich aus der zeitlichen Versetzung der Umfrage (2002/2003) und der Erstellung des Literaturteils (2005) ergeben.

Bei der Auswertung der Fragebögen ergaben sich Probleme. Von dem ursprünglichen Design der Delfi-Analyse musste abgewichen werden. Nach der Auswertung der ersten Befragungsrunde wurde festgestellt, dass der Umfang der Befragung auf wenig Akzeptanz stieß. Aus diesem Grunde wurden die Anzahl der Fragen für die zweite Befragungsrunde reduziert, wobei jedoch darauf geachtet wurde, dass für die Zielrichtung der Befragung relevanten Teile auch in der zweiten Runde - Interviews - abgefragt wurden.

Ein weiteres Problem der Auswertung bestand darin, dass keine statistische Methode angewendet werden konnte. Dies hing zum einen mit der geringen Anzahl der Antworten pro Frage zusammen. Zum anderen kam erschwerend hinzu, dass die Einschätzung der Relevanz der Fragen durch die Experten anhand einer qualitativen Skala erfolgte. Aufgrund dieser beiden, im Zusammenhang mit statistischen Methoden, begrenzenden Parametern ist nach Rücksprache mit den Experten der statistischen Abteilung der Bundesforschungsanstalt für Ernährung und Lebensmittel (BFEL), Standort Kiel, auf die Verwendung statistischer Methoden verzichtet worden. Die Auswertung ist anhand von prozentualen Einschätzungen erfolgt. 
Der zweite Punkt der Methodendiskussion ist die Frage nach dem Stellenwert der Studentenbefragung sowie nach der Verwendbarkeit deren Ergebnisse im Rahmen dieser Arbeit. Die Gruppe der Studenten kann nicht einfach gleichgesetzt werden mit der Gruppe der Verbraucher in Deutschland. Dies ist aus mehreren Gründen nicht möglich. In den Kategorien Alter, Einkommen, Bildung usw. ist eine solche Gruppe sicherlich nicht repräsentativ. Hinzu kommt, dass die Gruppe der Studenten alle Agrarwirtschaft studieren und insofern überdurchschnittlich gut über den Bereich der Tierproduktion informiert sein dürften.

Aufgrund dieser besonderen Voraussetzungen sollte man erwarten, dass die Ergebnisse der Befragungen dieser Gruppe sich eher mit den Einschätzungen der Experten bzw. der Fachwissenschaft als mit den Bewertungen durch den Verbraucher decken würden. Dies war jedoch nicht der Fall. In vielen Bereichen deckten sich die Antworten der Studenten eher mit der Einschätzung der Experten über den Verbraucher. Dies betrifft insbesondere den Bereich der ethischen Gesichtspunkte und den damit verbundenen möglichen Meideverhalten. Während sie z.B. genau wie die Verbraucher Freilufthaltung eher positiv beurteilten, hatten gerade die Experten auf die Risiken dieser Haltungsform ausdrücklich hingewiesen.

Innerhalb der Ergebnisse der Studentenbefragung gab es dann noch den interessanten Aspekt der Unterscheidung der Antworten zwischen den Studenten $(\mathrm{mL})$ und den Studenten (oL). Insbesondere bei den tierschutzrelevanten Fragen wichen die Antworten zum Teil auffällig ab. Während die Studenten $(\mathrm{mL})$ Verfahren wie das Kupieren als notwendig ansahen, lehnten die Studenten (oL) diese Methoden wesentlich stärker ab.

Das Ziel der Arbeit ist die Herausarbeitung der realen als auch der ethischen Risiken der Produktionskette Fleischerzeugung. In Bezug auf die ethischen Risikopunkte ist eine der Kernthesen, dass die zunehmende Distanzierung zwischen der Erzeugung von Fleisch und den Verbrauchern die Kommunikation von Risiken immer mehr erschwert. Genau dieses lässt sich an einzelnen Punkten sehr gut mit Hilfe der Studentenbefragung zusätzlich belegen. Dabei muss jedoch darauf hingewiesen werden, dass es bei der Verwendung der Umfrageergebnisse dann wirklich um die Veranschaulichung von Aussagen und Ergebnisse der Expertenbefragung sowie der Fachwissenschaft geht und nicht um die Darstellung einer auf Repräsentativität ausgerichteten Umfrage.

Als letzter Punkt soll an dieser Stelle das Problem der verschiedenen Zeitebenen der Arbeit angesprochen werden. Die Expertenbefragung sowie die Studentenbefragung wurden 2002/2003 durchgeführt und ausgewertet. Aufgrund von verschiedenen Umständen konnte der Literaturteil erst 2005 beendet werden. Trotz des zeitlichen Abstandes kann man davon ausgehen, dass die Expertenbefragung auch heute noch in den meisten Bereichen die wesentlichen Haltungen und Einstellungen der Expertenschaft widerspiegelt. Darüber hinaus bietet sich zugleich die Möglichkeit abzugleichen, inwieweit die Trends von vor drei Jahren, sich inzwischen entwickelt haben.

\subsection{Zucht}

Die extreme Zucht auf Fleischanteile hat ein hohes ethisches Risikopotenzial und Auswirkungen auf die Produktqualität. Bei dem Einsatz der zur Zeit am Markt gängigen Rassen und genetischen Konstruktionen wird es in den nächsten Jahren kaum Änderungen geben. Dieses ist angesichts des schrumpfenden genetischen Potenzials zu diskutieren. 
Eine einseitige Zucht auf einen hohen Fleischanteil mit extrem ausgeprägten Körperteilen, ist ethisch bedenklich. Direkt haben allerdings Bilder über Tiere mit extrem ausgeprägten Körperteilen keinen nachweisbaren Rückgang beim Fleischkonsum bewirkt. Auswirkungen auf den Fleischkonsum hatte eher ein mangelndes Wasserhaltevermögen beim Schweinefleisch. Fleisch, das in der Pfanne schrumpft, hat beim Verbraucher zu Meidereaktionen und zu einer stärkeren Beachtung der Fleischqualität in der Wissenschaft und Wirtschaft geführt.

Bei der Diskussion über die Zucht ist zu bedenken, dass der Verbraucher immer sensibler auf Bilder im Zusammenhang mit dem lebenden Tier reagiert und in Zukunft ein Meideverhalten nicht auszuschließen ist. Dieses wird untermauert durch die Ergebnisse der Befragung. Sowohl Experten als auch Studenten gehen von einer zunehmenden Sensibilisierung der Verbraucher aus und schließen nicht aus, dass es zu einem Meideverhalten beim Fleischkonsum kommen kann. In Richtung eines möglichen Meideverhaltens weist auch die Beurteilung der Studenten (o.L.), die mit ca. 45\% bei Missständen zu einem Meideverhalten neigen. Sicher spielt hierbei die wachsende Entfremdung von der Landwirtschaft eine Rolle (ALVENSLEBEN, 1998B). Eine weitere wichtige Rolle beim Risikoempfinden der Verbraucher spielen die Medien. Durch deren Darstellung können Risikowahrnehmungen verstärkt oder gemindert werden (vergl. Kapitel 2.1.3).

In der Befragung wurde der Begriff „Qualzucht“ verwendet um den Tierschutzaspekt stark zu betonen. Die Meinung der Experten über die Zucht auf einen extremen Fleischansatz (Qualzucht) ist sehr unterschiedlich. In der mündlichen Befragung reichen die Antworten von Zustimmung bis totaler Ablehnung des Sachverhaltes. Nach WICKE ET AL. (2001); BIZERAY ET AL. (2002) und IDEL u. MATHES (2004) hat die Zucht auf hohe Fleischanteile Auswirkungen auf die Konstitution der Tiere und ist ethisch zum Teil bedenklich. Auf Grund unterschiedlicher Meinungen ist es notwendig, messbare Standards zur Beurteilung von Qualzucht zu entwickeln. Hierbei ist das Benennen von eindeutigen Parametern allerdings nicht ganz einfach. Zum Beispiel werden die von den Experten genannten Punkte wie Verluste, Fortpflanzungsfähigkeit, Bewegungsverhalten u.a. auch stark durch Fütterung und/oder Haltung beeinflusst. Weiterer Forschungsbedarf ist vorhanden.

Die durch einen hohen Fleischansatz bedingten Qualitätseinbußen beim Fleisch sind bekannt und deren Ursachen weitestgehend erforscht. Zur Verbesserung der Produktqualität wird in der Schweinezucht das MHS-Gen in den Vaterlinien reduziert (V. LENGERKEN ET AL., 1998; WICKE ET. AL., 2001). Obwohl man die Ursachen für die Qualitätseinbußen kennt, ist in naher Zukunft nicht damit zu rechnen, dass sich in der Fleischerzeugung die Zuchtrichtungen ändern werden bzw. ein größeres Rassenspektrum genutzt wird (vergl. BRANSCHEID, 2004). Das produzierte Fleisch entspricht überwiegend den Anforderungen des Marktes (z.B. Magerkeit, Produktionskosten, technologische Eigenschaften).

Die Abnahme des genetisch verfügbaren Potenzials ist ein weiteres Problem, das durch die einseitige Zucht auf Leistung verursacht wird. Die von den Experten geschätzte, weitere Abnahme der Rassen bzw. genetischen Konstruktionen ist angesichts der abnehmenden Biodiversität (vergl. GEH, 2001; BARTH ET AL., 2004) kritisch zu sehen. Schon heute werden zur Erzeugung von Fleisch nur sehr wenige Rassen eingesetzt. Hat man es bei den Rindern noch mit Rassen zu tun, werden bei den Schweinen (GENRES, 2003) und vor allem bei Geflügel (ANONYMUS, 2004D) nur genetische Konstruktionen in der Fleischerzeugung eingesetzt. Durch die Konzentration auf wenige Rassen besteht die Gefahr, dass weitere Rassen aussterben und der Genpool weiter schrumpft. Wichtiges genetisches Potenzial geht auf diese Weise verloren. 
Zum Erhalt von gefährdeten Rassen kann die Hybridzucht aber auch eine Chance sein. Aufgrund vorhandener Probleme wird unterschiedliches Genmaterial eingesetzt um die Zucht positiv zu beeinflussen. Man greift dabei auch auf gefährdete Rassen zurück. Zum Beispiel hat in der Schweinezucht die Rasse Duroc, aufgrund ihres positiven Einflusses auf den intramuskulären Fettgehalt, an Bedeutung gewonnen (V. LENGERKEN ET AL., 1998; WICKE ET. AL., 2001). Nach Einschätzung der FAO gehören Durocschweine allerdings noch zu den gefährdeten Rassen (TGRDEU, 2005).

Bei den heutigen Zuchtzielen ist ein Umdenken sowohl aus ethischen als auch aus Gründen der Produktqualität notwendig. Einige der heutigen extremen Zuchtrichtungen sind in Hinblick auf den Tierschutz als bedenklich einzustufen. Sowohl eine extreme Zucht als auch eine mangelhafte Produktqualität bergen nach wie vor ein Risikopotenzial, das zu einem Konsumverzicht führen kann. Bei einer Änderung von Zuchtzielen könnten dann auch zurzeit nicht kommerziell genutzte Rassen an Bedeutung gewinnen und somit zum Erhalt einer genetischen Vielfalt beitragen. Eine Änderung des Zuchtziels wird es aber nur dann geben, wenn finanzielle Einbußen bzw. Anreize den Aufwand rechtfertigen. Verbraucher und Wirtschaft müssten dann aber ihre Anforderungen an das Produkt Fleisch ändern.

\subsection{Gentechnologische Produktionsverfahren}

Gentechnologische Produktionsverfahren bergen sowohl ein ethisches Risikopotenzial als auch Risiken in der Produktsicherheit.

Der Anbau von gentechnisch veränderten Pflanzen (gv-Pflanzen) steigt weltweit rasant an (JAMES, 2004) und in der Tierfütterung werden gv-Pflanzen (Soja) schon heute eingesetzt. Zurzeit hat der weltweite Anbau und die Verfütterung von gv-Soja keinen nachweisbaren Einfluss auf den Fleischkonsum. Das könnte sich bei einer kommerziellen Zulassung von gvPflanzen in Deutschland ändern. Denn in den gentechnischen Verfahren sehen sowohl die Experten als auch die Studenten ein hohes ethisches Risikopotenzial. Aus diesem Grunde rechnen auch 65\% der Experten damit, dass der kommerzielle Anbau zu einem Meideverhalten des Verbrauchers bei Fleisch führen kann. Hierbei wird die Kommunikation von Risiken und Nutzen entscheidend für das Verbraucherverhalten sein. Allerdings beurteilt ALVENSLEBEN (1999B) eine Erhöhung der Verbraucherakzeptanz durch Information und Aufklärung eher skeptisch. Eine höhere Akzeptanz kann erst dann erwartet werden, wenn Produkte mit einem deutlich erkennbaren Verbrauchernutzen angeboten werden. Dieser Nutzen kann häufig allerdings nur subjektiv ermittelt werden und ist zurzeit nicht erkennbar (AlVENSleben, 1999B; LehNER, 1999; INSTitut Für DeMOSKOPIE AlLENSBACH, 2002).

Weitere Schwierigkeiten entstehen, da die Meinung des Verbrauchers über die Gentechnologie nicht gleichzusetzen ist mit den naturwissenschaftlich nachweisbaren und ökonomisch belegbaren Vor- und Nachteilen. Hinzu kommt, dass häufig auch in der Wissenschaft ein Dissens besteht, wie einzelne Ergebnisse zu interpretieren sind und welche Gewichtung die Ergebnisse erfahren. Um eine möglichst hohe Akzeptanz beim Verbraucher zu erreichen, ist es notwendig, sowohl die naturwissenschaftlich-technischen als auch die ethischmoralischen Aspekte in die Risikoanalyse und Risikobewertung einzubeziehen (GAISSER ET AL., 2000). Ebenso muss die Information der Öffentlichkeit verbessert werden. Äußerungen von Verbrauchern zu elementaren Sachverhalten zeigen immer wieder gravierende Kenntnismängel (ANONYMUS, 2004B). 
Im Gegensatz zu einer bedingten Akzeptanz des Verbrauchers von gentechnischen Verfahren in der Pflanzenproduktion wird der Einsatz bei Tieren zu einem Meideverhalten führen. Sowohl die Experten als auch die wissenschaftliche Literatur (ALVENSLEBEN, 1999B; LEHNER, 1999; GAISSER ET AL., 2000; ENGEL, 2002; SCHIEMANN, 2002 u.a ) sehen in gentechnisch veränderten Tieren ein hohes ethisches Risiko. Allerdings werden nach JANY (2000B) transgene Tiere in der Landwirtschaft voraussichtlich in den nächsten 20 Jahren keine Bedeutung in der Lebensmittelproduktion erlangen.

Neben dem ethischen Risiko werden weitere Risiken durch gv-Pflanzen unterschiedlich bewertet. Nach Meinung der Experten gehen von gv-Pflanzen keine bzw. nur geringe Gesundheitsgefahren aus. Dieses wird von verschiedenen Wissenschaftlern bestätigt. Sie begründen die Meinung damit, dass gv-Pflanzen gut untersucht sind und sehr strengen Sicherheitsbestimmungen unterliegen. Eine gesundheitliche Unbedenklichkeit muss vor der Markteinführung wissenschaftlich nachgewiesen sein (BODENMÜLLER, 2000, ENGEL, 2002; JANY u. KIENER, 2000B; JAMES, 2002; SCHIEMANN, 2002).

Obwohl gentechnisch veränderte Produkte gut untersucht sind, sind heute noch nicht alle Risiken bekannt. Zum Beispiel wird von FLACHOWSKY ET AL. (2000), SCHIEMANN (2002) und SMALLA (2003) nicht ausgeschlossen, dass es zu Einkreuzungen von transgenen Eigenschaften in andere Organismen kommen kann. Auch GAISSER ET AL. (2000) und NISCHWITZ (2004) sehen Risiken bei gentechnisch veränderten Pflanzen. Z.B. wird aus den USA von sogenannten „Superunkräutern“ berichtet, die gegen den Einsatz von Pestiziden resistent sind (NISCHWITZ, 2004). Es ist auch nicht auszuschließen, dass Insekten durch genveränderte Pflanzen negativ beeinflusst werden (BODENMÜLLER, 2000).

Aufgrund der derzeitigen Wissenslage ist zurzeit eine vollständige Klärung der Risiken durch Gentechnik nicht möglich und es besteht weiterhin Forschungsbedarf. Dieses bedingt, dass eine Akzeptanz der Gentechnik durch den Verbraucher auch in Zukunft nur schwer zu erreichen ist. Allerdings werden auch zunehmend die Chancen der Gentechnologie vermehrt wahrgenommen. Der Verbraucher nimmt eher eine positive Grundhaltung zu Gentechnologien ein, wenn diese zur Bekämpfung von Krankheiten und zur Erkennung und Verhinderung von Erbschäden eingesetzt werden. Erfolge in diesen Bereichen könnten dazu führen, dass der Einsatz von Gentechnologien in anderen Bereichen (z.B. die Landwirtschaft) immer mehr akzeptiert werden (INSTITUT FÜR DEMOSKOPIE ALLENSBACH, 2002).

\subsection{Zoonosen}

Keime mit hoher Bedeutung in der Lebensmittelproduktion sind Salmonellen, Campylobacter und coliforme Keime. Diese Keime werden von den Experten genannt und gehören zu den am häufigsten Krankheiten auslösenden Keimen (RKI, 2004). Auffällig an den Ergebnissen der Befragung ist, dass die Yersiniose bei der Beurteilung der Experten nur eine geringe Bedeutung erlangt und auch keine steigende Bedeutung für die Zukunft gesehen wird. Nach Zahlen des RKI (2004) sind Yersinien, in Deutschland, der dritthäufigste Auslöser für Zoonoseerkrankungen. NEUBAUER ET AL. (2001) publizieren, dass die Yersiniose in manchen Regionen der USA eine ebenso große Bedeutung hat wie die Salmonellose. Ebenso auffällig bei den Ergebnissen der Expertenbefragung ist, dass dem Bakterium Listeria monocytogenes eine sehr große Bedeutung von Seiten der Experten beigemessen wird. Erkrankungsfälle durch Listerien treten allerdings im Vergleich zu Salmonellen und Campylobacter nur sehr selten auf (KOCH, 2003; RKI, 2004). 
Neben den oben genannten Zoonosen sehen die Experten zukünftig eine steigende Bedeutung bei den Staphylokokken, dem Botulismus-Erreger und bei Influenza-Viren. Viren spielen allerdings mit nur $2 \%$ bei den durch Nahrungsmittel verursachten Erkrankungen eine insgesamt untergeordnete Rolle. Trotz dieser geringen Bedeutung (bei Nahrungsmittel bedingten Erkrankungen) ist die Nennung der Influenza durch die Experten bemerkenswert, weil die Befragung vor dem Erscheinen der Aviären Influenza in China (2003/2004) durchgeführt worden ist. Die Einschätzung der Experten, dass parasitäre Erkrankungen kaum noch eine Rolle spielen, deckt sich mit den Veröffentlichungen des RKI (2004).

Um Zoonosen langfristig in der Nahrungskette zu reduzieren, muss die Bekämpfung auf allen Stufen erfolgen. Am Beispiel der Salmonellen sollen Eintragsschwerpunkte entlang der Produktionskette Fleisch diskutiert werden. Hierbei handelt es sich in erster Linie um technische Prozesse, zu denen der Verbraucher keinen Bezug hat. Aus diesem Grund erfolgt keine Risikobetrachtung aus der Sicht der Verbraucher.

\subsubsection{Futtermittelproduktion}

Eintragsschwerpunkte von pathogenen Keimen in die Futtermittelproduktion sind Futtermittelausgangserzeugnisse, schlechte Hygiene bei der Produktion und Lagerung und der Eintrag über Schadnager und Vögel. Allerdings wird die Bedeutung der Futtermittel als Überträger unterschiedlich bewertet. Die Aussagen von DORN u. SCHWARZER (1991), BISPING (1993) und KLARMANN (2001) spiegeln die Meinung der Experten wieder, dass Futtermittel zwar eine mögliche Infektionsquelle sind, aber nicht überbewertet werden dürfen. Dagegen sind SCHLÜTER ET AL. (1992) und KÖHLER (1993) anderer Meinung. Sie sehen in mit Salmonellen kontaminierten Futtermitteln eine der Hauptinfektionsquellen für Geflügelbestände. Auch LÖHREN (2004) spricht sich für Maßnahmen aus. Zum Beispiel sollten Rohwarenlieferanten, die mehrfach durch z.B. positive Salmonellenproben aufgefallen sind, gesperrt bzw. gezwungen werden, Maßnahmen zur Salmonellenreduzierung durchzuführen.

Nach Angaben der PDV (2004) lag die Salmonellenbelastung von Futtermittelausgangserzeugnissen bei 3,6\%. Dieser Anteil ist zwar nicht sehr hoch, aber es ist Handlungsbedarf vorhanden. Dieser ergibt sich auch aus der Prämisse, dass es sich um Unternehmen handelt, die sich den Hygienevorschriften des GMP+-Regelwerkes unterworfen haben.

Im Produktionsprozess von Futtermitteln bergen alle Stellen Risiken, an denen es zu einer Anhaftung bzw. Ablagerung von Staub und Futtermittelresten kommen kann. Wenn es an diesen Stellen zusätzlich zum Eindringen von Feuchtigkeit oder zur Kondenzwasserbildung kommt, herrschen gute Bedingungen für pathogene Keime. LÖHREN (2004) sieht vor allem im Kühler anhaftendes Material als ein hohes Risiko an. Aber auch mangelnde Hygiene in den Förderwegen und im Mischer können erhebliche hygienische Probleme hervorrufen.

In den letzten Jahren haben sich Hygienebedingungen in der deutschen Futtermittelproduktion verbessert. Gefördert wird diese Entwicklung durch die Einführung von Qualitätsmanagementsystemen (z.B. QS, GMP). Allerdings werden auch Schwachstellen deutlich. Von den Mitarbeitern werden die Anforderungen der Qualitätsmanagementsysteme zum Teil nur schwer akzeptiert. Umfangreiche Reinigungen erfolgen teilweise nur kurz vor den Audits. Die Unternehmer kennen häufig die spezifischen Hygieneschwachstellen ihrer Anlagen nicht.

Als ein weiterer Eintragsweg von pathogenen Keimen gelten Schadnager, Vögel usw. Ebenso wie die Experten fordern auch HENSGEN (2004) und LÖHREN (2004) eine konsequente Bekämpfung von Schadnagern, Vögeln etc. in Futtermittelbetrieben. Diese 
Maßnahmen gehören zu den Grundprinzipien der Bekämpfung von pathogenen Keimen. Ebenso ist die Schadnagerbekämpfung in allen Managementsystemen (z.B. QS, GMP+, EUREP-GAP, IFS u.a.) einer der Grundbausteine der Programme. In der Futtermittelproduktion erfolgt die Bekämpfung allerdings häufig durch eigene Mitarbeiter, die nicht immer über die dafür notwendige Ausbildung verfügen. An dieser Stelle ist noch Handlungsbedarf vorhanden.

Obwohl die Einführung von Qualitätsmanagementsystemen im Bereich der Futtermittelproduktion schon einen Fortschritt darstellt, reicht das allein nicht aus. Sowohl bei der Schulung der Mitarbeiter als auch bei der Identifikation von hygienisch kritischen Stellen besteht in der Futtermittelproduktion nach wie vor ein großer Handlungsbedarf.

\subsubsection{Landwirtschaft}

Problemschwerpunkte, die in der Urproduktion diskutiert werden, sind Hygienemängel und die Übertragung von Zoonosen durch Tierkontakte und Freilandhaltung.

Ähnlich wie die Experten sehen SINELL (1998A), FEHLHABER (2002) und BAARS (2003) in der landwirtschaftlichen Tierhaltung eine der Haupteintragsquellen von pathogenen Keimen in die Lebensmittelkette. Die Autoren stellen zum Teil heraus, dass sich die Situation des Infektionsdruckes durch den landwirtschaftlichen Betrieb in den letzten Jahren nicht nennenswert verbessert hat. Das hat nach SINELL (1998A) und HAMMER (2001) zur Folge, dass sich der Eintrag von pathogenen Keimen in der Stufe der Schlachtung nicht verhindern lässt. Als eine der Ursachen für eine Belastung der Urproduktion können die von den Experten genannten Hygienemängel (Abbildung 5.10-4) angesehen werden. LÖHREN (2004) sieht bei Geflügel ähnlich wie die Experten die hauptsächlichen Schwachpunkte in der Reinigung und Desinfektion. Dieses begründet er mit den in den Betrieben häufig vorhandenen baulichen Unzulänglichkeiten, wie z.B. Ritzen und Fugen. Die von LöHREN (2004) gemachten Aussagen können aber auch auf die Rinder- und Schweinehaltung übertragen werden. HeEschen (2003), Meyer (2004) und Pfannhauser (2005) sehen in einer mangelnden Personal- und Seuchenhygiene Risiken. VAN DER WOLFF ET AL. (1999) und MEYER (2004) bestätigen, dass der Fütterungshygiene eine erhebliche Bedeutung beim Eintrag und der Vermehrung von Keimen zukommt. Aufgrund der zurzeit noch nicht befriedigenden Situation besteht gerade bei der Umsetzung von Hygienemaßnahmen ein verstärkter Handlungsbedarf.

Eine Unterbrechung der Infektkette kann durch systematisches und konsequent durchgeführtes Hygienemanagement erreicht werden. Dieses zeigen die Maßnahmen in der Geflügelproduktion. Hier konnten nach SAULI (2004) gute Erfolge erzielt werden. In der Schweinefleischproduktion werden seit 2000 der Hygienestatus im Rahmen des QSSystems erfasst. 14.3\% der kategorisierten Betriebe haben den Salmonellenstatus 2 und 4,9\% der Betriebe den Salmonellenstatus 3 (QS, 2006). Unter Berücksichtigung dieser Zahlen wird deutlich, dass weitere umfangreiche Maßnahmen notwendig sind.

Aber nicht nur eine mangelnde Hygiene führt zu einer Verbreitung von Zoonoseerregern. Eine weitere Quelle sind die Tiere selbst. In der konventionellen Tierhaltung werden Tiere häufig in größerer Stückzahl auf engem Raum gehalten, was eine Ausbreitung von Keimen begünstigt (KREUZER, 2002). Aber bei einem strikt angewendeten Hygienemanagement bietet die konventionelle Tierhaltung auch Vorteile: die Isolation der Tiere von der Außenwelt und die Möglichkeit, die Stalleinrichtungen vor jeder Neubelegung zu desinfizieren. 
Ebenso wie die Experten sehen auch PIETSCHMANN u. HAFEZ (2002), BAARS (2003) und METHNER (2004) ein Risiko in der Anreicherung von pathogenen Keimen und Zoonosen in der Freilandhaltung von Schweinen und Geflügel. Auch FEHLHABER (2003A) fordert angesichts der Zoonosenproblematik und der damit verbundenen Verbrauchergefährdung, die Vor- und Nachteile von ökologischen Haltungssystemen sorgfältig zu prüfen. Nach PetermanN u. MAIWORM (2004) sind in diesen Haltungssystemen Krankheiten beobachtet worden, die jahrelang nicht mehr aufgetreten waren. Inwieweit durch ein besseres Management Defizite in der Freilandhaltung ausgeglichen werden können, kann nach heutigem Stand der Wissenschaft nicht abschließend beurteilt werden. Hier ist nach wie vor Forschungsbedarf vorhanden.

Unumstritten ist, dass die Freilandhaltung am ehesten das Idealbild der Landwirtschaft verkörpert. Nach ALVENSLEBEN (1999A) wird dieses Idealbild durch die Medien geprägt. Auch entspricht diese Haltungsform dem nach ADAM (2001) gestiegenen Anspruchs des Verbrauchers nach Tier- und Umweltschutz. Wie wenig die Risiken der Freilandhaltung bekannt sind, kann an der Einschätzung der Beurteilung der Studenten betrachtet werden. Während über $60 \%$ der Studenten $(\mathrm{mL})$ Gefahren in der Freilandhaltung für Mensch und Tier sehen, sind das in der Gruppe der Studenten (oL) nur ca. 38\%. An diesem Ergebnis lässt sich belegen, wie schwer es ist, Risiken dem Verbraucher zu kommunizieren. Man muss dabei berücksichtigen, dass aufgrund der Studienrichtung diese Studenten einen überdurchschnittlichen Bezug zur Landwirtschaft haben. Nach ALVENSLEBEN (2002A) haben nur $11 \%$ der Verbraucher ein reales Bedürfnis, darüber informiert zu werden, wie unsere Lebensmittel hergestellt werden (vergl. auch Kapitel 2.2).

Die Bekämpfung von Zoonosen in der Urproduktion wird weiter ein Problem bleiben. Wichtig ist, dass in den Betrieben vermehrt der hygienische Status festgestellt wird. Hierzu sollten aber nicht nur der Salmonellenstatus erfasst werden. Ebenso ist es wichtig, andere Zoonosen - die in der Nahrungskette von Bedeutung sind - zu berücksichtigen. Denn gerade im Hinblick auf die Einschätzung der Experten, dass Zoonosen in Zukunft an Bedeutung gewinnen, muss deren Bekämpfung in der Urproduktion verbessert werden.

Interessant wird es sein, die Entwicklung der Hygienesituation in der landwirtschaftlichen Urproduktion nach dem Inkrafttreten der neuen EU-Hygieneverordnungen (VO EU 852/2004 u. VO EU 853/2004) zum 01.01.2006 zu beobachten. Zwar wird für den landwirtschaftlichen Betrieb kein HACCP-Konzept gefordert, aber die Betriebe sind erstmalig zu der Erarbeitung einer Risikobetrachtung verpflichtet. Nach den neuen Verordnungen müssen außerdem Tiere vor der Schlachtung vom Bestandstierarzt untersucht werden, und dem Schlachthof muss 24 Stunden vor der Schlachtung ein entsprechendes tierärztliches Attest vorliegen.

\subsubsection{Schlachtung}

In der Schlachtung sind mikrobiologische Risiken bekannt und werden umfangreich überwacht. Sowohl von den Experten als auch in der Literatur werden das Schlachttier und das Personal als Haupteintragsquellen angesehen. Als ein weiteres Übertragungsrisiko wird Kreuzkontaminationen im Schlachtband genannt.

Eine Unterbrechung der Infektkette an der Lebendviehannahme ist nur sehr schwer möglich. Tiere, die gesund erscheinen, verbreiten durch Tierkontakt und Fäkalien pathogene Keime im gesamten Wartestall (SEIDLER, 1998; REUTER, 2003; WIESE, 2003). Ebenso macht das meist fehlende Wissen über den Hygienestatus der Tiere ein striktes Hygienemanagement 
unmöglich (SINELL, 1998A; FEHLHABER, 2003A). Ansätze in diese Richtung bietet das Salmonellenmonitoring bei Schweinen im Rahmen des QS-Systems. Allerdings werden in den meisten Fällen die erhobenen Daten nicht für konkrete Maßnahmen genutzt. Eine Schlachtung der Tiere nach ihrem Salmonellenstatus oder Sanktionen gegenüber dem Lieferanten handhaben nur wenige Schlachthöfe.

Mängel in der persönlichen Hygiene und Schlachtfehler sind Eintragswege von Mikroorganismen durch das Personal (vergl. hierzu z.B. SEIDLER, 1998; REUTER, 2003; LeITLINIE BADEN-WÜRTEMBERG, 2004; HENSGEN, 2004). Aufgrund der technischen Prozesse ist in der Geflügelschlachtung die Gefahr von Hygienefehlern geringer als in der Rinder- oder Schweineschlachtung (WIESE, 2003).

Das Hygieneverständnis des Personals in den einzelnen Betrieben ist sehr unterschiedlich. Hygieneschulungen sind zwar auf den Schlachthöfen gesetzlich vorgeschrieben, aber die Qualität der Schulungen ist sehr unterschiedlich. Probleme gibt es zumeist aufgrund der teilweisen mangelhaften Qualität der Schulungen. Darüber hinaus wird bei den Schulungen nicht genügend darauf geachtet, dass ein Teil der Mitarbeiter aufgrund seiner Herkunft nur mangelhaft Deutsch spricht. Sie können die auf Deutsch durchgeführten Schulungen nicht verfolgen und daher auch nicht umsetzen.

Inwieweit Kreuzkontaminationen als ein weiteres Hygienerisiko auftreten können, ist stark abhängig von dem Hygienestatus der Schlachttiere und dem Personal. Einmal in den Schlachtprozess eingeschleppte Keime verbreiten sich entlang aller Stationen des gesamten Schlachtprozesses. Besonders kritisch werden der Bereich der Entnahme des Magen- und Darmtraktes, das Brühen und das Stechen, aber auch eine veraltete oder unsachgemäß eingesetzte Technik gesehen.

Hygieneprobleme in der Schlachtung werden trotz umfangreicher Kontrollen auch weiter auftreten. Die Experten schätzten für die zukünftige Entwicklung ein, dass es zu einem Rückgang der Probleme in diesem Bereich kommen wird. Um dieses Ziel wirklich erreichen zu können, sind in erster Linie konsequente Maßnahmen in der Urproduktion notwendig. Erfolge lassen sich am schnellsten durch Bonus-Malus-Systeme erreichen. Aber auch in Bezug auf die Personalhygiene ist eine Verbesserung des Schulungsstandards notwendig. Hierbei sollte beachtet werden, dass die Schulungen in der Muttersprache der Mitarbeiter erfolgen sollte.

\section{$6.5 \quad$ Tierseuchen}

Nach Meinung der Experten wird durch den weltweiten Handel mit Tieren und tierischen Produkten die Gefahr von Seuchen bzw. die Einschleppung von neuen Keimen zunehmen. Dieses Risiko wird auch von KREISSL-DÖRFLER (2002) gesehen. Die wesentliche Gründe für diesen Trend sehen die Experten in den unterschiedlichen hygienischen Niveaus und in der weltweiten Verbreitung länderspezifischer Erreger. Aber auch die oft mangelhafte Meldung von Seuchenfällen und die zum Teil sehr oberflächlichen Grenzkontrollen erleichtern das Ausbreiten von Krankheiten (ANONYMUS, 2002A).

Es muss in diesem Zusammenhang auch betont werden, dass das Einschleppen von Erregern aus dem Ausland zwar ein wesentlicher Faktor für die zunehmende Seuchengefahr ist, aber nicht der einzige. Eine nicht zu unterschätzende Bedeutung haben auch die heutigen Tierhaltungsformen. Aufgrund der extrem hohen Bestandsdichten in manchen 
Regionen ist die Anfälligkeit und die Verbreitungsgeschwindigkeit wesentlich höher als noch vor ca. 30 Jahren. Die Anfälligkeit der Tierhaltung hat z.B. der letzte große Seuchenzug der Maul- und Klauenseuche 2001/2002 gezeigt. Nach NORDHOFF (1998) mehren sich die Meinungen, die Bestandsdichten in manchen Regionen zu reduzieren. In den Niederlanden wurden z.B. die Schweinebestände per Gesetz reduziert. Aber auch der Aspekt einer Zunahme der Freilandhaltung muss, im Zuge der Seuchenproblematik, diskutiert werden. Denn gerade durch die Kontaktmöglichkeiten mit der Außenwelt ist die Gefahr einer Kontamination mit Keimen oder Viren besonders hoch. Nach PETERMANN u. MAIWORM (2004) wurden in Beständen von im Freiland gehaltenen Hennen Erkrankungen beobachtet, die seit Jahren nicht mehr aufgetreten sind.

Impfungen im Seuchenfall oder zur Vorbeugung von Seuchen werden sowohl von den Experten als auch in der Literatur kontrovers diskutiert. Für das Impfen sprechen vor allem ethische Aspekte und Tierschutzaspekte. Durch eine Impfung kann unter Umständen die Erkrankung der Tiere und somit das Ausbreiten der Krankheit verhindert werden. Aus Sicht des Tierschutzes wird dadurch ein Leiden der Tiere verhindert. Ein andere Vorteil ist, dass auf Massentötungen zur Eingrenzung der Seuche größtenteils verzichtet werden kann. Allein während des Seuchenzugs der Maul- und Klauenseuche 2001 wurden bis zu 10 Millionen Tiere getötet (BMVEL, 2001).

Gegen den Einsatz von Impfstoffen spricht, dass gerade bei den Tierseuchen häufig nicht nur ein Serotyp für eine bestimmte Krankheit verantwortlich sein kann, sondern verschiedene. All diese Serotypen könnten dann nicht durch eine einzige Impfung abgedeckt werden (KREISSL-DÖRFLER, 2002). Ebenso spricht dagegen, dass es zu Impfdurchbrüchen kommen kann (BAUMGARTNER, 2001; ANONYMUS, 2002E), bzw. sich der Erreger im Tier vermehrt, ausgeschieden wird und sich unerkannt weiterverbreiten kann (HEESCHEN, 2004).

Ein nicht zu unterschätzender Grund, der ebenfalls gegen das Impfen spricht, ist der wirtschaftliche Aspekt. Länder, in denen geimpft werden darf, gelten nicht als erregerfrei und unterliegen daher Handelsbeschränkungen. Dieses hat zur Folge, dass dadurch viele Märkte verschlossen bleiben. Länder, die als erregerfrei gelten, haben dann das Recht, die Einfuhr zu beschränken. Aufgrund von wirtschaftlichen Erwägungen werden zum Teil auch Notimpfungen verhindert, da viele Länder vom Export abhängig sind.

Gerade bei der Diskussion um Impfungen im Seuchenfall treffen die ethischen und die wirtschaftlichen Erwägungen aufeinander. Unter Berücksichtigung der Themenstellung, - Ermittung der realen Risiken und der Punkte, die von Verbraucher kritisch gesehen werden - spricht vieles für ein Impfen der Tiere. Solange es Alternativen gibt, werden Bilder von Massentötungen beim Verbraucher immer Unverständnis auslösen und dieses könnte leicht zu spontanem oder organisiertem Meideverhalten führen.

Vorbeugende Impfungen bzw. Prophylaxen, z.B. gegen Durchfallerreger u.a., erfolgten vor dem 1.1.2006 häufig mit antibiotisch wirkenden Mitteln. Nach dem BFR (2003A) und der FAO ET AL. (2003) haben sich z.B. durch die medikamentöse Bekämpfung von Zoonosenerregern beim Tier Resisitenzen beim Menschen gegen antimikrobielle Wirkstoffe entwickelt. Nach MCKELLAR (1999) und HAKENBECK (2001) wird damit das anwendbare Spektrum von Antibiotika in der Humanmedizin kontinuierlich reduziert.

Gerade aufgrund der vorabbeschriebenen Gefahr, ist nach Einschätzung der Experten, die Zunahme von Prohylaxen und Schutzimpfungen kritisch zu sehen. Zwar sind Schutzimpfungen und Prophylaxen wichtige Maßnahmen zur Gesunderhaltung der Tiere, aber sie 
sollten nicht eingesetzt werden, um den Infektionsdruck bei großen Bestandsdichten bzw. Beständen zu senken. Es müssen vielmehr zwingend Maßnahmen eingeleitet werden, die die Produktions- und Haltungsbedingungen selbst verbessern, so dass eine Infektionsgefahr im Vorfeld verringert wird. Hierzu müssen umfangreiche Hygienemaßnahmen in der Urproduktion erfolgen.

\subsection{Rückstände und Kontaminanten}

Rückstände und Kontaminanten müssen auf zwei Ebenen diskutiert werden. Die erste Ebene beinhaltet die Einstellung der Verbraucher zu Rückständen. Auf der zweiten Ebene muss die reale Situation mit den Eintragswegen über das Futtermittel und über die Tierhaltung diskutiert werden.

Bei der Rückstandsdiskussion muss dem Verbraucher verdeutlicht werden, dass es keine „Null-Toleranz" bei Rückständen gibt. Ebenso wie die Experten sehen POTTHAST (2002) und SANTARIUS (2004), dass es bei Rückständen keine Null-Toleranzen gibt. Aufgrund der immer feineren Nachweisverfahren werden heutige „Null-Werte“ durch modernere Geräte morgen zu messbaren Größen. Dadurch kann der gefundene Rückstand zu einer „potentiellen“ Gesundheitsgefahr werden. Besonders wichtig ist es deshalb zu kommunizieren, dass ein in einem Lebensmittel gefundener Rückstand nicht gleichbedeutend mit einem Risiko ist. Allerdings verläuft nach KROKER (2003) gerade die Diskussion bezüglich Rückstände sehr emotional. Der Verbraucher ist nur schwer für wissenschaftliche Bewertungen zugänglich.

Die Kommunikation des Risikos „Rückstand“ stößt aufgrund von unterschiedlichen Problemwahrnehmungen von Wissenschaft/Wirtschaft und Verbraucher schnell an ihre Grenzen (vergl. Kapitel 2.1.3). Ein wichtiger Schritt in Richtung einer ausgewogenen Risikokommunikation ist das Festlegen von nachvollziehbaren, wissenschaftlich begründeten Grenzwerten. Aber auch die Darstellung und Kommunikation von Chancen und Risiken der Ursachen für die Rückstände (z.B. Pflanzenschutz, Einsatz von Medikamenten) ist in der Risikokommunikation mit dem Verbraucher notwendig.

Unumstritten ist, dass einer der Haupteintragswege von Rückständen und Kontaminanten in die Nahrungsmittelkette das Futtermittel ist. Für viele Rückstände aus Futtermitteln sind technische oder menschliche Unzulänglichkeiten die Ursache. Der Weg der Rückstände in die Futtermittel erfolgt hierbei auf verschiedene Weise. Zum einen sind die Rückstände zu diskutieren, die über den Anbau, durch Pflanzenschutzmaßnahmen und Düngung und über importierte Futtermittel in die Nahrungskette gelangen. Zum anderen müssen Rückstände betrachtet werden, die über fehlerhafte Verfahren in das Futtermittel gelangen.

Ein Eintrag von Rückständen in Futtermittel erfolgt sowohl über Altlasten, Pflanzenschutzmittel als auch über Müllkompost und Klärschlamm. Die von den Experten vorausgesagte Verbesserung der Rückstandssituation lässt sich anhand der Literatur belegen. Nach den Jahresstatistiken des BMVEL (2004B; 2005A) ist der Anteil an unzulässigen und unerwünschten Stoffen gesunken. Ebenso wie die Experten sieht auch HEESCHEN (2003) ein Problem durch Organochlorverbindungen, da diese Stoffe in der Umwelt persistent sind und sich in der Nahrungskette kumulieren. Nach dem BMU (2001) ist die Dioxinbelastung der Lebensmittel in erster Linie auf Futtermittel zurückzuführen. Allerdings nahm nach BLÜTHGEN ET AL. (2000) die Aufnahme des Menschen von Organochlorverbindungen durch tierische Lebensmittel von 1992-1999 stark ab. Neben den Organochloverbindungen werden Schwermetalle als ein Problem angesehen. Nach SCHENKEL (2001) hat sich der Eintrag von 
Schwermetallen in die Umwelt deutlich reduziert. Die Belastung bei Fleisch und Fleischerzeugnissen wird von HONIKEL U. HECHT (1999) als gering eingestuft.

Die Befürchtung der Experten und der Studenten, dass Rückstände aus importierten Futtermitteln steigen werden, kann weder bestätigt noch dementiert werden. Anhand von Untersuchungsergebnissen über Rückstände von Pflanzenschutzmitteln im Getreide sind kaum Unterschiede festzustellen (BVL, 2002; 2003; EU 2001A, 2002C; 2003C). Nach SCHWIND (2000) und dem BMVEL (2005A) werden Organochlorverbindungen (z.B. PCBs) und Aflatoxin B1 häufig in Verbindung mit importierten Futtermitteln genannt. Allerdings werden nur wenige Rückstände von PCBs und Aflatoxin B1 in Futtermitteln gefunden. (BMVEL, 2005A).

Ein Risiko durch Rückstände aus Müllkompost und Klärschlämmen sehen sowohl $43 \%$ der Experten als auch StAdeLmanN (2001) und BerGs (2004). Positiv wird die Rückstandsituation für Schwermetalle bewertet. Probleme gehen eher von organischen Schadstoffen aus. Nach StAdELMANN (2001) und BeRGS (2004) verbessert sich auch hier die Rückstandssituation. Auf Grund einer unzureichenden Datenlage und einer Kumulation der Rückstände sind organische Schadstoffe weiterhin ein Problem.

Weitere Wege für Rückstände in Futtermitteln sind eine falsche Trocknungstechnik bzw. ein falscher Brennstoff und Verschleppungen im Produktionsprozess. Durch Trocknungsverfahren verursachte Probleme werden auch von HECHT (2003) gesehen. Gerade der Einsatz von Direktbefeuerungsanlagen führt zu Rückständen. Die Notwendigkeit, Direktbefeuerungsanlagen besser zu überwachen, wird sowohl im Ernährungs- und agrarpolitischen Bericht 2004 der Bundesregierung (ANONYMUS, 2004I) unterstrichen und in den Qualitätsmanagementsystemen QS und GMP+ gefordert.

Die unterschiedliche Bewertung der Anlagensicherheit durch die Experten lässt sich anhand der Literatur nachvollziehen. Ein Großteil der Futtermittelproduktion erfolgt in kleinen bis mittelständischen Betrieben. Diese Betriebe produzieren zum Teil alle Futtermittel (Rind, Schwein, Geflügel) über eine Linie (STRAUCH, 2002). Sowohl nach IFF (2001 u. 2002) als auch nach STRAUCH (2002) ist auf einer solchen Anlage eine verschleppungsfreie Produktion nicht möglich, da immer Rückstände in der Anlage verbleiben. Durch eine konsequente Überwachung und dem Erfassen des Verschleppungsniveaus lässt sich das Risiko zwar minimieren, aber nicht ausschalten. Durch die Zugabe von kritischen Stoffen im sogenannten „End of Line“ Verfahren kann ein Verschleppungsrisiko vermindert werden. Ebenso erfolgt eine Reduzierung der Verschleppung durch die Trennung der Mischfutterproduktion nach Tierarten (HELLWIG, 2003; BODE, 2004).

Die Einschätzung der Experten, dass es durch Mykotoxine im Fleisch zu keiner Gefährdung des Endverbrauchers kommt, findet sich auch in der Literatur wieder. Verschiedene Autoren haben keine gesundheitsgefährdenden Gehalte an Mykotoxinen im Fleisch festgestellt (vergl. ElmAdFA u. BuRger, 1999; GolL, 1993; KUIPER-GOOdMANN, 1999; WEIDENBÖRnER, 1999; DÄNICKE u. OldENBURG, 2000; DÄNICKE ET AL., 2002; MÜNSTER ET AL., 2002A). Nach GOLL (1993) und KUIPER-GOODMANN (1999) findet eine Anreicherung der Mykotoxine vornehmlich in den Organen und im Blut statt. MüNSTER ET AL. (2002A) schätzen die Gefahr, dass Menschen durch Lebensmittel tierischer Herkunft Ochratoxin A aufnehmen, geringer als 5\% ein. Die Hauptgefährdung des Menschen durch Mykotoxine erfolgt durch pflanzliche Nahrungsmittel (SINELL, 1998A; MüNSTER ET AL., 2002A).

Die abweichende Meinung von 50\% der befragten Experten bezüglich der Filterfunktion des Tieres steht damit im Widerspruch zur Literatur. Eine mögliche Begründung für diese Ein- 
schätzung kann sein, dass die Experten nicht nur die Filterfunktion in Bezug auf das Fleisch berücksichtigt haben, sondern auch andere tierische Produkte (z.B. Milch, Eier) mit einbezogen haben. Bei Milch ist ein "Carry over" von Aflatoxinen in das Produkt nachgewiesen worden (WEIDENBÖRNER, 1999; RANDEL, 2002). Im Gegensatz zu Fleisch und Innereien wird bei mykotoxinbelasteter Milch von einer Verbrauchergefährdung ausgegangen (WEIDENBÖRNER, 1999).

Das Risikoempfinden von Futterzusatzstoffen aus wissenschaftlicher Sicht und aus Verbrauchersicht wird von den Experten unterschiedlich bewertet. Sie schätzen, dass Kräutermischungen von der Wissenschaft wesentlich kritischer gesehen werden als vom Verbraucher. Umgekehrt ist die Einschätzung bei Prebiotika, Enzymen und synthetischen Aminosäuren. Diese Stoffe sind den meisten Verbrauchern unbekannt. Dadurch kann der Verbraucher auch nur schwer beurteilen, ob von den Stoffen ein Risiko ausgehen könnte. Wie in Kapitel 2.2 beschrieben werden nach JUNGERMANN und SOLVIC (1993) und ALVENSLEBEN (2002B) unbekannte Sachverhalte eher mit Risiken verbunden als bekannte und häufig deshalb abgelehnt. Bekannte Sachverhalte, die auch in einem anderen Zusammenhang positiv gesehen werden (Kräutermischungen), beurteilt der Verbraucher dagegen eher positiv. Mit diesem Ansatz lässt sich die Einschätzung der Experten der oben genannten Stoffe aus der Sicht des Verbrauchers erklären. Ähnlich dürfte auch die Bewertung der Studenten zu beurteilen sein. Denn der Anteil der Studenten (oL) schätzen Prebiotika, Enzyme und synthetische Aminosäuren kritischer ein als die Studenten (mL). Kräutermischungen werden von beiden Studentengruppen positiv bewertet. Die Experten beurteilen dagegen die Kräutermischungen aus wissenschaftlicher Sicht wesentlich negativer. Über Kräutermischungen liegen nach SCHMITZ ET AL. (2003) noch relativ wenige wissenschaftliche Erkenntnisse vor. Eine exakte Risikoeinschätzung ist somit nicht möglich.

Die kritische Einschätzung der Hormone, antibiotischen Stoffe und Kokzidostatika von allen Gruppen war zu erwarten. Diese Stoffe werden auch in der Literatur kontrovers diskutiert. Zum Beispiel werden Hormone grundsätzlich vom Verbraucher kritisch gesehen. Nach Veröffentlichungen von MISSURA (2000) und der EU (2002B) ist wissenschaftlich weder die Unbedenklichkeit noch ein eindeutiges Risiko beim ordnungsgemäßen Einsatz von Hormonen bewiesen. MEYER (1999) verweist darauf, dass Fleisch aus der europäischen Bullenmast höhere natürliche Hormongehalte aufweist als das Fleisch von Ochsen, das in den USA unter dem Einsatz von Hormonen produziert wurde. Nach seiner Meinung liegen die Risiken in der Hormonmast in falsch applizierten Implantaten und dem Einsatz von nicht zugelassenen Mitteln. Hierdurch kann es zu Rückständen kommen.

Rückstände werden immer wieder in Nahrungsmitteln gefunden. In vielen Fällen haben die gefundenen Rückstände keine gesundheitliche Bedeutung. Gerade bei solchen Funden ist es wichtig, dem Verbraucher den Sachverhalt nachvollziehbar zu kommunizieren. Diese Kommunikation muss unter Berücksichtigung der Unterschiede in der Problemwahrnehmung von Wissenschaft und Verbraucher erfolgen. Allerdings wird es auch immer wieder zu Rückstandsfunden kommen, die gesundheitliche Risiken bergen. Hier ist es notwendig, schnell und umfassend zu reagieren. Verbraucher sollten in solchen Fällen frühzeitig in die Kommunikation einbezogen werden. 


\subsection{Landwirtschaftliche Tierproduktion}

Alle Diskussionspunkte in der Urproduktion besitzen sowohl ein ethisches als auch ein wissenschaftlich begründetes Risikopotential. Kritisch diskutiert werden sollen die Tierhaltungsformen mit den sich daraus entwickelnden Verhaltensstörungen sowie den Eingriffen am Tier, um diese auszugleichen. In diesem Rahmen wird auch die Kastration behandelt, die ebenfalls einen massiven Eingriff am Tier darstellt. Ein weiterer Diskussionspunkt sind die Folgen von BSE.

Die Einschätzung der Experten, dass die heutigen Tierhaltungsverfahren vom Verbraucher als ethisch bedenklich betrachtet werden, stützen SIES und MAHLAU (1997). In ihrer Befragung assoziieren Verbraucher mit dem Wort „Tierhaltung“ in erster Linie negative Eigenschaften wie Masse, nicht artgerecht und Verhaltensstörungen. Diese Bedenken sind in der gesamten EU vorhanden (ALVENSLEBEN, 2001B; 2002D; HARPER, 2001). Die Geflügelmast wird am negativsten beurteilt. Auch die Studenten schätzen die Geflügelmast am negativsten ein. Auffällig ist, dass nur $4 \%$ der Studenten (oL) die Geflügelhaltung für tiergerecht halten.

Dass die heutigen Tierhaltungsverfahren Defizite aufweisen, wird nicht bestritten. Trotzdem ist die extrem negative Bewertung der Verfahren nicht gerechtfertigt. Beanstandungsgründe bei Kontrollen sind häufig keine Beschäftigungsmöglichkeiten, keine Alarmanlagen, fehlende Notstromaggregate, fehlende Aufzeichnungen über veterinärmedizinische Behandlungen, bauliche Mängel im Stall und Mängel bei der Tierkontrolle (WITTKOWSKI u. HERMÜLHEIM, 2001; BMVEL, 2005B). Beanstandungen wie Kannibalismus, kranke Tiere und Überbelegung der Ställe werden nicht genannt. Dieses heißt aber nicht, dass die genannten Missstände nicht in der Tierhaltung vorkommen. Gerade Bilder über Kannibalismus, Überbelegungen und kranke Tiere haben zum negativen Image der konventionellen Tierhaltung beigetragen. Dass solche Zustände nicht akzeptiert werden dürfen und verfolgt werden müssen, steht außer Frage. Durch die einseitige Darstellung in den Medien erhalten aber negative Bilder eine besondere Gewichtung. Der Verbraucher differenziert häufig nicht mehr in seinen Wahrnehmungen. Latent vorhandene Zweifel über die Sicherheit unserer Nahrungsmittel werden wieder aktiviert (ALVENSLEBEN, 2002C; RÖHR ET AL., 2002B).

Der Einschätzung der Experten, dass die heutigen Haltungssysteme Technopathien fördern, kann in der Literatur belegt werden (vergl. DLG, 2000; BESTMAN, 2002; MCADIE u. KELLING, 2002; u.a.). HUNTER ET AL. (2001) bestätigen, dass die heutigen Haltungsformen von Mastschweinen und -rindern Kannibalismus und Schwanzbeißen fördern. Diese Verhaltensstörungen werden zum Teil durch Eingriffe am Tier reduziert, was aus der Sicht des Tierschutzes kritisch zu hinterfragen ist (BMVEL, 2002).

Die Experten vermuten überwiegend, dass ein Verbot von Maßnahmen wie Enthornen, Kupieren oder Schnabelkürzen in der Zukunft kommen wird. Dies wird an die heutigen Haltungsbedingungen enorme Anforderungen stellen. Nach dem Bericht der BEZIRKSREGIERUNG WESER-EMS (2005) kann unter den heutigen Produktionsbedingungen und dem Einsatz der heutigen Genetiken in der Putenmast nicht auf das Schnabelkürzen verzichtet werden. Aber auch in der Schweine- und Rinderhaltung müssen, zur Verringerung der Verhaltensstörungen, die Haltungsbedingungen verändert werden. Nach HUNTER ET AL. (2001) ist das Schwanzkürzen zwar eine wirksame Methode gegen Schwanzbeißen, aber durch solche Maßnahmen dürfen keine Haltungsdefizite ausgeglichen werden. Genau wie die Experten nennen BARTUSSEK (2001), HUNTER ET AL. (2001) und BADERTSCHER (2003) 
ausreichende Beschäftigungsmöglichkeiten, ein gutes Stallklima, optimale Futterzusammensetzung als Vorbeugemaßnahmen gegen Schwanzbeißen.

Allerdings haben nicht nur die Betriebe der konventionellen Tierhaltung Probleme mit Verhaltensstörungen. BESTMAN u. WAGENAAR (2003) haben in Untersuchungen festgestellt, dass auch in Bio-Betrieben, bei denen die Tiere z.B. in kleineren Herden gehalten werden, die Besatzdichten geringer sind und die Tiere über Auslauf im Freien verfügen, ebenfalls Probleme mit Federpicken haben.

Standards für Haltungsbedingungen werden sowohl vom Gesetzgeber als auch im Rahmen von freiwilligen Vereinbarungen vorgeschrieben bzw. vorgegeben (vergl. DLG, 2000; BEZIRKSREGIERUNG WESER-EMS, 2005; BMVEL, 2005B; QS, 2005G u.a.). Staatliche Kontrollen werden durchgeführt, können aber aufgrund der Vielzahl an landwirtschaftlichen Betrieben nur einen kleinen Teil abdecken. Die Forderung der Experten nach mehr Kontrollen erfolgt zurzeit hauptsächlich durch die Umsetzung und Etablierung von Qualitätsmanagementsystemen in der Urproduktion. Zum Beispiel wurden im Rahmen von QS über 50.000 landwirtschaftliche Betriebe (Stand 01.09.2005) überprüft. Ebenso ist davon auszugehen, dass sich die Kontrolle der Tierschutzregelungen, ab dem 01.01.2007 im Rahmen von Cross Compliance, positiv auf den Kontrollumfang auswirken wird.

Insgesamt müssen Maßnahmen gegen Verhaltensstörungen konsequenter umgesetzt werden. Hierbei ist zu beachten, dass diese nicht ausschließlich aufgrund der Haltungsbedingungen entstehen, sondern zum Teil auch genetischen Ursprungs sind. Zum Beispiel stellen KJAER ET AL. (2001) fest, dass Federpicken durch gezielte genetische Selektion vermindert werden kann. Ebenso stellen MCAdIE u. KELLING (2002) und BestMAN u. WAGENAAR (2003) u.a. fest, dass die Ursache für Federpicken auch genetisch bedingt ist. Nach WICKE ET AL. (2001) und BIZERAY ET AL. (2002) beruhen Beinschäden beim Geflügel zum größten Teil auf züchterischen Entwicklungen in Richtung schnellen Wachstums. Verstärkt werden die Beinschäden allerdings auch durch die intensiven Haltungsformen.

Interessant ist, dass die Experten aufgrund der ethischen Relevanz bei Enthornen, Kupieren oder Schnabelkürzen von einem Verbot ausgehen, in einem Beibehalten der Kastration aber kein Problem gesehen wird. Alle diese Eingriffe lösen nachweislich Schmerzempfindungen bei den Tieren aus (FAULKNER u. WEARY, 2000; TAYLOR ET AL., 2001; HAY ET AL., 2003; HESTER u. SHEA-MOORE, 2003 u.a.). Nach §5 Abs. 1 des Tierschutzgesetzes ist aber an einem Wirbeltier ein mit Schmerzen verbundener Eingriff verboten. Allerdings konnte im Rahmen der Befragung nicht festgestellt werden, ob sich die Bewertung der Kastration auf die heutigen Bedingungen (ohne Betäubung) bezieht oder ob zukünftig von einer Betäubung ausgegangen wird. Die Alternativen zur herkömmlichen Kastration werden allerdings von den Experten genauso negativ bewertet wie die Kastration.

In anderen Ländern werden schon Alternativen zur Kastration ohne Betäubung angewendet. Nach LUY u. HILDEBRAND (2003) werden in Irland, Großbritannien, Portugal und Spanien die Ferkel fast gar nicht mehr kastriert. Allerdings ist nach BAUMGARTNER ET AL. (2004) und der EFSA (2004) in diesen Ländern auch die Abneigung gegen den Ebergeruch wesentlich geringer. Eine Schlachtung der Eber vor der Geschlechtsreife hätte, wie von den Experten angemerkt, wirtschaftliche Konsequenzen. Die Tiere wären leichter und der Ertrag aus den Schlachttieren geringer. Eine Immunokastration wird nach BAUMGARTNER ET AL. (2004) schon in Australien und Neuseeland in der Praxis angewendet. Hierbei ist DUNSHEA ET AL. (2001), ebenso wie die Experten, der Meinung, dass ein solches Verfahren in Hinblick auf 
den Verbraucher eher kritisch zu sehen ist. Zwar erleiden die Tiere keine Schmerzen, ihnen wird aber ein Serum gespritzt, dass das Wachstum der Hoden verhindert. Nach GUTZWILLER (2003) ist eine Kastration unter Lokalanästhesie unter Praxisbedingungen möglich und die Injektion kann von derselben Person durchgeführt werden, die das Ferkel hält. Allerdings dauert die gesamte Kastration aufgrund der Wirkungszeit des Lokalanästhetikums doppelt so lange wie ohne Betäubung.

Vor allem wirtschaftliche Aspekte spielen eine Rolle, warum weiter an der heutigen Kastrationsmethode festgehalten wird. Aus Tierschutzgründen ist ein Beibehalten der Kastration ohne Betäubung allerdings zu überdenken. Alternativen müssen unter Berücksichtigung des Tierschutzgedankens, der Wirtschaftlichkeit und der Bedenken der Verbraucher wissenschaftlich bewertet werden. Eindeutige Aussagen zu den Alternativen fehlen für den deutschen Markt zurzeit noch. Da in Deutschland der Tierschutz einen immer größeren Stellenwert erhält, ist zu vermuten, dass ein Kastrationsverbot ohne Betäubung in Deutschland eingeführt werden wird.

Gerade bei tierischen Futtermitteln und Risikomaterialien, die mit BSE in Verbindung stehen, zeigt sich die Diskrepanz zwischen Verbrauchereinstellung und wissenschaftlicher Einschätzung. Die meisten Experten sehen keine Gefahr durch tierische Futtermittel. Hierbei muss aber angeführt werden, dass die Einschätzung unter der Voraussetzung getroffen wurde, dass die Futtermittel von BSE getesteten und für den menschlichen Verzehr freigegebenen Tieren stammt, bei denen das Risikomaterial ordnungsgemäß entfernt und entsorgt wurde. Ebenso wie die Experten halten auch MÄNNER und SIOMON (2001) und KAMPHUES (2004) Tiermehl, unter den oben beschriebenen Bedingungen, für unbedenklich. Eine sichere Trennung zwischen der Rinder- und Schweinefutterproduktion bzw. Fütterung ist nach den Experten aber eine notwendige Voraussetzung. Diese sichere Trennung zwischen der Rinder- und Schweinefutterprofuktion bzw. Fütterung wird auch von MÄNNER u. SIMON (2001) und KAMPHUES (2004) verlangt. Probleme bei einer Wiederzulassung von Tiermehlen werden von den Experten in erster Linie in der Kommunikation gesehen.

Vergleicht man die Art und Weise, wie die Diskussionen über den Einsatz von Tiermehlen in der Fütterung geführt werden, mit der Risikowahrnehmung und -bewertung von JUNGERMANN u. SOLVIC (1993) und ALVENSLEBEN (1999A) so wird schnell deutlich, dass die meisten der dort angesprochenen Kriterien, die für eine Akzeptanz notwendig sind, nicht erfüllt werden. Dies betrifft vor allem die Elemente Freiwilligkeit, Kontrollierbarkeit, Verantwortlichkeit und die Undeutlichkeit von Risiken, die alle nicht erfüllt werden (vergl. Kapitel 2.1.3, 2.2.1). Hinzu kommt nach ALVENSLEBEN (1999A), dass der Verbraucher durch das Überangebot an Lebensmitteln gesättigt ist und dadurch wesentlich kritischer auf Abweichungen von seinen Vorstellungen reagiert. Dieses hängt mit der wachsenden Entfremdung von der heutigen Landwirtschaft und den dadurch hervorgerufenen Wahrnehmungsverzerrungen zusammen. Unterstützt wird diese Entwicklung durch Medienberichte, die einzelne Ereignisse verstärken und damit insgesamt dazu beitragen, dass das Grundvertrauen sinkt. Deshalb zeigen die BSE-Krise und vor allem der Einsatz von tierischen Futtermitteln alle klassischen Momente, die nach ALVENSLEBEN (1999B) zu einer zunehmenden Verbraucherverunsicherung führen (vergl. auch Kapitel 2.2.1).

Aber auch die ethischen Bedenken werden durch den Einsatz von Tiermehlen stark angesprochen (VAN DEN DAELE, 1993). Inwieweit aber eine offene Kommunikation diesen Teufelskreis durchbrechen könnte, ist im Rahmen dieser Arbeit nicht zu beurteilen. 
Positiv an einer Wiederzulassung von Tiermehlen in der Fütterung von Nichtwiederkäuern sehen die Experten die Verbesserung der Versorgung der Tiere. BERK u. SCHULZ (2000) und BRAUNGART ET AL. (2002) sehen positive Effekte vor allem in der Eiweiß- und Aminosäurenversorgung und HERTER ET AL. (2001), BRAUNGART ET AL. (2002) und RODEHUTSCORD (2002) in der Phosphorversorgung.

Ebenso wie in der Fütterung sehen die Experten auch in der alternativen Entsorgung von tierischen Futtermitteln kaum eine Gefahr. Allerdings kann nach heutigem, wissenschaftlichen Stand eine Gefahr nicht 100\%tig ausgeschlossen werden. Das BFR (2005A) beurteilt z.B. die Gefahr, dass BSE-Erreger in die Nahrungskette gelangen als sehr gering, schließt es aber nicht aus. Ebenso sehen HEESCHEN (2004) und HERTER ET AL. (2004) eine Möglichkeit des Eintrags durch Dünger. Ein anderer wichtiger Eintragsweg ist, laut der Experten, die Verschleppung innerhalb der Futterproduktion. Dieser Weg wird auch in der Literatur kritisch gesehen (KIENZLE ET AL., 2004). Aus diesem Grund sollten bei einer Wiederzulassung tierischer Futtermittel nicht nur getrennte Produktionswege gefordert werden, sondern auch ein generelles Produktionsverbot von Wiederkäuerfutter und Nichtwiederkäuerfutter in einem Werk vorgeschrieben werden. Ebenso dürfen Tierhalter, die Wiederkäuer und Monogastraden halten, kein Tiermehl enthaltenes Futter einsetzen. Wogegen allerdings alle Kontrollen und Vorschriften relativ machtlos sind, ist der bewusste, kriminelle Einsatz von Tiermehl. In solchen Fällen ist es wichtig, zu kommunizieren, dass es sich um Einzelfälle handelt.

\subsection{Umgang mit dem lebenden Tier vor der Schlachtung}

Trotz umfangreicher Standards kommt es immer wieder zu Negativmeldungen über Tiertransporte. Aufgrund des hohen Einflusses auf die Produktqualität sollten alle Beteiligten daran interessiert sein, den Transport so schonend wie möglich durchzuführen.

Genau wie von den Experten werden auch von der Politik und den Tierschutzverbänden strengere und umfassendere Regelungen für den Tiertransport gefordert. Die Forderungen beziehen sich auf Transportzeiten, Wartezeiten, erlaubte Zuladungen, Ausstattung der Fahrzeuge sowie die Qualifikation des Personals. Während die Experten die Be- und Entladevorgänge und das Klima für besonders kritisch halten, sehen die Studenten in der Transportzeit ein größeres Risiko. Lange Transportzeiten werden auch vom Verbraucher (Einschätzung der Experten) als tierschutzwidrig eingestuft und abgelehnt.

Innerhalb der EU sind relativ umfangreiche gesetzlichen Regelungen für den Transport von Tieren verfasst worden. Nach WERNER ET AL. (2005) werden diese aufgrund von Meinungsverschiedenheiten zwischen den einzelnen EU-Ländern in naher Zukunft jedoch noch nicht in Kraft treten. Uneinigkeit besteht vor allem bezüglich der Transportzeiten für Tiere. Etwa die Hälfte der Länder will die Transportzeiten auf acht Stunden begrenzen. Die anderen Länder möchten die bestehenden Regelungen beibehalten (ANONYMUS, 2004C). Uneinig sind sich auch die Experten bei der Entwicklung der Transportzeiten bis zum Jahr 2015. Zwar geht der überwiegende Teil der Experten von einer Abnahme der Transportzeiten aus. Aber immerhin gehen 29\% der Experten von einer Zunahme der Transportzeiten aus.

Die Beurteilung, der Experten dass die Klimabedingungen im Fahrzeug einen starken Einfluss auf die Zahl der Transporttoten und die Fleischqualität haben, werden von TROEGER ET AL. (1999), DEWEY ET AL. (2004) und NIJDAM ET AL. (2004) bestätigt. In der EU sind 1999 Standards für Klimabedingungen bei Tiertransporten erarbeitet worden. Forderungen nach 
klimatisierten Fahrzeugen, für Tiertransporte, sind noch nicht vorhanden, gehen aber in diese Richtung. Die Belüftungssysteme sollten in der Lage sind, die Klimabedingungen während des gesamten Transportes so zu gestalten, dass die festgelegten Grenzwerte für die jeweilige Tierart nicht überschritten werden.

Die Notwendigkeit einer Verkürzung der Transportzeit kann in der vorliegenden Arbeit nicht beantwortet werden. Ein Einsatz von klimatisierten Fahrzeugen bei langen Strecken und extremen Witterungen ist aus Gesichtspunkten des Tierschutzes zu fordern. Allerdings darf bei der Diskussion nicht vergessen werden, dass der Anteil an Transporttoten in den letzten Jahren kontinuierlich gesunken ist und bei unter einem Prozent liegt (AlvarEZ, 1981; BAYLISS u. HINTON 1990; GREgORY u. AUSTIN, 1992; DANISH, 2004; WERNER ET AL., 2005). Solange Tiertransporte nicht unter extremen Bedingungen (Hitze, Kälte, Schwüle) erfolgen und auch die anderen Parameter wie Ladedichte, Fahrstil und Umgang mit den Tieren optimal befolgt werden, sind die heutigen Belüftungssysteme ausreichend.

Der Umgang mit den Tieren bei den Transporten sowie bei der Lebendviehhandhabung auf dem Schlachthof werden als einer der kritischsten Punkte durch die Experten genannt. Hierbei ist der unsachgemäße Umgang mit den Tieren sowohl aus der Sicht des Verbrauchers als auch aus Tierschutzgründen und in Bezug auf die Produktqualität kritisch zu betrachten. Ein hohes ethisches Potenzial im Umgang mit den Tieren und einen wesentlichen Einfluss auf die Produktqualität sehen auch WOLTERSDORF (1994), TROEGER ET AL. (1998), LAMBOOIJ (2000) und GRANDIN (2004). Unumstritten ist, dass der Treiber, durch sein Verhalten einen entscheidenden Einfluss auf die Belastung der Tiere ausübt. Um diese Belastungen möglichst gering zu halten, müssen die Treiber über umfangreiche ethologische Kenntnisse und praktische Erfahrungen verfügen (FISCHER, 1994). Nach Meinung der Experten ist der Sachkundenachweis für Personen, die lebende Nutztiere handhaben, zwar eine wichtige Voraussetzung für den sachgemäßen Umgang mit den Tieren. Sie sehen hier aber noch Handlungsbedarf. Um den Umgang mit den Tieren zu verbessern, werden regelmäßige und auch praktische Schulungen gefordert. Sowohl bei den Transporten als auch auf dem Schlachthof sehen TROEGER ET AL. (1998), Defizite im Umgang mit den Tieren. Mangelnde Erfahrungen oder Kenntnisse über das Fluchtverhalten der Tiere werden häufig mit massiven Treibvorgängen bzw. durch den Elektrotreiber als Treibhilfe ausgeglichen. Gerade der Elektrotreiber ist aus Tierschutzgründen und aufgrund seiner negativen Auswirkungen auf die Produktqualität kritisch zu sehen (FISCHER, 1994; GRANDIN, 1996; TROEGER ET AL., 1998). Ein komplettes Verbot des Elektrotreibers ist aber nach FISCHER (1994) auch nicht zu empfehlen. Wenn andere Treibhilfen versagen, ist der gezielte Einsatz des Elektrotreibers sicher als das kleinere Übel zu sehen.

Aber nicht nur Personalfehler führen zu einer Belastung der Tiere. Bauliche Mängel bei den Treibgängen und in den Ruhebereichen sowie hohe Lärmpegel führen, nach der Einschätzung der Experten, zu einer Belastung der Tiere. Die Stallanlagen auf dem Schlachthof sind allerdings erst in den letzten Jahren in das Interesse der Schlachthofbetreiber gerückt. Auf vielen Schlachthöfen sind schon umfangreiche Umbaumaßnahmen oder Neubauten durchgeführt worden, um den Stallbereich tiergerechter zu gestalten. Nach SCHÄFFER u. V. BORELL (2002) sind allerdings kaum objektive Kontrollkriterien bzw. Bewertungsverfahren für den Bereich der Lebendviehandhabung vorhanden. Nach der Meinung der Autoren fehlen z.B. einheitliche Grenzwerte für das Stallklima und den Lärmpegel. Über die Besatzdichte in den Ruhebuchten gibt es unterschiedliche Meinungen. Gerade laute und hochtönige Geräusche rufen nach GRANDIN (1996) häufig Meidereaktionen beim Tier hervor. 
Aber auch eine falsche Beleuchtung, Zugluft oder falsche gestaltete Treibwege hindern die Tiere häufig daran, sich in eine gewünschte Richtung zu begeben. Als ein geeignetes Überwachungskriterium zur Beurteilung des Umgangs mit den Schlachttieren sehen sowohl GRANDIN (2001A) als auch SCHÄFFER u. V. BORELL (2005) die Vokalisation der Tiere. Allerdings gilt die Vokalisation zurzeit noch nicht als Überwachungskriterium auf den Schlachthöfen. Auf vielen Schlachthöfen dürfte die Vokalisation der Tiere nur schwer festzustellen sein, da die Grundlautstärke im Wartebereich so hoch ist, dass eine klare Zuordnung von Geräuschen nicht erfolgen kann. Gerade bei der Lebendtierhandhabung ist auf den Schlachthöfen noch ein enormer Umdenkprozess notwendig. Denn nur durch eine Aufwertung des Bereichs der Lebendviehhandhabung lassen sich die Mängel beheben.

Aus der Sicht der Experten werden Betäubungsverfahren grundsätzlich vom Verbraucher kritisch beurteilt. Hierbei ist nicht das System entscheidend, sondern die ordnungsgemässe Durchführung der Betäubung. Bei einer unzureichenden Betäubung bzw. einer nicht sachgerechten Durchführung der Betäubung ist jedes Verfahren kritisch zu bewerten (falscher Ansatzpunkt der Elektroden, ungenügende Betäubungszeit bei $\mathrm{CO}_{2}$, falsche $\mathrm{CO}_{2}$ Konzentration usw.). Genau wie von den Experten werden in der Literatur weniger die Betäubungsverfahren an sich als kritisch gesehen als die Fehler, die bei einer nicht sachgemäßen Durchführung entstehen (VON HOLLEBEN ET AL., 2002; MACHOLD ET AL., 2003; LUY u. HILDEBRAND, 2004). Die einzelnen Betäubungsverfahren werden je nach Autor in der Literatur unterschiedlich beurteilt. Hierbei herrschen, wie es das Beispiel Schweinebetäubung besonders deutlich macht, unterschiedliche Ansichten darüber, welche der Methoden die bessere ist (HOLST, 2002; MACHOLD ET AL., 2003; SCHÄFFER u. V. BORELL, 2004B). Bei einer ordnungsgemäß durchgeführten Betäubung ist keine der Methoden als kritisch zu betrachten.

\subsection{Rückverfolgbarkeit}

Von den Experten werden auf allen drei Produktionsstufen Schwachpunkte in der Rückverfolgbarkeit gesehen. Die Rückverfolgbarkeit auf das einzelne Tier, wie von einzelnen Experten gefordert, wäre der Idealfall. Diese ist zwar anzustreben, aber nach dem BLL (2001 u. 2003) kann der Detaillierungsgrad in der Rückverfolgbarkeit immer nur ein Kompromiss sein. Eine Rückverfolgung auf den einzelnen Produzenten bei Futtermitteln oder auf das Tier ist aus Kostengründen und organisatorischen Gründen meistens nicht möglich. Am ehesten ist dieser Idealfall sowohl nach der Meinung der Experten sowie auch nach den Meinungen von AdAM (2001); EU-KOMISSION (2003A) und SCHÜTT-ABRAHAM (2003) auf der Stufe der Schlachtung zu erreichen. In den Stufen der Futtermittelproduktion und der Landwirtschaft sind dagegen wesentlich größere Schwierigkeiten vorhanden. Hier werden über die verschiedenen Handelspartner auf den Stufen kleine Teilmengen bzw. Tiergruppen zu größeren handelsfähigen Mengen bzw. Tiergruppen zusammengefasst, die nicht mehr dem Ursprungsbetrieb zugeordnet werden können. Diese Zusammenfassung stellt die Rückverfolgbarkeit und die Möglichkeit einen Produktrückruf durchzuführen, jedoch nicht grundsätzlich in Frage. Denn solange die Rücknahme oder der Rückruf der Chargen eines nicht sicheren Lebensmittels sichergestellt ist, obliegt es dem einzelnen Lebensmittelunternehmer, wie weit er seine Chargen eingrenzt. Nach dem BLL (2003) ist davon auszugehen, dass die Unternehmen allein aus betriebswirtschaftlichen Gründen, unter Berücksichtigung der betrieblichen Verhältnisse und technischen Machbarkeiten und einer 
Kosten-Nutzen-Abwägung das System der Rückverfolgbarkeit, so detailliert wie möglich gestalten.

Trotzdem sind gerade in der Umsetzung die Probleme sichtbar. Die Prozesse sind heute häufig vielschichtig. Grundvoraussetzung für eine sichere Rückverfolgbarkeit ist eine exakte Datenerfassung und Dokumentation. Hierbei müssen besonders die Schnittstellen (z.B. Übergang zwischen verschiedenen Verantwortungsbereichen) beachtet werden. Aus Sicht der Experten sind die Schwachstellen der Rückverfolgbarkeit eine schlechte oder ungenaue Datenerfassung und Dokumentation. Probleme treten zum Beispiel in der Futtermittelproduktion auf. Aufgrund von produktionstechnischen Bedingungen ist dort eine sehr detaillierte Rückverfolgbarkeit nicht immer möglich. Als Beispiel sei die Getreideerfassung genannt: Viele kleine Partien werden zu einer großen, handelbaren Charge zusammengefasst. Durch den Einsatz von praktikablen Systemen lassen sich viele Schwachstellen reduzieren. Eine Hilfe bei der Rückverfolgbarkeit bieten die bereits bestehenden Systeme, wie technische Kennzeichnungsmöglichkeiten und die Erfassung von biometrischen Daten (vergl. Kapitel 2.4.13). Dadurch lässt sich die Fehlerquote minimieren. Aus Kostengründen haben sich allerdings die technischen und biometrischen Lösungen in der Futtermittelproduktion und in der Landwirtschaft nur in wenigen Betrieben durchgesetzt.

Nach wie vor gibt es auf allen Stufen der Produktionskette Fleisch Probleme bei der Rückverfolgbarkeit. Systematische und nachvollziehbare Systeme etablieren sich erst langsam am Markt. Aber auch bei den Mitarbeitern fehlt häufig noch das Verständnis für die Notwendigkeit der Rückverfolgbarkeit und der damit verbundenen Dokumentation. Ein häufig angeführtes Argument für eine mangelhafte Dokumentation ist, dass die Datenerfassung zu zeitaufwendig ist. Hierbei und in Bezug auf die Fehlerquellen in der Dokumentation bieten die technischen Möglichkeiten sicher eine Hilfe. Bevor diese aber nicht finanziell interessant werden, wird die Dokumentation nach wie vor per Hand erfolgen. Um die Akzeptanz zu steigern ist es allerdings notwendig, möglichst einfache Systeme zu implementieren, damit der Dokumentationsaufwand für den Mitarbeiter handhabbar bleibt. Es ist häufig sinnvoller den Detaillierungsgrad zu reduzieren, um dafür aber schlüssige und nachvollziehbare Informationen zu erhalten. Allerdings wird der Detaillierungsgrad der Rückverfolgbarkeit in dem Maße steigern, wie es sich lohnt, automatische Systeme einzusetzen.

\subsection{Kontrollen}

Trotz Kontrollen kommt es immer wieder zu Skandalen. Sowohl die Experten als auch FEHLHABER (1999) und PÖCKER ET AL. (2004) halten eine Kombination aus innerbetrieblichen und staatlichen Kontrollen für notwendig. Bei der Diskussion über Kontrollen und dem Kontrollumfang darf aber nicht vergessen werden, dass sich Sicherheit nicht „erkontrollieren“ lässt (Fehlhaber, 2000; KANTELberG u. Dilly, 2002; PÖCKER ET AL., 2004). Einen positiven Einfluss, auf die von den Experten genannten Schwachstellen (zu geringer Kontrollumfang, mangelnde schlüssige Kontrollsysteme), haben die auf allen Stufen vermehrt an Bedeutung gewinnenden Qualitätsmanagementsysteme.

Zum Beispiel werden auf der Futtermittelstufe Rückstandskontrollen vorgeschrieben (QS, 2005B).Aber auch ein HACCP-System zur Risikoidentifikation war schon vor der gesetzlichen Forderung Voraussetzung für die Zertifizierungen nach QS oder GMP+. Nach Meinung der Experten können viele Risiken durch ein funktionierendes und systematisches 
HACCP-Konzept begrenzt werden. Diese Einschätzung entspricht dem neuen EuLebensmittelrecht, das ab dem 01.01.2006 in Kraft ist.

Trotz der positiven Einschätzung vom HACCP-System dürfen die zurzeit in einigen Firmen praktizierten HACCP-Konzepte nicht überbewertet werden. In den einzelnen Betrieben ist die Qualität der Umsetzung sehr unterschiedlich. Ein Teil der Betreibe hat für den eigenen Betrieb spezifische Risikoanalysen erstellt und lenkt seine Prozesse entsprechend der Analyse. In anderen Betrieben wird auf vorgefertigte Analysen für die jeweilige Branche zurückgegriffen, die die speziellen Risiken des eigenen Betriebs nicht oder nur unzureichend berücksichtigt. Ursachen für dieses Verhalten sind häufig ein mangelndes Wissen der Mitarbeiter über HACCP-Systeme. Gerade bei den innerbetrieblichen Kontrollen ist ein enormer Schulungsbedarf vorhanden. Dieser Schulungsbedarf bezieht sich zum einen auf das Erstellen entsprechender Risikoanalysen, zum anderen aber auch auf die Durchführung der Kontrollen selbst.

Auch sind nach AlVENSLEBen (1999A, 2000A) und MeLCHERS (2003) Qualitätsmanagementsysteme, Zertifizierungen und/oder Qualitätssiegel keine ausreichenden Instrumente um das Verbrauchervertrauen zu gewinnen. Sie sind nützliche Instrumente um das Risiko des Anbieters zu verringern, in negative Schlagzeilen verwickelt zu werden oder zur Rationalisierung der Erzeugung. 


\section{$7 \quad$ Schlussfolgerungen}

Die ermittelten Hauptrisikopunkte aus der Studie sind in allen drei Produktionsstufen identisch. Mangelnde Hygiene, mangelnde Qualifikation des Personals und teilweise Unkenntnis der Prozessabläufe führen immer wieder zu Risiken.

Zur Beherrschung von Risiken sind aber auch eine umfassende Dokumentation und Kontrollen notwendig. Gerade in den Stufen Landwirtschaft und Futtermittelwirtschaft gibt es Defizite. Hilfestellungen bei der Umsetzung von Dokumentationsanforderungen und Kontrollen bieten die Anforderungen der sich auf allen Stufen etablierenden Managementsysteme. In Verbindung mit einem systematischen und umfassenden HACCP-System lassen sich viele Risiken minimieren, wenn nicht sogar ausschalten. Über das HACCP-System hinaus muss gewährleistet sein, dass die Produzenten auf der Basis eines allgemeinen Qualitätssicherungssystems für die Einhaltung aller gesetzlichen Vorschriften sorgen.

Darüber hinaus sollte in der vollständigen Kette der Fleischproduktion ein wirksames Risikound Krisenmanagement aufgebaut werden. Beides sind Führungsaufgaben und erfordern ein schnelles und eindeutiges Handeln.

Krisen lassen sich bei einem so sensiblen Produkt wie Fleisch auch mit den umfassendsten Kontrollsystemen nicht vermeiden und werden immer wieder auftreten. Die Grundursache dafür ist u.a. auch die beachtliche opportunistische Triebkraft der Branche, die vor die Gesamtheit schädigenden Aktionen vielfach nicht zurückschreckt. Dies wird zusätzlich durch Kosteneinsparungen im öffentlichen Dienst indirekt gefördert. Jedoch haben Einzelfirmen, die mit geschlossenen Programmen arbeiten, durchaus die Möglichkeit, sich von einem generalisierten Krisenablauf abzukoppeln, wenn im Voraus Konzepte festgelegt werden, wie auf eine Unregelmäßigkeit zu reagieren ist. Ungeplante ad-hoc-Reaktionen führen zu widersprüchlichen Aussagen und unkoordiniertem Handeln, die den Eindruck der Unsicherheit eher noch verstärken.

Verbraucher reagieren auf alle Aspekte, die den Tierschutz (sachgemäßer Umgang mit den Tieren) betreffen, und auf Rückstände jeglicher Art empfindlich. Je nach Darstellung in den Medien kann dieses zu einem Meideverhalten führen. Hierbei ist nicht die reale Gefahr entscheidend, sondern die Bewertung erfolgt rein subjektiv. Dieses Verhalten wird vor allem durch eine wachsende Entfremdung zur Urproduktion gefördert.

Als Voraussetzung eines wirksamen Risikomanagements lassen sich aus der Auswertung wissenschaftlicher Literatur, den Ergebnissen der Befragung und Gespräche folgende Empfehlungen ableiten:

- In der Futtermittelproduktion ist es wichtig, die Verfahren weiter zu optimieren. Hierbei sollte vor allem ein Augenmerk auf die Trocknungstechnik und die Anlagensicherheit gelegt werden. An beiden Stellen herrscht in der heutigen Futtermittelproduktion Handlungsbedarf.

- Rückstände und Kontaminanten sind prinzipiell unvermeidbar. Eine Dokumentation aller eingesetzten Futterkomponenten sowie geeignete Monitoringsysteme zur Überwachung können das Risiko eindämmen. Probleme wird es weiterhin bei der Kommunikation von Rückständen geben. Die Diskussion bezüglich der Rückstände verläuft meistens sehr emotional und der Verbraucher ist schwer für wissenschaftliche Bewertungen zugänglich. An dieser Stelle ist enormer Handlungsbedarf vorhanden. 
- Fleischvermarkter haben keinen direkten Einfluss auf den Einsatz von gentechnisch veränderten Futtermitteln. Mit Aussagen, dass Tiere garantiert nicht mit genveränderten Futtermitteln gefüttert wurden, muss sehr kritisch umgegangen werden. Vor allem bei Mais und Soja kann deren Einsatz nicht ausgeschlossen werden.

- Für den Abnehmer empfiehlt es sich, darauf zu achten, dass die Futtermittelhersteller in den Ursprungsländern nach EU-Anforderungen und den Grundsätzen eines HACCPKonzeptes produzieren.

- Extreme Merkmalsausprägungen haben einen negativen Einfluss auf die Produktqualität und führen zu tierschutzrelevanten Problemen. Bei dem Einsatz von bestimmten Rassen und Kreuzungen sollte darauf geachtet werden, dass extreme Merkmalsausprägungen vermieden werden. Ebenso sollte die Produktqualität in der Zucht eine stärke Beachtung finden.

- Bis auf die Immunkastration spielen bei den alternativen Verfahren zur heutigen Kastration vor allem wirtschaftliche Aspekte eine Rolle. Aus Tierschutzgründen ist ein Beibehalten der Kastration ohne Betäubung allerdings zu überdenken. Alternativen müssen unter Berücksichtigung des Tierschutzgedankens, der Wirtschaftlichkeit und der Bedenken der Verbraucher wissenschaftlich bewertet werden. Da in Deutschland der Tierschutz einen immer höheren Stellenwert erhält, ist zu vermuten, dass ein Kastrationsverbot ohne Betäubung auch in Deutschland wahrscheinlich wird.

- Gentechnisch modifizierte oder geklonte Tiere werden in naher Zukunft nicht für die Fleischproduktion in Deutschland zur Verfügung stehen. Bei einem potentiellen Einsatz muss von einem Meideverhalten der Verbraucher ausgegangen werden. Selbst mit Hilfe wissenschaftlich fundierter Erkenntnisse über die Unbedenklichkeit solcher Produkte wird eine Akzeptanz nur schwer zu erreichen sein.

- Pathogene Keime in der Urproduktion gelten als Risiko für die gesamte Produktionskette. Die Überwachung durch entsprechende, auf Einzelprobleme abzielende Monitoringsysteme (z.B. Salmonellen, EHEC) wäre ideal. Aber auch durch schlüssige Hygienekonzepte könnte die Situation verbessert werden. Diese fehlen aber zurzeit noch in vielen landwirtschaftlichen Betrieben.

- Das Verbot von antimikrobiellen Stoffen als Leistungsförderer ist positiv zu bewerten, da Resistenzen gegen diese Stoffe zu einer Gefahr für den Menschen geworden sind. Das Verbot stellt allerdings hohe Anforderungen an die heutigen Tierhaltungssysteme und das Tiermanagement. Es müssen zwingend Maßnahmen erfolgen, die die Produktionsund Haltungsbedingungen verbessern, so dass eine Infektionsgefahr im Vorfeld verringert wird. Hierzu müssen vor allem umfangreiche Maßnahmen in der Urproduktion erfolgen.

- Die Diskussion bezüglich der Bestandsdichten einiger Gebiete in Deutschland ist sicher noch nicht beendet. Bei möglichen steigenden Seuchenfällen würde diese an Brisanz gewinnen. Ebenso wird, in Bezug auf die Seuchenproblematik, die Freilandhaltung eine wichtige Rolle spielen. Hier stellt sich die Frage, ob die vorhandenen Vorkehrungen ausreichend sind oder ob die Tiere im Falle einer möglichen Seuchengefahr tierschutzgerecht in Stallungen oder geschlossenen Systemen unterzubringen sind. Aber auch der weltweite Handel und ein ansteigender Tourismus bergen Risiken, die Seuchenausbrüche erleichtern. 
- Gerade bei der Diskussion um Impfungen im Seuchenfall treffen die ethischen und die wirtschaftlichen Erwägungen aufeinander. Unter Berücksichtigung der Themenstellung - Ermittlung der realen Risiken und der Punkte - die von Verbraucher kritisch gesehen werden, spricht vieles für ein Impfen der Tiere. Bilder von Massentötungen werden, solange es Alternativen gibt, beim Verbraucher immer Unverständnis auslösen. Man muss aber auch bedenken, dass Impfungen oder Prophylaxen nicht dazu eingesetzt werden dürfen, um Missstände im Tiermanagement auszugleichen. Sinnvoll sind Impfungen und Prophylaxen, die für die Gesunderhaltung der Tiere und zur Begrenzung der Seuchengefahr wichtig sind.

- Beim Einsatz von Fleisch von Tieren aus Freilandhaltung sollte beachtet werden, dass es namentlich bei Schwein und Geflügel zu hygienischen Problemen (Parasiten) kommt. Wenn diese auch nicht zu gesundheitlichen Konsequenzen für den Verbraucher führen müssen, entspricht dies nicht der „Verkehrserwartung“ bei solchen Produkten.

- Bei Importen von Tieren und Fleisch sollte auf die hygienischen Bedingungen in den Ursprungsländern bzw. Ursprungsbetrieben geachtet werden. Ggf. sollten Empfehlungen für Kontrollen und deren Umfang erfolgen. Hierzu ist auch die Prüfung der Zuverlässigkeit von Herkunftsnachweisen unerlässlich.

- Maßnahmen mit Tierschutzrelevanz müssen klar geregelt und kontrolliert sein. Hierzu sollten überprüfbare Bedingungen festgelegt werden, die sich auf das Alter, in dem diese Maßnahmen durchzuführen sind, auf die Zuständigkeit und Durchführungsbedingungen und auf die Schulung der für die Verrichtung Verantwortlichen beziehen. Besonders zu beachten sind die Kastration, das Kupieren, das Enthornen, das Schnabelkürzen, Tiertransporte und die Behandlung des Tieres vor der Tötung.

- Die Wiederzulassung von tierischen Futtermitteln wird zu Problemen führen, wenngleich diese sich auch auf die Versorgung monogastrischer Tiere positiv auswirken. Eine Vorreiterrolle ist in diesem Punkt für Fleischvermarkter nicht anzuraten.

- Ein Einsatz von klimatisierten Fahrzeugen bei langen Tiertransporten und extremen Witterungen ist aus Gesichtspunkten des Tierschutzes zu fordern. Allerdings darf bei der Diskussion nicht vergessen werden, dass der Anteil an Transporttoten in den letzten Jahren kontinuierlich sinkt. Solange Tiertransporte nicht unter extremen Bedingungen (Hitze, Kälte, Schwüle) erfolgen und auch die anderen Parameter wie Ladedichte, Fahrstiel und Umgang mit den Tieren optimal befolgt werden, sind die heutigen Belüftungssysteme ausreichend.

- Eine Aufwertung des Bereichs der Lebendviehhandhabung auf dem Schlachthof ist erforderlich. Messbare Überwachungskriterien für den ethisch unbedenklichen Umgang mit den Tieren vor der Schlachtung werden entwickelt. Die Messung der Vokalisation der Tiere ist hierzu geeignet. Allerdings dürfte auf vielen Schlachthöfen die Vokalisation der Tiere nur schwer festzustellen sein. Die Grundlautstärke in den Ställen ist so hoch, dass keine klare Zuordnung von Geräuschen erfolgen kann. Gerade bei der Lebendtierhandhabung ist auf den Schlachthöfen noch ein enormer Umdenkprozess notwendig.

- Bedarfsgegenstände werden als Risikopotenzial leicht außer Acht gelassen, können aber ebenfalls verborgene Risiken mit sich bringen. $\mathrm{Zu}$ beachten sind Verpackungsmaterialien, Gegenstände der Bearbeitung und das Umfeld der Lagerung. Bedarfsgegenstände dürfen nur aus geprüften Materialien hergestellt sein. 
- Auf allen Stufen sollten umfassende Hygienekonzepte existieren und darauf geachtet werden, dass das Personal regelmäßig geschult wird. Die Schulungen sollten die Grundlagen der Hygiene und der Produktionsverfahren sowie aktuelle Informationen über Risiken beinhalten. Ein wesentlicher weiterer Bestandteil von Hygienekonzepten ist eine systematische Schädlingsbekämpfung. Sowohl in der Futtermittelproduktion als auch in der Landwirtschaft sind hier Defizite vorhanden.

- Der Zukauf von Waren sollte aus Firmen erfolgen, die in branchenübergreifende Systeme integriert sind. Man muss aber darauf achten, dass die Kette durchgängig rückverfolgbar bis zum Futtermittelanbau ist. Dies gilt ganz besonders für Importfuttermittel, aber auch für Futterkomponenten, die in geringen Anteilen zugemischt werden. Kriterien, die über die gesetzlichen Forderungen hinausgehen, sollten genauso sorgfältig kontrolliert werden wie die gesetzlichen Vorgaben.

- Eine systematische und umfassende HACCP-Risikoanalyse sollte auf allen Stufen durchgeführt und umgesetzt werden.

- Auf allen Stufen werden sich der Hygienestatus, die Rückverfolgbarkeit und die Sicherheit der Produktionsbedingungen bis zum Jahr 2015 verbessern. Für die Vermarkter von Endprodukten ist es wichtig, an diesem Entwicklungsprozess richtungsweisend teilzunehmen. 


\section{$8 \quad$ Zusammenfassung}

Das Ziel der vorliegenden Arbeit ist zum einen die Identifikation und zusammenhängende Darstellung von Risiken in der Produktionskette Fleisch von der Futtermittelproduktion bis zum Ende der Schlachtung. Zum anderen sollen aber auch die Risiken, die vom Verbraucher als kritisch angesehen werden, identifiziert und dargestellt werden. Der Begriff Risiko beinhaltet daher sowohl physische, objektiv nachweisbare Risiken als auch subjektiv vom Verbraucher empfundene Risiken, die sich auf das Konsumverhalten auswirken können.

Zu diesem Zweck werden verschiedene Sachverhalte der gegenwärtigen Fachdiskussion und Fachliteratur dargestellt. Im Anschluss daran werden die Ergebnisse einer Expertenbefragung vorgestellt, in deren Verlauf 40 ausgewiesene Experten der Produktionskette Fleisch befragt wurden. Das Ziel der Befragung war die Ermittlung der wichtigsten Risikopunkte, die bei der Vermarktung von Frischfleisch zurzeit und in der Zukunft eine Rolle spielen werden. Zusätzlich wurden 64 Studenten des Fachbereichs Agrarwissenschaft befragt, die ausgewählte Themen einschätzen sollten.

Die Expertenbefragung erfolgte in zwei Stufen. In einer schriftlichen Befragung sollten die Experten bestimmte Sachverhalte und Statements beurteilen. Anschließend erfolgten auf der Basis der Fragenauswertung mündliche Interviews, in denen die Experten zu unklar gebliebenen Sachverhalten erneut Stellung beziehen sollten. Außerdem wurden die Experten aufgefordert, zu dem Entwicklungsstand bestimmter Risikopunkte Prognosen bis zum Jahr 2015 abzugeben.

Die Ergebnisse belegen, dass stufenübergreifende Defizite heute und auch in Zukunft eine wichtige Rolle spielen bzw. spielen werden. Hierzu zählen Hygiene einschließlich der Rückstände bei Futtermitteln und im Endprodukt, die Dokumentation auf allen Stufen und die Rückverfolgbarkeit von Futtermittelkomponenten und Endprodukten über die Stufen hinweg. Trotzdem gehen die Experten überwiegend von einem abnehmenden Problemdruck aus. Nur einzelne Punkte werden in Deutschland mittel- und langfristig weiter zu diskutieren sein: gentechnisch veränderte Organismen, der Einsatz tierischer Futtermittel in der Fleischerzeugung, die extreme Leistungszucht, importierte Erreger von Tierkrankheiten, einschließlich der Zoonosen, hygienische und toxikologische Kontrolle von Drittlandwaren und lange Tiertransporte. Die Lösung dieser Probleme dürfte vor allem mit vertikal integrierten Produktionssystemen zu erreichen sein. 


\section{$9 \quad$ Summary}

This work at hand has two aims. On the one hand it aims at identifying and presenting coherently the risks of the line of meat production from a scientific point of view. On the other hand it also aims at identifying and presenting those risks which might be considered as critical from the consumer's point of view and which might influence her or his behavior as a consumer. Therefore the term risk within the context of this work comprises both the scientifically verifiable risks as well as the risks which are only subjectively perceived by the consumer.

For this purpose many different circumstances and facts are presented in this work which is based upon the current scientific literature and discussion. Then this work presents the results of 40 interviews with expelled experts of the line of meat production. The interviews were aimed at defining the most important risks which have to considered in the field of fresh meat marketing. Additionally 64 students, who were studying agriculture at the university, were interviewed as well. They were supposed to evaluate certain topics from the their view.

The interviews of the experts were carried out in two steps. First the experts were asked to fill out questionnaires in which they had to evaluate several facts and statements. Their answers were used as the basic material for the second round of interviews which were carried out as oral interviews. In these interviews the experts were asked to evaluate facts and statements once more whose evaluation had remained unclear after the first round of interviews. Additionally they were asked to evaluate certain facts according to the development of their relevance until the year 2015.

The results prove that risks which occur on several levels of the line of meat production do not only play a particularly important role today but will continue to play an important in the future as well. These risks comprises the fields of hygiene including the problem of the residues in the fodder and in the end product, the documentation throughout the whole line of production and last but not least the traceability of the fodder components as well as the end products. Nevertheless the majority of experts assume that the pressure of most of these problems will be decreasing in the next few years. There are only a few points which will have to be discussed in the medium-range and long-range future. These risks comprise genetically engineered organisms, the usage of animal fodder in the line of meat production, the extreme high output breeding, the imported causes of animal diseases including the zoonosis, hygienic and toxic controls for products imported from countries of the Third World and animal transports which take a long time. The solutions of these problems should be primarily achieved by the implementation of vertical integrated production systems. 


\section{$10.1 \quad$ Literaturverzeichnis}

ADAM, F. (2001). Qualitäts-Management auch in der Schweineproduktion. SUS 3/2001: 1417.

AICHHOLZER, G. (2002). Das ExpertInnen-Delphi: methodische Grundlagen und Anwendungsfeld Technology Foresight. In: Das Experteninterview, Theorie, Methode, Anwendung. BOGNER, A.; LITTIG, B.; MENZ, W. (Hrsg.): Opladen 133-154.

ALTMANN, M.; KIRCHHEIM, U.; SCHÖBERLEIN, L.; WÄHNER, M.; WICKE, M.; FISCHER, K. (2005). PSE-Status bei marktkonformen Schweinen - Ergebnisse eines Monitorings in verschiedenen Schlachtbetrieben Deutschlands. Fleischwirtschaft 85 7/2005: 101104.

ALVAREZ, U. (1981). Diagnostische Erfahrungen bei der Anwendung des Geflügelfleischhygienegesetzes. Arch. Lebensmittelhygiene 32: 163 - 165.

ALVENSLEBEN, R. VON (1998A). Strategien zur Verbesserung des Image der Landwirtschaft. Quelle: www.uni-kiel.de/agrarmarketing/Lehrstuhl/HT.htm (aufgerufen am 05. 08.2005).

ALVENSLEBEN, R. VON (1998B). Risikowahrnehmung des Verbrauchers: Woraus resultiert die Verunsicherung? Schriftenreihe des Bundes für Lebensmittelrecht und Lebensmittelkunde e.V. 127: 28-43.

ALVENSLEBEN, R. VON (1999A). Die Fleischwirtschaft von morgen: Anforderungen von Gesellschaft und Verbrauchern. Lohmann Informationen 1/1999: 25-30.

ALVENSLEBEN, R. VON (1999B). Verbraucherakzeptanz von gentechnisch veränderten Lebensmitteln. Quelle: http://www.uni-kiel.de/agrarmarketing/Lehrstuhl/hst99.pdf (aufgerufen am 05.08.2005).

ALVENSLEBEN, R. VON (2000A). Was will der deutsche Verbraucher? Produzieren Deutschlands Bauern für den Markt?. Internationales Forum Agrarpolitik 1999. Bonn DBV.

ALVENSLEBEN, R. VON (2001A). Der landwirtschaftliche Zukunftsbetrieb in der EU. RWASommertagung am 28.6.2001 in Graz.

ALVENSLEBEN, R. VON (2001B). Agrarprodukte und Agrarmärkte in der Europäischen Union unter besonderer Berücksichtigung von Ökoprodukten, Tierschutzaspekten und Produkten aus der Region. 11. ZUFO-Umweltsymposium zum Thema „Neue Wege in der Landwirtschaft" am 26./27.11.2001. Münster.

ALVENSLEBEN, R. VON (2001C). Verbraucherverhalten. Referat aus der Sitzung der Arbeitsgruppe "Qualitätssicherung" der niedersächsischen Kommission "Zukunft der Landwirtschaft - Verbraucherorientierung" am 4.5.2001. Hannover.

ALVENSLEBEN, R. VON (2002A) Verbraucherbild - Verbraucherverantwortung - Verbraucher. Zeitschrift für Lebensmittelrecht 2/2002: 139-150.

ALVENSLEBEN, R. VON (2002B) Lebensmittelskandale und Ökoprodukte, Wie Verbraucher Qualität und Risiken wahrnehmen. Lohmann Informationen 4/2002: 27-35. 
ALVENSLEBEN, R. VON (2002C). BSE, Nitrofen und der Ökolandbau. „Grüne Illusionen“ in der Frankfurter Allgemeinen Zeitung Nr. 131 vom 10.06. 2002: 11.

ALVENSLEBEN, R. VON (2002D). Wie sieht der Verbraucher die Nutztierhaltung?. Analysen - Perspektiven - Konsequenzen Vortrag anlässlich der Einweihung des Fortbildungszentrums der Agrar- und Veterinär-Akademie (AVA) am 18.10. 2002. Horstmar-Leer.

ALVENSLEBEN, R. VON (2002E). Das Bild der Landwirtschaft in den Köpfen von Verbrauchern und Journalisten. Vortrag anlässlich der 546. Sitzung des Päsidiums des Deutschen Bauernverbandes am 07.10.2002 in Dinklage.

ALVENSLEBEN, R. VON (2002F). Neue Wege in der Tierhaltung Verbraucheransichten und -einsichten. Vortrag anlässlich der KTBL-Tagung am 10.04.2002 in Potsdam

ALVENSLEBEN, R. VON; KAFKA C. (1999). Grundprobleme der Risikokommunikation und ihre Bedeutung für die Land- und Ernährungswirtschaft. In: Schriften der Gesellschaft für Wirtschaft- u. Sozialwissenschaften des Landbaus e.V., Band 35: 57-64.

ANIL, M.; HARBOUR, D. (2001). Current stunning ang slaughter methods in cattle and sheep. Fleischwirtschaft 81 11/2001: 123-124

ANLAGE 5 FMV (2004). Grüne Broschüre, Das geltende Futtermittelrecht 15/2004.

ANONYMUS (2001). Schlußfolgerungen und Empfehlungen. Runder Tisch in Brandenburg zur Rinderkrankheit BSE. Ministerium für Landwirtschaft, Umweltschutz und Raumordnung: 52-60.

ANONYMUS (2002A). Wirtschaftliche und tierseuchenrechtliche Aspekte der letzten Maulund Klauenseuchenkrise in der EU. Quelle: http://www.europarl.eu.int/meetdocs/ committees/fiap/20020617/468130DE.pdf (aufgerufen am 05.08.2005)

ANONYMUS (2002B). Ein Plus für den Verbraucherschutz, Herkunftsnachweis mit kleiner Gewebeprobe fördert Lebensmittelsicherheit. Fleischwirtschaft 82 4/2002: 29.

ANONYMUS (2002C). Gegenüberstellung unterschiedlicher Leistungsmittler. Landwirtschaftsblatt Weser-Ems 29 vom 19. Juli 2002: 37.

ANONYMUS (2002D). Rinderschlachtung: Eine Alternative zum Bolzenschuss. Fleischwirtschaft 05/2002: 23-33.

ANONYMUS (2002E). Wirtschaftliche und tierseuchenrechtliche Aspekte der letzten Maulund Klauenseuchenkrise in der EU. DT\468130DE.doc, PE 315.459/rev. Europäisches Parlament Generaldirektion Wissenschaft Direktion A Abteilung Landwirtschaft, Regionalpolitik, Verkehr und Entwicklung: 3.

ANONYMUS (2003). Alle Beteiligten in der Verantwortung, Die EU Mitgliedstaaten sollen neue Leitlinien für die gute Transportpraxis erstellen. Fleischwirtschaft 83 8/2003: 16.

ANONYMUS (2004A). Der Unternehmer ist verantwortlich; Fragen und Antworten zu den zusammengefassten Hygieneanforderungen an Lebensmittel. Fleischwirtschaft 84 5/2004: 84-85.

ANONYMUS (2004B) Gentechnisch veränderte Lebensmittel. In: Brennpunkt Lebensmittelsicherheit 3/2004. Behrs Verlag.

ANONYMUS (2004C). Heftiger Streit um Tiertransportzeiten. Quelle: fleischwirtschaft.de, (aufgerufen am 23.01.2005). 
ANONYMUS (2004D). Agrobiodiversität entwickeln!, Handlungsstrategien und Impulse für eine nachhaltige Tier- und Pflanzenzucht. Positionspapier für eine nachhaltige Tierund Pflanzenzucht, Verbundprojekt „Agrobiodiversität entwickeln“. Quelle: www.agrobiodiversitaet.net (aufgerufen am 14.05.2005).

ANONYMUS (2004E). Der DOT-Code reicht bis zum Teilstück. Fleischwirtschaft 84 5/2004: 101-103.

ANONYMUS (2004F). Identifikation durch Retina-Scanning, Die Netzhaut als neue Lösung für die Identifikation und Nachverfolgung von Nutztieren. Fleischwirtschaft 84 7/2004: 17.

ANONYMUS (2004G). Mängel bei BSE Test. Food \& Hygiene 03/2004: 2.

ANONYMUS (2004H). BSE-Test-Skandal: Chronologie und Risikomanagement. Food \& Hygiene, 02/2004: 3.

ANONYMUS (2004I). Futtermittelsicherheit und Überwachung. Ernährungs- und agrarpolitischer Bericht 2004 der Bundesregierung, Teil C, Maßnahmen,: 55-56. Quelle: http://www.kompetenznetze.de (aufgerufen am 01.11.2005).

ANONYMUS (2005). Informationsbroschüre für die Empfänger von Direktzahlungen über die anderweitigen Verpflichtungen (Cross Compliance). Niedersächsisches Ministerium für den ländlichen Raum, Ernährung, Landwirtschaft und Verbraucherschutz.

AUMANN, J. VERREET, J. (2002). Mykotoxine in der Pflanzenproduktion - der Einfluss der Produktionsfaktoren. In: Quasi, erster Arbeitsbericht der Arbeitsgruppe Lebensmittelqualität und -sicherheit der Agrar- und Ernährungswissenschaftlichen Fakultät der Christian-Albrechts-Universität zu Kiel, vorgelegt am 01. Februar 2002: 25-33. Quelle: http://www.quasi.uni-kiel.de/download/Bericht_QUASI_2002.pdf (aufgerufen am 15.05. 2004).

BAARS, S. (2003). Deutsche Gesellschaft für Züchtungskunde. Eine Herausforderung an Wissenschaft und Praxis. Tiergerechte Haltung und sichere Produkte. Quelle: www. innovations-report.de (aufgerufen am 15.05.2004).

BADERTSCHER, R. (2003). Tierwohl und Wirtschaftlichkeit: ein Widerspruch?. Beitrag zur GEWISOLA 2003 in Hohenheim, 43. Jahrestagung der GEWISOLA.

BANDICK, N. (2003). Fleischgewinnung unter dem Gesichtspunkt der BSE. Fleischwirtschaft 83 7/2003: 107-108.

BARTH, R.; BILZ, M.; BRAUER, R.; CLAUSEN, J.; DROSS, M.; HEINECKE, C.; IDEL, A.; ISELE, J.; KOHLSCHÜTTER, N.; MATHES, M.; MEYER, A.; PETSCHOW, U.; VÖGEL, R.; WALTER, S.; WISSEN, M.; WOLFF, F.; WUNDERLICH, U. (2004). Agrobiodiversität entwickeln!, Handlungsstrategien für eine nachhaltige Tier- und Pflanzenzucht. Institut für ökologische Wirtschaftsforschung, Öko-Institut, Schweinsfurt-Stiftung, Freie Universität Berlin, Landesanstalt für Großschutzgebiete.

BARTON-GADE, P.; VON HOLLEBEN, K.; VON WENZLAWOWICZ, M. (2001). Animal welfare and controlles atmosphere stunning (CAS) of poultry using mixtures of carbon dioxide and oxygen. World's Poultry Science Journal 57: 189-200.

BARTUSSEK, H. (2001). Möglichkeiten zu geeigneter Beschäftigung von Schweinen. BAL Gumpensteiner Bautagung 2001, "Stallbau - Stallklima - Verfahrenstechnik": 49-57. 
BAUMGARTNER, J.; BINDER, R.; HAGMÜLLER, W.; IBEN, C.; SCALA, U.S.; WINCKLER, C. (2004). Aktuelle Aspekte der Kastration männlicher Ferkel 2. Mitteilung: Alternativmethoden zur chirurgischen Kastration und zusammenfassende Bewertung, Wiener Tierärztliche Monatsschrift 8/2004.

BAUMGARTNER, W. (2001). Tierseuchen- Information zur MKS, Maul- und Klauenseuche (MKS; Foot-and-mouth disease, FMD). Quelle: http://www.vu-wien.ac.at/PH/MKS.htm (aufgerufen am 05.08.2005).

BAYLISS, P.A.; HINTON, M.H. (1990). Transportation of broilers with special references to mortality rates. Appl. Anim. Behav. Sci. 28: 93 - 118.

BDF, Bundesverband Deutscher Fleischrinderzüchter und -halter (2003). Statistischer Jahresbericht, 2003: Verteilung der Herdbuchtiere nach Rassen. Quelle:http://www.bdf-web .de/Inhalte/Statistiken/JB2003/Verteilung_Hbt_Rassen.pdf (aufgerufen am 17.05.05).

BECKER, T. (1999). Gentechnik und Verbraucher. Landinfo 3/1999: 20-26. Ministerium ländlicher Raum. Baden-Württemberg

BENNER, E. (2004A). Staatliche Pull-Strategien und Verbraucherverhalten - Zur staatlichen Förderung des ökologischen Landbaus; Online-Fachzeitschrift "Ländlicher Raum" 1/2004. Quelle: http://www.laendlicherraum.at/ (aufgerufen am 05.08.2005).

BENNER, E. (2004B). Grenzen einer umwelt- und qualitätsorientierten Gestaltung der Gemeinsamen Agrarpolitik. GEWISOLA, Vortrag anlässlich der 44. Jahrestagung der GEWISOLA vom 27.-29. Sep. 2004 . Quelle: www.gewisola.de (aufgerufen am 15.8. 2004).

BERG, E.P. (1998). Critical Points Affecting Fresh Pork Quality within the Packing Plant. Quality \& Safety. Quelle: www.meatscience.org/Pubs/factsheets/qscritpointspack.pdf (aufgerufen am 17.10.2005).

BERG, E.P. (2001). Running Head: Effect of stress on meat yield and quality, Influence of stress on composition and quality of meat, poultry and meat products. University of Missouri, Columbia, MO 65211, Quelle: http://www.fass.org/fass01/pdfs/Berg.pdf (aufgerufen am 10.01.2005).

BERGMANN K. (2000). Der verunsicherte Verbraucher: Neue Ansätze zur unternehmerischen Informationsstrategie in der Lebensmittelbranche. Heidelberg: Springer Verlag.

BERGS, C. (2004). Perspektiven der landwirtschaftlichen Klärschlammverwertung. Rostock 23.09.2004. Bundesministerium für Umwelt, Naturschutz und Reaktorsicherheit Referat WA II 4 Siedlungsabfälle, Quelle: http://www.bwkbund.de/kongresse/bundeskongress 2004/Bergs_bwk -23-09-2004.pdf

BERK, J.; COTTIN, E. (2003). Einfluss von Herkunft und Haltungsformen auf Tiergesundheit, Tieraktivität und Tibiale Dyschondroplasie (TD) bei männlichen Puten. In: FAL-Jahresbericht, 2003: 102-103.

BERK, A.; SCHULZ, E. (2000). Ernährungsphysiologische Bewertung von Tiermehlen, Dietary and physiological evaluation of meat and bone meal. Institut für Tierernährung, Bundesforschungsanstalt für Landwirtschaft (FAL). Quelle: http://www.stn-vvtn.de/ archiv /berk.pdf. (aufgerufen am 02.08.2005). 
BERTLING, L. (2006). Vier Jahre Zeit für die Einführung. EU Kommission hat Übergangsregelungen für die Verordnungen des Hygienepakets festgelegt. Fleischwirtschaft 86 4/2006: 47-48.

BESTMAN, M. (2002). Betriebsmanagement verhindert Federpicken - Erfahrungen aus den Niederlanden. Louis Bolk Institut. Berater-Rundbrief 3/2003. Stiftung Ökologie \& Landbau, 17: 19

BESTMAN, M.; WAGENAAR, J.P. (2003). Farm level associated with feather pecking in organic laying hens. Livestock Production Science 80: 133-140.

BEZIRKSREGIERUNG WESER-EMS (2005). Tierschutzrelevante Mindestanforderungen für die intensive Putenmast. Bezirksregierung Weser-Ems -Tierschutzdienst Niedersachsen-. Quelle: http://cdl.niedersachsen.de/blob/images/C12602914_L20.pdf (aufgerufen am 10.04.2006).

BFR (2003A). Antibiotika, Im Tierbestand restriktiv einsetzen. Fleischwirtschaft 83 6/2003: 13.

BFR (2003B). Erste Ergebnisse des Internationalen Symposiums über Risikoanalyse der Antibiotikaresistenz. Bericht des BfR vom 13.11.2003. Quelle: http://www.bfr.bund.de/ cm/232/erste_ergebnisse_des_internationalensymposiums_ueber_risikoanalyse_der_ antibiotikaresistenz.pdf (aufgerufen am 20.03.2005)

BFR (2003C). Antibiotika-Resistenz bei Keimen in der Fleischproduktion zu hoch. Bericht des BfR vom 01.04.2003. Quelle: http://www.bfr.bund.de/cms5w/sixcms/detail.php/2040 (aufgerufen am 05.04.2006)

BFR (2005A). Knochenfragmente in Zuckerrübenschnitzel. Quelle: http://www.bgvv.de/cm /208/knochenfragmente_in_zuckerruebenschnitzeln.pdf (aufgerufen am 03.08.2005).

BFR (2005B). Geflügelschlachthygiene: Anpassen an die Gegebenheiten vor Ort. Fleischwirtschaft 85 2/2005: 29.

BGVV (1998) Alte Keime - neue Risiken. BGVV Pressemitteilungen 14/1998, 12.06.1998.

BGVV (2002A). Zusammenfassende gesundheitliche Bewertung von Nitrofen, Stellungnahme des BgVV vom 6. Juni 2002. Quelle: http://www.bgvv.de/cm/218/ zusammenfassende_gesundheitliche_bewertung_von_nitrofen.pdf, (aufgerufen am 05.08.2005).

BGVV (2002B). Acrylamid in Lebensmitteln - ernstes Problem oder überschätzte Gefahr?. Ergebnisse einer Informationsveranstaltung des BgVV am 29. August 2002. Quelle: http://www.bfr.bund.de/cm/208/acrylamid_in_lebensmitteln_ernstes_problem_oder_ue berschaetzte_gefahr.pdf (aufgerufen am 05.08.2005).

BIDNER, B.S (2003). Factors Impacting Pork Quality And Their Relationship To Ultimate pH. Ph.D. Thesis. Department of Animal Science, University of Illinois at UrbanaCampaign, IL.

BIHAN-DUVAL, E.LE (2004). Genetic variability within and between breeds of poultry technological meat quality; Genetische Unterschiede zwischen und innerhalb von Linien in technologischen Kriterien der Geflügelfleischqualität. World's Poultry Science Journal, Vol. 60, Nummer 3: 331-339. 
BINDER R.; HAGMÜLLER, W.; HOFBAUER, P.; IBEN, C.; SCALA, U.S.; WINCKLER, C.; BAUMGARTNER, J. (2004). Aktuelle Aspekte der Kastration männlicher Ferkel - 1. Mitteilung: tierschutzrechtliche Aspekte der Ferkelkastration. Wien. Tierärztl. Mschr. 91: 178-183.

BINDER R.; HAGMÜLLER, W.; HOFBAUER, P.; IBEN, C.; SCALA, U.S.; WINCKLER, C.; BAUMGARTNER, J. (2004). Aktuelle Aspekte der Kastration männlicher Ferkel - 2. Mitteilung: Alternativmethoden zur chirurgischen Kastration und zusammenfassende Bewertung. Wien. Tierärztl. Mschr. 91: 198-209.

BISPING, W. (1993). Salmonellen in Futtermitteln. Deutsche tierärztliche Wochenschrift 100: 262-263.

BIZERAY, D.; ESTEVEZ, I.; LETERRIER, C.; FAURE, J.M. (2002). Influence of increased enviromental complexity, performance, and level of fearfulness in broilers. Poultry Science 81: 767-773.

BLAHA, T. (1993). Die Ausbreitungsdynamik von Salmonellen in Tierbeständen. Deutsche tierärztliche Wochenschrift 100: 262-263. Berlin München.

BLAHA, T. (2001). Salmonellenbekämpfung beim Schwein. Quelle: http://www.vetline.de/ download/vkn_01_11.pdf (aufgerufen am 05.08.2005).

BLAHA, T. (2004). Wohlstand verändert die Sicht auf Landwirtschaft und Ernährung. Nutztierpraxis Aktuell 9, 6/2004.

BLL (2001). Leitfaden Rückverfolgbarkeit, Die Organisation der Rückverfolgbarkeit von Produkten in der Lebensmittelkette. Bund für Lebensmittelrecht und Lebensmittelkunde. Bonn.

BLL (2003). Stellungnahme zu den rechtlichen Vorgaben im Hinblick auf das Gebot der Rückverfolgbarkeit in Artikel 18 der Verordnung (EG) Nr. 178/2002. Bund für Lebensmittelrecht und Lebensmittelkunde e.V., Quelle: www.bll-online.de (aufgerufen am 26.07.2005).

BLÜTHGEN,A.; RUOFF, U.; TEUFEL, P. (2000). Abschätzung und Steuerung des Risikos für den Verbraucher. Forschungsreport 2/2000: 25-27.

BMU (2001) Bundesministerium für Umwelt, Naturschutz und Reaktorsicherheit. Höchstgehalte für Dioxine in Lebensmitteln. Mitteilung des Bundesministerium für Umwelt, Naturschutz und Reaktorsicherheit vom 06.12.2001.

BMVEL (1999). Gutachten zur Auslegung von $\S 11 \mathrm{~b}$ des Tierschutzgesetztes (Verbot von Qualzüchtungen), erschienen am 02.06.1999

BMVEL (2001). Memorandum der Bundesrepublik Deutschland zur zukünftigen MKSVorbeuge- und Bekämpfungspolitik. Quelle: http://www3.verbraucherministerium.de/ index-BFFF935801414141B526525A4603338B.html (aufgerufen am 05.08.2005).

BMVEL (2002). Zukunft der Tierhaltung, Bericht der gleichnamigen Arbeitsgruppe im Bundesministerium für Verbraucherschutz, Ernährung und Landwirtschaft.

BMVEL (2003). Tierschutzbericht 2003, „Bericht über den Stand der Entwicklung des Tierschutzes“. BMVEL 321-0869-1/8. 
BMVEL (2004D). Jahresstatistik 2004 über die amtliche Futtermittelüberwachung in der Bundesrepublik Deutschland mit Erläuterungen. Quelle: http://www.verbraucherministerium.de/data/0006DFD0B91F1300A3A26521C0A8D816.0.pdf (aufgerufen am 05.08. 2005).

BMVEL (2005A). Jahresstatistik 2004 über die amtliche Futtermittelüberwachung in der Bundesrepublik Deutschland mit Erläuterungen. Quelle:http://www.verbraucherministerium.de/data/0006DFD0B91F1300A3A26521C0A8D816.0.pdf (aufgerufen am 05.10.2005).

BMVEL (2005B). Tierschutzbericht 2005, Bericht über den Stand der Entwicklung des Tierschutzes“. Quelle: http://www.verbraucherministerium.de (aufgerufen am 15.10. 2005).

BODE, H. (2004). Methoden der Fütterungsarzneimittelherstellung. Quelle: http://xtra.belapharm.com/news/symposium2004/vortrag_bode.pdf, (aufgerufen am 05.08.2005).

BODENMÜLLER, K. (2000). Biologische, konventionelle und gentechnische Anwendungen in der Landwirtschaft - gesundheitliche und ökologische Aspekte. Quelle: http://www. internutrition.ch/in-news/mediainfo/dl/biogenst.doc (aufgerufen am 05.08.2005). Schweiz. InterNutrition - Schweizerischer Arbeitskreis für Forschung und Ernährung.

BOGNER, A.; MENZ, W. (2002). Expertenwissen und Forschungspraxis: die modernisierungstheoretische und die methodische Debatte um die Experten. In: Das Experteninterview, Theorie, Methode, Anwendung. BOGNER, A.; LITTIG, B.; MENZ, W (Hrsg.): 7-31. Opladen.

BOLSCHER, J.J.M. (2001). Transponder löst Strichcode ab, RFID-Technologie für die lückenlose Lieferkette von der Tierhaltung bis zum Verbraucher. Fleischwirtschaft 81 10/2001: 58-60.

BORCHERS, K. (2002a). Transmissible spongioforme Encephalopathien (TSE): Der Erreger und seine Eigenschaften. Fleischwirtschaft 82 7/2002: 110-112.

BORCHERS, K. (2002b). Transmissible Spongioforme Enzephalopathien: Alte Krankheit mit neuer Brisanz. Deutsche tierärztliche Wochenschrift 115: 81-90. Berlin München.

BRANSCHEID, W. (1998). Marketing von Fleisch und Qualitätsmanagement. In: Fleisch- und Fleischwaren Band 1. BRANSCHEID, W.; HONIKEL, K.O.; VON LENGERKEN, G.; TROEGER, K. (Hrsg.): 39-83

BRANSCHEID, W. (2004). Zukunftsperspektiven für Fleisch und Fleischerzeugnisse. Fleischwirtschaft 84 5/2004: 213-217.

BRANSCHEID, W.; GREINACHER, A.; DOBROWOLSKI, A. (2000). Markenfleischprogramme beim Schwein, Leistungen, Trends und Unterschiede. Fleischwirtschaft, 83 12/2000: 44-48.

BRANSCHEID, W.; HAHN, G.; WICKE, M (2004A). Qualität von Putenfleisch, Probleme und Gegenmaßnahmen. Fleischwirtschaft 84 11/2004: 109-112.

BRAUNGART, M.; NIKOV, K.; RIVIÈRE, A.; SOTH, J. (2002). Biologische Kreisläufe und Nährstoffmanagement. Quelle: http://www.epea.com/pdf_files/ans_beitrag_EPEA_06_ 09_2002.pdf (aufgerufen am 02.08.05). 
BRC (2005). BRC Globaler Standard Lebensmittel. British Retail Consortium.

BROOKES, G. (2002). The farm level impact of using Bt maize in Spain. Quelle: http://www. pgeconomics.co.uk/pdf/bt_maize_in_spain.pdf (aufgerufen am 05.08.2005). Brookes West, Jasmine House, Canterbury Rd, Elham, Canterbury, Kent, UK, CT4 6UE.

BROOKES, G. (2004). Verhaltensanomalien beim Schwein. Nutztierpraxis Aktuell 8, 3/2004. Quelle: http://www.ava1.de/pdf/artikel/schweine/brooks.pdf (aufgerufen am 02.08.05).

BRUHN, M. (2003). Die Entwicklung der Nachfrage nach Bioprodukten unter besonderer Berücksichtigung des Nitrofen-Geschehens und der Einführung des staatlichen Biosiegels. Arbeitsbericht Nr. 21, Institut für Agrarökonomie der Universität Kiel: 23.

BTK (2003). Stellungnahme zum Vorschlag für eine Verordnung des Rates über den Schutz von Tieren beim Transport und allen damit zusammenhängenden Vorgängen sowie zur Änderung der Richtlinien 64/432/EWG und 93/119/EG des Rates. Quelle:

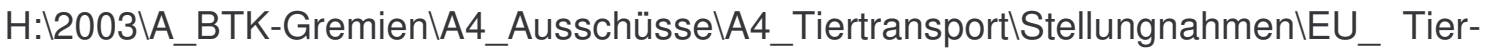
transportVO_9.03.doc (aufgerufen am 05.08.2005).

BVET (2003). Ferkelkastration: gleiche Absicht, anderer Weg. Mitteilungen, Medien- und Informationsdienst. Quelle: http://www.blw.admin.ch/aktuell/medien/d/030610.pdf (aufgerufen am 05.08.2005).

BVL (2001). Jahresbericht 2000-2001 zum Nationalen Rückstandskontrollplan. Quelle: http://www.bvl.bund.de/cln_027/nn_493682/DE/01__Lebensmittel/01_Sicherheit_Ko ntrollen/04_NRKP/nrkp_node.html_nnn=true (aufgerufen am 11.08.2005).

BVL (2002). Bericht 2002, Nationale Berichterstattung Pflanzenschutzmittel-Rückstände Ergebnisse aus Deutschland. Quelle: http://www.bvl.bund.de/nn_493682/DE/01_ __Lebensmittel/01__Sicherheit_Kontrollen/05_NB_PSM_Rueckstaende/01_nb_ psm/nbpsm_Bericht_2002.html (aufgerufen am 10.10.2005).

BVL (2003). Jahresbericht 2003 zum Nationalen Rückstandskontrollplan. Quelle: http://www.bvl.bund.de/cln_027/nn_493682/DE/01__Lebensmittel/01__Sicherheit_Ko ntrollen/04_NRKP/nrkp_node.html_nnn=true (aufgerufen am 11.08.2005).

BVL (2004). Jahresbericht 2004 zum Nationalen Rückstandskontrollplan. Quelle: http://www. bvl.bund.de/cln_027/nn_493682/DE/01__Lebensmittel/01_Sicherheit_Kontrollen/04_ _NRKP/nrkp_node.html_nnn=true (aufgerufen am 11.08.2005. Berlin).

CORON (1991). Der Grosse Coron, Band 6 (Es-Frie). München.

COUNCIL OF THE EUROPEAN COMMUNITIES (1996). Council Directive 96/22/EC of 29 April 1996 concerning the prohibition on the use in stockfarming of certain substances having hormonal or thyrostatic action and of beta-agonists. Official Journal of the European Community, L125.

DAHMS, S. (2003). BSE: Inzidenzen-regional, zeitlich, Prognosemodelle. Fleischwirtschaft 83 7/2003: 111-114.

DÄNICKE, S.; OLDENBURG, S. (2000). Risikofaktoren für die Fusariumtoxinbildung in Futtermitteln und Vermeidungsstrategien bei der Futtermittelerzeugung und Fütterung. Landbauforschung Völkerode, Sonderheft 216. 
DÄNICKE, S.; GÄDEKEN, D.; UEBERSCHÄR, K.-H.; MEYER, U.; SCHOLZ, H. (2002). Effects of Fusarium-toxin contaminated wheat in ruminant nutrition. 24. MykotoxinWorkshop Berlin - Marienfelde 03. bis 05. Juni 2002, Abstracts der Vorträge und Poster. Bundesinstitut für gesundheitlichen Verbraucherschutz und Veterinärmedizin und Gesellschaft für Mykotoxinforschung e.V: 31-32

DANISH (2002). Unabhängige Kontrolle in der dänischen Schweineproduktion. DANISH. Danske Slagterier.

DANISH (2003). QSG Handbuch. Quelle: http://www.danskeslagterier.dk/smcms/Danish _Deutsch/Qualitatssicherung/QSG_Handbuch/Index.htm?ID=329 (aufgerufen am 01.02.2005).

DANISH (2004). DANISH Qualitätssicherungs-Garantie. DANISH Report.

DE BOT, P.; DAHLMANS, H.; ENTHOVEN, H.; HEIJNEN, G.; MUL, A. (2004). Study into drying processes for animal feed matirials and HACCP. Qualitätsreihe Nr. $101 \mathrm{der}$ pdv (Productschap Diervoeder). Product Board Animal Feed.

DEFRA (2002) Department for Environment Food and Rural Affairs. Zoonoses Report, United Kingdom 2002. Quelle: http://www.defra.gov.uk/animalh/diseases/zoonoses/ zoonoses_reports/zoonoses2002.pdf (aufgerufen am 05.08.2005).

DEHNE, H.-W.; OERKE, E.-C.; STEINER, U. (2001) Mykotoxine in Getreide und Getreideprodukten - Fusarium-Befall an Weizen. 1. Workshop, QUASI der Agrar- und Ernährungswissenschaftlichen Fakultät der Christian-Albrechts-Universität zu Kiel. Quelle: http://www.quasi.uni-kiel.de (aufgerufen am 05.08.2005)

DEWEY, C.; HALEY, C.; WIDOWSKI, T.; FRIENDSHIP, R. (2004). Factors Associated with In-Transit Losses. London Swine Conference - Building Blocks for the Future 1-2 April 2004:51-54.

DIN 10503 (1998). Lebensmittelhygiene. DIN Deutsches Institut für Normung. Beuth-Verlag.

DLG Merkblatt 321 (2000). Tiergerechtheit auf dem Prüfstand. Quelle: http://www.dlg-test.de/ pbdocs/merkblatt/dlg-merkblatt_321.pdf (aufgerufen am 05.08.2005).

DLG (2001). Verbraucherschutz in der Land- und Ernährungswirtschaft, Leitlinien und Maßnahmen zur Neuorientierung. Deutsche Landwirtschaftliche Gesellschaft.

DORN, P.; SCHWARZER, C. (1991). Salmonellen-Situation in Futtermitteln am Beispiel Geflügel; Band 3. Proc. VIII Intern. Kongress für Tierhygiene, Leipzig, 20.-24.08.1991: 986-990.

DRUSCH, S.; RANDEL, G.; SCHWARZ, K. (2002). Mykotoxine in der Lebensmittelverarbeitung. Quasi, erster Arbeitsbericht zur Lebensmittelqualität und - sicherheit: 49-57.

DUNSHEA, F. R.; COLANTONI, C.; HOWARD, K.; MCCAULEY, I.; JACKSON, P. ;LONG, K. A.; LOPATICKI, S.; NUGENT, E.A.; SIMONS, J.A., WALKER, J.; HENNESSY, D.P. (2001). Vaccination of boars with a $\mathrm{GnRH}$ vaccine (Improvac) eliminates boar taint and increases groth performance. Journal of Animal Science 79: 2524-2535.

DVT (2004A). Jahresbericht 2003/2004. Quelle. http://www.dvtiernahrung.de/img/medien/JB03-04-Gesamt-72dpi.pdf (aufgerufen am 05.08.2005). 
DVT (2004B). Erklärungen zur Gentechnikfreiheit sind unseriös, Position des DVT, 18.02.2004. Quelle: http://www.dvtiernahrung.de (aufgerufen am 05.08.2005).

EFSA (2004). Welfare aspects of the castration of piglets, Scientific Report of the Scientific Panel for Animal Health and Welfare on a request from the Commission related towelfare aspects of the castration of Piglets. The EFSA Journal (2004) 91, Question NEFSA-Q-2003-091.Quelle: www.efsa.eu.int/science/ahaw/ahaw_opinions/512/report _ahaw03_ej91_pigcast_v2_en1 (aufgerufen am 05.08.2005).

EFSA (2005). EFSA provides update on avian influenza and food safety. Quelle: www.efsa.eu.int/press_room/press_release/1193/comm_pr_avian_flu_update_200510 26_en1.pdf (aufgerufen am 24.10.2005).

ELLENDORFF, F. (2003). Nachhaltigkeit für intakte Umwelt. Fleischwirtschaft 83 3/2003: 6468.

ELLERBROEK, L (1997). Zusammenfassung des Sachverständigengespräches. Erfordernisse und Möglichkeiten in der Schlachtgeflügel- und Geflügelfleischuntersuchung, Protokoll eines Sachverständigengespräches am 20./21. November 1997 im BgVV:. 52-57.

ELLERBROEK, L.; WICHMANN-SCHAUER, H.; HAARMANN, M. (1999). Untersuchungen zum Vorkommen von Salmonellen bei deutschem Nutzgeflügel und Geflügelfleisch. Quelle: http://www.bgvv.de/sixcms_upload/media/91/fober_salmonelgeflugel.pdf (aufgerufen am 05.08.2005).

ELLNER, F.M. (2002). Mykotoxine in Getreide - Zwischenbilanz nach vier Jahren Monitoring. 53. Deutsche Pflanzenschutztagung. Bonn. Mitt. Biol. Bundesanstalt. Land- Forstwirtschaft: 73-74.

ELMADFA, I.; BURGER, P. (1999). Expertengutachten zur Lebensmittelsicherheit Ochratoxin A. Im Auftrag des Bundeskanzleramts. Institut für Ernährungswissenschaften der Universität Wien.

EMNID (2002). Ergebnisse der TNS-EMNID Umfrage zum Thema Verbraucherschutz für EOS Gallup Europe. Flash-EB 117: Consumer Survey.

ENDER, K.; AUGUSTINI, C. (1998). Schlachttierwert von Rind und Kalb. In: Fleisch- und Fleischwaren Band 1. BRANSCHEID, W.; HONIKEL, K.O.; VON LENGERKEN, G.; TROEGER, K. (Hrsg.): 165-203.

ENGEL, K.-H. (2002). Strategien zur Bewertung der gesundheitlichen Unbedenklichkeit gentechnisch veränderter Lebensmittel. BLL-Workshop "Gentechnik im Lebensmittelbereich“ - Stand der Wissenschaft - 21. Februar 2002: 47-55.

ENGELHARDT, G. (1999). Mykotoxine - Giftige Stoffwechselprodukte von Schimmelpilzen. Quelle. http://www.Ife.bayern.de/lebensmittel/mykotox.html (aufgerufen am 05.08. 2005).

EU ENTSCHEIDUNG 2001/471/EG (2001). Entscheidung der Kommission von 08.Juni 2001 über Vorschriften zur regelmäßigen Überwachung der allgemeinen Hygienebedingungen durch betriebseigene Kontrollen. Amtsblatt der Europäischen Gemeinschaft Nr. L 165/48 vom 21.06.2000: 0048-0053. 
EU, European Commission (1999). Standards for the Microclimate inside Animal Transport Road Vehicles. Sanco/B3/AW/R13/1999, Report of the Scientific Committee on Animal Health and Animal Welfare, Adopted 8 December 1999.

EU, European Commission (2000A). Opinion of the scientific committee on annimal nutrition on the Dioxin contamination of feedingstuffs an their contribution to the contamination of food of animal origin. Adopted on 06.11.2000. Directorate C - Scientific Opinions C3 - Management of scientific committees II; scientific co-operation and networks.

EU, European Commission (2000). Weissbuch zur Lebensmittelsicherheit. KOM (1999) 719 endg. Brüssel.

EU, European Commission (2001A). Annexb to Monitoring of Pesticide Residues in Products of Plant Origin in the European Union, Norway and Iceland, 1999 Report. Annex to SANCO/397/01 final.

EU, European Commission (2002B). Review of previous SCVPH opinions of 30 April 1999 and 3 May 2000 on the potential risks to human health from hormone residues in bovine meat and meat products. Opinion of the scientific committee on veterinary measures relating to public health.

EU, European Commission (2002C). Annex to Monitoring of Pesticide Residues in Products of Plant Origin in the European Union, Norway, Iceland and Lichtenstein, 2000. Report. April 2002. Annex to SANCO/687/02 final.

EU, European Commission (2003A). Zusammenfassender Bericht über eine Reihe von in allen Mitgliedstaaten im Jahr 2002 durchgeführten Inspektionsbesuchen zur Beurteilung der Durchführung von Kontrollen der Rückverfolgbarkeit und der Etikettierung von Rindfleisch und Rinderhackfleisch. GD (SANCO)/9505/2003.

EU, European Commission (2003B). Vorschlag für eine Verordnung des Rates über den Schutz von Tieren beim Transport. KOM (2003) 425 endgültig Nr. 2003/0171 (CNS).

EU, European Commission (2003c). Annex to Monitoring of Pesticide Residues in Products of Plant Origin in the European Union, Norway, Iceland and Lichtenstein, 2001 Report. March 2003. Annex to SANCO/20/03 final.

EU, European Commission (2004). Leitlinien für die Anwendung der Artikel 11, 12, 16, 17, 18, 19, und 20 der Verordnung (EG) Nr. 178/2002 über das allgemeine Lebensmittelrecht, Schlussfolgerungen des ständigen Ausschusses für die Lebensmittelkette und Tiergesundheit. ABI. L 165 vom 30.4.2004, S.1. Berichtigung veröffentlicht im Amtsblatt Nr. L 191 vom 28.5.2004: 1. Quelle: http://www.awt-feedadditives.de/Der_Verband. 2.0.html (aufgerufen am 22.07.2005).

EU, European Commission (2005). Fahrplan für die TSE-Bekämpfung. Quelle: http://europa. eu.int/comm/food/food/biosafety/bse/roadmap_de.pdf (aufgerufen am 05.08.2005).

FAO; WHO; OIE (2004). Joint FAO/OIE/WHO 2nd Workshop on Non-human Antimicrobial Usage and Antimicrobial Resistance: Management Options, Oslo, Norway, 15-18 March 2004, Executive Summary. Quelle: http://www.who.int/foodsafety/publications /micro /en/exec.pdf (aufgerufen am 05.08.2005).

FAULKNER, P.M.; WEARY, D.M. (2000). Reducing pain after dehorning in dairy calves. Diary Science 83: 2037-2041. 
FAULL, W.B.; HUGES, J.W.; CLARKSON, M.J.; DOWNHAM, D.Y.; MANSON, F.J.; MERRITT, J.B.; MURRAY, R.D.; RUSSELL, W.B.; SUTHERST, J.E.; WARD, W.R. (1996). Epidemiology of lameness in dairy cattle: the influence of cubicles and indoor and outdoor walking surfaces. Vet Rec. 139: 130-136.

FEHLHABER, K. (1997). Vom Tier stammende Produkte- Verbraucheransprüche an Qualität und Sicherheit. Stand und Möglichkeiten zur umweltgerechten und artgemäßen Erzeugung von tierischen Produkten. Leipziger Universitätsverlag.

FEHLHABER, K. (1998). Die Lebensmittelhygiene-Verordnung aus fachlicher Sicht; Kurzfassung eines Vortrags vom Seminar 8. Hygiene-Forum, 12./13.03.1998 in Hamburg. HACCP Infoservice. Quelle: www.haccp.de/wissen.htm (aufgerufen am 05.08.2005).

FEHLHABER, K. (1999). Zur Lebensmittelüberwachung in Deutschland - Tradition und Gegenwart. RFL-Rundschau für Fleischhygiene und Lebensmittelüberwachung 51, Heft 1:27-30.

FEHLHABER, K. (2000). Bedeutung der Lebenduntersuchung, Möglichkeiten, Grenzen, Alternativern. Jahrestag DVG-Fachgruppe Schweinekrankheiten, 2.-3.3.2000, Hannover:18-21

FEHLHABER, K. (2003A). Mikrobiologische Risiken, Aktuelle Aspekte der Verbreitung von Zoonosen über vom Tier stammende Lebensmittel. Nutztierpraxis Aktuell 7: 30-41.

FEHLHABER, K. (2003B). Prämortale Belastung - mikrobielle Risiken. Vortrag beim AfT Frühjahrssymposium 2003. Quelle: http://aft-online.net/index.php?subsite=subsite 6 (aufgerufen am 15.10.2005).

FINCHER, M.G.; GIBBONS, W.J.; MAYER, K.; PARK, S.E. (1956). Diseases of cattle. American Veterinary Puplications, Inc., Evansten, Illionis: 399-416.

FISCHER, K. (1987). Qualitätsabweichungen bei Rindfleisch. In: Kulmbacher Reihe, Band 7, Rindfleisch Schlachtkörperwert und Fleischqualität: 235-268.

FISCHER, K. (1994). Transport zur Schlachtstätte. In: Kulmbacher Reihe, Band 13, Schlachten von Schwein und Rind: 22-42.

FLACHOWSKY, G. (2000). Tierernährung und Produktqualität. Forschungsreport 1/2000: 18-21.

FLACHOWSKY, G. (2002). Bewertung der Lebensmittelsicherheit - Beiträge der FAL zur weiteren Verbesserung . Landbauforschung Völkenrode FAL Agricultural Research 1 52: 1-7. FAL Bundesforschungsanstalt für Landwirtschaft.

FLACHOWSKY, G.; AULRICH, K.; BÖHME, H.; DAENICKE, R. (2000). Transgene Kost fürs liebe Vieh?, Fütterungsversuche mit gentechnisch veränderten Futtermitteln. Forschungsreport 1/2000: 32-35.

FLEISCHHYGIENEGESETZ (FIHG), (2003). BGBL. I S, 1243, vom 30. Juni 2003: 2-23.

FRANK, C.; ALPERS, K. (2003). Infektionen mit EHEC beim Menschen in Bericht über die epidemiologische Situation der Zoonosen in Deutschland für 2002, Übersicht über die Meldungen der Bundesländer. Quelle: http://www.bfr.bund.de/cms/media.php/95/ bericht_ueber_die_epidemiologische_situation_der_zoonosen_in_deutschland_fuer_2 002.pdf: 160-164 (aufgerufen am 05.08.2005). 
FRIES, R. (2002A). BSE im Vereinigten Königreich und in der EU. Fleischwirtschaft 82 7/2002: 104-106.

FRIES, R. (2002B). Epidemiologische Aspekte der BSE. Fleischwirtschaft 82 8/2002: 98-101.

FRIES, R. (2003). TSE - das Phänomen und die Folgen. Fleischwirtschaft 82 6/2003: 104105.

FRIESTAD, M.; WRIGHT, P. (1994). The Persuasion Knowledge Model: How People Cope with Persuasion Attemps. Journal of Consumer Research, Inc, Vol. 21, June 1994: 131.

FRIESTAD, M.; WRIGHT, P. (1995). Persuasion Knowledge: Lay People's and Researchers' Beliefs about the Psychology of Advertising. Journal of Consumer Research, Inc, Vol. 22, June 1995: 62-74.

FUCHS, E.; BINDER, E. M.; SCHATZMAYR, G.; HEIDLER, D.; KRSKA, R. (2002). Analytical evalution of a trial feeding ochratoxin A to pigs. 24. Mykotoxin-Workshop, BerlinMarienfelde, 03. bis 05. Juni 2002. BgVV.

GAISSER, S.; HÜSING, B.; ZIMMER, R. (2000). Gentechnik bei Pflanzen ("Grüne Gentechnik"). Frauenhofer Institut Systemtechnik und Innovationsforschung. Quelle: http://www.isi.fhg.de/bt/Pflanzen.pdf (aufgerufen am 05.08.2005).

GAREIS, M. (2000). Aspekte der Lebensmittelsicherheit aus wissenschaftlicher Sicht Mikrobiologische Risiken in Sachen Lebensmittel, Symposium. Wie sicher sind unsere Lebensmittel? 23./24. Februar 2000 in Königswinter: 45-63.

GARTUNG, J.; UMINSKI, K.; HARTWIG, M. (2004). Hähnchenmast in Deutschland. Auszug aus unveröffentlichtem Bericht: „Investitionsbedarf in der Junggeflügelmast" Nr. 379/2003 der FAL im Auftrag des KTBL, Stand Juni 2004. Quelle: http://www.ktbl.de /federvieh/haehnchenmast.pdf (aufgerufen am 05.08.2002).

GASKELL, G.; ALLUM, N.; STARES, S. (2003). Europeans and Biotechnology in 2002, Eurobarometer 58.0. Quelle: http://europa.eu.int/comm/public_opinion/archives/eb/ebs _177_en.pdf (aufgerufen am 05.08.2005).

GEH (2001). Gesellschaft zur Erhaltung alter und gefährdeter Haustierrassen e.V.. Über 1.800 Nutztier-Rassen weltweit vom Aussterben bedroht. Quelle: www.g-e-h.de/aktuell /presse/presse2.htm (aufgerufen am 16.05.05).

GENRES (2003). Nationaler Bericht Deutschlands als Beitrag zum Bericht der FAO über den Zustand tiergenetischer Ressourcen der Welt mit einem nationalen Fachprogramm zur Erhaltung und nachhaltigen Nutzung tiergenetischer Ressourcen in Deutschland. Quelle: http://www.genres.de/tgr/nationales_fachprogramm/pdf_version/nfp-tgr gesamttext (aufgerufen am 05.08.2005).

GERIGK, K. (1992). Einführung in die Problematik - Begriffsbestimmung, Wesen und Definitionen. Akademie für Tierärztliche Fortbildung Schriftenreihe. Interdisziplinäres Symposium Salmonellose am 16./17. November 1992 in Bonn. Bad Godesberg: 1-6

GESCHKA, H. (1977). Delphi. Langfristige Prognosen, In: Möglichkeiten und Methoden der Langfristprognostik komplexer Systeme. BRUCKMANN, G. (Hrsg.): 42-45. Würzburg/ Wien.

GLÄSER, J.; LAUDEL, G. (2001). Experteninterviews und qualitative Inhaltsanalyse. UTB. 
GLODEK, P. (1988). Züchterische Bearbeitung von Antagonismen zwischen Leistung und Gesundheit beim Schwein. Tierärztl. Praxis, Suppl. 3: 11-15

GOLL, M. (1993). Aspekte der Mykotoxinproblematik in der Tierproduktion. Lohmann Informationen Juli-September: 1-4.

GRANDIN, T. (1990). Design of loading facilities and holding pens. Appl. Anim. Behav. Sci. 28: 187 - 201.

GRANDIN, T. (1996). Animal Welfare in Slaughter Plants. Presented at the 29th Annual Conference of American Association of Bovine Practitioners. Proceedings. Quelle: http: //aamp.com/documents/AnimalWelfareinSlaughterPlants_001.pdf: 22-26 (aufgerufen am 10.10.2005)

GRANDIN, T. (2000). Perspectives on transportation issues: The importance of having physically fit cattle and pigs. Quelle: http://www.grandin.com/behaviour/perspectives. transportation.issues.html (aufgerufen 15.10.2005).

GRANDIN, T. (2001A). Livestock-handling quality assurance. Journal of Animal Science, 79: E239-E248. Quelle: www.asa.org/jas/jas0916.pdf (aufgerufen am 18.10.2005).

GRANDIN, T. (2001B). Animal Welfare during Transport and Slaughter. Research Consortium Sustainable Animal Production. Quelle: http://agriculture.de/acms1/conf6/ ws5atransport.htm (aufgerufen am 15.10.2005).

GRANDIN, T. (2002A). Welfare of Pigs During Transport. Swine Welfare fact sheer,Vol.1, NO. 3, 2002 National Pork Board • Des Moines, lowa USA.

GRANDIN, T. (2002B). Handling Pigs for Optimum Performance on the Farm and in the Slaughter Plant and Methods to reduce PSE and bloodsplash. Manitoba Swine Seminar, Volume 16, Sharing Ideas and Information for Efficient Pork Production. Quelle: http://www.gov.mb.ca/agriculture/livestock/pork/\#2004 (aufgerufen am 17.10. 2005).

GRANDIN, T. (2004). Animal Welfare and Humane Slaughter. Quelle: www.grandin.com /references /humane.slaughter.html, Updated November 2004 (aufgerufen am 20.20. 2005).

GRANDIN, T. (2005). Animal Welfare Evaluation of Gas Stunning (Controlled Atmoshere Stunning) of Chickens and other Poultry. Quelle: www.grandin.com/gas.Stunning. poultry.eval.html (aufgerufen 23.10.2005).

GREGORY, N.G.; AUSTIN, S.D. (1992). Causes of trauma in broilers arriving dead at processing plants. Vet. Record. 131: 501 - 503.

GS1 (2005). EAN. Quelle: http://www.gs1-germany.de/internet/content/produkte/ean/index _ger.html (aufgerufen am 26.07.2005).

GUARDIA, M.D.; ESTANY, J.; BALASCH, S.; OLIVER, M.A.; GISBERT, M.; DIESTRE, A. (2004). Risk assessment of PSE condition due to pre-slaughter conditions and RYR1 gene in pigs. Meat Science 67: 471-478

GUTZWILLER, A. (2003). Kastration von Ferkeln unter Lokalanästhesie. Agrarforschung, Journal of Swiss Agricultural Research 10(01): 10-13. 
HÄDER, M. (2000). Die Expertenauswahl bei Delphi-Befragungen. ZUMA-How-to-Reihe, Nr.5.

HÄDER, M.; HÄDER, S. (1994). Die Grundlagen der Delphi-Methode, Ein Literaturbericht. ZUMA-Arbeitsbericht Nr. 94/02. Mannheim.

HÄDER, M.; HÄDER, S. (1998). Neuere Entwicklungen bei der Delphi-Methode, Literaturbericht II. ZUMA-Arbeitsbericht Nr. 98/05. Mannheim.

HAFEZ, M. (1997). Probleme der Haltungs- und Zuchtbedingten Erkrankungen bei Puten. In: Protokolldienst 5/97 Evangelische Akademie Bad Boll, Verwirklichung des Tierschutzes in der Nutztierhaltung, Fachtagung vom 1. - 3. März in Bad Boll 1996

HAKENBECK, R. (2001). Populationsstrukturen und Resistenzentwicklung bei humanpathogenen Bakterien: Oropharynx aus molekularer Sicht. Bad Honnef-Symposium 2001, Resistenzentwicklung: Ökologie, Epidemiologie und Prävention (Development of Resistance: Ecology, Epidemiology and Prevention). Paul-Ehrlich-Gesellschaft für Chemotherapie e.V.

HAMMER, P. (2001). Zoonosenproblematik bei Lebensmitteln. 1. Workshop, QUASI der Agrar- und Ernährungswissenschaftlichen Fakultät der Christian-Albrechts-Universität zu Kiel. Quelle: http://www.rz.uni-kiel.de/ (aufgerufen am 05.08.2005): 39-44.

HAMPEL, J. (1999). Die Europäische Öffentlichkeit und die Gentechnik, Einstellungen zur Gentechnik im internationalen Vergleich. Akademie für Technikfolgenabschätzung in Baden-Württemberg, Arbeitsbericht Nr. 111

HAMPEL, J.; PFENNIG, U. (2001). Gentechnik in der öffentlichen Meinung, Unterschiedliche Entwicklung in Europa. IA-Informationen 2/2001: 34-35. Akademie für Technikfolgenabschätzung in Baden Württemberg.

HAMPEL, J.; RENN, O. (1999). Gentechnik in der Öffentlichkeit, Wahrnehmung und Bewertung einer umstrittenen Technologie. Campus Verlag Frankfurt/New York.

HARPER, G. (2001). Art und Ausmaß der Tierschutzbedenken von Verbrauchern. Centre for Food Economics Research Department of Agricultural and Food Economics The University of Reading United Kingdom. Quelle: http://www.uni-kiel.de/agrarmarketing /EU/gembrudt.PDF (aufgerufen am 05.08.2005).

HARPER, G.; HENSON, S. (2001). Consumer Concerns about Animal Welfare and the Impact on Food Choice1. EU FAIR CT98-3678 Final Report. Quelle: http://europa. eu.int/comm/food/ animal/welfare/eu_fair_project_en.pdf (aufgerufen am 10.04.2005).

HARTUNG, M. (2002). Bericht über die epidemiologische Situation der Zoonosen in Deutschland für 2001, Übersicht über die Meldungen der Bundesländer. BgVV-Hefte 6/2002. Berlin. Bundesinstitut für gesundheitlichen Verbraucherschutz und Veterinärmedizin.

HARTUNG, M. (2003). Bericht über die epidemiologische Situation der Zoonosen in Deutschland für 2002, Übersicht über die Meldungen der Bundesländer. Quelle: http://www.bfr.bund.de/cms/media.php/95/bericht_ueber_die_epidemiologische_situati on_der_zoonosen_in_deutschland_fuer_2002.pdf (aufgerufen am 05.08.2005). 
HAUENHORST, E. (2005). Vernetzte Strukturen sind unerlässlich, EU-Konzepte und ihre Konsequenzen für die amtliche Überwachung und Untersuchung. Fleischwirtschaft 85 3/2005: 14-16.

HAY, M.; VULIN, A.; GÉNIN, S.; SALES, P.; PRUNIER, A. (2003). Assesment of pain induced by castration in piglets: behavioural and physiological responses over the subsequent 5 days. Appl. Anim. Behav. Sci. 82: 210-218.

HDE (2004). International Food Standard, Standard zur Beurteilung von Eigenmarkenlieferanten, Version 4. Berlin. HDE-Hauptverband des Deutschen Einzelhandels e.V.

HECHT, H. (1997). Belastung von Fleisch und Fleischerzeugnissen mit umweltbedingten Rückständen. Mitteilungsblatt der Bundesanstalt für Fleischforschung Kulmbach: 235246.

HECHT, H. (2003). Stellungnahme zur Herstellung von Trockengrün. Arbeitsgruppe "Carryover" unerwünschte Stoffe in Futtermitteln beim Bundesministerium für Verbraucherschutz, Ernährung und Landwirtschaft. Quelle: www.dvtiernahrung.de/img/medien/ CarryO-Gruppe-Trocknung.pdf (aufgerufen am 15.08.05).

HEESCHEN, W. H. (2003). Lebensmittel schützen - eine integrierte Konzeption!, - Neue Erkenntnisse und Aktualisierungen -. Brennpunkt Lebensmittelsicherheit 7/2003. Behrs Verlag.

HEESCHEN, W. H. (2004). BSE - Stand der Kenntnisse und des Risikomanagements. Brennpunkt Lebensmittelsicherheit, 9/2004: 11-22. Behrs Verlag.

HEESCHEN, W. H. (2006) Vogelgrippe - Aviäre Influenza. Fokus Lebensmittelsicherheit 3/2006. Behrs Verlag.

HELLWIG, E.-G. (2003). So bringt man Medikamente gesetzeskonform ans Tier. Nutztierpraxis Aktuell 5/2003..

HENSEL, A. (2003). Risiko-Ranking für mehr Transparenz. Fleischwirtschaft 83 7/2003: 8-9.

HENSGEN, M. (2004). HACCP in der Fleischverarbeitung, Leitfaden für die praktische Umsetzung. Behrs Verlag.

HERTER, U.; KÜLLING, D.; BECKER; D; VAN SLOOTEN, K.; BRÜSCHWEILER, B.; DOHERR, M.; FROSSARD, R.; HEIM, D.; HETT, A.; HUGUENIN, O.; KUPPER, T.; PERLER, L.; SCHMIDT, J.; WIDMER, F.; ZARN, J. (2001). Risikoanalyse zur Abfallverwertung in der Landwirtschaft, Teil 1 Grobbeurteilung. Reckenholz, Zürich. Eidgenössische Forschungsanstalt für Agrarökologie und Landbau FAL.

HESSE, D. (1999). Tiergerechte Nutztierhaltung, Projekte zum Themenbereich Wechselwirkungen zwischen "Tier und Technik" sowie "Tier und Umwelt". FAL- Jahresbericht 1999, Bericht des Instituts für Tierschutz und Tierhaltung: 141-142.

HESTER, P.Y.; Shea-Moore, M. (2003). Beak trimming egg-laying strains of chickens. World's Poultry Science Journal 59: 458-476.

HIEPE, TH.; ECKERT, J.; GÜRTLER, H. (1998). Nahrungsketten, Risiken durch Krankheitserreger, Produkte der Gentechnologie und Zusatzstoffe?. Karl F. Haug Fachbuchverlag. 
HILDEBRAND, G. (2002). Lebensmittelsicherheit: vermeintliche und reale Risiken. Bundesgesundheitsblatt, Gesundheitsforschung, Gesundheitsschutz 45/2002: 473.

HOFMANN K. (1973). Was ist Fleischqualität?. Fleischwirtschaft 53 1973: 485.

HOLST, S. (2001). CO2 stunning of pigs for slaughter, Practical guidelines for good animal welfare. 47th International Congress of Meat Science and Technology, Krakow, Poland.

HOLZAPFEL, W.; BAUMGART, J.; HEESCHEN, W.; V. RHEINBABEN, F.; ZSCHALER, R. (2004). HACCP. In: HOLZAPFEL, W. (Hrsg.). Lexikon Lebensmittel -Mikrobiologie und -Hygiene. 3. Auflg. 129-130. Behrs Verlag.

HOLZHEU, F.; WIEDEMANN, P.M. (1993). Perspektiven der Risikowahrnehmung. In: Risiko ist ein Konstrukt: Wahrnehmungen zur Risikowahrnehmung. BECKER, U.; DUNWOODY, S.; HOLZHEU, F. ET AL. (Hrsg.): 9-20. Knesebeck Verlag.

HONIKEL, K.-O. (1998). Rückstände und unerwünschte Substanzen (Schadstoffe), Definition der Begriffe. In: Fleisch- und Fleischwaren, Band 1. BRANSCHEID, W.; HONIKEL, K.O.; VON LENGERKEN, G.; TROEGER, K. (Hrsg.).: 507-508.

HONIKEL, K.-O. (2001). BSE, Menschen- und Rinderwahn. Fleischwirtschaft 81 12/2001: 11-14.

HONIKEL, K.-O.; HECHT, H. (1999). Schadstoffe in Fleisch und Fleischwaren. In:. Lebensmittel tierischer Herkunft in der Diskussion. KLUTHE, R (Hrsg.): 39-46.

HORN, D. (2005). Amtliche Kontrollen in Europa ab 2006, Erläuterungen zur KontrollVerordnung (EG) Nr. 882/2004. Fleischwirtschaft 85 5/2005: 112-114.

HUIS IN'T VELD, J.H.J.; MULDER, R.W.A.W.; SNIJDERS, J.M.A. (1994). Impact of animal husbandry and slaughter technologies on microbial contamination of meat: Monitoring and Control. Meat Science 36: 123 - 154.

HUNTER, E.J.; JONES, T.A.; GUISE, H.J.; PENNY, R.H.C.; HOSTE, S. (2001). The relationship between tail biting in pigs, docking procedure and other management practices. The Veterinary Journal 161: 72-79.

IDEL, A.; MATHES, M. (2004). Die falschen Ziele, Warum die Tierzucht ökologisiert werden muss. Quelle. www.agrobiodiversitaet.net (aufgerufen am 05.08.2005).

IFAV (2003). Markenfleischprogramme in der EU. Quelle: http://www.ifav.de/ve/Vergangenheit/vergangenheit.html (aufgerufen am 05.08.2005).

IFF (1998). Internationale Forschungsgemeinschaft Futtermitteltechnik e.V. Modellierung des Feststoffmischprozesses für die Mischfutterherstellung. AiF-Nr.: $10934 \mathrm{~N}$ Bearbeitungszeitraum: 01.12.1996-30.11.1998. Quelle: www.iff-braunschweig.de/ergebnisse (aufgerufen am 05.08.2005).

IFF (2001). Internationale Forschungsgemeinschaft Futtermitteltechnik e.V. Untersuchungen zur Reduzierung von Verschleppungen und Kreuzkontaminationen bei der Herstellung von Vormischungen und Mischfutter. AiF-Nr.: 12165 N Bearbeitungszeitraum: 01.10.1999-30.06.2001. Quelle: www.iff-braunschweig.de/ergebnisse (aufgerufen am 05.08.2005). 
IFF (2002). Internationale Forschungsgemeinschaft Futtermitteltechnik e.V. Untersuchungen zur Reduzierung von Verschleppungen und Kreuzkontaminationen bei der Herstellung von Vormischungen und Mischfutter. AiF-Nr.: $12589 \mathrm{~N}$ Bearbeitungszeitraum: 01.09.2000-31.08.2002. Quelle: www.iff-braunschweig.de/ergebnisse (aufgerufen am 05.08.2005).

IKB (2004A). Qualitätshandbuch niederländisches Schweinefleisch. aktualisierte Fassung November 2004. Quelle: http://www.hollandmeat.nl (aufgerufen am 01.02.2005).

IKB (2004B). Die Schweinefleischproduktion in den Niederlanden (aktualisierte Fassung November 2004). Quelle: http://www.hollandmeat.nl/default.aspx?cid=30\&lcid=de-DE, (aufgerufen am 05.08.2005). Informationsbüro der Niederländischen Fleischwirtschaft.

INSTITUT FÜR DEMOSKOPIE ALLENSBACH (2002). Verändertes Meinungsklima gegenüber der Gentechnologie. Quelle: http://www.animal-health-online.de/drms/rinder /gentech.pdf (aufgerufen am 05.08.2005).

JAMES, C. (2002). Preview, Global Status of Commercialized Transgenic Crops: 2002. ISAAA Briefs, No. 27-2002.

JAMES, C. (2004). PREVIEW: Global Status of Commercialized Biotech/GM Crops: 2004. ISAAA Briefs, No. 32-2004 Quelle: http://www.isaaa.org (aufgerufen am 05.08.2005).

JANY, K.-D. (2000A). Gentechnik in der Ernährung - unnötiges Risiko oder notwendige Hilfe? (1. Teil). Ernährungsmed. 22000: 13-17.

JANY, K.-D. (2000B). Gentechnik in der Ernährung - unnötiges Risiko oder notwendige Hilfe? (2. Teil). Ernährungsmed. 3/2000: 15-19.

JANY, K.-D.; KIENER, C. (2000A). Lebensmittelproduktion - Quo vadis?, Gennahrung ist die Realität, auch auf unserem Teller. Quelle: http://www.bfa-ernaehrung.de/Bfe-Deutsch /Information/FAZ-deutsch.pdf (aufgerufen am 05.08.2005).

JANY, K.-D.; KIENER, C. (2000B). Gentechnik - Ein Beitrag zur nachhaltigen Produktion neuer Lebensmittel, Novel Foods - Designer Foods - Functional Foods. Quelle: http:// www.bfa-ernaehrung.de/Bfe-Deutsch/Information/e-docs/nachhaltigkeit.pdf (aufgerufen am 05.08.2005).

JUNGERMANN, H.; SOLVIC, P. (1993). Charakteristika individueller Risikowahrnehmungen. In: Risiko ist ein Konstrukt: Wahrnehmungen zur Risikowahrnehmung. BECKER, U.; DUNWOODY, S.; HOLZHEU, F. (Hrsg.).: 89-107. Knesebeck Verlag.

KAADEN, O.-R.; EICHHORN, W.; EBBAUER, S. (2001). Viren in Lebensmitteln tierischer Herkunft, Sachstand und quo vadis. Deutsche Veterinärmedizinische Gesellschaft e.V., Arbeitskreis: Ernährung, Garmisch-Partenkirchen, Germany. 23./24.09.01: 9-20.

KAMPHUES, J. (2004). Nebenproduktverwertung in der Tierernährung- von ihren Anfängen bis zum Weißbuch zur Lebensmittelsicherheit. Quelle: http://www.saria.de/Saria_de/de/ Pressemitteilung/01_07_2004.php (aufgerufen am 05.08.2005).

KANTELBERG, J.; DILLY, P. (2002). Zwischen Lebensmittelsicherheit und Vertrauen, Vor lauter Sicherheitsangst kann der Genuss verlorengehen / Führen die wuchernden neuen Vorschriften und Zertifikate in eine "Sicherheitsfalle"?. Gordian: 149-150. Nahrungs- und Genussmittel-Fachverlag. 
KEMP, R. (1993). Risikowahrnehmung: Die Bewertung von Risiken durch Experten und Laien - ein zweckmäßiger Vergleich. In: Risiko ist ein Konstrukt: Wahrnehmungen zur Risikowahrnehmung. BECKER, U.; DUNWOODY, S.; HOLZHEU, F. (Hrsg.).: 109-127. Knesebeck Verlag.

KEPPLINGER, H.M.; HABERMEIER, J. (1996). Ereignisserien. Was kann man nach spektakulären Ereignissen über die Wirklichkeit wissen?. In: Markt, Macht und Medien, Band 23. MAST, C. (Hrsg.): 261-272. Konstanz

KEPPLINGER, H.M., KNIRSCH, K. (2002): Erlaubte Übertreibungen, In: Medien und Mittler sozialer Kommunikation. NAWRATIL, U.; SCHÖNHAGEN, P.; STARKULLA, H. (Hrsg.). Leipzig

KIEFER, S. (2002). Vertragliche Bindungen - starke Kette, Beim Qualitätsmanagement in der Fleischwirtschaft gibt der Geflügelsektor den Ton an. Fleischwirtschaft 82 10/2002: 7073.

KIEFER, S.; JAHN, G.; SPILLER, A. (2003). Schluß mit dem Durcheinander. DLG-Mittleilungen 10/2003: 64-65.

KIENZLE, E.; CLAUSS, M.; WOLF, E.; SCHWENGLER, F.; KLEE, E.; SAUTER-LOUIS, C.; CHAHER, E.; STOLLE, A.; KOCH, U.; HOLTBECKER, J.; KRETZSCHMAR,A.; LEDERER, R.; GIESE, A.; WICHMANN, H.-E.; BÖHM, R.; STRATHMANN, F.-W.; MEYER, C.; THALMEIER, K.; SCHAUDINN, M. (2004). Risikoanalyse im Zusammenhang mit dem Auftreten von BSE einschließlich einer Untersuchung zum Vorkommen von vCJD in Bayern, Abschlussbericht. Studie des LMU-München im Auftrag des Bayrischen Staatsministeriums für Umwelt, Gesundheit und Verbraucherschutz. Quelle: http://home.hetnet.nl/ mad.cow/archief/2004/may04/risikobayern.pdf (aufgerufen am 03.08.2005).

KJAER, J. B.; SORENSEN P.; SU, G. (2001). Divergent selection on feather pecking behaviour in laying hens (Gallus gallus domesticus). Appl. Anim. Behav. Sci. 71: 229239.

KLARMANN, D. (2001). Nachweis von Salmonellen in Futtermitteln, Ergebnis der Untersuchungen im Laborbereich Tiergesundheit des Institutszentrums der Landwirtschaftskammer Weser-Ems in den Jahren 1997 bis 1999. Quelle: http://www.lwkwe.de/pdf/dreijahresberichtsalmonellen97-99lwblatt.pdf (aufgerufen, 26.10.2005).

KNAURS (1981). Knaurs Lexikon, Band 1. Knaur.

$\mathrm{KOCH}$, J. (2003). Listeriose-Erkrankungen des Menschen in Bericht über die epidemiologische Situation der Zoonosen in Deutschland für 2002, Übersicht über die Meldungen der Bundesländer. Quelle: http://www.bfr.bund.de/cms/media.php/95/bericht_ueber_ die_epidemiologische_situation_der_zoonosen_in_deutschland_fuer_2002.pdf: 188189 (aufgerufen am 05.08.2005).

KOCH, J.; ALPERS, K.; AMMON, A. (2002). Infektionen mit Salmonellen beim Menschen. Bericht über die epidemiologische Situation der Zoonosen in Deutschland für 2001, Übersicht über die Meldungen der Bundesländer. BgVV-Hefte 6/2001: 23-26.

KÖHLER, B. (1993). Beispiele für die Anreicherung von Salmonellen in der Umwelt. Deutsche tierärztliche Wochenschrift 100: 264-274. Berlin München. 
KÖHLER, H. (2002). Mykotoxine und ihr Einfluss auf die Immunreaktionen. Abstract BandFortbildungsveranstaltung für den öffentlichen Gesundheitsdienst. 13. bis 15. März 2002. Berlin. BfArM, BgVV, BZgA, PEI, RKI, UBA.

KÖLLING, H. (1997). Schlachtbandgeschwindigkeit und seine Auswirkungen auf die Möglichkeiten zur Geflügelfleischuntersuchung. In: ELLERBROEK, L, Erfordernisse und Möglichkeiten in der Schlachtgeflügel- und Geflügelfleischuntersuchung, Protokoll eines Sachverständigengespräches am 20./21. November 1997 im BgVV: 27-29.

KOMPENDIUM GENTECHNOLOGIE UND LEBENSMITTEL (2000). Kompendium Gentechnologie und Lebensmittel. InterNutrion - Schweizerischer Arbeitskreis für Forschung und Ernährung. Quelle: www.genius-biotech.de (aufgerufen am 05.08.2005).

KOTLER, P.; BIEMEL, F. (1992). Marketing- Management, Analyse, Planung, Umsetzung und Steuerung, Kapitel 9: 381-408. C.E. Poeschel Verlag.

KREIENBROCK, L.; SCHÄL, J.; BEYERBACH, M.; ROHN, K.; GLASER, S.; SCHNEIDER, B. (2004). EpiLeg - Orientierende epidemiologische Untersuchung zum Gesundheitsstatus in Legehennenhaltungen verschiedener Haltungssysteme. Quelle: http:// www.tiho-hannover.de/einricht/bioepi/forschung/forschungsprojekte/ibei_26 (aufgerufen am 05.08.2005).

KREISSL-DÖRFLER, W. (2002) Bericht zur Bekämpfung der Maul- und Klauenseuche in der Europäischen Union im Jahr 2001 und zu künftigen präventiven Maßnahmen zur Vermeidung und Bekämpfung von Tierseuchen in der Europäischen Union 2002/2153 (INI). EUROPÄISCHES PARLAMENT A5-0405/2002. Quelle: http://www.kreissldoerfler.de/politik/Bericht\%20abgestimmtDE.pdf (aufgerufen am 05.08.2005).

KREITMEIR, A. (2004). Qualitätsmanagementsysteme und Salmonellenbekämpfung. Bayrische Landesanstalt für Landwirtschaft Institut für Ernährungswirtschaft und Markt. Quelle. http://www.Ifl.bayern.de/iem/qualitaetssicherung/08734/ (aufgerufen am 05.08. 2005).

KREUZER, G. (2002). Mikrobiell und parasitär bedingte Lebensmittelvergiftungen. RFL, Rundschau für Fleischhygiene und Lebensmittelüberwachung, 7/2002 54: 147-150.

KRIETER, J. (2001). Salmonellen in der Zucht- und Produktionsstufe beim Schwein. 1. Workshop, QUASI der Agrar- und Ernährungswissenschaftlichen Fakultät der Christian-Albrechts-Universität zu Kiel. Quelle: http://www.quasi.uni-kiel.de/: 31-38. (aufgerufen am 05.08.2005).

KRÖCKEL, L.; HECHELMANN, H. (1998) Mikrobiologie der Kühlung, Kühllagerung und Fleischreifung. In: Kulmbacher Reihe 15, Kühlen, Zerlegen, Kühllagerung, Reifung: Einfluß auf die Fleischqualität: 35-57

KROKER, R. (2003). Kompaktwissen Tierarzneimittelrückstände. Behrs Verlag.

KRUSE, S. (2005). Die neue Futtermittelhygieneverordnung der Europäischen Gemeinschaft. Quelle: http://cdl.niedersachsen.de/blob/images/C13032428_L20.pdf (aufgerufen am 25.10.2005).

KÜHNE, D. (2003). Verbraucher-Akzeptanz von Eberfleisch. Fleischwirtschaft 83 6/2003: 94. 
KÜHNEN, V.; GOLDBACH, H. E. (2004). Schwermetallbilanzen verschiedener Betriebstypen: Eintragswege, Flüsse, Minderungspotential. Landwirtschaftliche Fakultät der Universität Bonn, Schriftenreihe des Lehr- und Forschungsschwerpunktes USL: 118.

KUIPER-GOODMANN, T. (1999). Approaches to the Risk Analysis of Mycotoxins in the Food Supply. Third joint FAO/WHO/UNEP inmternational converence on mycotoxins Tunis, Tunisia, 3-6 March 1999. Quelle: ftp://ftp.fao.org/es/esn/food/myco7a.pdf (aufgerufen am 05.08.2005).

KUMMER, B. (2001). Klärschlammentsorgung - Beitrag zu einer nachhaltigen Kreislaufwirtschaft?. bvse- Bundesverband Sekundärrohstoffe und Entsorgung e.V. Quelle: http://www.bvse.de/service/fachauf/btrbeitrag.php3?id=16.

KÜNZI, N.; STANZINGER, G. (1993). Zuchtprogramme u. Schwein. Allgemeine Tierzucht, 382-409 und 99-114. Ulmer.

LAMBOOIJ, E. (2000). Transport Of Pigs. In: Livestock Handling and Transport. GRANDIN, T. (Hrsg.): 275 - 296. CABI Publishing New York

LAMBOOIJ, E.; GERRITZEN, M.A.; ENGEL, B.; HILLEBRAND, S.J.W.; LANKHAAR, J.; PIETERSE, C. (1999). Behavioural responses during exposure of broiler chickens to different gas mixtures. Appl. Anim. Behav. Sci. 62: 255-265.

LEHNER, P. (1999). Gentechnik aus der Sicht des Konsumenten, Gründe für die Ablehnung - Kriterien für die Akzeptanz. Fachartikel INFORM 1/99. Quelle: http://www.akwien.at /dat/6144_2.pdf (aufgerufen am 05.08.2005).

LEITLINIE BADEN-WÜRTTEMBERG (2004). Baden-Württembergische Leitlinie für eine gute Hygiene-Praxis in Schlacht-, Zerlegungs- und Fleischverarbeitungsbetrieben. Quelle: htp://www.mlr.baden-wuerttemberg.de/mlr/bse/Leitlinien03-05-04.pdf (aufgerufen am 15.10.2005).

LEHESKA, J.M.; D.M. WULF und R.J. MADDOCK (2003). Effects of fasting and transportation on pork quality development and extent of post-mortem metabolism. Journal of Animal Science 81: 3194-3202

LIPPMANN, C.; LUSKY, K.; HENKLEIN, P.; SASSE, M.; FISCHER, L.; SCHADE, R.; DOBERSCHÜTZ, K. D. (2004). Untersuchungen zum Nachweis von TSE mittels Antikörpern, Möglichkeiten und Grenzen des Einsatzes am lebenden Tier. Fleischwirtschaft 84 7/2004: 97-99.

LMHV - Lebensmittelhygiene-Verordnung (1997). Verordnung über Lebensmittelhygiene . BGBI. I 1997: 2008 97a; 21.5. 2001: 959.

LÖHREN, U. (2004). Tagungsbericht, 22. Jenaer Symposium - Zoonosen des Geflügels, Teil 1. Bundesgesundheitsbl - Gesundheitsforsch -Gesundheitsschutz 2004: 41-50.

LÜCKER, E. (2002) 12 Auflg. Yes, I'm still eating beef!. Fleischwirtschaft 82 12/2002: 8-9.

LUY, J.; HILDEBRAND, G. (2003). Das Problem der Ferkelkastration, Ebergeruch - Schnittstelle von Tierschutz und Lebensmitteltechnologie. Fleischwirtschaft 83 11/2003: 2226. 
LUY, J.; HILDEBRAND, G. (2004). Die Tiertötung - seit über zwei Jahrtausenden ein Problemfall abendländischer Philosophie. Symposium: Ethische und ethnische Aspekte bei der Auswahl und der Herstellung von Lebensmitteln 2002: 7-23. Gesellschaft Deutscher Lebensmitteltechnologen e.V.. Buckenhüskes.

MACHOLD, U.; TROEGER, K.; MOJE, M. (2003). Gasbetäubung von Schweinen. Fleischwirtschaft 83 10/2003: 109-114.

MAIER, A.; SCHIER, A. (2004A). Integrierte Bekämpfung von Ährenfusariosen an Winterweizen in Baden-Württemberg, 1. Teil: Der Einfluss von Bodenbearbeitung, Fruchtfolge, Düngung, Sortenwahl und Strohmanagement auf das Auftreten von Ährenfusarien. Vortrag 16-1. 54. Deutsche Pflanzenschutztagung in der Universität Hamburg, Posterdemonstration, Quelle: http://www.bba.de/veranst/dpst/vortraegekomplett. pdf (aufgerufen am 05.08.2005).

MAIER, A.; SCHIER, A. (2004B). Integrierte Bekämpfung von Ährenfusariosen an Winterweizen in Baden-Württemberg, 2. Teil: Der Einfluss von Fungiziden auf das Auftreten von Ährenfusarien. Vortrag 16-2. 54. Deutsche Pflanzenschutztagung in der Universität Hamburg, Posterdemonstration. Quelle: http://www.bba.de/veranst/dpst/ vortraege-komplett.pdf (aufgerufen am 05.08.2005).

MÄNNER, K.; SIMON, O. (2001). BSE: Anmerkungen aus der Sicht der Tierernährung. Runder Tisch in Brandenburg zur Rinderkrankheit BSE: 32-47. Ministerium für Landwirtschaft, Umweltschutz und Raumordnung.

MÄNNL, M. (1994). Betäubung und Entblutung von Schwein und Rind. In: Kulmbacher Reihe, Band 13, Schlachten von Schwein und Rind: 62-83.

MATHES, M.; IDEL, A. (2004). Kapitel 2: Entwicklung von Agrobiodiversität und Zucht bei Pflanzen und Tieren. Agrobiodiversität entwickeln! Handlungsstrategien für eine nachhaltige Tier- und Pflanzenzucht. Institut für ökologische Wirtschaftsforschung, Öko-Institut, Schweinsfurt-Stiftung, Freie Universität Berlin, Landesanstalt für Großschutzgebiete. Quelle: www.agrobiodiversitaet.net: 34-37 (aufgerufen am 14.05.2005).

MCADIE, T. M.; KEELING, L. J. (2002). The social transmission of fether pecking in laying hens: effects of enviroment and age. J. App. Anim. Behav. Sci. 75: 147-159.

MCKELLAR, Q.A. (1999). Antibiotics and resistance in farm animals. Nutrition \& Food Science 4/1999: 178-180.

MELCHERS, C.B. (2003). Erwartungen des Verbrauchers zu Tierhaltung und Lebensmittelsicherheit. Deutsche tierärztliche Wochenschrift 110 8/2003: 319-323. Berlin München.

METHNER, U. (2004). 22. Jenaer Symposium - Zoonosen des Geflügels, Tagungsbericht, Teil 1. Bundesgesundheitsbl - Gesundheitsforsch - Gesundheitsschutz 47/2004: 4150 .

METTENLEITER, T. C. (1998). Viren und Prionen in der Nahrungskette. Nahrungsketten, Risiken durch Krankheitserreger, Produkte der Gentechnologie und Zusatzstoffe?. 9198. Karl F. Haug Fachbuchverlag. 
MEYER, C. (2004). Qualitative und quantitative Risikofaktoren für die Einschleppung und Verbreitung von Salmonellen in unterschiedlichen Produktionsverfahren beim Schwein. Dissertation an der Außenstelle für Epidemiologie der Tierärztlichen Hochschule Hannover und dem Institut für Tierzucht und Tierhaltung der Christian-AlbrechtsUniversität zu Kiel. Tierärztliche Hochschule Hannover.

MEYER, H. H. D. (1999). Anabole Hormone. Fleischwirtschaft 79 7/1999: $19-20$.

MISSURA, M. (2001). Hormonrückstände in Rind- und Kalbfleisch. BVET Magazin 4/2001: 24-27

MOJE, M. (2004). Große Lücke schließen; Schlachten: Momentaufnahme und Ausblick. Fleischwirtschaft 84 12/2004: 10.

MORTIMORE, S.; WALLACE, C. (2000). Eine Einführung in das System von HACCP. In: HACCP, Die praktische Umsetzung. MORTIMORE, S. ; WALLACE, C. (Hrsg.).: 1-13. Behrs Verlag.

MÜLLER, H. (2001). Maul- und Klauenseuche (MKS) - Wissenschaftliche Grundlagen. Universität Leipzig. Veterinärmedizinische Fakultät Institut für Virologie. Leipzig.

MÜLLER, H. E. (2002). Lebensmittelinfektionen und -vergiftungen, Klinik, Therapie und gesetzliche Grundlagen zur Verhütung. Behrs Verlag.

MÜNSTER, Y.; BLANK, R.; WOLFFRAM, S. (2002A). Ochratoxin A. Erster Arbeitsbericht der Arbeitsgruppe Lebensmittelqualität und -sicherheit QUASI. Quelle: http://www.quasi. uni-kiel.de/download/Bericht_QUASI_2002.pdf: 16-24 (aufgerufen am 05.08.2005).

MÜNSTER, Y.; BLANK, R.; WOLFFRAM, S. (2002B). Mykotoxine in der Tiernährung. Erster Arbeitsbericht der Arbeitsgruppe Lebensmittelqualität und -sicherheit QUASI. Quelle: http://www.quasi.uni-kiel.de/download/Bericht_QUASI_2002.pdf: 35-48 (aufgerufen am 05.08.2005).

NEUBAUER, H.; SPRAGUE, L.D.; SCHOLZ, H.; HENSEL, H. (2001). Nutztiere - Fachinformation, Yersinia enterocolitica: 2. Bedeutung beim Menschen. Deutsche tierärztliche Wochenschrift 11. Quelle: http://www.vetline.de/facharchiv/nutztiere/fachinformation/ yersinia_enterocolitica.htm (aufgerufen am 06.05.2002).

NIJDAM, E.; ARENS, P.; LAMBOOIJ, E.; DECUYPERE, E.; STEGEMAN, J.A. (2004). Factors Influencing Bruises and Mortality of Broilers During Catching, Transport, and Lairage; Einflussfaktoren auf Verletzungen und mortabilität bei Hähnchen während des Fangens, des Transportes und der Wartezeit. Poultry Science 83: 1610-1615.

NISCHWITZ, G. (2004). Sondierungsstudie "Gentechnikfreie Regionen in Deutschland". Quelle: http://www.bfn.de/09/nischwitzioew.pdf (aufgerufen am 01.04.2005).

NORTHOFF, E. (1998). FAO: In Europa besteht weiter die Gefahr von Tierseuchen. Informationsdienst Wissenschaft. Quelle. http://idw-online.de/public/zeige_pm.html? pmid=167 (aufgerufen am 05.08.2005).

OFROU (2001). Merkblatt betreffend Anforderungen an Fahrzeuge für den Transport von Tieren. Bundesamt für Straßen, 30-25.2 Bon/Kue, Bern, 11.Mai 2001. Quelle: http:// www.astra.admin.ch/media/pdfpub/2001-05-11_72_f.pdf 
OLAH, P.A.; ELIJAH, L.M.; DOCKTER, M.R.; DOETKOTT, C.; MILLER, Z.; LOGUE, C.M. (2004). Comparision of antimicrobial resistance in Salmonelle and Campylobacter isolated from turkeys in zhe Midwest USA. Food Microbiol. 21: 779-789.

PDV (Productschap Diervoeder) (2003A). GMP-Richtlinien für die Konzeption und Organisation von Rückrufaktionen, GMP 24, 12.11.2003. Quelle: www.pdv.nl (aufgerufen am 05.08.2005).

PDV (Productschap Diervoeder) (2003B). Richtlinien für Tracking \& Tracing nach GMP für den Tierfuttersektor 2002, GMP27; 12.11.2003. Quelle: www.pdv.nl (aufgerufen am 05.08.2005).

PDV (Productschap Diervoeder) (2004). Evaluierung von Salmonellen in Futtermitteln 2003. Qualitätsreihe Nr. 98. Marktverband Tierfutter.

PDV (Productschap Diervoeder) (2005A). GMP-Standard für den Straßentransport im Tierfuttersektor, GMP07; 24.05.2005. Quelle: www.pdv.nl (aufgerufen am 05.08.2005).

PDV (Productschap Diervoeder) (2005B). GMP-Standard für Handel und Produktion von Mischfutter, GMP02; 16.02.2005. Quelle. www.pdv.nl (aufgerufen am 05.08.2005).

PESTEMER, W.; BUHR, L.; HEIDEL, W.; RÖBER, K.-C.; BROSCHEWITZ, B. (2003). Aufklärung der Kontaminationspfade von Nitrofen. Nachrichtenblatt des Deutschen Pflanzenschutzdienstes: 45-50. Quelle: www.bba.de (aufgerufen am 10.12.2004)

PETERMANN, S.; MAIWORM, K. (2004). Sichere Lebensmittel und alternative Haltungsformen beim Geflügel - ein Widerspruch?. 22. Jenaer Symposium - Zoonosen des Geflügels, Tagungsbericht, Teil 1, Bundesgesundheitsbl - Gesundheitsforsch Gesundheitsschutz 2004/47: 41-50.

PETERSEN, U. (2001). Carry over Forschung aus der Sicht der Gesetzgebung. 30 Jahre Carry over Forschung, BAFF, Vom Blei im Splitter zum Dioxin im Trester: 95-107.

PETTY, R.E.; CACIOPPO, J.T. (1986). The Elaboration-Likelihood-Model of persuasion .In: Advances in Experimental Social Psychology. BERKOWITZ, L. (Hrsg.) Bd. 19: 123.205. New York. Academic Press.

PFANNHAUSER, W. (2005). Standards in der Agrarwirtschaft: öffnen sie neue Produktsegmente, sichern sie bestehende Produkte ab?. In: Jahrbuch der österreichischen Gesellschaft für Agrarökonomie, Band 14. DARNHOFER, I.; PÖCHTRAGER, S.; SCHMID, E. (Hrsg.): 1-5.

PIETSCHMANN, C.; HAFEZ, H. M. (2002). Von Salmonellen und glücklichen Hühnern, Der Spagat zwischen artgerechter Haltung und Verbraucherschutz. Fundiert. Quelle: www.elfenbeinturm.net/archiev/2002a/kampf4.html (aufgerufen am 05.08.2005).

PINGEL, H.; WICKE, M.; VON LENGERKEN, G. (1998). Gewinnung und Qualität von Geflügelfleisch. In: Fleisch- und Fleischwaren, Band 1. BRANSCHEID, W.; HONIKEL, K.O.; VON LENGERKEN, G.; TROEGER, K. (Hrsg.): 301-338.

PÖCKER, C.; SCHULZE ALTHOFF, G. ;PETERSEN, B.; BLAHA, T. (2004). "Risikoorientierte Fleischuntersuchung" Ein Informations- und Entscheidungsmodell. Fleischwirtschaft 84 03/2004:113-116.

POTTHAST, K. (1981). Chemie der Fette und Fettbegleitstoffe. In: Kulmbacher Reihe Band 2, Beiträge zur Chemie und Physik des Fleisches: 19-37. 
POTTHAST, V. (2002). Nulltoleranz - Realität oder Wunschdenken. 2. DVT-Jahrestagung, Futtermittel in Lebensmittelqualität - zwischen Theorie und Praxis. Quelle: www.dvtiernahrung .de/img/medien/Lang-Potthast.pdf (aufgerufen am 10.04.2005).

PUDEL, V. (2002). Der mündige Verbraucher: Zwischen Gefühl und Vernunft?. anlässlich des 4. Ernährungs-Symposiums der Heinz Lohmann Stiftung ,Agrar- oder Verbraucherwende? Wer bestimmt den Markt?" am 19. und 20. Juni 2002 in Königswinter / Bonn, Quelle: http://www.fnl.de/aktuelles/artikel.html\#pudel (aufgerufen am 15.10.2003).

QAIM, M.; VIRCHOW, D. (1999). Macht Grüne Gentechnik die Welt satt? - Herausforderungen für Forschung, Politik und Gesellschaft -. Gutachten für die Friedrich-EbertStiftung, Quelle: http://www.zef.de/download/articles/GrueneGentechnik-FES.PDF (aufgerufen am 05.08.2005).

QS (2005a). Bereits über 1.000 Systempartner im QS-System (01.01.2005). Quelle: www.qs.info (aufgerufen am 05.08.2005).

QS (2005b). Leitfaden Futtermittelwirtschaft, 1.03.2005. Quelle: www.q-s.info (aufgerufen am 05.08.2005).

QS (2005c). Checkliste Futtermittelwirtschaft, 1.09.2005. Quelle: www.q-s.info (aufgerufen am 05.10.2005).

QS (2005d). Leitfaden Salmonellenmonitoring, 7.09.2005. Quelle: www.q-s.info (aufgerufen am 05.08.2005).

QS (2005e). Checkliste zur neutralen Kontrolle - Schlachtung/Zerlegung - Schwein, 1.09.2005. Quelle: www.q-s.info (aufgerufen am 05.10.2005).

QS (2005f). Leitfaden zur Produktion von Hack- und Druschfrüchten, 1.09.2005. Quelle. www.q-s.info (aufgerufen am 05.10.2005).

QS (2005g). Leitfäden Landwirtschaft Schwein, Geflügel u. Rind, 1.09.2005. Quelle: www.qs.info (aufgerufen am 05.10.2005).

QS (2006). Kategorisierte Betriebe im Salmonellenmonitoring -Stand 09.05.2006-. Telefonische Auskunft, Herr May, QS Mitarbeiter verantwortlich für das Salmonellenmonitoring

QUASI (2002). Mykotoxine. Quelle: http://www.quasi.uni-kiel.de/download/Bericht_QUASI_ 2002.pdf; Quasi, erster Arbeitsbericht (aufgerufen am 05.08.2005).

RANDEL, G. (2002). Aflatoxine. Quasi, erster Arbeitsbericht der Arbeitsgruppe Lebensmittelqualität und -sicherheit der Agrar- und Ernährungswissenschaftlichen Fakultät der Christian-Albrechts-Universität zu Kiel, vorgelegt am 01. Februar 2002. Quelle: http:// www.quasi.uni-kiel.de/download/Bericht_QUASI_2002.pdf: 9 (aufgerufen am 15.05. 2004).

RANGNICK, U.-B. (2005). mündliche Auskunft. Informationsbüro der Niederländischen Fleischwirtschaft, Düsseldorf.

RENN, O.; KASTENHOLZ, H.; BRÜGGEMANN, A.; GRAY, P.; HENSCHEL, C.; ROHRMANN, B.; WIEDEMANN, P. (2000). Risk Communication Chemical Product Risks. An OECD Background Paper. OECD-Workshop Berlin, Germany: 18-20. 
RENN, O.; HAMPEL, J. (2002). Gentechnik: Von der Akzeptanz zur Akzeptabilität. BMVEL, Fachtagung Was ist Sache in der Grünen Gentechnik?, 19. und 20. April 2002, Bad Neuenahr. Quelle: http://www.gruee-gentechnik.de/dgg/Doku_Fachtagung/Renn_ vortrag.pdf (aufgerufen am 05.08.2005).

REUTER, G. (2003). Mikrobiologie des Fleisches. In: Mikrobiologie der Lebensmittel, Fleisch - Fisch Feinkost. WEBER, H. (Hrsg.): 1-111. Behrs Verlag

REWERTS, I. (1998). Aufklärung der Verbraucher auf dem Gebiet der Ernährung. 17. Hülsberger Gespräche. Quelle: http://www.schaumann-stiftung.de/deutsch/4/pb3.php3: 132-141 (aufgerufen am 10.08.2005).

RIEDER, P.; Anwander Phan-huy, S. (1998). Soziale und ökonomische Risiken der Gentechnologie im Agrarsektor. Agrarwirtschaft und Agrarsoziologie 1/98: 77-95, Quelle: http://www.zil.ethz.ch/publications/members/soziooekonomische_Risiken_der_ Gentec (aufgerufen am 05.08.2005).

RKI, Robert Koch Institut / BgVV, Bundesinstitut für gesundheitlichen Verbraucherschutz u. Veterinärmedizin / PEI, Paul-Ehrlich-Institut / BfArM, Bundesinstitut für Arzneimittel u. Medizinprodukte (2001). 44. Auflg. Die bovine spongioforme Enzephalopathie (BSE) des Rindes und deren Übertragbarkeit auf den Menschen. Bundesgesundheitsbl Gesundheitsforsch - Gesundheitsschutz 5•2001, 44: 421-431.

RKI, (2004). Robert Koch Institut. Aktuelle Statistik meldepflichtiger Infektionskrankheiten. Epidemiologisches Bulletin. Quelle: http://www.rki.de/INFEKT/EPIBULL/2004/02_04. PDF (aufgerufen am 05.08.2005).

RL (EU) Nr. 64/433/EWG. Richtlinie 64/433/EWG des Rates über die gesundheitlichen Bedingungen für die Gewinnung und das Inverkehrbringen von frischem Fleisch.

RL (EU) Nr. 72/462/EWG. Richtlinie Nr. 72/462/EWG des Rates vom 12. Dezember 1972 zur Regelung viehseuchenrechtlicher und gesundheitlicher Fragen bei der Einfuhr von Rindern und Schweinen und von frischem Fleisch aus Drittländern. Amtsblatt Nr. L 302 vom 31.12.1972: 0028-0054.

RL (EU) Nr. 91/628/EWG. Richtlinie Nr. 91/628/EWG des Rates vom 19. November 1991 über den Schutz von Tieren beim Transport sowie zur Änderung der Richtlinien 90/425/EWG und 91/496/EWG. Amtsblatt Nr. L 340 vom 11.12.1991: 0017-0027.

RL (EU) Nr. 92/102/EWG. Richtlinie 92/102/EWG des Rates vom 27. November 1992 über die Kennzeichnung und Registrierung von Tieren. Amtsblatt Nr. L 355 vom 05.12.1992: 0032-0036.

RL (EU) Nr. 93/43/EWG. Richtlinie Nr. 93/43/EWG des Rates vom 14. Juni 1993 über Lebensmittelhygiene. Amtsblatt Nr. L 175 vom 19.07.1993: 0001/0011.

RL (EU) Nr. 93/119/EG. Richtlinie 93/119/EG des Rates vom 22. Dezember 1993 über den Schutz von Tieren zum Zeitpunkt der Schlachtung oder Tötung. Amtsblatt. Nr. L 340/21, geändert durch VO (EG) Nr. 806/2003 vom 14. 4. 2003 Amtsblatt. Nr. L 122/1 und VO(EG) Nr. 1/2005 vom 22. 12. 2004 Amtsblatt. Nr. L 3/1.

RL (EU) Nr. 95/29/EG. Richtlinie Nr. 95/29/EG des Rates vom 29. Juni 1995 zur Änderung der Richtlinie Nr. 91/628/EWG über den Schutz von Tieren beim Transport. Amtsblatt Nr. L 148 vom 30.06.1995: 0052-0063. 
RODEHUTSCORD, M. (2002). Phosphorversorgung vom Schwein: Sorgsamer Umgang mit begrenzten Reserven. Quelle: http://www.landw.uni-halle.de/lfak/inst/iew/P-Schwein. pdf (aufgerufen am 05.08.2005).

RÖHR, A.; ALVENSLEBEN, V. R.; MÜLLER, M.J. (2002A). Teil 3: Verbraucherinformation durch Risikobewertung und -kommunikation bei Lebensmitteln. QUASI, Erster Arbeitsbericht der Arbeitsgruppe Lebensmittelqualität und -sicherheit: 123, 127.

RÖHR, A.; MÜLLER, M.J.; ALVENSLEBEN, V. R. (2002B). Verbraucherschutz und Risikowahrnehmung - wir brauchen eine differenzierte Vorgehensweise. QUASI, Zweiter Arbeitsbericht der Arbeitsgruppe Lebensmittelqualität und -sicherheit, Schriftenreihe der Agrar- und Ernährungswissenschaftlichen Fakultät der Universität Kiel, Heft 97: 60-68.

SAMITZ, G. (2000). Lebensmittelsicherheit am Beispiel Fleisch, Von der Urproduktion bis zum Verzehr. Internistische Praxis: 461-462. München Marseille.

SANDMAN, PH. D. (1993). Responding to Community Outrage: Strategies for Effective Risk Communication. American Industrial Hygiene Association.

SANTARIUS, K. (2004). 25 Jahre Arzneimittelrückstände. Fleischwirtschaft 84 1/2004: 112115.

SAULI, I. (2004). Neuer Ansatz zur Evaluierung der Salmonellenüberwachung. BVETMagazin 3/2004; Schweizer Zoonosenbericht 2003: 20-22.

SCANGA, J.A.; MCKEITH, F.K.; SAVELL, J.W.; BELK, K.E.; GRIFFIN, D.B.; WRIGHT, L.I.; STETZER, A.J.; PERSON, R.C.; LONERGAN, S.M.; POWELL, T.H.; MEISINGER, D.J.; SMITH, G.C. (2003). Benchmarking Value in the Pork Supply Chain: Quantitive Strategies and Opportunities to Improve Quality. Final Report to the National Pork Board by Colorado State University, University of Illinois at Urbana, Texas A\&M University and lowa State University to the American Meat Science Association, Savoy, IL.

SCHADE, C.; EISELE, T.; PALBERG, N. (2003). Aktive Schädlingskontrolle. Behrs Verlag.

SCHÄFFER, D.; VON BORELL, E. (2002). Tiergerechtheit: Handling von Schlachtschweinen. Fleischwirtschaft 82 11/2002: 51-56.

SCHÄFFER, D.; VON BORELL, E. (2004a). Treiber treffen häufig nicht richtig; Der Treibhilfeneinsatz beim Eintritt der Schweine in Elektrobetäubungsanlagen. Fleischwirtschaft 84 7/2004: 21-24.

SCHÄFFER, D.; VON BORELL, E. (2004b). Tiergerechtheit: Handling von Schlachtschweinen, 5. Kontrollpunkte für die CO2-Betäubung-Checklisten für die Beurteilung. Fleischwirtschaft 84 02/2004: 22-29.

SCHÄFFER, D.; VON BORELL, E. (2005). Vokalisation von Schlachtschweinen. Fleischwirtschaft 85 9/2005: 37-44.

SCHEEDER, M. R. L. (1992). Untersuchungen der Fleischbeschaffenheit verschiedener Muskeln von Jungmastrindern. Dissertation, FOSVW der Universität Göttingen.

SCHENKEL, H. (2001). Am Anfang standen die Schwermetalle. In: 30 Jahre Carry over Forschung, Vom Blei im Splitter zum Dioxin im Trester, Zusammenstellung einer 
Vortragsreihe am 19./20. Juni 2001, Bundesanstalt für Fleischforschung Kulmbach: 519.

SCHEPER, U. (1999). Das Image der Landwirtschaft bei Meinungsmultiplikatoren. Dissertation Kiel, Shaker Verlag.

SCHIEMANN, J. (2002). Bewertung der Umweltauswirkungl gentechnisch veränderter Pflanzen. BLL-Workshop "Gentechnik im Lebensmittelbereich" - Stand der Wissenschaft - 21. Februar 2002: 56-65.

SCHLENKER, G. (2003). TSE - Stoffkreisläufe in der Tierproduktion. Fleischwirtschaft 82 6/2003: 112-114.

SCHLÜTER, A.; BEYER, C.; BEYER, W.; HAGELSCHUER, I.; GEUE, C. (1992). Epidemiologische Untersuchungen in großen Geflügelanlagen am Beispiel der Infektion mit unterschiedlichen Salmonella-Serovaren; Vol. 2. Prox 3rd World Congress "Foodborne Infections and Intoxikations", Berlin 16.-19.06.1992: 1004-1009

SCHMIDT, K.; GERVELMEYER, A. (2003). WHO Surveillance Programme for Control of Foodborne Infections and Intoxications in Europe, 8th Report 1999-2000. Quelle: http://www.bgvv.de/cms/detail.php?template=internet_de_index_js (aufgerufen am 05.08.2005).

SCHMITZ, S.; HISS, S.; SAUERWEIN, H. (2003). Einsatz von Immunstimulatoren als Alternative zu Leistungsförderern in der Schweinemast. Forschungsbericht Nr. 111 des Institut für Physiologie, Biochemie und Hygiene der Tiere der Friedrich-WilhelmsUniversität Bonn. Lehr- und Forschungsschwerpunkt „Umweltverträgliche und Standortgerechte Landwirtschaft“, Landwirtschaftliche Fakultät der Rheinischen Friedrich-Wilhelms-Universität Bonn.

SCHÖPE, M. (2005). Die Rolle der bäuerlichen Landwirtschaft aus volkswirtschaftlicher Sicht. Beitrag im Rahmen der II. Bayerisch-Österreichischen Strategietagung Bauern, Märkte, Leben Visionen für Europa 2020, Landshut, 31.03 bis 01.042005.

SCHRAMM, M.; SPILLER, A. (2003). Farm-Audit- und Farm-Advisory-System. Quelle: http://wwwuser.gwdg.de/ uaao/spiller/publikationen/publikationen.htm (aufgerufen am 05.08.2005).

SCHROETER, A.; DORN, CH.; HELMUTH, R. (2003). Bericht des Nationalen Veterinärmedizinischen Referenzlabors für Salmonellen im Jahr 2002 in Bericht über die epidemiologische Situation der Zoonosen in Deutschland für 2002, Übersicht über die Meldungen der Bundesländer. Quelle: http://www.bfr.bund.de/cms/media.php/95/ bericht_ueber_die_epidemiologische_situation_der_zoonosen_in_deutschland_fuer_2 002.pdf: 133-139 (aufgerufen am 05.08.2005).

SCHÜTT-ABRAHAM, I. (1999). Tierschutzgerechte Betäubung von Schlachtgeflügel - Teil I: Elektrobetäubung. EG-Seminar "Tierschutz", 24.08-02.09.1999, Dublin. Quelle: http://www.schuett-abraham.de/schlachtung/bet.htm (aufgerufen am 20.10.2005).

SCHÜTT-ABRAHAM, I. (2003). TSE im Schaf / BSE im Schaf?. Fleischwirtschaft 83 6/2003: 106-108.

SCHÜTTE, A.; WENZLAWOWICZ, M.V.; MICKWITZ, G.V. (1994). Tiertransporte und Fleischqualität bei Schweinen. Fleischwirtschaft 74: 126-132. 
SCHWIND, K.-H. (2000). Zum Carry-over Verhalten von Toxaphen. Forschungsreport 2/2000: 22-24.

SCHWIND, K.-H. (2004). Umweltkontaminanten im Lebensmittel Fleisch: Wie viel und woher?. Mitteilungsblatt BAFF 163: 39-50.

SCHWIND, K.-H.; HECHT, H. (2004). Dioxin in Futter- und Lebensmitteln, Ein Paradebeispiel für Carry over-Vorgänge und ihre Folgen. 39. Kulmbacher Woche, 04./05. Mai 2004.

SEIDLER, D, (1998). Fleischgewinnung/Frischfleisch. In: HACCP in der Praxis. SINELL, H.J.; MEYER, H. (Hrsg.): 175-211. Behrs Verlag.

SIES, S.; MAHLAU, G. (1997). Das Image der Landwirtschaft - Ergebnisse von Assoziationstests. Arbeitsbericht Nr. 6, Lehrstuhl für Agrarmarketing. Institut für Agrarökonomie, Universität Kiel.

SINELL, H.-J. (1998a). Situation der Ernährung in Deutschland -mikrobiologische Aspekte-. 17. Hülsenberger Gespräche 1998. Quelle: http://www.schaumann-stiftung.de/deutsch/ 4/pb3.php3: 22-35 (aufgerufen am 10.08.2005).

SINELL, J. (1998b). Grundlagen Entwicklung und Begriffe. In: Lebensmittelsicherheit: HACCP in der Praxis. SINELL, J.; MEYER, H. (Hrsg.): 5-28. Behrs Verlag.

SMALLA, K. (2003). Risikobewertung von Markergenen transgener Pflanzen. Forschungsreport 1/2003: 37-39.

SMULDERS, F. J. M.; UPMANN, M. (2000). Verminderung der bakteriellen Belastung auf frischem Fleisch. Fleischwirtschaft 80 9/2000: 32-35.

STADELMANN, F. X. (2001). Klärschlamm: Dünger oder Abfall. EWAG, Infotag 2001. Quelle. http://www.eawag.ch/events/infotag01/ (aufgerufen am 05.08.2005).

STAHLBERG, D.; FREY, D. (1993). Das Elaboration-Likelihood-Model von Petty und Cacioppo. In: Theorien der Sozialpsychologie, Band 1: Kognitive Theorien. FREY, D.; IRLE, M. S. (Hrsg.).: 327-361.

STAMPER, E. (2004). Animal transportation - An industry perspective. Global conference on animal welfare: an OIE initiative Paris, 23-25 February 2004: 77-78. OIE, European Commission.

STATISTISCHES BUNDESAMT (2003). Land- und Forstwirtschaft, Fischerei. Betriebe mit ökologischem Landbau. Fachserie 3/ Reihe 2.2.1. Wiesbaden

STAUFENBIEL, R. (2001). Die Ursachen von BSE- Ein Überblick über die ätiologischen Theorien zu BSE. Runder Tisch in Brandenburg zur Rinderkrankheit BSE: S. 10-21. Ministerium für Landwirtschaft, Umweltschutz und Raumordnung.

STEINBACH, G.; KROLL, U. (1999). Salmonellainfektionen in Schweinebeständen - Zu ihrer Epidemiologie und Bedeutung für Erkrankungen des Menschen. Deutsche tierärztliche Wochenschrift 106: 282-288. Berlin München. 
STETTER, B (2004). Einführung in das neue EU-Recht. EU-Recht und Fleischhygiene, Leitlinie für Wirtschaft, Handwerk und Überwachung in Baden-Würtemberg, Fachtagung in Brackenheim am 25.11.2004. Quelle: www.mlr.baden-wuerttemberg. de/mlr (aufgerufen am 17.10.2005). Ministerium für Ernährung und Ländlichen Raum Baden-Württemberg.

STRACKE, R. (2000). Schlachtdaten per Modem abrufen - können die Mäster profitieren?. SUS 3/2000: 8-11.

STRAUCH, W. (2002). Wie realistisch ist eine verschleppungsfreie Mischfutterproduktion?. 2. DVT-Jahrestagung, Futtermittel in Lebensmittelqualität - zwischen Theorie und Praxis. DVT, Deutscher Verband Tiernahrung e.V.

SZEWZYK, R.; FEUERPFEIL, I.; LÓPEZ-PILA, J.; SCHMIDT, R. (2001). Übertragung von resistenten Bakterien und von Resistenzgenen zwischen Wasser- Boden - Mensch. Bad Honnef-Symposium 2001, Resistenzentwicklung: Ökologie, Epidemiologie und Prävention (Development of Resistance: Ecology, Epidemiology and Prevention). PaulEhrlich-Gesellschaft für Chemotherapie e.V.

TAUSCHER, B.; BRACK, G.; FLACHOWSKY, G.; HENNING, M.; KÖPKE, U.; MEIERPLOEGER, A.; MÜNZING, K.; NIGGLI, U.; PABST, K.; RAHMANN, G.; WILLHÖFT, C.; MAYER-MIEBACH, E. (2003). Bewertung von Lebensmitteln verschiedener Produktionsverfahren. Statusbericht 2003. Schriftenreihe des BMVEL. Reihe A: Angewandte Wissenschaft, Heft 499.

TAYLOR, A. A.;WEARY, D. M.;LESSARD, M.;BRAITHWAITE, L. (2001). Behavioural responses of piglets to castration: the effect of piglet age. Appl. Anim. Behav. Sci. 73: 32-43.

TEUFEL, P.; HONIKEL, K.O.; WELLING, M. (2000). Essen: eine riskante Sache?. Forschungsreport 2/2000: 4-5.

TGRDEU (2005). Zentrale Dokumentation Tiergenetischer Ressourcen in Deutschland. ZADI, Zentralstelle für Agrardokumentation und -information. Quelle: http://www. genres.de/CFDEV/tgrdeu/index.htm (aufgerufen am 17. Mai 2005).

TIERSCHUTZGESETZ (1998). (BGBI. IS. 1105) vom 25. 05. 1998.

Tierschutztransportverordnung, Verordnung zum Schutz von Tieren beim Transport. TierSchTrV - Tierschutztransportverordnung, Fassung vom 11.6.1999. BGBI. I Nr. 31 1999: 1337; 29.10.2001: 2785 Art. 378.

TRANSGEN (2005a). Weltweiter Anbau von gv-Pflanzen, 2004. Quelle: www.transgen.de (aufgerufen am 31.03.2005).

TRANSGEN (2005b). Wo ist Gentechnik drin?. Quelle: http://www.transgen.de (aufgerufen am 02.04.2005).

TRANSGEN (2005c). Fisch. Quelle: http://www.transgen.de/gentechnik/fische/145.doku.html (aufgerufen am 02.04.2005).

TROEGER, K. (1998). Fleischgewinnung und Behandlung. In: Fleisch- und Fleischwaren, Band 1. BRANSCHEID, W.; HONIKEL, K.O.; VON LENGERKEN, G.; TROEGER, K. (Hrsg.): 363-408. 
TROEGER, K. (2002). Rinderschlachtung: Blutentzug sofort nach Stromfluss-Ende. Fleischwirtschaft 82 06/2002: 22-25.

TROEGER, K. ; VON LENGERKEN, G.; BRANSCHEID, W. (1998). Schlachttiertransport. In: Fleisch- und Fleischwaren, Band 1. BRANSCHEID, W.; HONIKEL, K.O.; VON LENGERKEN, G.; TROEGER, K. (Hrsg.): 339-357.

TROEGER, K.; SCHURR, B.; WACHSMANN, G.; KOLB, R.; BEHRSCHMIDT, M. (2002). Vorbeugende Maßnahmen gegen eine mögliche BSE-Gefährdung, Alternative Methoden zur medianen Längsspaltung bei der Schlachtung von Rindern. Fleischwirtschaft 82 10/2002: 129-135.

V. LENGERKEN, G.; WICKE, M.; FISCHER, K. (1998). Schlachttierwert des Schweines. In: Fleisch- und Fleischwaren, Band 1. BRANSCHEID, W.; HONIKEL, K.O.; VON LENGERKEN, G.; TROEGER, K. (Hrsg.): 205-240.

VAN BORELL, E.H (2001). The biology of stress and its application to livestock housing and transportation assessment. Journal of Animal Science 79 (E. Suppl.): E260 - E267.

VAN DEN DAELE, W. (1993). Hintergründe der Wahrnehmung von Risiken der Gentechnik: Naturkonzepte und Risikosementik. In: Risiko ist ein Konstrukt: Wahrnehmungen zur Risikowahrnehmung. BECKER, U.; DUNWOODY, S. HOLZHEU, F. (Hrsg.): 89-107. Knesebeck Verlag.

VAN DER WOLF, P.J.; BONGERS, J.H.; ELBERS, A.R.; FRANSSEN, F.M.; HUNNEMANN, W.A. (1999). Salmonella infections in finishing pigs in the Netherlands: bacteriological herd prevalence, serogroup and antibiotic resistance of isolates and risk factors for infection.. Vet. Microbiol. 67/4 4: 263 - 275.

VelARDE, A.; GISPERT, M.; FAuCitANO, P.; ALONSO, P.; MANTECA, X.; DIESTRE, A. (2001). Effects of the stunning procedure and the halotane genotype on meat quality and incidence of haemorrhages in pigs. Meat Science 58: 313-318.

VELLEMANN, S. G.; ANDERSON, J. W.; COY, C. S.; NESTOR, K. E. (2003). Effect of selection for growth rate on muscle damage during turkey breast muscle development. Poultry Science 82: 1069-1074.

VEMMER H.; W. HEESCHEN; A. BLÜTHGEN (1991): Übergang polychlorierter Biphenyle(PCB) aus Futtermitteln in Gewebe von Mastschweinen. 2. Mitteilung: Der mengenmäßige Übergang von PCB aus dem Futter in das Mastschwein. Landbauforschung Völkerrode 2, 74-81

VEMMER H.; W. HEESCHEN; A. BLÜTHGEN; R. DAENICKE (1993): Übergang polychlorierter Biphenyle (PCB) aus Futtermitteln in Gewebe von Mastbullen. 2. Mitteilung: PCB-Gehalte in den einzelnen Geweben der Mastbullen in Abhängigkeit von der PCB-Konzentration des Futters. Landbauforschung Völkerrode 1, 27-33

VERFVERBG - VERFÜTTERUNGSVERBOTSGESETZ (2001). Gesetz über das Verbot des Verfütterns, des innergemeinschaftlichen Verbringens und der Ausfuhr bestimmter Futtermitte, vom 29. März 2001. BGBI. 2001 I: 463, 226. 8.8.2002: 3116. 
VERREET, J.; AUMANN, J. (2001). Mykotoxinproblematik in der Pflanzenproduktion. 1. Workshop, Quasi der Agrar- und Ernährungswissenschaftlichen Fakultät der ChristianAlbrechts-Universität zu Kiel. Quelle: http://www.quasi.uni-kiel.de/: 12-17 (aufgerufen am 05.08.2005).

VO (EU) Nr. 1255/97. Verordnung (EG) Nr. 1255/97 des Rates vom 25. Juni 1997 zur Festlegung gemeinschaftlicher Kriterien für Aufenthaltsorte und zur Anpassung des im Anhang der Richtlinie Nr. 91/628/EWG vorgesehenen Transportplans. Amtsblatt Nr. L 174 vom 02.07.1997: 0001-0006.

VO (EU) Nr. 1760/2000. Verordnung (EG) Nr. 1760/2000 des Europäischen Parlaments und des Rates vom 17. Juli 2000 zur Einführung eines Systems zur Kennzeichnung und Registrierung von Rindern und über die Etikettierung von Rindfleisch und Rindfleischerzeugnissen. Amtsblatt der Europäischen Gemeinschaft Nr. L 204 vom 11.08.2000: 0001-0010.

VO (EU) Nr. 1774/2002. Verordnung (EG) Nr. 1774/2002 des Europäischen Parlaments und des Rates vom 3. Oktober 2002 mit Hygienevorschriften für nicht für den menschlichen Verzehr bestimmte tierische Nebenprodukte. Amtsblatt Nr. L 273 vom 10.10.2002: 0001-0095.

VO (EU) Nr. 178/2002. Verordnung vom 28.Januar 2002 zur Festlegung der allgemeinen Grundsätze und Anforderungen des Lebensmittelrechts, zur Errichtung der Europäischen Behörde für Lebensmittelsicherheit und zur Festlegung von Verfahren zur Lebensmittelsicherheit. Amtsblatt Nr. L 031 vom 01.02.2002: 0001-0024.

VO (EU) Nr. 1782/2003. Verordnung (EG) Nr. 1782/2003 des Rates vom 29.September 2003 mit gemeinsamen Regeln für Direktzahlungen im Rahmen der Gemeinsamen Agrarpolitik und mit bestimmten Stützungsregelungen für Inhaber landwirtschaftlicher Betriebe und Amtsblatt der Europäischen Gemeinschaften Nr. L 270/01 vom 21.10.2003: 0001-0069.

VO (EU) Nr. 1829/2003. Verordnung (EG) Nr. 1829/2003 des Europäischen Parlaments und des Rates vom 22. September 2003 über genetisch veränderte Lebensmittel und Futtermittel. Amtsblatt der Europäischen Gemeinschaft Nr. L 268/1 vom 18.10.2003: 0001-0023.

VO (EU) Nr. 1830/2003. Entscheidung der Kommission von 22. September 2003 über die Rückverfolgbarkeit und Kennzeichnung von genetisch veränderten Organismen und über die Rückverfolgbarkeit von aus genetisch veränderten Organismen hergestellten Lebens- und Futtermitteln. Amtsblatt der Europäischen Gemeinschaft Nr. L 268/24 vom 18.10.2003: 0024-0028.

VO (EU) Nr.183/2005. Verordnung (EG) Nr. 183/2005 des Europäischen Parlaments und des Rates vom 12. Januar 2005 mit Vorschriften für die Futtermittelhygiene. Amtsblatt der Europäischen Union Nr. L 35/1.

VO (EU) Nr. 1831/2003. Verordnung (EG) Nr. 1831/2003 des Europäischen Parlaments und des Rates vom 22. September 2003 über Zusatzstoffe zur Verwendung in der Tierernährung (Text von Bedeutung für den EWR). Amtsblatt Nr. L 268 vom 18.10.2003: 0029-0043. 
VO (EU) Nr. 2160/2003. Verordnung (EG) Nr. 2160/2003 des Europäischen Parlaments und des Rates vom 17. November 2003 zur Bekämpfung von Salmonellen und bestimmten anderen durch Lebensmittel übertragbaren Zoonoseerregern. Amtsblatt der Europäischen Gemeinschaft Nr. L 325 vom 12.12.2003: 0001-0015.

VO (EU) Nr. 852/2004. Verordnung (EG) Nr. 852/2004 des Europäischen Parlaments und des Rates vom 29.April 2004 über Lebensmittelhygiene. Amtsblatt der Europäischen Gemeinschaften Nr. L 139/1 vom 30.04.2004: 0001-0054.

VO (EU) Nr. 853/2004. VERORDNUNG (EG) Nr. 853/2004 des europäischen Parlaments und des Rates vom 29. April 2004 mit spezifischen Hygienevorschriften für Lebensmittel tierischen Ursprungs. Amtsblatt der Europäischen Union Nr. L 139/55: 0055-0205.

VO (EU) Nr. 854/2004. Verordnung (EG) Nr. 854/2004 des Europäischen Parlaments und des Rates vom 29.April 2004 mit besonderen Verfahrensvorschriften für die amtliche Überwachung von zum Verzehr bestimmten Erzeugnissen tierischen Ursprungs. Amtsblatt der Europäischen Gemeinschaft Nr. L 139/206 vom 30.04.2004: 0206-0319.

VO (EU) Nr. 882/2004. Verordnung (EG) Nr. 882/2004 des Europäischen Parlaments und des Rates vom 29. April 2004 über amtliche Kontrollen zur Überprüfung der Einhaltung des Lebensmittel- und Futtermittelrechts sowie der Bestimmungen über Tiergesundheit und Tierschutz. Amtsblatt Nr. L 165 vom 30.04.2004: 0001-0141.

VON HOLLEBEN, K.; SCHÜTTE, A.; VON WENZLAWOWICZ, M.; BOSTELMANN, N. (2002). Betäubung: Handlungsbedarf am Schlachthof. Fleischwirtschaft 82 05/2002: 28-30.

VON RICHTHOFEN, I.-B. Freiin. (2003). Beobachtungen zum Verhalten von Schlachtrindern auf Ferntransporten, Dissertation, Tierärztliche Hochschule Hannover

WALLENBURG, J. (1998). Begrüßung zu den 17. Hülsenberger Gesprächen 1998. 17. Hülsenberger Gespräche 1998. Quelle: http://www.schaumann-stiftung.de/deutsch /4/pb3.php3: 7-9 (aufgerufen am 10.08.2005).

WARNANTS, N; VAN OECKEL, M.J.; BOUCQUE, CH.V. (1998). Effect of Incorporation of Dietary Polyunsaturated Fatty Acids in Pork Backfat on the Quality of Salami. Meat Science 49: 435-445.

WARRISS, P. D.; BROWN, S. N. (1983). The influence of pre-slaughter fasting on carcass and liver yield in pigs. Livestock Prod. Sci. 10: 273 - 282.

WARRISS, P. D.; BROWN, S. N. (1994). A survey of mortality in slaughter pigs during transport and lairage. Vet. Record. 134: 513 - 515.

WARRISS, P.D. (1998). The Welfare of slaughter pigs during transport. Animal Welfare 7: 365-381.

WASSMUTH, R.; ACHILLES, W.; OPITZ VON BOBERFELD, W.; ZEEB, A.; HERMANN, HJ.; GLOZE, M. (2002). Ganzjährige Freilandhaltung von Rindern. In: KTBL (Hrsg.): KTBL-Schrift 409

WEBER, H. (1996). Betriebshygiene und Qualitätssicherung. In: Mikrobiologie der Lebensmittel Grundlagen. MÜLLER, G.; WEBER, H. (Hrsg.): 407-529. Hamburg. 
WEGNER-HAMBLOCH, S. (2004). Rückverfolgbarkeit in der Praxis, Artikel 18 u. 19 der VO (EG) Nr. 178/2002 schnell und einfach umgesetzt. Behrs Verlag.

WEIDENBÖRNER, M. (1999). Lebensmittel-Mykologie. Behrs Verlag.

WEISE, E. (2003). Mikrobiologie des Geflügels. In: Mikrobiologie der Lebensmittel, Fleisch Fisch Feinkost. Weber, H. (Hrsg.).: 563-643. Behrs Verlag.

WERNER, C.; REINERS, K.; WICKE, M. (2005). Mortalitätsraten beim Transport von Schlachtschweinen. Fleischwirtschaft 85 9/2005: 133-136.

WHO (2000). WHO responds to new challenges in food safety. Press Releases 2000 $\mathrm{WHO} / 4$.

WICKE, M.; VON LENGERKEN, G.; FIEDLER, I.; ALTMANN, M.; ENDER, K. (1991). Einfluß der Selektion nach Merkmalen der Muskelstruktur des M. longissimus auf Belastungsempfindlichkeit und Fleischbeschaffenheit beim Schwein. Fleischwirtschaft 71: 437442.

WICKE, M.;HAHN, G.; MAAK, S.; VON LENGERKEN, G. (2001). Nachhaltigkeit in der Fleischerzeugung; Der Aspekt physiologischer Wachstumsgrenzen bei Schweinen und Geflügel. Fleischwirtschaft 81 9/2001: 125-128.

WIEDEMANN, P.M. (1999). Risikokommunikation: Ansätze, Probleme und Verbesserungsmöglichkeiten. Heft 70. Arbeiten zur Risikokommunikation. Programmgruppe Mensch, Umwelt, Technik (MUT). Forschungszentrum Jülich GmbH.

WIEDEMANN, P. M.; CLAUBERG, M. (2003) Risikokommunikation (Bericht der Programmgruppe Mensch, Umwelt, Technik). Forschungszentrum Jülich $\mathrm{GmbH}$.

WIESNER, E.; RIBBECK, R. (1991). Wörterbuch der Veterinärmedizin. Fischer Bd. 2.

WIKIPEDIA (2005). WIKIPEDIA, Die freie Enzyklopädie. Quelle: http://de.wikipedia.org/ wiki/Experte (aufgerufen am 20.10.2005).

WIKIPEDIA (2006). WIKIPEDIA, Die freie Enzyklopädie. Quelle: http://de.wikipedia.org/wiki/ Hazard_Analysis_and_Critical_Control_Points (aufgerufen am 25.03.2006).

WILDNER, S. (1998). Die Tierschutzproblematik im Spiegel von Einstellungen und Verhaltensweisen der deutschen Bevölkerung. Arbeitsbericht 9: 50 Institut für Agrarökonomie der Universität Kiel.

WITTE, W.; KLARE, I. (1999). Antibiotikaresistenz bei bakteriellen Infektionserregern. Bundesgesundheitsblatt 42: 8-16.

WITTKOWSKI, G.; HERMÜHLHEIM, A. (2001). Bericht über die Kontrollen im Rahmen des Programms "Offene Stalltür" im Jahre 2000. Tiergesundheitsamt Bayern e.V.

WOLFF, J. (2001). Es begann mit dem Aflatoxin: Die zunehmende Bedeutung der Mykotoxine in der Futter- und Nahrungskette. In: 30 Jahre Carry over Forschung, Vom Blei im Splitter zum Dioxin im Trester. Zusammenstellung einer Vortragsreihe am 19./20. Juni 2001, Bundesanstalt für Fleischforschung Kulmbach: 41-56.

WOLFF, J.; GAREIS, M.; SCHEUER, R.; ENGEL, G.; BRESCH, H.; ROSNER, H.; MAJERUS, P.; SCHNEIDER, R. (1996). Belastung des Verbrauchers und der Lebensmittel mit Ochratoxin A - Proceedings. 18. Mykotoxin Workshop, Kulmbach, 10.12. Juni 1996: 103-107. 
WOLTERSDORF, W. (1994). Aufstallung vor dem Schlachten. In: Kulmbacher Reihe, Band 13, Schlachten von Schwein und Rind: 43-61.

YIANNIKOURIS, A.; JOUANY, J.P. (2002). Mycotoxins in feeds and their fate in animals: a review. Anim. Res. 51: 81-99.

ZDS, ZENTRALVERBAND DER DEUTSCHEN SCHWEINEPRODUKTION E.V. (2003). Schweineproduktion 2002 in Deutschland: 67, 72-75.

ZERL (2005). Untersuchungen im Rahmen des Nationalen Rückstandskontrollplanes 2004, Tabelle , Überblick. Bundesamt für Verbraucherschutz und Lebensmittelsicherheit (BVL). Quelle: http://www.bvl.bund.de/cln_027/nn_493680/DE/01_Lebensmittel/01 Sicherheit_Kontrollen/04_NRKP/01__berichte_nrkp/tabellen2004,templateld=raw, $p$ roperty=publicationFile.pdf/tabellen2003.pdf (aufgerufen am 11.08.2005).

ZMP (2004). Wettbewerb am Geflügelmarkt wird härter, Zwischen Globalisierung und nationalem Anspruch. ZMP-Nachrichten: 10.12.2004. Quelle: http://www.zmp.de/news/ NewsContent.asp?Datald=0\&DatumsZahl=20041217\&rPos=7 (aufgerufen am 05.08.2005).

ZMP BILANZ (2004a). ZMP-Bilanz Vieh und Fleisch, 2004.

ZMP BILANZ (2004b). ZMP-Bilanz Eier und Geflügel, 2004. 


\section{$10.2 \quad$ Tabellenverzeichnis}

Tabelle 2.1-1: Die 7 Grundsätze des HACCP-Konzeptes nach dem Codex Alimentarius. ....................7

Tabelle 2.3-1: Beispiele für Qualitätsmanagementsysteme und ihre Einsatzbereiche.........................15

Tabelle 2.4-1: $\quad$ Nutzen/Vorteile und Risiken/Nachteile von gentechnischen Verfahren (Lehner, 1999; Quaim u. Virchow, 1999; Flachowsky et al., 2000; Jany, 2000a, 2000b; Kompendium Gentechnologie und Lebensmittel, 2000; Nischwitz, 2004).

Tabelle 2.4-2: Bedeutende Keime für lebensmittelbedingte Erkrankungen (MüLLER, 2002; HARTUNG, 2003; RKI, 2004).

Tabelle 2.4-3: Verlässlichkeit der Bewertung und Prävalenz von Salmonellen (1998 - 2000) in jedem Produktionsschritt (SAULI, 2004).

Tabelle 2.4-4: Wichtige Zoonoseerreger und deren Resistenzen auf bestimmte Stoffe (BFR, 2003B).

Tabelle 2.4-5: Anteil der resistenten Salmonellen-Isolate verschiedener Herkünfte 2000-2002 (SCHROETER ET AL., 2002 gekürzt)

Tabelle 2.4-6: Eintragspfade Schwermetalle (SCHENKEL, 2001)

Tabelle 2.4-7: Ablagerungsorte verschiedener Schwermetalle im Organismus landwirtschaftlicher Nutztieren (SCHENKEL, 2001 abgeändert)

Tabelle 2.4-8: Pfanzenschutzmittelrückstände in Getreide (BVL, 2002, 2003; EU, 2001A, 2002C, 2003c).

Tabelle 2.4-9: Verfahrenstechnische Risiken in der Tierproduktion.

Tabelle 2.4-10: Ursachen und Maßnahmen bei Technopathien, Ethnopathien und Haltungsfehlern. ....53

Tabelle 5.1-1: Entwicklung der zur Fleischproduktion eingesetzten Rassen/Genetikvielfalt zum Jahr 2015.

Tabelle 5.1-2: Verzichtverhalten von Studenten auf Fleisch bei Bekanntwerden von Missständen. ...84

Tabelle 5.1-3: $\quad$ Entwicklung des Problembewusstseins der Verbraucher im Hinblick auf Qualzucht zum Jahr 2015

Tabelle 5.2-1: $\quad$ Einschätzung der Entwicklung des Einsatzes von genveränderten Pflanzen in der Fütterung von Masttieren zum Jahr 2015.

Tabelle 5.2-2: $\quad$ Einschätzung der Entwicklung des Problembewusstseins der Verbraucher in Hinblick auf genveränderte/geklonte Tiere zum Jahr 2015

Tabelle 5.3-1: Einschätzung der Entwicklung der Anzahl der Erkrankungen durch Zoonosen zum Jahr 2015.

Tabelle 5.3-2: $\quad$ Einschätzung der Entwicklung des Vorkommens von pathogenen Keimen, die über das Futtermittel das Nahrungsmittel Fleisch kontaminieren, zum Jahr 2015.

Tabelle 5.3-3: Einschätzung der Entwicklung der Übertragung von Krankheitskeimen durch Schädlingsbefall zum Jahr 2015.

Tabelle 5.4-1: Einschätzung der Entwicklung der Gefahr des Eintrags von Keimen/ Krankheitserregern aus dem Ausland zum Jahr 2015. 
Tabelle 5.4-2: Einschätzung des Auftretens von anzeige- und meldepflichtigen Krankheiten zum Jahr 2015.

Tabelle 5.4-3: Einschätzung der Entwicklung des Einsatzes von Resten aus der Lebensmittelproduktion in der Tierfütterung zum Jahr 2015.

Tabelle 5.4-4: Beurteilung der Frage: Halten Sie es für sinnvoll, Reste aus der Lebensmittelproduktion in der Tierfütterung einzusetzen?

Tabelle 5.4-5: Einschätzung der Entwicklung des Einsatzes von Prophylaxen/ Schutzimpfungen zum Jahr 2015.

Tabelle 5.4-6: Einschätzung der Studenten bezüglich der Notwendigkeit des Einsatzes von Prophylaxen/Schutzimpfungen.

Tabelle 5.5-1: Einschätzung der Entwicklung des Vorkommens von Mykotoxinen, die über das Futtermittel das Nahrungsmittel Fleisch kontaminieren, zum Jahr 2015.

Tabelle 5.5-2: Einschätzung der Entwicklung über das Vorkommen von Rückständen aus Pflanzenschutzmitteln im Futtermittel zum Jahr 2015.

Tabelle 5.5-3: Einschätzung der Entwicklung des Vorkommens von Rückständen von in der EU verbotenen Stoffen in importierten Futtermitteln zum Jahr 2015.

Tabelle 5.5-4: Einschätzung der Entwicklung über das Vorkommen von Rückständen aus Müllkompost/Klärschlamm im Futtermittel zum Jahr 2015.

Tabelle 5.5-5: Einschätzung der Entwicklung des Vorkommens von Rückständen (un-) erlaubter Futterzusatzstoffe und Umweltkontaminanten zum Jahr2015.

Tabelle 5.5-6: Einschätzung der Entwicklung der potentiellen Gefahr der Schadstoffabgabe durch Bedarfsgegenstände zum Jahr 2015.

Tabelle 5.5-7: Einschätzung der Entwicklung der potentiellen Gefahr der Fremdkörper zum Jahr 2015.

Tabelle 5.6-1: Einschätzung der Entwicklung der Anlagensicherheit (Verschleppungsgrad) im Herstellungsprozess Futtermittel zum Jahr 2015......

Tabelle 5.6-2: Einschätzung der Entwicklung der Kontamination des Futtermittels bei der Lagerung zum Jahr 2015.

Tabelle 5.6-3: Einschätzung der Entwicklung des Auftretens von Defiziten beim Transport von Futtermitteln zum Jahr 2015.

Tabelle 5.6-4: Beurteilung der Experten über die Auswirkung tierischer Futtermittelkomponenten auf die Fleischqualität.

Tabelle 5.6-5: Einschätzung des Einsatzes und der Sicherheit von tierischen Futtermitteln zum Jahr 2015.

Tabelle 5.7-1: Einschätzung der Bedeutung von Maßnahmen wie Kupieren, Enthornen, Schnabelkürzen zum Jahr 2015.

Tabelle 5.7-2: Antworten zu der Frage: Haben Sie Bedenken gegen Maßnahmen wie Kupieren, Enthornen und Schnabelkürzen?

Tabelle 5.7-3: Einschätzung des Auftretens von Technopathien zum Jahr 2015. 109 
Tabelle 5.7-4: $\quad$ Entwicklung der Fleischproduktion von kastrierten Tieren zum Jahr 2015. 110

Tabelle 5.7-5: Antworten zu der Frage: Sehen Sie Gefahren in der Freilandhaltung von Geflügel und Schweinen für den Menschen oder das Tier?

Tabelle 5.7-6: Einschätzung der Entwicklung des Anteils an Freilandhaltung bei Geflügel und Schweinen zum Jahr 2015.

Tabelle 5.7-7: Antworten zu der Frage: Halten Sie die heutigen Haltungssysteme in der Tiermast für tiergerecht?

Tabelle 5.8-1: Einschätzung der Entwicklung von Tiertransporten in vollklimatisierten Fahrzeugen zum Jahr 2015.

Tabelle 5.8-2: $\quad$ Einschätzung der Entwicklung der Transportzeit für Schlachttiere zum Jahr 2015......115

Tabelle 5.9-1: Einschätzung über das Auftreten von Behandlungs- und Haltungsdefiziten im Bereich Tierannahme/Wartestall.

Tabelle 5.9-2: $\quad$ Entwicklung von Defiziten bei der Betäubung zum Jahr 2015.

Tabelle 5.9-3: Beurteilung der Studenten, welche Betäubungsverfahren der Verbraucher akzeptieren könnte.

Tabelle 5.9-4: $\quad$ Beurteilung einzelner Prozesse in der Schlachtung zum Jahr 2015.

Tabelle 5.9-5: Einschätzung der Sensibilität des Verbrauchers bezüglich BSE-Risikomaterial zum Jahr 2015.

Tabelle 5.9-6: Einschätzung der Entwicklung von Defiziten bei der Gewinnung von Nebenprodukten und Innereien zum Jahr 2015.

Tabelle 5.9-7: Entwicklung der Verzehrmengen von Innereien zum Jahr 2015.

Tabelle 5.9-8: Einschätzung über das Auftreten von Defiziten bei der Fleischkühlung zum Jahr 2015.

Tabelle 5.9-9: Einschätzung von Defiziten beim Fleischtransport und in der Zerlegung zum Jahr 2015.

Tabelle 5.9-10: Einschätzung von Defiziten bei der Fleischverpackung zum Jahr 2015. 122

Tabelle 5.10-1: Einschätzung über das Auftreten mangelhafter Hygiene im Produktionsprozess Futtermittel zum Jahr 2015.

Tabelle 5.11-1: Einschätzung über das Auftreten von Hygienemängeln im landwirtschaftlichen Betrieb zum Jahr 2015.

Tabelle 5.11-2: Einschätzung über die Futterhygiene im landwirtschaftlichen Betrieb zum Jahr 2015.125

Tabelle 5.11-3: Einschätzung über das Auftreten mangelhafter Reinigungshygiene im Schlacht- bzw. Zerlegebetrieb zum Jahr 2015.

Tabelle 5.11-4: Einschätzung über das Auftreten mangelhafter Personalhygiene zum Jahr 2015......126

Tabelle 5.11-5: Beurteilung der Personalhygiene in der Fleisch verarbeitenden Industrie. .................126

Tabelle 5.12-1: Entwicklung des Detaillierungsgrades bei der Rückverfolgbarkeit. ...... 127

Tabelle 5.12-2: Einschätzung der Maßnahmen bezüglich der Kennzeichnung im landwirtschaftlichen Betrieb zum Jahr 2015. . 
Tabelle 5.13-1: Entwicklung des Kontrollumfangs bei Futtermitteln zum Jahr 2015. 129

Tabelle 5.13-2: Antworten auf die Frage: Halten Sie die Schlachttieruntersuchung und Fleischuntersuchung in Deutschland für sicher?

Tabelle 5.13-3: Entwicklung des Umfangs von Untersuchungen, die ergänzend zur Schlachtkörperuntersuchung durchgeführt werden, zum Jahr 2015.

Tabelle 5.13-4: Einschätzung der Gefahrenkategorie durch Fleischskandale im Ausland...................131

Tabelle 5.13-5: Einfluss von Fleischskandalen im Ausland auf die Tierart...........................................131

Tabelle 5.13-6: Entwicklung von Problemen durch Fleischimporte zum Jahr 2015.

Tabelle 5.13-7: Beurteilung der Sicherheit von Fleisch aus Deutschland und von Fleisch, das aus Drittländern zugekauft wurde.

Tabelle 12.1-1: Bevorzugte Rassen/genetische Konstruktionen von Rindern.....................................207

Tabelle 12.1-2: Bevorzugte Rassen/genetische Konstruktionen von Schweinen.................................207

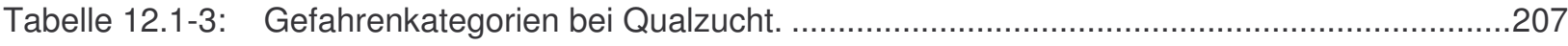

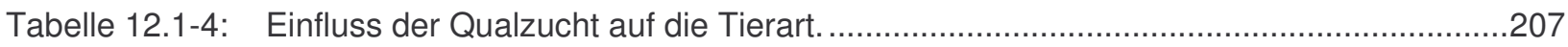

Tabelle 12.1-5: Einschätzung der Gefahrenkategorien bei genveränderten Pflanzen. .......................207

Tabelle 12.1-6: Einschätzung der Gefahrenkategorien bei genveränderten/geklonten Tieren. ...........208

Tabelle 12.1-7: Einschätzung des Einflusses von genveränderten/geklonten Verfahren auf die

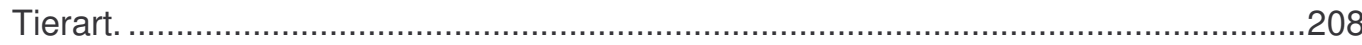

Tabelle 12.1-8: Einschätzung der Gefahrenkategorien bei Feldkeimen...........................................208

Tabelle 12.1-9: Einschätzung des Einflusses von Feldkeimen auf die Tierart....................................208

Tabelle 12.1-10: Einschätzung des Einflusses von Zoonosen auf die Tierart. ......................................208

Tabelle 12.1-11: Einschätzung der Gefahrenkategorien durch Schädlingsbefall. ...............................208

Tabelle 12.1-12: Einschätzung des Einflusses von Schädlingsbefall auf die Tierart............................208

Tabelle 12.1-13: Einschätzung der Gefahrenkategorien bei in Deutschland z.Zt. nicht vorkommenden Keimen/Krankheitserregern, die durch den weltweiten Handel eingeschleppt werden.208

Tabelle 12.1-14: Einschätzung des Einflusses von in Deutschland z.Zt. nicht vorkommenden Keimen/ Krankheitserregern auf die Tierart. 209

Tabelle 12.1-15: Einschätzung der Gefahrenkategorien bei dem Einsatz von Resten aus der Lebensmittelproduktion als Futtermittel. 209

Tabelle 12.1-16: Einschätzung des Einflusses von Resten aus der Lebensmittelproduktion als Futtermittel auf die Tierart...... 209

Tabelle 12.1-17: Einschätzung der Gefahrenkategorien bei dem Verzicht Einsatz von Prophylaxen/ Schutzimpfungen. 209

Tabelle 12.1-18: Einschätzung des Einflusses von Resten aus der Lebensmittelproduktion als Futtermittel auf die Tierart.

Tabelle 12.1-19: Kritische Verfahrensschritte in Ländern aus denen exotische Futtermittel importiert werden. 
Tabelle 12.1-20: Einschätzung der Gefahrenkategorien bei Futtermitteln, die durch mit Altlasten verunreinigte Böden, durch Klärschlamm oder durch Pflanzenschutzmittel aus importierten Futtermitteln kontaminiert wurden.

Tabelle 12.1-21: Einschätzung des Einflusses von durch mit Altlasten, Rückständen aus Klärschlamm oder durch Pflanzenschutzmittel aus importierten Futtermitteln verunreinigten Futtermittel auf die Tierart.

Tabelle 12.1-22: Einschätzung der Gefahrenkategorien bei Rückstände durch Kunststoff- materialien.210

Tabelle 12.1-23: Einschätzung der Gefahrenkategorien bei Fremdkörpern. 210

Tabelle 12.1-24: Einschätzung des Einflusses der Fremdkörpergefahr auf die Tierart. 210

Tabelle 12.1-25: Einschätzung der Gefahrenkategorien durch Reinigungsmittel bei Transportfahrzeugen.

Tabelle 12.1-26: Einschätzung des Einflusses von Rückstände von Reinigungsmitteln in Transportfahrzeugen auf die Tierart.

Tabelle 12.1-27: Einschätzung der Gefahrenkategorien durch Kupieren, das Enthornen, das Schnabelkürzen etc. und Kastration.

Tabelle 12.1-28: Einschätzung des Einflusses auf durch Kupieren, das Enthornen, das Schnabelkürzen etc. und Kastration die Tierart.

Tabelle 12.1-29: Einschätzung der Gefahrenkategorien bei einer Belastung des Bodens durch die Freilandhaltung bei Geflügel und Schweinen.

Tabelle 12.1-30: Einschätzung der Gefahrenkategorie durch Massentierhaltung................................211

Tabelle 12.1-31: Einfluss der Massentierhaltung auf die Tierart.......................................................211

Tabelle 12.1-32: Einschätzung der Gefahrenkategorie durch Technopathien in heutigen Haltungssystemen

Tabelle 12.1-33: Einfluss von Technopathien in heutigen Haltungssystemen auf die Tierart...............212

Tabelle 12.1-34: Einschätzung der Gefahrenkategorien durch Tiertransporte...................................212

Tabelle 12.1-35: Einschätzung des Einflusses von Transporten auf die Tierart..................................212

Tabelle 12.1-36: Einschätzung der Gefahrenkategorien durch eine hohe Bandgeschwindigkeit. 212

Tabelle 12.1-37: Einschätzung der Gefahrenkategorien zu der These: Der Verzicht auf tierische Futtermittelkomponenten wirkt sich auf die Zusammensetzung des Fleisches aus.....212

Tabelle 12.1-38: Einschätzung der Studenten bezüglich des Einsatzes und der Sicherheit tierischer Futtermittel an Geflügel und Schweine zum Jahr 2015.

Tabelle 12.1-39: Einschätzung des Einflusses der Aufbereitungsmethoden bei tierischen Futtermitteln auf die Tierart.

Tabelle 12.1-40: Einschätzung der Gefahrenkategorien bei der Entsorgung der Ursprungsstoffe tierischer Futtermittel (Kadaver, Schlachtabfälle usw.).

Tabelle 12.1-41: Einschätzung des Einflusses bei der Entsorgung der Ursprungsstoffe tierischer Futtermittel (Kadaver, Schlachtabfälle usw.)auf die Tierart.

Tabelle 12.1-42: Einschätzung der Gefahrenkategorien bei BSE-Risikomaterial. 213 
Tabelle 12.1-43: Einschätzung des Einflusses von BSE-Risikomaterial auf die Tierart. 213

Tabelle 12.1-44: Teile vom Schlachttierkörper und Bearbeitungsmethoden des Schlachttieres, die kritisch zu bewerten sind.

Tabelle 12.1-45: Einschätzung der Gefahrenkategorie von mangelhafter Hygiene in der Futtermittelproduktion.

Tabelle 12.1-46: Einschätzung des Einflusses von mangelhafter Hygiene in der Futtermittelproduktion.

Tabelle 12.1-47: Einschätzung der Gefahrenkategorien bei mangelhaftem Hygieneprogramm in landwirtschaftlichen Betrieben.

Tabelle 12.1-48: Einschätzung des Einflusses mangelhafter Hygieneprogramme im landwirtschaftlichen Betrieb auf die Tierart.

Tabelle 12.1-49: Einschätzung der Gefahrenkategorien durch mangelhafte Personalhygiene in einem Lebensmittel herstellenden Betrieb.

Tabelle 12.1-50: Einschätzung des Einflusses durch mangelhafte Personalhygiene in einem Lebensmittel herstellenden Betrieb auf die Tierart.

Tabelle 12.1-51: Einschätzung der Gefahrenkategorie, die durch eine mangelhafte Dokumentation und daraus bedingte mangelhafte Rückverfolgbarkeit im landwirtschaftlichen Betrieb entstehen kann.

Tabelle 12.1-52: Einschätzung einer mangelhaften Rückverfolgbarkeit im landwirtschaftlichen Betrieb bei der Tierart.

Tabelle 12.1-53: Einschätzung der Gefahrenkategorie bei einer mangelhaften Schlachttierkörperuntersuchung.

Tabelle 12.1-54: Einfluss einer mangelhaften Schlachttierkörperuntersuchung auf die Tierart. 215

Tabelle 12.1-55: Antworten zu der Frage: Halten Sie eine staatliche oder eine private Kontrolle für sicherer?

Tabelle 12.1-56: Übersicht der durchschnittlichen Kompetenzen der Experten pro Fachgebiet. 215

Tabelle 12.1-57: Von den Studenten bevorzugte Fleischarten. 215

\subsection{Abbildungsverzeichnis}

Abbildung 2.1-1: Risikobegriff, Risikowahrnehmung und Risikobewertung.....

Abbildung 2.1-2: Unterschiedliche Beurteilungen von Risiken durch Experten und Verbraucher (RÖHR ET AL., 2002B).

Abbildung 2.2-1: Dominierende Fragen in Bezug auf Lebensmittel sowie Aufgaben für Politik und Agrarforschung nach den 2. Weltkrieg (FLACHOWSKY, 2002 ergänzt).

Abbildung 2.4-1: Produktionskette Rindfleisch.

Abbildung 2.4-2: $\quad$ Carry over in im vom Tier stammenden Lebensmitteln (SCHWIND u. HECHT, 2004). .38 
Abbildung 2.4-3: Einflüsse auf die Mykotoxinproduktion in Futtermitteln, die Mykotoxinbelastung von Nutztieren sowie mögliche Rückstände in den Lebensmitteln tierischer Herkunft (DÄNICKE u. OLDENBURG, 2000).

Abbildung 2.4-4: $\quad$ Mittelwerte der Anteile verendeter Schweine oder von Tieren mit Kreislaufproblemen, Frakturen oder Lahmheiten im Jahr 2003 in Abhängigkeit von der durchschnittlichen Transportdauer $(n=319.005)$. Säulen mit unterschiedlichen Buchstaben $(a, b)$ zwischen den Jahren unterscheiden sich signifikant $(P<0,05)$ (WERNER ET AL., 2005).

Abbildung 5.1-1: Antworten auf die These: Die Zucht auf einen hohen Fleischanteil ist eine Form der Qualzucht.

Abbildung 5.2-1: $\quad$ Ergebnisse zur These: Der Verbraucher steht heute genveränderten Pflanzen sehr kritisch gegenüber. Inwieweit diese Veränderungen eine Auswirkung über das Futtermittel auf das Produkt Fleisch haben, ist heute noch ungewiss. Bitte nennen Sie Ihre Einschätzung zu genveränderten Pflanzen.

Abbildung 5.2-2: Ergebnisse zur These: Genveränderte/geklonte Tiere werden in der Zukunft für die Fleischproduktion eingesetzt.

Abbildung 5.3-1: Heutige/zukünftige Bedeutung von Zoonosen: Welche Zoonosen sehen Sie, die heute eine Rolle spielen, bzw. die an Bedeutung gewinnen?

Abbildung 5.3-2: Beurteilung der Frage: Wird das Risiko einer Erkrankung des Menschen durch Zoonosen in Deutschland steigen?

Abbildung 5.3-3: Beurteilung der These: Schädlinge, Vögel und Haustiere sind in fast allen Futtermittel herstellenden Betrieben zu finden.

Abbildung 5.4-1: Beurteilung der These: In Deutschland z.Zt. nicht vorkommende Keime/ Krankheitserreger werden durch den weltweiten Handel eingeschleppt......

Abbildung 5.4-2: $\quad$ Beurteilung der These: Reste aus der Lebensmittelproduktion sind als Futtermittel geeignet.

Abbildung 5.4-3: Beurteilung der These: Auf Prophylaxen/Schutzimpfungen zum Erhalt des gesundheitlichen Status darf nicht verzichtet werden.

Abbildung 5.5-1: Beurteilung der These: Feldkeime werden in der Nahrungskette eine Rolle spielen.

Abbildung 5.5-2 Einschätzung der Mykotoxine, die in der Nahrungskette von Bedeutung sind, in \% der Nennungen.

Abbildung 5.5-3: Einschätzung der These: In Drittländern sind häufig Pflanzenschutzmittel und/oder Mittel zur Behandlung von Futtermitteln zugelassen, die in Deutschland verboten sind. Bitte beurteilen Sie die Gefahr, dass sich Rückstände von solchen Mitteln im Futter anreichern.

Abbildung 5.5-4: Beurteilung der These: Eine Belastung der Futtermittel durch mit Altlasten verunreinigten Böden wird auch in Zukunft vermehrt vorkommen.

Abbildung 5.5-5: Rückstände im Müllkompost und Klärschlamm führen über eine Anreicherung im Fleisch zu einer gesundheitlichen Gefährdung des Menschen. 
Abbildung 5.5-6: Einschätzung der Experten $(\mathrm{N}=13)$ und der Studenten $(\mathrm{N}=62)$ bezüglich der Unbedenklichkeit von Futterzusatzstoffen.

Abbildung 5.5-7: Einschätzung, welche Rückstände von Umweltkontaminanten, mit denen das Tier im landwirtschaftlichen Betrieb in Berührung kommt, in Zukunft vermehrt eine Rolle spielen, in \% der Nennungen...

Abbildung 5.5-8: Beurteilung der These: Kunststoffmaterialien können Schadstoffe abgeben und führen beim Einsatz in Lebensmittelbetrieben zu Kontaminationen.

Abbildung 5.5-9: Beurteilung der These: Fremdkörper werden häufiger im Produkt Fleisch gefunden. Untersuchungen auf Fremdkörper im Produkt Fleisch (Metall, Glas usw.) werden zurzeit gar nicht oder nur begrenzt durchgeführt.

Abbildung 5.6-1: Einschätzung der kritischen Punkte, die bei der Lagerung von Futtermitteln eine Rolle spielen, in \% der Nennungen......

Abbildung 5.6-2: Beurteilung der These: Rückstände von Reinigungsmitteln in Transportfahrzeugen werden zu einem „potentiellen Problem“ in der Tierproduktion.

Abbildung 5.6-3: Beurteilung der These: Der Verzicht auf tierische Futtermittel-komponenten wirkt sich auf die Zusammensetzung des Fleisches aus.

Abbildung 5.6-4: Mögliche Wege, wie tierische Futtermittel in die Nahrungskette gelangen, in \% der Nennungen.

Abbildung 5.6-5: Beurteilung der These: Die Entsorgung der Ursprungsstoffe, die zur Herstellung von tierischen Futtermitteln verwendet wurden, ist nicht ausreichend gesichert.....106

Abbildung 5.7-1: Beurteilung der These: Durch die Verankerung des Tierschutzgedankens im Grundgesetz hat der Tierschutz einen andern Stellenwert erlangt. Hierdurch ist die Wahrscheinlichkeit hoch, dass das Kupieren, das Enthornen, das Schnabelkürzen etc. zukünftig verboten werden.

Abbildung 5.7-2: Beurteilung der These: Die heutigen Haltungssysteme begünstigen Technopathien.

Abbildung 5.7-3: Beurteilung der These: Die Kastration von Tieren wird vom Verbraucher aus Tierschutzgründen sehr kritisch gesehen. Ein Beibehalten der Kastration kann zu Problemen führen.

Abbildung 5.7-4: Kritische Punkte in der Mast von Tieren, die einen Einfluss auf die Produktqualität haben, und die Beurteilung, die aus der Sicht des Verbrauchers in der Kommunikation als kritisch zu bewerten sind, in \% der Nennungen.

Abbildung 5.7-5: Beurteilung der These: Durch Freilandhaltung bei Geflügel und Schweinen kommt es zu einer starken Belastung des Bodens......

Abbildung 5.7-6: Beurteilung der These: Die Anzahl der Tiere pro Stallgebäude und oder pro Betrieb (Einschränkung der Massentierhaltung) sollte begrenzt werden.

Abbildung 5.8-1: Beurteilung der These: Tiertransporte werden vom Verbraucher zunehmend abgelehnt. Ein langer Transport ist aus der Sicht der meisten Verbraucher tierschutzwidrig. 
Abbildung 5.8-2: Kritische Punkte beim Transport und die Beurteilung der Punkte durch die Studenten bezüglich der Bedenklichkeit aus der Sicht der Verbraucher, in \% der Nennungen.

Abbildung 5.9-1: Bauliche Mängel und Managementfehler für den Bereich Tierannahme/ Wartestall, die die Produktqualität negativ beeinflussen und Einschätzung der Punkte, die in der Kommunikation durch den Verbraucher kritisch gesehen werden, in \% der Nennungen.

Abbildung 5.9-2: Mängel beim Zutrieb bzw. Einhängen der Tiere zur Betäubung, in \% der Nennungen.

Abbildung 5.9-3: Kritische Punkte im Schlachtband, in \% der Nennungen.

Abbildung 5.9-4: Beurteilung der These: Aufgrund der Bandgeschwindigkeiten ist beim Geflügel und beim Schwein die einzelne Schlachttierkörperuntersuchung nicht gewährleistet.

Abbildung 5.9-5: Beurteilung der These: Rückstandslos lässt sich das BSE-Risikomaterial nicht entfernen.

Abbildung 5.10-1: Beurteilung der These: Die Hygiene in der Futtermittelproduktion ist vielfach mangelhaft.

Abbildung 5.10-2: Punkte in der Futtermittelproduktion, bei denen die Gefahr von Hygienemängel besteht, und Einschätzung der Punkte durch die Experten und die Studenten, die durch den Verbraucher kritisch gesehen werden könnten, in \% der Nennungen. ..123

Abbildung 5.11-1: Beurteilung der These: Hygieneprogramme im landwirtschaftlichen Betrieb sind häufig mangelhaft.

Abbildung 5.11-2: Hygienemängel im landwirtschaftlichen Betrieb und Einschätzung der Punkte durch die Experten, wo es zu einer Gefährdung der Verbraucher kommen kann, in $\%$ der Nennungen.

Abbildung 5.11-3: Beurteilung der These: Die Personalhygiene ist einer der wesentlichsten kritischsten Punkte in einem Lebensmittel herstellenden Betrieb.

Abbildung 5.12-1: Beurteilung der These: In den meisten landwirtschaftlichen Betrieben ist die Dokumentation mangelhaft. Hierdurch ist eine schlüssige Rückverfolgbarkeit meist ausgeschlossen.

Abbildung 5.12-2: Schwachstellen in der Rückverfolgbarkeit, in der Landwirtschaft und Beurteilung der Punkte, die in der Kommunikation mit dem Verbraucher kritisch zu bewerten sind, in \% der Nennungen.

Abbildung 5.13-1: Beurteilung der These: Die Qualität der Schlachttierkörperuntersuchung leidet, wenn diese in Eigenverantwortung durch den Schlachthof durchgeführt wird.

Abbildung 5.13-2: Untersuchungen, die ergänzend zur amtlichen Untersuchung erfolgen sollen, in $\%$ der Nennungen.

Abbildung 5.13-3: Beurteilung der These: Durch Fleischskandale im Ausland kommt es zu einem Meideverhalten bei Fleisch in Deutschland.

Abbildung 5.13-4: Kontrollen, die bei Waren aus Drittländern durchgeführt werden sollten, bewertet nach Wichtigkeit, in \% der Nennungen. 
Abbildung 12.2-1: Feldkeime die in der Nahrungskette von Bedeutung sind, in \% der Nennungen. ...216

Abbildung 12.2-2: Mykotoxine die in der Zukunft in der Nahrungskette von Bedeutung sind, in \% der Nennungen

Abbildung 12.2-3: Einschätzung der Rückstände von Futterzusatzstoffen im Fleisch nach Nennungen.

Abbildung 12.2-4: Einschätzung welche Rückstände aus importierten Futtermitteln die in die Nahrungskette gelangen können, in \% der Nennungen.

Abbildung 12.2-5; $\quad$ Einschätzung der Wahrscheinlichkeit der Stoffe/Verbindungen die durch Altlasten in der Nahrungskette gelangen können, in \% der Nennungen.

Abbildung 12.2-6: Mittel/Futterzusatzstoffe zur Leistungssteigerung, in \% der Nennungen.

Abbildung 12.2-7: Einschätzung der Wahrscheinlichkeit von Rückständen aus Müllkompost und Klärschlamm, in \% der Nennungen.

Abbildung 12.2-8: Einschätzung der Studenten ( $N=62)$ bezüglich der Unbedenklichkeit von Futterzusatzstoffen.

Abbildung 12.2-9: $\quad$ Einschätzung der Rückstände von Umweltkontaminanten, mit denen das Tier im landwirtschaftlichen Betrieb in Berührung kommt, die sich im Produkt Fleisch (Fett) anreichern können, in \% der Nennungen

Abbildung 12.2-10: Einschätzung der kritischen Punkte, die bei der Herstellung von Futtermitteln eine Rolle spielen, in \% der Nennungen

Abbildung 12.2-11: Einschätzung der kritischen Punkte beim Transport von Futtermitteln, in \% der Nennungen

Abbildung 12.2-12: Technopathien, in \% der Nennungen. 220

Abbildung 12.2-13: Betäubungsverfahren, die durch den Verbraucher als kritisch bewertet werden, in $\%$ der Nennungen.

Abbildung 12.2-14: Kritische Punkte bei der Fleischkühlung, in \% der Nennungen

Abbildung 12.2-15: Kritische Punkte bei der Fleischverladung/Transport und die Beurteilung der Punkte durch die Studenten bezüglich der Bedenklichkeit aus der Sicht der Verbraucher, in \% der Nennungen.

Abbildung 12.2-16: Kritische Punkte bei der Zerlegung und die Beurteilung der Punkte durch die Studenten bezüglich der Bedenklichkeit aus der Sicht der Verbraucher, in \% der Nennungen.

Abbildung 12.2-17: Kritische Punkte der Fleischverpackung und die Beurteilung der Punkte durch bezüglich der Bedenklichkeit aus der Sicht der Verbraucher, in \% der Nennungen.

Abbildung 12.2-18: Beurteilung der These: Tierische Futtermittel stehen zu Zeit stark in der Kritik und deren Verfütterung ist verboten. Bitte nennen Sie Ihre Einschätzung, ob durch geeignete Aufbereitungsmethoden von tierischen Futtermitteln, bei der Verfütterung an nicht Wiederkäuer, keine Gefahren für den Menschen ausgeht. 
Abbildung 12.2-19: Kritische Punkte bei der Fütterungshygiene von Masttieren und Einschätzung der Punkte, durch die Experten und Studenten, die in der Kommunikation durch den Verbraucher kritisch gesehen werden, in \% der Nennungen.

Abbildung 12.2-20: Kritische Punkte bei der Reinigung von Schlacht- und Zerlegebetrieben, in \% der Nennungen.

Abbildung 12.2-21: Kritische Punkte in der Personalhygiene, in \% der Nennungen. .225

Abbildung 12.2-22: Kritische Punkte bei der Rückverfolgbarkeit von Futtermitteln, in \% der Nennungen. 225

Abbildung 12.2-23: Kritische Punkte bei der Rückverfolgbarkeit in der Schlachtung und Zerlegung, in $\%$ der Nennungen. 226

Abbildung 12.2-24: Kritische Punkte bei der internen Kontrolle von Futtermitteln, in \% der Nennungen226

Abbildung 12.2-25: Kritische Punkte bei der externen Kontrolle von Futtermitteln, in \% der Nennungen.

Abbildung 12.2-26: Kontrollen die bei Waren aus Drittländern durchgeführt werden sollten, in \% der Nennungen. 227

Abbildung 12.2-27: Wohn- bzw. Arbeitsorte der befragten Experten in Deutschland 228 


\section{Anhang}

\subsection{Anhang Tabellen}

Tabelle 11.1-1: $\quad$ Bevorzugte Rassen/genetische Konstruktionen von Rindern.

\begin{tabular}{lc}
\hline Rasse & Nennungen $(\mathrm{n}=30)$ \\
\hline Angus/Anguskreuzungen & $23 \%$ \\
Limousin & $23 \%$ \\
Herford & $13 \%$ \\
Fleckvieh & $10 \%$ \\
Gelbvieh/Gelbviehkreuzungen & $7 \%$ \\
Braunvieh & $7 \%$ \\
Blonde d'Aquitane & $7 \%$ \\
Simmentaler Kreuzungen Fleischrasse & $3 \%$ \\
Uckermärker & $3 \%$ \\
Charolais & $3 \%$ \\
\hline
\end{tabular}

Tabelle 11.1-2: $\quad$ Bevorzugte Rassen/genetische Konstruktionen von Schweinen.

\begin{tabular}{lc}
\hline Rasse & Nennungen $(\mathrm{n}=30)$ \\
\hline Duroc/Landschwein // dt/nl x Duroc & $23 \%$ \\
Hybriden aus stresssanierten Linien (100\% MHS-NN) & $18 \%$ \\
Kreuzungstiere & $18 \%$ \\
PIC & $14 \%$ \\
BHZP & $9 \%$ \\
Edelschwein x Large Withe & $5 \%$ \\
Landrasse x Edelschwein & $5 \%$ \\
Schaumannzucht & $5 \%$ \\
Pietrainkreuzungen & $5 \%$ \\
\hline
\end{tabular}

Tabelle 11.1-3: $\quad$ Gefahrenkategorien bei Qualzucht.

\begin{tabular}{ccccccc}
\hline & chemisch & mikrobiologisch & physikalisch & sensorisch & $\begin{array}{c}\text { ernährungs- } \\
\text { physiologisch }\end{array}$ & ethisch \\
\hline $\mathrm{N}=22$ & 1 & 0 & 1 & 10 & 7 & 21 \\
$\%$ & 5 & 0 & 5 & 46 & 32 & 96 \\
\hline
\end{tabular}

Tabelle 11.1-4: $\quad$ Einfluss der Qualzucht auf die Tierart.

\begin{tabular}{cccc}
\hline & Rind & Schwein & Geflügel \\
\hline $\mathrm{N}=22$ & 13 & 20 & 21 \\
$\%$ & 59 & 91 & 96 \\
\hline
\end{tabular}

Tabelle 11.1-5: $\quad$ Einschätzung der Gefahrenkategorien bei genveränderten Pflanzen.

\begin{tabular}{ccccccc}
\hline & chemisch & mikrobiologisch & physikalisch & sensorisch & $\begin{array}{c}\text { ernährungs- } \\
\text { physiologisch }\end{array}$ & ethisch \\
\hline $\mathrm{N}=23$ & 3 & 6 & 0 & 3 & 9 & 18 \\
$\%$ & 13 & 26 & 0 & 13 & 39 & 78 \\
\hline
\end{tabular}


Tabelle 11.1-6: Einschätzung der Gefahrenkategorien bei genveränderten/geklonten Tieren.

\begin{tabular}{ccccccc}
\hline & chemisch & mikrobiologisch & physikalisch & sensorisch & $\begin{array}{c}\text { ernährungs- } \\
\text { physiologisch }\end{array}$ & ethisch \\
\hline $\mathrm{N}=22$ & 2 & 2 & 1 & 5 & 6 & 20 \\
$\%$ & 9 & 9 & 5 & 23 & 27 & 91 \\
\hline
\end{tabular}

Tabelle 11.1-7: Einschätzung des Einflusses von genveränderten/geklonten Verfahren auf die Tierart.

\begin{tabular}{cccc}
\hline & Rind & Schwein & Geflügel \\
\hline $\mathrm{N}=23$ & 15 & 20 & 18 \\
$\%$ & 65 & 87 & 78 \\
\hline
\end{tabular}

Tabelle 11.1-8: $\quad$ Einschätzung der Gefahrenkategorien bei Feldkeimen.

\begin{tabular}{ccccccc}
\hline & chemisch & mikrobiologisch & physikalisch & sensorisch & $\begin{array}{c}\text { ernährungs- } \\
\text { physiologisch }\end{array}$ & ethisch \\
\hline $\mathrm{N}=17$ & 4 & 14 & 0 & 1 & 6 & 0 \\
$\%$ & 24 & 82 & 0 & 6 & 35 & 0 \\
\hline
\end{tabular}

Tabelle 11.1-9: $\quad$ Einschätzung des Einflusses von Feldkeimen auf die Tierart.

\begin{tabular}{cccc}
\hline & Rind & Schwein & Geflügel \\
\hline $\mathrm{N}=16$ & 9 & 12 & 9 \\
$\%$ & 56 & 75 & 56 \\
\hline
\end{tabular}

Tabelle 11.1-10: $\quad$ Einschätzung des Einflusses von Zoonosen auf die Tierart.

\begin{tabular}{cccc}
\hline & Rind & Schwein & Geflügel \\
\hline $\mathrm{N}=24$ & 12 & 23 & 23 \\
$\%$ & 50 & 96 & 96 \\
\hline
\end{tabular}

Tabelle 11.1-11: $\quad$ Einschätzung der Gefahrenkategorien durch Schädlingsbefall.

\begin{tabular}{ccccccc}
\hline & chemisch & mikrobiologisch & physikalisch & sensorisch & $\begin{array}{c}\text { ernährungs- } \\
\text { physiologisch }\end{array}$ & ethisch \\
\hline $\mathrm{N}=24$ & 4 & 22 & 0 & 1 & 3 & 2 \\
$\%$ & 17 & 96 & 0 & 4 & 13 & 9 \\
\hline
\end{tabular}

Tabelle 11.1-12: $\quad$ Einschätzung des Einflusses von Schädlingsbefall auf die Tierart.

\begin{tabular}{cccc}
\hline & Rind & Schwein & Geflügel \\
\hline $\mathrm{N}=24$ & 14 & 21 & 22 \\
$\%$ & 61 & 91 & 96 \\
\hline
\end{tabular}

Tabelle 11.1-13: Einschätzung der Gefahrenkategorien bei in Deutschland z.Zt. nicht vorkommenden Keimen/Krankheitserregern, die durch den weltweiten Handel eingeschleppt werden.

\begin{tabular}{ccccccc}
\hline & chemisch & mikrobiologisch & physikalisch & sensorisch & $\begin{array}{c}\text { ernährungs- } \\
\text { physiologisch }\end{array}$ & ethisch \\
\hline $\mathrm{N}=22$ & 1 & 22 & 0 & 0 & 17 & 18 \\
$\%$ & 18 & 100 & 0 & 0 & 23 & 18 \\
\hline
\end{tabular}


Tabelle 11.1-14: Einschätzung des Einflusses von in Deutschland z.Zt. nicht vorkommenden Keimen/Krankheitserregern auf die Tierart.

\begin{tabular}{cccc}
\hline & Rind & Schwein & Geflügel \\
\hline $\mathrm{N}=22$ & 16 & 20 & 21 \\
$\%$ & 73 & 91 & 96 \\
\hline
\end{tabular}

Tabelle 11.1-15: Einschätzung der Gefahrenkategorien bei dem Einsatz von Resten aus der Lebensmittelproduktion als Futtermittel.

\begin{tabular}{ccccccc}
\hline & chemisch & mikrobiologisch & physikalisch & sensorisch & $\begin{array}{c}\text { ernährungs- } \\
\text { physiologisch }\end{array}$ & ethisch \\
\hline $\mathrm{N}=22$ & 6 & 17 & 2 & 7 & 10 & 6 \\
$\%$ & 27 & 77 & 9 & 32 & 46 & 27 \\
\hline
\end{tabular}

Tabelle 11.1-16: Einschätzung des Einflusses von Resten aus der Lebensmittelproduktion als Futtermittel auf die Tierart.

\begin{tabular}{cccc}
\hline & Rind & Schwein & Geflügel \\
\hline $\mathrm{N}=23$ & 6 & 21 & 14 \\
$\%$ & 26 & 91 & 61 \\
\hline
\end{tabular}

Tabelle 11.1-17: $\quad$ Einschätzung der Gefahrenkategorien bei dem Verzicht Einsatz von Prophylaxen/Schutzimpfungen.

\begin{tabular}{ccccccc}
\hline & chemisch & mikrobiologisch & physikalisch & sensorisch & $\begin{array}{c}\text { ernährungs- } \\
\text { physiologisch }\end{array}$ & ethisch \\
\hline $\mathrm{N}=19$ & 2 & 14 & 0 & 0 & 2 & 5 \\
$\%$ & 11 & 74 & 0 & 0 & 11 & 26 \\
\hline
\end{tabular}

Tabelle 11.1-18: Einschätzung des Einflusses von Resten aus der Lebensmittelproduktion als Futtermittel auf die Tierart.

\begin{tabular}{cccc}
\hline & Rind & Schwein & Geflügel \\
\hline $\mathrm{N}=21$ & 16 & 19 & 20 \\
$\%$ & 76 & 91 & 95 \\
\hline
\end{tabular}

Tabelle 11.1-19: Kritische Verfahrensschritte in Ländern aus denen exotische Futtermittel importiert werden.

\begin{tabular}{lc}
\hline Verfahrensschritte & $\mathrm{N}=6$ \\
\hline Transport & 5 \\
Lagerung & 5 \\
Art der Trocknung (Direktbefeuerung, Sonnentrocknung) & 4 \\
Mangelnde Hygiene in der Ernte und Verarbeitung & 3 \\
HACCP- wird nicht angewendet & 3 \\
klimatische Verhältnisse, hohe Luftfeuchtigkeit & 1 \\
\hline
\end{tabular}


Tabelle 11.1-20: $\quad$ Einschätzung der Gefahrenkategorien bei Futtermitteln, die durch mit Altlasten verunreinigte Böden, durch Klärschlamm oder durch Pflanzenschutzmittel aus importierten Futtermitteln kontaminiert wurden.

\begin{tabular}{lccccccc}
\hline & $\%$ & chemisch & $\begin{array}{c}\text { mikro- } \\
\text { biologisch }\end{array}$ & physikalisch & sensorisch & $\begin{array}{c}\text { ernährungs- } \\
\text { physiologisch }\end{array}$ & ethisch \\
\hline Altlasten & $\mathrm{N}=19$ & 17 & 4 & 3 & 1 & 6 & 4 \\
Klärschlamm & $\%$ & 90 & 21 & 16 & 5 & 32 & 21 \\
& $\mathrm{~N}=22$ & 18 & 10 & 0 & 4 & 6 & 9 \\
Pflanzen- & $\%$ & 82 & 46 & 0 & 18 & 27 & 41 \\
schutzmittel & $\mathrm{N}=$ & 16 & 6 & 1 & 1 & 10 & 4 \\
\hline
\end{tabular}

Tabelle 11.1-21: Einschätzung des Einflusses von durch mit Altlasten, Rückständen aus Klärschlamm oder durch Pflanzenschutzmittel aus importierten Futtermitteln verunreinigten Futtermittel auf die Tierart.

\begin{tabular}{lcccc}
\hline Experten & & Rind & Schwein & Geflügel \\
\hline Altlasten & $\mathrm{N}=19$ & 9 & 12 & 9 \\
& $\%$ & 90 & 84 & 74 \\
Klärschlamm & $\mathrm{N}=21$ & 16 & 19 & 16 \\
& $\%$ & 76 & 91 & 67 \\
Pflanzenschutz- & $\mathrm{N}=19$ & 14 & 19 & 18 \\
mittel & $\%$ & 74 & 100 & 95 \\
\hline
\end{tabular}

Tabelle 11.1-22: $\quad$ Einschätzung der Gefahrenkategorien bei Rückstände durch Kunststoffmaterialien.

\begin{tabular}{ccccccc}
\hline & chemisch & mikrobiologisch & physikalisch & sensorisch & $\begin{array}{c}\text { ernährungs- } \\
\text { physiologisch }\end{array}$ & ethisch \\
\hline $\mathrm{N}=23$ & 19 & 4 & 5 & 7 & 2 & 2 \\
$\%$ & 29 & 17 & 22 & 30 & 9 & 9 \\
\hline
\end{tabular}

Tabelle 11.1-23: $\quad$ Einschätzung der Gefahrenkategorien bei Fremdkörpern.

\begin{tabular}{ccccccc}
\hline & chemisch & mikrobiologisch & physikalisch & sensorisch & $\begin{array}{c}\text { ernährungs- } \\
\text { physiologisch }\end{array}$ & ethisch \\
\hline $\mathrm{N}=27$ & 2 & 4 & 24 & 9 & 5 & 6 \\
$\%$ & 7 & 15 & 89 & 33 & 19 & 22 \\
\hline
\end{tabular}

Tabelle 11.1-24: $\quad$ Einschätzung des Einflusses der Fremdkörpergefahr auf die Tierart.

\begin{tabular}{cccc}
\hline & Rind & Schwein & Geflügel \\
\hline $\mathrm{N}=26$ & 24 & 25 & 20 \\
$\%$ & 92 & 96 & 77 \\
\hline
\end{tabular}

Tabelle 11.1-25: Einschätzung der Gefahrenkategorien durch Reinigungsmittel bei Transportfahrzeugen.

\begin{tabular}{ccccccc}
\hline & chemisch & mikrobiologisch & physikalisch & sensorisch & $\begin{array}{c}\text { ernährungs- } \\
\text { physiologisch }\end{array}$ & ethisch \\
\hline $\mathrm{N}=21$ & 17 & 7 & 2 & 5 & 3 & 1 \\
$\%$ & 81 & 33,3 & 9,52 & 23,8 & 14,3 & 4,76 \\
\hline
\end{tabular}


Tabelle 11.1-26: Einschätzung des Einflusses von Rückstände von Reinigungsmitteln in Transportfahrzeugen auf die Tierart.

\begin{tabular}{cccc}
\hline & Rind & Schwein & Geflügel \\
\hline $\mathrm{N}=19$ & 11 & 17 & 17 \\
$\%$ & 58 & 90 & 90 \\
\hline
\end{tabular}

Tabelle 11.1-27: Einschätzung der Gefahrenkategorien durch Kupieren, das Enthornen, das Schnabelkürzen etc. und Kastration.

\begin{tabular}{lccccccc}
\hline & $\%$ & chemisch & $\begin{array}{c}\text { mikro- } \\
\text { biologisch }\end{array}$ & physikalisch & sensorisch & $\begin{array}{c}\text { ernährungs- } \\
\text { physiologisch }\end{array}$ & ethisch \\
\hline Enthornen, & $\mathrm{N}=21$ & 0 & 3 & 2 & 2 & 2 & 19 \\
Kupieren, usw. & $\%$ & 0 & 14 & 10 & 10 & 10 & 91 \\
Kastration & $\mathrm{N}=23$ & 3 & 1 & 1 & 13 & 2 & 16 \\
& $\%$ & 13 & 4 & 4 & 57 & 9 & 70 \\
\hline
\end{tabular}

Tabelle 11.1-28: Einschätzung des Einflusses auf durch Kupieren, das Enthornen, das Schnabelkürzen etc. und Kastration die Tierart.

\begin{tabular}{lcccc}
\hline Experten & & Rind & Schwein & Geflügel \\
\hline Enthornen, & $\mathrm{N}=22$ & 15 & 19 & 21 \\
Kupieren, usw. & $\%$ & 71 & 86 & 96 \\
Kastration & $\mathrm{N}=23$ & 8 & 23 & 2 \\
& $\%$ & 35 & 100 & 9 \\
\hline
\end{tabular}

Tabelle 11.1-29: Einschätzung der Gefahrenkategorien bei einer Belastung des Bodens durch die Freilandhaltung bei Geflügel und Schweinen.

\begin{tabular}{ccccccc}
\hline & chemisch & mikrobiologisch & physikalisch & sensorisch & $\begin{array}{c}\text { ernährungs- } \\
\text { physiologisch }\end{array}$ & ethisch \\
\hline $\mathrm{N}=21$ & 9 & 17 & 3 & 1 & 2 & 2 \\
$\%$ & 43 & 81 & 14 & 5 & 10 & 10 \\
\hline
\end{tabular}

Tabelle 11.1-30: $\quad$ Einschätzung der Gefahrenkategorie durch Massentierhaltung.

\begin{tabular}{ccccccc}
\hline & chemisch & mikrobiologisch & physikalisch & sensorisch & $\begin{array}{c}\text { ernährungs- } \\
\text { physiologisch }\end{array}$ & ethisch \\
\hline $\mathrm{N}=19$ & 6 & 8 & 2 & 2 & 2 & 13 \\
$\%$ & 32 & 42 & 11 & 11 & 11 & 68 \\
\hline
\end{tabular}

Tabelle 11.1-31: $\quad$ Einfluss der Massentierhaltung auf die Tierart.

\begin{tabular}{cccc}
\hline & Rind & Schwein & Geflügel \\
\hline $\mathrm{N}=21$ & 9 & 20 & 20 \\
$\%$ & 43 & 95 & 95 \\
\hline
\end{tabular}

Tabelle 11.1-32: $\quad$ Einschätzung der Gefahrenkategorie durch Technopathien in heutigen Haltungssystemen.

\begin{tabular}{ccccccc}
\hline & chemisch & mikrobiologisch & physikalisch & sensorisch & $\begin{array}{c}\text { ernährungs- } \\
\text { physiologisch }\end{array}$ & ethisch \\
\hline $\mathrm{N}=20$ & 2 & 5 & 7 & 2 & 2 & 11 \\
$\%$ & 10 & 25 & 35 & 10 & 10 & 55 \\
\hline
\end{tabular}


Tabelle 11.1-33: Einfluss von Technopathien in heutigen Haltungssystemen auf die Tierart.

\begin{tabular}{cccc}
\hline & Rind & Schwein & Geflügel \\
\hline $\mathrm{N}=20$ & 13 & 19 & 20 \\
$\%$ & 65 & 95 & 100 \\
\hline
\end{tabular}

Tabelle 11.1-34: $\quad$ Einschätzung der Gefahrenkategorien durch Tiertransporte.

\begin{tabular}{ccccccc}
\hline & chemisch & mikrobiologisch & physikalisch & sensorisch & $\begin{array}{c}\text { ernährungs- } \\
\text { physiologisch }\end{array}$ & ethisch \\
\hline $\mathrm{N}=27$ & 6 & 8 & 12 & 12 & 1 & 20 \\
$\%$ & 22 & 30 & 44 & 44 & 4 & 74 \\
\hline
\end{tabular}

Tabelle 11.1-35: Einschätzung des Einflusses von Transporten auf die Tierart.

\begin{tabular}{cccc}
\hline & Rind & Schwein & Geflügel \\
\hline $\mathrm{N}=26$ & 21 & 26 & 20 \\
$\%$ & 81 & 100 & 77 \\
\hline
\end{tabular}

Tabelle 11.1-36: Einschätzung der Gefahrenkategorien durch eine hohe Bandgeschwindigkeit.

\begin{tabular}{ccccccc}
\hline & chemisch & mikrobiologisch & physikalisch & sensorisch & $\begin{array}{c}\text { ernährungs- } \\
\text { physiologisch }\end{array}$ & ethisch \\
\hline $\mathrm{N}=25$ & 5 & 20 & 3 & 7 & 3 & 7 \\
$\%$ & 20 & 80 & 12 & 28 & 12 & 28 \\
\hline
\end{tabular}

Tabelle 11.1-37: Einschätzung der Gefahrenkategorien zu der These: Der Verzicht auf tierische Futtermittelkomponenten wirkt sich auf die Zusammensetzung des Fleisches aus.

\begin{tabular}{ccccccc}
\hline & chemisch & mikrobiologisch & physikalisch & sensorisch & $\begin{array}{c}\text { ernährungs- } \\
\text { physiologisch }\end{array}$ & ethisch \\
\hline $\mathrm{N}=16$ & 4 & 1 & 0 & 9 & 9 & 5 \\
$\%$ & 25 & 6 & 0 & 56 & 56 & 31 \\
\hline
\end{tabular}

Tabelle 11.1-38: Einschätzung der Studenten bezüglich des Einsatzes und der Sicherheit tierischer Futtermittel an Geflügel und Schweine zum Jahr 2015.

\begin{tabular}{lcccccc}
\hline Studenten & & nein & eher nein & neutral & eher ja & Ja \\
\hline Einsatz & $\mathrm{N}=64$ & 12 & 20 & 7 & 14 & 11 \\
alle & $\%$ & 19 & 31 & 11 & 22 & 17 \\
Studenten $(\mathrm{mL})$ & $\mathrm{N}=40$ & 7 & 8 & 5 & 11 & 9 \\
& $\%$ & 17 & 20 & 13 & 28 & 23 \\
Studenten $(\mathrm{oL})$ & $\mathrm{N}=24$ & 5 & 12 & 2 & 3 & 2 \\
& $\%$ & 21 & 50 & 8 & 13 & 8 \\
Sicherheit & $\mathrm{N}=64$ & 7 & 21 & 18 & 12 & 6 \\
alle & $\%$ & 11 & 33 & 28 & 19 & 9 \\
Studenten $(\mathrm{mL})$ & $\mathrm{N}=40$ & 4 & 10 & 10 & 11 & 5 \\
& $\%$ & 10 & 25 & 25 & 28 & 13 \\
Studenten $(\mathrm{oL})$ & $\mathrm{N}=24$ & 3 & 11 & 8 & 1 & 1 \\
& $\%$ & 13 & 46 & 33 & 4 & 4 \\
\hline
\end{tabular}


Tabelle 11.1-39: Einschätzung des Einflusses der Aufbereitungsmethoden bei tierischen Futtermitteln auf die Tierart.

\begin{tabular}{cccc}
\hline & Rind & Schwein & Geflügel \\
\hline $\mathrm{N}=15$ & 1 & 12 & 12 \\
$\%$ & 7 & 80 & 80 \\
\hline
\end{tabular}

Tabelle 11.1-40: Einschätzung der Gefahrenkategorien bei der Entsorgung der Ursprungsstoffe tierischer Futtermittel (Kadaver, Schlachtabfälle usw.).

\begin{tabular}{lccccccc}
\hline Experten & & chemisch & $\begin{array}{c}\text { mikro- } \\
\text { biologisch }\end{array}$ & physikalisch & sensorisch & $\begin{array}{c}\text { ernährungs- } \\
\text { physiologisch }\end{array}$ & ethisch \\
\hline Entsorgung & $\mathrm{N}=16$ & 4 & 11 & 2 & 1 & 2 & 9 \\
& $\%$ & 25 & 69 & 13 & 6 & 13 & 56 \\
\hline
\end{tabular}

Tabelle 11.1-41: $\quad$ Einschätzung des Einflusses bei der Entsorgung der Ursprungsstoffe tierischer Futtermittel (Kadaver, Schlachtabfälle usw.)auf die Tierart.

\begin{tabular}{lcccc}
\hline Experten & & Rind & Schwein & Geflügel \\
\hline Entsorgung & $\mathrm{N}=14$ & 7 & 11 & 12 \\
& $\%$ & 50 & 79 & 86 \\
\hline
\end{tabular}

Tabelle 11.1-42: $\quad$ Einschätzung der Gefahrenkategorien bei BSE-Risikomaterial.

\begin{tabular}{ccccccc}
\hline & chemisch & mikrobiologisch & physikalisch & sensorisch & $\begin{array}{c}\text { ernährungs- } \\
\text { physiologisch }\end{array}$ & ethisch \\
\hline $\mathrm{N}=27$ & 4 & 15 & 2 & 0 & 2 & 12 \\
$\%$ & 15 & 56 & 7 & 0 & 7 & 44 \\
\hline
\end{tabular}

Tabelle 11.1-43: $\quad$ Einschätzung des Einflusses von BSE-Risikomaterial auf die Tierart.

\begin{tabular}{cccc}
\hline & Rind & Schwein & Geflügel \\
\hline $\mathrm{N}=27$ & 26 & 2 & 2 \\
$\%$ & 96 & 7 & 7 \\
\hline
\end{tabular}

Tabelle 11.1-44: Teile vom Schlachttierkörper und Bearbeitungsmethoden des Schlachttieres, die kritisch zu bewerten sind.

\begin{tabular}{clcl}
\hline Experten & \multicolumn{1}{c}{ Kategorie } & Anzahl der Nennungen & $\%$ \\
\hline \multirow{3}{*}{$\mathrm{N}=21$} & Rückenmark & 16 & 26 \\
& Kopf & 17 & 27 \\
& Zunge & 1 & 2 \\
& Milz & 6 & 10 \\
& Mandeln & 4 & 6 \\
Därme & 3 & 5 \\
& Lunge & 2 & 3 \\
Lymphe + Knoten & 4 & 6 \\
& Peyersche Platten & 2 & 3 \\
& alles Nervengewebe & 4 & 6 \\
& Thymus & 2 & 3, \\
& Separatorenfleisch & 1 & 2 \\
\hline
\end{tabular}


Tabelle 11.1-45: Einschätzung der Gefahrenkategorie von mangelhafter Hygiene in der Futtermittelproduktion.

\begin{tabular}{ccccccc}
\hline & chemisch & mikrobiologisch & physikalisch & sensorisch & $\begin{array}{c}\text { ernährungs- } \\
\text { physiologisch }\end{array}$ & ethisch \\
\hline $\mathrm{N}=17$ & 9 & 17 & 3 & 4 & 4 & 3 \\
$\%$ & 53 & 100 & 18 & 24 & 24 & 18 \\
\hline
\end{tabular}

Tabelle 11.1-46: Einschätzung des Einflusses von mangelhafter Hygiene in der Futtermittelproduktion.

\begin{tabular}{cccc}
\hline & Rind & Schwein & Geflügel \\
\hline $\mathrm{N}=17$ & 12 & 16 & 16 \\
$\%$ & 71 & 94 & 94 \\
\hline
\end{tabular}

Tabelle 11.1-47: Einschätzung der Gefahrenkategorien bei mangelhaftem Hygieneprogramm in landwirtschaftlichen Betrieben.

\begin{tabular}{ccccccc}
\hline & chemisch & mikrobiologisch & physikalisch & sensorisch & $\begin{array}{c}\text { ernährungs- } \\
\text { physiologisch }\end{array}$ & ethisch \\
\hline $\mathrm{N}=23$ & 4 & 22 & 0 & 3 & 5 & 6 \\
$\%$ & 17 & 96 & 0 & 13 & 22 & 26 \\
\hline
\end{tabular}

Tabelle 11.1-48: $\quad$ Einschätzung des Einflusses mangelhafter Hygieneprogramme im landwirtschaftlichen Betrieb auf die Tierart.

\begin{tabular}{cccc}
\hline & Rind & Schwein & Geflügel \\
\hline $\mathrm{N}=23$ & 14 & 22 & 21 \\
$\%$ & 61 & 96 & 91 \\
\hline
\end{tabular}

Tabelle 11.1-49: Einschätzung der Gefahrenkategorien durch mangelhafte Personalhygiene in einem Lebensmittel herstellenden Betrieb.

\begin{tabular}{ccccccc}
\hline & chemisch & mikrobiologisch & physikalisch & sensorisch & $\begin{array}{c}\text { ernährungs- } \\
\text { physiologisch }\end{array}$ & ethisch \\
\hline $\mathrm{N}=28$ & 7 & 28 & 3 & 7 & 3 & 7 \\
$\%$ & 25 & 100 & 11 & 25 & 11 & 25 \\
\hline
\end{tabular}

Tabelle 11.1-50: Einschätzung des Einflusses durch mangelhafte Personalhygiene in einem Lebensmittel herstellenden Betrieb auf die Tierart.

\begin{tabular}{cccc}
\hline & Rind & Schwein & Geflügel \\
\hline $\mathrm{N}=27$ & 25 & 26 & 27 \\
$\%$ & 93 & 96 & 100 \\
\hline
\end{tabular}

Tabelle 11.1-51: Einschätzung der Gefahrenkategorie, die durch eine mangelhafte Dokumentation und daraus bedingte mangelhafte Rückverfolgbarkeit im landwirtschaftlichen Betrieb entstehen kann.

\begin{tabular}{ccccccc}
\hline & chemisch & mikrobiologisch & physikalisch & sensorisch & $\begin{array}{c}\text { ernährungs- } \\
\text { physiologisch }\end{array}$ & ethisch \\
\hline $\mathrm{N}=10$ & 6 & 7 & 1 & 0 & 3 & 4 \\
$\%$ & 60 & 70 & 10 & 0 & 30 & 36 \\
\hline
\end{tabular}


Tabelle 11.1-52: Einschätzung einer mangelhaften Rückverfolgbarkeit im landwirtschaftlichen Betrieb bei der Tierart.

\begin{tabular}{cccc}
\hline & Rind & Schwein & Geflügel \\
\hline $\mathrm{N}=16$ & 9 & 14 & 13 \\
$\%$ & 56 & 88 & 81 \\
\hline
\end{tabular}

Tabelle 11.1-53: Einschätzung der Gefahrenkategorie bei einer mangelhaften Schlachttierkörperuntersuchung.

\begin{tabular}{ccccccc}
\hline & chemisch & mikrobiologisch & physikalisch & sensorisch & $\begin{array}{c}\text { ernährungs- } \\
\text { physiologisch }\end{array}$ & ethisch \\
\hline $\mathrm{N}=20$ & 5 & 17 & 4 & 6 & 3 & 4 \\
$\%$ & 25 & 85 & 20 & 30 & 15 & 20 \\
\hline
\end{tabular}

Tabelle 11.1-54: Einfluss einer mangelhaften Schlachttierkörperuntersuchung auf die Tierart.

\begin{tabular}{cccc}
\hline & Rind & Schwein & Geflügel \\
\hline $\mathrm{N}=23$ & 18 & 22 & 22 \\
$\%$ & 78 & 96 & 96 \\
\hline
\end{tabular}

Tabelle 11.1-55: Antworten zu der Frage: Halten Sie eine staatliche oder eine private Kontrolle für sicherer?

\begin{tabular}{|c|c|c|c|c|c|c|c|c|c|c|}
\hline \multirow[t]{2}{*}{ Studenten } & & \multicolumn{3}{|c|}{$\begin{array}{c}\text { Studenten gesamt } \\
\mathrm{N}=64\end{array}$} & \multicolumn{3}{|c|}{$\begin{array}{c}\text { Studenten }(\mathrm{mL}) \\
\mathrm{N}=40\end{array}$} & \multicolumn{3}{|c|}{$\begin{array}{c}\text { Studenten (oL) } \\
\mathrm{N}=24\end{array}$} \\
\hline & & staatl. & privat & beide & staatl. & privat & beide & staatl. & privat & beide \\
\hline Landwirtschaft & $\%$ & 48 & 49 & 3 & 45 & 53 & 3 & 52 & 43 & 4 \\
\hline Futtermittelpro. & $\%$ & 62 & 34 & 3 & 55 & 42 & 3 & 74 & 22 & 4 \\
\hline Schlachtung & $\%$ & 57 & 34 & 3 & 53 & 45 & 3 & 65 & 30 & 4 \\
\hline Zerlegung & $\%$ & 54 & 43 & 3 & 58 & 39 & 3 & 48 & 48 & 4 \\
\hline
\end{tabular}

Tabelle 11.1-56: Übersicht der durchschnittlichen Kompetenzen der Experten pro Fachgebiet.

\begin{tabular}{lllc}
\hline & Offene Fragen & Geschlossene Fragen & Gesamt \\
\hline Landwirtschaft & 2,16 & 2,16 & 2,16 \\
Schlachtung & 2,20 & 2,20 & 2,20 \\
Futtermittel & 1,97 & 2,03 & 1,99 \\
\hline
\end{tabular}

Tabelle 11.1-57: $\quad$ Von den Studenten bevorzugte Fleischarten.

\begin{tabular}{lcccc}
\hline & & Studenten gesamt & Studenten $(\mathrm{mL})$ & Studenten $(\mathrm{oL})$ \\
$\mathrm{N}=64$ & $\mathrm{~N}=40$ & $\mathrm{~N}=24$ \\
\hline Schweinefleisch & 29 & 73 & 74 & 71 \\
Rindfleisch & 30 & 65 & 77 & 46 \\
Hähnchenfleisch & 8 & 32 & 21 & 50 \\
Putenfleisch & 6 & 25 & 15 & 42 \\
Schaffleisch & 2 & 8 & 5 & 13 \\
Keines & 3 & 6 & 8 & 4 \\
\hline
\end{tabular}


11.2 Abbildungen

Abbildung 11.2-1: Feldkeime die in der Nahrungskette von Bedeutung sind, in $\%$ der Nennungen.

\section{- Fuminozine \\ $\square$ Ochratoxin \\ $\square$ Zeraleon \\ $\square$ Fusarien \\ $\square D O N$ \\ $\square$ Aflatoxin \\ $\square$ Vomitoxin (DON) \\ $\square$ Mykotxine \\ $\square$ Mutterkornalkaloid \\ $\square$ Schwärzepilztoxine \\ $\square$ Citrinin \\ $\square$ Trichotheane}

口Ergotamin

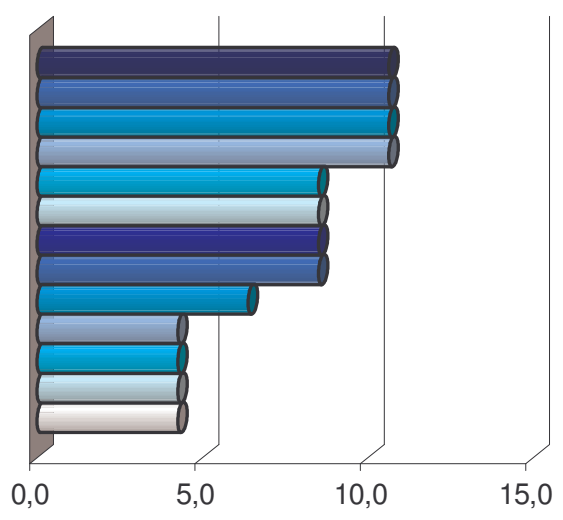

Abbildung 11.2-2: Mykotoxine die in der Zukunft in der Nahrungskette von Bedeutung sind, in $\%$ der Nennungen.
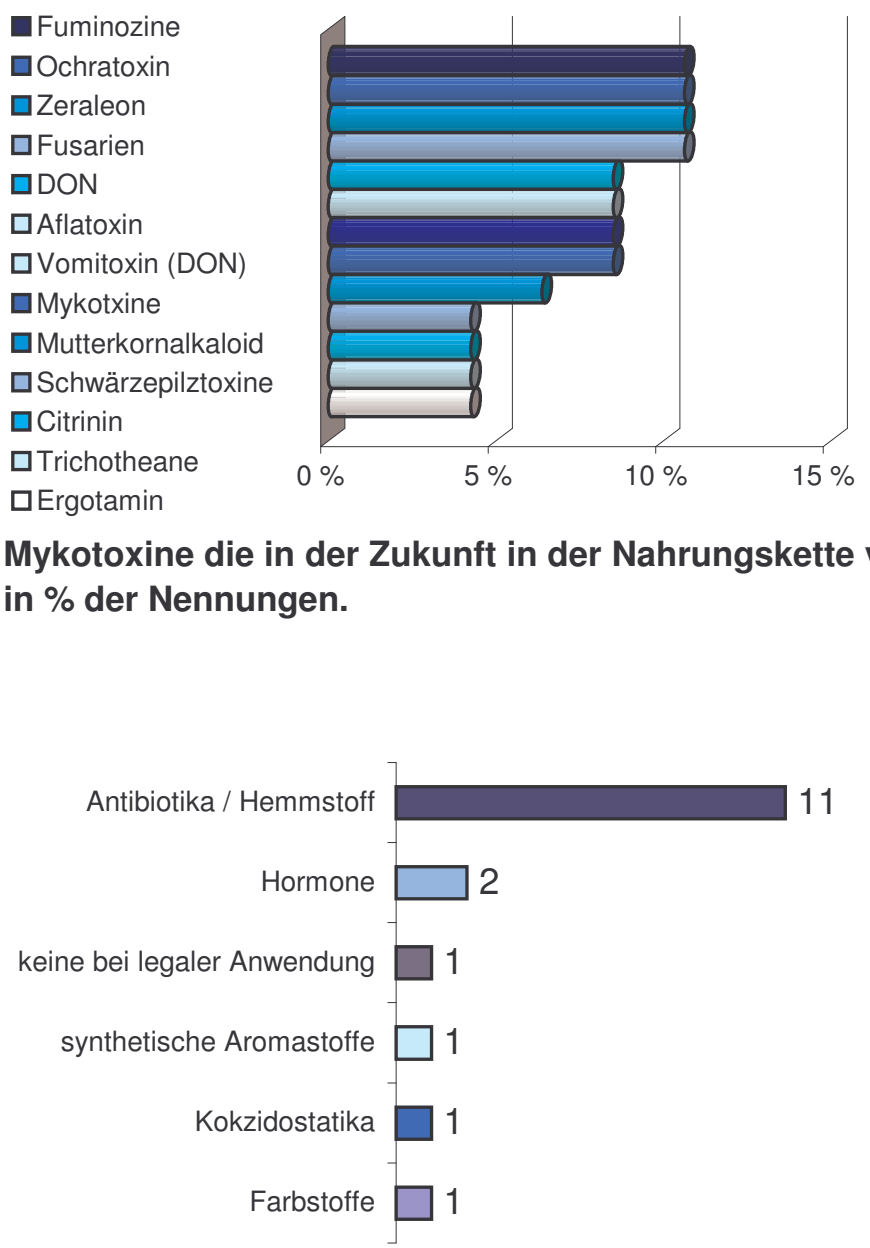

Abbildung 11.2-3: Einschätzung der Rückstände von Futterzusatzstoffen im Fleisch nach Nennungen. 


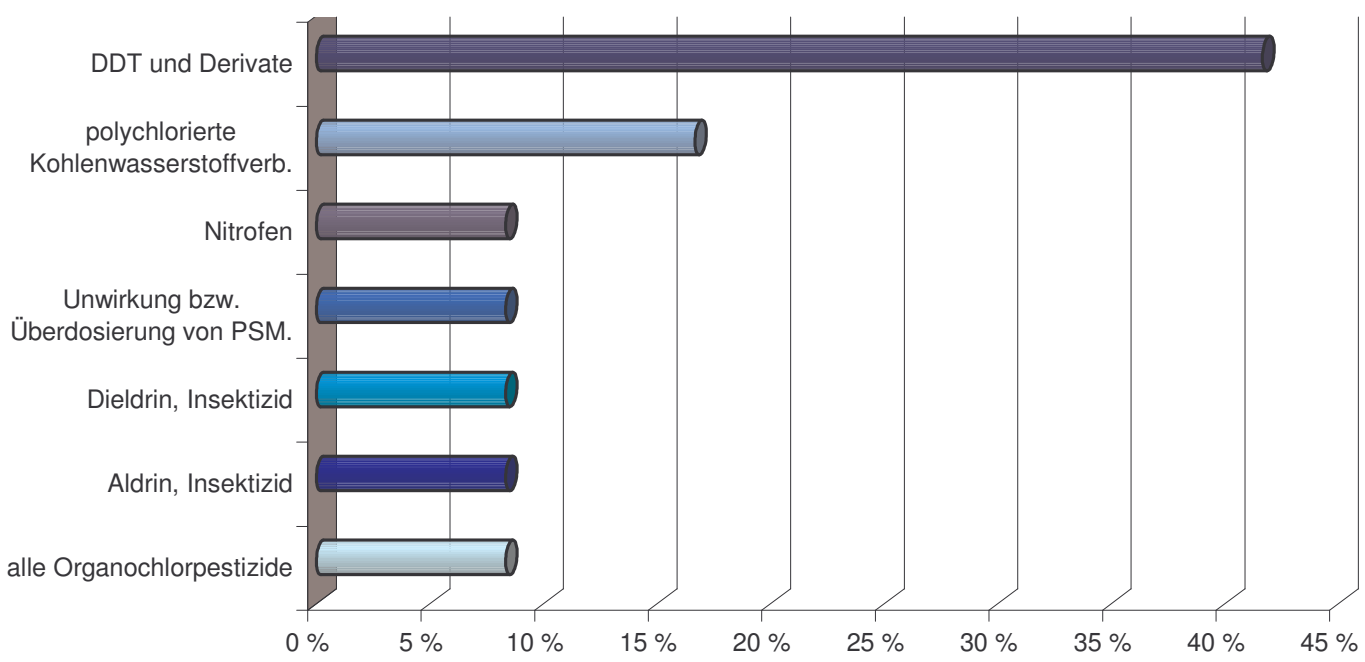

Abbildung 11.2-4: Einschätzung welche Rückstände aus importierten Futtermitteln die in die Nahrungskette gelangen können, in \% der Nennungen.

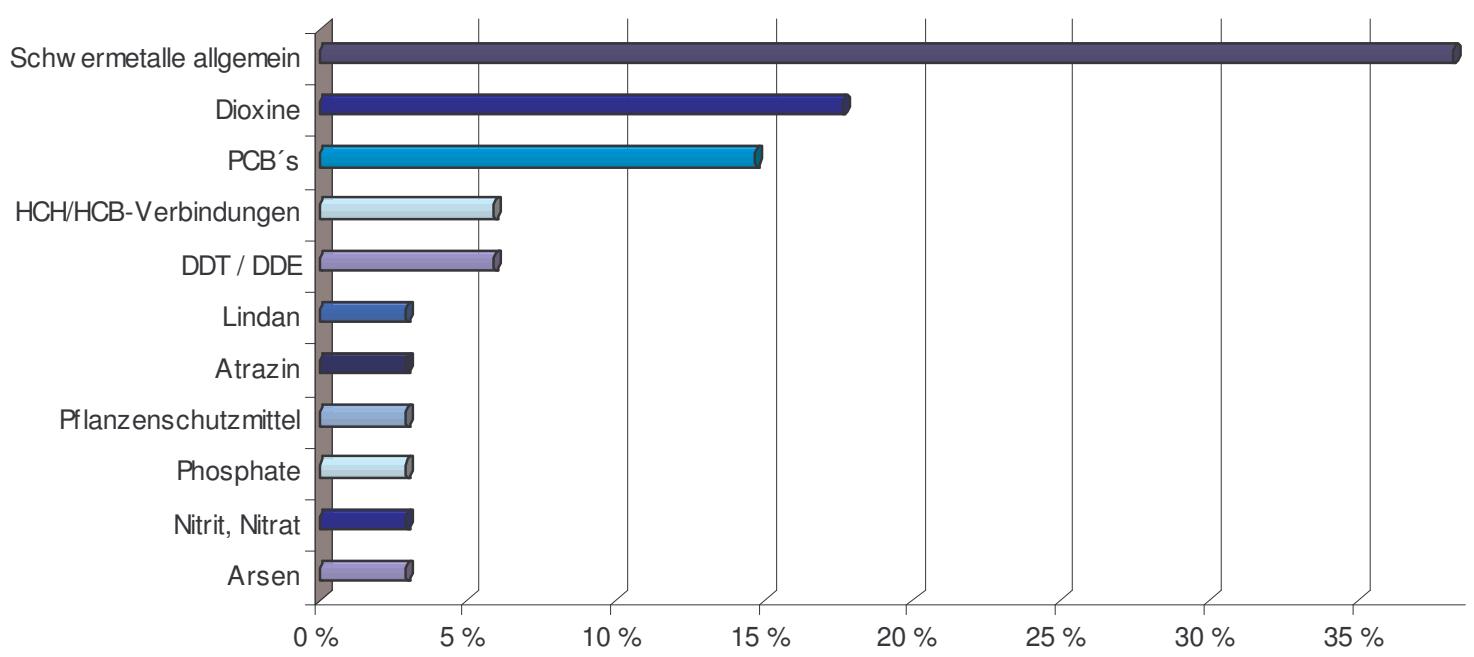

Abbildung 11.2-5; $\quad$ Einschätzung der Wahrscheinlichkeit der Stoffe/Verbindungen die durch Altlasten in der Nahrungskette gelangen können, in \% der Nennungen.
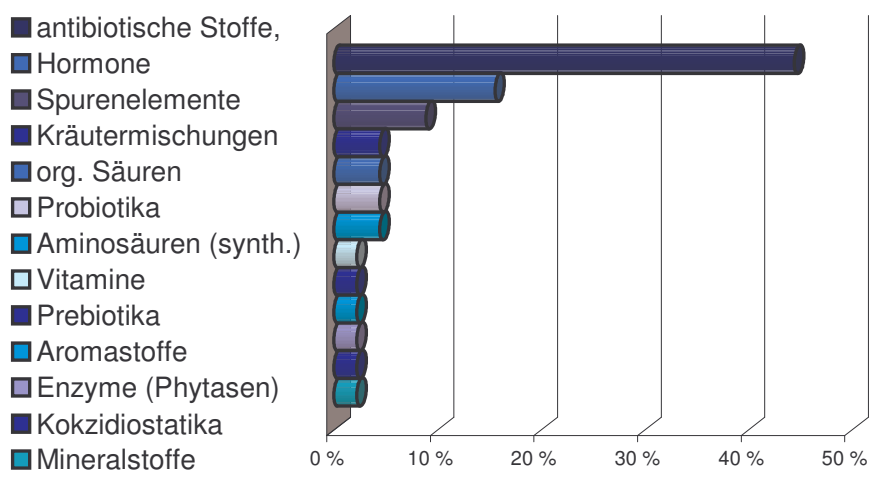

Abbildung 11.2-6: Mittel/Futterzusatzstoffe zur Leistungssteigerung, in \% der Nennungen. 


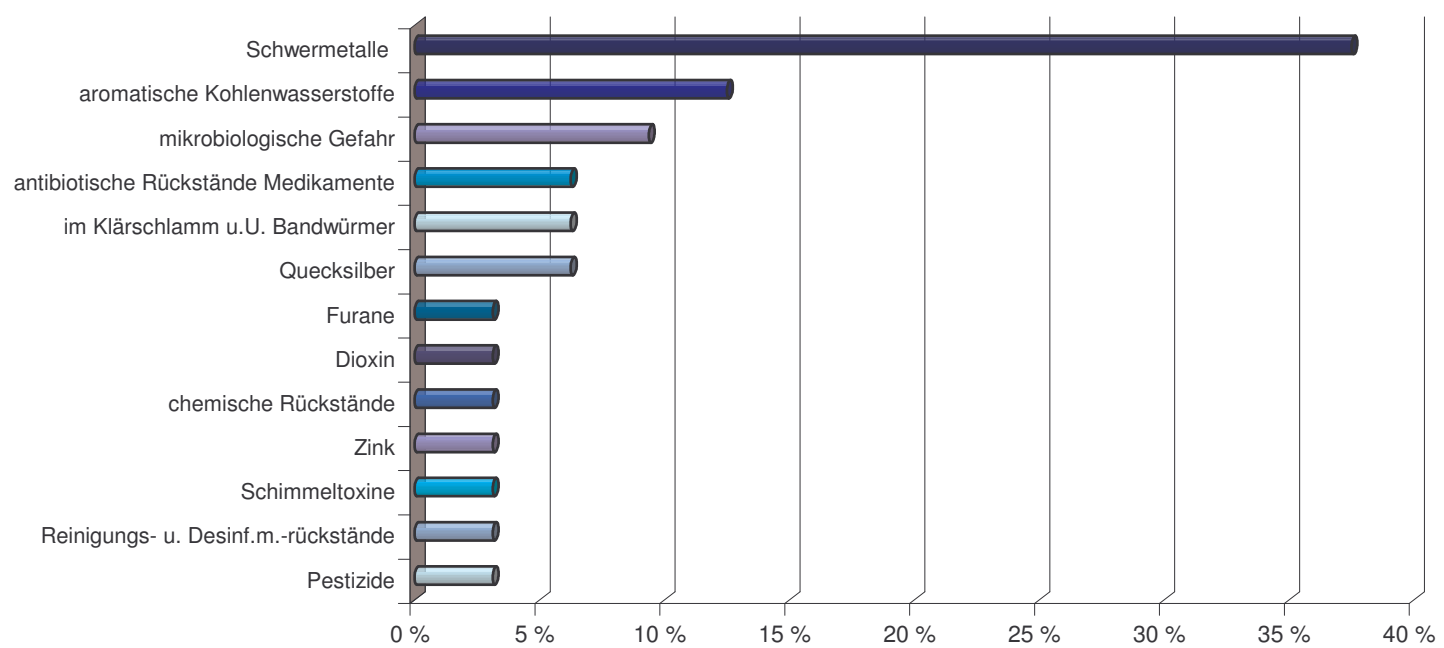

Abbildung 11.2-7: Einschätzung der Wahrscheinlichkeit von Rückständen aus Müllkompost und Klärschlamm, in \% der Nennungen.

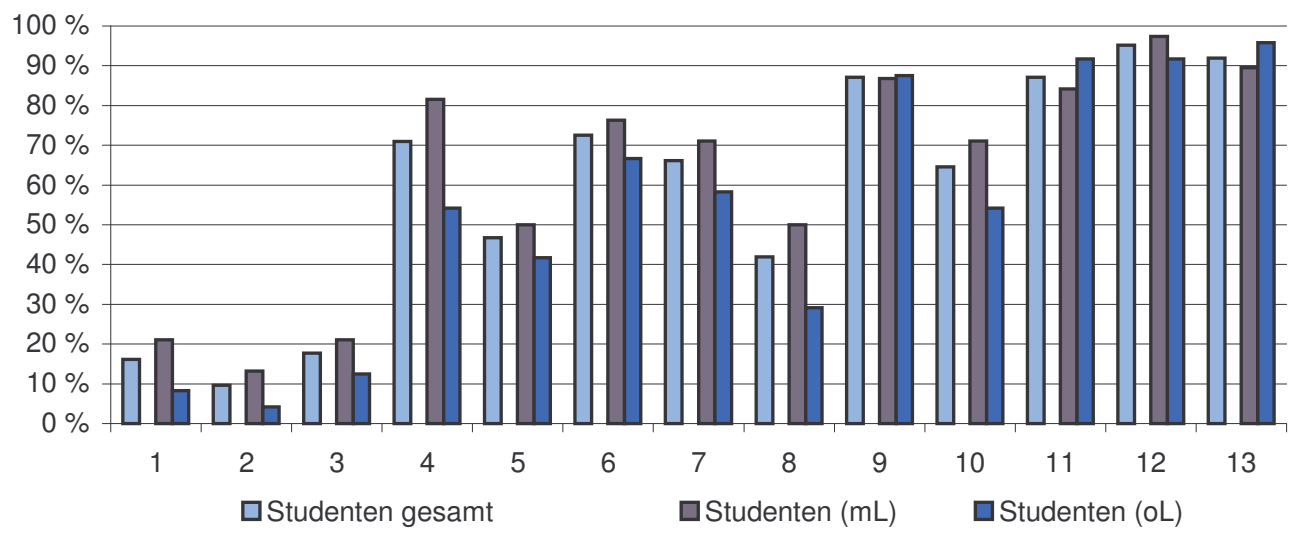
1. antibiotische Stoffe
2. Hormone
3. Kokzidostatika
4. Enzyme/Phytasen
5. Prebiotika
6. Aminosäuren (synth.)
7. Probiotika
8. Aromastoffe
9. Spurenelemente
10. org. Säuren
11. Kräutermischungen
12. Mineralstoffe
13. Vitamine

Abbildung 11.2-8: Einschätzung der Studenten ( $\mathrm{N}=62)$ bezüglich der Unbedenklichkeit von Futterzusatzstoffen. 

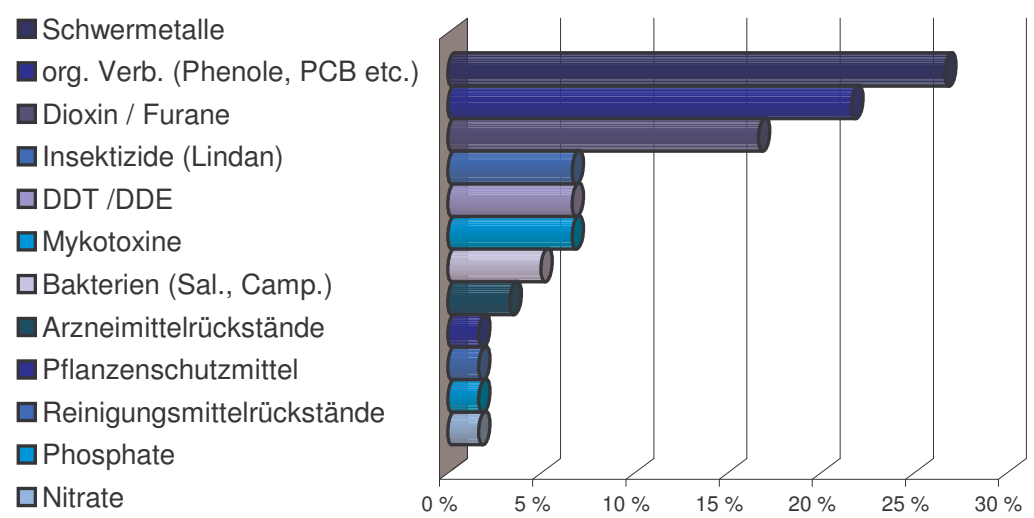

Abbildung 11.2-9: Einschätzung der Rückstände von Umweltkontaminanten, mit denen das Tier im landwirtschaftlichen Betrieb in Berührung kommt, die sich im Produkt Fleisch (Fett) anreichern können, in \% der Nennungen.

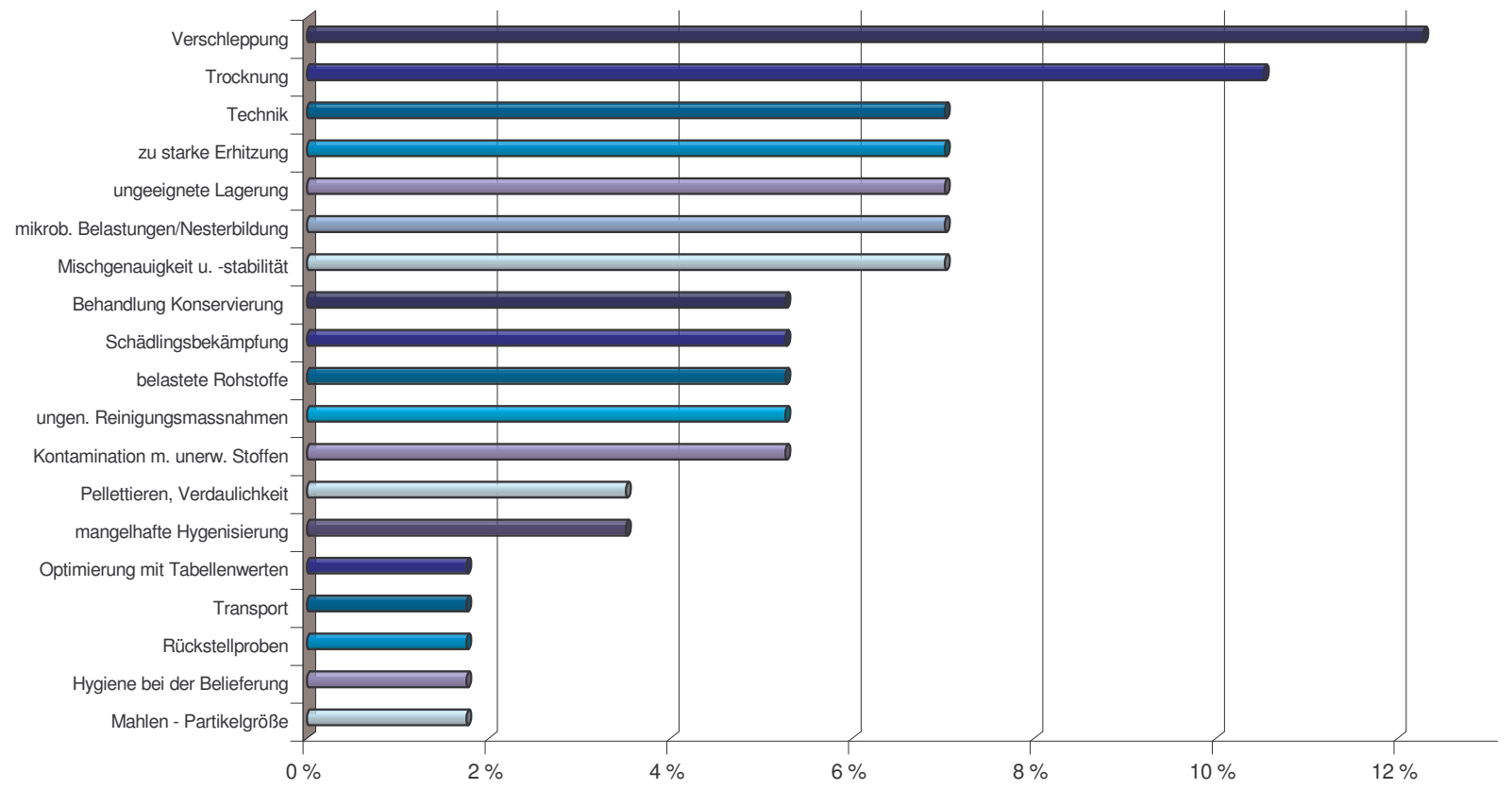

Abbildung 11.2-10: Einschätzung der kritischen Punkte, die bei der Herstellung von Futtermitteln eine Rolle spielen, in \% der Nennungen. 


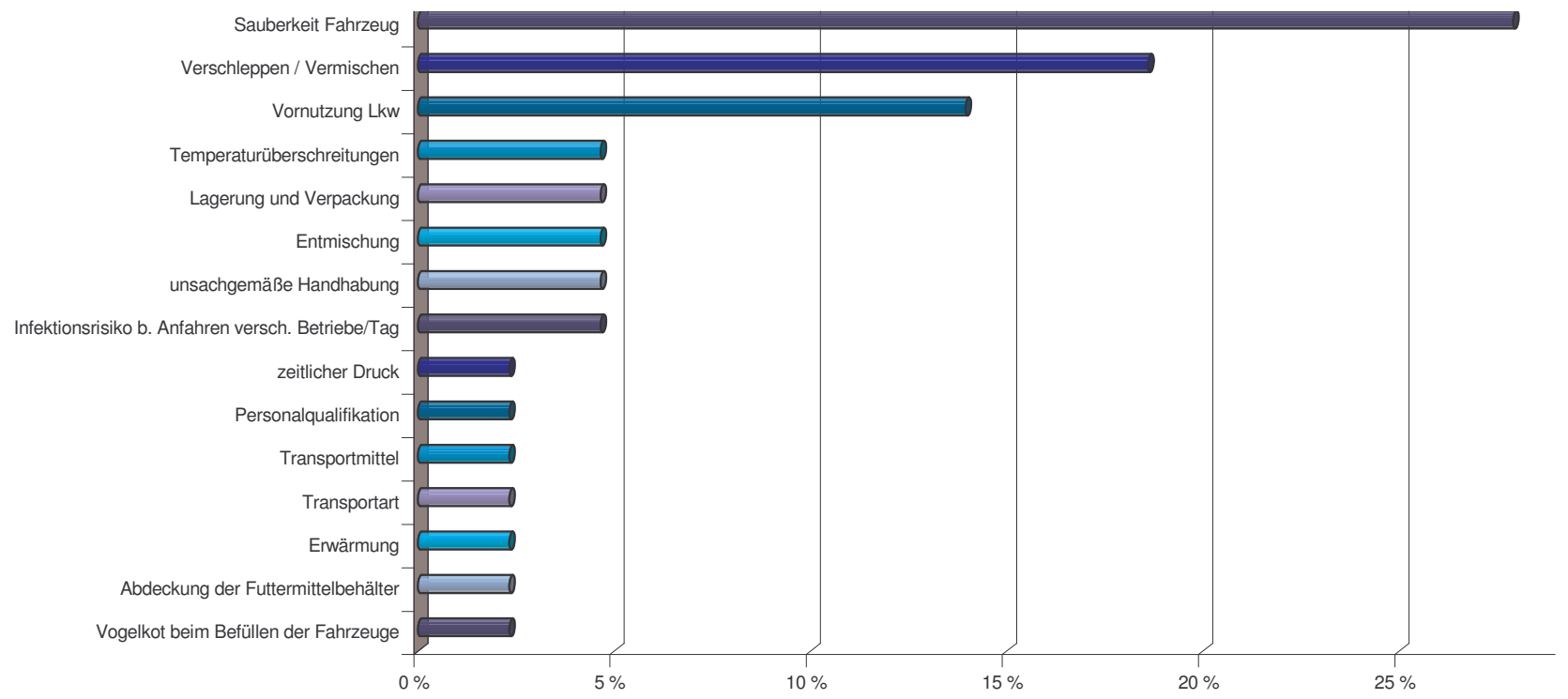

Abbildung 11.2-11: Einschätzung der kritischen Punkte beim Transport von Futtermitteln, in $\%$ der Nennungen.

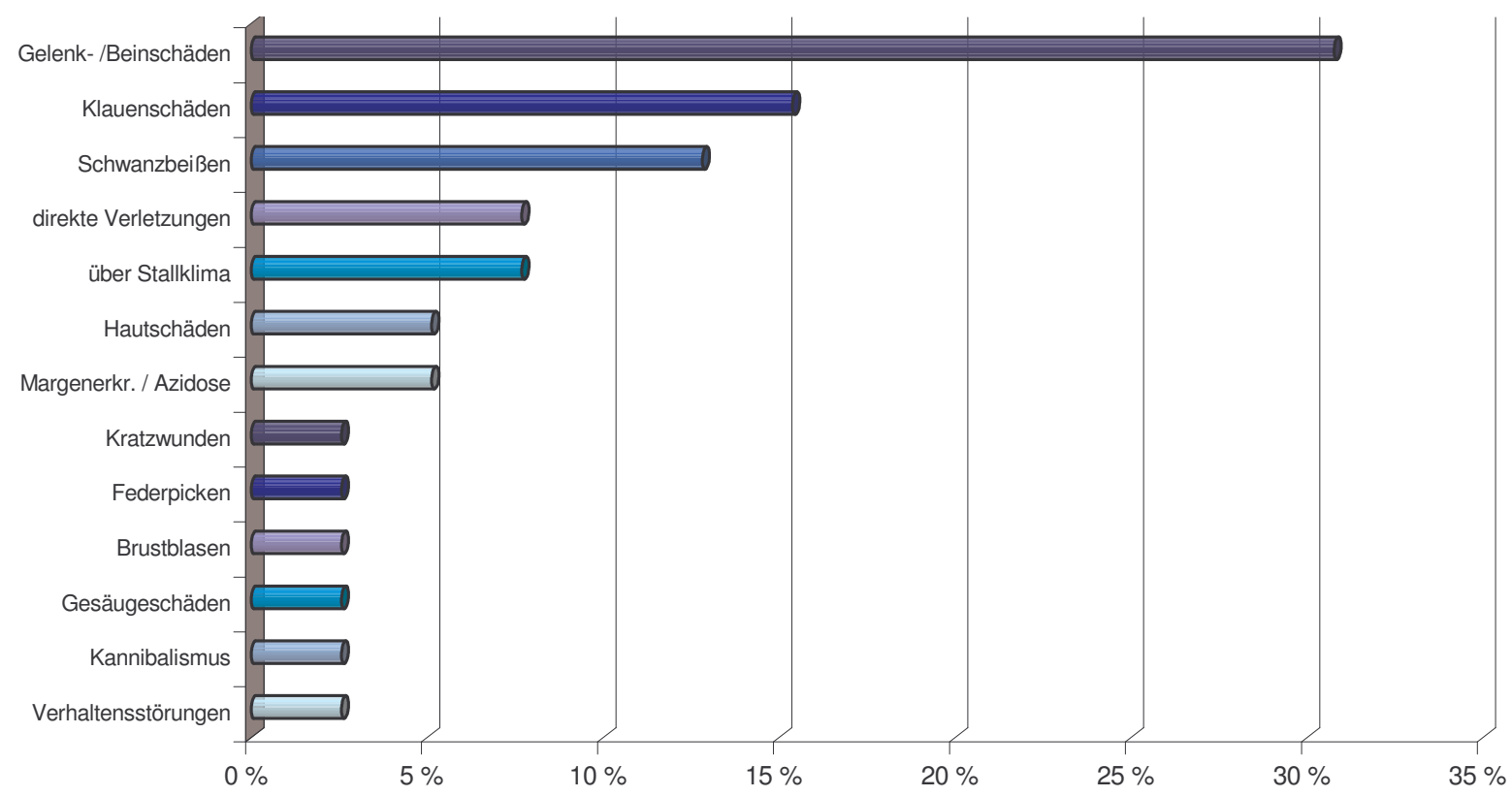

Abbildung 11.2-12: Technopathien, in \% der Nennungen. 


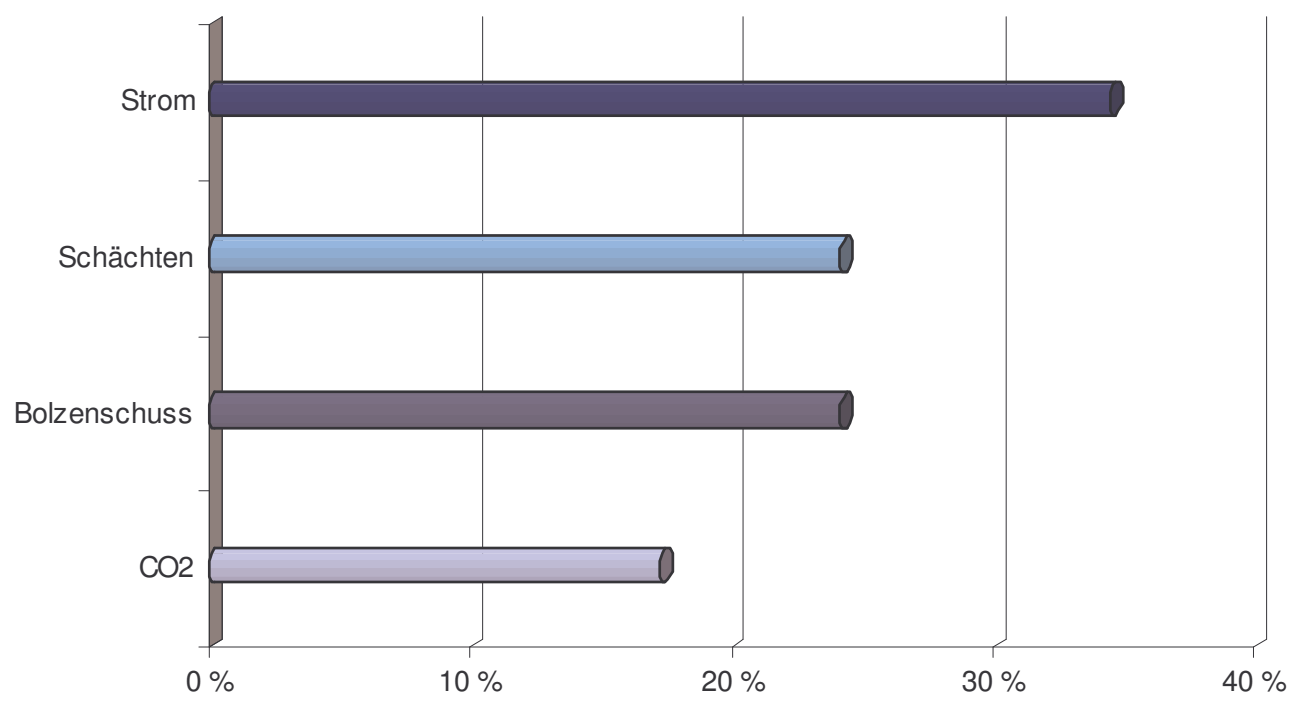

Abbildung 11.2-13: Betäubungsverfahren, die durch den Verbraucher als kritisch bewertet werden, in \% der Nennungen.

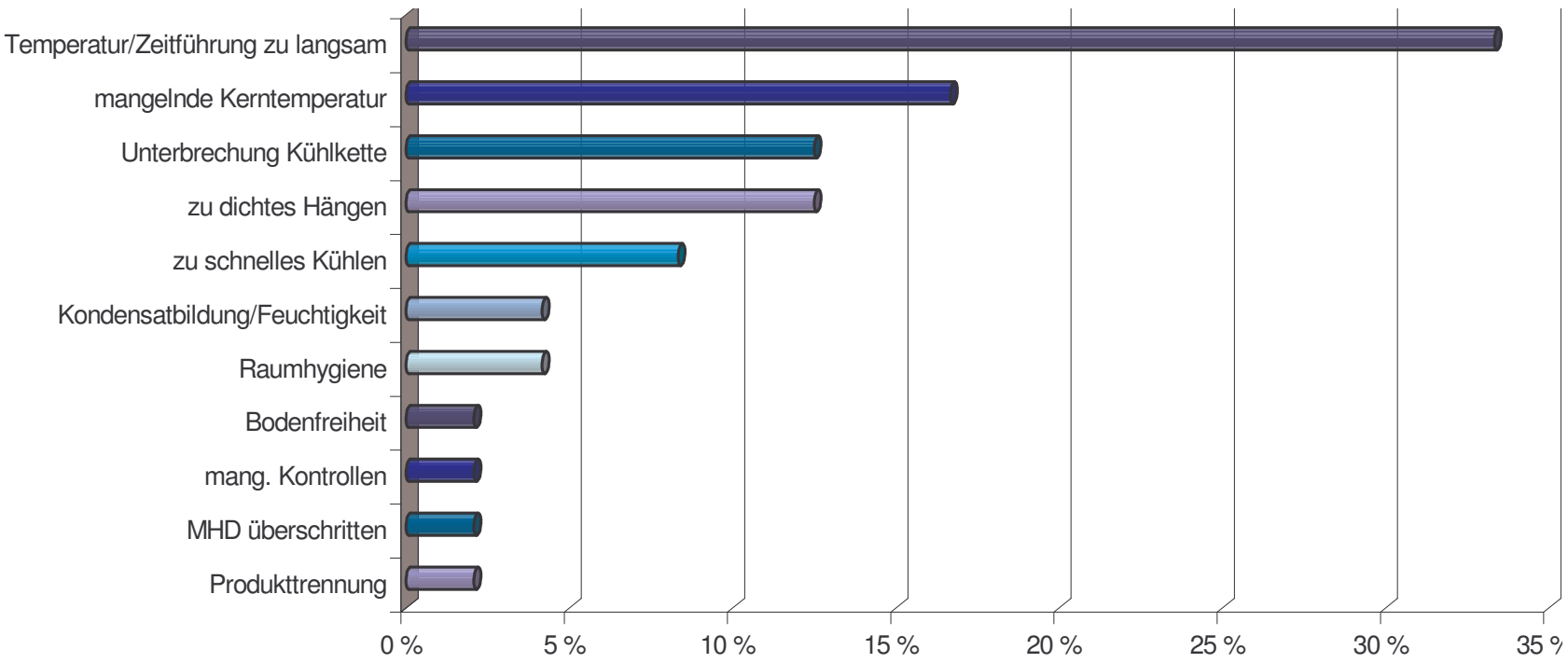

Abbildung 11.2-14: Kritische Punkte bei der Fleischkühlung, in \% der Nennungen. 


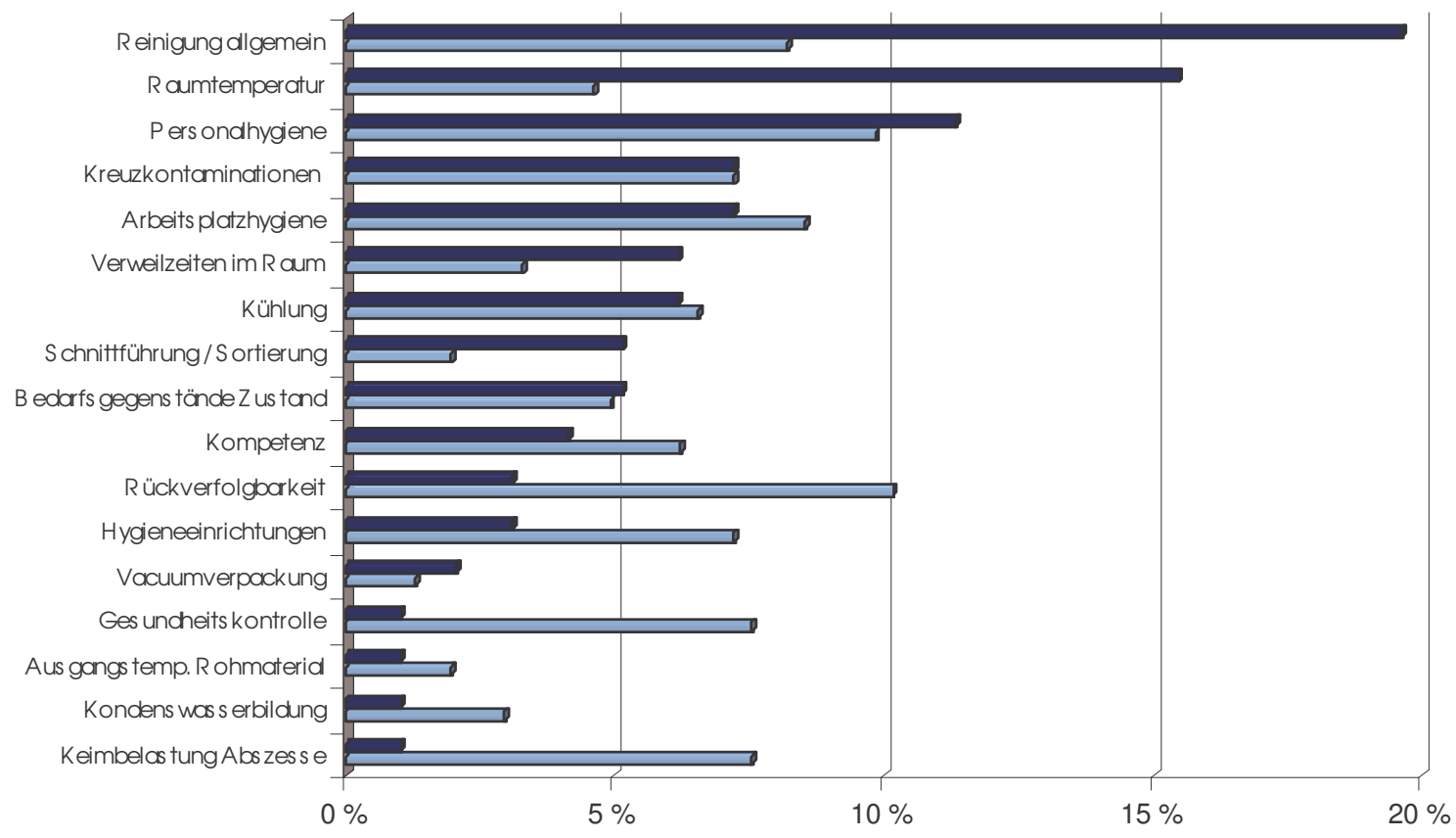

$\square$ Studenten: Kritische Punkte aus der Sicht des Verbrauchers Experten: Kritische Punkte

\section{Abbildung 11.2-15: Kritische Punkte bei der Fleischverladung/Transport und die Beurteilung der Punkte durch die Studenten bezüglich der Bedenklichkeit aus der Sicht der Verbraucher, in \% der Nennungen.}

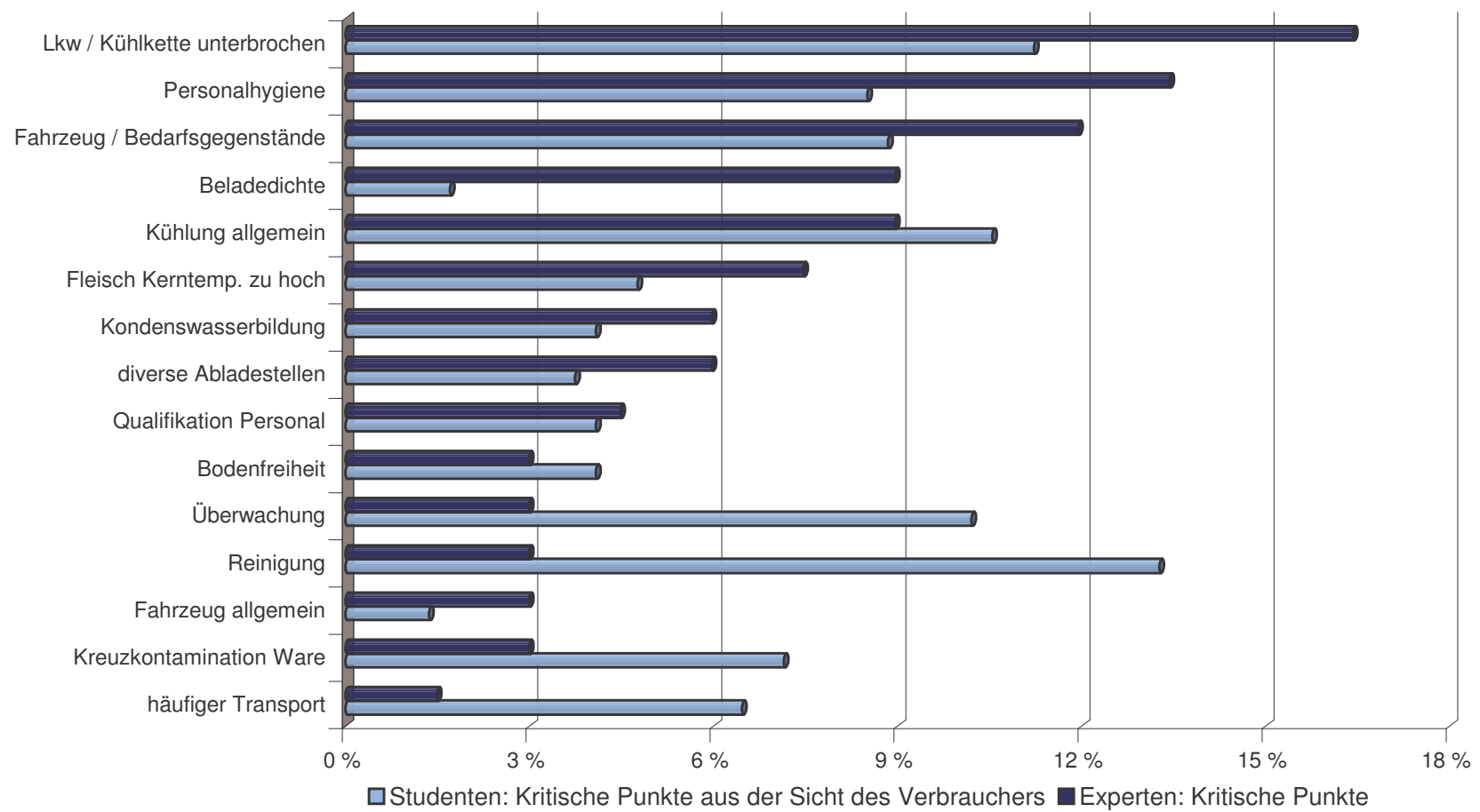

Abbildung 11.2-16: Kritische Punkte bei der Zerlegung und die Beurteilung der Punkte durch die Studenten bezüglich der Bedenklichkeit aus der Sicht der Verbraucher, in \% der Nennungen. 


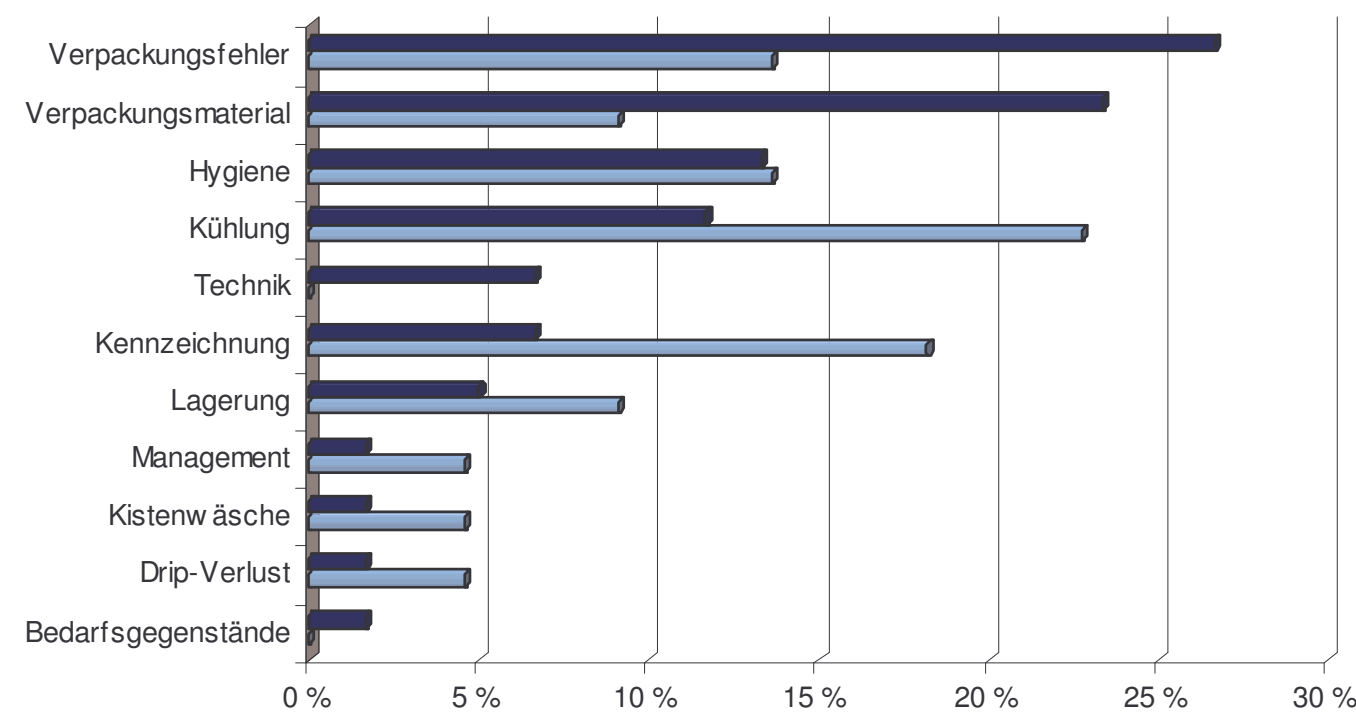

$\square$ Experten: Kritische Punkte aus der Sicht des Verbrauchers $\square$ Experten: Kritische Punkte

\section{Abbildung 11.2-17: Kritische Punkte der Fleischverpackung und die Beurteilung der Punkte durch bezüglich der Bedenklichkeit aus der Sicht der Verbraucher, in \% der Nennungen.}

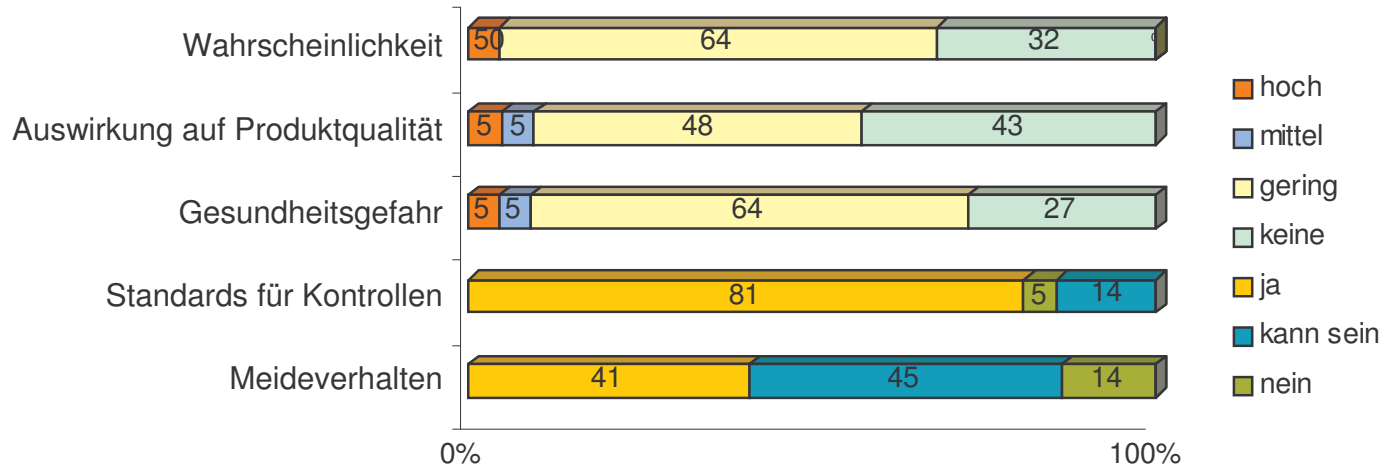

Abbildung 11.2-18: Beurteilung der These: Tierische Futtermittel stehen zu Zeit stark in der Kritik und deren Verfütterung ist verboten. Bitte nennen Sie Ihre Einschätzung, ob durch geeignete Aufbereitungsmethoden von tierischen Futtermitteln, bei der Verfütterung an nicht Wiederkäuer, keine Gefahren für den Menschen ausgeht. 


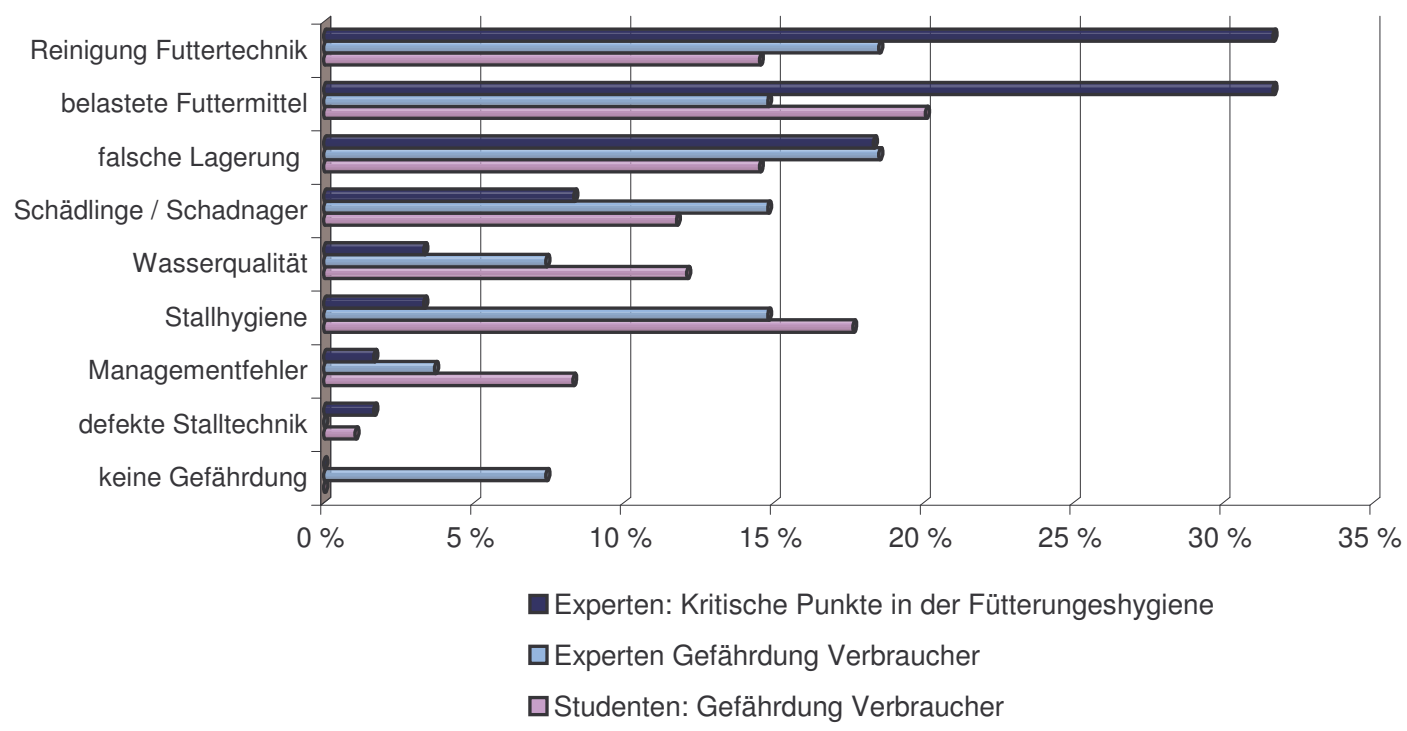

Abbildung 11.2-19: Kritische Punkte bei der Fütterungshygiene von Masttieren und Einschätzung der Punkte, durch die Experten und Studenten, die in der Kommunikation durch den Verbraucher kritisch gesehen werden, in \% der Nennungen.

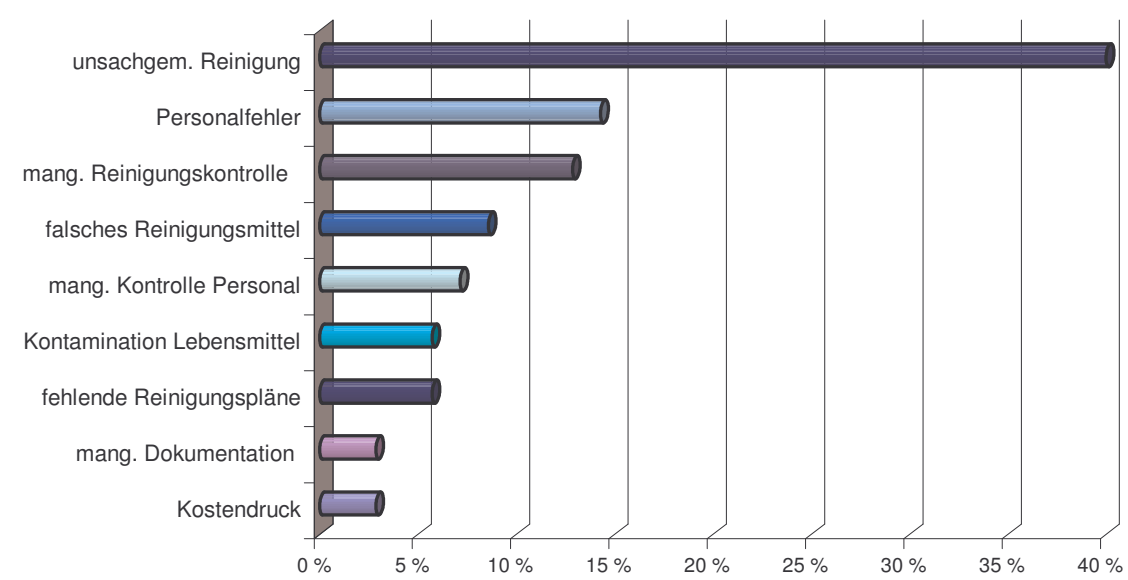

Abbildung 11.2-20: Kritische Punkte bei der Reinigung von Schlacht- und Zerlegebetrieben, in $\%$ der Nennungen. 
Mitarbeiter

$\square$ Arbeitskleidung

口Personalschulung

$\square$ Gesundheit

口Arbeitsgeräte

$\square$ Stand der Technik nicht umgesetzt

Kontrollen

口Akkordarbeit

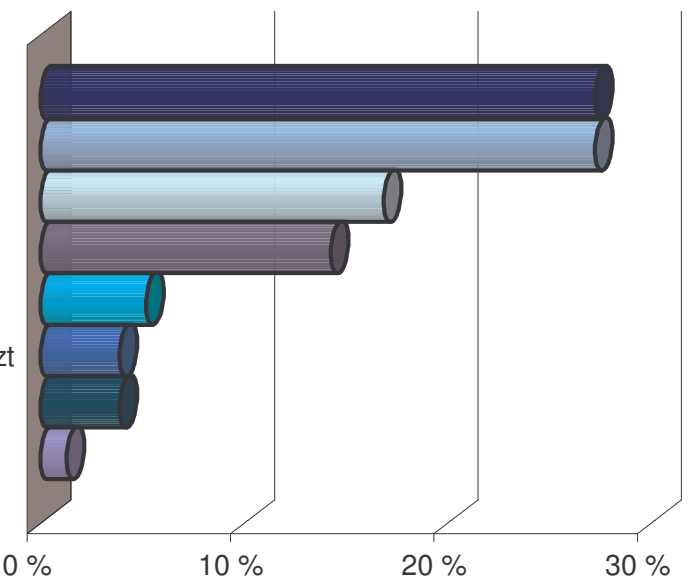

Abbildung 11.2-21: Kritische Punkte in der Personalhygiene, in \% der Nennungen.

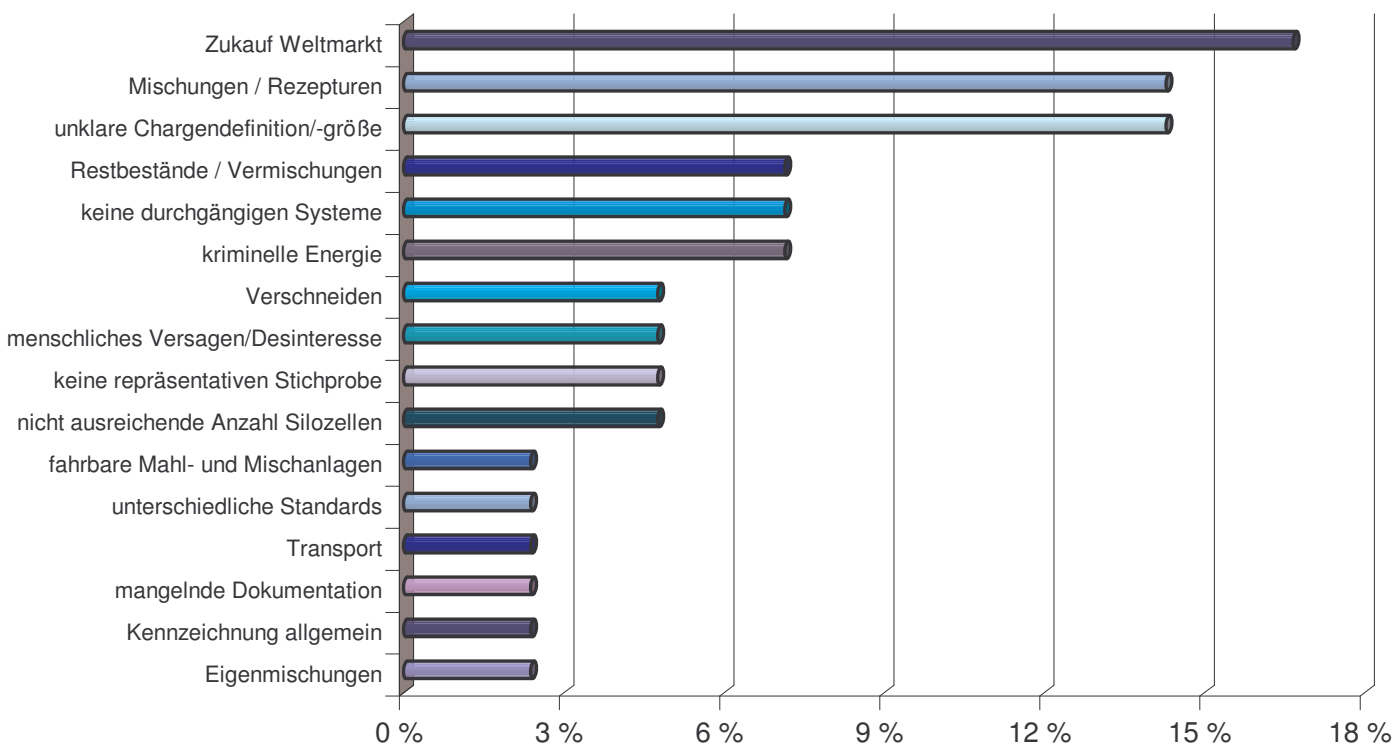

Abbildung 11.2-22: Kritische Punkte bei der Rückverfolgbarkeit von Futtermitteln, in \% der Nennungen. 


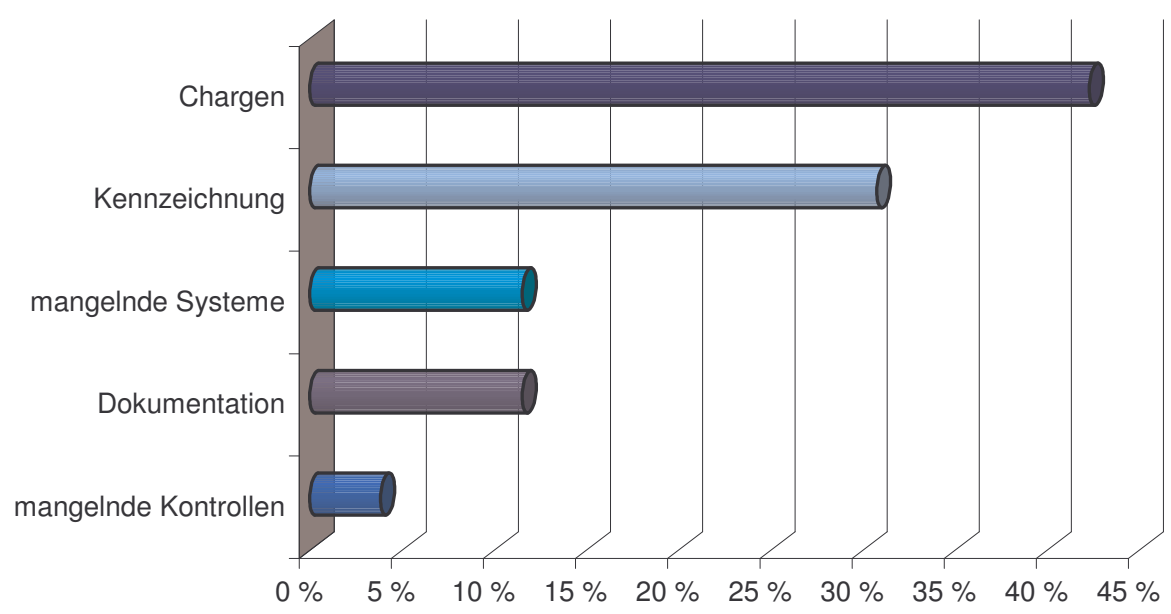

Abbildung 11.2-23: Kritische Punkte bei der Rückverfolgbarkeit in der Schlachtung und Zerlegung, in \% der Nennungen.

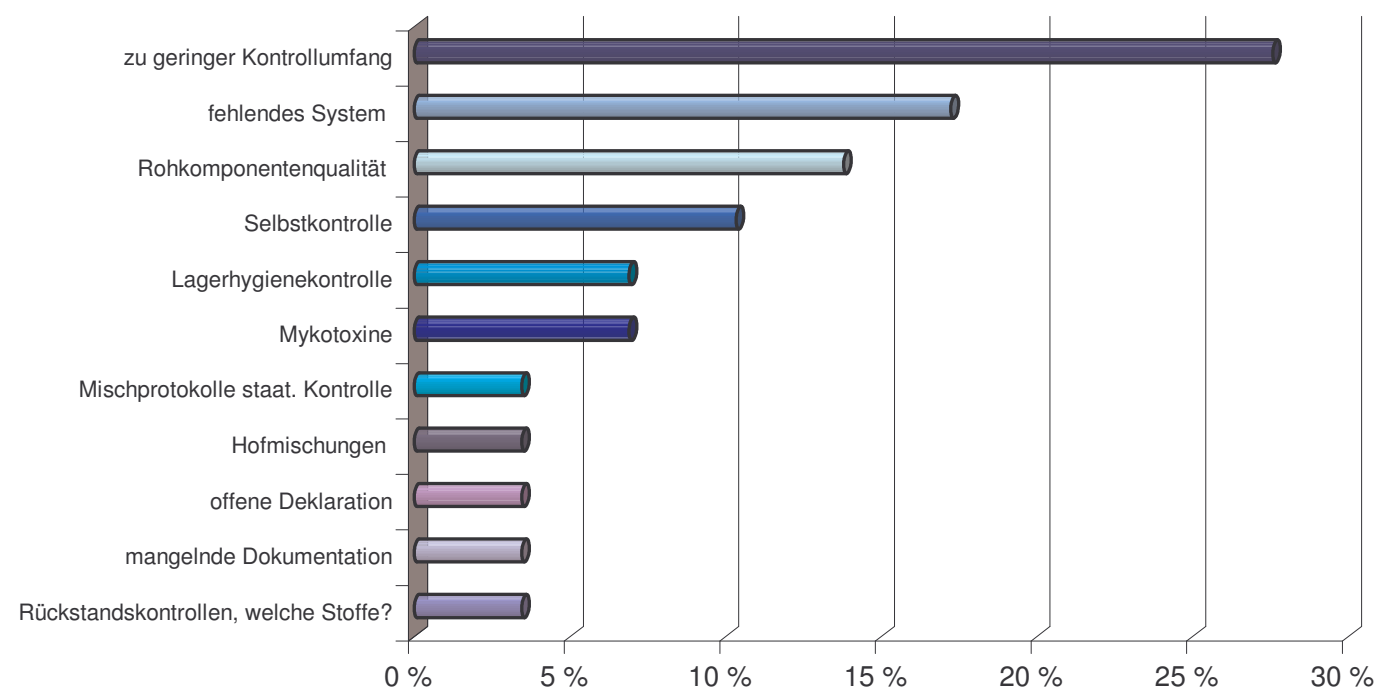

Abbildung 11.2-24: Kritische Punkte bei der internen Kontrolle von Futtermitteln, in \% der Nennungen 


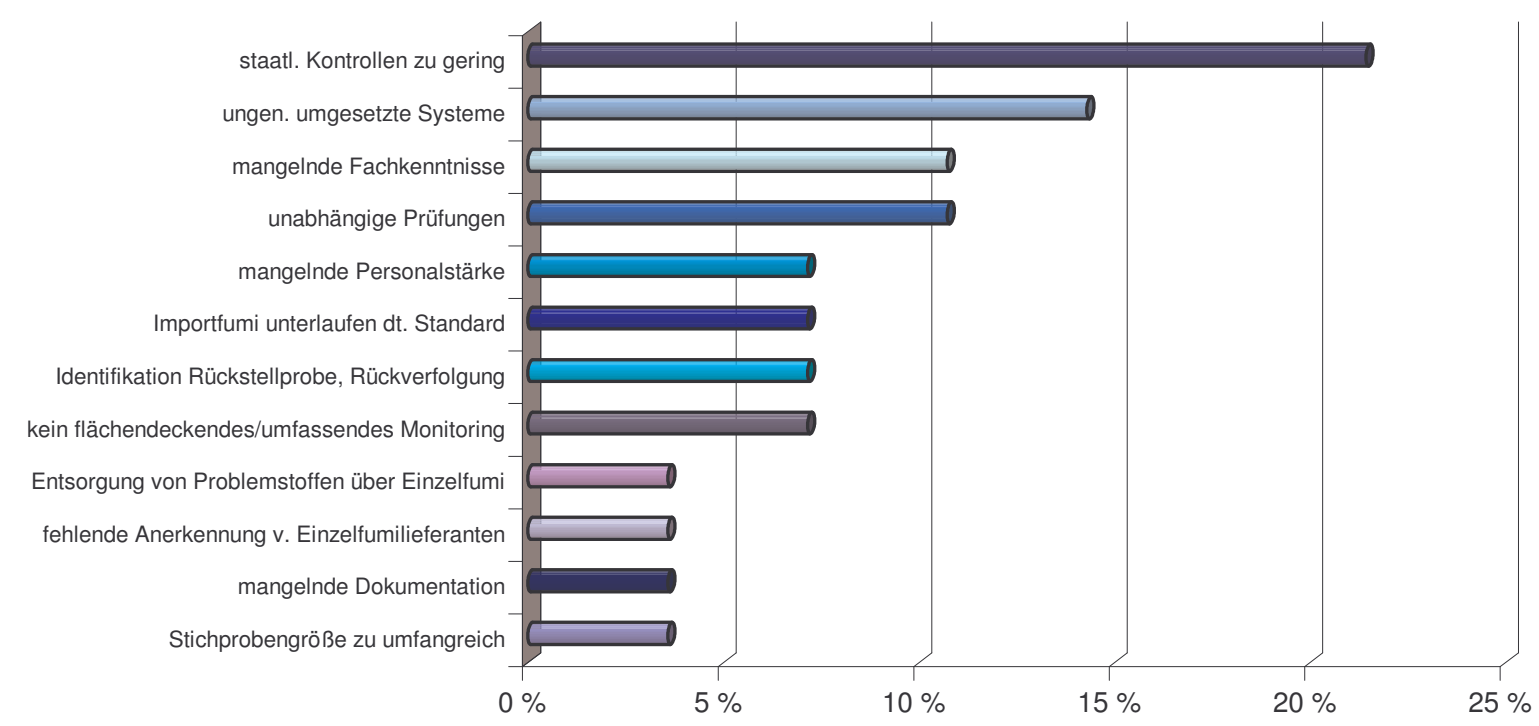

Abbildung 11.2-25: Kritische Punkte bei der externen Kontrolle von Futtermitteln, in \% der Nennungen

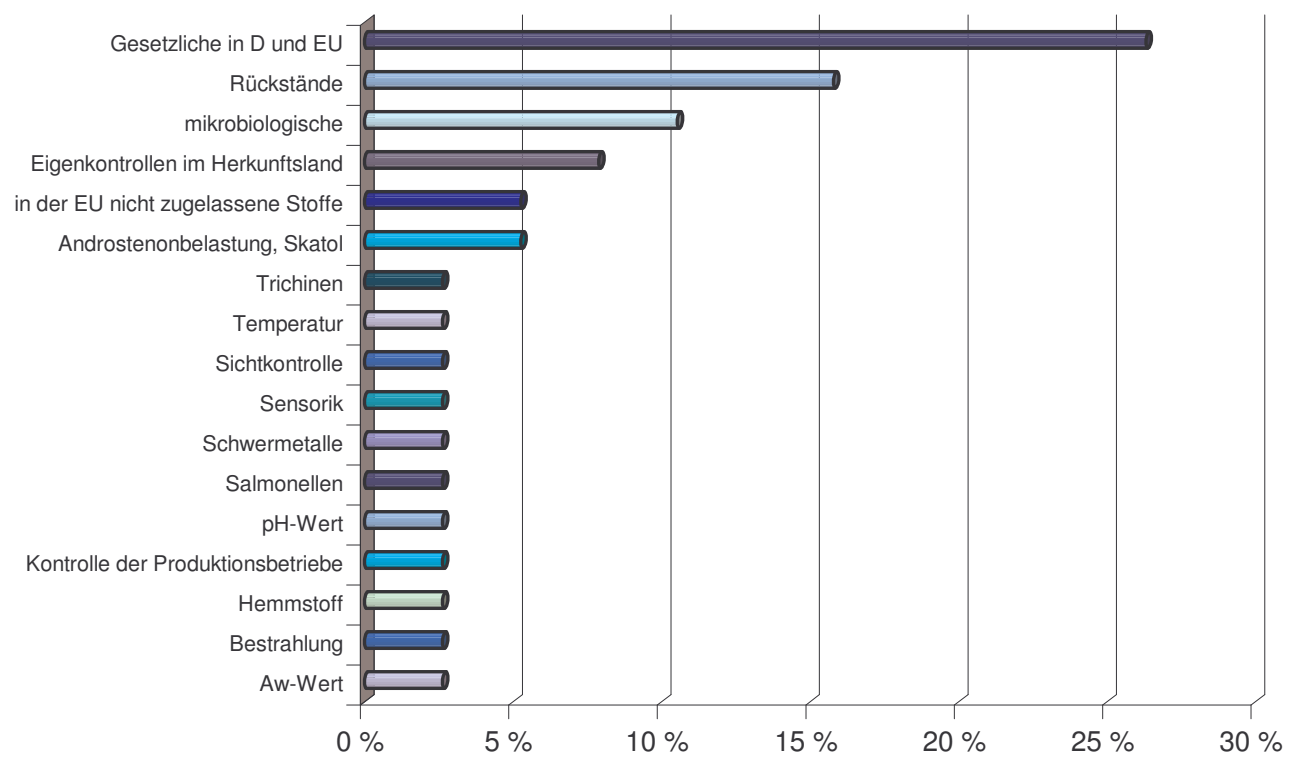

Abbildung 11.2-26: Kontrollen die bei Waren aus Drittländern durchgeführt werden sollten, in \% der Nennungen. 


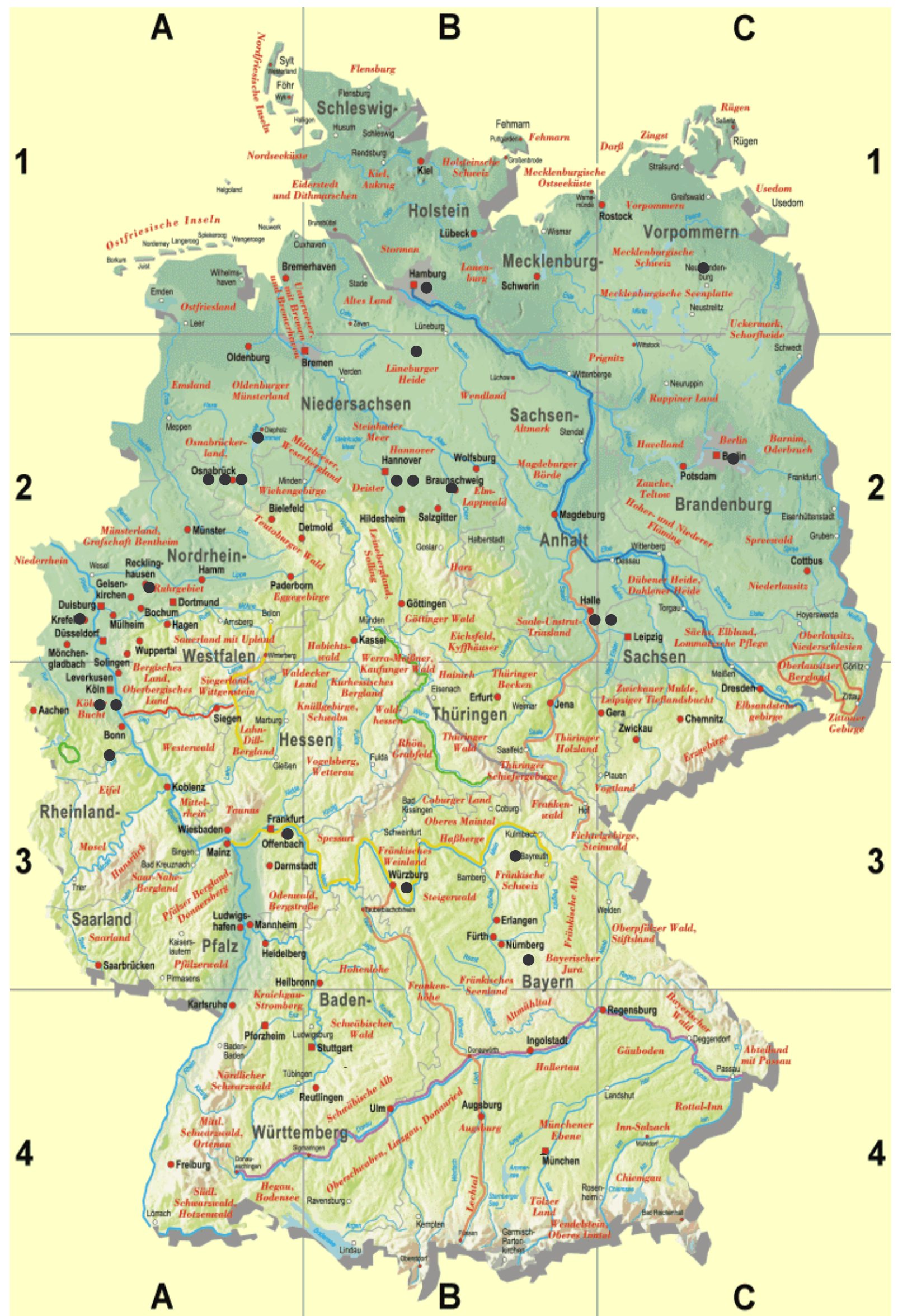

Abbildung 11.2-27: Wohn- bzw. Arbeitsorte der befragten Experten in Deutschland. 


\section{Erklärung}

Ich habe die vorliegende Dissertation selbständig und nur unter Verwendung der angegebenen Literatur und Hilfsmittel angefertigt.

Rastede, den 16. März 2006

Ute Röbken 


\section{Werdegang:}

Persönliche Daten:

Ute Röbken

geb. am 24.07.67 in Oldenburg (Oldb.)

Familienstand: verheiratet

Schulausbildung: $\quad 4$ Jahre Grundschule, 2 Jahre Orientierungsstufe, 4 Jahre Realschule, 3 Jahre Gymnasium, Abschluß:

Abitur

Berufsausbildung: $\quad$ 1986-1988 Ausbildung zur Landwirtin

Hochschulausbildung: $\quad$ 1988-1992 Studium an der Universität Gießen

Fachbereich Agrarwissenschaften

Fachrichtung Tierproduktion

Abschluß: Diplom-Ingenieurin

Berufliche Tätigkeiten: 1993 bis 1997 Tätigkeiten in der Qualitätssicherung, in der Fahrzeugdisposition und im Regionalverkauf in der Lebensmittelbranche. Firmen: Bruno Rosenboom Versandschlachterei und Kutscheid-Fleisch

Arbeitsschwerpunkte: Aufbau, Einführung und Weiterentwicklung von Markenfleischprogrammen, Entwicklung eines Hygienekonzeptes nach HACCP, Kontrolle der Hygiene, Aufbau einer Qualitätssicherung

Seit 1998 bis heute selbständig, Beratung für Managementsysteme für Hygiene- und Managementsysteme nach internationalen Standards.

Arbeitsschwerpunkte: Aufbau von Managementsystemen nach DIN EN ISO 9001-2000, DIN EN ISO14000, HACCP, GMP+ (Futtermittel), International Food Standard (IFS), Qualität und Sicherheit (QS), Hygieneschulungen, Hygienekontrollen in den Betrieben, Auditierung von Managementsystemen vom 17.09.2001 bis 15.09.2004 Anstellung als wissenschaftliche Mitarbeiterin im Forschungs- und Studienzentrum für Veredelungswirtschaft in Weser-Ems der Uni Göttingen, Professur für Produktkunde - Qualität tierische Erzeugnisse

Zusatzqualifikationen: Ausgebildete Auditorin, Zugelassen für: DIN EN ISO 9001-2000, Rindfleischetikettierung, GMP+, IFS, BRC und QS Öffentlich bestellte und vereidigte Sachverständige für Pferde und Rinder (Zucht, Haltung, Fütterung), Landwirtschaftskammer Weser-Ems

Rastede, den 11.05.2006 


\section{Danksagung}

Die Anfertigung und erfolgreiche Vollendung dieser Dissertation beruht nicht nur auf der eigenen Arbeit, sondern auf der Mitarbeit und Hilfsbereitschaft vieler netter Menschen, die mir in dieser Zeit zur Seite standen und bei denen ich mich an dieser Stelle ganz herzlich bedanken möchte.

Ich danke zunächst Herrn Prof. Dr. Michael Wicke für die Betreuung meiner Arbeit. Für die Übernahme des Zweitgutachtens bedanke ich mich bei Herrn Prof. Dr. Wolfgang Branscheid. Für die Überlassung des Themas möchte ich mich bei der CMA und der Firma Aramak bedanken.

Den Mitarbeitern der Bundesforschungsanstalt für Ernährung und Lebensmittel (BFEL) in Kiel gilt mein Dank bezüglich der kritischen Beratung und der Entscheidungsfindung für eine Methode zur Auswertung meiner Daten.

Besonderen Dank gelten Ulf Legis und Magret Hagen. Beide haben mir mit kompetenten Anregungen und in konstruktiven und kritischen Diskussionen hilfreich und aufbauend zur Seite gestanden.

Ferner danke ich allen Freunden und Kollegen, die durch ihre Hilfsbereitschaft und moralische Unterstützung zum Gelingen dieser Arbeit beigetragen haben. 